


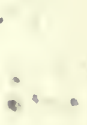



Digitized by the Internet Archive in 2008 with funding from Microsoft Corporation 

MENDEL'S

PRINCIPLES OF HEREDITY 
CAMBRIDGE UNIVERSITY PRESS

zlonoon: FETTER LANE, E.C.

C. F. CLAY, MANAGER

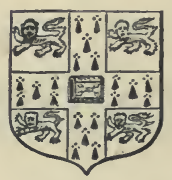

ALSO

Zondon: H. K. LEWIS, 136, GOWER STREET, W.C.

Ilondon: WILLIAM WESLEY \& SON, 28, ESSEX STREET, STRAND

שDinburgb: 100, PRINCES STREET

Berlin: A. ASHER AND CO.

Zlipzig: F. A. BROCKHAUS

ferow gork: G. P. PUTNAM'S SONS

Gomban and Calcutta: MACMILLAN AND CO., LTD. 


\section{'}




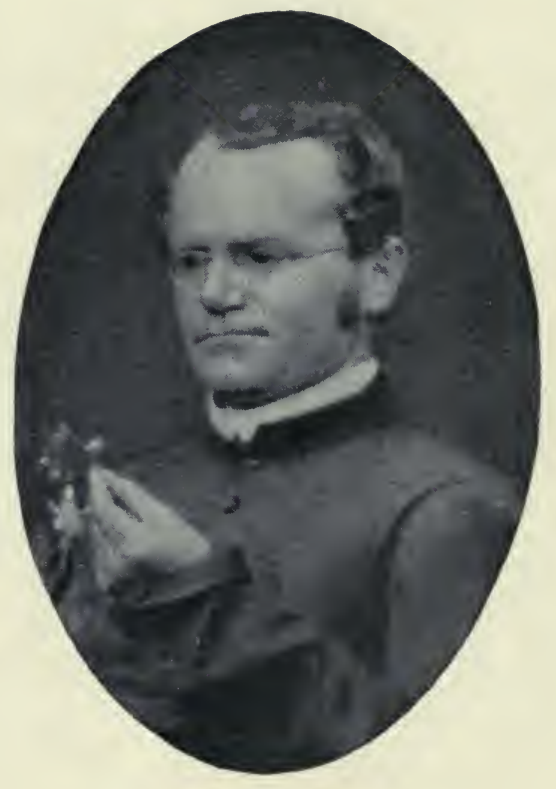

GREGOR MENDEL, I866

Enlarged from a group of the brethren of the Königskloster 


\section{MENDEL'S}

\section{PRINCIPLES OF HEREDITY}

BY

W. BATESON, M.A., F.R.S., V.M.H.

HONORARY FELLOW OF ST JOHN'S COLLEGE,

DIRECTOR OF THE JOHN INNES HORTICULTURAL INSTITUTION

Cambridge :

at the University Press

I 9 I 3 


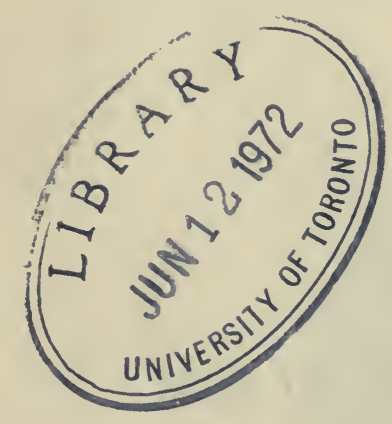

Published March 1909

Reprinted August 1909

Third Impression with Additions 1913

CH
366
33
013
con 5 


\section{PREFACE}

THE object of this book is to give a succinct account 1 of discoveries in regard to Heredity made by the application of Mendel's method of research. Following the clue which his long lost papers provided we have reached a point from which classes of phenomena hitherto proverbial for their seeming irregularity can be recognized as parts of a consistent whole. The study of Heredity thus becomes an organised branch of physiological science, already abundant in results, and in promise unsurpassed.

A translation of Mendel's two papers, together with a biographical note, is appended. The translation of the first, based on a draft prepared for the Society by $\mathrm{Mr}$ C. T. Druery, was printed in the Royal Horticultural Society's Journal, rgor. With modifications I published it separately in 1902, giving a brief summary of Mendelism as then developed, under the title Mendel's Principles of Heredity: $A$ Defence. The object of that publication was to put Mendel's work before the English speaking peoples and to repel the attack which the late Professor Weldon had recently made on Mendelian methods and the conclusions drawn from them. The edition was at once sold out, but I did not reprint the book. As a defence it had served its purpose. Moreover the progress of experiment with the extension of Mendelian conceptions was rapid, and the account of those conceptions there given was in some important respects soon out of date. In particular my view of the nature of compound factors was shown to be unnecessarily complex and largely incorrect. Though 
obviously in a subject fast extending under the influence of many workers there can be no finality, yet at the present time our knowledge of the main facts has reached a definite stage, and a useful and relatively permanent presentation of the phenomena can be attempted.

The range and diversity of facts, zoological and botanical, from which the material is drawn are so wide that it has been difficult to present them adequately within a moderate compass. Many of the types studied might singly provide the subject of a treatise, and the temptation to annotative excursion has been very great; but the course which seemed most useful was to admit only such detail as had a clear significance in the exposition of principle, or as a suggestion for further inquiry. The reader therefore will understand that if he turns to the original records specified he will almost always find information, perhaps important, which is omitted here.

In the original plan of the book it was intended to discuss somewhat fully the bearing of the new facts on the great problems of Biology, but it is perhaps more fitting that these theoretical considerations should be detached from a presentation of the concrete phenomena. In 1907 I had the honour of delivering the Silliman Lectures in Yale University, and I then took these wider aspects of Genetics as my theme, showing the bearing of the new knowledge on current theory, especially on that of Evolution, and the nature of Variation. The substance of these lectures I propose to publish separately with amplifications, and on the present occasion allusion to these matters has been restricted to the briefest possible indication of the lines of thought which Mendelism inevitably suggests.

A chapter dealing with practical applications of Mendelian principles has been introduced. Such applications will probably far exceed any limits we can yet perceive. Among them we must foresee not merely advances in the art of 
breeding animals and plants, but a control over the destiny of our own species. These things are spoken of in their place. To prevent disappointment, however, it must be at once admitted that for fanciers Mendelism can as yet do comparatively little. "Fancying" provides the chief interest in life for thousands of persons in this country. It is an occupation with which the scientific naturalist should have more sympathy than he has commonly evinced. If the scientific world had kept in touch with the operations of the "fancy" much nonsense which has passed into scientific orthodoxy would never have been written. The study of Mendelian phenomena will do something to bring about a fruitful interchange of experience. But for the "fancy" our work can as yet do two things only. First, in the study of the workings of the Mendelian system it will provide a most fascinating pursuit, which if followed with assiduous care may at any moment lead to some considerable advance in scientific knowledge. Secondly, the principles already ascertained will be found of practical assistance in the formation of new breeds and may save many mistakes and waste of time. But applied to the business of breeding winners in established breeds they cannot materially help, for almost always the points which tell are too fine to be dealt with in our analysis.

In a work of this kind an author must necessarily speak of various subjects on which his knowledge can be superficial only, and I trust that if inaccuracies have been introduced, readers will be good enough to send me corrections.

Much and varied assistance has been given me by many persons. Such help on special points has been acknowledged in the text, but a fuller and more prominent acknowledgment is due to my colleagues. Without their cooperation there would have been, so far as Cambridge is concerned, but meagre contributions to record. In the early days of Mendelism, and before, Miss E. R. Saunders 
collaborated with me. A beautiful series of results, especially relating to the heredity of Stocks (Matthiola), has been the fruit of her labours exclusively. Not only have these results greatly advanced our knowledge of genetic phenomena, but I think that at a time when Mendelism was, in England.at least, regarded with suspicion, the obvious precision of her work and the persistence of her advocacy did much to convince the scientific world of the reality of our assertions.

In I 904 I had the good fortune to gain Mr R. C. Punnett as a partner. Since that date we have worked in close collaboration, and the work that we have thus done has been in every sense a joint product, both as regards design, execution, and interpretation of results. Though for the presentation of the views contained in this book I am solely responsible, those that apply to the subjects of our own work are often his, or have been arrived at in consequence of interchange of ideas with him.

On some points of general physiology I' have received useful suggestions and criticism from Mr F. F. Blackman, and in this respect I am also especially beholden to Miss F. M. Durham.

The Plates of Sweet Peas and Mice are photographic reproductions, on the whole very accurate, of coloured drawings most kindly made for me by Miss Wheldale. The Plate of Primula flowers is taken from an excellent coloured photograph by Mr Waltham*. For Fig. 9 I am obliged to the New Phytologist.

For several years past I have had an exceptional opportunity of seeing breeding conducted on a large scale

* Since the word "magenta," often used in English for the description of a colour, is not understood on the Continent, I may say that it means a purplish or bluish red, as distinguished from a crimson or pink red. On Plate VI, Figs. 8, 9, I 4, I5, 20, 2 I represent shades of magenta, while Figs. 2, 7, 13,19 are true reds. 
through the great kindness of Messrs Sutton of Reading, who have given me the privilege of watching such parts of their work in raising varieties as seemed especially instructive, with unrestricted access to their pedigree books. From this I have derived much profit, and many hints which have formed the starting point for fuller experiment. My hearty thanks are due to them for this important assistance.

\section{W. BATESON.}

Grantchester, Cambridge.

February, 1909.

Note to the third impression.

In the past three years the progress of Mendelian analysis has been very rapid, and certain chapters of this book, especially those dealing with Coupling and Repulsion, and with the Heredity of Sex, are in essential respects out of date. Knowledge of these subjects is at present in a transitional stage, and I have endeavoured in a series of brief Appendixes to acquaint the reader with the nature of the principal advances made, while awaiting an opportunity of rewriting the book.

I am obliged to Professor Arnold Lang and to Mr C. C. Hurst for calling my attention to errors, which, with some others, have been corrected.

W. B.

November, I9I2. 



\section{CONTENTS}

\section{PART I.}

CHAPTER

I. INTRODUCTORY.

Introductory-Some pre-Mendelian Writings-Mendel's DiscoveryDominant and Recessive-Segregation. Allelomorphism-Homozygote and Heterozygote. Purity of Type.

II. The Material Investigated . . . . . . I8

List of Structural Characters in Plants and Animals-List of Types in which the inheritance of Colour has been studied-Preliminary Deductions - Dominance and heterozygous characters-Mendel's system distinguished from that of Galton.

III. Numerical Consequences and Recombinations • •

Representations of the $\mathrm{F}_{2}$ Generation and Novelties due to Re-combination of Factors-Compound Characters-Combs of Fowls-Heterostylism-White Flowers from Red $\times$ Cream.

IV. Heredity of Colour . . . . . . .

Factors determining Colours: the Ratio 9:3:4-The "Presence and Absence" Hypothesis. Fpistatic and Hypostatic Factors-Colours of Mice-Pied Types-A Dominant Piebald.

V. Heredity of Colour (continued) . • • •

Albinos giving Coloured Offspring; Reversion on Crossing-Various Kinds of Whites-Stocks-Orchids-Pigeons-Fowls-Primula.

VI. Heredity of Colour (continued) . . . . .

Eye-Colours. Variations in Colour of the Iris-Deficiency of EyePigments in some Coloured Types.

VII. Heredity of Colour (continued) • . • •

The Genetics of Yellow Pigments in certain Animals. Yellow Mice not breeding true-The Case of Basset Hounds and the "Law of Ancestral Heredity." Relation of this Principle to Mendelian Rules.

VIII. Heredity of Colour (continued) . • . •

Various Specific Phenomena in Colour-Inheritance. Relation of Colour to Hoariness in Stocks. Miscellaneous Cases. Colour of a Special Part controlling that of other Parts-Summary and DiscussionSubtraction-Stages. 
IX. Gametic Coupling and Spurious Allelomorphism • ${ }_{4} 8$

Pollen-Shape and Flower-Colour. Axil-Colour and Sterile AnthersHooded Standard and Flower-Colour in Sweet Peas.

X. Heredity AND SeX . . . . . . . . I64

Evidence from Breeding Experiments. Bryonia-Sex-limited Heredity. The Horns of Sheep-Colour-Blindness-Sex and Spurious Allelomorphism. The Currant Moth-The Cinnamon Canary-The Silky Fowl-Aglia tau-Cytological Evidence--Summary.

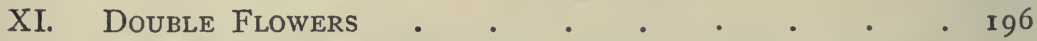

Miscellaneous Cases. Recessive and Dominant Doubling- "Hose-inHose" Flowers-The Special Case of Double Stocks.

XII. Evidence as to Mendelian Inheritance in Man

Normal Characters-Diseases and Malformations. Dominants-Sexlimited Dominants-Recessives-Notes on collecting Evidence.

XIII. INTERmediates Between Varieties and the "Pure

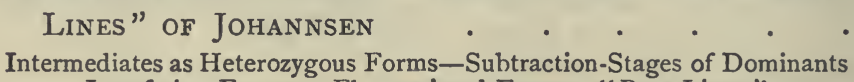
-Interfering Factors-Fluctuational Forms-"Pure Lines."

XiV. Miscellaneous Exceptional and Unconformable PheNOMENA

Crosses breeding true without Segregation. Parthenogenetic or Apogamic Forms. Hieracium-Sexual Forms-Numerical Aberrations -Irregularities of Dominance-Alternation of Generations-Maternal Characters in certain Seeds.

XV. Biological Conceptions in the Light of Mendelian Discoveries .

Nature of Units-Nature of Segregation-Moment of SegregationDifferentiation of Parts compared with Segregation-Reversion and Variation. "Bush" and "Cupid" Sweet Peas-Mendelian Segregation and Species-Discontinuity in Variation-Mendelism and Natural Selection.

XVI. Practical Application of Mendelian Principles

Meaning of Pure-bred-Rogueing-Raising Noveltres-A Practical Example-Unfixable Types-Technical Methods-Sociological Application.

Appendixes .

\section{PART II.}

I. Biographical Notice of Mendel

2. Translation of the Paper on Hybridisation . . 335

3. Translation of the Paper on Hieracium . . . 380

BIBLIOGRAPHY

INDEX OF SUBJECTS

INDEX OF AUTHORS 


\title{
LIST OF .ILLUSTRATIONS
}

\author{
PORTRAITS OF MENDEL.
}

In 1866

About 1862 .

About 1880 .
Frontispiece

to face p. 309

to face p. 3I 7

\section{COLOURED PLATES.}

Plate I. Lepidoptera . to face p. 44

"II. Mice . . . . . . . between $p p$. 80-8I

"III. Reversion in Sweet Peas . . . between pp. 93-94

„ IV. Fowls . . . . . . . . to face $p$. 103

„. V. Spurious Allelomorphism in Sweet Peas betzeen pp. I54-I 55

"VI. Heredity of Colour in Primula Sinensis between pp. 294-295

\section{FIGURES.}

FIGURE

PAGE

I. Tall and "Cupid" dwarf Sweet Peas . . . . 9

i. Diagram showing consequences of Segregation . . . I

3. Inheritance of seed-characters in Pea . . . . I5

4. Branched and unbranched forms in Stocks (Matthiola) . 20

5. Hooded and normal Barley . . . . . . 2 I

6. Heredity in Wheat . . . . . . . . 23

7. Fern-leaf and palm-leaf in Primula Sinensis . . . 24

8. Two-row and six-row Barley . . . . . . 27

9. Starch-grains in Peas. . . . . . . . 29

ro. Round and wrinkled seed in Maize . . . . . 30

II. Down-colour in Chickens . • . • • • • $5^{\mathrm{I}}$

12. Types of combs in Fowls . . . . . . . 6I

I3. Combs of newly-hatched Chickens . . . . . $\quad 62$

14. Descent of "homostyle" character in Primula . . . 69 
FIGURE

PAGE

I5. Diagram of $\mathrm{F}_{2}$ showing ratio $9: 3: 4 \quad . \quad . \quad . \quad$. 77

16. Diagram of $\mathrm{F}_{2}$ showing ratio $9: 7 \quad$. $\quad . \quad$. $\quad . \quad$. 89

17. Diagram of $\mathrm{F}_{2}$ in Sweet Pea showing ratio $27: 9: 28$. 9 I

I8. Pedigrees of eye-colour in Man . . . . . 108

19. Pollen grains of Sweet Peas . . . . . . I50

20. Heredity of horns in Sheep . . . . . . I7I

21. Heredity of a peculiar form of curly hair . . . 207

22. Descent of congenital lock of white hair . . . . 207

23. Brachydactylous hands . . . . . . . 2 II

24. Skiagram of hands . . . . . . . . 212

25. Hands of brachydactylous child . . . . . 2 I 3

26. Pedigree of brachydactylous family . . . . . 214

27. Descent from brachydactylous members . . . . 2 I4

28. Drinkwater's pedigree of brachydactyly . . . . 2 I5

29. Descent of prae-senile cataract . . . . . . 216

30. Another cataractous family . . . . . . 217

3I. Descent of stationary night-blindness - between $p p$. 220-22I

32. Descent of Colour-blindness . . . . . . 223

33. Ideal Scheme of descent of simple sex-limited condition, e.g. horns of Sheep . . . . . . 230

34. Tentative representation of descent of Colour-blindness . $23 \mathrm{I}$

35. Polish $\times$ Rivet Wheat. . . . . . . . 259

36. Seeds of Polish $\times$ Rivet Wheat . . . . . . 260

37. Reversion in height of Sweet Peas . . . . . ${ }_{2} 82$

38. Two types of dwarf Sweet Peas . . . . . 283 


\section{PART I}





\section{CHAPTER I}

\section{INTRODUCTORY. MENDEL'S DISCOVERY.}

Introductory-Some pre-Mendelian Writings-Mendel's Discovery-Dominant and Recessive-Segregation. Allelomorphism - Homozygote and Heterozygote. Purity of Type.

14 Among the biological sciences the study of heredity occupies a central position. Whether we be zoologists, botanists, or physiologists, the facts of heredity concern us. Upon this physiological function all the rest in some degree depend. Every advance in knowledge of that central function must affect the course of thought along each several line of biological inquiry.

Moreover though, as naturalists, we are not directly concerned with the applications of science, we must perceive that in no region of knowledge is research more likely to increase man's power over nature. The science of sociology, and in many of its developments the science of medicine also, must of necessity form working hypotheses respecting the course of heredity, and we cannot doubt that a perception of the truth in regard to the function of transmission will greatly contribute to the progress of these sciences. Lastly, to the industrial arts of the breeder of plants or animals, the knowledge we are attempting to provide is of such direct importance that upon this consideration no special emphasis is required. In studying heredity, therefore, we are examining a vital problem of no mean consequence, and those who engage in that work are happy in the thought that they are assisting one of the main advances in natural knowledge.

But though we may approach this study of geneticsto use the modern designation-from so many different sides, it is especially in their bearing on the problem of

E. H. 
the evolution of species that the facts have hitherto been most profitably investigated. It was in the attempt to ascertain the interrelationships between species that experiments in genetics were first made. The words "evolution" and "origin of species" are now so intimately associated with the name of Darwin that we are apt to forget that the idea of a common descent had been prominent in the minds of naturalists before he wrote, and that, for more than half a century, zealous investigators had been devoting themselves to the experimental study of that possibility. Prominent among this group of experimenters may be mentioned Koelreuter, John Hunter, Herbert, Knight, Gaertner, Jordan, Naudin, Godron, Lecoq, Wichura-men whose names are familiar to every reader of Animals and Plants under Domestication. If we could ask those men to define the object of their experiments, their answer would be that they were seeking to determine the laws of hereditary transmission with the purpose of discovering the interrelationships of species. In addition to the observation of the visible structures and habits of plants and animals they attempted by experiment to ascertain those hidden properties of living things which we may speak of as genetic, properties which breeding tests can alone reveal. The vast mass of observation thus accumulated contains much that is of permanent value, hints that if followed might have saved their successors years of wasted effort, and not a few indications which in the light of later discovery will greatly accelerate our own progress.

Yet in surveying the work of this school we are conscious of a feeling of disappointment at the outcome. There are signs that the workers themselves shared this disappointment. As we now know, they missed the clue without which the evidence so laboriously collected remained an inscrutable medley of contradictions.

While the experimental study of the species problem was in full activity the Darwinian writings appeared. Evolution, from being an unsupported hypothesis, was at length shown to be so plainly deducible from ordinary experience that the reality of the process was no longer doubtful. With the triumph of the evolutionary idea, curiosity as to the significance of specific differences was 
satisfied. The Origin was published in 1859. During the following decade, while the new views were on trial, the experimental breeders continued their work, but before I 870 the field was practically abandoned.

In all that concerns the problem of species the next thirty years are marked by the apathy characteristic of an age of faith. Evolution became the exercising-ground of essayists. The number indeed of naturalists increased tenfold, but their activities were directed elsewhere. Darwin's achievement so far exceeded anything that was thought possible before, that what should have been hailed as a long-expected beginning was taken for the completed work. I well remember receiving from one of the most earnest of my seniors the friendly warning that it was waste of time to study variation, for "Darwin had swept the field."

Parenthetically we may notice that though scientific opinion in general became rapidly converted to the doctrine of pure selection, there was one remarkable exception. Systematists for the most part kept aloof. Everyone was convinced that natural selection operating in a continuously varying population was a sufficient account of the origin of species except the one class of scientific workers whose labours familiarised them with the phenomenon of specific difference. From that time the systematists became, as they still in great measure remain, a class apart.

A separation has thus been effected between those who lead theoretical opinion and those who by taste or necessity have retained an acquaintance with the facts. The consequences of that separation have been many and grievous. To it are to be traced the extraordinary misapprehensions as to the fundamental phenomena of specific difference which are now prevalent.

If species had really arisen by the natural selection for impalpable differences, intermediate forms should abound, and the limits between species should be on the whole indefinite. As this conclusion follows necessarily from the premisses, the selectionists believe and declare that it represents the facts of nature. Differences between species being by axiom indefinite, the differences between varieties must be supposed to be still less definite. Consequently the conclusion that evolution must proceed by insensible 
transformation of masses of individuals has become an established dogma. Systematists, entomologists or botanists for example, are daily witnesses to variation occurring as an individual and discontinuous phenomenon, but they stand aside from the debate; and whoever in a discussion of evolutionary theory appeals to the definiteness of varietal distinctions in colour for instance, or in form, as recognizable by common observation without mechanical aid, must be prepared to meet a charge of want of intelligence or candour. This is no doubt a passing phase and will end so soon as interest in the problems of evolution is combined with some knowledge of variation and heredity.

Genetic experiment was first undertaken, as we have seen, in the hope that it would elucidate the problem of species. The time has now come when appeals for the vigorous prosecution of this method should rather be based on other grounds. It is as directly contributing to the advancement of pure physiological science that genetics can present the strongest claim. We have an eye always on the evolution-problem. We know that the facts we are collecting will help in its solution; but for a period we shall perhaps do well to direct our search more especially to the immediate problems of genetic physiology, the laws of heredity, the nature of variation, the significance of sex and of other manifestations of dimorphism, willing to postpone the application of the results to wider problems as a task more suited to a maturer stage. When the magnitude and definiteness of the advances already made in genetics come to be more generally known, it is to be anticipated that workers in various departments of biology will realise that here at last is common ground. As we now know, the conceptions on which both the systematists and the speculative biologists have based their methods need complete revision in the light of the new facts, and till the possibilities of genetic research are more fully explored the task of reconstruction can hardly be begun. In that work of exploration all classes of naturalists will alike find interest. The methods are definite and exact, so we need not fear the alienation of those systematists to whom all theoretical inquiry is repulsive. They are also wide in their scope, and those who would turn from the details of classification 
as offering matter too trivial for their attention may engage in genetic inquiries with great confidence that every fragment of solid evidence thus discovered will quickly take its place in the development of a coordinated structure.

\section{Some pre-Mendelian Writings.}

Of the contributions made during the essayist period three call for notice: Weismann deserves mention for his useful work in asking for the proof that "acquired characters"-or, to speak more precisely, parental experience-can really be transmitted to the offspring. The occurrence of progressive adaptation by transmission of the effects of use had seemed so natural to Darwin and his contemporaries that no proof of the physiological reality of the phenomenon was thought necessary. Weismann's challenge revealed the utter inadequacy of the evidence on which these beliefs were based. There are doubtless isolated observations which may be interpreted as favouring the belief in these transmissions, but such meagre indications as exist are by general consent admitted to be too slight to be of much assistance in the attempt to understand how the more complex adaptative mechanisms arose. Nevertheless it was for the purpose of elucidating them that the appeal to inherited experience was made. Weismann's contribution, though negative, has greatly simplified the practical investigation of genetic problems.

Though it attracted little attention at the time of its appearance, an honourable place in the history of our science must be accorded to the paper published by de Vries (1889) under the title Intracellulare Pangenesis. This essay is remarkable as a clear foreshadowing of that conception of unit-characters which is destined to play so large a part in the development of genetics.

The supreme importance of an exact knowledge of heredity was urged by Galton in various writings published during the period of which I am speaking. He pointed out that the phenomena manifested regularity, and he made the first comprehensive attempt to determine the rules they obey. It was through his work and influence that the existence of some order pervading the facts became generally 
recognized. In 1897 he definitely enunciated his now famous "Law" of heredity, which declared that to the total heritage of the offspring the parents on an average contribute $\frac{1}{2}$, the grandparents $\frac{1}{4}$, and the great-grandparents $\frac{1}{8}$, and so on, the total heritage being taken as unity. To this conclusion he had been led by several series of data, but the evidence upon which he especially relied was that of the pedigrees of Basset Hounds furnished him by the late Sir Everett Millais. In that instance the character considered was the presence or absence of black in addition to yellow and white. The colours were spoken of as tri-colour and non-tri-colour, and the truth of the law was tested by the average numbers of the respective colours which resulted from the various matings of dogs of known ancestral composition. These numbers corresponded so well with the expectations given by the law as to leave no reasonable doubt that the results of calculation were in general harmony with natural fact.

There are features in this important case which need special consideration, and to these I will return. Meanwhile we may note that though there was admittedly a statistical accord between Galton's theory and some facts of heredity, yet no one familiar with breeding or even with the literature of breeding could possibly accept that theory as a literal or adequate presentation of the facts. Galton himself in promulgating it made some reservations; but in the practice of breeding, so many classes of unconformable phenomena were already known, that while recognizing the value of his achievement, we could not from the first regard it as more than an adumbration of the truth. As we now know, Galton's method failed for want of analysis. His formula should in all probability be looked upon rather as an occasional consequence of the actual laws of heredity than in any proper sense one of those laws.

Of the so-called investigations of heredity pursued by extensions of Galton's non-analytical method and promoted by Professor Pearson and the English Biometrical school it is now scarcely necessary to speak. That such work may ultimately contribute to the development of statistical theory cannot be denied, but as applied to the problems of heredity the effort has resulted only in the concealment of 
that order which it was ostensibly undertaken to reveal. A preliminary acquaintance with the natural history of heredity and variation was sufficient to throw doubt on the foundations of these elaborate researches. To those who hereafter may study this episode in the history of biological science it will appear inexplicable that work so unsound in construction should have been respectfully received by the scientific world. With the discovery of segregation it became obvious that methods dispensing with individual analysis of the material are useless. The only alternatives open to the inventors of those methods were either to abandon their delusion or to deny the truth of Mendelian facts. In choosing the latter course they have certainly succeeded in delaying recognition of the value of Mendelism, but with the lapse of time the number of persons who have themselves witnessed the phenomena has increased so much that these denials have lost their dangerous character and may be regarded as merely formal.

\section{Rediscovery of Mendel: his Method.}

With the year I900 a new era begins. In the spring of that year there appeared, within a few weeks of each other, the three papers of de Vries, Correns, and Tschermak, giving the substance of Mendel's long-forgotten treatise. Each of these three writers was able from his own experience to confirm Mendel's conclusions, and to extend them to other cases. There could therefore, from the first, be no question as to the truth of the facts. To appreciate what Mendel did the reader should refer to the original paper*, which is a model of lucidity and expository skill. His success is due to the clearness with which he thought out the problem. ${ }^{\top}$ Being familiar with the works of Gaertner and the other experimental breeders he surmised that their failure to reach definite and consistent conclusions was due to a want of precise and continued analysis. In order to obtain a clear result he saw that it was absolutely necessary to start with pure-breeding, homogeneous materials, to consider each character separately, and on no account to confuse the different generations together. Lastly he realised

\footnotetext{
* See Part II.
} 
that the progeny from distinct individuals must be separately recorded. All these ideas were entirely new in his day. When such precautions had been observed he anticipated that a regular result would be attainable if the experiments were carried out on a sufficient scale.

After several preliminary trials he chose the edible Pea (Pisum sativum) for his subject. Varieties in cultivation are distinguished by striking characters recognizable without trouble. The plants are habitually self-fertilised, a feature which obviates numerous difficulties.

Following his idea that the heredity of each character must be separately investigated, he chose a number of pairs of characters, and made crosses between varieties differing markedly in respect of one pair of characters. The case which illustrates Mendelian methods in the simplest way is that in which heredity in respect of height was studied. Mendel took a pair of varieties of which one was tall, being 6-7 feet high, and the other was dwarf, $\frac{3}{4}$ to $I \frac{1}{2}$ feet. These two were then crossed together. In peas this is an easy operation. The unbroken anthers can be picked out of a bud with a pair of fine forceps and the pollen of the plant chosen for the father may be at once applied to the stigma of the emasculated flower. The cross-bred seeds thus produced grew into plants which were always tall, having a height not sensibly different from that of the pure tall variety. In our modern terminology such a cross-bred, the first filial generation, is called $F_{1}$. From the fact that the character, tallness, appears in the cross-bred to the exclusion of the opposite character, Mendel called it a dominant character; dwarfness, which disappears in the $F_{1}$ plant, he called recessive.

The tall cross-bred, so produced, in its turn bore seeds by self-fertilisation. These are the next generation, $F_{2}$. When grown up they prove to be mixed, many being tall, some being short, like the tall and the short grandparents respectively. Fig. I shows such an $F_{2}$ family in the Sweet Pea. Upon counting the members of this $F_{2}$ generation it was discovered that the proportion of talls to shorts exhibited a certain constancy, averaging about three talls to one short, or in other words, 75 per cent. dominants to 25 per cent. recessives. 
These $F_{2}$ plants were again allowed to fertilise themselves and the offspring of each plant was separately sown. It was then found that the offspring, $F_{3}$, of the recessives
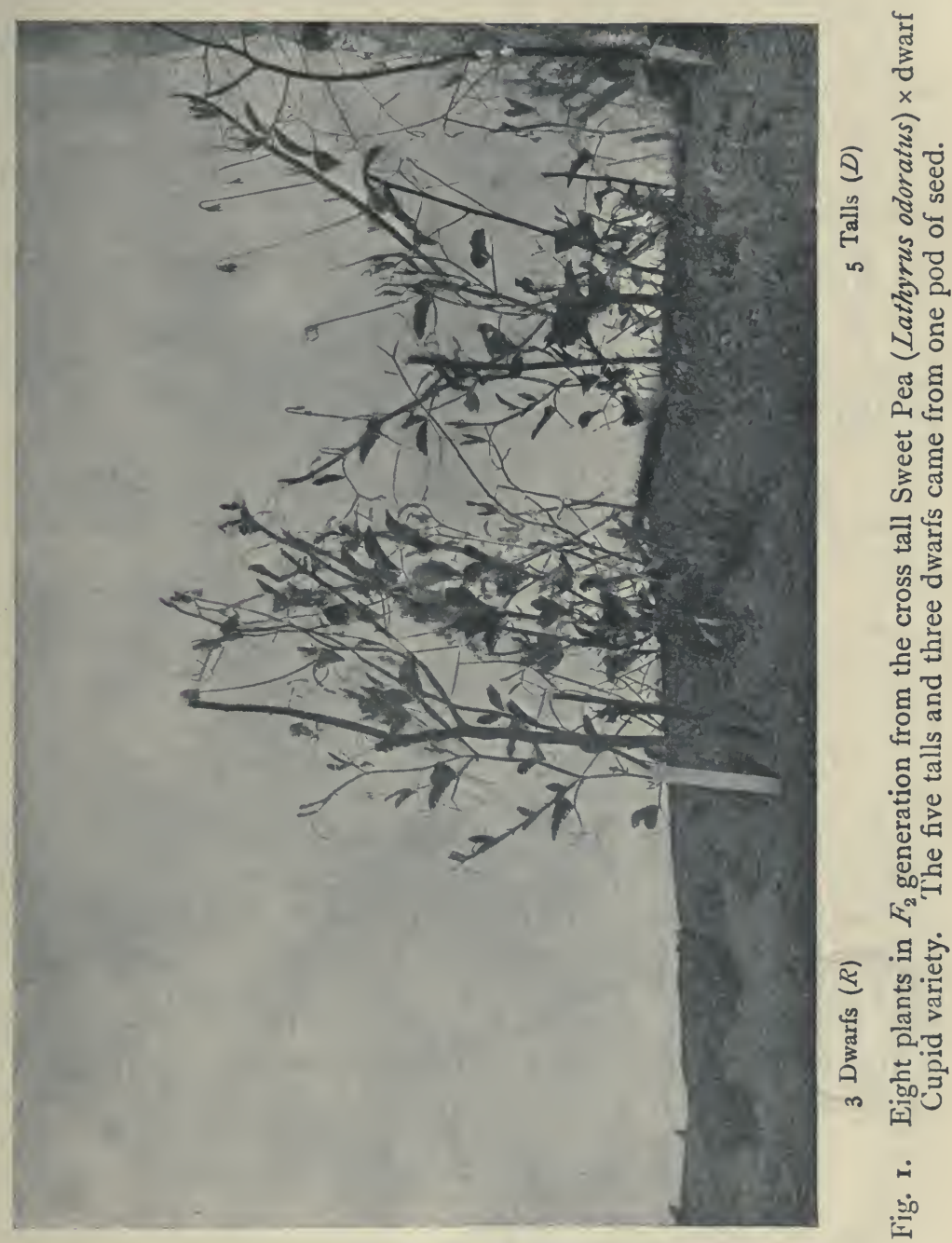

consisted entirely of recessives. Further generations bred from these recessives again produced recessives only, and therefore the recessives which appeared in $F_{2}$ are seen to 
be pure to the recessive character, namely, in the case we are considering, to dwarfness.

But the tall $F_{2}$ dominants when tested by a study of their offspring $\left(F_{3}\right)$, instead of being all alike (as the dwarfs or recessives were), proved to be of two kinds, viz.

(a) Plants which gave a mixed $F_{3}$ consisting of both talls and dwarfs, the proportion showing again an average of three talls to one dwarf.

(b) Plants which gave talls only and are thus pure to tallness.

The ratio of the impure $(a)$ plants to the pure $(b)$ plants was as 2 to $\mathrm{I}$.

The whole $F_{2}$ generation therefore, formed by selffertilisation of the original hybrid consists of three kinds of plants :

or $\begin{array}{cc}25 \% & 50 \% \\ 3 \text { dominants } & 25 \% \\ \text { pure dominants impure dominants } & \begin{array}{l}25 \\ \text { pure recessives }\end{array}\end{array}$

\section{Segregation. Allelomorphism.}

The conclusion which Mendel drew from these observations is one which will suggest itself to any one who reflects on the facts. The result is exactly what would be expected if both male and female germ-cells of the cross-bred $F_{1}$ were in equal numbers bearers of either the dominant $(D)$ or recessive $(R)$ character, but not both. If this were so, and if the union of the male and female germ-cells occurs at random, the result would be an $F_{2}$ family made up of

$$
{ }_{3} \underbrace{D D:{ }_{25} D R:{ }_{25} R D}_{3}:{ }^{25} R R .
$$

But, as the first cross showed, when $D$ meets $R$ in fertilisation the resulting individual is in appearance $D$; therefore $F_{2}$ appears as $3 D: I R$. The results of the $F_{3}$ 
generation are in exact agreement with this suggestion: for the $R$ plants give $R$ only; and of the $D$ plants onethird give $D$ only, while two-thirds give the same mixture, ${ }_{3} D:$ I $R$, which was produced by $F_{1}$ (Fig. 2. I).

The descent may be represented diagrammatically thus :

Parents

Tall $(T T) \times$ Short $(t t)$

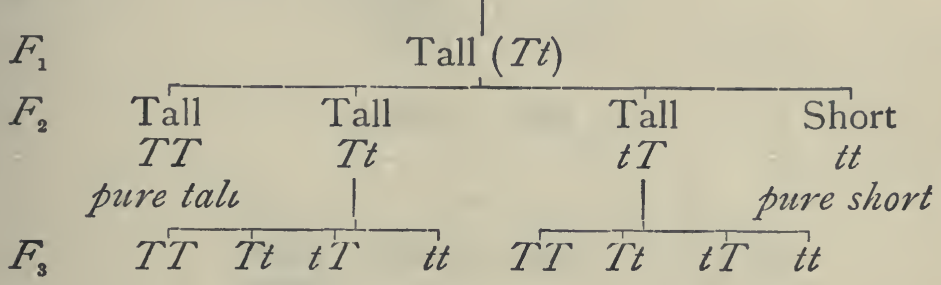

Now since the fertilised ovum or zygote, formed by the original cross, was made by the union of two germ-cells or gametes bearing respectively tallness and dwarfness, both these elements entered into the composition of the original $F_{1}$ zygote; but if the germ-cells which that zygote eventually forms are bearers of either tallness or dwarfness, there must at some stage in the process of germ-formation be a separation of the two characters, or rather of the ultimate factors which cause those characters to be developed in the plants. This phenomenon, the dissociation of characters from each other in the course of the formation of the germs, we speak of as segregation, and the characters which segregate from each other are described as allelomorphic, i.e. alternative to each other in the constitution of the gametes (Fig. 2).

That this is the true account was proved by further experiments which Mendel made by crossing the $F_{1}$ with pure dominants and with pure recessives. For $D R \times D D$ gave an offspring all dominant in appearance, though in reality consisting of both $D R$ plants and $D D$ plants, on an average in equal numbers. On the other hand $D R \times R R$ gives an equal number of dominants and recessives, of which the dominants are all $D R$ plants, and the recessives are all pure recessives. These various experiments illustrate the composition of the four simple types of Mendelian families, which may be set out thus: 

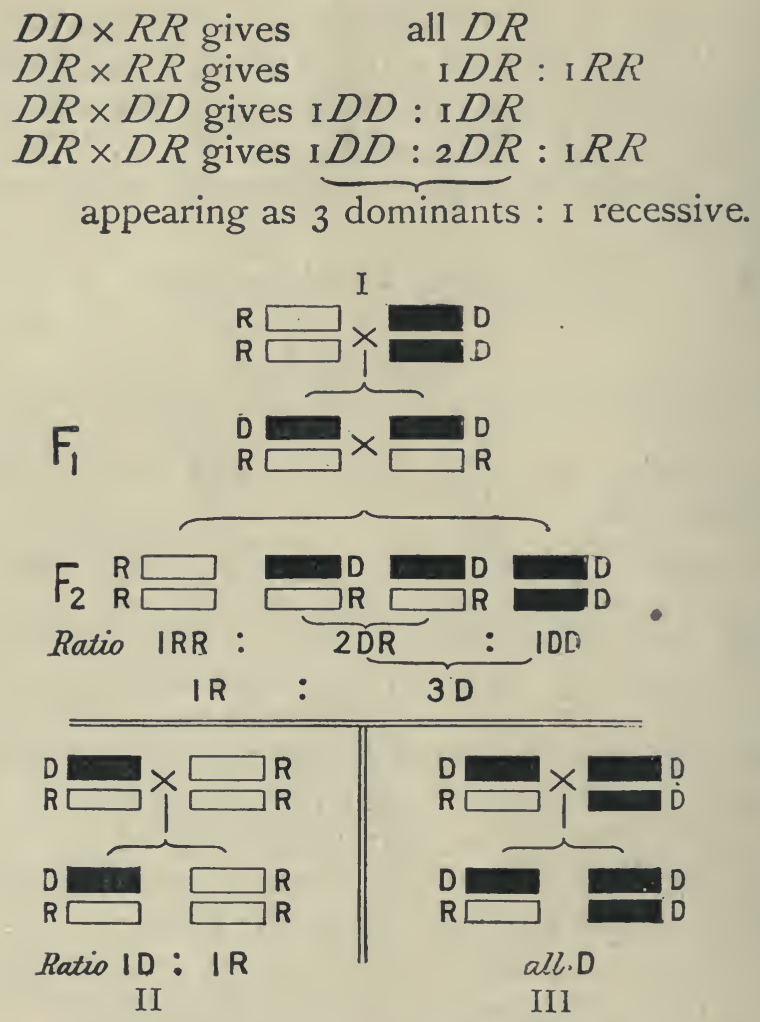

Fig. 2. Diagrams showing numerical consequences of segregation.

I. The mating $D D \times R R$, and $D R \times D R$. II. The mating $D R \times R R$. III. The mating $D R \times D D$.

The way in which these ratios are produced may be easily represented by means of a number of draught-men. Pairs of draughts then represent zygotes; single draughts represent germ-cells. That there is a propriety in representing zygotic or somatic cells as double structures and germ-cells as single structures will be evident to biologists ; for we know that each somatic nucleus in plants and animals is a double structure, containing twice the number of chromosomes present in each mature germ-cell. Two black draughts may then be taken to represent a pure black individual, two white draughts a white individual. When they are crossed together $F$, is represented by a black 
draught and a white one (Fig. 2. I). Supposing the black to be a dominant the fact may be represented by putting it on the top. When segregation of the allelomorphs, blackness and whiteness, takes place in gameto-genesis, the germ-cells of the cross-bred are again bearers of blackness or of whiteness, and it may readily be shown experimentally that the results of their various random combinations give rise to the ratios stated above.

The fact of segregation was the essential discovery which Mendel made. As we now know, such segregation is one of the normal phenomena of nature. It is segregation which determines the regularity perceptible in the hereditary transmission of differences, and the definiteness or discontinuity so often conspicuous in the variation of animals and plants is a consequence of the same phenomenon. Segregation thus defines the units concerned in the constitution of organisms and provides the clue by which an analysis of the complex heterogeneity of living forms may be begun.

There are doubtless limits beyond which such analysis cannot be pursued, but a vast field of research must be explored before they are reached or determined. It is likely also that in certain cases the units are so small that no sensible segregation can be proved to exist. As yet, however, no such example has been adequately investigated; nor, until the properties and laws of interaction of the segregable units have been much more thoroughly examined, can this class of negative observations be considered with great prospect of success.

The dominance of certain characters is often an important but never an essential feature of Mendelian heredity. Those who first treated of Mendel's work most unfortunately fell into the error of enunciating a "Law of Dominance" as a proposition comparable with the discovery of segregation. Mendel himself enunciates no such law. Dominance of course frequently exists. The consequences of its occurrence and the complications it introduces must be understood as a preliminary to the practical investigation of the phenomena of heredity, but it is only a subordinate incident of special cases, and Mendel's principles of inheritance apply equally to cases where there is no dominance 
and the heterozygous type is intermediate in character between the two pure types.

To the detection of the genetic system of any given case it is however necessary that the results of combinations should be sensibly regular. When, as occasionally happens, a character may sometimes behave as a dominant and sometimes not, we have as yet no satisfactory means of further analysis. These irregularities in dominance may confidently be attributed to the disturbing effects of other factors or of conditions, but the detection of such unknown factors must be a long and perhaps impossible task.

Mendel applied his method to the following seven distinct pairs of characters in peas, and found that in each the inheritance was similar. The dominant character is put first.

I. Height: whether tall or short.

2. Distribution of flowers on the stem: whether arranged along the axis of the plant, or bunched together at the top so as to form a false umbel*.

3. Colour of unripe pod: whether a shade of green or bright yellow.

4. Shape of pod: whether simply inflated, or deeply constricted between the seeds, i.e. as in "sugarpeas" or "Pois sans parchemin."

5. Colour of seed-skin: whether various shades of grey or brown, with or without violet spotting, or white. The "grey" skins are always associated with coloured flowers and almost always with a purple or red mark in the axils.

6. Colour of cotyledons : whether yellow or green.

7. Shape of seeds : whether rounded or wrinkled.

It will be observed that the first five are plant-characters. In order to see the result of crossing, the seeds must be sown and allowed to grow into plants. The last two characters belong to the seeds themselves. The seeds of course are members of a generation later than that of the plant which bears them. Thus when a cross is made the

* This is a fasciated and semi-monstrous form. 
resultant seeds are $F_{1}$, showing the dominant character yellowness or roundness, but the seed-skins are maternal tissue. Such $F_{1}$ seeds grow into $F_{1}$ plants and bear $F_{2}$ seeds which show the typical mixture of dominants and recessives in the pods (Fig. 3). In each case Mendel's

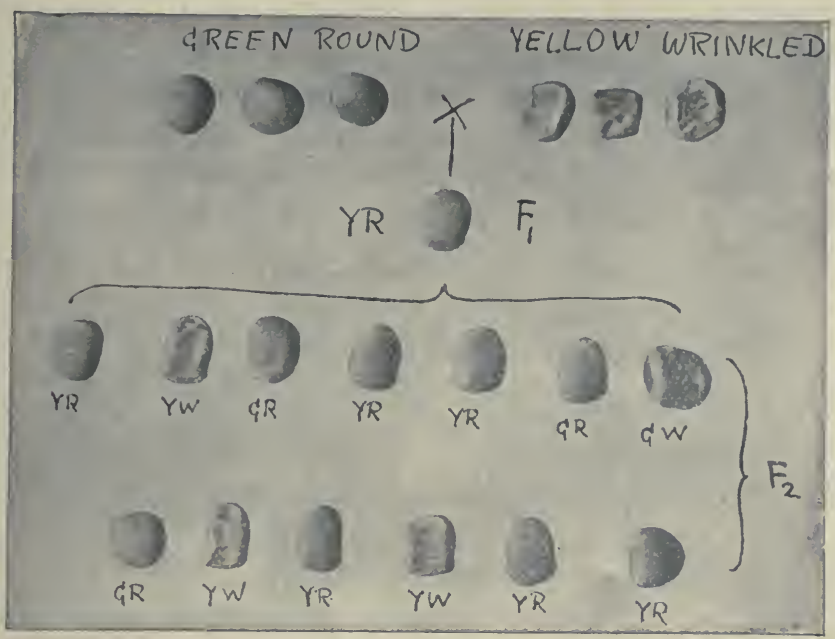

Fig. 3. Inheritance of seed-characters in Pea. The seed of a green round variety fertilised by pollen of a yellow wrinkled variety are yellow and round $\left(F_{1}\right)$. The reciprocal cross would give the same result. Two pods of $F_{2}$ seed borne by the $F_{1}$ plant are shown. There were 6 yellow round, 3 green round, 3 yellow wrinkled, I green wrinkled.

observations have been substantially confirmed by later observers, and the operation of similar processes has now been recognized in a long series of most diverse characters in both animals and plants.

\section{Consequences of Segregation: Homozygote and Heterozygote.}

Before considering the various extensions of Mendelian research, it may be well to indicate in general terms the chief significance of the facts. The first conception to which we are led is that of unit-characters, units because they may be treated as such in the cell-divisions of gametogenesis. It is evidently upon some process of 
qualitative segregation occurring in one or more of these cell-divisions that allelomorphism depends. The opposite members of each pair of characters being allelomorphic to each other, every zygote*, or individual produced in fertilisation, must, in respect of any such pair, be either a homozygote, that is to say, a zygote formed by the union of two gametes each bearing the same allelomorph, as $A A$ and $a a$, or a heterozygote formed by the union of two germs bearing different allelomorphs, as $A a$. Therefore in respect of any pair of allelomorphic characters, the individuals composing the whole population are of three kinds only:

I. Homozygotes of the form $A A$,

2. Homozygotes of the form $a a$,

3. Heterozygotes of the form $A a$.

The gametes are of two kinds only, $A$ and $a$. Each kind of homozygote is pure to the character of the gametes which compose it.

\section{Purity of Type.}

Purity of type thus acquires a precise meaning. It is dependent on gametic segregation, and has nothing to do with a prolonged course of selection, natural or artificial.

All this is of course consonant with the visible facts that have been discovered by the cytologists, in so far as the nucleus of each somatic cell is a double structure, while the nucleus of each gametic cell is a single structure. It is, in my judgment, impossible as yet to form definite views as to the relations of the various parts of the cell to the function of heredity. The details of cytology and their interpretation are beyond our present province, but this much is certain: that when in these discussions we idealize the characters as borne by the gamete in an unpaired state and by the zygote in a paired state, we make no assumption which is not in full accord with histological appearances.

From the fact that the development of characters in animals or plants depends on the presence of definite units

* In botany the term zygote is usually restricted to the single cell which results from the process of fertilisation, but by a natural extension the word may be used for the individual which develops by somatic divisions from that cell. 
or factors in their germ-cells, the paradox at once follows that an organism may be pure-bred in respect of a given character though its parents were cross-bred in the same respect. Purity depends on the meeting of two gametes bearing similar factors, and when two similarly-constituted gametes do thus meet in fertilisation, the product of their union is pure. The belief, so long prevalent, that purity of type depends essentially on continued selection is thus shown to have no physiological foundation.

Similarly it is evident that an individual may be pure in respect of one character and cross-bred or impure in respect of others.

As a consequence of the application of Mendel's principles, that vast medley of seemingly capricious facts which have been recorded as to heredity and variation is rapidly being shaped into an orderly and consistent whole. A new world of intricate order previously undreamt of is disclosed. We are thus endowed with an instrument of peculiar range and precision, and we reach to certainty in problems of physiology which we might have supposed destined to continue for ages inscrutable.

After such a discovery it is obvious that old ideas must be revised. Systematists debating the limits of "specific rank" or the range of variability, morphologists seeking to reconstruct phylogenetic history, physiologists unravelling the interaction of bodily functions, cytologists attempting to interpret the processes of cell-division-each of these classes of naturalists must now examine the current conceptions of his study in the light of the new knowledge. The practical breeder of animals or plants, basing his methods on a determination of the Mendelian units and their properties, will in many of his operations be able to proceed with confidence and rapidity. Lastly, those who as evolutionists or sociologists are striving for wider views of the past or of the future of living things may by the use of Mendelian analysis attain to a new and as yet limitless horizon. 


\section{CHAPTER II}

THE MATERIAL INVESTIGATED.

List of Structural Characters in Plants and AnmalsList of Types in which the inheritance of Colour has been. studied-Preliminary Deductions-Dominance and heterozygous characters-Mendel's system distinguished from that of Galton.

Heredity following the general rules described in the last chapter has been witnessed in a great diversity of animals and plants. The characters already proved to follow such rules show an equal diversity. The following is a list of some of them. Adequately to represent the facts respecting each of these cases lengthy description would be needed. In regard to several of them occurrences which do not readily fall into line have been recorded. Of these some are probably due to errors of observation or mistakes of various kinds, but a few will doubtless prove to be genuine exceptions to rule and may constitute points of departure for fresh and more extended research. In the outline of the phenomena, which is all that this book can profess to offer, it seemed best to restrict as far as possible the enumeration of these details, which can only be thoroughly appreciated by reference to the original papers; but such annotations as appeared necessary either in elucidation of the phenomena or by way of incentive to further work are briefly given with references to the original sources. These annotations will be better understood after the later chapters have been read.

In the following list when one character is conspicuously dominant it is put first, but in several cases the dominance is imperfect.

\section{Plants.}

I. Tallness and dwarfness. Peas (Pisum) and Sweet Peas (Lathyrus odoratus). Runner and French Beans (Phaseolus).

As regards Peas the facts have been recorded by Mendel (I95), Tschermak (269, 270 , \&c.), R.E.C.* (20). When varieties differing

* R.E.C. stands throughout for Reports to the Evolution Committee of the Royal Society, giving an account of the experiments of W. Bateson, E. R. Saunders and R. C. Punnett. Other contributors to these Reports are mentioned by name. 
greatly in height are used, dominance is complete, and the two parent forms are represented as three to one in $F_{2}$. No clear exception has yet been observed. Peas (Pisum) exist in a vast number of distinct horticultural varieties which can roughly be classified as tall (about 5-6 ft.), half-dwarfs (about $4 \mathrm{ft}$.), dwarfs (about 9 ins. to $3 \mathrm{ft}$.). The genetic relations of the half-dwarfs to the others are not fully explored, and further investigation will probably lead to the discovery of important facts. The cross halfdwarf $\times$ tall giving tall as dominant has produced some extreme dwarfs in $F_{2}$, doubtless by recombination (q.v.), R.E.C. 20 , p. 69. The cross half-dwarf $\times$ dwarf has given intermediates in $F_{1}$ (ibid.).

The cross between tall and dwarf "Cupid" Sweet Peas gives complete dominance of tallness and simple segregation in $F_{2}$, "Cupids" indistinguishable from the original "Cupid" parent reappearing (Fig. I).

Phaseolus has been investigated especially by Tschermak (278) who records some apparently anomalous results. de Vries (298, II. p. 76) states that he found that extracted $F_{2}$ dwarf Antirrhinum did not breed true, but threw plants of various heights. The experiment should be repeated.

2. Branching habit and the unbranched habit. Sunflower (Helianthus, Shull, 24I) and Cotton (Balls, 6). The branched form of Stock (Matthiola incana) is dominant to the unbranched Brompton type. In $F_{2}$ the unbranched type reappears, but the ratio has not been determined (Fig. 4). E. R. Saunders (unpublished).

3. The straggling habit of both the tall and dwarf "Cupid" Sweet Peas, and the much-branched erect habit of the "Bush" Sweet Peas (R.E.C. 22).

The relation of these two types to each other is not altogether simple. As described (q.v.) $F_{1}$ from Cupid $\times$ Bush is a reversionary form exactly like the normal tall variety. Neither the tall varieties nor the Cupids show the profuse branching of the Bush Sweet Peas which gives them their peculiar appearance. This is evidently recessive to the unbranched condition, and the fact thus stands out in contrast to those observed in the case of Sunflower and Cotton. But in the Sweet Pea we have the additional complication that the factor which represses the excessive branching by its presence gives increase of height. The tall and the Bush differ from each other in respect of this factor only. It is present in the tall but absent from the Bush. In the cross between Bush and Cupid two pairs of factors are concerned as explained in the passage referred to.

4. Hairiness and glabrousness. Lychnis. Matthiola (Stocks). Wheat.

The case of Lychnis has been studied by de Vries (288) and R.E.C. (19). In crosses between fully hairy and glabrous strains the discontinuity is complete. Various forms intermediate in hairiness may nevertheless be found wild and are by no means rare. Silene inflata 
often exists in two forms, hairy and glabrous, growing side by side, and doubtless their genetic relations are the same as those found for the corresponding varieties of Lychnis. In this species a third form is found with hairs on the edges only (1 2 ).

The case of Matthiola is important and presents features of special interest, R.E.C. ( $19,20,2 \mathrm{r}$, see also Correns, 6r). Between thoroughly hoary and glabrous strains the discontinuity is absolute, and the glabrous
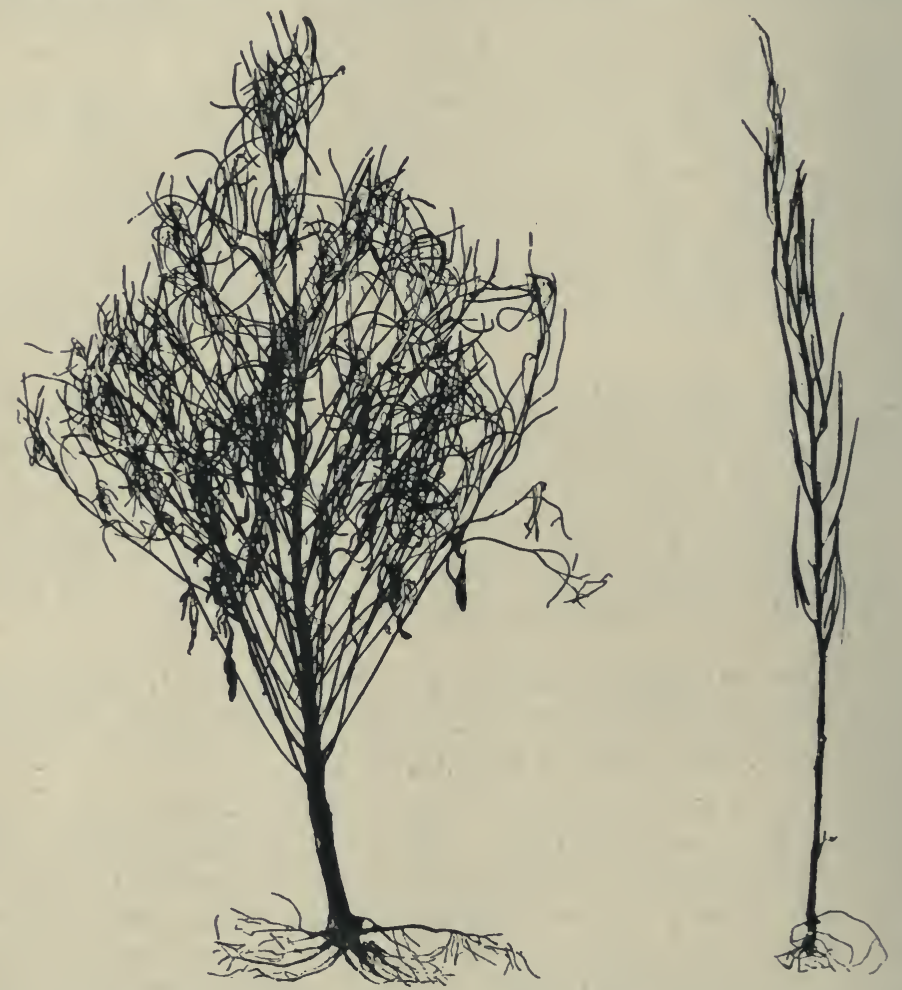

Fig. 4. Matthiola. Branched and unbranched forms in $F_{2}$. A photograph of Miss Saunders' plants, the leaves removed. (Supplied by Miss Killby.)

are entirely destitute of hairs. The dominance is complete and homozygotes cannot be distinguished from heterozygotes. A third, or "half hoary" form exists, which is glabrous or nearly so on the upper surface only. Its behaviour has not been fully investigated ( 19, p. 33.)

The genetics of hairiness in wheat have been studied by Spillman (247), T'schermak (270), Biffen (27). The heterozygotes are sometimes intermediate in hairiness.

The Peach and the Nectarine are probably related to each other as hairy dominant and glabrous recessive.

Peculiar results are recorded in Cotton (Balls, 6). 
5. Prickliness and smoothness of fruits. Datura. (R.E.C. 19, 20.) Ranunculus arvensis (20).

The case of Datura is interesting from the fact that it sometimes has mosaic fruits, one quarter or one half being prickly and the rest smooth. This is perhaps to be regarded as indicative of segregation occurring among zygotic cells (see Chap. xv.).

Ranunculus arvensis has three types, spiny, tuberculated, and smooth. The first is a simple dominant. Tuberculated $\times$ smooth gave $F_{1}$ partially spiny (2 I, P. 55).

6. Absence of glands (Matthiola incana) on leaves was dominant to presence of glands (M. sinuata) (R.E.C. 2O, p. 40).

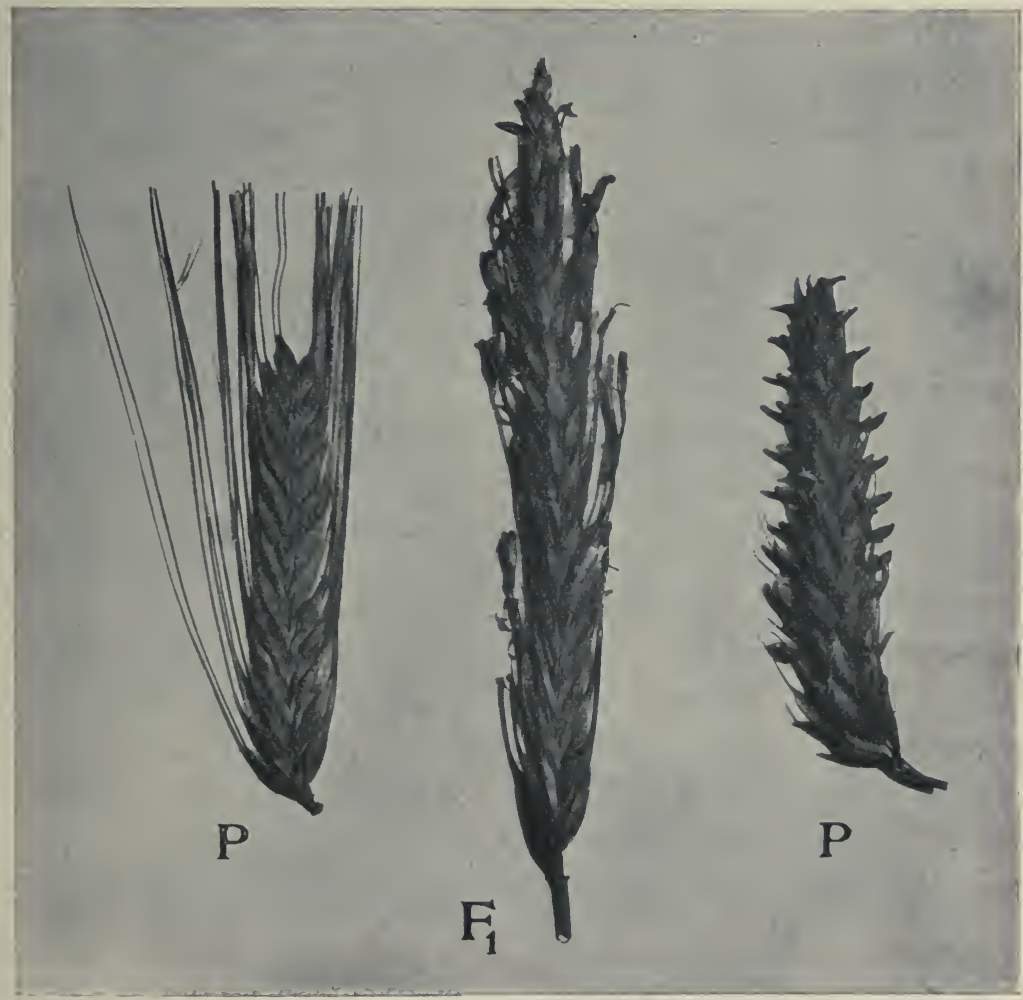

Fig. 5. Cross between a normally awned Barley and a variety with "hooded" awns. $P, P$, the parents. $F_{1}$ shows partial dominance of hoods. The increase in length of ear is noticeable. The case also illustrates the result of crossing a 2-row type with a 6-row type, showing dominance of the former. (From Professor Biffen's specimens,) 
7. Rough and smooth foliage. Wheat. Biffen (27).

8. Keeled glumes and rounded glumes. Wheat. Ibid.

9. Beardless and bearded ears. Wheat. Ibid. Also Spillman (247) and Tschermak (270).

Most, if not all, of the "beardless" varieties exhibit a slight and variable amount of awn especially on the uppermost spikelets (Fig. 6).

I0. The "hoods" or "Kapuzen" characteristic of certain Barleys show a partial dominance over the normal type. These hoods, Professor Biffen states, are, structurally, aborted florets (Fig. 5). Tschermak (270), Biffen (30).

I I. Hollow and solid straw. Wheat. Biffen (27).

This is a structural character of an interesting kind, and one upon which the commercial value of straw very largely depends. It was shown that many factors were concerned in the production of the stem-characters; and in $F_{2}$ by the recombination of these factors a great variety of straws appeared.

I 2. Blunt and pointed pods. Pisum. Tschermak (271), R.E.C. (20). Phaseolus. Tschermak (272).

The dominance in this case is complete. Some varieties exist in both a blunt and a pointed type (e.g. Sutton's Continuity). The nature of these cases is discussed later.

13. Lax and dense ears of Wheat and Barley give different results according to the varieties used. Sometimes $F_{1}$ is lax, sometimes it is intermediate (Spillman, 247; Biffen, 27, 28). See Fig. 6 . In Barley an increase in earlength has been observed (Fig. 5).

I4. Development of fibrous parchment-like lining to pods, and the absence of the same which constitutes the "sugar peas." Pisum. In Phaseolus (kidney-beans), where similar types occur, the evidence is that the dominance is reversed (Emerson, I 20, I 21 ).

This is one of the features originally investigated by Mendel. He regarded the parchmented type as a dominant. In our experiments $F_{1}$ has always had some parchment but the quantity is so much reduced as to cause the heterozygote to have a very distinct appearance (R.E.C. 20).

I 5. Much serrated and little serrated edges of leaves. Urtica (cp. Phyteuma, Correns, 70, p. 197). This cross 
was described by Correns (77) who gives a striking diagram representing his results. The cross was made between two forms known as Dodartii and pilulifera, which were

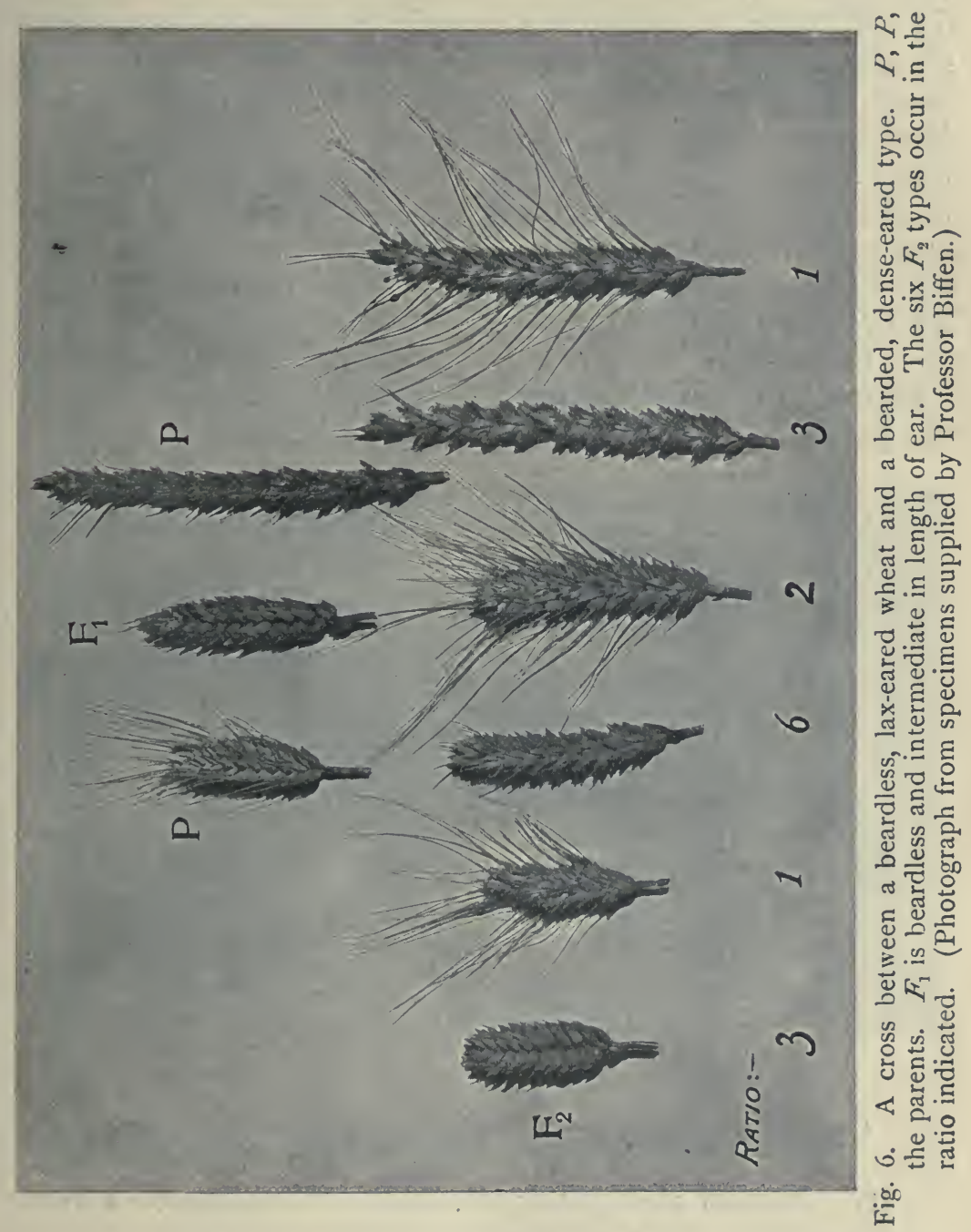

regarded by Linnaeus as distinct species. The almost entire-leaved Dodartıi has been treated by later authors as a variety of pilulifera. 
16. Palmatifid or "palm-leaf" and pinnatifid or "fern-leaf." Primula Sinensis (Fig. 7).

The fern-leaved form arose in English horticulture about 1860 as a variation from the normal type. I have had opportunities of seeing its genetic behaviour on a large scale at Messrs Sutton's, and many experiments have been made with it by $\mathrm{Mr}$ R. P. Gregory in conjunction with me. Dominance is usually complete, but at Messrs Sutton's I have seen on two occasions strains containing plants of intermediate leaf-shape, which were presumably heterozygous, for the two types occurred on sister-plants. The leaf-shape is entirely independent of the colours and other features of the plant, and can be transferred bodily from one colour-type to another. Messrs Sutton's varieties "Mont Blanc" and "Sirdar," for example, are sold both in the palm-leaved and in the fern-leaved forms.

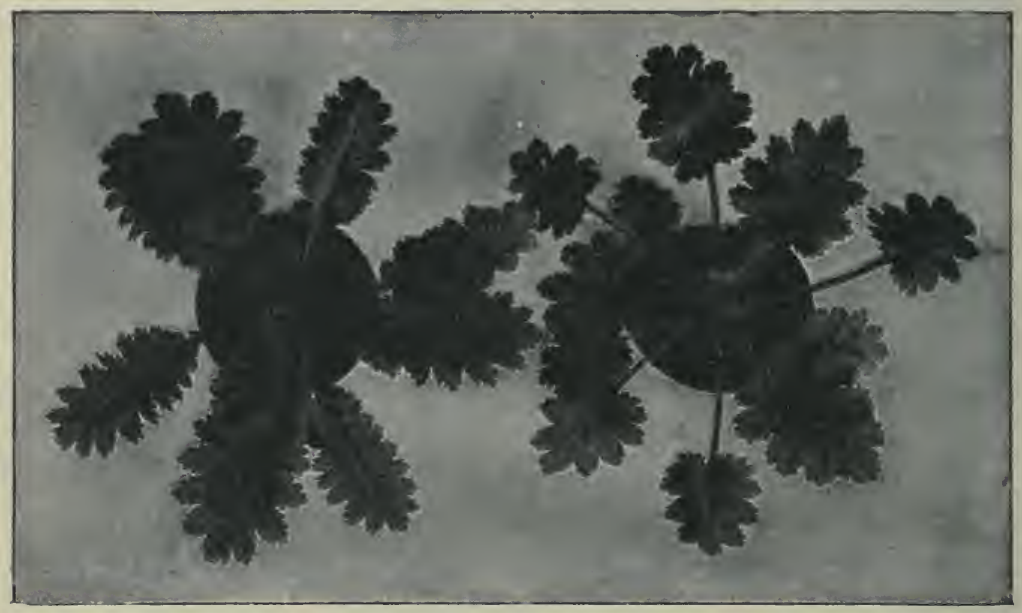

Fern-leaf $(R)$

Palm-leaf $(D)$

'Fig. 7. The two types of leaf found in Primula Sinensis.

17. Leaves and petals normal or laciniated. Chelidonium majus. de Vries (290) and (298), I. p. I34.

This case is interesting in comparison with No. 15. In the Nettle, serration is a dominant, while here laciniation is a recessive. A careful study of the physiological distinction between the two processes would probably lead to important results (cp. Leake, I7O, on leaves of Cotton). 
18. Certain leaf characters in Capsella bursa-pastoris. Shepherd's Purse. (Unpublished work of Shull ; about to appear as a publication of the Carnegie Institution.)

I 9. Various characters in the seed of Cotton. Balls (6).

Many of these are of great commercial importance. Balls (6) gives the following list :

Dominant.

Long staple.

Regular distribution.

Coloured lint.

Silky lint.

More fuzz.
Recessive.

Short staple.

Irregular distribution.

White lint.

Harsh lint.

Less fuzz.

He says that all the desirable characters are dominant, and that hence the chances of picking out a stable form by common selection are very small. Individual selection must be adopted.

20. Biennial habit and annual habit. Hyoscyamus. Correns (73).

More research on the relations of annuals to biennials is greatly to be desired. Points of the highest physiological interest are involved. In connection with root crops also some questions of commercial importance are raised. In R.E.C. (19, p. I35) I ventured to suggest that the persistence of "runners" which go to seed in such plants as Beet and Mangel may be due to want of individual selection of pure dominants, and in view of Correns' observation the probability of this suggestion is increased.

21. Normal stem and fasciated stem associated with peculiar distribution of inflorescences. Peas (Pisum). Mendel (195), R.E.C. (20). In our experience various intermediates occur in $F_{2}$.

22. Susceptibility to rust-disease (Puccinia glumarum) and resistance to the same. Wheat. Biffen $(27,29)$.

This is perhaps one of the most important instances to which Mendelian method has yet been applied. Using a variety very susceptible to rust and another practically immune to its attacks Biffen found that $F_{1}$ was not perceptibly less attacked than the rusty type. $F_{2}$ showed ordinary segregation, and the green, resistant plants, standing among the yellow rusty ones, formed a very striking spectacle. The recessives bred true and their progeny has remained rust-proof. It has not yet been shown to what the resistance is due. Working with Professor Biffen, Miss Marryat (193) found that the rust-hyphae are checked after entering the stomata of the resistant plants. If, as may be suspected, the resistance is due to the presence of some anti-toxin, the dominance of "susceptibility" must be taken to indicate that the formation of the anti-toxin is prevented by the presence of a factor in the dominant forms, a conclusion which may lead to definite progress in the physiology of disease-resistance. 
23. Flat standard and hooded standard in the flower of Sweet Pea. R.E.C. (22). See Plate V. Some very curious phenomena have been observed in this case, which are described in Chap. Ix. The type known as "Snapdragon," perhaps an extreme form of hood, is also a recessive to the flat type. R.E.C. $(20$, p. 83$)$.

24. Imbricated petals and stellate or "star"-type. Primula Sinensis. Observations made at Messrs Sutton's and experiments of R. P. Gregory with W. Bateson. (See Fig. I4.)

25. The monstrous condition of the calyx in which it resembles the corolla, seen in "hose-in-hose" Campanula is an imperfect dominant to the normal. Associated with this homoeotic variation, the female organs are more or less completely sterile in certain strains. Correns (76). This subject is discussed in Chap. XI.

26. Abortion of the female organs in the lateral florets of Barley, as found in the 2-rowed types, and the complete or hermaphrodite development of the florets, as in the 6-rowed types (Fig. 8). Tschermak (270) and (275), p. I I. Biffen (30).

This case is somewhat complex. There are three types. (I) Six-row, in which 3 perfect hermaphrodite florets are developed in each spikelet. All set seed and the result is that the ear has 6 rows of seeds. (2) Types in which the lateral florets have anthers but no female organs. (3) The "Abyssinian" type in which the lateral florets contain neither male nor female organs. The types (2) and (3), being able to make seeds only in the central florets of each spikelet, alike develop two rows of seeds. When (I) is crossed with (3), $F_{1}$ is like (2); and (2) crossed with (3) gives $F_{1}$ also like (2). Some further complexities have been observed, but in general it appears that the dominant factor has the power of partially preventing the formation of the reproductive organs in the lateral florets. The facts may perhaps be interpreted as bearing on the phenomenon of Sex.

Tschermak (275) describes crosses between a 2-row and a "4-row" type. From Professor Biffen I understand that the latter is in reality a lax-eared 6-row type. $F_{1}$ is 2-row, and in $F_{2}$ the ratio is 12 2-row: 3 "4-row": I 6-row. This is a special case of the ratio $9: 3: 3: 1$, laxear and 2-row being dominants. Tschermak and Shull (242) regard it as an illustration of the effects of a latent factor. 
27. The two-celled type of fruit is dominant to the many-celled type in Tomato. Price and Drinkard (22 I).

The case is one of the few in which the genetic behaviour of a meristic or divisional feature has been investigated apart from any complexity introduced by differentiation.

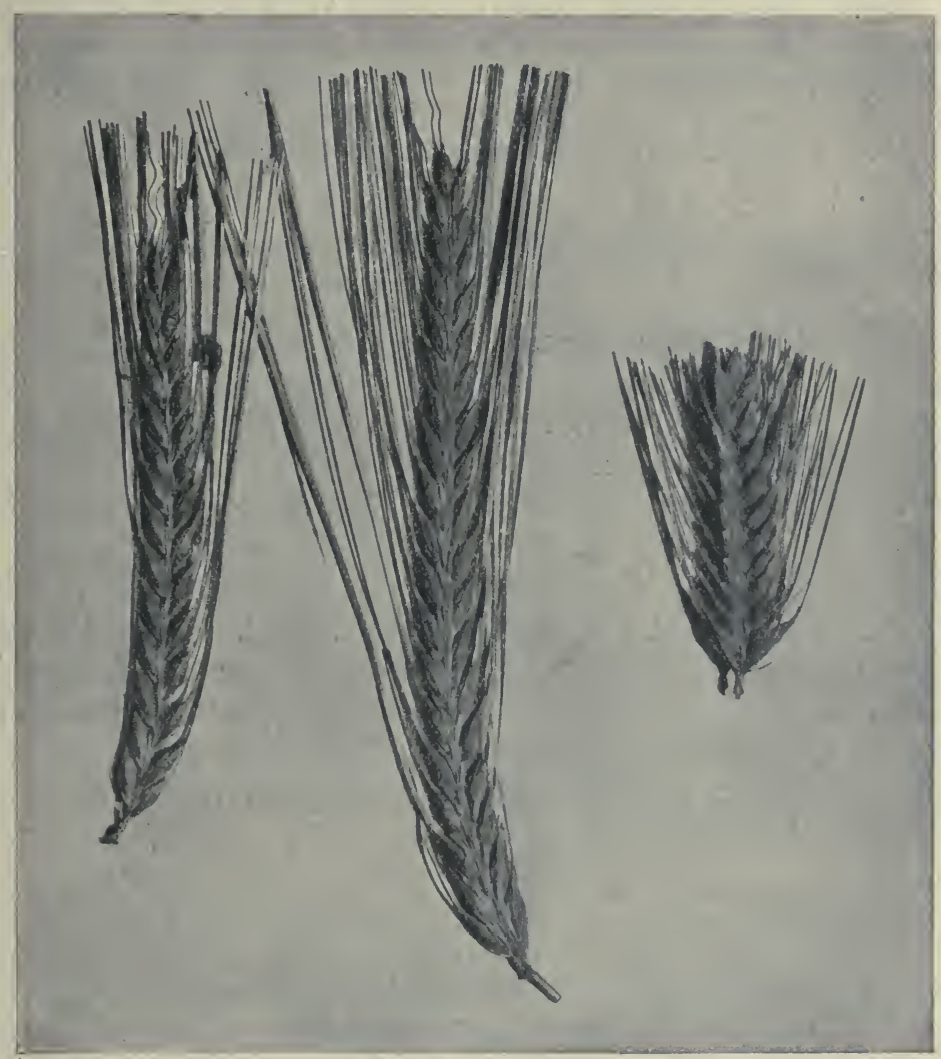

Fig. 8. Cross between Abyssinian 2-row Barley and a club-headed 6-row type. The middle figure shows $F_{1}$. The length of ear is increased. (From Professor Biffen's specimens.)

28. Style short, associated with large pollen grains, constituting the "thrum" type, and style long, associated with small pollen grains constituting the "pin-eyed" type. Primula Sinensis and acaulis. Bateson and Gregory (I7).

The short styled type has been found in the homozygous condition in $P$. Sinensis but not yet in $P$. acaulis. For the relations of these types to the "homostyled" form, see p. 68. Dominance is complete. 
29. Long style and short style in Oenothera. (This difference is probably quite distinct in nature from ordinary heterostylism as seen in Primula, \&c.) de Vries (290). The same fact has been observed by Balls (6) in Cotton.

30. Normal long pollen grains with three pores, and rounded pollen grains usually with two pores. Sweet Pea (Lathyrus odoratus). R.E.C. (20, 21, 22). See Fig. 19.

3I. Normal anthers and sterile anthers. Sweet Pea. R.E.C. $(20,21,22)$.

With regard to these two last features numerous complications occur, which are described in later chapters.

32. Roundness of seed connected with the presence of starch in large elongated simple grains, and wrinkledness of seed connected with the presence of peculiar compound starch-grains. Pisum.

This is one of the most familiar of Mendel's original examples (see Fig. 3). It has been re-investigated by many observers. Correns (60); Tschermak (269, \&c.); R.E.C. (20); Hurst (155); Lock (172, I 73). The $F_{1}$ seeds made by fertilising an emasculated flower of a wrinkled variety with pollen from a round variety, or vice versa, are generally ordinary round seeds, and $F_{2}$ shows the common ratio 3 round : I wrinkled, the two types being mixed in the pods of the $F_{1}$ plants.

Among the multitude of varieties of peas now cultivated there is a great diversity both of rounds and of wrinkleds. The interrelations of these several types, even as regards seed-shape, have as yet been imperfectly explored. The degree to which the wrinkles are formed is fairly uniform for any one type, but the various types show different degrees of wrinkling. The differences obviously depend chiefly on the chemical and physical properties of the reserve-materials in the cotyledons, and an analysis of these peculiarities might lead to further discoveries.

Gregory (I34) found that the starch in round peas occurs chiefly as large elongated simple grains, whereas in wrinkled peas it is in the form of small grains of irregular shape which are often compounded together (Fig. 9). Darbishire (94) added the interesting fact that in $F_{1}$ the grains are intermediate, many being large and simple, but round instead of elongated, with an admixture of compound grains. He confirmed also Denaiffe's observation* that wrinkled take up more water than round, but he found that $F_{1}$ is intermediate in this respect, and he suggests that the size, the

* Denaiffe, Les Pois poiagers, p. 9. 
shape of the grains, and their simple or compound nature, may be governed by distinct factors. He regards the absorptive power as again separable from these features.

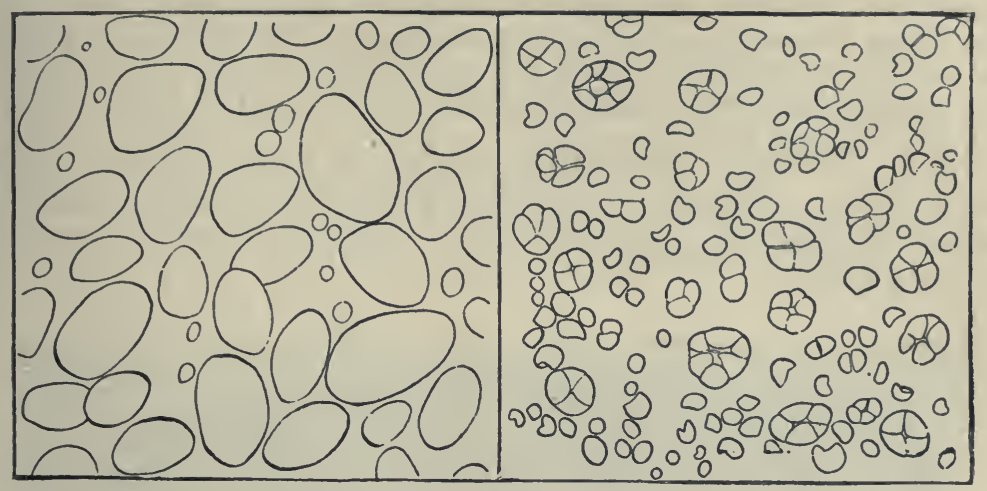

Round and Indent.

Wrinkled.

Fig. 9. Outlines of starch grains in the different types of peas. The wrinkled contains many compound grains. (Fron Gregory.) Magnification the same in both figures.

A third type of pea, of which the purple sugar-peas (sans parchemin) are a good instance, may be described as "indent." These also have large, simple starch grains. Such seeds are of irregular flattened shape and may be confounded with true wrinkled peas. Their properties are entirely different, and the two sorts must be carefully distinguished. One of their special properties will be discussed in a later chapter, but here it must suffice to say that their genetic properties are essentially those of round peas. Much confusion has been introduced by want of care in distinguishing these types.

Intermediates, which on casual sorting, cannot be classed either as round or wrinkled, sometimes occur. Some of the round types (e.g. Victoria Marrow) contain a large proportion of such seeds. Their peculiarity is almost certainly due to environmental influence, though obviously the liability to this affection may be transmitted. When such seeds are found in $F_{2}$ from a cross between thoroughly round and wrinkled varieties, the pitting, when it exists, generally affects all the round seeds of the pods in which it occurs. With experience such pitting can immediately be recognized as distinct from the true genetic wrinkling, and in our experiments the results of a further sowing have repeatedly confirmed the judgment made by inspection of the seeds.

A complete account of all the phenomena would run to great length. The interrelations of round and wrinkled seeds are to be recommended as offering perhaps the most favourable example for an investigation of the chemical nature of a genetic factor. The wrinkling is evidently the consequence of a particular method of drying, and this must depend on the nature of the reserve-materials. A first step would be to determine the relative amounts of sugar and starch in the two chief types. It is natural 
to suppose that the wrinkled peas are those in which the transformation of sugar into starch has gone less far than in the round peas; but, as much starch is formed in the wrinkleds, one ferment having this transformative power must be present in them. Hence we are led to suppose that in the round pea a second ferment is present which can carry the process further. As offering an attractive problem in physiological chemistry the phenomena are recommended to those who have the requisite skill to investigate them.

33. Starchy endosperm giving a full, rounded seed, and sugary endosperm giving a shrivelled and wrinkled seed. Maize. Fig. Io. de Vries (290); Correns (63); Lock (172, I 74).

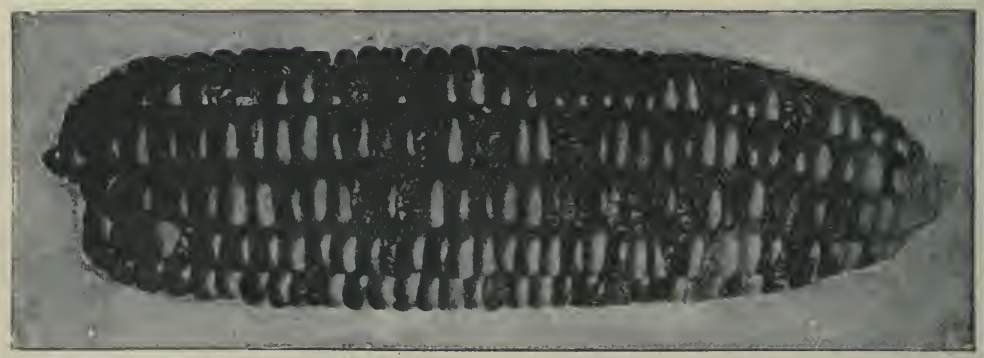

Fig. I0. A cob of Maize (Zea mays) borne by an $F_{1}$ plant from the cross round $\times$ wrinkled, fertilised with its own pollen, showing the mixture of round (dominant) and wrinkled (recessive) seeds. (From a specimen given by Dr Webber.)

Of the various Mendelian experiments this is one of the most demonstrative. Dominance is perfect so far as external observation goes. Correns records a remarkable excess of round seeds as recurring with great constancy in certain families when $F_{1}$ is self-fertilised (see later).

It often happens that pollen from one variety of maize is blown by the wind to the stigmas of another variety. If this pollen possesses a dominant factor capable of affecting the seed, seeds exhibiting it are formed. If for instance pollen from a round maize is blown on to a wrinkled or sugar-corn, round seeds will be formed among the normally wrinkled seeds. When formerly it was supposed that the endosperm, which contains the reservematerials, was a maternal structure, the change in the seed was regarded as an influence exerted by the embryo on the maternal tissues. The effects of such influences were called by Focke "Xenia." There are a few examples of such influence which may. with probability be regarded as genuine*; but since the discovery of the fact that the endosperm of maize results from a double fertilisation effected by the second nucleus of the

* The phenomena are discussed by Darwin, An. and Plts., ed. II. I885, I. pp. 428-433. It seems likely that in some of these instances the factor introduced by the pollen-grain can influence or infect tissues in contact with the embryo. 
pollen-tube, cases like that of maize are not strictly to be classed as Xenia (see Correns, 58).

34. Glutenous and starchy endosperms. Wheat. Biffen

Professor Biffen's researches respecting these important features are not yet completed. The glutenous, translucent, hard type has definite dominance over the opaque, soft, starchy type.

35. Single flowers usually behave as dominants to doubles, as in Stocks, Primula, \&c. In Carnations the doubleness dominates.

The most extensive researches on the genetics of doubleness are those of Miss Saunders in the case of Stocks (Matthiola), R.E.C. (20-23). The peculiar phenomena discovered are discussed in a separate chapter (q.v.).

36. In Phaseolus hypo-geal cotyledons are dominant to epi-geal. Various intermediates in $F_{2}$. Tschermak (278, p. 54).

This list and that which follows make no pretension to completeness. Those features are enumerated which either seem of special interest, or have been studied with some thoroughness. Indications respecting many more are to be found in the original papers (see especially for Peas and Phaseolus the writings of Tschermak and Lock; for Cotton, Balls; for Oenothera, \&c., de Vries, and Macdougal (186); for Wheat and Barley, Biffen, and Tschermak; for Maize, Correns, and Lock; for various plants, Correns, and de Vries).

In the genus Brassica numerous crosses have been studied by Sutton (262). In his experiments it was found, among other important results, that the bulbing of the Swede, Turnip, and Kohl Rabi disappeared completely in crosses with non-bulbing Kales, and that in $F_{2}$ imperfect bulbing reappeared. Professor Biffen, who is continuing work on the same lines, tells me that in regard to these and similar characters cultural conditions play a great part, and lead to curious and conflicting results. 


\section{Animals. Structural Characters.}

MAN.

A considerable number of diseases and malformations have been shown to behave usually as dominants. A few conditions may be said, more doubtfully, to behave as recessives. The subject of human inheritance is discussed in Chap. xIr. Of normal characteristics, eye-colour is the only one yet studied (Hurst, I6I)* sufficiently to justify a positive statement as to the existence of a Mendelian system of descent.

\section{CatTle.}

37. Absence of horns in polled breeds of Cattle is dominant to the presence of horns (R.E.C. 19; Spillman, 246).

In sheep the inheritance of horns is sex-limited (q.v.), and from evidence given me by Mr E. P. Boys-Smith I suspect that this is true in the case of Goats also.

\section{HORSE.}

38. There is little doubt that the gait known as "pacing" is recessive to the ordinary trotting gait in the American trotters. Trotters bred together may produce pacers, but hitherto I have found no authentic instance of genuine natural pacers, when mated together, producing trotters. Correspondents have sent me word of several apparent exceptions to this rule, but all on inquiry have proved to be erroneous. In the pacing gait the two legs of the same side of the body are moved together or nearly so, while in trotting the foreleg of one side moves almost with the hind leg of the other. Horses may be trained with more or less success to adopt either gait, but the distinction between natural pacers and natural trotters is a fairly sharp one (I6). The physiological nature of the difference is quite obscure, but presumably it is of nervous origin.

\section{Mouse.}

39. From time to time mice are found hairless, with the skin thrown up into corrugated folds. Experimenting with such mice Mr Archibald Campbell found the condition to

\footnotetext{
* See also Davenport (107).
} 
be a recessive, the presence of normal fur being a dominant. The fur grows at first normally and falls off as maturity is reached. Of $\mathrm{I}_{2} F_{2}$ mice 3 lost their hair. I am indebted to $\mathrm{Mr}$ Campbell for information respecting this interesting case, and for living specimens. The attempt to breed the recessives together failed, but in Gaskoin's case* naked parents produced young like themselves. From his account it appears that the young which he observed never grew their hair, but the fact is not absolutely certain from the description. [See also Campbell, Zoologist, r 907, p. I, P1.]

40. The normal condition and the "waltzing" habit in Japanese mice. The waltzers exhibit a peculiar vertiginous movement of the head when they come out into the light, and spin often with extreme rapidity, running after their tails till apparently exhausted.

Our knowledge of this case is derived from Von Guaita (I35) and Darbishire (90). The dominance of the normal type is complete, and in $F_{2}$ waltzers reappear. The $F_{2}$ numbers obtained by Darbishire were $45^{8}$ normals, 97 waltzers, where the expectation is 4 I 6 normals, I 39 waltzers. The deficiency may perhaps indicate a complication, but more probably it is due to the greater delicacy of the abnormal mice, which was so great that all attempts to breed them together were unsuccessful.

\section{Rabbit, Guinea-Pig.}

4I. Normal short hair and the long "Angora" hair Rabbit, Guinea-pig, and doubtless Cat (see Hurst, 157 ; Castle, 45 and 48; Sollas, unpublished; Castle and Forbes, 55).

Castle (48), p. 64, gives important details as to the physiological nature of the distinction between the normal and "Angora" hair, which he regards as resulting from a special method of growth.

42. The rough or rosetted condition of the coat in the Guinea-pig dominates over the normally smooth condition (Castle, 48 ; Sollas, unpublished).

Castle found occasionally that animals partially rosetted occurred in $F_{2}$.

* For references see Bateson, Materials for Study of Variation, I894. p. 56. A good figure is given by Gaskoin, Proc. Zool. Soc. 1856 .

B. $\mathrm{H}$. 
43. Polydactylism occurred in a Guinea-pig, the offspring of normal parents, and ran an irregular course in its subsequent descent (Castle, 49).

CAT.

44. The abbreviated tail of the Manx Cat is a dominant (more or less imperfect) to the normal tail (see Anthony, 2 ; Hind, I 5 I ; Davenport, 98; Kennel, I66,a). Godron (Mém. Ac. Stanislas, 1865) records a similar observation in the Dog.

45. Polydactylism is almost certainly dominant; but, as in other types, irregularities doubtless occur.

Fowls.

For the study of heredity Fowls are especially well suited. In addition to their many colour-characteristics the various breeds present a great range and variety of structural features.

Among the long series of offspring which hens of the more fertile breeds produce, the descent of these characteristics can be watched in families of ample length. The chief papers dealing with Fowls are R.E.C. (19-22); Hurst (I56); Davenport (IOI). The following is a list of the principal facts already elicited as to the behaviour of these structural features but much remains to be done.

46. Various shapes of comb, for example the rose comb and the pea comb, are both dominant to the single comb. The double or longitudinally split condition is also dominant to the unsplit.

See pp. 6r-7. Many of the finer details in regard to the heredity of comb-shapes are not yet clear. The classification of the comb-types in the newly-hatched chickens is generally very easy, but in occasional strains forms intermediate between the pea and the single occur in $F_{2}$, which may probably be due to subtraction-stages of the pea factor $(q . v$.$) . Some of the$ singles extracted in $F_{2}$ from various crosses have lateral "sprigs"-as fanciers say. It is not impossible that these irregular processes are due to additional minor factors, but they are subject to so much fluctuation that their descent would be very difficult to trace. The comb of the Silky fowl is a rose, $+a$ trifid element which causes its posterior end to be divided into three irregular points. In $F_{2}$ from Silky $\times$ Single, regular rose combs are produced in those individuals which have the rose factor without this trifid element.

Attention may be called to the dominance of the median splitting of the comb found in certain breeds, for the facts may have a bearing on the genetics of meristic characters. Splitting of the comb may occur in one of 
several distinct ways. It may affect mainly the anterior portion, or the posterior. The split combs of established breeds have possessed ordinary dominance; but a form of posterior splitting somewhat like that of the $F_{1}$ from Breda $\times$ Single occurred apparently as a mutation among extracted singles, and exhibited a curious genetic behaviour suggesting irregularity of dominance (20, pp. 108 and $\mathrm{II}_{3}$ ).

47. The normally webbed feathers are dominant to the peculiar feathers of the Silky fowl.

48. Extra toe is usually dominant to the normal fourtoed condition, but exceptions occur.

This irregularity of dominance is exhibited by all cases of polydactylism yet studied in birds or mammals. It seems to be a property of certain strains. Some families run a perfectly regular Mendelian course, others contain members with only the normal four toes, which are yet capable of transmitting the extra toe. The numbers in such families are not favourable to the suggestion that the irregularity is caused by a definite disturbing factor.

49. Crest is dominant to no crest.

$F_{2}$ may contain individuals with crests far larger than those of the parent crested breed, a fact which suggests that in breeds with small crests (e.g. Silky) the full development of the crest is kept in check by some other factor.

50. Feathered leg partially dominates over clean leg.

Both Hurst $\left(\mathrm{r}_{5} 6\right)$ and Davenport found dominance very irregular. $F_{1}$ is intermediate, and traces of leg-feathering are occasionally seen in the offspring of clean-legged birds.

51. "Frizzling," or turning back of the feathers, is dominant to the plain straight feathers of the normal.

52. Normal size of feathers on the hocks, or tibio-tarsal region, is dominant to elongation of these feathers to form quills - the "Vulture-hock" of fanciers.

53. Muff, or tuft of feathers at sides of the bill and throat, as in Faverolles, is dominant to no muff, as in ordinary breeds.

54. Imperfect development of coccyx and tail-feathers with absence of tail, as in "Rumpless" fowls, is dominant to the normal development of those parts. Davenport (IOI) and Amer. Nat. XLIV. p. I 34.

The case is exactly comparable with that of the Manx Cat. Davenport's later paper shows that this is the right interpretation of the facts. It is also in harmony with the observation given by Darwin in An. and Plts. ed. 2, II. p. 4 . 
55. Certain breeds (Houdan, Polish, Breda) have an extraordinary development of the nostril, which is patulous, with alae horizontal instead of curving downwards. This peculiarity is recessive to the normal (Davenport, IOI). Hurst has observed the same thing and Mr Punnett and I have similar evidence from the Breda. Davenport states that in his experience the "high" nostril is never combined with a fully developed comb.

56. The tendency to go broody and sit on eggs dominates over the absence of this instinct, characteristic of several Mediterranean breeds. There is probably segregation in regard to these two dispositions, but this cannot yet be asserted positively.

In regard to fertility as measured by egg-production there is as yet no clear evidence.

57. The loud and penetrating shrieks which the cocks (and to a less degree the hens) of an Egyptian breed give out when caught, were reproduced almost exactly by the $F_{1}$ generation from a cross with a non-shrieking breed. Though numerical data in regard to such a character are scarcely attainable, there is little doubt of the segregation as evidenced by $F_{2}$.

\section{Pigeons.}

58. The normal foot is dominant to the webbed condition of the toes which sometimes occurs as an abnormality (Staples-Browne, 254).

Mr J. L. Bonhote tells me that in his experiments webbed birds have produced normal offspring. He is making further experiments with this family.

59. The "shell," or turning-back of the head-feathers of the Nun is dominant to the normal plain head (ibid.).

6o. Birds with normal, I 2 -feathered tails crossed with the many-feathered Fantail give intermediate numbers in $F_{1}$. In $F_{2}$ I 2 -feathered tails reappear, but, so far, no real Fan has come from the cross-breds. $\mathrm{Mr}$ Staples-Browne, to whom I am indebted for this information, will publish a complete account of his evidence. He tells me that the extracted I 2 -feathered birds do not breed true, but may throw birds with 13 or 14 feathers. 


\section{Canaries.}

6I. Crest is dominant to plain-head, as the non-crested condition is called by fanciers (R.E.C. I9, p. I 3 I ; Davenport, I05).

The type of crest which fanciers admire consists of feathers neatly laid down over the head. To produce such birds crested individuals are bred with plain-heads, and it is clear that the exhibition type of crest is a heterozygous form. When crested birds are bred together it is said that an ugly, standing crest frequently is produced, and presumably this is the homozygous type of crest. The mating of two crested parents is by several authors said to give rise to some bald birds. Other writers (e.g. Blakston) have ridiculed this statement, and formerly I was inclined to regard it as a mere exaggeration, but Davenport in his recent paper mentions bald heads as sometimes occurring among his crested birds. He has kindly supplemented his published account with the statement that the bald patch is an area "on the back of the head varying from four to six millimetres in diameter practically without feathers and remaining featherless throughout life. The crest, however, on top of the skull remains perfectly evident, and often baldness can only be detected by blowing the feathers." In no case was such a bald patch found in a plain-head.

The bald patch on the occiput is recognized by Blakston (Cage Birds, p. 104) as a property of crested birds, and presumably the "balds" alleged to come from the mating of two crests are birds homozygous for crestfactor, in which the crest stands up and allows the bald patch to be seen. Davenport had a crested bird without any bare patch, and he found that the feathering in this region was due to a separate dominant factor.

\section{Animals and Plants in which Colour-Characters have been shown to have a Mendelian Inheritance.}

The phenomena of colour-inheritance are complicated in several ways. Some of these complications which are of great importance and interest will be considered in subsequent chapters. It is, however, convenient to enumerate the genera in which Mendelian heredity has been observed in order to illustrate the scope of the principle. The following list of genera contains the chief of those in which heredity according to a Mendelian system has been shown to occur. In some of them as the result of extensive research many Mendelian features of colour have been discovered, and the existence of numerous colourfactors is demonstrated. In others only one such factor for colour has been detected. 


\section{Plants.}

Antirrhinum (Snapdragon).

Atropa.

Brassica (Turnips and Swedes).

Clarkia.

Coreopsis.

Datura.

Gossypium (Cotton).

Helianthus (Sunflower).

Hordeum (Barley).

Hyoscyamus (Henbane).

Lathyrus (Sweet Pea).

Lychnis.

Matthiola (Stocks).

Mirabilis.
Orchids (several genera).

Papaver.

Phaseolus.

Phyteuma.

Pisum.

Polemonium.

Primula.

Salvia.

Solanum (Tomato).

Triticum (Wheat).

Verbascum (Mullein).

Viola.

Zea (Maize, Indian Corn).

\section{Animals.}

Man.

Mice.

Rats.

Rabbits.

Guinea-pigs.

Horse.

Pigs.

Sheep.

Cattle.

Cats.
Fowls.

Pigeons.

Canaries.

Axolotl.

Lepidoptera, various (Silkworm ; $A$ braxas grossulariata; Angerona prunaria, \&c.).

Coleoptera (Lina; Leptinotarsa ; Crioceris).

Helix.

For the convenience of readers acquainted with the phenomena in outline and desirous of pursuing the subject further the following brief annotations are placed here. Until the chemistry of pigmentation is better understood, a comparison between the behaviour and properties of the several types cannot be instituted with much confidence.

Antirrhinum. Wheldale $(3 \circ 3)$ has shown that the lowest or hypostatic factor dominant" to albino gives yellow in the "lips" of the flower; the addition of various other factors produces anthocyan reds which superposed on the yellow give deep crimson red colour. A second series of reds, more purplish or magenta in tint (colour of wild $A$. majus), results from addition of a factor which in absence of anthocyans gives an ivory colour. This ivory is epistatic to yellow. It is remarkable that the lowest anthocyan factor gives red in the tube with a tinge in the lips, while the addition of the next above it gives the self-coloured flower.

There is also a white-tubed type of each colour-combination ("Delila" of de Vries, 298).

All the factors except that for yellow and ivory can be carried by the albino. Among the reds several heterozygous combinations can be recog. nized. The heredity of striping is still under investigation.

Atropa Belladonna. The normal dark-fruited type is dominant to yellow-fruited (de Vries, 290 ; Saunders, 19). 
Brassica. White chromoplasts dominant to yellow in Swedes and Turnips (Sutton, 262).

Clarkia elegans. Common magenta-red dominant to salmon pink (Bateson and Punnett).

Coreopsis tinctoria. Ordinary yellow type dominant to var. brunnea with brown flowers (de Vries, 290). The brown flowers like those of Cheiranthus (Wall-flower) are no doubt due to presence of much dark anthocyan, and the case is probably one in which the development of little anthocyan dominates over the development of much anthocyan (cp. Lathyrus, Primula, \&c.).

Datura. Purple in flower or stem dominant to white flower and green stem (de Vries, 290; Saunders, I9).

Gossypium (Cotton). Dominance of many colour-characters in plant, flower, and seed (Balls, 6). $\quad F_{2}$ details not yet published.

Helianthus. Purple disk dominant to yellow disk (Shull, 24r).

Hordeum (Barley). Black pigment in paleae dominant to its absence (Tschermak, 270 ; Biffen, 30).

Hyoscyamus niger annuus $\times H$. niger pallidus were found by Correns (69) to give $F_{1}$ flowers of intermediate tint.

Lathyrus (Sweet Pea). Anthocyan colours dominant. Purples dominant to reds. Colour depends on two complementary factors. Yellow chromoplasts recessive to colourless. Facts fully described in later chapters. Plants with coloured flowers have dark seed-coats. Whites have seed-coats colourless.

Lychnis. $\quad F_{1}$ between $L$. diurna and $L$. vespertina has flowers of intermediate tint ranging through many grades (see de Vries, 290; Correns, 69; R.E.C. 19). Segregation imperfectly studied.

Matthiola (Stocks). Colours as in Sweet Pea (R.E.C. I9-2I ; Tschermak, 278; Correns, 6r). For colours of seeds see R.E.C. I9.

Mirabilis. Colours consist of a complex series of reds and yellows, the interrelations of which are not yet clear (see Correns, 67, 74, 77). Miss Marryat's experiments (unpublished) prove the existence of a number of heterozygous forms.

Orchids. Dominance of anthocyan colour in Cypripedium is clear. In that genus it results from union of two complementary factors (Hurst, Gard. Chron. 1908, I. p. I 73). As regards distribution of colour the facts are complex, but several indications of Mendelian distribution have been recognized (Hurst, I 53, I60). See p. 96.

Papaver. Presence of dark purple spot at base of petals dominant to the absence of such colour (de Vries, 290).

Phaseolus. The elaborate researches of Tschermak $(271-3,275,278)$ have demonstrated the existence of numerous factors controlling the colour of the flowers and seed-coats in $P$. vulgaris, $P$. multiflorus and their hybrids. The flower-colours are purples, reds, and white, with a bicolour form of the red ("Painted Lady"). Colour has not yet been produced by union of 
two whites, but on the analogy of the Sweet Pea such a result may be attainable. Otherwise the same rules apply generally. At least two sets of pigments take part in coloration of seed-coats: (I) brown, (2) purple. Whiteflowered plants have seed-coats unpigmented, and the bicolour flowers go with parti-coloured half-white seeds. The development of purple in the coats and the pattern in which it is deposited depend on various factors which can be carried by the albino. Various complications were met with (see originals). The cross between the two species showed some degree of sterility.

Similar results were obtained by Emerson (120, 12 I). The seed-coats of heterozygous plants in some cases were distinguishable. $\mathrm{He}$ also found green pods dominant to yellow pods (cp. Pisum, p. 14).

Further facts, with a scheme elucidating some of the curious ratios which the seed-colours may exhibit (e.g. I8: I $8: 6: 6: \mathrm{I} 6)$ in $\mathrm{F}_{2}$, are given by Shull (242).

Phyteuma Halleri (dark violet) $\times P$. spicatum (white) gave two types in $F_{1}$, 5 plants being bright blue with violet tinge, 4 violet (Correns, 70 ).

Pisum (Edible Peas). Flower-colours of three types. (I) Purple. Standard a pale purplish white; wings deep chocolate purple. (2) Pink. Standards pinkish white; wings a fine salmon-pink. (3) White. $F_{2}$ containing all three types is the usual $9: 3: 4$ in order named. Mark in axils of leaves, if present, is purple in (I), red in (2), absent in whites. Tschermak experimented with a purple strain without the axil-mark, and found that, as in Sweet Peas, the factor for that character can be carried by the albino.

Seed-coats colourless or greenish in white-flowered plants. In plants with coloured flowers one or more of three distinct kinds of pigments always present: (I) a purple, occurring in spots, (2) a brown, distributed either generally over the surface, or in bands (as in Maple peas), (3) an insoluble greenish grey, distributed over the whole testa. Neither (I) nor (2) can be developed in the absence of (3), but traces of (2) may sometimes be seen in white-flowered plants. There are separate factors for ( 1$),(2)$, and (3), of which (I) and (2) may be carried by the whites (Lock, I76).

Cotyledon-colours are yellow, and green. Yellow is a dominant, and heterozygotes are indistinguishable from homozygous dominants. In rare cases green has been seen as exceptions in $F_{1}$, but these are probably due to abnormal conditions. Many modern varieties have cotyledons patched with green and yellow. Genetically these are greens which show a special liability to bleaching. If protected while ripening they remain green.

Colours of Pisum have been chiefly studied by Mendel (195); Tschermak (269, $27 x-3)$; Correns (60); R.E.C. (20); Lock (I 72, I 73, I 75-6); Hurst (1 55).

Polemonium. Correns (70) found the white var. of $P$. coeruleum dominant to the yellow of $P$. flavum; and the blue type of coeruleum $\times$ flavum gave $F_{1}$ blue. It may be inferred that the yellow of flavum is a chromoplast colour, and that the blue anthocyan dominated as usual. Hybrids sterile.

Primula. $P$. Sinensis exists in a long series of colour-types the relations of which are still being investigated by $R$. P. Gregory in con- 
junction with me. Some of the more striking facts are referred to in later chapters. White flowers with green stem constitute an albino, recessive to all colours. The magenta shades have a factor epistatic to crimson and pink. Blue is hypostatic to all the red shades. The whites which have red or reddish stems are dominant whites, showing only a pale shade or tinge of colour in $F_{1}$. Deep colours cannot appear on stems that are not red except in the white-edged "Sirdar" (q.v.).

Salvia Horminum. Purple, red, white, related as in Pisum, \&c. Saunders, R.E.C. (20).

Triticum (Wheat). Red chaff is dominant to white chaff. Grey chaff is epistatic to red and dominant to white. Tschermak (270); Biffen (27); Spillman (247). (24r).

Verbascum blattaria. Yellow (a sap-colour) dominant to white. Shull

Viola. White is recessive to colour (de Vries, 290) in $V$. cornuta. The brown seed-colour of $V$. papilionacea is dominant to buff of $V$. hirsutula, and the purple of the capsule of hirsutula to the absence of purple in papilionacea (Brainerd, 4r).

Zea (Maize). Yellow endosperm dominant to white. Blue in aleurone layer an irregular dominant to absence of blue. (Definite exceptions are frequent.) Red pericarp, a plant-character, dominant to absence of red. The relations of the striped types have not been clearly determined. Correns (63); Lock (172, 1 74).

\section{Man.}

\section{Colours of Animals.}

Eye-colour (q.v.), Hurst (I6I), Davenport (ro7).

Albinism $(q \cdot v$.$) is doubtless recessive, but in man its descent is complex$ and has not yet been elucidated.

Red hair is recessive to dark hair and perhaps to ordinary brown (see Hurst, 162).

\section{Cattle.}

Red-roan is a heterozygote of red and white (Wilson, $3 \mathrm{II}$ ); and blueroan is similarly related to black and white. Spillman (249) suggests that black is dominant to red.

\section{Cats.}

Red is dominant to black in males. Tortoiseshell is the corresponding form of the heterozygote in females. Doncaster (rog). Dilution-types, blue, and cream, recessive to saturated colours.

As to eye-colour see Przibram (224).

Mice, Rats, Rabbits, Guinea-pigs.

Colours fully discussed in later chapters. Chief papers by Allen (r); Bateson (10); Castle $(48,53)$; Crampe $(83, a)$; Cuénot $(84-9)$; Darbishire (90); Durham (r 16); Hurst (157); Mudge (204). Albino recessive in all cases. A piebald type dominant to self-colour exists in mice (Durham, I 16$)$ and in rabbits, the so-called "English" variety (Hurst). 


\section{Horse.}

Chestnut recessive to bays and browns. Relations of these two dominants to each other not clear. Hurst ( $\left.15^{8}\right)$.

Pigs.

Several notes published by Spillman $(249,25 \mathrm{I}-2)$. White is usually a dominant to colour in domesticated races * but piebalds are frequent in $F_{1}$. The relation of black to red is not yet clear. The white belt, characteristic of certain breeds, is, according to Spillman, due to a complementary pair of factors which may be separately carried by self-blacks. He makes the interesting suggestion that the appearance of the belt may be a "reversion" to a condition like that of the Indian Tapir $(25 \mathrm{I})$.

The colour of the wild boar is dominant to the red of Tamworth and segregates normally from it $\left(25^{2}\right)$. The wild colour is presumably due to an "agouti" factor like that of the rodents.

\section{Sheep.}

From such fragments of evidence as I can find it seems that the white of ordinary sheep is not, as in the pig, a dominant to colour, but a recessive. From Darwin's record (An. and Plts. II. p. 4) of the appearance of all black sheep from a cross between white Southdown ewes and a Spanish ram with two black spots, it may perhaps be inferred that the black colour is due to complementary factors.

Black face and white face give a speckled face in the heterozygote. The dark ring round the eyes depends on a separable factor (Wood, 3 I2).

\section{Fowls.}

Colours very complicated and genetics imperfectly understood. Whites are of various kinds, one being dominant and at least two recessive. Colour depends on complementary factors which may be borne by whites. Black is an imperfect dominant to black-red. Brown-red a dominant to black-red (Fig. II). Blue is a heterozygous colour of black and a splashed white. The red and yellow pigments of the black-red cock may be replaced by white, thus giving the Silver Duckwing, but in the hen the red of the breast is not thus replaced, and the Duckwing hen differs from the blackred in having the yellow of hackle and mantle replaced by white. These replacements may occur as consequence of recombination in $F_{2}$ from crosses between white and coloured breeds, whence it is to be inferred that the replaced reds and yellows depend on a special factor. Pencilling is a dominant to its absence, and various mottlings are also dominant. The descent of colour is influenced in some cases by sex in ways not yet clear, and in both sexes heterozygous types occur.

The relations of the buff of Cochin (and of other breeds derived from it) to other colours are not yet known.

As regards colour of the down, the brown striped condition is dominant to the pale brown down associated, ior example, with Wheaten. Both types of down may occur in the same breed (e.g. Indian Game), and the fact seems to have no relation to the adult plumage. R.E.C. $(21,22)$.

* Mr Staples-Browne has given me confirmatory evidence. 
The black body-pigmentation of the Silky is a dominant, but may be inhibited by another factor, the descent of which is sex-limited. See Chap. x.

Another sex-limited descent is to be found in the relations of Cuckoo to black, but the details have not been ascertained (Spillman, 249, 253,a).

The daw-eye is recessive to red. The dark iris is usually a dominant to red.

Red ear-lobe is an imperfect dominant to white. R.E.C. (I9).

Principal papers dealing with these features are R.E.C. (I9-22), Hurst (I 56); Davenport (IOI).

\section{Pigeons.}

Black is dominant to blue. Relations of red and yellow not clear. Black and blue are dominant to white of Fantail ; heterozygotes generally, if not always, having some white. Chequering dominant to its absence. The white rump of the Rock-pigeon is dominant to blue rump (StaplesBrowne, 255).

\section{Canaries.}

Presence of black, as in green and pied types, dominant to absence of black as in the various yellows and cinnamons. The pink eye of cinnamons is recessive to black eye, with a sex-limited inheritance. There are probably several heterozygous colours, but in order to determine these the genetic interrelationships of the various Yellows, Jonque, Mealy, must be worked out. The "cap" and lacing of the Lizard are dominants. (Noorduijn, 2I3-5; Davenport, I05; F. M. Durham, unpublished.)

\section{Axolotl.}

Crosses between normal and albino gave dominance of pigmentation. Subsequent generations showed remarkable and as yet unique features. In $F_{2}$ dark larvae to white larvae were $3: \mathrm{I}$; but the white $F_{2}$ larvae, though remaining red-eyed, acquired a certain amount of pigment, sometimes distributed as a metameric chequering. No thorough albino occurred in $F_{2}$. When however these chequered albinos were bred with a true albino, the latter was found dominant and true albinos were produced (Häcker, I 43-4). Häcker compares this case with the phenomena seen in Mice, \&c., but there is an essential distinction in the fact that in all other instances true albinos come in $F_{2}$ and in the dominance of the true albinism over the chequered character. It would be interesting to see whether the development of pigment in the $F_{2}$ whites is in any way dependent on conditions.

\section{LEPIDOPTERA.}

Bombyx mori (Silkworm). Brown colour seen in a dark variety of the moth was proved to be an imperfect dominant (Coutagne, 83, p. I 22).

The larvae have many colour-types. Coutagne used a dark "moricaud" variety, a variety with transverse stripes, and ordinary white larvae. Both the coloured types are dominant to white, but when the dark self-colour factor and the stripe-factor are present in the same larva the stripes show on the dark ground-colour (ibid. p. 142). Toyama (268) also made many experiments with the colours of the larvae. He found striping a dominant over plain white. In certain $F_{2}$ families from striped $\times$ white a new pale 
form was produced (p. 349). The ratio of striped : common marked white : pale unmarked white is as Shull points out (242) I $2: 3:$ I (actually $1463,363,126)$. I incline to interpret this as signifying that the I 2 striped were in reality of two kinds, in the ratio $9: 3$, but that the distinction between the common and the pale was not easy to detect in the striped class. On this view the striped parent was a "pale." Shull regards the paleness as "latent" in both parents.

As regards the colour of silk there is a complication. In Toyama's experiments yellow was always a dominant to white. Coutagne sometimes obtained this result, but (l.c. p. 123) the white of a race called "Blanc des Alpes" proved to be dominant to yellow.

Abraxas grossulariata (Currant Moth). The type is dominant to var. lacticolor (q.v.), Doncaster (III, I I4). See Plate I, figs. I, 2. The peculiarities of this case are discussed in connection with Sex.

Mr L. W. Newman has been good enough to send me information as to a cross between $A$. grossulariata and the var. varleyata. This is a nearly black suffused form (see Porritt, Ent. Rec. xv. p. Io). $F_{1}$ was typical grossulariata, and in $F_{2}$ there were 24 typical and 7 varleyata $(40,3 q)$.

Angerona prunaria. The dark-banded var. sordiata dominant to the normal, reticulated type. In the heterozygotes the lighter bands are more or less reticulated. Doncaster (III). See Plate I, figs. 7-ro.

Xanthorhoe ferrugata. The form with purplish band is dominant to that with black band. Prout, L. B. (223).

Hemerophila abruptaria: the dark var. may be inferred to be a dominant to the type, from the experiments of Harris (147). Plate I, figs. 5, 6.

Amphidasys betularia (Peppered Moth). The normal is almost certainly recessive to the black, or doubledayaria form (see, for example, the records of Main and Harrison, I92). Plate I, figs. 3, 4.

Triphaena comes: the reddish form is recessive to the melanic (see Bacot, 3, and Prout, 222 ).

Callimorpha dominula: red of the hind wings is dominant to yellow. Standfuss (253, b, p. 222). Mr L. W. Newman has kindly given me information that he bred 34 reds and ro yellows in $F_{2}$.

Aglia tau: type is recessive to the dark form lugens. Standfuss $(253, b$, p. 3I I), (see Chap. x, for the sex-distribution of these varieties).

Lasiocampa quercus (Oak-egger). The heredity of colours of the hairs of the larvae has been investigated by Bacot (4) and Warburg (302). Several varieties were studied and their genetic interrelationships are not altogether certain, but it appeared that red fur of var. sicula was dominant to the white of the var. meridionalis. When English and French races were crossed, various blend-forms were produced in $F_{1}$.

\section{Coleoptera.}

Most of the observations thus far made relate to Phytophaga. Complications were met with in all the cases investigated by Miss McCracken. I have not been able clearly to understand the exact procedure followed in the matings and must refer the reader to the original papers. 


\section{Plate I}

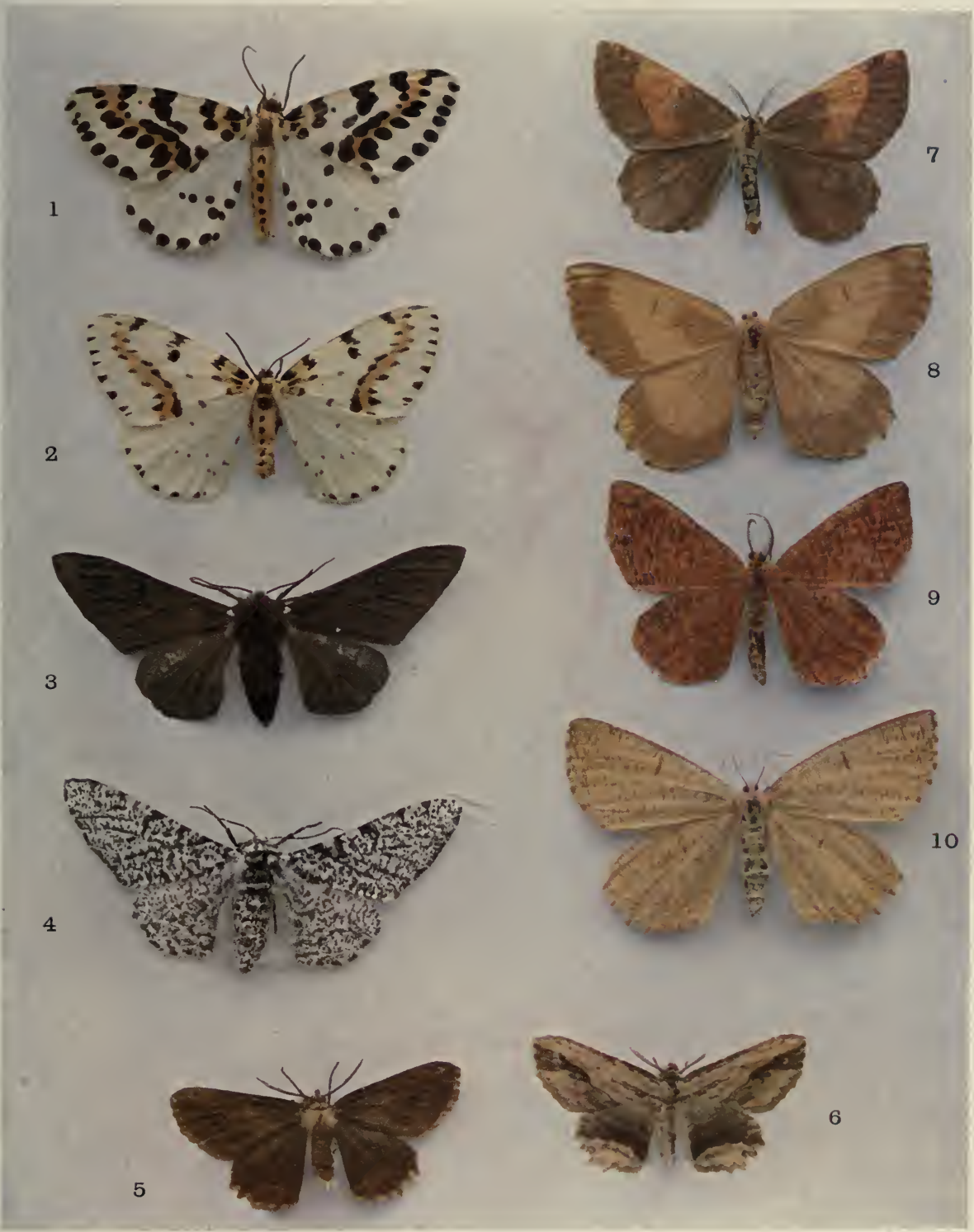

1. Abraxas grossulariata.

2. Ditto var. lacticolor. 3. The var. Doubledayaria of 4. Amphidasys betularia.

5. Dark var. of 6. Hemerophila abruptaria. 7. and 8. Male and female var. sordiata of 9. and 10. Angerona prunaria, male and female 

Lina lapponica. Two forms occur, one having elytra spotted with black on a brown ground, while in the other the elytra are entirely black. The latter is recessive, and the formation of the brown pigment in the ground is thus due to a dominant factor. McCracken (189, 199).

Melasoma (Lina) scripta. A totally black form was found to be recessive to the spotted type. Two intermediate conditions may occur, one of which may be a homozygous type (see original). McCracken (I9I).

Gastroidea dissimilis. Two forms, either deep blue-black, or shiny bright green. The latter is recessive. Curious complication as regards numerical results. McCracken (190).

Leptinotarsa decemlineata (Colorado potato beetle). Evidence obtained by Tower (266, pp. 275-9) indicates that a variety called by him pallida behaves as a recessive. The var. melanothorax of the species $L$. multitaeniata also proved to be a recessive to its type (ibid. pp. 284, 292, 293). Various other more complex phenomena are recorded $(q \cdot v$.).

Crioceris asparagi (Asparagus beetle). Each elytron has three yellow areas or spots on a blue-black ground. The upper spot is sometimes united to the middle one. This condition proved to be recessive to that in which the spots are separate, but all intermediate conditions occur [being presumably heterozygous]. Lutz (182).

Mollusca.

Helix hortensis and H. nemoralis. The unbanded variety is dominant to the banded types in both the species, sometimes completely, sometimes partially. Generally also red ground-colour is dominant to yellow or brown, but this effect may diminish with age of the hybrid individual. Lang (167-9). For details as to hybrids between the two species see (I69).

As regards dominance of colours very little in the way of general rule can yet be predicated, nor till the chemistry of pigments is much better understood is it likely that such general rules will be discovered. It may, however, be remarked that actual albinism, the total absence of pigmentation, is always, so far as we know, a recessive character in both animals and plants. Curious cases nevertheless are known both in animals and plants where a partial whiteness, which we should a priori imagine to be a kind of albinism, behaves as a dominant*. Another fact of a somewhat paradoxical nature is to be seen in the behaviour of some of the very deep colours, red and purples, characteristic of the flowers and other parts of some garden strains. These more intense colours both in Primula Sinensis, in the Stock, and in the Sweet Pea (and doubtless

* See Chap. v. 
Cyclamen) are recessive to the paler and more commonplace tints*.

Though in the case of colours in plants which are due to the development of pigmented sap, albinos are recessive to the coloured types, the yellow or cream colour due to the presence of yellow chromoplasts is recessive to the colourless condition of the chromoplasts. Hence we find, what at first seems paradoxical, that white flowers are dominant over cream-coloured flowers. Yellow dependent on $s a p$-colour is dominant to the corresponding white.

With regard to the behaviour of black pigment, which might naturally be supposed to have similar genetic properties in the various animals, no quite. satisfactory general rule can be laid down. The presence of black pigment is commonly dominant to the absence of black, as in the racehorse, where chestnut, namely the absence of black "points" is recessive to the presence of such "points" as in bays and browns. Most cases, however, such as that of the mouse, and other animals in which black pigment exists intimately mixed with other pigments are not so simple as this and involve special problems. In so far as the features of those cases can be expressed in the simple terminology hitherto used, these blacks must be classed as recessive to the normal colours. Further particulars will be given in the chapters on Colour.

\section{Preliminary Deductions from Mendelian Phenomena.}

It will be observed that animals and plants, as such, do not show any difference in their manner of heredity. Inheritance on simple Mendelian lines may be followed by characters of very diverse kinds, such as height, shape, chemical constitution, colour, and several structural features. In view of such a list the important question arises whether there is any distinct category or class of characters to which the Mendelian system does not apply. Various possible limitations may be discovered when the phenomena have been more fully examined, but it may be stated at once that no such class of characters has hitherto been identified.

* In Antirrhinum Miss Wheldale finds that the deeper magentas are recessive to the ordinary magentas, but in the crimson-red series the paler are recessive to the deeper tints. 
As yet only one example of a character which can at all readily be interpreted as meristic in nature has been shown to have a Mendelian inheritance. This is the case of the reduction in number of the human phalanges in brachydactyly. We speak of a character as "meristic" when it manifests itself in respect of the number of parts into which the body or one of its organs is divided. Meristic characters are in several ways distinguishable from other features of bodily organisation. The physiological occurrences which result in meristic variations are in all likelihood distinct from those which produce substantive changes, and exceptional interest would attach to any investigation of the genetic properties of such variations. Polydactylism is of course a meristic feature, but it may involve something more than a divisional change, pure and simple, since change in the number of digits is usually accompanied by change in the distribution of differentiation. A case in which the disturbance of differentiation is not so evident is provided by the cross between Oxalis tetraphylla, much cultivated in Germany as Gliicksklee, and one of the forms with three leaflets. This cross was partially investigated by Hildebrand*, who used $O$. latifolia. He found that the 3 -fold character was an imperfect dominant, the leaves being 3 -fold with the exception of occasional 4 -fold leaves which appeared for the most part at the flowering period. The hybrids were fully fertile, but their progeny has not been studied. Satisfactory meristic cases from which all confusing elements are eliminated must be rare, but it is greatly to be hoped that they will now be searched for. It is most desirable that cases of difference in the groundplan numbers of some radial type will be found amenable to experimental tests. Here the problem may be seen in a somewhat simplified form on account of the elimination of serial differentiation $\dagger$.

* Hildebrand, Jenaische Ztsch. f. Naturze. I889, xxiIr. N. F. xvi. p. 56.

$\dagger$ Since this paragraph was set up Price and Drinkard's (22 I) evidence has been published showing the dominance of two chambers in the fruit of the tomato over the many-chambered condition. More evidence as to such cases would be welcome.

Drinkwater's recent discovery as to the bones of the brachydactylous fingers, showing that the middle phalanx is actually formed as a distinct bone which afterwards unites with the distal phalanx, raises considerable doubt whether the variation in that case is meristic after all. 
Respecting the genetics of one most interesting class of variations evidence is scanty. This is right- and lefthandedness. From Mayer's* observations on Partula (Gastropod) we learn that parents of either twist may bear young of either twist. The numbers in the uteri were so small that the absolute numbers are insignificant, and it may be an accident that no mixture of types was found in any one uterus. Lang $\dagger$ bred numerous left-handed Helixpomatia with each other and obtained thousands of young, all right-handed, which in their turn again produced exclusively right-handed offspring. Direction of twist is a fundamental meristic phenomenon, being, as Crampton and Conklin have proved, determined as early as the first cleavage-plane in the egg; and great light on the problems of cell-division might perhaps be obtained if the inheritance of these differences could be determined. The only case we have attempted to study, that of Medicago, in which the fruits are right- or left-handed spirals according to species, proved unworkable, perhaps on account of the minute size of the flower and the roughness of the manipulations.

Lutz (I8I) has collected facts as to the inheritance of the mode in which the hands are clasped, whether the right or left thumb is placed uppermost. No definite result was obtained, but effects of heredity were somewhat marked, though neither condition bred true. Lutz kindly tells me that a full analysis was made, taking families separately.

When the Mendelian principles were first rediscovered the suggestion was made that though the system might apply to the unions of pure races, there was no certainty that such rules apply to the uncontrolled matings of natural forms. The objection was not one which was likely to have weight with those who had an acquaintance with genetic phenomena, but it had undoubtedly an effect in postponing general recognition of the importance of Mendel's discovery. Categorical proof of the invalidity of this objection is now provided by one of the cases referred to above-that which concerns the heterostylism of Primula. It is scarcely doubtful that in the Primrose nearly every plant.arises by the "legitimate" union of long- and short-

* Mayer, A. G., Mem. Mus. Comp. Zool. Harvard, xxvi. No. 2, I902.

† Lang, A., Vierteljahrs. d. nat. Ges. Zürich, 1896, and 168, p. 42, together with information kindly sent in a letter. 
styled individuals. Yet the long-styled are always pure. Moreover, all the short-styled plants hitherto tested have proved to be simple heterozygotes, giving equality of longs and shorts when bred with longs. Hitherto no pure $D D$, viz. short-styled plant, has been found in the case of the Primrose, but no difficulty has been met with in raising pure short-styled plants of Primula Sinensis. Besides this example of Mendelian heredity manifested by a wild type several of the examples of colour-inheritance in insects relate to wild species.

The circumstance that a character has not been previously bred pure does not, so far as is known, in any way influence the mode of transmission of that character. For instance, in the breeding of thoroughbred race-horses the heredity of chestnut colour is that of an ordinary recessive*, though the various colours, bay, brown, and chestnut have been indiscriminately united together in the breed. No difference is manifested between colour-inheritance of chestnuts which have had many chestnut ancestors in recent generations, and those that have no chestnut progenitor in the nearer degrees. The same is true for some of the colour-cases seen in Lepidoptera which had not been the subject of any previous selection. A remarkable example of an obviously Mendelian inheritance in a wholly wild form is that of the eye-colour of the Owl-Athene noctuat.

Abundant examples of characters breeding true, though newly-constituted, will be provided by those cases in which a novelty of structure is brought suddenly into existence by the occurrence of fresh combinations. In spite of their recent origin, such new combinations have just the same genetic properties and powers of transmission that are possessed by the types of long-selected breeds.

The suggestion hazarded by several writers that a distinction may be drawn between inter-racial and intra-racial heredity has no foundation in fact.

* Mr Hurst, who first elucidated the colour-inheritance of race-horses, found that according to the records, chestnuts of various ancestries have exclusively chestnut offspring with about $\mathrm{I} \%$ of exceptions, which are very possibly due to error in the returns (see later).

† Giglioli, Ibis, 1903, p. I. (See later, p. I 10.)

B. H. 


\section{Dominance: the Heterozygote Character.}

The character of the heterozygote, the "hybrid character" of Mendel, gives no indication as to the system by which the parental characters are transmitted. The expressions, "blended" inheritance, "particulate" inheritance and so on, terms formerly devised by Galton for describing the zygotic appearances, are now seen to be descriptive not so much of the mode of transmission as of the consequences of certain groupings of special allelomorphs; and as it is obviously preferable in all possible cases to use the ultimate descriptions reduced to terms of gametic composition, such terms are now seldom requisite. Dominance must be discussed more fully when other facts have been set forth, and in this preliminary notice of the more salient features of the phenomena it will be enough to point out that dominance is no inseparable attribute of Mendelian inheritance. The essential phenomenon is segregation.

The occurrence of dominance is often an assistance to the investigator and may greatly simplify the analysis of the various generations. Seldom however is dominance uniformly complete, and in certain cases, as those of the combs in poultry, where dominance is quite definite, it is still possible for an observer thoroughly familiar with the material to distinguish the homozygous dominants from the heterozygous with fair certainty. Provided the recessives as a class can be identified the application of Mendelian analysis is almost equally easy whether the heterozygotes show definite dominance or some intermediate condition.

The statement made by de Vries that dominance is an attribute of the phylogenetically older character has not been borne out by more extended investigation. In the lists given above many examples to the contrary occur. No one, for instance, can doubt that the various types of dominant comb (rose, pea, \&c.) in fowls and the colour called "Brownbreasted" have arisen since domestication. This colourexample is illustrated by Fig. I I, where the distinction between the striped Black-red type and the almost unicolorous Brown-red, or "Brown-breasted" type is shown. The striped type is practically that of the wild Gallus bankiva, but the unicolorous type of down-colour is a com- 
plete dominant. Males raised as $F_{1}$ between the two pure breeds are in their adult plumage almost intermediate, but the $F_{1}$ hens are indistinguishable from the pure Brown-red hens.

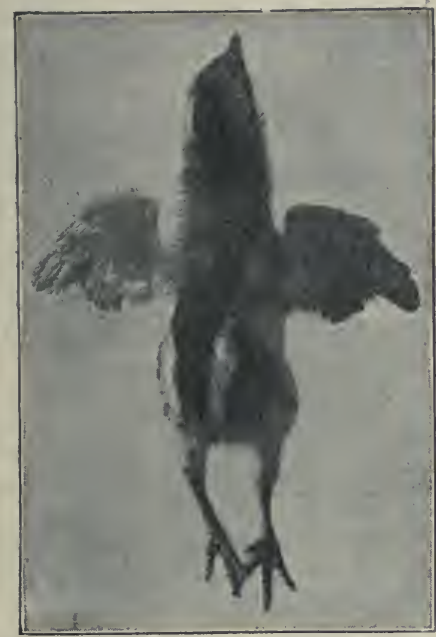

A

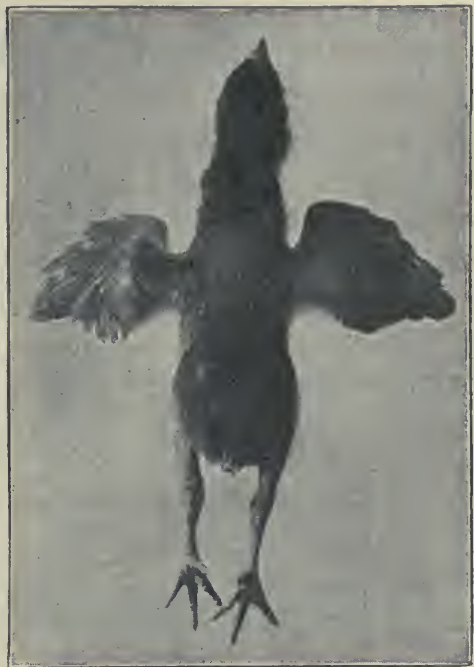

B

Fig. I I. Two newly-hatched chickens in $F_{2}$ generation from the cross Brown-red Game Bantam $\times$ Black-red. $A$ is the Black-red type having dark stripes on a light ground. $B$ is the Brown-red (or "Brown-breasted") type, a dark, almost unicolorous, blackish brown. $A$ is the recessive and $B$ is the dominant. The whole $F_{2}$ family consisted of $5^{8}$ like $B$ and 8 like $A$.

As an example in which the heterozygotes are intermediate the inheritance of colour in the Andalusian fowl may be taken. Andalusians are in general colour what fanciers call blue-namely a diluted black. In the cocks the hackles and saddle-feathers are full black, and the feathers of the breast are edged or "laced" with black. The hens are blue, laced with black more or less, all over. This breed is recognized by the fanciers as never breeding true to colour. When blue is bred with blue three colours are produced, blacks, blues, and a peculiar white* splashed with grey. Experimenting with this breed we have found

* These splashed whites are quite distinct from actual whites. They are in reality coloured birds as regards composition, and their down-colour is a faint bluish, very like that of the White Rosecomb bantam. 
that the numbers from blue $\times$ blue average about I black: 2 blues: I splashed white. Both the blacks and the whites extracted from blues breed true to their respective types, black $\times$ black giving all blacks; white $x$ white giving all whites again. When however black is bred with one of these whites the offspring are all blues. There is thus no doubt that the blue is the heterozygous form, while the gametes bear either the blackness, or the whiteness. Obviously in such a case, continued selection of blues will not make them breed true. This can only come to pass if it shall be found possible to get a blue bird in the gametes of which the blue or intermediate character is carried as a definite factor. These results may be represented in tabular form thus :
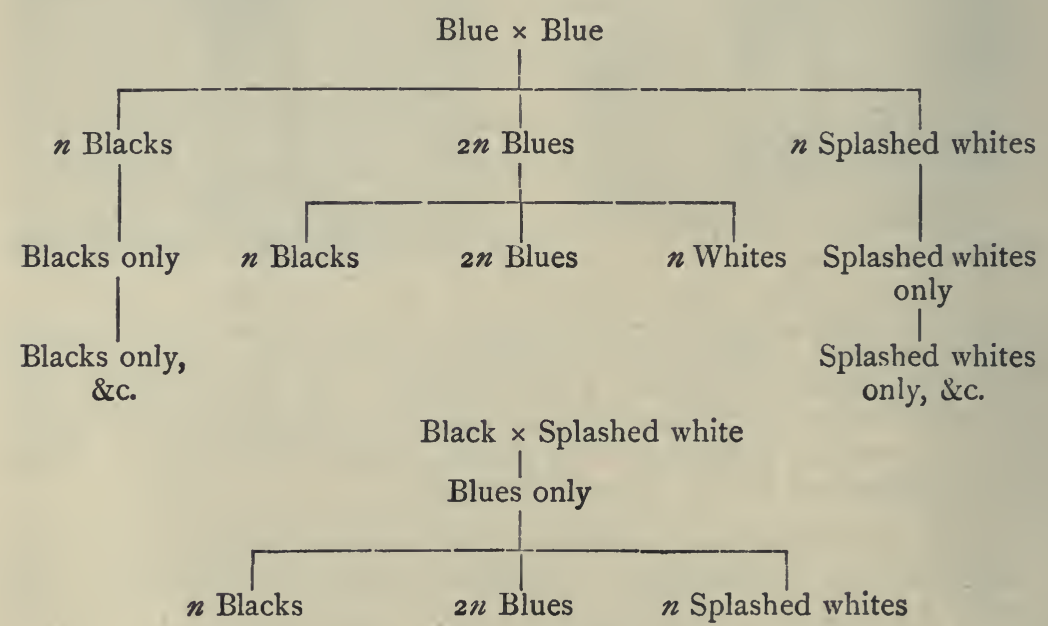

Such a case as this shows well what Mendel meant by the "hybrid-character." It is that character, or appearance, or quality, which is produced by the meeting of the opposite allelomorphs of the same pair in one zygote or individual. The hybrid-character is a thing apart, which must always be separately determined by experiment. Sometimes it is indistinguishable from the dominant, sometimes, as here, it is an appearance recognizably distinct from that of either dominant or recessive.

Prof. J. Wilson ( 3 I I ) has shown that a similar rule probably holds in the case of shorthorn colours, where red- 
roan is a heterozygous character, caused by the meeting of the factors for red and white*. The blue-roan so often seen in the cross between black Aberdeen-Angus cattle and white shorthorns is presumably the corresponding heterozygote form for black and white.

With further knowledge of the details and closer examination of material probably many such cases will be found. Darbishire, for example, has lately shown that though it is usually impossible in the case of peas to tell pure round seed from the heterozygous rounds, by external appearances, yet on microscopical examination the two classes can be distinguished at once by the different structure of the starch grains (94).

Many such cases where dominance is imperfect are now known. This phenomenon has no bearing on the more important question of the degree of perfection with which segregation is accomplished. The supposition that dominance was an essential phenomenon of Mendelism was of course a delusion. Imperfection of dominance does not even obscure the application of Mendelian analysis. The cases in which difficulty does arise are those in which dominance is irregular and the recessive class cannot be distinguished with certainty. In the fowl, for instance, the extra toe is usually a dominant, but in some strains there is irregularity, and birds without the extra toe may nevertheless transmit it. So also the blue colour of maize seeds, though usually a dominant, may sometimes be carried on by seeds which appear white (Lock, I 74). Even in these examples, however, there is no reason to think that such irregularities are indications of imperfect segregation. It is not impossible that they may be ascribed to interference caused by the presence of other factors in various combinations, and sometimes, no doubt, to disturbance by external conditions.

All observations point to a conclusion of great importance, namely that a dominant character is the condition

* Further information based on a long series of observations of the Sittytown herd of Shorthorns has since been published by Mr Robert Bruce in Breeder's Gazette, 25 Nov. I 908. Full statistics are given, affording with rare exceptions evidence strongly confirmatory of Prof. Wilson's views. I am obliged to $\mathrm{Mr}$ Alexander Bruce for a copy of this paper. 
due to the presence of a definite factor, while the corresponding recessive owes its condition to the absence of the same factor. This generalisation, which so far as we yet see, is applicable throughout the whole range of Mendelian phenomena, renders invaluable assistance in the interpretation of the phenomena of Heredity. The green pea, for instance, owes its recessive greenness to the absence of the factor which, if present, would turn the colouring matter yellow, and so forth. With the examination of further evidence the significance of this principle will become readily apparent.

\section{Mendel's System distinguished from that of Galton.}

From the outline of the evidence now set forth the essential aims and methods of Mendelian inquiry will have been understood. By this method we reach reality and concrete fact among phenomena that had become almost proverbial for their irregularity. The key to the problems of genetics and, as we confidently believe, to that of Species also, lies in the recognition of the character-units, or factors as we often call them. Their allelomorphism is a phenomenon of gametogenesis, and is a consequence of those attractions and repulsions by which the germinal celldivisions are effected. Discontinuity in variation-to use the word variation in its old, comprehensive sense-results from the existence of these units. We recognize therefore that this discontinuity-Galton's "Organic Stability"-is ultimately dependent on the physiology of gametogenesis, and not as we formerly supposed on some feature in the physiology of zygotes. How this simple conclusion was missed we may in vain surmise. The discovery at one stroke replaces all previous disquisitions regarding the laws of inheritance. The magnitude of the discovery and the novelty of its consequences have indeed delayed general recognition of its truth. To this may have been due the curious fact that the famous Nägeli failed altogether to realise the importance of Mendel's work. Nägeli was of course especially devoted to the study of heredity, and even made it the subject of elaborate mathematical treatment. As we now know, he was in correspondence with Mendel, 
from whom he received a considerable series of letters and illustrative specimens ( 197 ). These must have utterly failed to arouse his interest, for when in I 884, the year of Mendel's death, he published his great treatise on heredity, no reference was made to Mendel or his work. That this neglect was due to want of comprehension is evident from a passage where he describes an experiment or observation on cats, which as it happens, gave a simple Mendelian result. The Angora character (recessive) disappeared in a cross with a certain common cat whose hair-character is, as we now know, dominant. The cross-breds were mated together and the Angora character reappeared in one individual among a litter of common cats*. This typically Mendelian fact was thus actually under Nägeli's own observation, but from the discussion which he devotes to the occurrence it is clear that Mendel's work must have wholly passed from his memory, having probably been dismissed as something too fanciful for serious consideration.

It may be useful to specify the distinctive features of Mendelian inheritance which differentiate the cases exhibiting it from those to which Galton's system of calculation -or any other systems based on ancestral compositioncan apply.

(I) In Mendelian cases, in which the characters behave as units, the types of individuals considered with respect to any pair of allelomorphic characters are three only, two being homozygous and one heterozygous; while according to such a system as Galton's the number of possible types is regarded as indefinite.

(2) The Mendelian system recognizes that purity of type may be absolute, and that it may arise in individuals of the $F_{2}$ or any later generation bred from heterozygotes. The views based on ancestry regard purity of type as relative, and arising by the continued selection of numbers of individuals.

(3) In Galton's system no account is taken of dominance, a phenomenon which plays so large a part in the practical application of any true scheme of heredity.

These distinctions are so definite and striking that at first sight it seemed likely that the two methods might be * C. Näge
1884 , p. 199 . 
applicable to two physiologically distinct classes of phenomena. It was anticipated that some characters, or possibly even some forms of life, might follow the one system and others the other.

The results of further researches make this supposition increasingly improbable; and though undoubtedly there are cases which cannot yet be subjected to Mendelian analysis, it is fairly certain that there is no large group of facts in heredity to which the Galtonian system or any modification of it exclusively applies.

There are however numerous examples where the arithmetical results predicable by either system are nearly or quite the same, though further breeding would of course reveal that even in these cases the applicability of the Galtonian method was only superficial.

The first aim of genetics must now be to determine the magnitude, number and ultimately the nature of those units which together make up the visible fact we call heredity; and so to discover the consequences of their several combinations in zygosis or fertilisation. For the power thus to formulate our purpose and for the development of a method by which it may be successfully purșued we are beholden to Mendel's genius.

The difficulty which some feel in realising the significance of Mendelism arises from the habit of looking on the bodies of animals and plants as single structures. So soon as the mind becomes thoroughly accustomed to the fact that all individuals, at least those of the higher and more familiar types, are double, it becomes easy to think in Mendelian terms, and the world of gametes, whose pairings have brought into existence the individuals we see, comes naturally and persistently before the mind. Henceforth we have to penetrate behind the visible appearances of the individual, and endeavour to reconstruct first those processes of cell-division which produced the germ-cells or gametes, distributing the characters or factors among them according to definite systems; and then the subsequent process of union of those gametes pair by pair, in fertilisation to form zygotes, each developing and manifesting in its development those properties of structure, instinct and conduct conferred upon it by that particular complement of factors which its two original gametes contained. 


\section{CHAPTER III}

NUMERICAL CONSEQUENCES AND RECOMBINATIONS.

Representations of the $F_{2}$ Generation and Novelties due to Re-combination of Factors-Compound CharactersCombs of Fowls-Heterostylism - White Flowers from Red $\times$ Cream.

THE unity of characters being recognized, we may next examine some of the statistical consequences of this phenomenon. In order to determine the number of units and allelomorphic pairs which are concerned in any practical case, we have to be guided first by the visible statistical composition of $F_{2}$; and next by such tests of the gametic constitution of the several $F_{2}$ individuals as can be made by breeding from them.

To those who are familiar with algebraical methods, the employment of $F_{2}$ numbers to discover the number of terms in the gametic series may present small difficulty, but to others the following graphic method of demonstration may be of service. For the introduction of this system, which greatly simplifies difficult cases, I am indebted to Mr Punnett.

Take the simplest example, of one pair of allelomorphs, say Tall $(T)$ and Dwarf $(t)$. The parent zygotes of the pure strains are then $T T$ and $t t$. Their gametes are $T, T$, and $t, t$, respectively. The $F_{1}$ heterozygote is $T t$, and its gametes are all either $T$ or $t$ in equal numbers. As this is true both of the female germs and the male germs, there are four possible combinations. Make therefore four 
squares, representing the female gametes or germ-cells, similar cells being placed vertically, thus:

\begin{tabular}{|c|c|}
\hline$T \%$ & $t \uparrow$ \\
\hline$T q$ & $t \uparrow$ \\
\hline
\end{tabular}

Similarly the male germs may be represented by four squares :

\begin{tabular}{|c|c|}
\hline$T$ ठิ & $T$ ठิ \\
\hline$t$ ชิ & $t$ ठ \\
\hline
\end{tabular}

Here the similar germs are placed horizontally instead of vertically. If therefore the first set of squares be superposed on the second, all the four possible zygotic combinations are represented, thus:

\begin{tabular}{|c|c|}
\hline $\begin{array}{l}T q \\
T \text { वे }\end{array}$ & $\begin{array}{l}t q \\
T \text { ठ }\end{array}$ \\
\hline $\begin{array}{c}T \text { o } \\
t \delta\end{array}$ & $\begin{array}{l}t q \\
t \delta\end{array}$ \\
\hline
\end{tabular}

Thus there are I $T T+2 T t+\mathrm{I} t t$; and since $T$ is dominant, the visible appearance of $F_{2}$ is

$$
3 T: \mathrm{i} t \text {. }
$$

We may next deal similarly. with the case of two allelomorphic pairs, $A a$ and $B b$. We shall now require 16 squares. Writing each set of four similar female germs in vertical rows and the corresponding sets of male germs in horizontal rows, keeping to the same order, the sixteen possible combinations are represented. In each square the 
upper expression indicates the nature of the female gamete, the lower one that of the male gamete :

\begin{tabular}{|c|c|c|c|}
\hline$A B$ & $A b$ & $a B$ & $a b$ \\
$A B$ & $A B$ & $A B$ & $A B$ \\
\hline$A B$ & $A b$ & $a B$ & $a b$ \\
$A b$ & $A b$ & $A b$ & $A b$ \\
\hline $\begin{array}{c}A B \\
a B\end{array}$ & $\begin{array}{c}A b \\
a B\end{array}$ & $\begin{array}{c}a B \\
a B\end{array}$ & $\begin{array}{c}a b \\
a B\end{array}$ \\
\hline$A B$ & $A b$ & $a B$ & $a b$ \\
$a b$ & $a b$ & $a b$ & $a b$ \\
\hline
\end{tabular}

The constitution of each of the sixteen types which are produced in $F_{2}$ is thus displayed, and since $A$ is dominant over $a$, and $B$ over $b$, the visible appearance of $F_{2}$ is

$$
9 A B: 3 A b: 3 a B: 1 a b \text {. }
$$

Similarly when three pairs of factors are concerned, $A a, B b, C c$, the $F_{1}$ type will exhibit them all, and be in appearance $A B C$. In $F_{2}$ there must then be eight visibly distinct types, and the ratios in which they severally appear will be as follows :

$$
\begin{aligned}
27 A B C+9 a B C+9 A B c+9 A b C+ & 3 A b c+3 a B c \\
& +3 a b C+1 a b c .
\end{aligned}
$$

I am obliged to a mathematical friend for the following scheme by which the number of types and the ratios in which each will appear are given for any number of pairs of factors, one factor of each pair being dominant and the other recessive.

$$
\begin{aligned}
& 4=3+I \\
& I 6=(3+I)^{2}=3^{2}+3+3+I=9+3+3+I \\
& 64=(3+I)^{3}=3^{3}+3 \cdot 3^{2}+3 \cdot 3+1=27+27+9+1 \\
& 256=(3+I)^{4}=3^{4}+4 \cdot 3^{3}+6 \cdot 3^{2}+4 \cdot 3+I \\
&=8+27+27+27+27 \\
&+9+9+9+9+9+9 \\
&+3+3+3+3 \\
&+I
\end{aligned}
$$


So in general

$$
\begin{aligned}
4^{n}= & 3^{n} \\
& +3^{n-1}+3^{n-1}+\ldots \ldots n \text { times } \\
& +3^{n-2}+3^{n-2}+\ldots \ldots \frac{1}{2} n(n-1) \text { times } \\
& +3^{n-3}+3^{n-3}+\ldots \ldots \frac{1}{6} n(n-1)(n-2) \text { times } \\
& +\& c .
\end{aligned}
$$

Compound Characters and Novel Types produced by Re-combinations.

Thus far we have dealt only with cases in which the characters of each allelomorphic pair have independent effects on the visible appearance of the zygotes. In peas, for instance, we have seen a pair of characters, tallness and dwarfness, producing their effects quite independently of other pairs of characters, such as those which determine the flower colour or the seed-shapes. Each can be separately perceived by its effects, and the presence of the one in no way influences the development of the other. Such are the imaginary characters $A, a$, and $B, b$, whose distribution is represented above, and the four types produced by their several combinations are each distinguished without difficulty, as $A B, a B, A b, a b$.

We now pass to a class of cases manifesting greater complexity. The essential phenomenon in these cases is that definite characters are produced by the mutual interaction of factors belonging to distinct allelomorphic systems. Such interactions, as we now know, are of the greatest importance in heredity, and the progress of genetics will consist largely in disentangling the elements to which these combination-effects are due.

We may speak of characters thus produced as compound characters. The nature of such compound characters is well exemplified by phenomena which have been observed respecting the inheritance of several types of combs seen in various breeds of poultry, and as these cases are illustrative of many others I propose to consider them in some detail. 


\section{The Combs of Forvls.} types:

In the first place we are concerned with the following

Single comb. The high, serrated comb which is familiar to everybody is called by fanciers a single comb (Fig. I $2 A$ ). It is the type found in Gallus bankiva and I believe all the wild species, and we are fairly safe in regarding it as the primitive or original form from which all the others have been derived. It is the characteristic comb of Leghorns, Minorcas, and many other breeds.
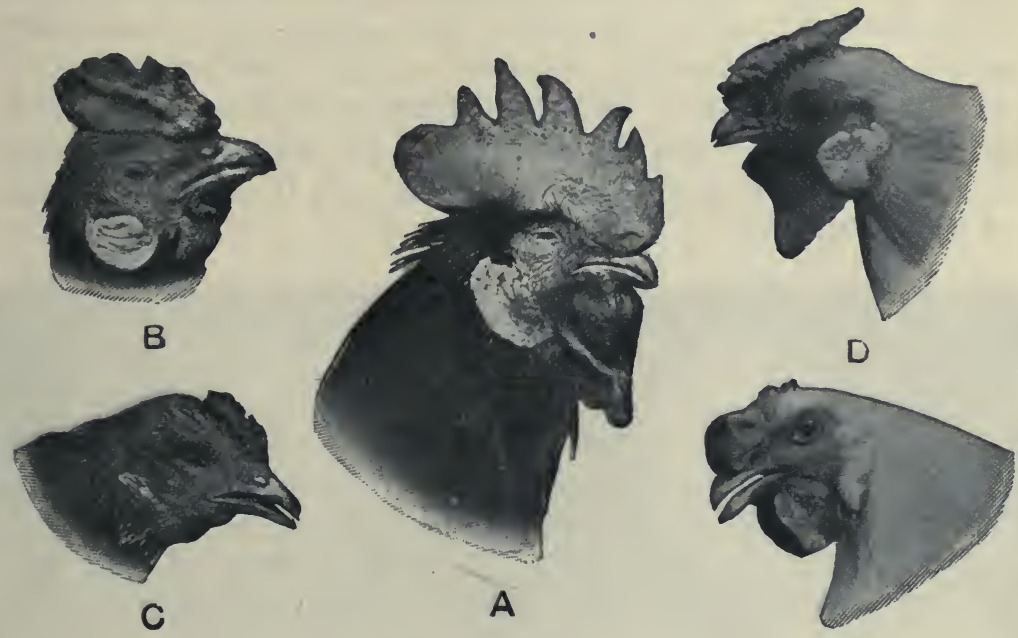

E

Fig. I 2. Various types of combs in Fowls.

A. Single Comb : cock.

B. Pea Comb : cock.

C. Pea Comb : hen.

D. Rose Comb : (Bantam) cock.

$E$. Walnut comb in a young cock. This is the type in Malays, and can be produced by crossing Rose $\times$ Pea.

Pea Comb. This comb (Fig. i $2 B, C$ ) differs from the single in being much lower and closer to the head. It has no spike-shaped serrations, but merely rounded lumpy projections along the middle line. In addition to these there is a characteristic development of similar lumps or tubercles on each side usually uniting to form a more or less definite 
lateral ridge. These two lateral ridges together with the median one constitute the three ridges commonly spoken of as the essential feature of the pea comb. The size and details of development of these combs differ a good deal with individuals and with strains. Pea comb is especially characteristic of Indian Game, Aseel, and the Brahma breeds.

The $F_{1}$ from pea $\times$ single is pea, that character manifesting a definite dominance. The heterozygous pea combs are generally higher than the pure pea and may usually, though not always, be distinguished from them. Sometimes the heterozygous pea comb is so large and has the ridges so ill-defined that it approaches the single type, but combs which cannot at once be referred to one class or the other are extremely rare. The distinction is especially sharp in the case of the newly hatched chicks, becoming somewhat less marked with later development. $F_{2}$ from this cross is of the usual form, 3 pea : I single.

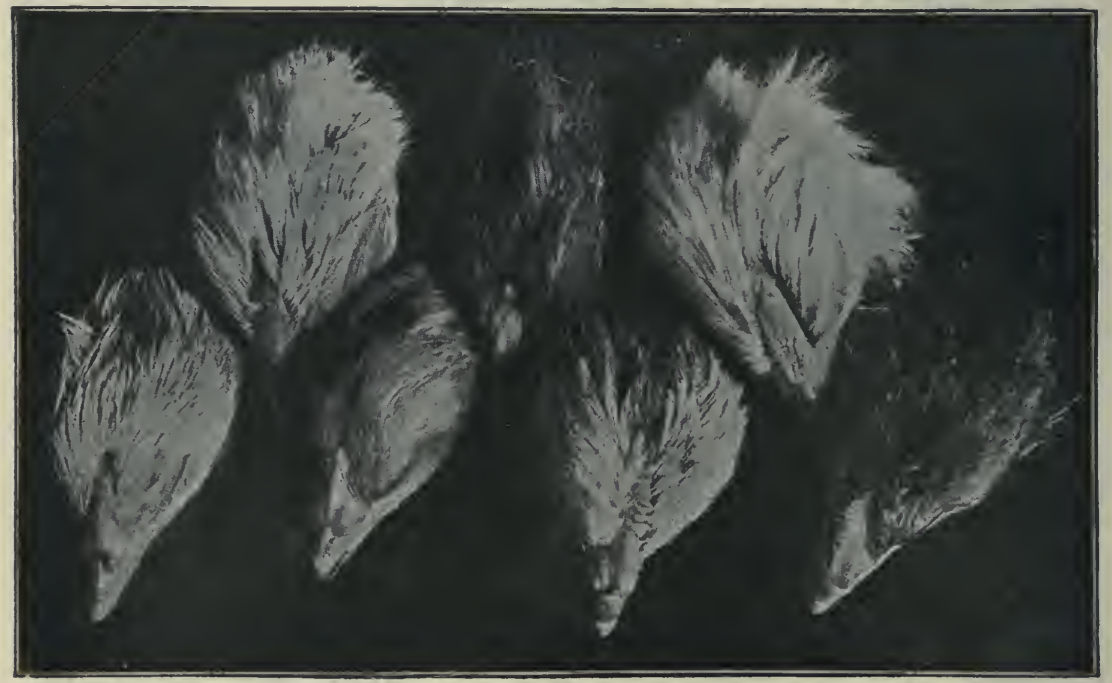

Fig. 13. The combs as they appear in newly hatched chickens.

In the top row from left to right:-Walnut comb in a lightcoloured bird, showing the peculiar band of hairs; ditto in a darkcoloured bird; Rose comb. ditto.

In lower row from left to right :-Pea comb; ditto; Single comb; 
Rose Comb. The next type to be taken into account is the rose comb, which consists of a triangular mass of small spikes or papillae. The apex of the triangle, called the peak or pike, points backwards and is free from the head. Such a comb is characteristic of Hamburghs, Rose-combed Dorkings and many other breeds. A particular form of rose comb with the pike curved downwards is a peculiarity of Wyandottes.

$F_{1}$ from rose $\times$ single is rose. The dominance of the rose comb is very definite, and it is frequently quite impossible to distinguish pure rose from the heterozygous type containing single. $F_{2}$ consists of the usual 3 rose : I single.

Rose $\times$ Pea. When a pure rose-combed bird is crossed with a pure pea the resulting comb is very different from either. It has no distinct papillae like the rose, or ridges like the pea. In the newly hatched chick the region of the comb is covered with a nearly flat or somewhat wartylooking skin. At the beginning of the posterior third there is generally a most curious band of bristles or hairs crossing the comb. Some $F_{1}$ birds have hairs scattered over the posterior part of the comb, either with or without a definite transverse band. The hairs usually increase in quantity and definiteness as maturity approaches. It seldom happens that a rose-pea ("walnut") bird has none of these hairs, though with age they may get worn off. As the chicken grows, the skin of the comb itself increases in size and becomes more or less corrugated. Such corrugations may become very large in males and are especially developed anteriorly. In this region the comb is often widened so as to form a lobe on each side, but the part behind the band remains single, so that the whole comb has a 3 -lobed appearance when seen from above. One of the corrugations very often appears as a furrow separating the flatter posterior lobe trom the anterior and more elevated part of the comb. It is from the corrugated surface of this comb that it is called by fanciers a "walnut" comb. The only pure breed in which such a comb occurs is the Malay. In Fig. I 3 the hairs can be seen fairly well in the top left-hand bird, which has light plumage. In the bird next to it the plumage is dark and the band of hairs is not so distinct in the photograph. 
It was not a little surprising to see so striking and characteristic a structure as the walnut or Malay comb appear with strict regularity as the product of two such dissimilar parents as the rose and the pea. Not only is the general appearance of the walnut quite distinct from these, but the presence of the hairs constitutes a feature of absolute difference, for no hairs are found on combs of the usual types. Were the walnut, rose, pea, and single combs found as characteristics of wild birds no naturalist would hesitate to regard them as four distinct specific characters; and even as the special properties of domesticated birds I suppose they would by many be regarded as evidence of long-continued selection. Nevertheless, as will be seen, these four forms stand to each other in a simple genetic relation and the fact suggests wide possibilities in regard to many hitherto unexceptionable differences "of specific value" recognized among animals and plants.

The interpretation of the facts was at first by no means easy, and I am sorry to have been responsible for the promulgation of a quite erroneous suggestion regarding them. Further knowledge of kindred phenomena has, however, made the elucidation of this case now perfectly clear and simple.

To return to the experimental results. Having found that rose $\times$ pea gives walnut, the next thing to be done was to test the genetic properties of birds thus produced. This was done in two ways (I) by breeding the walnuts together, (2) by breeding them with singles. In what follows, the names may be abbreviated thus: $R$, rose ; $P$, pea; $R P$, walnut; and $S$, single.

Experiment showed that $R P \times R P$ gave an $F_{2}$ family $R P, R, P$, and $S$. The appearance of $S$, which was not known to have been put in, is not at first sight intelligible. Repeated trials proved that the ratio in which these combs appeared was

$$
{ }_{9} R P:{ }_{3} R:{ }_{3} P:{ }_{1} S \text {. }
$$

It was further proved by experiment that the $R$ birds were either pure $R$ or contained the recessive $S$, but gave no more $P$ or $R P$; that the $P$ birds similarly could only give $P$, or $P$ and $S$; while the $S$ were all pure to that character. 
Consistent with this result were the offspring obtained from first-cross $R P \times S$, for this mating gave the ratio

$$
\text { I } R P: \text { I } R: \text { I } P: \text { I } S \text {. }
$$

As it was already established that $R$ and $P$ were each dominant to $S$, the inference was certain that the gametes produced by the $F_{1}(R P)$ birds were $R P, R, P$, and $S$ in equal numbers.

The whole series of phenomena may be represented as due to the combinations of two pairs of allelomorphic characters or factors, namely

I. Rose (domt) $R$, absence of rose (rec.) $r$.

2. Pea (domt) $P$, absence of pea (rec.) $p$.

\begin{tabular}{|c|c|c|c|}
\hline $\begin{array}{l}R P \\
R P \\
\\
\text { walnut } \\
\text { pure }\end{array}$ & $\begin{array}{c}R P \\
R p \\
\text { walnut } \\
\text { giving rose }\end{array}$ & $\begin{array}{c}R P \\
r P \\
\\
\text { walnut } \\
\text { giving pea }\end{array}$ & $\begin{array}{c}R P \\
r p \\
\text { walnut } \\
\text { giving all } 4\end{array}$ \\
\hline $\begin{array}{c}R p \\
R P \\
\\
\text { walnut } \\
\text { giving rose }\end{array}$ & $\begin{array}{l}R p \\
R p \\
\text { rose } \\
\text { pure }\end{array}$ & $\begin{array}{c}R p \\
r P \\
\text { walnut } \\
\text { giving all } 4\end{array}$ & $\begin{array}{c}R p \\
r p \\
\text { rose } \\
\text { giving single }\end{array}$ \\
\hline $\begin{array}{c}r P \\
R P \\
\text { walnut } \\
\text { giving pea }\end{array}$ & $\begin{array}{c}r P \\
R p \\
\text { walnut } \\
\text { giving all } 4\end{array}$ & $\begin{array}{l}r P \\
r P \\
\\
\text { pea } \\
\text { pure }\end{array}$ & $\begin{array}{c}r P \\
r p \\
\text { pea } \\
\text { giving single }\end{array}$ \\
\hline $\begin{array}{c}r p \\
R P \\
\text { walnut } \\
\text { giving all } 4\end{array}$ & $\begin{array}{c}r p \\
R p \\
\text { rose } \\
\text { giving single }\end{array}$ & $\begin{array}{c}r p \\
r P \\
\text { pea } \\
\text { giving single }\end{array}$ & $\begin{array}{c}r p \\
r p \\
\text { single } \\
\text { pure }\end{array}$ \\
\hline
\end{tabular}

Reference to the diagram of combinations shows that the two "absences" of rose and pea respectively will meet once on an average in 16 times, and to such a meeting without doubt the appearance of the single combs as a novelty, in $F_{2}$, is to be ascribed.

In the early years of this experiment, knowing that $R$

B. H. 
and $P$ were allelomorphic to $S, I$ came to regard them as also allelomorphic to each other. This idea led to confusion, but we know now that no case justifies such an application of the principle of allelomorphism. A rose comb is not due to an elemental factor which can segregate from the pea comb factor. The two factors belong to distinct allelomorphic pairs and each in the gametogenesis of the heterozygote segregates from its own allelomorph, which is simply the absence of the factor in question. The single comb contains neither $R$ nor $P$, The rose comb is a single comb modified by the presence of $R$, while the pea comb is produced by the presence of $P$. We may therefore describe the rose as $R$ no $P$, and the pea as $P$ no $R$. It is convenient to use capital letters for dominants and small letters for recessives, the rose being thus written $R p$, and the Pea, $r P$. The walnut comb is the $R P$, while $r p$ gives the single.

The allelomorphism of the elements which go to the constitution of the shapes of combs in fowls may without doubt be carried very much further. For example there are indications that the size of the comb depends to some extent at least on other pairs of factors. Another curious set of phenomena, perhaps worth investigating further, may be studied in a cross between a single comb breed and the "Breda" fowl. The Breda is usually said to have "no comb." As a matter of fact it has two very minute tubercles which represent the comb. When this breed is crossed with the single comb, $F_{1}$ has what may be called a "double" single comb. It consists of two large lobes or leaves diverging outwards from a common base*. Such a comb is evidently due to the introduction by the Breda of a factor which may be called "bifidity." This factor acts on the large comb brought in by the single-combed parent and the result of the combination is a large, double comb. $F_{2}$ from this cross has not yet been raised, but there can be no doubt that it will contain members having the "no comb" of the Breda and the "absence of bifidity" of the singlecombed breed. Such birds probably have only a minute tubercle at the posterior end of the comb region.

* The two lobes sometimes unite anteriorly to a greater or less extent. 
$\mathrm{Mr}$ Hurst suggested to us that an excellent confirmation of the truth of the analytical method by which the composition of the rose and pea combs has been represented could be obtained by a cross between the rose comb and the Breda, which, as has been stated, has the bifidity-factor but practically no comb at all. The elements involved are
Rose, $R$.
No rose, $r$.
Comb present, $C$.
Comb absent, $c$.
Bifidity, $B$.
No bifidity, $b$.

The $F_{1}$ generation has the composition $R r, C c, B b$, namely a double rose comb. $\quad F_{2}$ generation contains a great variety of forms, of which those having $B$ and $C$, but no $R$, have the high bifid comb like that of the $F_{1}$ raised in the cross Breda $\times$ Single, and those which contain only $r$ and $b$ in combination with $C$ have single combs of the ordinary high type (Fig. I2). Both these kinds occurred in $F_{2}$, and their appearance is entirely confirmatory of the scheme of representation adopted. Since neither the rose nor the Breda have outwardly any suggestion of single comb in their appearance, were it not for a knowledge of Mendelian analysis such a result must have seemed utterly unaccountable.

When therefore we look at such an organ as the comb of a fowl and attempt to conceive its genetic properties, we have to remember that the structure as a whole may be composite in origin, and that the visible appearances and properties may result from the interaction of a number of distinct elements each transmitted independently in gametogenesis.

Everything however points to the conclusion that the number of these elements is finite, and that their properties are not beyond the reach of orderly analysis.

In the example of the walnut combs the interaction of two dominant factors is such that the 9 members of the $F_{2}$ series of 16 have a feature, the walnut comb, distinct from those of the original parents, while the I member of the series which contains neither dominant factor, also has a feature, the single comb, distinct from anything visibly 
introduced. The next case also illustrates the appearance of a novelty in $F_{2}$, but, as will be seen, it is one of the groups of 3 members which manifests it.

\section{Heterostylism in Primula.}

The dimorphic, heterostyled condition of Primula plants is well known and need not be described in detail. The two types are distinguished thus:

A. Thrum, or Short-styled type.

I. Style short, the stigma standing at the level of constriction of the tube.

2. Anthers at the mouth of the tube.

3. Pollen grains large.

B. Pin, or Long-styled type.

I. Style long, the stigma usually standing in the mouth of the tube.

2. Anthers at the level of constriction.

3. Pollen grains small.

Experiments made by Mr R. P. Gregory in conjunction with me showed that the inheritance of these two types is Mendelian*, the short-styled or thrum behaving as dominant. The long-styled, being recessive, always breed true to that type on self-fertilisation or when bred inter se.

In connection with this fact it is interesting to observe

* I know no authentic case of the presence of both long- and shortstyled flowers on the same plant. Such occurrences are frequently announced, but so far as I can discover the records are based on mistakes. In occasional flowers on long-styled plants, especially in the beginning of the flowering period, the style does not attain its proper length. Such flowers are through carelessness sometimes misdescribed as shortstyled. The anthers however are at the lower level, and the pollen-grains are small, so the essentially long-styled nature of these plants is quite clear.

The statement is also sometimes made that pin plants have produced thrum-eyed offspring without the intervention of a cross. This mistake is due to the appearance of a type with "exsert" anthers. Such anthers project from the mouth of the tube and give a thrum-like look to the flower. But careful examination shows that the anther-filaments are inserted at the lower level, and the pollen-grains are small. Such plants are therefore long-styled. 
that Primula Sinensis, which is preferred by fanciers in the pin form, was easily bred true to that type, and is always so maintained in good strains. For our experiments it was with considerable difficulty that we procured any thrum plants. On the other hand it was decided long ago that the Auricula and the Polyanthus for exhibition purposes must always be thrums, but though thrums have thus been largely selected for breeding, pin-eyed plants are continually reproduced, as must be expected, for the thrum is a dominant, and therefore liable to contain the recessive type.

The facts about to be described relate to an experiment made with a peculiar race of $P$. Sinensis grown by Messrs

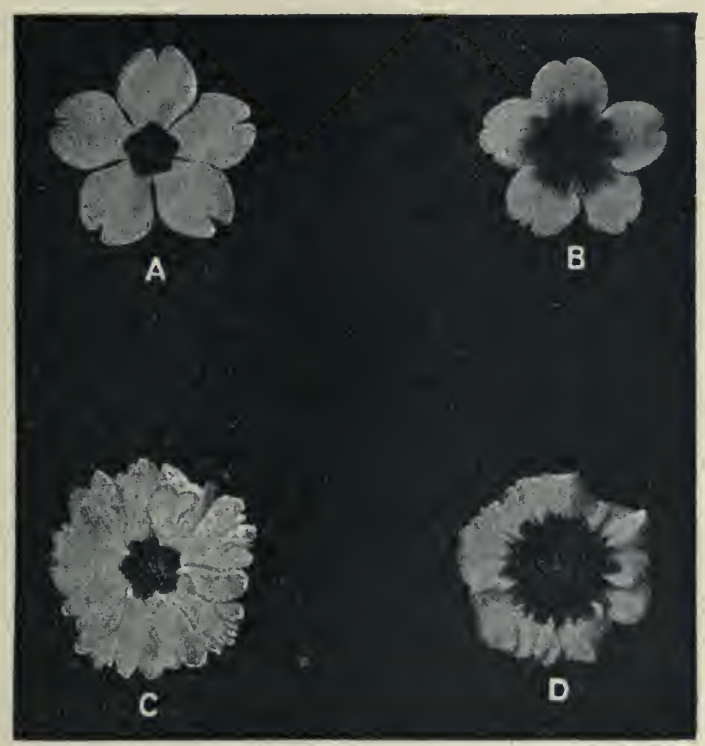

Fig. 14. Some of the types of flowers in $F_{2}$ from the cross short-style (thrum); small eye $\times$ homostyle; large eye.

$A$. The long-styled flower: with small eye.

$B$. The homostyled : large eye.

C. The short-styled: small eye.

$D$. The short-styled : large eye.

In the two upper flowers the corolla is of the "star" type. $D$ is the ordinary, imbricated type of Sinensis. $C$ is more or less intermediate in corolla-shape. This shape is the usual heterozygote formed between star type and Sinensis type. The corolla-shapes are of course quite independent of the style and "eye" characters. 
Sutton under the name "Primrose Queen." To the casual observer this race differs from an ordinary Primula in the fact that the yellow "eye," instead of forming a small and well-defined pentagon, is continued as a yellow flush extending far over the limb of the corolla. The anthers of these flowers stand at the lower level and the pollen-grains are small; but the style instead of projecting into the mouth of the tube stops at the anther-level, being thus practically the same length as in the short-styled type. Such a form of flower was called by Darwin homostyled.

Crosses were made between this homostyle race with the yellow flush and an ordinary thrum with the pentagonal or small eye. $F_{1}$ is thrum with the small eye, showing that the yellow flush is recessive. $F_{2}$ gave the following series:

9 thrum or short-style with small eye,

3 " " with the large eye,

3 ordinary pin or long-style with small eye,

I homostyle with the large eye like Primrose Queen.

The long-styled or pin type, which apparently was not put in, is evidently due to the re-combination of the characters. The two pairs of characters are
Thrum type (domt).
Small eye (domt).
Pin type (rec.).
Large eye (rec.).

The homostyle is the form which the pin type assumes when the large eye is developed; but when in $F_{2}$ the pin type meets the small eye, the ordinary pin or long-styled form is produced.

$F_{3}$ gave a simple and confirmatory result; for of the $F_{2}$ long-styled plants, some proved pure to the long-styled character, while others threw the recessive homostyle.

\section{White Flowers in $F_{2}$ from Red $\times$ Cream.}

Exactly comparable with the foregoing case is the paradoxical appearance of white-flowered individuals in the $F_{2}$ from the cross of a sap-coloured variety with a variety having cream-coloured flowers. For example, in Sweet Peas or Stocks (Matthiola) when a red-llowered type is crossed with a cream, $F_{1}$ is red, without any cream-colour. 
$F_{2}$ consists of 9 reds without cream, 3 reds with cream, 3 whites, I cream.

When the allelomorphs are correctly distinguished the significance of this series is obvious. The operations may be shown in a tabular form, thus.

Parents Red variety $\times$ Cream variety

Allelomorphs $\left\{\begin{array}{c|c}\text { Red sap }(D) & \text { Colourless sap }(R) \\ \text { Colourless corps. }(D) & \text { Yellow corpuscles }(R)\end{array}\right.$

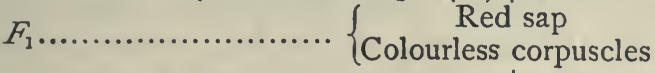

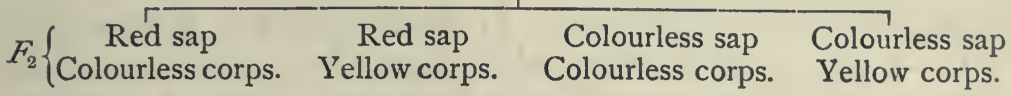

Appearance 9 Red 3 Red Cream 3 White $\quad$ I Cream

These cases of novelties resulting through a re-combination of the factors brought in by the original pure types are striking because it is not at first sight evident how the novelty has been produced. Generally speaking, however, the re-combinations form in $F_{2}$ a series of types many of which are obviously new combinations of features which could be recognized on inspection as present in the pure parents. Thus the cross between a bearded, rough chaff, red wheat, and a beardless, smooth chaff, white wheat give in $F_{1}$ a beardless, rough chaff, red. But in $F_{2}$ all the different possible combinations occur, such as bearded, smooth, red; beardless, rough, white ; bearded, rough, white, \&c., each in their appropriate numerical proportions. In the Guinea-pig, starting from albino, smooth coat, long hair, and crossing it with coloured, rough coat, short hair, $F_{1}$ is coloured, rough coat, short hair. But $F_{2}$ contains the various re-combinations of these three pairs of characters, such as albino, rough coat, short hair ; coloured, smooth coat, long hair, \&c. Thus by selecting any desired type in $F_{2}$ any of these new combinations can be fixed and perpetuated. Basing his procedure on a knowledge of the dominance or recessiveness of each character the breeder may thus guide his operations with certainty.

That this has been the mode by which most of the new breeds of domesticated plants and animals have been created is obvious. The traces of it remain in many cases. For 
instance, Sutton's "Nonpareil," one of the marrow peas, consists in about equal numbers of yellow-cotyledon seeds and green-cotyledon seeds. Like all the new peas it must have arisen at some definite moment by the selection of an individual yellow-seeded plant which was true for the various good qualities of Nonpareil-being homozygous for them in other words-but in cotyledon-colour it was heterozygous. As the diversity of colour was not thought objectionable it persists. If any one wishes to make an exclusively green-seeded Nonpareil, all he has to do is to take green seeds from a sack of that variety and sow them, saving the seeds they bear. If he desires the yellow type pure, he may similarly sow yellow seeds from the same sack. Most of these by now will presumably be pure to yellow, but some may not. By keeping the seeds of any plant which gives only yellow seeds a pure yellow Nonpareil will be constituted.

Similarly Sutton's "Continuity" is a pea which is allowed to be either pointed in pod or blunt. The variety is true in other respects and it is clear that its original progenitor was a plant homozygous for the peculiarities of Continuity but heterozygous in respect of the pod-shape. The pointed-pod plants would be found true for that character, since it is recessive, while the blunt-pod plants might or might not be true for it.

So in the Chinese Primrose, several varieties, e.g. Sutton's Mont Blanc, Sirdar, \&c., distinguished by peculiarities of colour have been fixed both in a palm-leaved and in a fern-leaved form, these having of course been saved in $F_{2}$ or later generations from a heterozygote in which the palm and fern-leaved characters were combined. In Sweet Peas, after the original dwarf "Cupid" was found-according to tradition a chance seedling among tall plants-it was easy to transfer the characteristic colour of the various tall types on to a Cupid foundation, and now any colour almost can be had either as a tall or as a Cupid.

\section{Possible Limits to Re-combination.}

These illustrations might be extended indefinitely. It will probably occur to many that there are limits to these possibilities of transference, and so undoubtedly there are. 
The detection of these limits is one of the more important tasks still awaiting us. Though on this head little can yet be asserted with confidence it is likely that such limitations are constituted in two distinct ways: First, from all we know of the capacities of animals and plants we must anticipate that some characters are incompatible in the same individual. For example in cattle the highest milkproduction is not to be found in the breeds which make the best beef. Meat-production and milk-production are to some extent alternative and can only be combined by compromising one quality or both. That such an alternative distribution is merely a result of allelomorphism seems on the whole unlikely, though certainly not impossible.

Then again we must surely expect that these transferable characters are attached to or implanted upon some basal organisation, and the attributes or powers which collectively form that residue may perhaps be distinguishable from the transferable qualities. The detection of the limits thus set upon the interchangeability of characters would be a discovery of high importance and would have a most direct bearing on the problem of the ultimate nature of Species. 


\section{CHAPTER IV}

\section{HEREDITY OF COLOUR.}

Factors determining Colours: the Ratio 9:3:4.-The "Presence and Absence" Hypothesis. Epistatic and Hypostatic Factors-Colours of Mice-Pied Types$A$ Dominant Piebald.

WITH regard to the application of the Mendelian system to problems of colour inheritance the evidence is now considerable. The fact that in both animals and plants albinism behaved as a recessive to colours was soon discovered. Several examples among plants are mentioned by de Vries in his first paper on this subject, and shortly after similar facts were recorded in regard to animals. Since in the course of a large range of experiments with many species of animals and plants no case to the contrary has been met with, it may perhaps be asserted as a general truth that pigmentation is always dominant to total absence of pigment*. When however we proceed to the investigation of the genetic properties of varieties which are so far deficient in pigment as to be called sometimes partial albinos, we find that various specific rules are followed and no uniformity of behaviour has yet been discovered. White fowls for instance are thus commonly spoken of as partial albinos, but the pigmentation of their eyes sharply distinguishes them from albinos which are destitute of pigment, and many of their genetic properties are found experimentally to be quite distinct from those of real albinos. The same is true of certain varieties of plants, which though varying from the specific type by possessing white flowers have yet some red or purple sap in the stem or elsewhere. With-

* The Axolotl is perhaps an exception. See p. 43. The fact that in plants colourless chromoplasts are dominant to yellow chromoplasts scarcely constitutes an exception, for the yellow of the chromoplasts is not pigment in the usual acceptation of that tern. 
out experiment no prediction can be made with confidence as to the behaviour of such types in their crosses.

Albinism being recessive in all ordinary cases, $F_{2}$ from the cross colour $\times$ albino contains I albino to 3 coloured members. As regards the characters of the dominant or coloured members various complications have to be considered.

In the simplest cases the coloured $F_{2}$ individuals are all of the same colour. For example on crossing a grey rabbit with an albino, $F_{1}$ is grey and $F_{2}$ may be 3 greys : I albino. But frequently it is found that in addition to the greys and albinos blacks appear in $F_{2^{2}}$ Repeated experiments, for example those of Hurst, have shown that in such families the $F_{2}$ ratio is

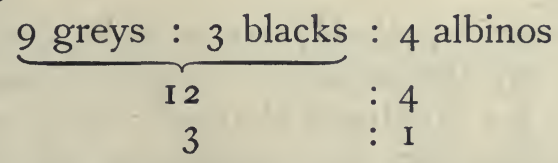

The relation of this ratio to the ordinary $9: 3: 3:$ I was first pointed out by Cuénot (86)*. As represented by him two pairs of allelomorphs are concerned, namely:

\section{Dominant.}

I. Colour $(C)$.

2. Grey determiner $(G)$.
Recessive.

Albinism $(A)$.

Black determiner $(B)$.

The presence of one or other of the determiners $G$ or $B$ is only perceptible when it exists in combination with the colour-factor. If $G$ is present together with $C$, the colour is grey; if $B$ is present with $C$ but without $G$, the colour is black. If a coloured individual contain both $G$ and $B$, being thus heterozygous in the second pair of factors, the colour is grey, for the effects of grey dominate. But since in the absence of colour $(C)$ neither determiner produces a perceptible effect, albinos may exist of the forms $A A G G$, $A A G B$, or $A A B B$, and without breeding tests it will not be possible to distinguish between these several forms. On crossing with a black of course each albino can be known by its effects. For $G G$ will then give greys only, $G B$ will give equal numbers of greys and blacks, while $B B$ albinos will give only blacks.

* Shortly before the publication of Cuénot's paper Mr R. H. Lock wrote to me from Ceylon with the same suggestion. 


\section{The "Presence and Absence" Hypothesis applied to the Case of Colour.}

So long as attention is restricted to crosses like these involving only two sorts of colours besides the albinos, the system suggested by Cuénot is adequate, but when a third colour has to be considered, as in the case of mice, some modification is required. The simplest notation by which these and other complex Mendelian phenomena can be expressed is provided by what is spoken of as the Presence and Absence hypothesis already illustrated in the case of the combs of fowls.

Mendel himself probably conceived of allelomorphism as depending on the separation of a definite something responsible for the dominant character from another something responsible for the production of the recessive character. It is however evidently simpler to imaginethat the dominant character is due to the presence of something which in the case of the recessive is absent. As yet there is no absolute proof that this mode of describing the facts is correct, - but everything points that way, and no phenomena have yet been encountered which cannot be thus formulated when their nature is understood. In cases where the pure dominants are recognizably distinct from the heterozygous dominants, it must naturally be supposed that two "doses" of the active factor are required, one from the paternal, and another from the maternal side, in order to produce the full effect.

Applying the presence and absence system to the case of the colours of rabbits, the first pair of allelomorphs can obviously be represented as

\section{Dominant.}

Recessive.
I. Presence of Colour $(C)$.
Absence of Colour (c).

The second pair we have so far spoken of as the grey determiner and the black determiner, regarding these two as allelomorphic to each other. But it is equally possible to describe them thus
2. Grey determiner $(G)$.
Absence of ditto $(g)$. 
Then in the case where grey $\times$ albino gives in $F_{2}$

$$
9 \text { grey : } 3 \text { black : } 4 \text { albino, }
$$

we simply have to regard $B$, the black determiner, ; as common to both parents, and the same numerical result is produced. Such a case may usefully be represented in a tabular form, thus :

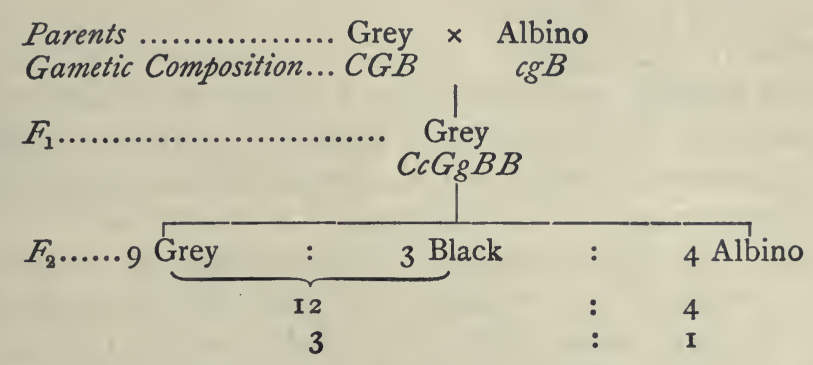

\begin{tabular}{|c|c|c|c|}
\hline $\begin{array}{l}\text { C G B } \\
\text { C G B } \\
\\
\quad \text { grey }\end{array}$ & $\begin{array}{l}\text { C G B } \\
\text { C G B } \\
\\
\quad \text { grey }\end{array}$ & $\begin{array}{l}C \text { g } B \\
C G B \\
\end{array}$ & $\begin{array}{l}\text { c g B } \\
\text { C G B } \\
\\
\\
\text { grey }\end{array}$ \\
\hline $\begin{array}{l}C G B \\
C G B\end{array}$ & $\begin{array}{l}c G B \\
c G B\end{array}$ & $\begin{array}{l}C g B \\
c G B\end{array}$ & $\begin{array}{l}\mathrm{cg} B \\
\mathrm{cGB}\end{array}$ \\
\hline grey & albino & grey & albino \\
\hline $\begin{array}{l}C G B \\
C g B\end{array}$ & $\begin{array}{l}c G B \\
C g B\end{array}$ & $\begin{array}{l}C g B \\
C g B\end{array}$ & $\begin{array}{l}\operatorname{cg} B \\
\operatorname{cg} B\end{array}$ \\
\hline grey & grey & black & black \\
\hline $\begin{array}{l}C G B \\
c g B\end{array}$ & $\begin{array}{l}c G B \\
c g B\end{array}$ & $\begin{array}{l}C g B \\
\operatorname{cg} B\end{array}$ & $\begin{array}{l}\operatorname{cg} B \\
\operatorname{cg} B\end{array}$ \\
\hline grey & albino & black & albino \\
\hline
\end{tabular}

Fig. I 5. Distribution of grey, black, and albino individuals in $F_{2}$ from the cross grey, $C G B$, with albino $c g B$, showing the meaning of the ratio 9 grey : 3 black : 4 albino. 
In this diagram the 9 squares containing $C, G, B$, are the 9 greys, the 3 squares containing $C$ and $B$ only are the 3 blacks and the 4 squares containing no $C$ at all are the 4 albinos.

Proceeding to the case of mice we write the composition as follows :
Grey
$C, G, B, C h$.
Black
$C, g, B, C h$.

Chocolate $C, g, b, C h$.

We thus regard the black mouse as one from which the grey determiner, $G$, has been removed. In the chocolate mouse the process of removal has been carried further and the black determiner, $B$, is also gone.

A proof that this system of representation is so far correct is obtained by crossing the grey mouse with the chocolate. Such a cross, if $G$ is not allelomorphic to $B$, must give blacks in $F_{2}$. This experiment has been lately carried out by Miss F. M. Durham, to whose work our knowledge of the genetics of mice is largely due. The result is that, as expected, $F_{2}$ does contain blacks, and though the numbers as yet obtained are small, there can be little doubt that the $F_{2}$ ratio is

\section{2 grey : 3 black : I chocolate.}

Some interesting questions arise in regard to the greys. Obviously we should expect 9 greys containing $G$ and $B$ +3 greys without $B$. Now fanciers are well aware of a distinction between two kinds of greys or "agoutis" as they are called. These are known as "golden agoutis" and "cinnamon agoutis," the former containing black pigment, the latter being without it. In the $F_{2}$ from grey $\times$ chocolate both these kinds of agoutis appear, and evidently the cinnamon agoutis are the expected greys wanting in the determiner $B$.

Thus far all is clear. Certain difficulties however remain unexplained. These will be described later. At this stage in the discussion it is convenient to notice that in view of the facts now stated the use of the term dominance must be more carefully restricted than has hitherto been necessary. When we speak of the colour as being dominant over the absence of colour we mean that if the colour is present it will appear, and that if the factor for 
colour is absent the individual will be devoid of colour. The term is thus used correctly to denote the relation between allelomorphic features belonging to the same pair. But confusion will be introduced if we extend the same term to the relationship between various determining factors which belong to distinct allelomorphic pairs.

Hitherto we have spoken of the determiner for such a colour as grey in rabbits and mice as "dominant" over the colours lower in the scale, such as black or chocolate. Nevertheless we are here dealing with a relationship quite different in order from that subsisting between the coloured and the albino. Pending a more precise knowledge of the nature of this relationship it will be enough to regard those factors which prevent others from manifesting their effects as higher, and the concealed factors as lower. In accordance with this suggestion the terms epistatic and hypostatic may conveniently be introduced. We shall then speak of the determiner for grey as epistatic to that for black; that for black as epistatic to the determiner for chocolate, and so on.

When the facts are thus clearly represented we perceive that the variation by which, for example, a black mouse came originally into existence, consisted in the omission of the determiner for grey. The chocolate mouse similarly owed its origin to the successive omission of the determiner for black.

The important question what the effect of the grey determiner, for example, actually is, remains undecided. A further serious difficulty also arises in regard to the relation of the colour yellow to the other colours. Neither of these points is yet satisfactorily understood in the case of mice. The recent papers of Castle (53) and of Hurst* have made the phenomena in rabbits comparatively clear, though even there, however, an unexplained difficulty remains. The special problems raised by the behaviour of yellow pigment in these animals will be discussed in a subsequent section $†$ (see Chap. viI).

* Read at the Internat. Congr. Zool. Boston, r907 : not yet published.

+ According to the number of factors involved and to the definiteness with which their several combinations can be distinguished, an indefinite variety of ratios may of course be produced in $F_{2}$ families. Some of the most interesting are those in which some of the heterozygous combinations can be distinguished from the homozygous dominants. (See for examples Shull, 242.) 
The $F_{2}$ ratio $9: 3: 4$, the significance of which we have been considering, is one which very frequently recurs in Mendelian analysis. For example, as Tschermak found, when a pink-and-white flowered eating pea ( $P$ isum sativum) is crossed with a white flowered type, $F_{1}$ is often of the original purple flowered type. Then $F_{2}$ will be

9 purple : 3 pink-and-white : 4 white.

Similarly pink Salvia Horminum $\times$ white may give $F_{1}$ purple, and $F_{2} 9$ purple : 3 pink : 4 white. In these cases the factor for the purpleness is of course brought in by the albino, but exactly the same $F_{2}$ may result from a cross between the purple type and an albino not carrying the factor for purpleness. All that is essential for the production of this ratio in $F_{2}$ is that $F_{1}$ should be heterozygous for two factors, of which one is perceptible whenever present, while the other needs the presence of the first in order that its own effects may be manifested. Such cases are very numerous and in practical breeding are to be looked out for continually. Care must be taken to distinguish them from families like those of the Andalusian fowl (p. $\left.5^{2}\right)$ in which the commonest term in the $F_{2}$ series is a heterozygous type. There the numbers will be $1: 2: \mathrm{I}$, which in a practical example may give results not obviously distinguishable from $3: 9: 4$. To decide between the two possibilities it is necessary to breed the $F_{2}$ types again. If neither of the scarcer types when bred inter se can throw the other, and the commoner type cannot be bred pure, the latter is a heterozygous type; but if one of the scarcer types can throw the other, then the ratio is presumably $9: 3: 4$, and in such a case it will be possible to raise a breed true to the type occurring as 9.

\section{Saturation and Dilution of Colours.}

Omitting yellow from our consideration, we thus recognize that in the mouse the colours, grey (agouti), black, chocolate, which the fur visibly presents, result from the interaction of several factors, and that these factors can in great measure be shown to be distributed in gametogenesis according to Mendelian allelomorphic systems. The ex- 
$=2$ 


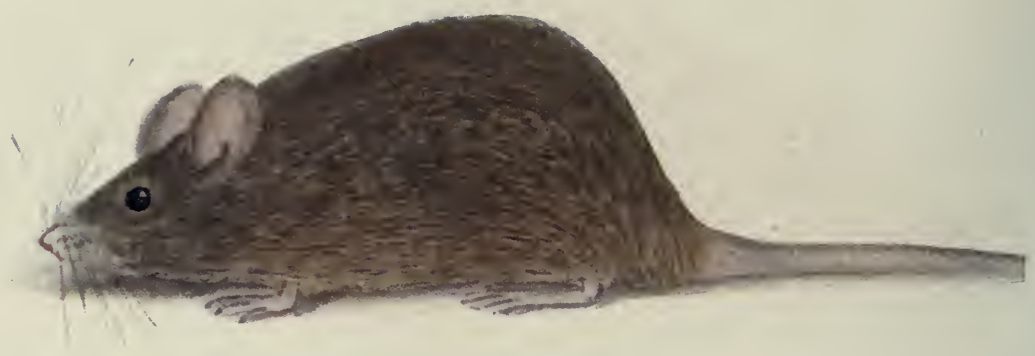

I. Agouti.

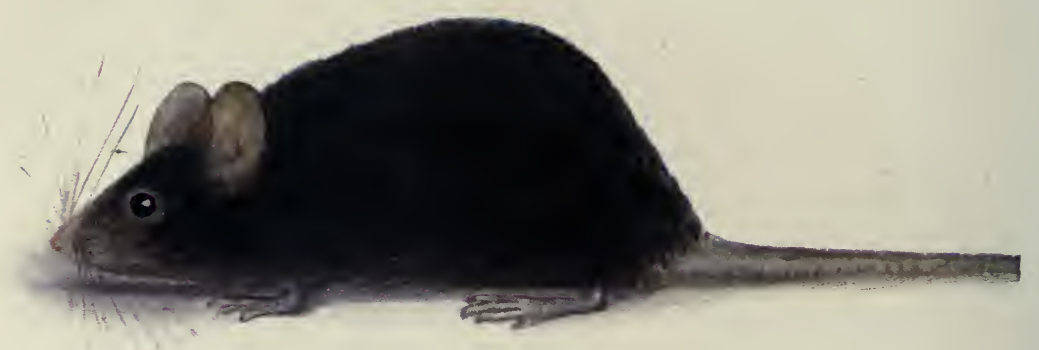

2. Black.

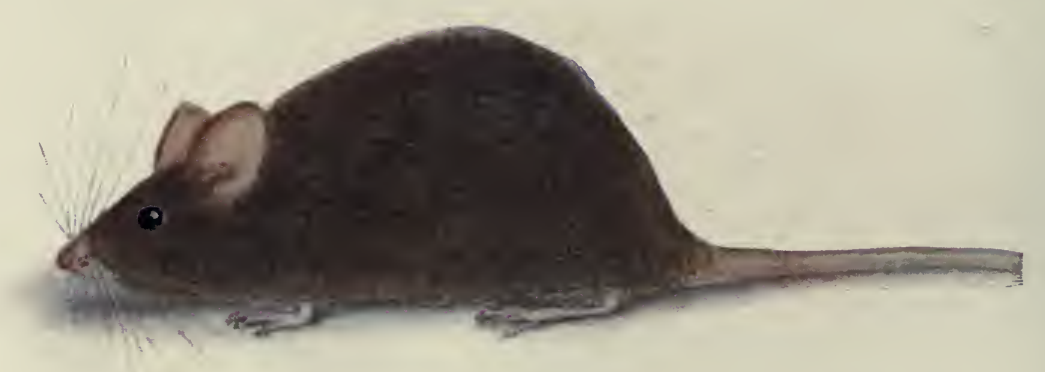

3. Chocolate. 
Plate II

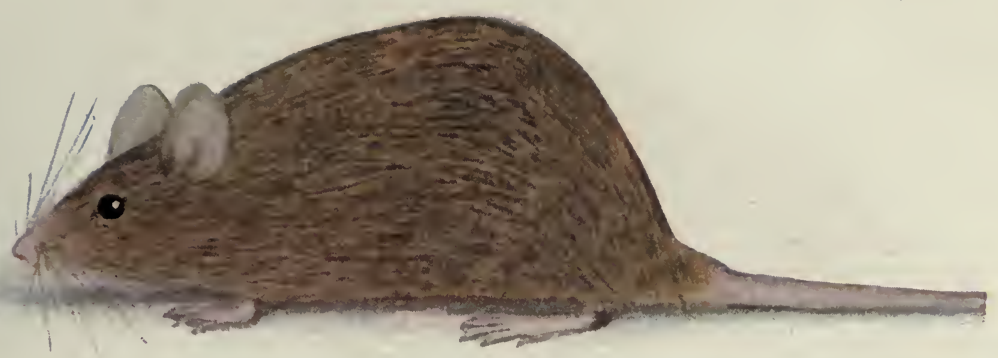

4. Cinnamon Agouti, viz. Agouti without black.

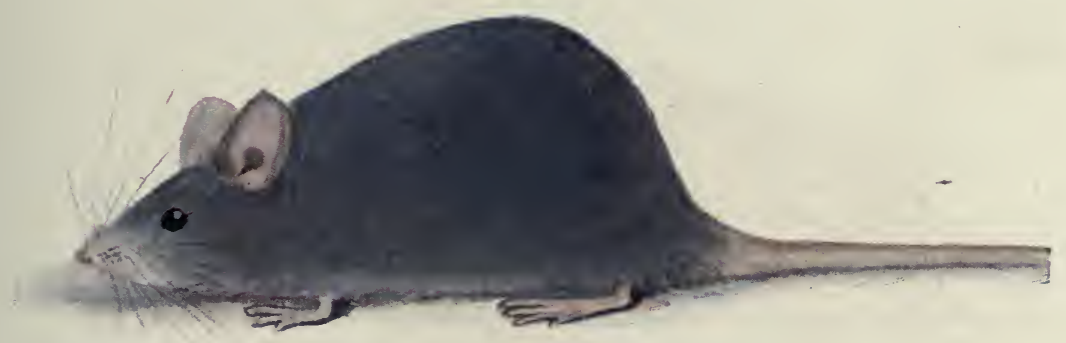

5. Blue := dilute black.
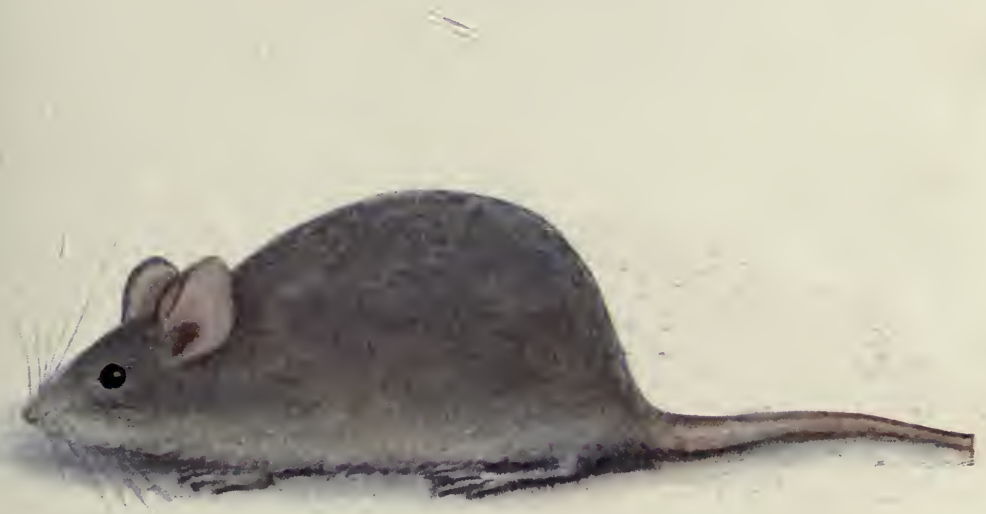

6. Silver fawn := dilute chocolate. 

periments of Miss Durham (II6) have shown that not only the particular pigment is thus constituted, but also that the intensity or degree of saturation in which it is formed can be represented as determined by similar factors.

For example the black colour may exist in the saturated condition, when the mouse is called black, or in a more dilute form, when it gives the "blue" appearance. Similarly the chocolate colour when diluted gives what fanciers call "silver-fawn." Experiment shows that the cross black $\times$ silver fawn gives exactly the same result (in $F_{1}$ and $F_{2}$ ) as blue $x$ chocolate. (See Plate II.) points.

The following are experimental results illustrating these

The allelomorphs concerned may be represented as $B$, the black determiner, $b$ the absence of $B$, leaving the colour chocolate. $D$ the dense or saturated condition of the colour, $d$, the absence of $D$, leaving the colour dilute. (In the case of the introduction of the albino we should have also to take cognizance of $C$, the presence of colour, $c$, its absence.).

The actual results may then be expressed in a tabular form.

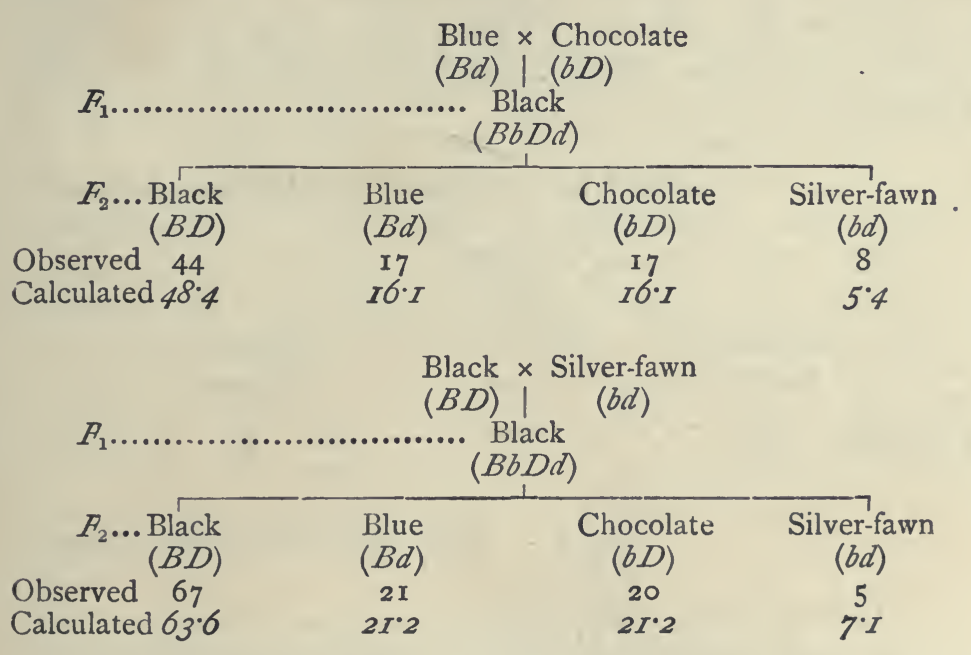

It is thus immaterial whether the factor for saturation is brought in together with the black determiner or with B. $\mathbf{H}$. 
the chocolate (more strictly, with the "absence of the black determiner"). So long as the same factors are introduced, the consequences in $F_{1}$ and the results of re-combination in $F_{2}$ are the same. But when the dilution is introduced from each side, $F_{2}$ is of course the usual 3 dominants : I recessive, thus :

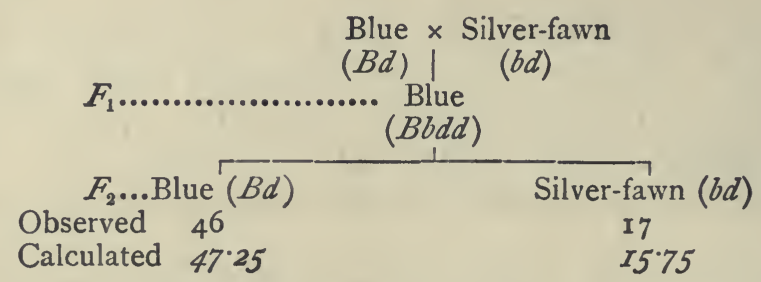

It is evident that an extracted albino cannot be carrying a determiner for a colour higher in the scale than that of its coloured parents. Moreover if the parents from which an albino is extracted are alike, and if they throw no offspring with colours other than their own. (besides the albinos), then the albinos so extracted must be all bearers of the determiner for their parental colour. If such albinos are crossed with forms of a colour lower in the scale than that borne by the albinos, $F_{1}$ must be of the colour determined by the albinos. For example, Miss Durham obtained the following:

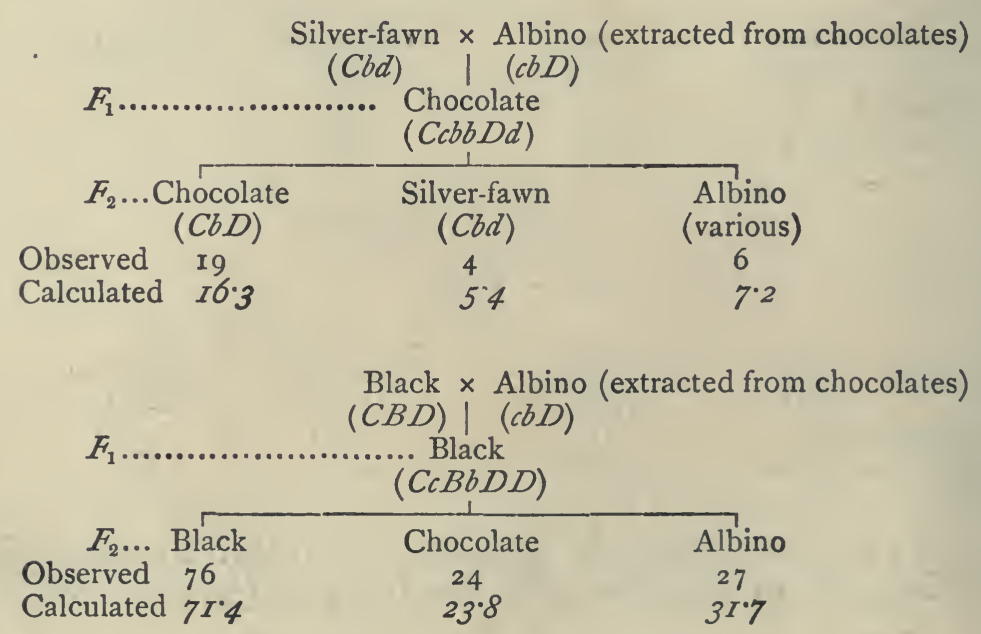


Blue $\times$ Albino (extracted from chocolates) $(C B d) \mid(c b D)$

$F_{1}$ .. Black

$(C c B b D d)$

$\begin{array}{cccccc}F_{2} \ldots \text { Black } & \text { Blue } & \text { Chocolate } & \text { Silver-fawn } & \text { Albino } \\ \text { (various) } & (C B D) & (C B d) & (C b D) & (C b d) & \text { I 2 } \\ \text { Obs. } & 33 & \text { Io } & 8 & 2 & 16 \cdot 2 \\ \text { Calc. } & 27.4 & 9 \cdot I & 9 . I & 3.0 & \end{array}$

On microscopical examination the dilution of the pigment seems to consist in a diminution in the number of the pigment granules, and not in a reduction of their size. It is interesting to notice that many different animals have varieties in which the dilution has proceeded to the same extent. For example the particular dilution of black which we call blue is known as characterizing definite varieties in both rabbits, cats and mice, not to mention other cases less certainly comparable.

As was mentioned in the description of the Andalusian fowl and its genetic features the blue colour of that breed is not comparable with the blues we have been discussing. In the mouse the blue is gametic, being a condition which can be carried by the germ cells, while in the Andalusian the blue is zygotic and depends on the collocation in one individual of one germ-cell bearing black with another which does not bear black. It is interesting, as exemplifying the danger of reasoning from analogy where genetic phenomena are concerned, that the blue-roan of cattle should not follow the same rules as the other mammalian blue varieties, but should conform rather to the Andalusian system. Of course blue roan is, even to the eye, not the homogeneous blue of the blue cat or mouse, but a mixture of white or whitish hairs among blue and black ones. Still on analogy we might have expected the blue of cattle to be capable of representation in the germ-cells, but the facts, so far as I can discover, afford no support to that supposition*.

* From such meagre evidence as I have obtained it is likely that the blue, or blue dapple, of Dachshunds and other hounds is also a heterozygous combination. As to the blue of Greyhounds and Great Danes I have no iniormation, but I suspect it to be a dilute black capable of being bred true. 


\section{Wholly Coloured and Pied Varieties.}

In the analysis of the relationship between the whole or self-coloured forms and the varieties which differ from them in having an admixture of white many curious and specific phenomena are met with. It is probably true to say that generally the whole-colour is dominant to the pied, but several examples to the contrary are already known. In all the cases yet studied the genetic properties of the pied types can be represented factorially by regarding the pattern or distribution of the colour as due to a distinct factor or to its absence. Where the whole-colour is a dominant, the presence of the factor must be taken as causing that distribution, so that in the absence of that factor the individual is pied. Conversely if the pied type is dominant the presence of the pattern-factor acts by restraining the distribution of the colour, and in the absence of the restraining factor the whole-colour prevails.

One of the clearest cases is that studied by Hurst in the rabbit, where the pattern known as "Dutch-marked" was proved to be a recessive. In the Dutch rabbit the hind quarters are coloured, for example with grey or black, while the front half of the body is white except for a patch of colour-grey or black as the case may be-surrounding the eyes and covering the ears. This pattern though fluctuating in minor respects is fairly definite and is at once distinguishable both from the self-colours and from the various other pied forms. The cross between a pure selfcolour and a Dutch gives $F_{1}$ nearly self-colour with $F_{2}$ consisting of 3 selfs : I Dutch in the ordinary way. An interesting feature is however to be observed in the fact that the heterozygotes between self and Dutch generally (? always) have some small amount of white collar, marked especially on the back behind the head. Apart from Mendelian experiment it might have been supposed that such a white mark showed that the animal contained some albino blood. Experiment shows on the contrary that the actual self-colours may be carrying albino as a recessive, while the small white mark is an indication of heterozygosis with Dutch pattern only.

The behaviour is in all respects as if the whole-colour 
pattern depended on the existence of a dominant. So also, exactly as in the case of dilution and saturation, an albino may carry either the whole-colour factor, or its absence. Consequently when a Dutch rabbit is crossed with an albino bearing self-colour, $F_{1}$ is self-colour and $F_{2}$ gives 9 self-colour : 3 Dutch : 4 albinos.

In rats there is a type of colouring which rather closely corresponds to the Dutch of rabbits. This has a "hood" of colour over the back of the head and shoulders continued down the back in a stripe which may either be entire or broken into spots. This behaves towards the self-colour just as does the Dutch in the rabbit; and just as in the rabbit the heterozygote between the self-pattern and this hooded type always has some white. Crampe $\left(8_{3}, a\right)$ observed this fact before the days of Mendelian analysis. He noticed that when a wild grey rat was crossed with an albino, $F_{1}$, as we call it, might be a real self-colour, or might have some white; but that subsequently hooded rats only occurred as offspring of those which had some white. Such rats, which are nearly whole-colour but have a little white, are known in the fancy as the "Irish" type. Experiments made by Doncaster and also by Mudge indicate that these may be again divided into two subordinate classes, distinguished by the amount and distribution of the white, and there is some evidence to show that these two types of Irish rats have distinct gametic compositions.

Though the Dutch rabbit and the hooded rat are each such clearly recognizable types, yet within these types there is great fluctuation, and it is practically certain that the fancier's ideal Dutch-pattern rabbit, with the demarcation between the colour and the white passing in a sharp transverse line across the middle of the animal, does not exist as a gametic entity. Such individuals of course come into existence from time to time, but selection will not fix their type. As Castle and MacCurdy ( 183 ) have shown in the case of rats, selection may nevertheless to a considerable extent be effective in producing hooded types with more colour, and with less colour, which are evidently gametic possibilities.

In mice no pied type exists which is quite so definite as the Dutch pattern of rabbits or the hooded type of rats, 
and by taking pains every gradation in amount of white could be found represented among fancy mice. Experience, however, soon shows that some at least of these are gametic types, while presumably others are the consequence of various heterozygous combinations. Cuénot's work with mice led him to the conclusion that in mice the several degrees of piedness are recessive to each other in the order of the amount of white, those with more white being recessive to those with less. In general terms this is a true account, but we have not found the rule to apply quite strictly even in mice, perhaps through the existence of the complication next to be considered. It should be remarked also that no general statement can be made as to dominance of self-colours over pied which is applicable to animals in general, and on a wide survey of the results of breeding many paradoxical occurrences are met with. Especially curious are the cases, by no means very rare, in which a cross between a domesticated and a wild animal, e.g. dog and wolf-has produced a partially pied offspring.

\section{Dominant Pied Types.}

So far we have been considering the behaviour of pied patterns recessive to the whole-coloured types. Though several points remain for investigation the genetic relations of these patterns are fairly clear. A remarkable complication has next to be mentioned. Both in the rabbit and the mouse it is now known that in addition to the pied types which are recessive there are others which are dominant to the whole-coloured form. Hurst has proved this for the variety of rabbit called "English pattern." This animal is white with spots of colour (black, grey, or otherwise) generally of small size on the sides of the body, a patch over the eyes, and a "chain" of spots sometimes nearly continuous down the middle of the back. In ideal specimens the spots should be of a special form and have a definite distribution, but neither of these features seems to be gametic. The English type is not now much in fashion and looks uncommon to those used to modern rabbits, but formerly it was very abundant. 
Hurst's experiments ( 160 ) showed that this pattern is an ordinary dominant to self-colour-a definite but most unexpected result.

More lately Miss Durham (I I6) has found a dominant, and doubtless analogous, pied type in mice. So far no criterion has been discovered which distinguishes this dominant pied pattern externally from the recessive ones, but in breeding the distinction is perfectly sharp. It is likely that the factor for this new dominant was brought into Miss Durham's strains by the introduction of the type called "black-eyed white," but the evidence is not perfectly clear on this point. As may be supposed, the combination of the dominant pied with the recessive pied in the same strain gave results which it was impossible to disentangle, though when each of these types was isolated the course of descent was pertectly clear. The case is interesting not merely as exemplifying a new kind of factor, but as illustrating a type of complication that may very possibly have to be reckoned with in other difficult and as yet incomprehensible sets of phenomena.

Another example of a pied condition dominant to selfcolour has been seen in our poultry experiments (22); but since in fowls some of the wholly white breeds are practically dominant in whiteness, it is only to be expected that some of the partial whites should also show dominance.

In plants, as in animals, no general rule can be laid down as to the dominance of self-coloured or parti-coloured flowers. In the Sweet Pea, for example, the old-fashioned "Painted Lady" (see Plate V) has a red standard and wings nearly white. It is thus a bi-colour type, but it is dominant to the self-coloured reds and pinks. On the other hand in Antirrhinum the self-coloured types are always dominant to the "Delilah" forms which have the lips coloured and the tube or throat white, as was first shown by de Vries. Miss Wheldale who has since worked on a large scale with Antirrhinum (303) found that for each shade of flower which exists as a self-colour, a corresponding Delilah or white-tubed type can be made which behaves to the corresponding self-colour as a simple recessive. 


\section{CHAPTER V}

\section{HEREDITY OF COLOUR--CONTINUED.}

Albinos giveng Coloured Offspring; Reversion on Crossing Various Kinds of Whites-Stocks-Orchids-PigeonsFowls-Primula.

WE have seen that albinos, both animal and vegetable, though devoid of pigments, may yet bear factors which are capable of determining the quality and distribution of colour when they meet with colour in the zygote. Particular colours may thus be due to the co-existence of several distinct determining factors, each with an independent distribution among the germ-cells. The grey of the rabbit for instance is caused by the presence (I) of a colourelement or elements; (2) of a factor which determines them to be the mixture we call grey, and not for instance, black or yellow. In the case of certain plants this analysis can be carried a step further, and the formation of colour at all in the flowers can be proved to depend on the co-existence of two complementary factors in the individual.

The first indication of this phenomenon was found in the fact that two plants each totally devoid of colour in the flowers and stems, and each breeding true to albinism, may when crossed together give purple flowers in $F_{1}$. The two white parents each contain a factor which, alone, is incapable of forming colour. Each of these factors is independently transmitted in gametogenesis, and thus in $F_{2}$ the ratio of coloured individuals to whites is $9: 7$. This proportion depends on the fact that a series of 16 individuals is necessary to exhibit all the possible combinations of germ-cells, for, as in any example of hybridisation involving two pairs of allelomorphs, there will be tour types of female cell and four types of male cell produced by $F_{1}$. Of these 
sixteen individuals 9 will contain both the dominant or present factors, while of the remaining 7 individuals, 3 will contain the one dominant, 3 will contain the other, and I will contain neither. There will therefore be 9 which are coloured and 7 which are albino. In the diagrammatic scheme $C$ and $R$ are the symbols representing the two complementary factors, $c$ and $r$ being their respective allelomorphic absences.

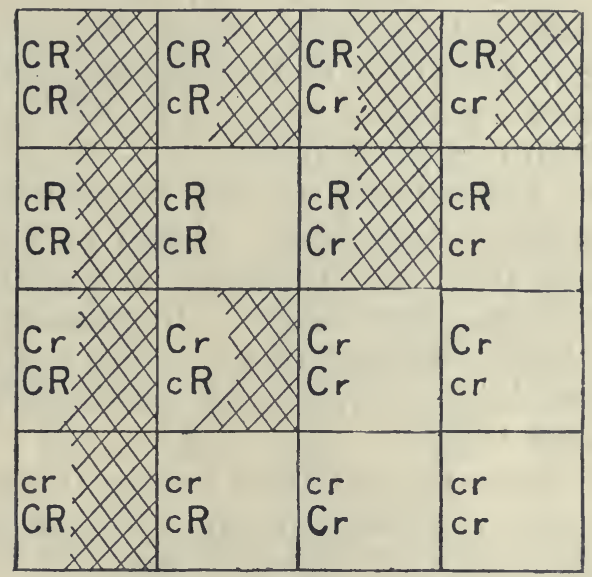

Fig. r6. Composition of the 9 coloured and 7 albıno offspring in $F_{2}$ from the cross between albino $C r$ with albino $c R$, showing the ratio 9 coloured : 7 albinos.

Two examples of this phenomenon have been studied in detail. The first is that provided by the Sweet Pea (Lathyrus odoratus) which has formed the subject of experiments carried on jointly by Mr Punnett and myself for some years.

The work was begun by crossing two white sweet peas belonging to the variety Emily Henderson. These plants were alike in every respect so far as could be perceived, excepting that the shapes of their pollen grains differed, the one having the normal long pollen grains of the species, while the other had roundish grains. The object of the experiment was to trace the descent of the pollen-character and at the beginning no question of colour was entertained. When $F_{1}$ was grown however it was clear that here was a 
remarkable opportunity of studying a reversion in colour due to crossing, for these plants instead of being white were purple like the wild Sicilian plant from which our cultivated sweet peas are descended.

The facts respecting the colour inheritance will now be given. With regard to the pollen-shape it must suffice at present to state that the long shape is a dominant and the round a recessive. The details as to the distribution of these two shapes among the $F_{2}$ individuals, which are interesting and have greatly aided the development of genetic theory, are given in the chapter dealing with the phenomenon of "gametic coupling." The present section is concerned with colour only.

When the reversionary $F_{1}$ generation was first seen its nature was entirely mysterious. When $F_{2}$ was raised from these $F_{1}$ plants the series consisted of a mixture of plants, some coloured and some white. In some cases the series of coloured plants consisted of two kinds only, purples like $F_{1}$, and a red bicolour type, the well-known old variety called "Painted Lady."

In other cases $F_{2}$ contained besides those mentioned, two quite distinct additional types of purples and two corresponding additional types of reds.

The phenomena, though, as will be shown, in reality very simple, presented superficially an appearance of great complexity. Further difficulties were met with in the fact which was soon discovered, that the cross between longpollened whites and round-pollened whites does not always give the coloured types, but may result in ordinary whites only.

It is unnecessary to go through the long series of steps by which the analysis of the phenomena was carried out. The meaning of the facts is now perfectly clear and they can all be arranged in one consistent scheme.

Of the two white parents originally used the one possessed one of the two factors we have called $C$ and $R$, the other introducing the other complementary factor. The meeting of these two elements produces colour in the flower. If no other epistatic factor is present their colour is red. As a matter of experiment however one of the parents, proved afterwards to be that which had the long pollen, did carry 
such an epistatic factor whose property is to make the colour purple, just as the factor $B$, in the mouse, makes the coat colour black. $F_{1}$ was therefore purple, and $F_{2}$ consisted of 27 purples : 9 reds : 28 whites, as shown below. The factor which determines the colour to be purple is represented as $B$, the blue factor.

The diagram (Fig. I7) exhibits in a tabular form the composition of the various $F_{2}$ plants. The distribution of the

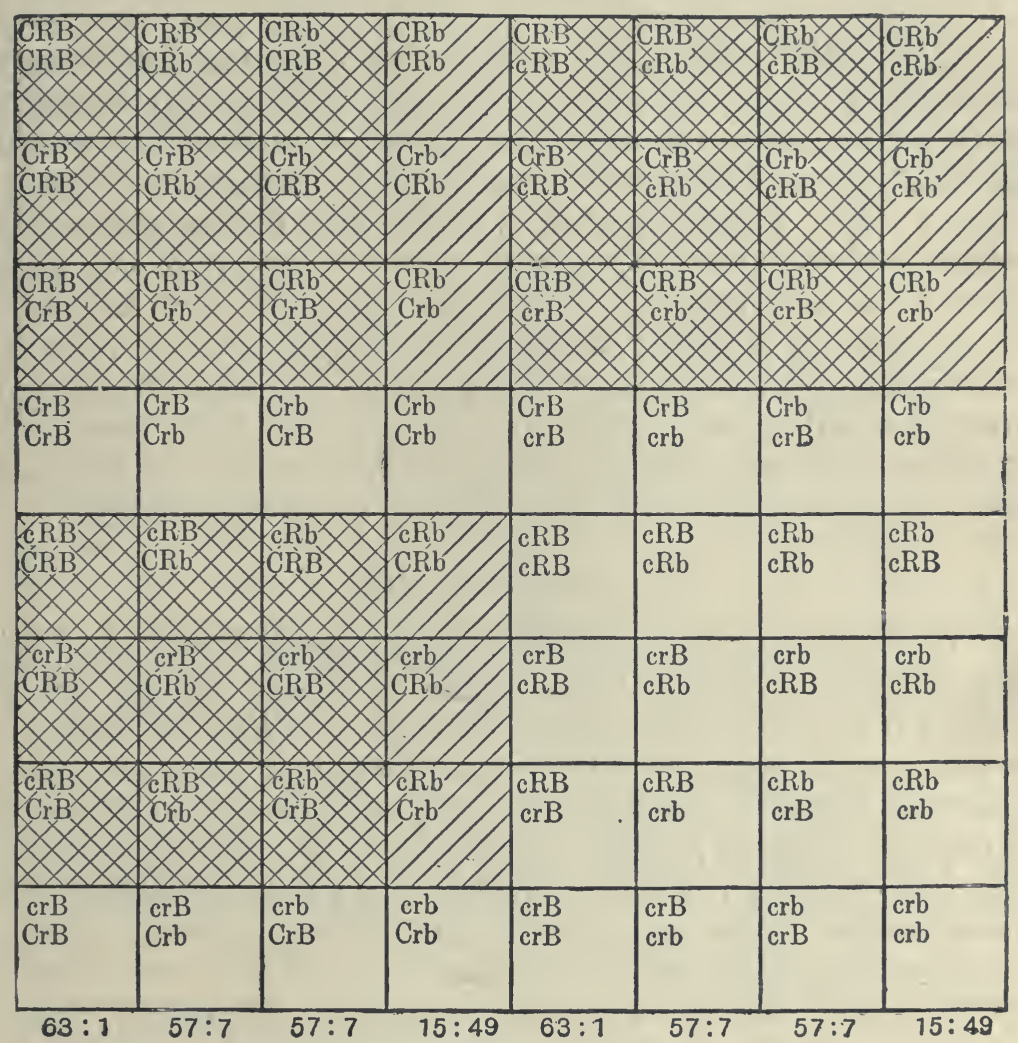

Fig. 17. The $F_{\text {a }}$ family from the cross white Emily Henderson Sweet Pea, long pollen $\times$ white ditto, round pollen. The 27 cross-hatched squares represent purple plants, all containing $B$. The 9 singlehatched squares without $B$ are the reds. The 27 squares lacking either $C$, or $R$, or both, are all whites of various compositions. The numbers at the foot show the ratio of long to round pollens in the types of individuals represented in the columns above. 
pollen-shapes among them will be the subject of further consideration (Chap. Ix).

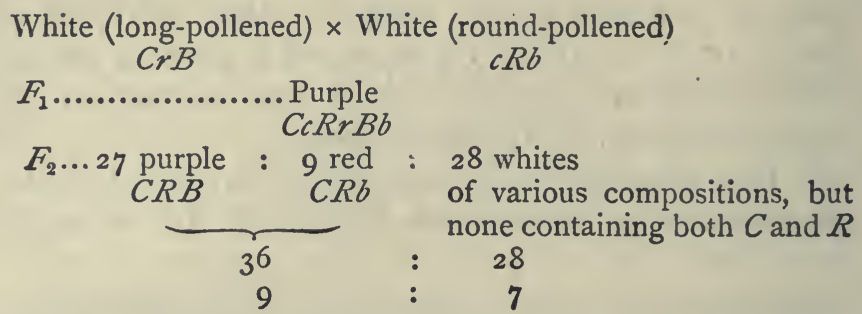

When these facts are made out we have no longer any difficulty in understanding how it can be that various results may follow the union of two white plants which would breed true to whiteness in perpetuity if left to self-fertilisation. For example, white $x$ white will always give only whites unless one of the complementary factors $C$ and $R$ is present in each parent. Whites of the form $C r b$ and $c R b$ when crossed together will give reds only, with 9 reds to 7 whites in $F_{2}$. Whites of the form $C r B$ and $c R B$ crossed together will give purple in $F_{1}$, and in $F_{2} 9$ purples : 7 whites, no reds being present in such a family because the blue factor $B$ occurs on each side of the parentage.

\section{Extracted Whites.}

We have so far represented each type of white used for a parent as if it were homozygous for those factors, but of course it may be heterozygous for one or more of them, in which case $F_{1}$ will contain a mixture of types. For example, $C C r r B b \times c c R R b b$ will give in $F_{1}$ purples and reds in equal numbers. Ccrrbb $\times c c R r b b$ will give in $F_{1}$ a family consisting of 3 whites : I red. Various other combinations are possible and most of them have now been met with in the course of experiment.

The fact that the extracted whites, that is to say, those which appear in $F_{2}$, have not in all cases the same properties as the original parental types is readily intelligible. In the early stages of the research it seemed strange that whereas the original long-pollened white crossed with the round-pollened white gave a coloured result, it was possible and indeed more usual to find whites exclusively produced by the cross of two extracted $F_{2}$ whites, long-pollened 
and round-pollened respectively, which outwardly were indistinguishable from the two original parents. As we now know, by the redistribution of factors a great variety of whites is in reality produced in $F_{2}$, and only those pairs which bear the complementary factors $C$ and $R$ can give coloured offspring. This apparent dissimilarity between the behaviour of the extracted forms and that of the pure types from which they are derived has been adduced as being inconsistent with Mendelian principles. When however the factorial composition of the various individuals is correctly represented it is evident that this multiplicity of composition in $F_{2}$ is what must be expected, and there is abundant evidence that such complications exist in all the plants and animals that have been experimentally studied on an adequate scale*.

\section{Subordinate Types in $F_{2}$.}

We have now to consider the meaning of the appearance of subordinate types in the purple and in the red classes which is a feature of some $F_{2}$ families. Of these there are frequently two. The first is characterized by having the wing-petals much more fully coloured than those of the $F_{1}$ purple, or of the Painted Lady. These distinctions are due to the operation of an epistatic factor for the lighter wing-colour. When this factor is absent the wings are dark, and the flower, if a purple, has the wings deep purple

* Factors which may thus exist without making their presence visible have been named by Tschermak "Cryptomeres." The term is open to the objection which zoologists especially will feel, that it may cause confusion owing to the fact that the series of words containing "mere" are now universally understood to refer to phenomena of division-or Meristic features. When the study of that part of genetics which is concerned with meristic variation is more fully developed this confusion will be aggravated. For the present moreover the expression "factor," qualified, if necessary, as unseen, seems sufficiently precise.

The words "latent" and "latency" must be applied in these cases with great care, if at all. Various writers have, for instance, spoken of the purple colour as "latent" in a white flower. This description is quite misleading, for the colour need not be present in such plants. The term "latent" is only admissible in application to the factors (as here, to the factor which can turn the colouring matters purple) not to the characters, except loosely to those which are actually present but hidden owing to the operation of some epistatic factor. 
instead of a light purplish blue. Similarly reds having the factor for light-wing are Painted Lady, if without it are of the type called Miss Hunt, with wings of a full pink.

Another pair of subordinate classes is due to the absence of a factor for whole-colour. When this factor is absent the purples become purple picotees, with an edging of colour on a white ground, and the reds become what we have called "tinged whites"; a type with a little pink in the standard only. The purple picotees, when the bud is just opening, have a purple edge to the standard and a blue edge to the wings, these edges being all that remains of the colours proper to these parts in whole-coloured purple. The "tinged white," which is really a red picotee, has the red of the Painted Lady standard reduced to a few pink lines in the centre of the standard. Both these picotee forms, like the picotees of tulips and most other flowers, acquire a more general flush of colour as the flower ages. These various types are illustrated in the coloured plate (Plate III), from which their peculiarities will be easily appreciated. Among the picotees of course there are two types of purples and two types of reds, differing in the presence or absence of the factor for light wing, but in forms so nearly white these differences are evasive, and we have not found it possible to classify the picotee class in respect of these distinctions.

It is to be observed that the simultaneous appearance of the subordinate types in each of the classes is a characteristic and necessary feature of the normal Mendelian processes. It would be impossible, for instance, to make a plant which could throw purple picotees in $F_{2}$ without red picotees, if the family contain both purples and reds. Conversely, if the plants throw purples and reds, and also whole-colours and picotees, then both kinds must be represented in both classes. In any examination of $F_{2}$ families, so soon as one pair of large classes can be recognized and one subordinate type perceived in one of them, search should be made in the other class in order to find the corresponding subordinate type in it also. If in one class a type exists which is never to be found in the other class though a long series has been examined, some complication is to be suspected, but whether the phenomenon is due to 
-

$\checkmark$ 

$\equiv$
$\frac{\pi}{0}$
$\frac{\pi}{\alpha}$
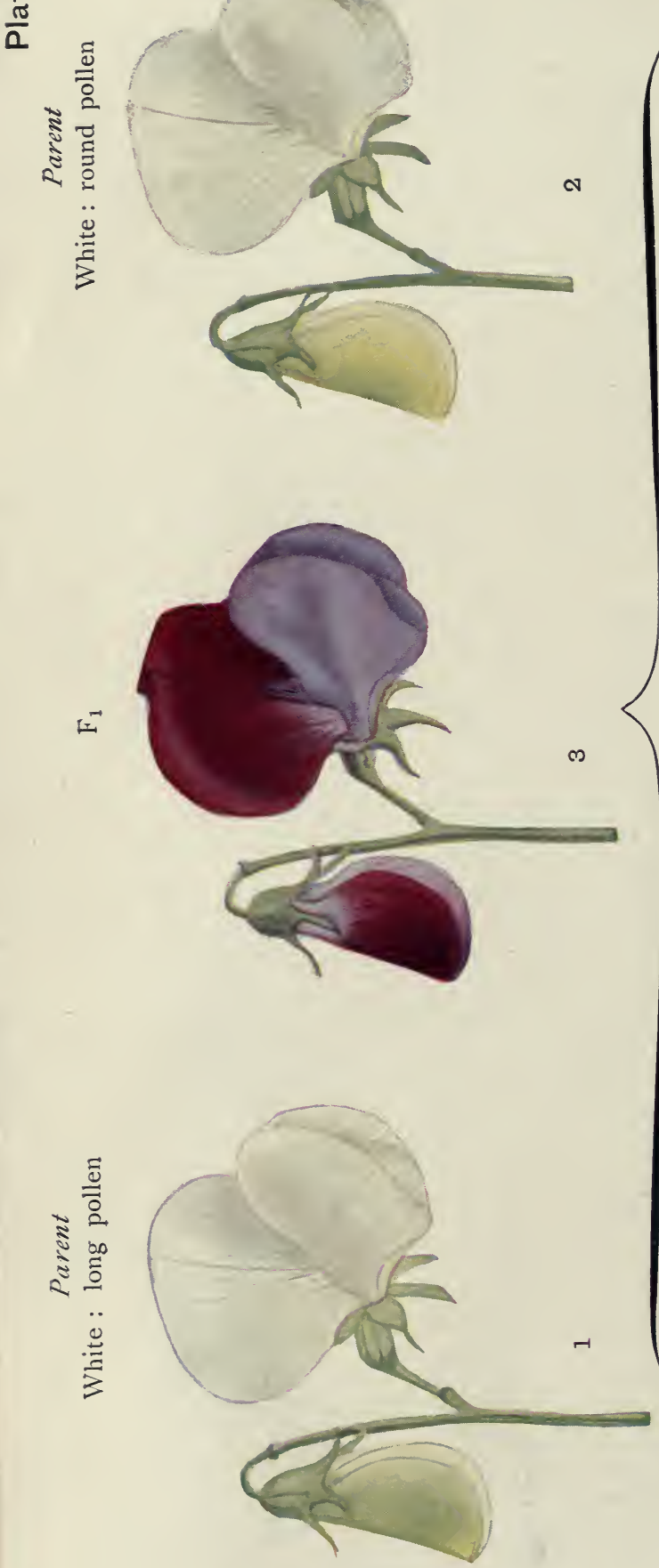
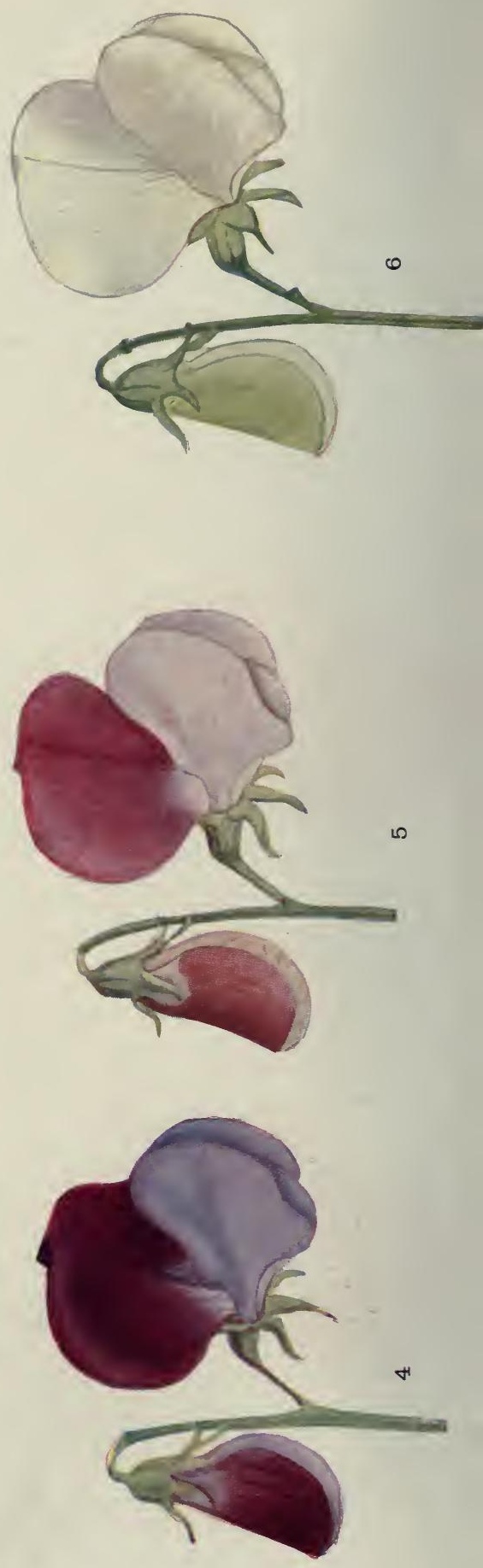

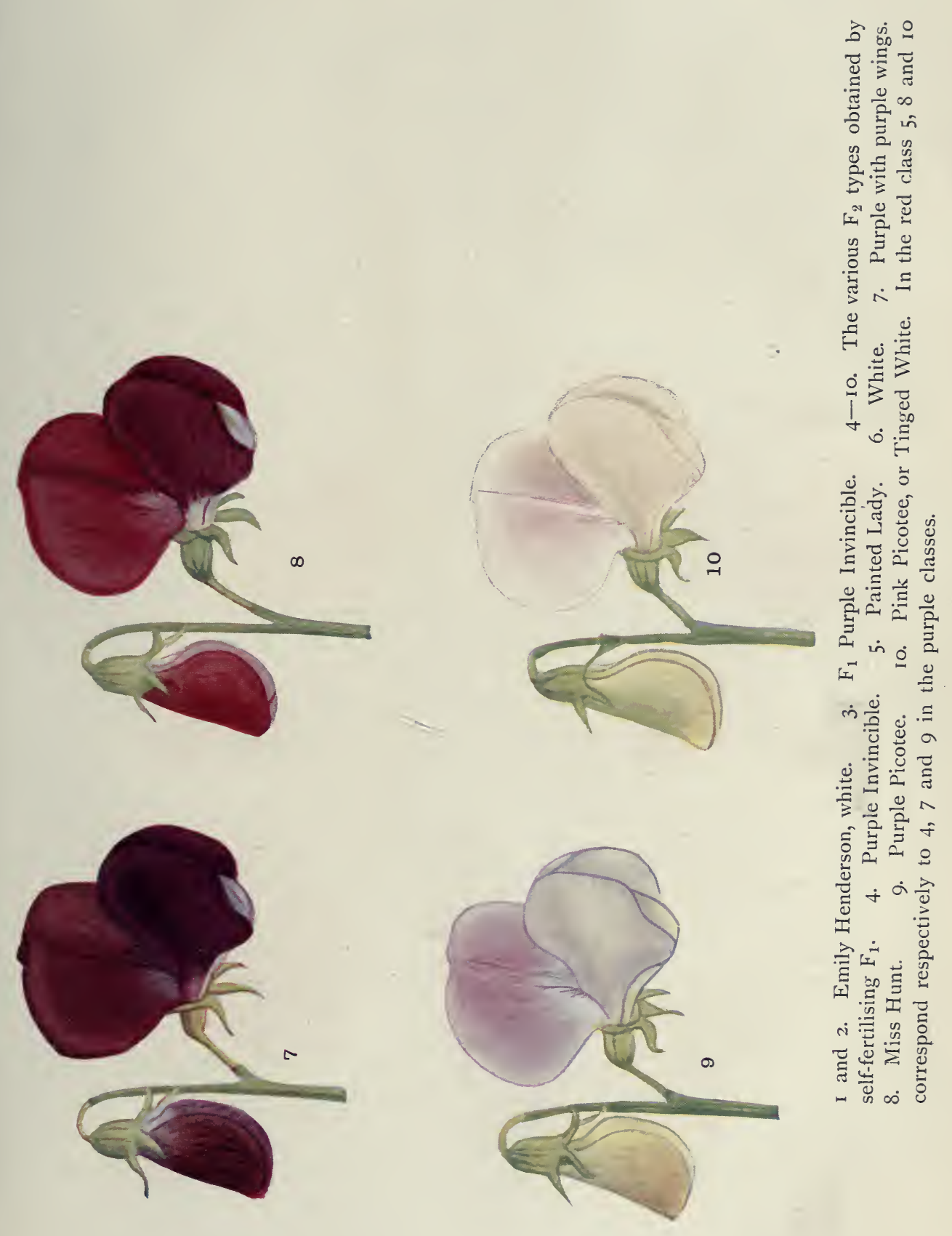

coupling or to some other cause can only be decided by the circumstances of the special case (see, for example, the case of Stocks, in which plants with hoary leaves are wanting in the classes without sap-colour in the flower (Chap. virI), and the distribution of the hooded standard among Sweet Peas (Chap. IX)).

\section{Colours of Stocks (Matthiola).}

The experiments of Miss E. R. Saunders have revealed a closely similar inter-relationship between the various colour-types in Stocks. There also coloured $F_{1}$ is produced by crossing two types both destitute of sap-colour. The two types giving this result are white and cream. The pale yellow of the cream is due not to sap-colour but to chromoplasts, and thus the two types are as regards sapcolour both albinos. In this case also $F_{1}$ has the common purple flower which we may regard as reversionary*, and in $F_{2}$ there appear both purples and reds. These results can be represented exactly like those in the Sweet Pea case. The sap-colour is due to the meeting of complementary factors, $C$ and $R$, derived from the two parents respectively. In the absence of the "blue" factor $B$, the colour produced by $C R$ is red, but when $B$ is present the colour is purple. As a matter of fact it can easily be proved that $B$ is introduced by the original white parent; for when a red is crossed with the white, $F_{1}$ is purple, but when a red is crossed with a cream, $F_{1}$ is red, showing that the cream is devoid of $B$, but that the white possesses it.

The numbers in $F_{2}$ are also on the plan described for the Sweet Pea. The total of sap-coloured plants to the total of non-sap-coloured (whites + creams) averages $9: 7$, and when the proportion of purples to reds is also represented the ratio is

27 purples : 9 reds : 28 whites and creams.

Just as in the Sweet Pea again there is a series of subordinate types among the purples in $F_{2}$, and a corresponding series of types among the reds. For example, there is a dilute form of purple which the horticulturists

* As will be described later, in this case when both parents have glabrous leaves, $F_{1}$ reverts to the hoary leaves of the original type. 
call "plum," and a dilute form of red which is known as "copper," \&c. Any of these colours may be on a white ground, or on a cream ground*.

\section{Albino Orchids giving Coloured $F_{1}$.}

In Orchids a series of facts has been observed which are exactly comparable with those described in the Sweet Peas and Stocks. There are several albino orchids in the genus Cypripedium. Of these some when crossed together give coloured offspring, and others crossed together give albinos only. Mr Hurst $\uparrow$ most kindly furnished me with a complete list of the results of crossing albino Orchids so far as they have been published. This list covers a very wide range of cases to which no reference can be made here. As regards Cypripedium most of the experiments were made by $\mathrm{Mr} \mathrm{N}$. C. Cookson of Wylam. I am much indebted to him and to his gardener $\mathrm{Mr} \mathrm{H}$. J. Chapman for information about them. For example:

C. callosum Sanderae $\times$ C. bellatulum album gives all offspring coloured ( 25 plants bred by $\mathrm{Mr}$ Cookson).

C. Lawrenceanum Hyeanum $\times C$. bellatulum album, all offspring coloured (Cookson).

On the other hand

C. Lawrenceanum Hyeanum $\times$ C. callosum Sanderae gives offspring all albinos (viz. C. "Maudiae") (raised by Messrs Charlesworth and independently by Baron Schröder).

If therefore we suppose that bellatulum album is carrying one of the complementary factors, say $C$, and that the other factor $R$ is present in callosum Sanderae and in Lawrenceanum Hyeanum, the results are correctly and consistently represented.

As regards the behaviour of the pure types however there is one occurrence to be recorded which cannot be explained. Of the albinos named above, one, callosum Sanderae has been self-fertilised and gave albinos only, as must be expected.

* Though all the various combinations occur it is probable that the distribution of white and cream is by no means a simple one, but the analysis of the inter-relationship between white and cream has not yet been worked out completely.

$\dagger$ See also Hurst, Gard. Chron. Feb. 6, 1909, p. 81. 
Larrenceanum Hyeanum when selfed, gave 14 albinos, but in addition, one coloured plant, which Mr Cookson tells me cannot be thought to have resulted from error. Such an occurrence is as yet unaccountable, but otherwise all is clear.

In addition to the cases given above there are many in which $C$. insigne Sanderianum and insigne Sanderae were used, but $\mathrm{Mr}$ Hurst tells me that of these the latter is certainly a tinged type, while the former has some dark hairs on its petals which may contain sap-colour. Some of the crosses with these types gave reversionary offspring, and others gave various whites.

The genus Cattleya provides another list of comparable cases. For instance

C. Mossiae Wageneri $\times$ C. Gaskelliana alba gave 4 albinos (Hye).

" $\quad \times$ C. intermedia alba gave I4 albinos (Holford).

C. Schröderae alba $\times$ C. intermedia alba gave coloured offspring (Cookson).

C. Gaskelliana alba $\times$ C. Harrisoniana alba gave 4 coloured plants (Thwaites).

" $\quad$ " $\quad \times$ C. Warneri alba gave 5 albinos and 2 coloured plants (Peeters).

$\times C$. Mendelii alba gave $2 F_{1}$ pink flush (Thwaites, Gard. Chron. I9IO, I. p. 62).

From these statements it may with great probability be inferred that the albinism of Mossiae Wageneri, Gaskelliana alba and intermedia alba is due to the absence of one factor (say $C$ ); that in Harrisoniana alba and Schröderae alba it is the other complementary factor which is wanting (say $R$ ); and that Warneri alba is heterozygous for the presence of one of them (being $C c$, on this scheme). It is to be hoped that some Orchid grower will make the various unknown combinations and extend the series.

There are, besides the cases enumerated, many instances in Cypripedium, Cattleya, and other genera, of various tinged types breeding true when self-fertilised and giving reversionary coloured offspring when crossed.

B. $\mathrm{H}$. 


\section{The Case of Antirrhinum (Snapdragon).}

The experiments of Miss Wheldale (303) on Antirrhinum show that in that plant the appearance of the red and magenta flower-colours is similarly dependent on the presence of two complementary factors. In one respect, however, this case differs from those which we have been considering; for whereas in them either of the two colourfactors, spoken of as $C$ and $R$, might be present in a truly white-flowered plant, it appears that in Antirrhinum one of the two factors produces either a bright yellow or a very pale "ivory" yellow in the flowers. The other complementary factor may be present in actual whites. Hence magentas or reds may be produced by the cross between white and yellow, or white and ivory, but hitherto crosses between actual whites have not given coloured offspring. If in the Sweet Pea for instance the presence of $C$ caused the flowers to be ivory instead of white the case would be like that of Antirrhinum. At first sight it might be supposed that the phenomenon seen in Matthiola (Stocks), where white $\times$ cream gives $F_{1}$ with anthocyan colours, was more strictly parallel, but in the Stock the cream is a plastid-colour, whereas in Antirrhinum the ivory is a true sap-colour.

It is a noticeable and rather unexpected feature in the case of Antirrhinum that the pale ivory is due to a factor epistatic on the bright yellow. The ivory is so pale that it might on casual examination be described as white; never theless it is definitely and completely dominant to the bright yellow type.

Ivory crossed with white gives $F_{1}$ magenta-red but yellow crossed with the same white gives $F_{1}$ crimson-red. The distinction between the two kinds of red is due to the factor which turns bright yellow into ivory.

A comparison between the Sweet Peas or Stocks on the one hand and Antirrhinum on the other, may be represented in tabular form. Using a terminology applicable to both cases let us designate the factors thus* :

Presence and absence of the "ferment." $F, f$.

$" \quad " \quad$ " $\quad$ " $\quad$ " $\quad$ "Chromogen." $C, c$.

* In the previous discussion of the Sweet Pea the factors $F$ and $E$ are designated $R$ and $B$ respectively. 
The following appearances will then be produced in the flowers by the various combinations.

$\begin{array}{ll}\text { Szeret Pea } & \text { Antirrhinum } \\ F c e \text { white } & \text { white } \\ f C e \text { white } & \text { yellow } \\ f c E \text { white } & \text { white } \\ f C E \text { white } & \text { ivory } \\ F C e \text { red } & \text { crimson-red } \\ F C E \text { purple } & \text { magenta-red. }\end{array}$

As regards the distribution of the pigments over the parts of the flower, many facts of importance have been discovered (see original papers). Several distinct factors are involved, and by careful analysis Miss Wheldale has been able to show that almost all the heterozygotes can be recognized and distinguished from the corresponding homozygotes. The degree to which it has been found possible to effect the genetic analysis of the types in Antirrhinum is very remarkable. At first sight the series of forms appears to consist of numberless intergrading tints, but by continued experiment Miss Wheldale has succeeded in disentangling the various genetic combinations and showing the factorial composition of almost all. Even as regards the inheritance of striping an approximate factorial representation has been worked out, though from the fact that striped types may occasionally have self-coloured offspring this part of the analysis must still remain incomplete.

Needless to say there is as yet no system of colourreproduction, whether by lithography or photography, which can be applied with accuracy sufficient to represent the various tones of colour, and for the most part the work is unpublished.

\section{Reversion on Crossing: the Nature of Variation.}

From the facts now before us a clear conception may be formed of the meaning of reversion occurring in consequence of a cross. This phenomenon is due to the meeting together of factors which are complementary to each other, and must be together present in order that the original or reversionary type may reappear. We have seen how in the mouse or rabbit the wild grey type is reproduced when 
a black is crossed with an albino bearing the factor $G$, greyness. Here the reversion is due to the meeting of this factor with the colour-element present in the black animal. The case of the Sweet Pea or the Stock, when two colourless types crossed together produce coloured $F_{1}$ is of exactly the same nature, except in so far as both of the complementary factors are in this case imperceptible until they meet each other. The occurrence of the ratio 9 coloured viz. reversionary : 7 uncoloured is a proof of the correctness of this mode of representing the facts.

When reversion is correctly represented we are led on to form some mental picture of the essential nature of a variation. It must occur by the omission or by the introduction of a factor.

\section{Reversion in Pigeons.}

In connection with the reversion produced by crossing two white individuals the famous case of pigeons may be considered, though in that example one parent was coloured.

Darwin was the first to draw attention prominently to such phenomena, and the experiments which he made consequent on a hint given by Boitard and Corbié have long been classical. In these experiments the blue colour with black bars resembling the colour of the wild rock pigeon (Columba livia) was reproduced as a result of crossing birds quite free from such characteristics. Darwin's experiments were very complicated*. He was concerned only with the question of origin, and to provide a qualitative demonstration that the peculiarities of the blue rock pigeon could be reproduced from modern varieties which had ostensibly lost them.

Mr Staples-Browne (255) has recently investigated the matter afresh in a simpler case. He crossed a Black Barb with a White Fantail, producing $F_{1}$ black with some white feathers. From such $F_{1}$ birds $F_{2}$ was produced, consisting of

Blacks.

Blacks
with white
feathers.

Blues.

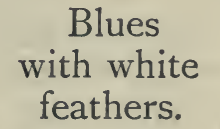

Whites.

* Animals and Plants, ed. 2, 1885, I. p. 207. 
The numbers were not sufficient to give the ratio definitely, but it was established by subsequent breeding that the whites bred true; that the blues did not throw blacks; but some at least of the blacks could throw blues. The blues breed true except in so far as they may throw whites*.

We have then the fact that a reversionary type was produced, but that it did not appear till $F_{2}$. The meaning of this is obviously that the blue cannot appear in $F_{1}$ because black is epistatic to it. The elements necessary to produce blue are all present in $F_{1}$, but in it black also is present which conceals the blue. After the re-combinations, some $F_{2}$ forms contain the blue group of elements without black, and these are therefore blue. We do not know in this case that the Black Barb itself did not contain all that is necessary to the production of blue, and it is thus possible that the reversion on crossing may here be only a phenomenon of re-combination. This could only be decided by a long statistical analysis.

Genetic Properties of White Types which are not Albinos (Forvls and Primula). Dominant and Recessive Whites.

Albinism, namely total deficiency of colour, as we have seen, is always recessive to the presence of colourt. In contrast with real albinos, the white forms which contain pigment in some part of their organisation show various phenomena of colour-inheritance. Among both animals and plants such types are known. For example, races of pigs, cats, mice, cattle, dogs and fowls exist in which the skin, hair or feathers are white, or nearly so, though the eye is pigmented. The "white" races of mankind perhaps belong to the same category in some respects. Our knowledge of the genetic behaviour of most of these types as regards pigmentation is still fragmentary, but what little is known points to the existence of considerable complications.

Respecting white poultry, however, several features have been ascertained which are interesting. In them we meet

* Apparently only those blacks and blues which had some white feathers were capable of throwing whites.

t In Axolotl (see p. 43) an alleged exception is recorded. 
a series of facts which perhaps, better than any other, illustrate the impossibility of understanding the significance of genetic phenomena without minute individual analysis.

In poultry we know at least four different sorts of white plumage, each with its own special properties. As far as external appearances of the adults go there is little or nothing which would lead an observer to suspect that the genetic powers or capabilities of these four types were entirely distinct in respect of colour.

In the first type the white acts as a dominant. Generally speaking when a purely white variety such as White Leghorn is crossed with a coloured variety, such as Indian Game or Brown Leghorn, $F_{1}$ is, in the newly hatched condition, white with a few specks of black. The black then present may be confined to a single hair, but we have met very few instances of total absence of such a speck or "tick" as it is called. As the down is replaced by feathers similar ticks of black and brown appear in them. The position and amount of the ticks in the feathers bear no obvious relation to the down-ticks. These $F_{1}$ birds, however, at all ages are substantially white birds, the amount of colour though definite, being almost always insignificant. Exceptions occur in the case of cocks, which sometimes have enough red in the wings to bring them into the category called by fanciers "pile," though they are of the usual white in the downy stage.

We may thus speak of White Leghorn as a dominant white. $F_{2}$ from such an $F_{1}$ generation consists of a great variety of colours, the relations of which have not been worked out.

But among the derivatives from various matings* which Mr Punnett and I have carried out white birds have been produced which are, so far as their whiteness is concerned, simple recessives to colour. Sometimes, but not always, they have ticks of grey colour. These recessive whites breed true to whiteness as the White Leghorns do, and no one looking at them would doubt for a moment that they were moderate specimens of that breed.

In addition to these the white of White Rosecomb

* These birds arose from a mixture of Brown Leghorn, White Dorking and Indian Game. See Rep. Evol. Ctee, III. p. 19. 

Plate IV

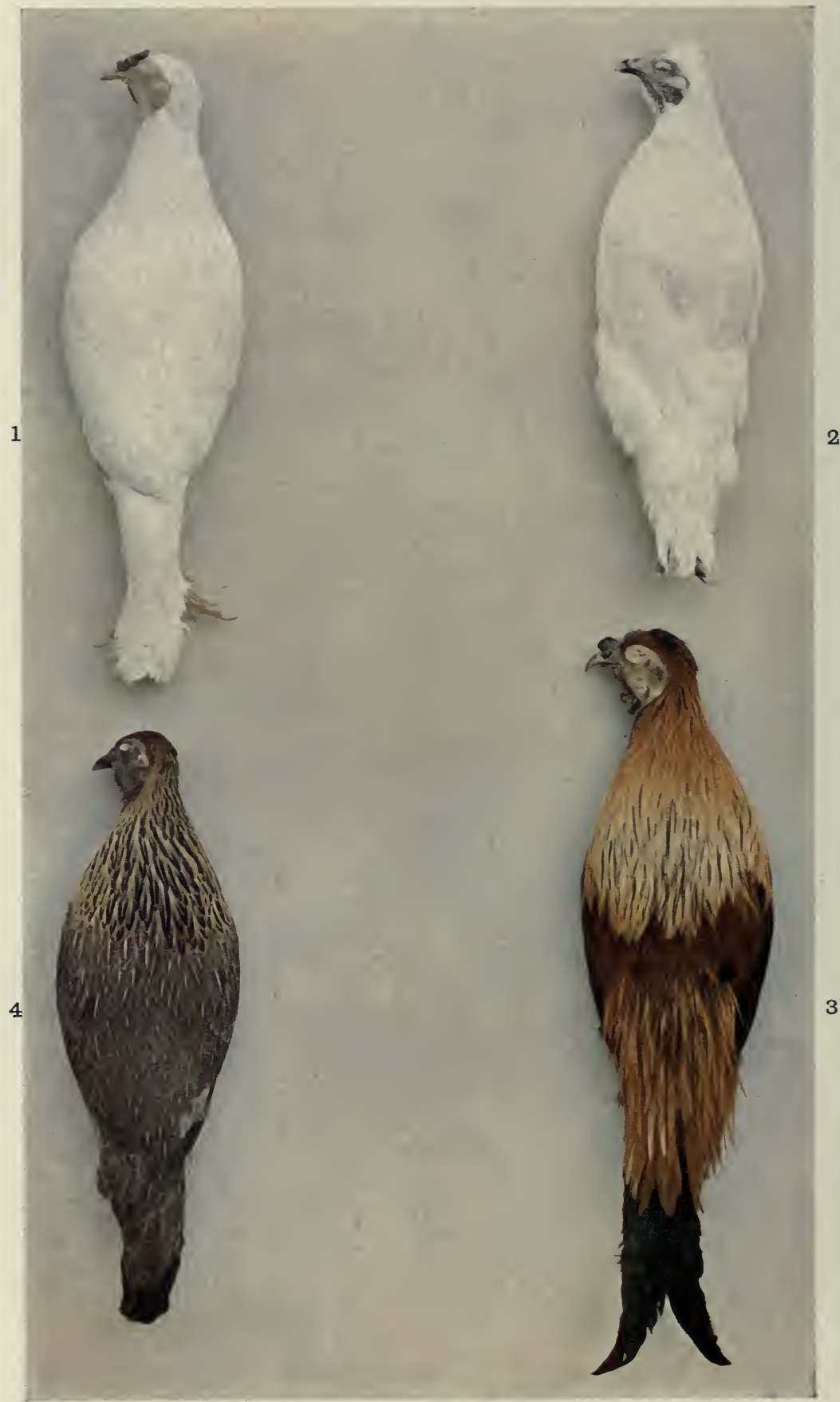

I. Hen of Recessive White strain.

2. Hen of Silky breed.

3 and 4 . Cock and hen, $F_{1}$ from the cross between Recessive White hen $\times$ Silky cock. 
Bantams is also a recessive. Strictly speaking perhaps this breed should not be called white. Though appearing white on casual inspection, more careful examination always, I believe, reveals the presence of one or more ticks of grey colour. These flecks of grey colour may be extremely minute, but in our experience are never absent. The down, too, of the breed is of a faint bluish or smoky colour, though there is no such smokiness in the adult plumage.

Lastly we have to consider the white of White Silky fowls, which is again a recessive to colour. The adult Silky is pure white as a rule, but like the White Rosecomb, the down of the chickens has some colour usually (though perhaps not always). The colour in this case however is buff, not blue or black, and it occurs on the sides of the head, in the region of the pale stripes of striped chickens, and on the rump.

To resume: there are four kinds of whites.

(I) Dominant white of White Leghorns. A pure white.

(2) Recessive white of our own derivative strain.

(3) Recessive white of Rosecomb Bantams.

(4) Recessive white of Silky fowls.

Crossed with coloured breeds such as Brown Leghorn

(1) gives white birds with a little colour.

$(2),(3)$ and (4) give wholly coloured birds with the pigmentation of the plumage as fully developed as in any coloured breeds.

The next point concerns the relations of the various Recessive whites with each other, and is of considerable interest. Our experiments have shown that the cross between Recessive whites (2) and the Silky (4) gives exclusively whole-coloured birds in $F_{1}$. In $F_{2}$ such birds give 9 whole-coloured to 7 whites. Of the 7 whites some have white down like the Recessive white (2), while others have buff in the down like the Silky (4)*. The appearance of coloured $F_{1}$ from two whites, with 9 coloured $: 7$ white in $F_{2}$, is obviously a phenomenon comparable with that which has been described in the Sweet Peas and Stocks. The two white types ( 2 and 4 ) evidently contain complementary

* The numerical relations of these two forms are not yet clear. 
factors which must be together present in the zygote in order that colour should be produced. The colour of the $F_{1}$ birds is practically Black-red, approaching closely to the plumage of Gallus bankiva (see Plate IV).

It might a priori be expected that since the White Rosecomb (3) has very distinct though light pigmentation in its down, a cross between this and the Silky (4) would be more likely to give a coloured $F_{1}$. This, nevertheless, is not the case. The cross between these two breeds is an ordinary white, showing that no reliance whatever can be placed on such considerations, and that experiment alone can decide what properties the several types possess.

The whiteness of the recessive whites is thus due to the absence of one of the elements needed for the development of colour. Analytical experiments show on the contrary that the white of the dominant white ( 1 ) is not due essentially to the absence of an element but to the presence of a factor which prevents the development of colour. Thus, if $X$ and $Y$ are the two complementary factors which produce colour, the one recessive white is $X y$ and the other is $x Y$. The dominant white (I) has the suppressing factor $S$, and as the result of many experiments it appears that the individuals of that breed have various compositions in respect of $X$ and $Y$, though always homozygous for $S$.

Hence it follows that when Dominant white (I) is crossed with Recessive white $(2), F_{1}$ is white; but $F_{2}$ may, and in our experience always does, give some coloured birds. The proportion in which the coloured $F_{2}$ birds appear must of course depend on the composition of the individual dominant white which was originally introduced, and various ratios occur. The numbers obtained show that of the dominant whites some are $X x Y y S S$, another contained only one of the colour-factors and so may be written $X x y y S S$. Probably all the possible combinations occur, but the experiment has not been carried far enough to prove that they do*.

* The difference in size between Recessive white (2) and the White Rosecomb is so considerable that the direct cross between these two cannot be made. But $F_{1}$ from White Rosecomb $\times$ Silky has been crossed with (2) and the offspring were in about equal numbers whites and browns. There is therefore no reasonable doubt that the factor carried by the Silky and by the Rosecomb is the same. Either both have $X$, or both $Y$. 
The facts thus take their places in a consistent scheme, and when the factors are correctly determined, all the observed properties of the several types can be represented. Some curious consequences follow which would have appeared very surprising both to evolutionists and to fanciers a few years ago. For example, if a pair of coloured birds throw very few whites the chances are large that these whites if mated together will breed true. On the other hand if a pair of coloured birds throw many whites, the chances are that such whites if bred together will have some coloured offspring, and it is not very unlikely that all their offspring will be coloured! For in the first case where the whites are few, they are probably $I$ in 4 , indicating that both parents are homozygous in either $X$ or $Y$, say $X$, and heterozygous in respect of the other factor $Y$. Therefore all the whites which come will be carrying $X$ and none will have $Y$. Therefore they will breed true to whiteness if mated together.

In the second case however, where the whites are many, they are probably 7 whites : 9 coloured. In such a case the parents must be heterozygous both in $X$ and in $Y$. Consequently some of the whites will be bearing $X$ and others $Y$, and there will be a good chance that if such whites are bred together these two complementary factors will again come into combination and coloured offspring be produced.

Among plants only one example of dominance of white flower-colour over the ordinary sap-colours has yet been investigated. This occurs in Primula Sinensis. In this species colour may be present either in the flowers, or in the stems and leaves, or in both. When white flowers occur on stems green and devoid of pigment, the white is recessive as in any other case of total albinism. There are however white varieties which have more or less red in the stems, and these when crossed with varieties having coloured flowers give $F_{1}$ with flowers white or nearly so. There is almost but not quite always a slight tinge of magenta-red in the petals of such $F_{1}$ plants, and the intensity of this colour increases with low temperatures but diminishes if the house be kept warm.

Such dominant whites, when crossed with the green- 
stemmed recessive whites, give a white-flowered $F_{1}$ with a reddish stem. The $F_{2}$ generation from this cross has contained a small number of plants with coloured flowers, but the ratio has not yet been determined. There is an obvious general agreement between this case and that described in fowls, and we may feel fairly sure that the Primula colours similarly depend on two colour-factors, and that the absence of colour in the dominant whites is due to the super-imposition of a third factor*.

* The white-flowered variety of Matthiola incana (Stock) is as regards its genetic behaviour, a coloured form. Moreover its flowers tinge on fading and its embryos have the deep green colour characteristic of purpleflowered types. Crossed with a self-coloured type it gives $F_{1}$.with purple flowers. Clearly therefore it is not, as some of the tinged forms of Primula Sinensis are, a dominant white. Miss Wheldale has suggested with some plausibility that the white $M$. incana is comparable with the picotee types of Sweet Peas. 


\section{CHAPTER VI}

\section{HEREDITY OF COLOUR-CONTINUED.}

Eye-Colours. Variations in Colour of the Iris-Deficiency of Eye-Pigments in some Coloured Types.

THE colours of eyes present some special genetic problems which are worth examining in detail.

Pigments are formed in several parts of the vertebrate eye, but chiefly in the choroid and the iris. In all normal eyes the choroid contains much deep black pigment, and to this fact the blackness of the pupil is of course due. The common differences in eye-colour with which we are familiar in man are not caused by changes in the colour of the pupil but by variations in the structure and pigmentation of the iris alone. Such differences, being conspicuous factors in individual and racial differentiation, have long attracted the attention of anthropologists. With the object of elucidating the heredity of eye-colours extensive pedigrees were collected by Galton, and biometrical studies of a similar kind have been published by Professor Pearson and his assistants. Various conclusions of a statistical nature have been based on these data, but as no critical analysis was attempted, such results are devoid of genetic importance. As we now know, moreover, the method of classification employed in collection was unfortunately defective. The categories were not sufficiently precise, and the material would give no reliable indications if analysis were attempted.

As the result of comprehensive investigation carried out in a single district on Mendelian lines, Hurst has succeeded in making an important advance*. Recognizing that such descriptions as "light," "dark," and so forth are

* Similar evidence has also been published by Davenport (107). 
too vague he instituted a more critical classification, which led to the discovery that the presence of brown prgment on the anterior surface of the iris behaves as a dominant to the absence of such pigment, which is a recessive character.

Parents without the pigment in question have exclusively children who are similarly without it, whereas persons possessing the brown pigment may be either homozygous or heterozygous in that respect, and their offspring follow the numerical rules with fair exactitude.

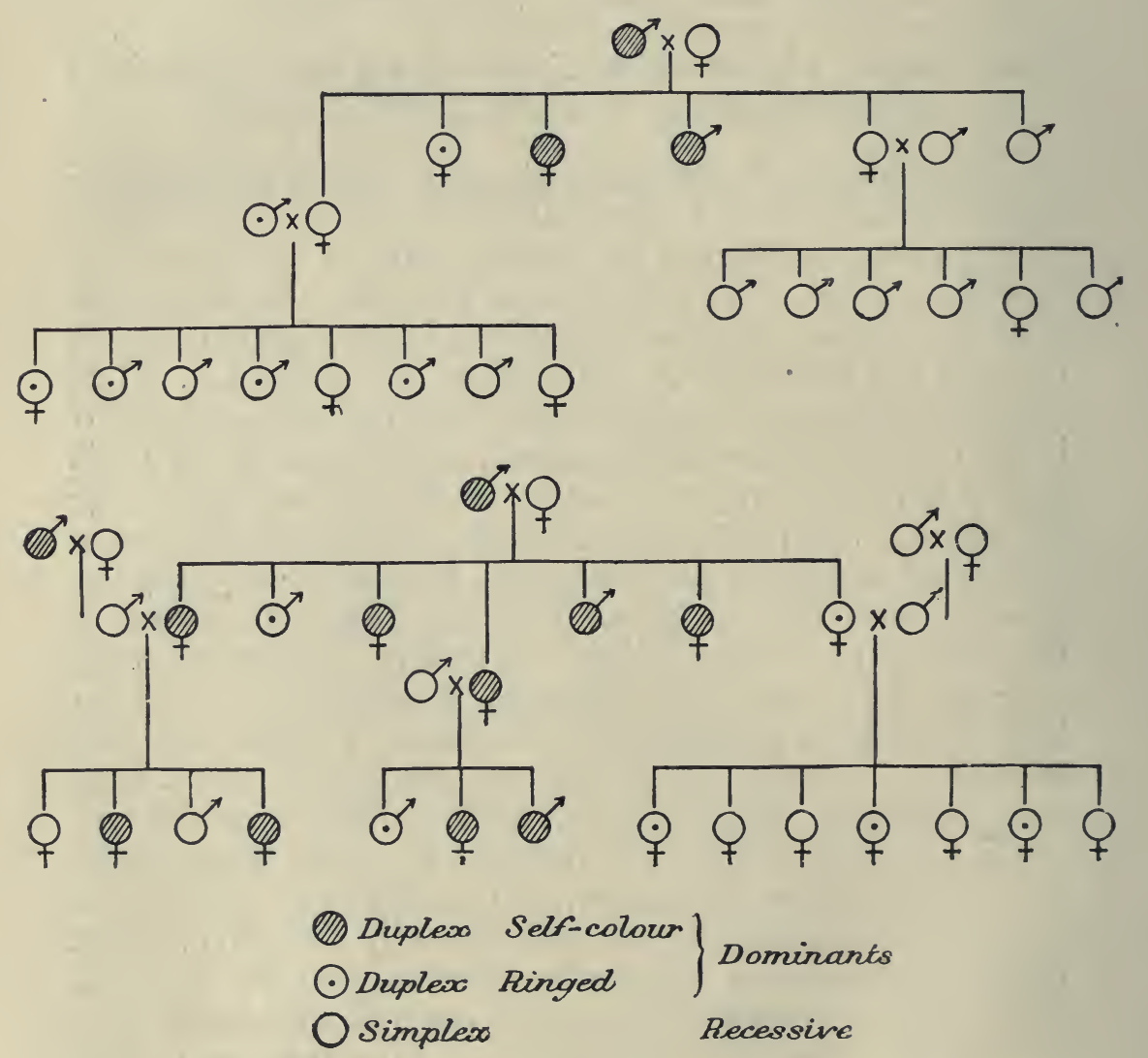

Fig. 18. Two pedigrees showing the descent of eye-colour (after Hurst).

"Brown" eyes are no doubt all dominants, and "blue" eyes are very often recessives; but, on the other hand, many eyes which would be called "light" have some of the 
pigment, and many eyes which would be called "dark" are without it. The differences between the non-pigmented "dark" iris and the non-pigmented "light" iris are caused by structural differences in the iris itself.

Apart from its scientific importance it will be evident that this discovery is of considerable practical interest as a contribution to human genetics. Much nevertheless remains to be done in the application of precise methods to the problem of human eye-colours. For example, amongst the dominants examined by him Hurst recognized two distinct types: (I) self-coloured irides in which the pigment is distributed over the whole surface of the iris, (2) ringed irides in which the pigment was absent from the periphery but existed as a ring round the pupil. The relations between these two types could not be satisfactorily determined. All that the evidence showed was that the "self" behaves as a dominant to the "ringed." But on analogy with other such cases we of course should expect that these distinctions were caused by distinct determining factors, either of which might be borne by the non-pigmented recessive without revealing its presence. If this were so, some of the nonpigmented should, on breeding with the ringed, have offspring with self-coloured irides, just as some albino rabbits on breeding with Dutch-marked rabbits may have selfcoloured offspring. Curiously enough, however, in an ample series of observations, Hurst met with no such occurrence, though examples of self parents producing the three kinds of offspring, self, ringed, and non-pigmented, were not rare. Here, at present, the matter rests*.

Hurst's observations relate entirely to the population of a small English village where naturally only a limited range of types would be met with. The inter-relations of the eye-colours of strongly marked racial types doubtless will provide an excellent field for the application of a similar analysis. It is likely too that such work would result in the discovery of the principles governing the association of certain eye-colours with particular colours of the hair, and

* Hurst has suggested with some plausibility that the absence of any self-coloured irides among the offspring of ringed $x$ non-pigmented may be attributed to the fact that the offspring examined were mostly schoolchildren. He thinks it not improbable that some of the ringed may subsequently develop into self-coloured irides. 
other characteristics. In all such inquiries the first step is to distinguish the critical differences which are treated as units in the constitution of the germ-cells.

In addition to these observations on man we have a few indications as to the heredity of iris-colours in birds.

The iris of the domestic breeds of fowls presents at least three types of coloration. The common colour in the full grown birds is a bright red, apparently due to the formation of a red pigment when the period of adult life is approached, the colour in earlier stages being a dull blackish green.

Malay fowls are peculiar in having a pale, yellowish white iris-the "daw-eye" of fanciers-which behaves as a recessive to the red iris. This eye may be found in birds of various colours and probably it always indicates an admixture of Malay blood*. I have seen, for instance, a daw-eye in a White Leghorn of a good strain. Here it undoubtedly cropped out as a recessive character among the normal red-eyed birds, and I happen to know that White Malays were used by a prominent White Leghorn breeder to increase the size of his strain.

Another peculiar type is seen in the nearly black iris of Andalusians and Silkies. In Andalusians we have found this eye imperfectly dominant to the red. The case of the Silkies is much more complex and the inheritance is disturbed by sex in the manner hereafter to be described.

In the Little Owl (Athene noctua), Giglioli has described the occurrence of a remarkable variation in eye-colour which is evidently an example of the appearance of a recessive in the wild state. The irides of the normal birds are yellow, but in the variety, of which several specimens were found in one district, the irides were black. The circumstances of the discovery which are related by Giglioli in detail $\dagger$ leave little doubt that the condition was recessive. It is a curious fact that, in all the aberrant individuals found, the distribution of the brown markings on the feathers also differed from that of the type. So distinct was the variety from the ordinary $A$. noctua that a new specific name was suggested for it until the fact that it could be bred from the type was established.

* Sir J. Bowring (Philippine Islands, I859, p. I 5 I) says that "white eyes" are preferred in fighting cocks. Probably these are the origin of our Malays and Aseels. $\dagger$ Giglioli, Ibis, 1903, p. 1 . 


\section{Deficiency of Eye-Pigment in some Coloured Types.}

In the eyes of actual albinos, destitute of pigment, the eye has the well-known pink appearance. This is due to the admission of light through the partially transparent iris. Generally speaking such great deficiency of ocular pigments occurs only in total albinos, but to this rule there are a few remarkable and interesting exceptions.

The best known example is the so-called Himalayan Rabbit*. In it the eyes look like those of the pure albino though having a trace of pigment. At birth Himalayans are white or nearly so, but soon the muzzle, ears, tail, and feet assume a deep chocolate-black. This type behaves as a recessive to the normal grey, and to black, and it is a normal dominant to the pure albino. Hurst however found that Himalayans, though obviously pigmented, may carry the factors for black and for self-colour, without any indication of such factors being manifested in their own colour or in its distribution. Recent experiments made by Punnett have given the same result.

The next example of the pink eye present in an animal not devoid of colour is seen in certain "Japanese Waltzing" mice.

The "waltzing" is a peculiar vertiginous spinning to which those animals are subject. I understand that the exact physiological nature of this movement has never been fully elucidated, but the condition has been shown to be often, if not always, associated with malformation of the auditory labyrinth. As already mentioned, the waltzing character behaves as a Mendelian recessive to the normal habit.

Such waltzing mice exist in various colours, some having ordinary black eyes, others pink eyes. Various colours also may occur in the coats and we have not yet complete

* Darwin gives (Animals and Plants, ed. 2, I885, I. p. I I3) facts as to the production of Himalayans by crossing Silver-greys. In the light of present knowledge it is no longer possible to accept these experiments as evidence of an original production of the variety. Presumably the case was only one of the reappearance of a recessive form, and indeed Darwin adds the remark that he had "recently been assured the pure Silver-greys of any such breed occasionally produce Himalayans." 
knowledge as to which of these colours are compatible with the pink eye and which are not.

Elaborate experiments were made by Darbishire (90) to test the inheritance of these mice. The waltzers used were all pink-eyed and bred true to that character. In general terms the coat-colour was fawn-and-white, but no detailed account of the pigments present has been published.

Crosses were made between these waltzers and ordinary albinos. The $F_{1}$ generation so produced were usually, as regards coat-colour, grey like wild mice, the shade being sometimes darker, sometimes lighter, together with more or less white. In a few families the colour was black. The albino parents being of miscellaneous origin, such diversity in $F_{1}$ colour is what we should now expect.

In respect of eye-colour the remarkable fact was observed that the $F_{1}$ mice were always black-eyed. In $F_{2}$ various coat-colours occurred, including a peculiar new type with exceedingly dilute pigment in the hair, spoken of as "lilac." Among these coat-colours the eye-colours were distributed according to systems not yet ascertained. All that can be clearly perceived is that the mice with agouti or full black in the coat-colour (whether pied with white or not) always had black eyes; the completely white individuals always had pink eyes; and lastly, the eyes of the "lilac" mice were always pink*. The facts were unfortunately presented by Darbishire in a form which makes further analysis impossible. A proper investigation of this series of phenomena would have greatly increased our knowledge of the genetics of pigmentation.

The important conclusion may nevertheless be confidently drawn that at least two factors are concerned in producing the black colour of the eye. Cuénot (88, p. II), as the result of his own experiments, identifies these with (I) colour in the coat; (2) a factor determining the blackness of the eye, which needs the coat-colour factor as its complement. This is no doubt a part of the truth, but

* Miss Durham (I 6 ) has shown that the pink eyes of these "lilac" mice and of the original pink-eyed waltzers are not wholly devoid of pigment, but contain traces both in the iris and choroid visible in nicroscopic sections. The eyes of the albino mice are absolutely without pigment. 
there are suggestions that it is not the whole, and that further complications have to be met. To discuss these in detail is beyond the scope of this work, but it seems likely that the degree of saturation or dilution of the coat-colour has to be considered.

There is evidently some intimate relation between the colour of the eye and the colour of the coat; for no mice have the coat-colour wild-grey ("agouti" of fanciers), black, or even blue, unless the eyes are black. The interesting fact has also been discovered that the actual nature of the pigment of the choroid differs in the different varieties*. Miss Durham finds that the chocolate mice always have chocolate eyes, not black, no black pigment being present in either iris or choroid. The same fact has been published by Castle (53) for the guinea-pig and was also noticed independently by Miss Sollas in guinea-pigs. From these observations it is to be inferred that either the coat-colour controls the eye-colour, or the colour of the eye controls that of the coat, but for various reasons it is not possible yet to declare positively which account is the right one.

Guinea-pigs differ from other animals in that their albino strains generally have small "smudges" of blackish pigment on some of the extremities, especially the tips of the ears, though the eyes are entirely without pigment. It is said that albino guinea-pigs occur without these smudges, but I have not seen one.

The Cinnamon Canary must also be noticed here. This variety on hatching has pink eyes, though as development proceeds, pigmentation supervenes. Many fanciers believe that they can recognize a difference between the eyes of Cinnamons and those of other varieties, but the distinction is not readily obvious. In plumage Cinnamons differ from other breeds in that the black pigment is absent and the feathers consequently have a brownish tint. Miss Durham, from her observations, concludes that the pigment which is developed in both eyes and feathers is chocolate, and that the Cinnamon is in fact a chocolate

* John Hunter has an important paper on this subject: "On the Colour of the Pigmentum of the Eye in different Animals" (Obs. on certain Parts of the Animal Economy, 1786, p. 199).

B. $\mathrm{H}$. 
variety of the Canary. The heredity of these characters has not yet been investigated by precise methods. Fanciers however are agreed that some very remarkable phenomena of sex-limitation occur, which will be described in connection with other instances of the same kind.

The only other example I know of a race which combines an albino eye with external colour is the breed of cats called "Siamese." These animals, which breed perfectly true, were introduced from Siam, where they have been kept for an indefinite period as pets of the royal household. Like the Himalayan rabbit, Siamese cats are born almost white, but the fur becomes a curious fawn with darker chocolate points on the ears and extremities. The eyes are more or less deficient in pigment throughout life. Some pigment however is formed in the eye, as is evidenced by the fact that the iris has a blue colour and is partially opaque.

From the little that is known respecting this group of cases it is clear that the genetics of eye-pigments offer many features of interest. We have seen first that variations in the colour of the iris may exhibit simple Mendelian heredity. Secondly when the general pigmentation of the eye is considered, it appears that the presence or absence of such pigment may follow Mendelian rules; but there is an inter-dependence between the factor or factors which produce the pigmentation of the eye and those which govern the pigmentation of the fur or feathers, such that the eye-colour must be regarded in certain cases (e.g. mice) as determined by two, or perhaps more, complementary factors. The actual nature of the pigment in the eye is also variable, black being replaced by chocolate in several types destitute of black in their hair. Lastly, there is a noticeable association of chocolate pigment, in hair or feathers, with the more or less non-pigmented eye, as in the Himalayan rabbit, the Siamese cat, and the Cinnamon canary.

The peculiar phenomena connected with Albinism in Man are mentioned later in connection with human heredity in general. 


\section{CHAPTER VII}

HEREDITY OF COLOUR-CONTINUED.

The Genetics of Yellow Pigments in certain Animals. Yellow Mice not breeding true-The Case of Basset Hounds and the "Law of Ancestral Heredity." Relation of this Principle to Mendelian Rules.

THE genetic relations of yellow and black pigments present so many difficulties that a separate chapter is needed for their consideration. There is one comparatively simple case, that of the thorough-bred or race horse, in which the presence of black behaves as a simple dominant to the colour known as chestnut. In practically all the other forms that have been investigated there is some complication. One of these is the celebrated example of the Basset Hounds, which played so prominent a part in connection with Galton's enunciation of the "Law of Ancestral Heredity."

In examining this section of the evidence which Mendelian analysis has provided we meet three facts of surprising novelty. First, that the relations of yellow to black and other colours may be entirely different in animals so nearly related as the rabbit and the mouse. Secondly, that in mice yellows never breed true, though in the rabbit, for instance, no such difficulty occurs. Thirdly, that in rabbits the presence of the factor for the grey or agouti type of coloration alters the appearance of the yellow animal, giving it a white belly and tail, whereas yellows destitute of this factor have these parts of a bluish colour. Such facts cannot of course be properly interpreted until the chemistry of pigmentation is more fully understood, but taken together they illustrate the extraordinary multiplicity of specific rules which genetic research reveals. 
When the hair of the wild house-mouse, for instance, is examined microscopically three kinds of pigment-granules can be distinguished, black, chocolate, and yellow. As Miss Durham pointed out (IO, p. 72), in a solution of potash the yellow granules dissolve at once; the chocolate dissolve, but more slowly; the black not at all. The pigments may co-exist in the same parts of a hair, but in the wild "grey" or agouti.colour there are bands in the individual hairs, where only yellow pigment can be seen.

The black mouse has black and also chocolate pigment, but no yellow. 'The chocolate has chocolate alone.

The yellow mouse may have exclusively yellow pigment, but in some yellows heterozygous with black, granules of the other pigments may be mixed with the more abundant yellow ones.

In the blue, the silver-fawn and the cream, the pigments are as in the black, the chocolate, or the yellow, respectively, but the number of granules is fewer, giving the wellknown appearance of dilution in colour.

The colours of rats, rabbits, guinea-pigs and horses, appear to be similarly made up of three presumably comparable sets of pigments. There are differences however both in the number of distinct pigmented types which exist, and in their genetic behaviour.

Rats.

In rats the only colours known are agouti, and black. Both types contain chocolate pigment as well as black, and agouti also contains yellow pigment; but no distinct chocolate or yellow varieties are known, and none of the dilute forms (blue, \&c.) have been recorded.

Rabitis.

Of the cases in rodents where yellow exists as a separable variety that of the rabbit is the simplest. Numerous colour-types of the rabbit are known, but in our present inquiry we are immediately concerned with the following: 1. Agouti, or wild grey, 2. Black, 3. Yellow. The question whether a chocolate rabbit can be formed is important. It appears that no such type is known to fanciers but $\mathrm{Mr}$ Punnett saw, in the possession of a French breeder, rabbits 
which seemed to him to be chocolate. No examination of the pigments of these rabbits has been made.

Agouti, as we know from the experiments of Hurst and others, is epistatic to black. Next, Castle (53) has shown and Hurst independently discovered* the fact that the yellows are of two distinct types. A. Yellows with white bellies and tails, which always bear the agouti factor, $G$. B. Yellows with blue bellies and tails, which do not contain $G$. The latter have been spoken of as sooty yellows, and to English fanciers they are known as tortoise-shells. Respecting the pigments of these types full information is wanting. Miss Durham found that agouti certainly contains black, chocolate and yellow; that black contains black and chocolate; but hitherto the yellows have not been studied on a comprehensive scale, and all that we know is that yellows may contain both black and chocolate granules in addition to yellow. None have yet been seen to contain yellow only.

Following Castle, the genetic composition and behaviour of the several types may be represented thus:

$\begin{array}{ll}\text { Agouti (wild grey) } & G B Y . \\ \text { Black } & g B Y . \\ \text { Yellow } A \text {, white belly } & G b Y . \\ \text { Yellow } B \text {, blue belly } & g b Y .\end{array}$

This scheme is so far satisfactory, but two difficulties arise when an attempt is made to compare the phenomena seen in the rabbit with those observed in mice and guineapigs; for first the scheme takes no account of chocolate; and secondly, in the mouse yellow is dominant to black, not recessive as in the rabbit. There is however no doubt as to the essential facts, that yellow $A$ crossed with black gives agouti, while yellow $B$ crossed with black gives simply black dominant. A further confirmation of this symbolic representation is found in the fact observed by Castle that agouti $\times$ yellow $B$ gives blacks in $F_{2}$ as the scheme demands $\dagger$. The nature of the factor $G$ is obscure. Castle regards it as a pattern-factor governing the distribution of the colours; but a grey rabbit differs from a black

* Communicated to the Internat. Congress Zool. 1907: as yet unpublished.

$\dagger$ Compare the similar phenomena in mice, p. 78 . 
in more than pattern, for actual bands of yellow pigment are formed in the hairs. $G$ therefore is a factor which can make pigment yellow that would have been black in the absence of $G$, so that in a restricted sense it is a dominant yellow factor, but the relation of this yellow of agoutis to the yellow pigment of yellow varieties is quite uncertain.

There is no suggestion that yellows of both types cannot be bred pure (cp. Mice).

Guinea-Pigs.

In guinea-pigs no true yellow exists, but it is apparently represented by red. The work of Castle (53) and the experiments of Miss Sollas, as yet unpublished, show that when red is crossed with black the offspring are either (I) black-reds, viz. patched with black and red; or (2) agoutis, in which the black and the red are intimately mixed in the banded hairs. Families of three kinds may thus be produced. Red bred with black may give

(A) all agoutis,

(B) all black-reds,

(C) agoutis and also black-reds.

It seems practically certain that the agouti-factor, $G$, as we have called it, may be carried by the red, not by the black. Consequently case $(\mathrm{A})$ is that in which the red is homozygous for this factor. In case (B) the red is homozygous for its absence, and in case (C) the red is heterozygous for the same factor. There is no difficulty in breeding reds as a true strain, and the peculiar phenomenon about to be described in yellow mice does not occur in guinea-pigs.

In guinea-pigs a distinct chocolate type exists, but such animals according to Miss Sollas always have some red hairs. The relation of these to the other types is not yet fully understood.

Mice.

The facts seen in rabbits and guinea-pigs are in fair agreement with each other, but when these are compared with the phenomena now well ascertained in the case of mice, fundamental differences are perceived which make it 
impossible to bring the whole series of observations into one consistent scheme. For first, Cuénot (87) has shown that in the mouse yellow is dominant to black, instead of being recessive to it as in the rabbit. The dominance is so far incomplete that yellows heterozygous with black have often-perhaps always-more or less black pigment in their hairs, but in general appearance they are obviously yellow.

Next according to Cuénot's experience none of the yellow individuals in $F_{2}$ from a cross of yellow $\times$ black or yellow $\times$ agouti are pure to yellow*. All such extracted yellows when bred together throw agoutis or blacks respectively. This fact has led him to suggest that there may be some incompatibility which prevents two yellow-bearing gametes from uniting in fertilisation. These "impure" yellows bred together gave 232 yellows and 86 agoutis, a fairly close approximation to $3: \mathrm{I}$. The expectation on that ratio is $23^{\circ} 5: 79^{\circ}$. Cuénot, commenting on the numbers produced, remarks that since pure yellows are not formed, he would anticipate a diminution in the relative numbers of yellows, and that such a diminution actually occurs. But, as Mr Punnett has pointed out to me, there seems to be no valid reason for expecting a departure from the ratio $3: \mathbf{I}$; for the spermatozoa may be regarded as unlimited in number. Of the "non-yellow" ova half would be fertilised by "non-yellow" sperm, producing agoutis, the other half being fertilised by "yellow" sperm and producing yellows. The "yellow" ova would all, on Cuénot's hypothesis, be fertilised by "non-yellow" sperm, and give rise to yellows. The nett result would then be still 3 yellows: I agouti.

Of $8 \mathrm{I} \quad F_{2}$ yellows tested by Cuénot, all proved to be heterozygous, so that the reality of the abnormal phenomenon must be regarded as established. Observations made

* A fact of a different order, though perhaps having a bearing on the problem, was observed by Hurst in crosses between Belgian Hares (Rabbits) and Angoras. The Belgian is in colour an "agouti," or grey, but it is much yellower than the wild rabbit of the warrens. Hurst found that $F_{1}$ from such Belgians and Angoras (bearing $G$ ) was of the wild or warren type of grey, and that in $F_{2}$ no rabbit quite of the Belgian colour ever came. The greys in $F_{2}$ varied a little in the amount oi yellow, and it is possible that, as in other cases in which a parental type fails to recur in $F_{2}$, the absence may be ascribed to the rarity of a particular combination. 
by Miss Durham agree entirely with those of Cuénot She finds it impossible to obtain any mice pure to yellow. The experience of fanciers seems to be universal that yellows cannot be bred in a pure strain. Those with which she has experimented always throw either agoutis, blacks, or chocolates, and we may take it that yellows of both sexes are always heterozygotes formed by the meeting of a yellow and a non-yellow gamete*. In spite of their genetic composition the yellows which throw chocolates have only yellow pigment in their hairs, and no chocolate.

\section{Cats.}

In cats we meet a new complication in the inheritance of yellow, namely that it is disturbed by sexual dimorphism. The rarity of tortoise-shell males is well known, and Doncaster (I09) has produced evidence which makes it practically certain that in the immense majority of instances the female heterozygote of orange $\times$ black is tortoise-shell, namely, patched with orange and black (like the guinea-pig), but that the male heterozygote similarly produced is orange (see Chap. $x$, dealing with the heredity of Sex). The dilute types cream and blue are similarly related to blue tortoise-shell.

\section{Fowls.}

There are further indications of peculiarity in the genetics of yellow pigments in the case of birds. The coloration known as "Pile" in fowls is seldom bred for exhibition from two pile birds. The colour consists, in cocks, of orange-yellow or red in the hackles and wing-coverts combined with a white ground. The hens are white, with a chestnut or reddish yellow breast. In the down the chickens of both sexes have longitudinal stripes of light chestnut. In the adults, and generally in the chickens too, there is a small and variable amount of blackish grey ticking; which is of course considered a fault. Pile is a colour known in several breeds, but especially in Game, Game

* Besides this curious peculiarity, yellows show another remarkable feature in their frequent tendency to become excessively fat. Miss Durham has met with several such specimens and the fact is well known to fanciers. In her experience also yellows are more liable to sterility than other mice. 
Bantams, and Leghorns. It is said never to breed true, throwing ordinary black-reds in unascertained proportions. Presumably pile is a heterozygous combination, black-red being one of the constituents, but what the other pure form (or forms) involved may be, is quite uncertain. The whole question is still very obscure, and from preliminary experiments made by $\mathrm{Mr}$ C. Fryer it seems probable that two pairs of factors are concerned in addition to black-red.

For exhibition purposes piles are often crossed with black-reds, fanciers being under the impression that a brighter pile results from this mating. The fact suggests that there may be piles of several different compositions. Taking the known facts together the one clear deduction is that pile, or yellow if we may so call it, is dominant to black-red*.

\section{Recapitulation and Discussion of the Foregoing FACTS.}

The outstanding peculiarities of this group of phenomena are as follows :

I. The difference between the genetics of similar colours in allied forms.

In rabbits black is epistatic to yellow, giving (in the absence of $G$, the agouti factor) simply a black heterozygote. In guinea-pigs (where red represents yellow), the heterozygote of black and red is a patch-work, viz. tortoise-shell. In both rabbits and guinea-pigs when $G$ is present this heterozygote is agouti, the factor $G$ being introduced by the yellow parent.

In mice yellow is epistatic to black, giving, in the absence of $G$, a yellow heterozygote. We can form no surmise as to the nature and causation of this discrepancy between these types. It is to be observed that the recessive yellow of the rabbit, viz. the blue-bellied type lacking both $G$ and $B$, nevertheless has some black pigment in its blue fur, while the yellow mouse does not necessarily have any.

* The whole-coloured $b u f f$ of Cochins and some other breeds derived from them is quite distinct from pile. The genetics of this pigmentation are complex and little known as yet. 
the complicated phenomena elsewhere observed in regard to pigments ostensibly the same. The colours of race horses are recorded with great accuracy in Weatherby's General Stud Book. By a careful analysis of these returns Hurst was able to show that chestnut on the one hand is recessive to bays and browns, which are dominant. Chestnuts are distinguished from bays and browns by the fact that in their hair no black pigment is developed. Bays always have black in the mane, tail and fetlocks. Browns have more or less black in the same parts, and generally black pigment is distributed to some extent over the whole coat.

As the records show, there are only about I per cent. of alleged exceptions to the rule that chestnut $x$ chestnut produces chestnut exclusively. Of the dominants some are pure dominants and give-again with about I per cent. of exceptions-bays and browns only, whether the other parent is chestnut, or bay or brown. Other dominants are shown also to be $D R$ in constitution, giving, when bred with chestnuts, equal numbers of dominants and recessives.

With regard to the few exceptions of both kinds appearing in the Stud Book records some are demonstrably mistakes, and the actual frequency of exceptions must be considerably less than I per cent., if indeed there are any genuine exceptions at all. The relation of bays to browns has not yet been made out, and as the two classes grade into each other somewhat, the detection of their relationship would demand observations of a sort somewhat more critical than those which the Stud Book provides. So far however as the segregation of black from the absence of black is concerned, the case is simple and regular*.

* The inheritance of coat-colour in horses was the subject of an extensive investigation by Professor K. Pearson and his assistants (2 I 9 ). The records of the Stud Books were tabulated and investigated by the application of biometrical methods. Various propositions have been enunciated as the result of this inquiry, amongst others the statement that nothing corresponding to Mendel's principles appears in the case of horse-colours. The key to the phenomena was of course the fact that chestnuts mated with chestnuts breed true-with rare and dubious exceptions. It would seem at first sight impossible to devise a system of tabulation which could fail to disclose so prominent a feature. Nevertheless Professor Pearson's correlation-tables, which were compiled from the records of more than 6000 horses, were made in such a way that the colours of sire and dam could 
The time has not arrived for any attempt to analyse the relations of horse-colours in general. By microscopical methods Miss Durham has found in horses the three pigments, black, chocolate, and yellow seen in other types. The term chestnut is used somewhat loosely in describing colours, and though usually a chestnut is a horse possessing yellow pigment only, there is a dark type of chestnut, sometimes spoken of as liver-chestnut, which is actually chocolate*. Nothing is known of the genetic relation of this to the yellow chestnut, or of the respective properties of those various chestnuts distinguished by the colour of their manes and other subordinate differences.

Among thorough-bred or race horses, types intermediate between chestnut and the dominant bays and browns must be exceedingly rare, for though entries indicating doubt between bay and brown are rather common in the Stud Books, alternative designations are scarcely ever given in regard to chestnuts.

Among common horses and hackneys such animals though exceptional can be found by looking out for them, and one or two may usually be seen in a day's walk through London streets. I have no information as to their genetic capacities, but presumably they are due to dilution-stages of the black pigment, corresponding to those which in the mouse \&c. constitute the blue varieties. They may also be cases of imperfect dominance.

not be taken into account together. The tables thus provide answers to questions as to the probable colour of a foal by a chestnut sire, the dam's colour being taken as unknown; as to the probable colour of the brother or half-brother of a chestnut foal, when the colours of both sire and dam are taken as unknown; with solutions of other problems of equal significance. Since however the colours of both sire and dam are recorded, and must indeed have been actually extracted from the Stud Book for the purposes of the tabulations, the investigators, by refraining from an inspection of these data till they had been separated, placed themselves at a gratuitous disadvantage. The true nature of the inheritance was therefore not discovered.

The failure was due to want of analysis. The similar failure of biometrical methods to find the plain rule of inheritance in the case of human eye-colour was due to the same defect of method, though in that case further obscurity arose from the use of faulty and uncritical observations. for black.

* When very dense this type of colour might carelessly be mistaken 
the complicated phenomena elsewhere observed in regard to pigments ostensibly the same. The colours of race horses are recorded with great accuracy in Weatherby's General Stud Book. By a careful analysis of these returns Hurst was able to show that chestnut on the one hand is recessive to bays and browns, which are dominant. Chestnuts are distinguished from bays and browns by the fact that in their hair no black pigment is developed. Bays always have black in the mane, tail and fetlocks. Browns have more or less black in the same parts, and generally black pigment is distributed to some extent over the whole coat.

As the records show, there are only about I per cent. of alleged exceptions to the rule that chestnut $x$ chestnut produces chestnut exclusively. Of the dominants some are pure dominants and give-again with about I per cent. of exceptions-bays and browns only, whether the other parent is chestnut, or bay or brown. Other dominants are shown also to be $D R$ in constitution, giving, when bred with chestnuts, equal numbers of dominants and recessives.

With regard to the few exceptions of both kinds appearing in the Stud Book records some are demonstrably mistakes, and the actual frequency of exceptions must be considerably less than I per cent., if indeed there are any genuine exceptions at all. The relation of bays to browns has not yet been made out, and as the two classes grade into each other somewhat, the detection of their relationship would demand observations of a sort somewhat more critical than those which the Stud Book provides. So far however as the segregation of black from the absence of black is concerned, the case is simple and regular*.

* The inheritance of coat-colour in horses was the subject of an extensive investigation by Professor K. Pearson and his assistants (2 I 9 ). The records of the Stud Books were tabulated and investigated by the application of biometrical methods. Various propositions have been enunciated as the result of this inquiry, amongst others the statement that nothing corresponding to Mendel's principles appears in the case of horse-colours. The key to the phenomena was of course the fact that chestnuts mated with chestnuts breed true-with rare and dubious exceptions. It would seem at first sight impossible to devise a system of tabulation which could fail to disclose so prominent a feature. Nevertheless Professor Pearson's correlation-tables, which were compiled from the records of more than 6000 horses, were made in such a way that the colours of sire and dam could 
The time has not arrived for any attempt to analyse the relations of horse-colours in general. By microscopical methods Miss Durham has found in horses the three pigments, black, chocolate, and yellow seen in other types. The term chestnut is used somewhat loosely in describing colours, and though usually a chestnut is a horse possessing yellow pigment only, there is a dark type of chestnut, sometimes spoken of as liver-chestnut, which is actually chocolate*. Nothing is known of the genetic relation of this to the yellow chestnut, or of the respective properties of those various chestnuts distinguished by the colour of their manes and other subordinate differences.

Among thorough-bred or race horses, types intermediate between chestnut and the dominant bays and browns must be exceedingly rare, for though entries indicating doubt between bay and brown are rather common in the Stud Books, alternative designations are scarcely ever given in regard to chestnuts.

Among common horses and hackneys such animals though exceptional can be found by looking out for them, and one or two may usually be seen in a day's walk through London streets. I have no information as to their genetic capacities, but presumably they are due to dilution-stages of the black pigment, corresponding to those which in the mouse \&c. constitute the blue varieties. They may also be cases of imperfect dominance.

not be taken into account together. The tables thus provide answers to questions as to the probable colour of a foal by a chestnut sire, the dam's colour being taken as unknown; as to the probable colour of the brother or half-brother of a chestnut foal, when the colours of both sire and dam are taken as unknown; with solutions of other problems of equal significance. Since however the colours of both sire and dam are recorded, and must indeed have been actually extracted from the Stud Book for the purposes of the tabulations, the investigators, by refraining from an inspection of these data till they had been separated, placed themselves at a gratuitous disadvantage. The true nature of the inheritance was therefore not discovered.

The failure was due to want of analysis. The similar failure of biometrical methods to find the plain rule of inheritance in the case of human eye-colour was due to the same defect of method, though in that case further obscurity arose from the use of faulty and uncritical observations. for black.

* When very dense this type of colour might carelessly be mistaken 


\section{"Tricolour" and "Non-tricolour" in Basset Hounds: The Law of Ancestral Heredity.}

The question of the relation of the yellow and black pigments is raised in the celebrated case of the colours of Basset Hounds. The importance of that subject is due to the fact that it was from a study of the evidence in regard to Bassets that $\mathrm{Mr} \mathrm{F}$. Galton was led to enunciate his "Law of Ancestral Heredity" with confidence as one which "appears to be universally applicable to bi-sexual descent." The publication of that paper played a considerable part in the history of modern genetic research and it is necessary that we should consider the facts in some detail.

The colours of Bassets are two, the first spoken of as tricolour, consisting of black and yellow marks on a white ground; the second, non-tricolour, which differs from the first in having no black. It is said that dogs which cannot be easily referred to one or other of these two types do not occur, and they must certainly be very rare if they exist at all.

Mr Galton's investigation was based on data supplied to him by the late Sir Everett Millais, a keen fancier of the breed. This evidence consisted in records giving the number of offspring of each type which had occurred in families of various compositions. It was thus possible to compare the number of tricolour and non-tricolour dogs produced in the families with the number of the respective types distributed among their pedigrees. Galton's figures indicated that there was a close correspondence between these two numbers, so that it was possible, given the ancestral composition of the families, to predict with considerable accuracy the numerical proportions in which the respective types would appear. According to Galton's system the family was regarded as the production of all the ancestors. Each ancestor was supposed to contribute in his or her degree to this total heritage, the more immediate progenitors contributing more, and the remoterprogenitors less, according to a definite arithmetical rule. This rule was that the average contribution of each ancestor was to be reckoned

$\begin{array}{ll}\text { for each parent } & \text { I/4 } \\ \text { for each grandparent } & \text { I/I6 } \\ \text { for each great-grandparent } & \text { I/64 }\end{array}$


and so on, the total heritage being thus reckoned as unity. It will be observed that this scheme differs entirely from those based on Mendelian principles, inasmuch as every ancestor is, according to the Law of Ancestral Heredity, supposed to have some effect on the composition of each family in its posterity, and each recent progenitor is regarded as having a very sensible influence on these numbers.

Though no one with a knowledge of practical breeding could entertain the supposition that Galton's Law had the universality of application claimed for it, there was on the other hand no doubt that the Law had successfully expressed a variety of facts in which no order at all had been previously detected.

We have now to consider the meaning of this evidence in the light of modern knowledge. At the time that Galton's views were promulgated nothing was known of segregation. The supposition that any individual, whatever its own characters, was capable of carrying on and transmitting to its posterity any of the characters exhibited by its immediate progenitors, at all events, was generally received without question by biologists. According to that idea the number of classes of individuals differing in respect of their ancestral composition and transmitting powers is to be regarded as indefinitely large, whereas in all cases of sensible allelomorphism the number of classes of individuals is three only, two being homozygous and one heterozygous. The difference between the two schemes is thus absolute and irreconcileable.

When Mendelian phenomena were first recognized it was naturally supposed that some classes of cases would be found to conform to the Mendelian scheme and others to the Law of Ancestral Heredity. With the progress of research however almost all the cases to which precise analytical methods have been applied have proved to be reducible to terms of Mendelian segregation; and of those which have not already been so elucidated some, we may feel confident, if not all, will be eventually shown to be governed by similar rules. In discussing aberrant pheno. mena like those alleged in regard to the Bassets the first question to be settled is whether the racts are correctly 
reported. If either type is recessive we should naturally expect this to be the non-tricolour, which is without black. Unfortunately as the non-tricolours are not fashionable there were comparatively few matings between two parents of that colour. Nevertheless 4I dogs, offspring of such matings, are given, of these 20 being tricolour. Though the records were not made by scientific men or with a scientific purpose directly in view it is almost impossible to imagine that all these cases can depend on mistakes, and pending the production of new and direct evidence we must take the records as correct.

In the Theory and Practice of Rational Breeding (London, I 889), pp. 26 and 27, Sir Everett Millais gives one or two more notes bearing on this question. He says that in England there were then two strains of Bassets, the Couteulx and the Lane. "The Couteulx is as a rule a very perfectly marked tricolour, with the tan and black markings deeply accentuated. The Lane hounds, on the other hand, are very weak in markings if they happen to be tricolour, but as a fact they are far more generally found to be lemon and white." In another place he mentions that "in nearly every litter of pure Couteulx there is generally a lemon and white puppy."

If it were not that the genetic relations of yellow and black pigments are, as we have seen, so complicated and uncertain in other types, we might be inclined to attribute the alleged production of tricolours by non-tricolours to imperfect classification of "weak" tricolours, but in dealing with this group of phenomena no such suggestion can be hazarded with any confidence.

The strange fact that yellow mice are never pure naturally occurs to the mind in connection with the evidence as to Bassets. A comparison between the two cases cannot nevertheless be instituted at all easily; for in the Bassets yellow is evidently not usually a dominant, which it should be if the impurity of the non-tricolour is to be attributed to a state of things comparable with that existing in mice. At present the Basset phenomena must be regarded as definitely unconformable. Perhaps the most probable view of their nature is that they are an illustration of irregular dominance, but this cannot be asserted with much confidence. 
It is curious that the one example to which a partially correct system of analysis was applied before Mendelian methods were rediscovered, should have been of this remarkably exceptional order.

There is little réason to anticipate, as we once did, that a distinct group of cases obeying a Law of Ancestral Heredity will have to be recognized. That principle in certain cases gives an epitome of the consequences of the Mendelian process, and in all likelihood its applicability to any phenomena of natural inheritance is due to this fact. The Law of Ancestral Heredity takes of course no account of dominance, or of segregation with all the consequences it entails; but as describing the results to be witnessed among a population interbreeding at random, its predictions would frequently approximate to the truth. In particular it is to be observed that the arithmetical results of $D R \times R$ and of $D R \times D R$ are correctly predicted by the Law of Ancestral Heredity. Some of the phenomena of blending are also expressed with accuracy. Besides this, inasmuch as dominant individuals which have both parents dominants will in a mixed population frequently be pure dominants, statistical phenomena which could be mistaken for an effect of ancestral composition will often occur*.

For instance if purple Sweet Peas in $F_{2}$ were bred from promiscuously, $F_{3}$ would consist of purples and other colours, and the excess of purples in the mass would be greater than it was in $F_{2}$. If the $F_{3}$ purples were again separately saved, the proportion of purples in $F_{4}$ would be still greater, and so on. This gradual increase in the proportion of purples might carelessly be mistaken for a consequence of the fact that each generation had more purples in its ancestry. As we now know, that conclusion would be quite incorrect. The increase is in reality due to the appearance of actually pure purples in $F_{2}$ and in subsequent generations, and to the effect which their presence has on the composition of the population. The impure purples in each generation

* Darbishire's conclusions in regard to mice (Biometrika, 1904, III. pp. 23-5) were obviously based on a mistake of this kind, as he has. himself since admitted. (See Mem. Manchester Lit. Phil. Soc. r905,

No. 6, p. 7.)

B. $\mathrm{H}$. 
remain exactly in the same condition as at their first appearance, and the selection has merely resulted in a reduction of their number. If the purples were saved individually and their seeds separately sown, it would immediately be seen that some were pure, giving purples only, and that others were impure, the latter consisting of the various gametic types with which we are now familiar.

Since then we know that ancestral composition does not decide the constitution of such a population, and since individuals of identical parentage may have most divergent genetic properties, it is absurd to attempt to trace the workings of any Law of Ancestral Heredity among these phenomena.

The suggestion that methods based on unanalysed statistics have scientific value in the study of heredity can scarcely mislead those who have examined the facts. Professor Pearson and others committed to these methods have of late defended their position by arguing that there is no fundamental incompatibility between Laws of Ancestral Heredity and the conclusions of Mendelian analysis. The matter would not be worth notice were it not that the same proposition is being freely repeated by several writers seeking some convenient shelter of neutrality*. It is to be observed however that the supposition of an underlying harmony between Mendelian and biometrical results was not put forward by the biometricians until every possible means of discrediting the truth of Mendelian facts had been exhausted. Those attacks having failed, we are asked to observe that the Law of Ancestral Heredity was meant as a statement of a statistical consequence, and is not concerned with physiological processes. Mr Galton's views on this point are well shown in the following passage in which he explicitly appeals to the physiological process of gametogenesis as apparently occurring in the way which his Law requires. For in introducing the Law as applicable to Bassets (I 25, p. 403) he wrote:

"It should be noted that nothing in this statistical law contradicts the generally accepted view that the chief, if not

* See for example Darbishire, Mem. Manchester Lit. and Phil. Soc. 1905, No. 6 and 1906, No. II; and Professor J. A. Thomson, Heredity, I907, passim. 
the sole, line of descent runs from germ to germ and not from person to person. The person may be accepted on the whole as a fair representative of the germ, and being so, the statistical laws which apply to the persons would apply to the germs also, though with less precision in individual cases. Now this law is strictly consonant with the observed binary subdivisions of the germ cells, and the concomitant extrusion and loss of one-half of the several contributions from each of the two parents to the germ-cell of the offspring. The apparent artificiality of the law ceases on these grounds to afford cause for doubt; its close agreement with physiological phenomena ought to give a prejudice in favour of its truth rather than the contrary."

Had segregation been known to $\mathrm{Mr}$ Galton the Law of Ancestral Heredity would not have been promulgated. It is obvious that so soon as that phenomenon is recognized and appreciated, all question of useful or direct applicability of the Law of Ancestral Heredity is at an end. That method of representing the phenomena of Heredity and all modifications of it are based on the false assumption that any individual can transmit the characteristics. of any ancestor, and especially of any recent ancestor. When this conception was shown to be untrue, the structure which the biometricians have offered to the world as a scientific study of Heredity ceased to have meaning or value. Statistical examination of ancestral composition may, as we have seen, occasionally give a prediction in good correspondence with fact, but this is due to coincidence and not to any elements of truth in the ratiocination by which the prediction was reached.

As an attempt to compass the solution of an intricate problem by labour and ingenuity without proper data or equipment Mr Galton's work deserves long to be remembered. It stands out as a significant and stimulating event in the history of biology. 


\section{CHAPTER VIII}

HEREDITY OF COLOUR-CONTINUED.

Various Specific Phenomena in Colour-Inheritance. Relation of Colour to Hoariness in Stocks. Miscellaneous Cases. Colour of a Special Part controlling that of other Parts.-Summary and Discussion.-SubtractionStages.

Again and again in tracing the genetic properties of colours in animals and plants we encounter the phenomenon of a specific connection between certain colours and their modes of hereditary transmission on the one hand, and various apparently distinct physiological properties on the other. Colour, which systematists have often spoken of as one of the superficial or impermanent properties of organisms, seems thus to be bound up with fundamental phenomena of chemical economy. To treat this part of genetics with any fulness is not yet possible. As an illustration may be mentioned the curious result discovered by Miss Saunders in Matthiola, using the varieties known as "ten-week Stocks." These may be either "hoary," viz. covered with branching hairs forming a tomentum, or glabrous and destitute of hairs. When the hoary are crossed with the glabrous, hoariness is an ordinary dominant, giving 3 hoary : I glabrous in $F_{2}$.

But when certain glabrous strains are crossed together the $F_{1}$ form is hoary, reverting to the primitive type. This reversion never occurs when any of the many red or purple varieties are crossed together, but is universal when any of them are crossed with either the white or the cream-coloured glabrous strains. The purples and reds owe their colours to the presence of coloured sap. This coloured sap is not present in the whites, nor in the creams, whose colour is due to the existence of yellow plastids in the cells of their petals. 
$F_{2}$ from the cross, for instance, of purple $\times$ white contains sap-coloured and non-sap-coloured plants, and of these some are hoary and some glabrous. But none of the plants which come without coloured sap have any hairs on their leaves. A consideration of the case shows that the factor for hoariness is really introduced by the white-flowered glabrous plant, and that the glabrousness is due to the inability of the hoariness-factor to make the hairs grow in the absence of the factors for sap-colour. The facts may be represented thus, $C$ and $R$ representing, as before, the factors for sap-colour, and $H$ the factor for hoariness.

$$
\begin{aligned}
& \text { Purple glabrous } \times \text { White glabrous }
\end{aligned}
$$

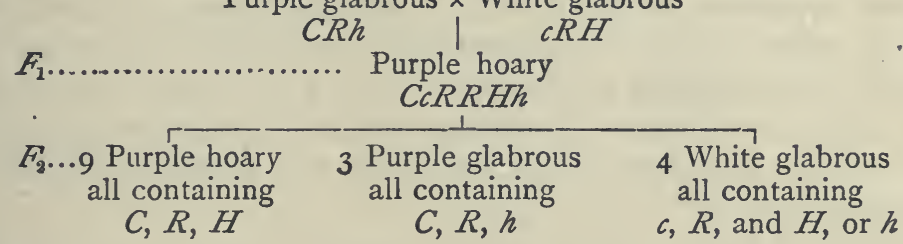

If cream glabrous be substituted for white glabrous the result is the same so far as sap-colour and hoariness are concerned, and in $F_{2}$ only those plants can be hoary which also have coloured sap.

Finally when cream and white glabrous types are crossed together, $F_{1}$ is purple and hoary, thus showing reversion in colour, owing to the meeting of the two complementary factors $C$ and $R$, one coming in from the cream and one from the white; and also reversion to hoariness because the hoariness-factor was really present all the while in both the cream and the white types, but was unable to show itself because one of the sap-colour elements was absent in each type. The heterozygosis of the two types brings together all the three elements $C, R$, and $H$, so the $F_{1}$ plants are both coloured and hoary.

The reason why hoary-leaved plants are never produced by crossing two types possessing coloured sap is at once apparent. For if the factor for hoariness were present in these types, they would be hoary.

As may well be supposed the disentangling of these results was a long and tedious process. The occurrences seemed at first contradictory, but after it had been ascer- 
tained that each kind of family was produced with regularity as the consequence of a particular kind of union, the work of bringing all these into one analytical scheme was only a matter of time. Much still remains to be done before the analysis will be complete for Stocks in general. For example, among the Brompton Stocks races occur which are hoary though devoid of sap-colour, and as yet we are not aware what condition or factor exists which there enables the hoariness-factor to assert itself.

In cases like these we get glimpses of the strict specific rules which govern the genetics of pigmentation. In the Sweet Pea again we have found that both variation in the pollen-shape and in the structure of the standard petal are closely related with the distribution of the factor which turns the colouring matter purple. There is every hope that in our further analyses these apparently trivial phenomena will serve as indications of the underlying processes.

Apart however from these curious inter-relations between colours and structural peculiarities, there are several remarkable specific phenomena to be seen in the genetic behaviour of colours. Of these some examples may be given as incentives to future experiment. General rules regarding colour-inheritance are scarcely to be expected as yet, for very little is known of the pigments of either animals or plants. Beyond the fact that albinism has always been found to be recessive to colour in both animals and plants no general proposition can be put forward with confidence. We believe also that yellow chromoplast-colour is always recessive to white or colourless chromoplast-colour, though the cases studied are not numerous enough to justify a general assertion. Stocks, Sweet Peas, Swedes and Turnips, Verbascum, may be cited as plants following this rule and no clear exception is yet known.

$\mathrm{Mr}$ Arthur Sutton tells me it is well known that when Swedes are being grown for seed, Turnips must not be allowed to flower near them, but that in growing turnipseed, no injury is done by the presence of flowering Swedes. The meaning of this is now clear. The Swedes are in general yellow-fleshed, their colour being due to yellow plastids. Turnips as a rule are white. If therefore the 
pollen of Turnips is carried by insects to the Swedes, the hybrid thus produced will be white-fleshed, and consequently attract attention, spoiling the uniformity of the crop. But as the white of the Turnip is a dominant, no visible effect is produced even though Swede pollen is brought by the insects to the Turnip flowers, for $F_{1}$ is white. In $F_{2}$ of course yellow Turnips would appear*, but as the roots are almost always eaten off, this result is scarcely ever reached by the farmer.

It might be expected perhaps that the blue and purple colours in flowers would always be dominant to the reds, but this is not so. In Stocks, Sweet Peas, Peas, and Salvia the purples are dominant. Probably the same is true for the blues of Delphinium, Cineraria and a good many more, but when we come to Primula Sinensis we find blue a recessive to the reds and magentas. Doubtless the chemistry of the blue pigment is there quite different.

In Solanum and Atropa black fruit is dominant to the yellow fruit, but in Bryonia the red fruit of $B$. dioica is dominant to the black fruit of $B$. alba. This paradox has been elucidated satisfactorily by Miss Wheldale. She tells me that the nature of the distinction between the two types is quite clear. In Atropa the black colour is due to the presence of a dark purple anthocyanin which like other pigments of the same kind is dominant to its absence. In Bryonia alba the black colour is caused by the presence of a little carotin in plastids, together with green chlorophyll undecomposed. In the red-berried Bryonia dioica the chlorophyll is decomposed (just as it is in the cotyledons of yellow-seeded Peas) and much carotin is present. Consequently, as may be expected, the presence of the decomposer of the chlorophyll is a dominant, as also is the abundant development of the carotin, and thus the black colour of the fruits is recessive to red.

In Rabbits, as has been stated above, yellow is a recessive to black, while in Mice it is a dominant.

The yellow varieties of many red Lepidoptera (Zygaena, Arctia, \&c.) are presumably recessivet, and the same is

* From Mr Sutton's experiments $(262)$. it seems however that $F_{1}$ is sterile.

+ Proved for Callimorpha dominula. See p. 44. 
apparently true of the yellow fruits, as compared with the red fruits of their corresponding types, and of the yellow flowers of some Composites (e.g. Gerbera)*.

In many types of flowers, e.g. Stocks, Primula, Sweet Pea, the very dark and more fully-coloured varieties are regularly recessive to the less dark types, whether purple or red. The same will almost certainly be proved for Cyclamen, Rose, Hollyhock, Dahlia, Carnation, Sweet William, and many more. In Antirrhinum Miss Wheldale finds that among magentas the darker are recessive to the common colours, but among the crimsons or reds the darker are dominant to the lighter.

The difficulties which preclude general statements in regard to the genetic relations of melanic types among animals have been illustrated in much that has gone before. The loose description "melanic varieties," common in the writings of systematists, covers a number of phenomena essentially distinct. For example there are melanic forms which owe their greater blackness to the presence of some dominant factor responsible for a greater deposit of black, or at least dark, pigment. In Fowls, for instance, black is at least partially dominant over the bankiva colour which fanciers call "Black-red." The dark brown variety called "Brown-breasted" is similarly a dominant. In Pigeons, as Staples-Browne has proved, black is dominant to the blue of the wild type. In the Horse the presence of black, as in bays and browns, is dominant over the absence of blacks as in chestnuts $†$.

On the contrary in Rabbits, Rats, Mice, \&c. the black variety is produced by the omission of the agouti-factor, $G$, from the wild type, and black thus is apparently a recessive. Even here however the presence of black pigment is dominant to its own absence. It would be interesting to know to which group the Cat belongs.

In Insects again no rule of universal application to

* That in Tomato yellow fruit is recessive to red was established by Hurst (160, p. II5). The case of Gerbera is given on the evidence of crosses made by Mr R. I. Lynch between red Gerbera Jamesoni and the yellow-flowered variety "Sir Michael."

$\uparrow$ The genetic relation of the totally or self-coloured black to the other horse-colours is not yet known. 
melanic varieties can be given. Of the melanic varieties of Moths which have been tested several are apparently dominant, more or less, to the normal or non-melanic types; but in the black Chrysomelid Beetle, Lina lapponica, investigated by Miss McCracken, the evidence shows plainly that the uniformly black type was recessive* to the normal which has black only in the form of spots. The common melanic varieties of the 2-spot Lady bird (Coccinella bipunctata) are probably also recessive to the ordinary red type.

The experiments of Standfuss interpreted according to the Mendelian system show that the dark variety lugens is dominant to the ordinary fulvous yellow type of Aglia tau (a Saturniid Moth). This case comes up for consideration in some detail with reference to the heredity of $\operatorname{Sex}(q . v$.$) .$

In Silkworms a melanic variety of the moth is an imperfect dominant to the normal, pale-coloured moth, giving a blend-form in $F_{1}$ (Coutagne, 83 ).

As regards the colour of the silk some interesting results have been obtained. Yellow silk was always found by Toyama (268) to be dominant to white, and this result was obtained by Coutagne in certain casest. For example the yellow race called "Var" was dominant in silk-colour to the white Japanese race used by Toyama and to the white "Bagdad" used by Coutagne; but Coutagne found the same yellow to be recessive to the white of two French races with which he experimented. Presumably this distinction is due to some idiosyncrasy on the part of the whites, analogous to what has been seen in fowls and Primula, but as to this nothing is known.

In crossing a yellow Siamese race with a white Japanese race, Toyama obtained a resolution-effect in $F_{2}$. Yellow $\times$ white generally gives yellow $F_{1}$ with 3 yellow: I white in $F_{2}$; but in this special case there were two new forms in $F_{2}$, a pale pinkish yellow, and a greenish white. This latter white could not always be satisfactorily distinguished from the pure whites, so the $F_{2}$ family has to be taken as $9: 3: 4$.

* There is a possible complication in this case.

+ Toyama states that the colour of the silk always corresponds to that of the abdominal legs of the larvae, and consequently it is not necessary to rear all the larvae up to the spinning stage in order to ascertain the colour of their cocoons. 
Observation, for example, gave $70: 21: 36$, the expectation being $72: 24: 32$. In certain other cases this resolutioneffect did not occur, though from analogy it might have been expected. Toyama regards the distinction as due to differences in the whites used, but it seems not impossible that it was really the yellows which possessed individual differences in this case. In either event there are difficulties to be faced, and on the evidence it is not clear which account is actually the more probable.

There are some illustrations of a principle by which the colour of one part of the organism may limit or control the possible colours of other parts.

In animals it is fairly certain that the eye-colour may act in this way, certain coat-colours being produced only if the eye be black, and others only if the eye be chocolate, but the facts are still somewhat obscure.

If 'the stem of the Chinese Primula be green and not red the deeper flower-colours cannot be developed in self-coloured types. A cross with a red-stemmed type, however pale in flower-colour, at once reveals the presence of the factors for the deep colours if they are there.

On the contrary, the white-edged types, such as Sutton's "Sirdar," though their flowers may be of a deep shade of purple or red, appear exclusively on stems which are green throughout except for a development of red colour at the collar or extreme base of the petioles. Such "Sirdars" cannot exist on a wholly-coloured stem. The stem may be parti-coloured in Primulas though the flower is whole coloured, but these special types of parti-coloured flower can only occur on a parti-coloured stem. In the $F_{2}$ series it is curious to see these deeply coloured, white-edged, flowers on stems apparently green, while none of their green-stemmed sisters with self-coloured flowers can bear a flower darker than pale salmon-pink.

Another striking example of the same phenomenon is to be seen in these Primulas, with the difference that there the want of a particular colour in the critical or "controlling" position is due to the dominance of a negative character, not to the absence of a complementary one. Certain deep red spots occur in some varieties, e.g. Sutton's "Crimson King" 
(a fine dark red), on the petals just external to the yellow eye (see Plate VI). These spots are never formed unless the stigma is red. When such a type is crossed with one having a green stigma, $F_{1}$ has a green stigma and no spots on the petals. In $F_{2}$ there are of course some with green stigmas and some with red, some with spots and some with no spots. But the distribution of these two characters shows that the combination green stigma + spot on petals does not occur. The stigma may be red though no spot be formed, but if the stigma be green, the spot is absent, though the factor for it may exist in the individual.

Formerly such cases might have been regarded as examples of "correlation," but that term is only applicable to them in a loose and quite incorrect sense.

Nothing so fully demonstrates the fundamental significance of colour in the economy of plants and animals as the strange series of phenomena that have been discovered in regard to the complex inter-relations between the genetic behaviour of certain kinds of pigmentation on the one hand and certain structural features on the other. In the chapters dealing with gametic coupling and with the heredity of Sex it will be shown that not only the factors governing structure, but also the factors which are the ultimate cause of sexual differentiation, may be distributed among the germ-cells according to systems which are modified and ordered in inter-dependence on the distribution of the factors for colour.

\section{Summary and Discussion of the Evidence as to the Genetics of Colour and Colour-Patterns.}

Since we have abundant proof that the development of colour and even of particular colours may be bound up with other features of morphological or physiological importance, it is clearly impossible to regard the genetics of colourcharacters as apart from the rest. A summary of the chapters dealing with that subject will nevertheless be useful at this point, and with this may be combined a brief discussion of essential points.

In many animals and plants colour has been shown experimentally to behave as if due to a single allelomorphic- 
factor. In three cases among plants (Sweet Pea, Stock, Orchids) however we already know that the production of colour (sc. sap-colour) requires the fortuitous concourse of two complementary factors which have independent distributions in gametogenesis, and individuals lacking either of these factors are completely devoid of colour.

In the light of this discovery we naturally ask whether it is not probable that the sap-colours of plants in general may not in reality be produced by pairs of complementary factors. It is tempting also to speculate on the possibility that the colours of animals may have a similar nature. At present however the objection holds that in no species of animal have two pure albinos been found to produce coloured offspring when mated together, and the $F_{2}$ ratio from the cross albino $\times$ coloured is always 3 coloured : I albino, never $9: 7$. But it may be suggested with great plausibility that this simply indicates that every individual, coloured or albino, contains one of the two factors, and the question whether colour is a single-or a double-factor character remains undecided. If a variation were to occur by which the supposed common factor was omitted from the composition of an albino, albinos bearing respectively each of the two factors could be raised, and nothing would then preclude the production of coloured individuals by crossing the two sorts of albinos together.

At first sight some of the facts related in regard to fowls seem to supply evidence of this kind. White Silky $\times$ a recessive white strain produces $F_{1}$ fully coloured. But neither parent is an albino in any strict sense, for both have eyes fully pigmented. As a matter of fact also the Silky breed, though quite white in plumage when adult, oftenperhaps always - has some buff colour in its down. The resemblance is therefore far from being complete.

Another case which suggests a similar interpretation is that of eye-colour in the mouse, for there black eyes result in $F_{1}$ from crossing certain pink-eyed mice having coloured coats with certain albinos. But here again one of the parents is obviously not albino, and as we now know from Miss Durham's observations, the eye of the coloured parent though ostensibly pink, really contains a small but definite amount of pigment. 
Animals and plants are alike in the fact that their colours, however produced, may be modified by the presence of additional factors. In each case therefore we must conceive of one lowest or hypostatic colour, and of epistatic factors superimposed on this which produce their several effects. In the Sweet Pea the lowest colour is red, which is turned to purple or blue if the factor having this power is present. Similarly in the mouse the lowest colour is chocolate, which becomes black if the black factor is added, and so on. The intensity and also the distribution or pattern of colours behave in descent as if they also were governed by such superimposed factors, though as will shortly appear, it is not certain that this mode of representation is strictly correct. However this may be, we are safe in regarding the pigmentation of animals and plants as a character usually resulting from the combined operations of several distinct factors, transmitted separately in heredity.

Applying conceptions which have lately become current in physiology Cuénot suggested that the determiners which modify colour in the mouse, for instance, may be distinct diastases acting on a single chromogen substance. In the present state of physiological chemistry it is, I suppose, impossible to speak with confidence as to the nature of the bodies concerned and we must keep an open mind. Nothing yet precludes the possibility that there may be one diastase responsible for the production of colour, and another set of bodies which, acting in the presence of the diastase and of the chromogen, determine the quality or shade of the colour*.

So in the mouse, the wild grey colour results from the joint action of at least three factors: (I) the colour, which, if no epistatic factor is present, would be chocolate; (2) a black determiner, which causes black pigment to appear; (3) the agouti-factor, $G$, which gives the hairs their banded appearance and also causes some yellow pigment to be formed in them. In rats a black variety exists because the factor $G$ may be absent, but no chocolate variety has been recorded because the factor for blackness has not yet fallen out. If

* As pointed out above, Cuénot's suggestion that in the case of mice the agouti-factor, $G$, is allelomorphic to the factor for blackness, $B$, is not an adequate representation of the phenomena. (See p. 76.) 
a chocolate rat were to be produced, then by crossing it with the wild grey type, blacks must occur in $F_{2}$, just as they are known to do in the case of the same mating in mice.

The facts compel the recognition of such a series of determining elements, and it is perhaps simpler to imagine these elements as distinct from the exciting cause, and additional to it, while remembering the possibility that they may in reality be only modifications of it.

Similarly in attempting to express the genetic interrelations of the several patterns of a colour as depending on the existence of definite factors, we have to bear in mind that we are only using a convenient symbolism. It is not incumbent on us to believe that there are any physiological substances which have the power of governing the distribution of the colour. Experiment shows that the power to cause the colour to be uniformly distributed as in the "self" type, or to be restricted to special regions of the body as in the Dutch rabbit, for instance, can be carried by the gametes, and that when these two possibilities are combined in heterozygosis, they segregate in gametogenesis.

This being so, the two possibilities may thus be represented symbolically as two factors, having regard to their effects on the configuration of the resulting zygote; but if we must attempt to imagine an answer to the question, wherein does the distinction between self pattern and Dutch pattern physiologically consist, we should, I suppose, refer it rather to differences in the distribution of one of the chromogenic factors than to the presence or absence of an additional element. In the self-coloured rabbit the two colour-producing elements are generally distributed over the skin, while in the Dutch rabbit either the chromogen or the diastase-if these be the critical substances-is restricted to certain areas. The colours in the pied animal thus come out in certain patches just as do lithographic colours upon the prepared parts of the stone when the ink is applied to the whole surface.

As the black rabbit or mouse is an animal from which the grey determiner, $G$, is absent, so the pied animal is one from which the self-coloured distribution is absent. Nevertheless the essential distinction between the two forms must surely be quantitative. In the self-coloured type one of the 
substances, say the chromogen, is distributed over the whole surface, but in the Dutch-marked, for example, it is reduced in quantity. The reduction however occurs in a fairly definite way, leading to the formation of a type having a recognizably distinct pattern. It does not seem an unreasonable speculation to suppose that we have here to deal with a condition in which the amount of the substance is insufficient to cover the whole region which it occupies in the self-coloured type, though why it should be restricted to one special region more than another it is impossible to say.

If the definite pied phases are to be thus regarded as representing quantitative diminution in the development of one of the determining substances, we may make a similar supposition in regard to the diluted colorations already mentioned in the case of mice. In the diluted colours the reduction in quantity, instead of diminishing the coloured area while keeping the intensity of the colour, is effected by diminishing the intensity of the colour while the totality of the distribution is retained. The black Dutch-marked mouse may thus be imagined to be a mouse in which one of the colour-factors exists in its full intensity, though there is not enough of it to cover the skin, while in the blue mouse the factor is generally distributed over the skin but in a dilute condition. In both cases alike the subtraction-stage as we may call it is a fairly definite stage in the reduction of the amount of pigment.

A physical analogy-doubtless imperfect, but nevertheless instructive-may be drawn from the way in which various oils distribute themselves over the surface of a liquid with which they do not mix, some forming circumscribed patches of greater thickness, which may be compared with the patches on the Dutch rabbit, others spreading in a thin layer over the whole surface, like the dilute colours spread over the whole coat. The analogy breaks down at the fact that in the oils the physical distinctions to which the different behaviours are due cannot be transferred from one oil to the other, whereas in the rabbit this is accomplished-a fact which entitles us to represent the several properties as distinct and transferable factors. Thus the results of the cross between a black-and-white Dutch- 
marked mouse and a self-coloured blue may be represented as due to the re-combinations of the two pairs of factors.

I. $S$, self colour. $\quad s$, its absence, viz. Dutch-marked.

2. $D$, dense colour. $d$, its absence, viz. dilute colour.

Black is common to both parents, and so need not be represented.

Dutch-marked black. $\quad \times \quad$ Blue

$s D$

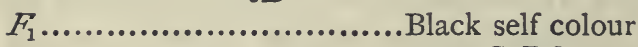

Sd

$\begin{array}{cccc}F_{2} \ldots \text { Black self colour } & \text { Black Dutch } & \text { Blue self colour } & \text { Blue Dutch } \\ 9 S D & 3 s D & 3 S d & \text { I } s d\end{array}$

The recognition of these subtraction-stages becomes important when we attempt to estimate the minimum number of factors necessary to produce the results we perceive. Symbolically the various subtraction-stages may be represented as depending on the removal of distinct factors, but physiologically they may be caused by special quantitative subtractions from one of the factors causing the production of colour, and consequently an economy of hypothesis may be made.

In exceptional cases the pattern which an albino form is carrying, if the expression be permitted, can be actually recognized by inspection though no real colour is developed. For example Lock noticed the following case in edible peas (Pisum sativum). Certain varieties of peas have brown anastomosing lines on the coats of the seeds. These peas are called maples in England (pois perdrix of French seedsmen). The maple skin occurs only in the seeds of strains which have coloured flowers. Such plants crossed with an ordinary white-flowered type having a plain seed-coat gave this result :

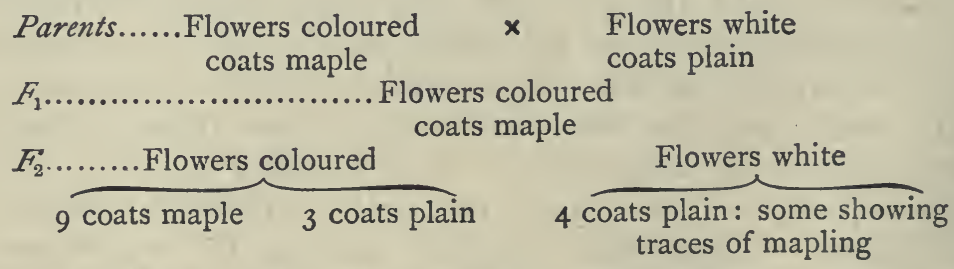

Among the white-flowered group in $F_{2}$ were some plants which bore seeds showing traces of the maple marking, not as brown lines, for no actual pigment seemed to exist, but as a damasked pattern showing where the mapling would have 
been if the plant had been a coloured one. Lock (176) has spoken of this faint pattern as the "ghost" of the mapling.

Mudge (204) has observed a very similar phenomenon in young albino rats. When the hair is short the coat may be seen to be similarly damasked, those parts which would be pigmented if the animal had pigment looking different in consistency from the rest.

What the exact difference between the hairs in these areas and the rest may be has not been ascertained, but evidently it must be a modification due to the existence of one of the factors for colour in those hairs. They are the parts prepared to develop colour if the other element were present in them. In black leopards and black kittens a similar damask effect can often be seen, the parts which in the spotted leopard or tabby cat would be light being distinguishable on careful examination. As it is not yet known whether black is dominant or recessive in these cases the exact meaning of these marks is uncertain.

Both in animals and plants there is satisfactory proof that whiteness, the absence of colour, may be due to partial or complete suppression of the pigment-factors and not merely, as in the albino, to their absence. This suppression is caused by a dominant, epistatic factor. White individuals containing such a factor are more or less totally dominant whites, whereas whites due to the absence of one or more pigmentation-factors are recessive whites. A cross between a dominant and a recessive white may obviously cause coloured individuals to appear in $F_{2}$, if the factors for pigmentation were introduced by the parental types ; for in $F_{2}$ there will be individuals lacking the suppressing factor.

Confusion has been introduced into these analyses by the use of the term "latency" in application to those factors which cannot be perceived without breeding tests. This difficulty has occurred especially in regard to albinos, though it pervades the whole system of factorial analysis. Albinos, for instance, in any species may have the most diverse factorial composition. All that is common to them is the absence of colour, i.e., if we adopt Cuénot's suggestion, of the chromogenic substance. The composition of each albino may be ascertained by crossing it with a coloured type and raising the $F_{2}$ generation. If the coloured type chosen be 
in all its characters hypostatic-or recessive, to use the simpler if less accurate term-to all the factors of the albino, the composition of the albino may be seen even from $F_{1}$. For instance, when bred to a pure black, a $G G$ albino will give greys only; a $G B$ albino will give equal numbers of greys and blacks; while a $B B$ albino will give blacks only. Conversely it is possible to manufacture by suitable matings albinos of each composition. For example, albinos extracted from chocolates can only bear the chocolate determiner. Those from black mice must all bear the black determiner if the families have been large and no chocolate has occurred. So, those from greys must all bear the determiner $G$, if in sufficient numbers no blacks or chocolates have been produced. With regard to pattern and saturation or dilution of colour the case is exactly the same. An albino from Dutch-marked parents cannot bear the self-colour factor, and one from blues cannot bear the saturation-factor.

It is this fact, that in most cases the albino will be bearing the determiner proper to the colour of the last coloured parent from which it was extracted, which has led several writers to speak of these colours or patterns as "latent" in the albino. This mode of expression is much to be regretted. There is no "latency" of black, or grey, or self-colour, as a whole in the albino. Certain factors which are essential to the production of those features may be present in any albino of unknown origin, but this fact does not in any way touch the question of the purity of the germ-cells, as has been quite erroneously suggested. Sulphate of copper is blue and chloride of copper is green, but it would be incorrect to speak of blue as latent in sulphuric acid, or of green as latent in hydrochloric acid; nor has the acid obtained from chloride of copper more of "greenness" in it than has the same acid obtained from sodium chloride.

Taking the evidence respecting the genetics of colour as a whole, though much remains which is obscure, as has been stated, especially in the discussion of yellow in the Rodents, there can be no reasonable doubt that with rare exceptions it will be found possible to express the whole series of phenomena as due to the combination and re-combination of a limited number of recognizable factors which are treated by the cell-divisions of gametogenesis as units. 
As the nature and properties of each of these units are successively determined, we cannot doubt that additions will be made to the number of examples, already not inconsiderable, in which a fixed interrelation can be proved to exist between the units which govern colour and those responsible for form and other physiological attributes.

In any attempt to picture the process of Evolution the group of genetic phenomena discovered in regard to colour has extreme value and interest. We thus are at once provided with clear illustrations which enable us to see the nature, if not as yet the causation, of Variation, and the significance of those particular Variations which we call reversionary. Such illustrations may well serve as testcases, by which the truth of evolutionary systems may be gauged. Though the result of these trials may largely prove destructive, the facts are not without a constructive bearing. One positive deduction cannot be overlooked: that the organism is so built up that definite additions to, or subtractions from its totality may readily be made by Variation, and that the consequence of such alteration of the ingredients may be recognizably definite, or to use another term, specific. 


\section{CHAPTER IX}

GAMETIC COUPLING AND SPURIOUS ALLELOMORPHISM.

\section{Pollen-Shape and Flower-Colour. Axil-Colour and Sterile Anthers-Hooded Standard and Flower-Colour in Sweet Peas.}

WE have now to consider one of the most curious and interesting developments of Mendelian research. In all the examples hitherto described the $F_{2}$ numbers have shown that when allelomorphs belonging to various pairs are simultaneously distributed among the gametes in the process of gametogenesis, the distribution is random, so that all possible combinations are represented by equal numbers of gametes. For example, in the case of the double heterozygote formed by crossing a yellow, round pea with a green, wrinkled variety, the gametes produced by the $F_{1}$ plant are in equal numbers bearers of
(I) yellow, round,
(2) yellow, wrinkled,
(3) green, round,
(4) green, wrinkled.

This fact is proved by the numbers $9: 3: 3: 1$ in which the several types of zygotes appear in $F_{2}$. The phenomena now to be described indicate a system of segregation taking place in such a way that gametes presenting certain such combinations occur with greater frequency than the others.

The example in which this state of things was first detected is that of the pollen-shapes of the Sweet Pea (Lathyrus odoratus). The experiments in that case were begun, as has already been described (p. 89), by crossing a white Emily Henderson having long pollen, with a white 
Emily Henderson having round pollen. The $F_{1}$ generation was a reversionary purple bicolor, with long pollen-for the long pollen-shape is dominant-and from this the $F$, generation was raised which consisted of

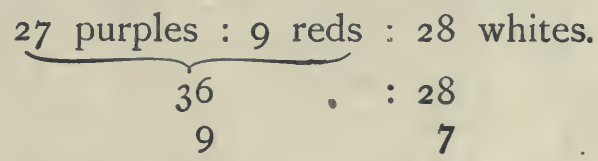

It has been shown that the interrelations of these several types proved that the colour in both purples and reds is due to the simultaneous presence in the zygote of two factors which we have called $C$ and $R$, and that the whiteflowered plants are those in which one or both of these factors are absent. The point, however, which now concerns us more immediately is the distinction between the purples and the reds. This, we have seen, is due to the presence or absence of a factor $B$, blue. Those coloured plants which have the factor $B$ are purple, those devoid of this factor $B$ are red-flowered.

We saw also that there were various subordinate classes among the purples with corresponding subordinate classes among the reds, each due to the possession or to the want of some special factor. These differences are similarly distributed among the purples and among the reds. For example if the family is one which contains picotee types, there are picotees among the purples and corresponding types among the reds, and in each colour-group the proportions are the same, averaging 3 fully-coloured to I picotee in the case of the purples and the same in the case of the reds.

When however the distribution of the pollen-characters, long and round, in a family containing purple, red, and white members is examined, it is found that taking the family as a whole the long-pollened plants are to the round-pollened as 3 to $I$ in the usual way. Among the white-flowered also there are 3 longs to $I$ round. But when the purples and the reds are separately studied, the numbers 3 long : I round are not found. On the contrary, in the purples there is a great excess of longs, which are to the rounds as about I 2 to I, while among the reds there is an excess of rounds, which are to the longs as about $3^{\circ} \cdot 2$ to $I$. 
The result of extensive counting shows that an approximation to the observed numbers would be produced by a gametic system of such a kind that the combinations of long pollen with blue factor, and round pollen with no blue factor
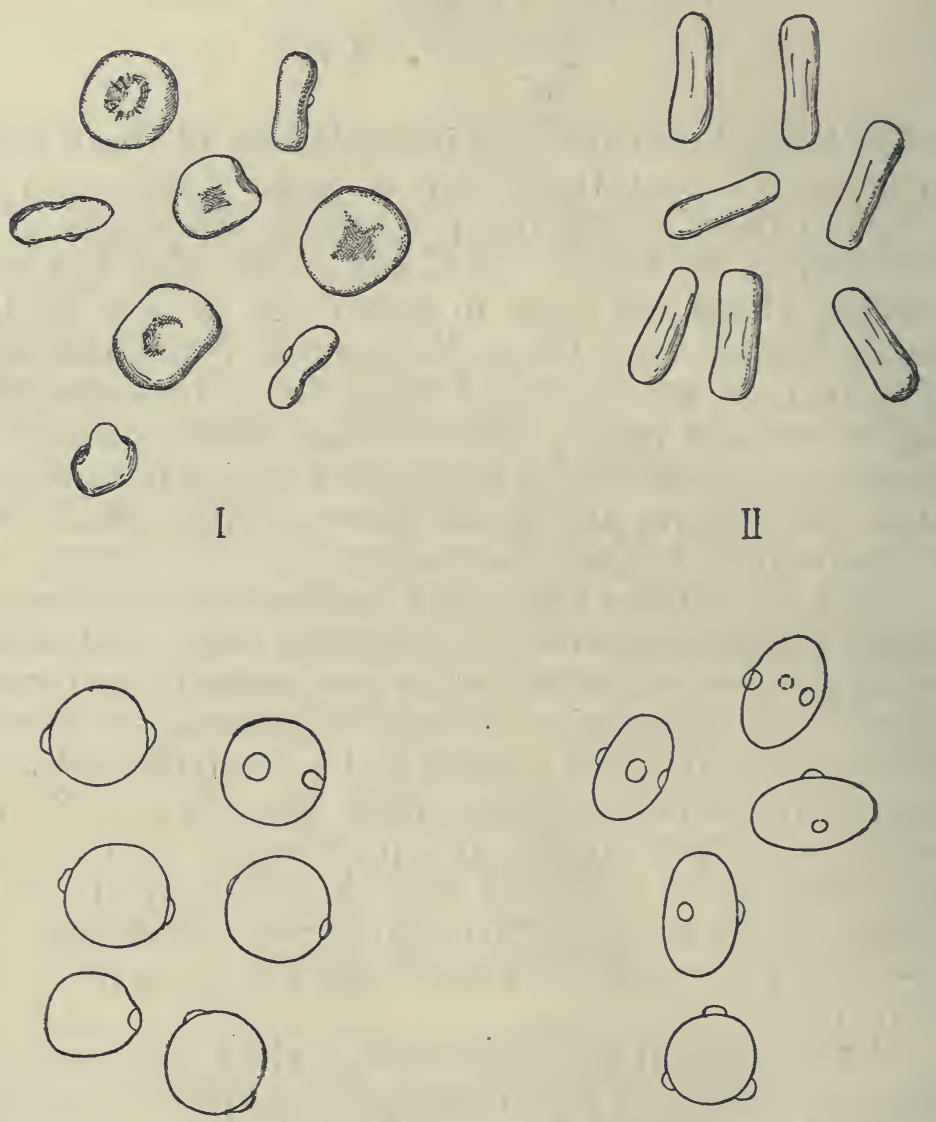

III

IV

Fig. 19. Pollen-grains of Sweet Pea.

The normal long (dominant) type is shown in II and IV: the peculiar round type (recessive) in I and III. The upper figures represent the dry condition. The lower figures show the appearance in sulphuric acid, which makes the pores visible. Three-pored and one-pored grains sometimes occur among the rounds, but they are usually two-pored. In IV one of the grains is seen end-on, and in I three of the disc-like grains are seen edge-wise. 
occur seven times as often as the other two possible combinations. We speak of this phenomenon as Gametic Coupling.

The term "coupling" is strictly applicable, because the association is between two dominant or "present" factors, here those for blue colour and long pollen. Abnormal distributions due to such coupling are to be carefully distinguished from those described later under the name "Spurious Allelomorphism," to which the term "coupling" should not be applied.

If the two pairs of factors are expressed thus :

\section{Dominant}

Blue colour $B$

Long pollen $L$
Recessive

Red colour $b$

Round pollen $l$

the gametic series is not

$$
\text { I } B L+\mathrm{I} B l+\mathrm{I} b L+\mathrm{I} b l \text {, }
$$

but

$$
7 B L+\mathrm{I} B l+\mathrm{I} b L+7 b l \text {, }
$$

or very nearly so.

Such a gametic series would give an $F_{2}$ family composed thus :

I 77 blue-long : I 5 blue-round : 15 red-long : 49 red-round.

Reference to Fig. I7 will show how these numbers are arrived at.

These ratios agree very nearly with those observed in actual experiments. For example the following series has

\begin{tabular}{|c|c|c|c|c|c|c|}
\hline \multirow{2}{*}{ Observed } & \multicolumn{2}{|c|}{ Purple } & \multicolumn{2}{|c|}{ Red } & \multicolumn{2}{|c|}{ White } \\
\hline & Long & Round & Long & Round & Long & Round \\
\hline
\end{tabular}
been produced.

The correspondence between calculation and observation is sufficiently close. Further experiments in which plants heterozygous in blue colour and in long pollen were crossed with red-flowered plants having round pollen make it practically certain that the series $7: 1: 1: 7$ expresses both the male and the female gametic series with approximate correctness.

Study of the $F_{3}$ families has proved conclusively that the abnormal distribution occurs only among the gametes of plants which are heterozygous both in the pollen characters 
and also as regards the factor $B$. Plants that are homozygous in either of these allelomorphs have the normal distribution of characters among their gametes, and they may be heterozygous in $C, R$, or in any of the other factors recognized in the Sweet Pea without any departure from the ordinary ratios being produced.

The gametic series has been spoken of as $7:$ I : I : 7 and these are the numbers which fit the observed result most closely, but attention should at once be called to the possibility that the series may in reality be $8:$ I : I : 8 . The observed numbers are too small to enable us as yet to discriminate between these two possibilities, though, as will be seen when the nature of coupling is discussed, the significance of the two series must be entirely different. It is however to be noticed that the series of gametes necessary to complete the whole system is thus either 16 , or $16+2$.

In the next two examples of such partial coupling the association is in groups of 32 , or $32+2$. Both these also occur in the Sweet Pea. The first concerns the peculiar sterility or contabescence of the anthers which has already been mentioned as a recessive character. The second factor is again a colour-factor. Among the various factors which control colour in the Sweet Pea is one which causes the appearance of a reddish purple spot in the axils of the leaves, referred to already as the dark-axil factor. When this factor is present (and the flowers are coloured) the axils are dark; when it is absent the axils are simply green as they always are in white-flowered plants*. At an early stage in the Sweet Pea investigation it was noticed that when a family contained plants differing in respect of sterility and fertility of anthers as well as in respect of dark and light axils, the plants with sterility in the anthers (having coloured flowers) were almost always light-axilled, and conversely the dark-axilled plants were almost always fertile in the anthers. In such families, among the white-flowered

* Dark axils sometimes exist in plants which have the flowers so nearly white as to pass for real whites. Probably in all such flowers a trace of colour is developed, and certainly in them the seed-coat is always black as it is in all the Sweet Peas with coloured flowers.

Plants raised from wild Sicilian seed were all purple bicolour in flowercolour, and nearly all had dark axils, but a few had light axils. 
plants, the sterile to the fertile were 3 to I without complication.

Statistical examination of these families on a large scale has shown that among the plants with coloured flowers the ratio of fertile with dark axil : fertile with light axil : sterile with dark axil : sterile with light axil, approaches closely to that which would be produced if the series of gametes bearing these four respective combinations were

$$
\text { I } 5 \text {. I : I : } 15 .
$$

The actual numbers observed were

\section{Dark axil}

$\begin{array}{rcc}\text { Fertile } & \text { Sterile } \\ 627 & 27 \\ \text { Expectation } & 637 & 27\end{array}$

Light axil

$\begin{array}{cc}\text { Fertile } & \text { Sterile } \\ \mathrm{r} 7 & 2 \mathrm{r} 4 \\ 27 & \mathbf{1 9 4}\end{array}$

\section{Repulsion or Spurious Allelomorphism.}

A third example of partial gametic coupling relates again primarily to the blue factor $(B)$ and the pollen-shapes, but in order to make clear the circumstances in which it occurs, another set of phenomena must first be described.

In the old types of Sweet Pea the standard is erect and has a small notch in the middle of its upper border. This is the natural shape of the wild flower. Of the modern types many are what is called "hooded." The standard in hooded forms turns forward and downward in various degrees, the amount varying with the type and also to some extent with the weather and the condition of the flower. The hooded standard differs also from the erect one in having little or no trace of the central notch. This difference causes the buds of the two types to be recognizably distinct before the flower opens, for in the hooded type the point of the folded standard projects sharply forward in front of the wings, while in the erect type this tip is rounded off by reason of the notch. (Plate V.)

The hooded standard also is sometimes distinguished by the existence of a sinus of variable size on each side of the standard, which thus has lateral lobes more or less well developed. These differences obviously point to a different distribution of the strains produced by the growth in the two types. The lateral sinus is not represented in the hooded flowers shown in Plate V. 
The hooded types may have a great diversity of colours, and fixed hooded varieties now exist in the purple, blue, red, pink, cream and other classes. It is nevertheless a remarkable fact that, so far as I am aware, none of the regular bicolour varieties ever have a really hooded standard. There is for instance no hooded type having the colour of the original purple, with its chocolate-purple standard and blue wings, nor can Painted Lady with standard red and wings nearly white be produced in a hooded shape. On the contrary the hooded types always have the standard and wings more nearly alike in colour, and there is the clearest evidence that in families ( $F_{2}$ and later generations) which contain original bicolour purples as well as hooded types, the hooded types corresponding to them are of the unicolorous kind known as "Duke of Westminster*."

From these facts it is evident that there is here some interdependence between the colour of the flower and its form. This interdependence is of course somatic, but as will be seen there is also a gametic connection between the phenomena of shape and colour.

The experiments bearing on these questions originated in a cross between the white, round-pollened Emily Henderson and a white, long-pollened hooded type known as Blanche Burpee. The Emily Henderson has an ordinary erect standard with the central notch.

$F_{1}$ produced from these two is a bicolour purple, with erect standard and long pollen, indistinguishable from the reversionary $F_{1}$ previously described as the offspring of the long and round whites. $F_{2}$ from such plants consists of the following types:
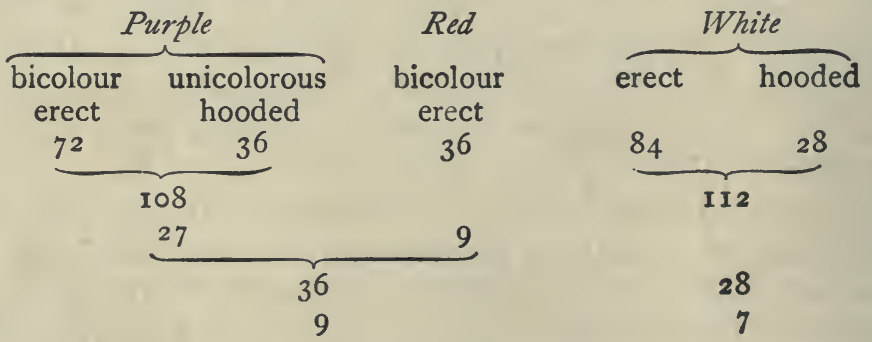

28

7

* Similarly if the bicolour purples with dark wings are present in the class with an erect standard, they are represented by "Duke of Sutherland" in the hooded class, viz. a deep unicolorous purple. 
$>$
$\stackrel{0}{\frac{\pi}{n}}$
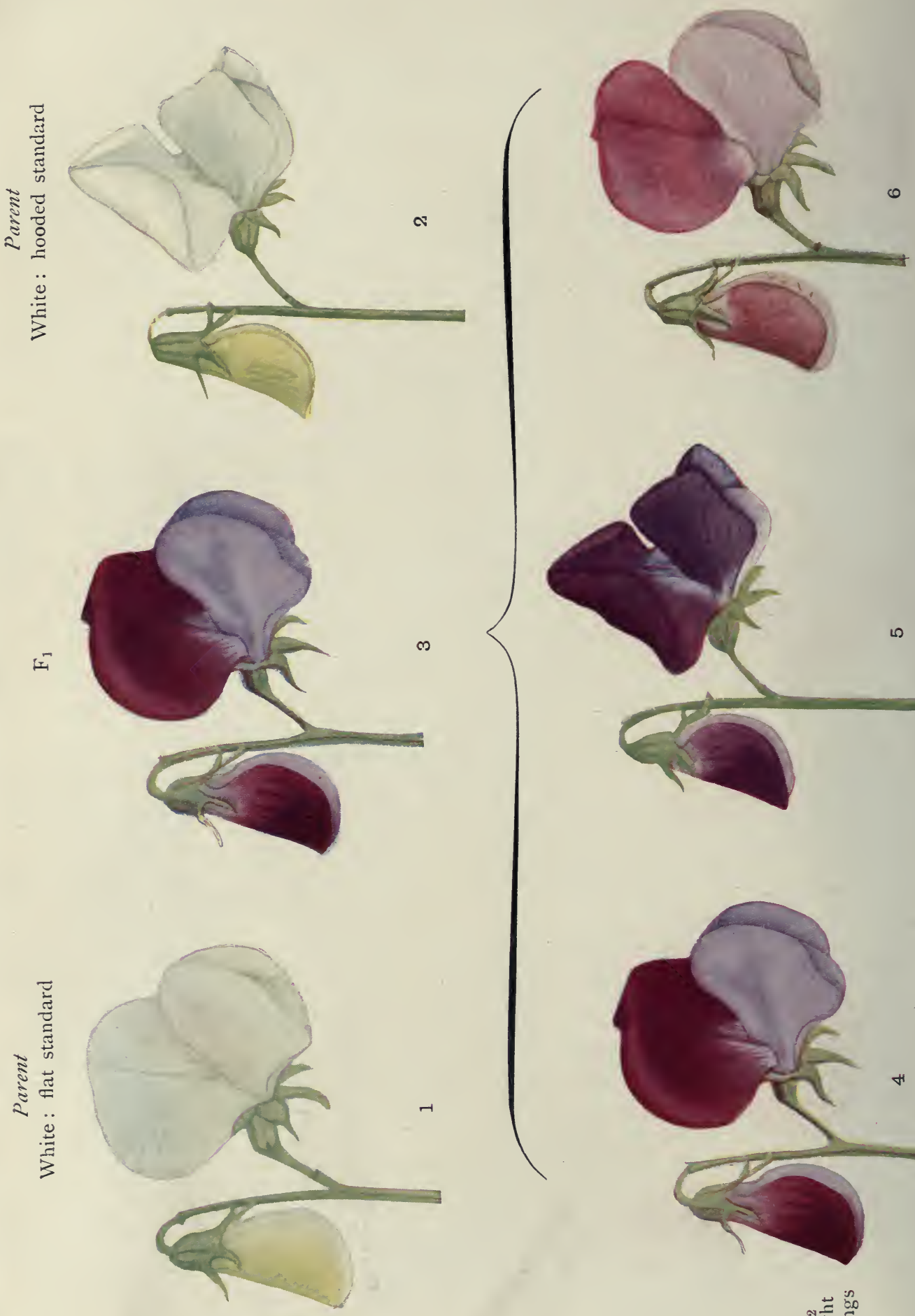

क

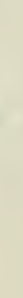



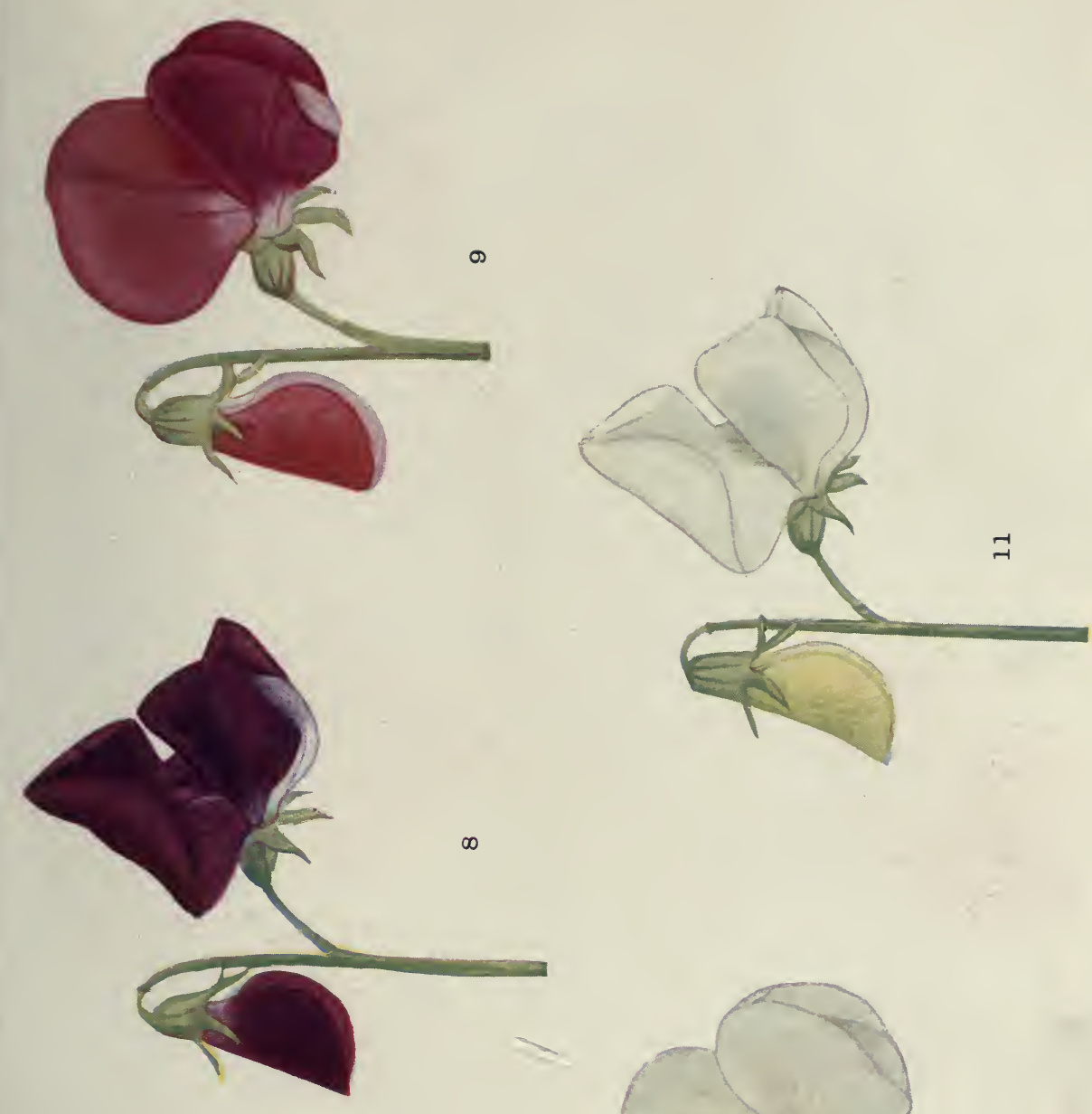

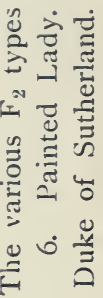

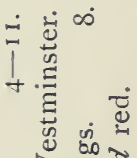

$\dot{-1} \stackrel{0}{\Xi}$

5०

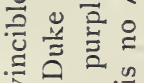

的进

政

ڤ.

ले त्री

m

ญำ

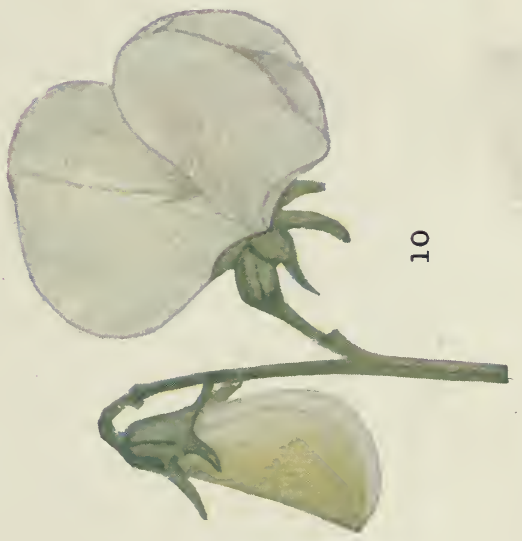

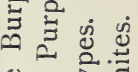

을

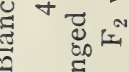

‥

क है है

䅉

5.

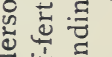

过灾

合总录

氙记

巫氞

- $\therefore$ 造

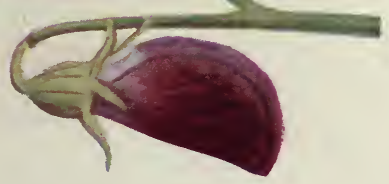

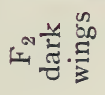



From these results it appears that the erect standard is dominant to the hooded. Next we have the remarkable feature that whereas the purples and the whites are both represented in the two classes erect and hooded, the reds are all erect. This fact indicates that those gametes which bear the factor for erect standard do not bear the factor $B$ which causes the purple or blue colour; and conversely the gametes which do not bear $E$, the erectness-factor, bear $B$. The gametes in respect of these two allelomorphic pairs have thus the composition $B e$, or $b E$, and as regards transmission of characters the effect is that which would be produced if $B$ were allelomorphic to $E$. In view of this curious fact it seems not impossible that we may be obliged hereafter to extend the conception of allelomorphism, and to recognize that factors concerned with features of organisation which seem to have no special physiological association, may be allelomorphic to each other.

Another curious result follows from the existence of this repulsion or spurious allelomorphism. If the hooded purples in $F_{2}$ be bred from, the $F_{\mathrm{s}}$ families will be either all hooded purples again or they will be such plants together with hooded whites. Similarly the red bicolours will give $F_{3}$ consisting either of red bicolours, or of red bicolours and erect whites. But the purple bicolours, must all by their constitution be heterozygous in both the factor for erectness and for blueness, and consequently their $F_{3}$ families contain hooded purples, erect bicolour purples, and erect bicolour reds in the ratio $I: 2:$ I (together with whites if the particular $F_{2}$ happens to be heterozygous in either $C$ or $R$, the fundamental colour-factors).

Such bicolour purples thus present the anomaly of being permanent heterozygotes, though in appearance they are actually the original type of Sweet Pea. Families may thus consist entirely of

\begin{tabular}{|c|c|}
\hline $\begin{array}{l}\text { Hooded } \\
\text { unicolorous purples }\end{array}$ & $\begin{array}{c}\text { Erect } \\
\text { bicolour purples }\end{array}$ \\
\hline I & 2 \\
\hline
\end{tabular}

and the bicolour purples in such families will give similar families again indefinitely.

On determining the pollen-characters of those families 
which contain erect and hooded, coloured and white, long and round pollens, it was found that in $F_{2}$ the distribution followed the system derived from the gametic series $7:$ I : I : 7 , but among the $F_{3}$ families derived from them several were found to exhibit coupling between the blue factor and long pollen according to the system $I 5: I: I: I 5$. For instance, to take the group of plants in which this was most evident, a certain $F_{3}$ plant with purple flower and erect standard together with eight similar plants (its offspring, in $F_{4}$ ) gave collectively

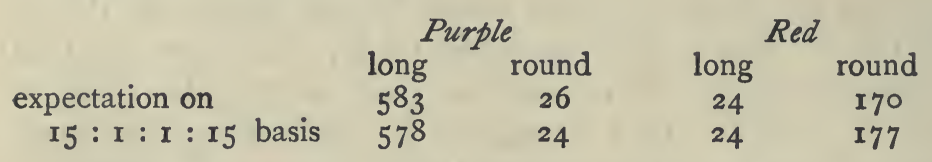

Here it is practically certain that the $I_{5}:$ I formula correctly expresses the gametic distribution. Nevertheless, though some of the offspring of $F_{2}$ gave such definite indications of the I5: I system others no less definitely followed the 7: I plan, and others again gave results so uncertain that it was impossible to assign them to either group with any confidence.

The facts are thus exceedingly complex, and all that can be stated is that coupling between the blue factor and the long pollen does certainly exist in certain families derived from a cross which involved the factors for erect and hooded standard; but that inasmuch as the $F_{2}$ distribution followed the 7: I plan*, the heterozygosis between erectness and hood cannot be the direct cause of this change in the coupling. Pending further analysis the distinction which decides whether the coupling shall follow the $7:$ I system or the I5: I system must be regarded as quite unknown, for examination of the various families has not revealed any consistent difference between them (see 22, pp. IO-I 3).

Since the $B$ factor is alternative to $E$, the erect standard, in the gametic composition, it follows that the zygotic com-

* $F_{2}$ here was

\begin{tabular}{ccccc} 
& \multicolumn{2}{c}{ Purple } & \multicolumn{2}{c}{ Red } \\
expectation on & long & round & long & round \\
$7: \mathbf{I}: \mathbf{I}: 7$ basis & 296 & $\mathbf{1 9}$ & 27 & 85 \\
& 295 & $\mathbf{2 5}$ & 25 & 82
\end{tabular}


bination of hooded standard with round pollen must be exceedingly rare in any of these families. If the gametic coupling is

7 Blue long + I Blue round + I Red long +7 Red round, the zygotic expectation is, in the simpler case where all the standards are erect,

I 77 Blue long + I 5 Blue round + I 5 Red long + 49 Red round.

But when the standards may be either erect or hooded, all the hooded plants are homozygous in $B$, and the expectation of a round-pollened plant occurring among the $B B$ class is only $\mathrm{I}$ in 64 . Observation agreed with this expectation, for in the $F_{2}$ families which certainly all followed the $7:$ I system, there were 83 hooded purple plants and of them one was round-pollened. The same expectation holds in regard to the hooded plants with white flowers, which also must all be $B B$. Unfortunately most of these were recorded before the importance of the question was appreciated, and in them the hoods were not noted. Of 17 plants of this class which were examined 16 were long and I was round.

Similarly, when the coupling is on the $15:$ I $:$ I $:$ I 5 system the hooded purples will be still rarer, and should occur only as I in 256 of their class. Among the 9 families which definitely followed the I5: I plan, one hooded round occurred among 209 plants of the hooded class.

\section{Discussion of the Physiological Significance of Gametic} Coupling and Spurious Allelomorphism*.

The significance of the phenomena just described lies in the fact that they demonstrate the existence of a complex interrelation between the factorial units. This interrelation is such that certain combinations between factors may be more frequent than others. The circumstances in which this interrelation is developed and takes effect we cannot as yet distinguish; still less can we offer with confidence any positive conception as to the mode in which it is exerted. Part I.

* For recent discoveries regarding these phenomena see Appendix to 
The time has not yet come for such an analysis to be attempted. Nevertheless we can scarcely forbear from considering some of the possibilities which suggest themselves.

In spurious allelomorphism the outward facts are comparatively simple. Two dominant, or "present" factors, behave as if in the cell-divisions of gametogenesis they repelled each other, and we must suppose that this repulsion is exerted at some definite cell-division, such that one factor passes into one daughter-cell and the other factor into the other. The dividing cell being $A a B b$, the daughter-cells are respectively $A b$ and $a B$. Though as yet only one case has been definitely proved to follow this system, the evidence in that case is very positive. Moreover when the facts of sexual inheritance come to be related, a group of cases will be described which conform so precisely with this type-example of spurious allelomorphism that it is practically certain that this case is not a solitary example, but one which typifies a category of genetic phenomena. It may therefore be taken that repulsion-or, more strictly, a relation which can be represented as repulsion-may exist between factors belonging to distinct allelomorphic pairs.

The state of things which results in gametic coupling is much more obscure. The association of characters here is quite distinct from the association of characters produced by spurious allelomorphism. In gametic coupling the dominant factors are associated together, while in spurious allelomorphism the dominant factors are dissociated from each other. If the coupling were total, so that all the gametes were either $A B$ or $a b$, just as in spurious allelomorphism they are all either $A b$ or $a B$, we might naturally suppose the one phenomenon to be the converse of the other. The one might then be represented as an effect of attraction just as the other may be represented as the result of repulsion between the two dominant factors. So far, however, as experiment has yet gone, we have no certain case in which the coupling is complete. There are no doubt instances of features apparently distinct which are nevertheless transmitted in collocation. In the Sweet Pea, for instance, the deep brown or blackish pigmentation of the seed-coat occurs only in plants with some colour in the flower, but these two features may thus be supposed to 
depend on one allelomorph, not on two. To prove the existence of complete coupling it would be necessary to show that features elsewhere known to depend on separate allelomorphs, could on occasion be linked in a complete union. Whether such a state of things is possible we do not know. There is no a priori reason for supposing that it is impossible.

The arithmetical series in which the numbers occur is the only guide as to the nature of the process, and obviously this is quite insufficient. The existence of the $7: 1$ systems and of the I $5: 1$ systems naturally suggests the possibility that a system based on $3:$ I may exist. We might then arrange the systems in a series thus*:

\begin{tabular}{|c|c|c|c|c|c|}
\hline \multirow[b]{2}{*}{ No coupling } & \multicolumn{3}{|c|}{ Gametes } & \multicolumn{2}{|c|}{ Tọtal in } \\
\hline & I $A B$ & I $A b$ & г $a B$ & I $a b$ & \\
\hline $3: 1$ & $3 A B$ & I $A b$ & I $a B$ & $3 a b$ & \\
\hline $7: \mathbf{I}$ & $7 A B$ & I $A b$ & I $a B$ & $7 a b$ & \\
\hline I $5: I$ & I $5 A B$ & I $A b$ & I $a B$ & ${ }^{1} 5 a b$ & \\
\hline
\end{tabular}

Hitherto, though some dubious indications of such a series have been seen, there is no clear case of coupling on the system $3: \mathrm{I}$.

It is not easy to conceive any probable system of symmetrical cell-divisions or dichotomies which would produce the series $7: I$ and $I 5: I$. If the segregation of characters were not all completed at one cell-division we might of course imagine a scheme which would give the system $8+i+I+8$, thus .

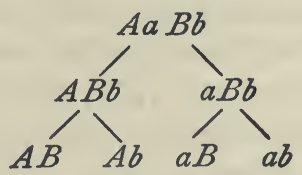

after which, if the cells $A B$ and $a b$ each divided again

* The $F_{2}$ numbers resulting from these couplings are as follows:

$A B \cdot A b \cdot a B \cdot a b$.

\begin{tabular}{|c|c|}
\hline $\begin{array}{l}3: I: I: 3 \\
7: 1: 1: 3\end{array}$ & $\begin{array}{r}41: 77: 7: \\
177: \leq 5: 15: \\
179\end{array}$ \\
\hline I5:I: I : I5 & $737: 3^{I}$ \\
\hline
\end{tabular}

If $n$ be half the number of gametes needed to express the whole series of couplings in a given case, then the four $F_{2}$ numbers are given by the formula

$$
3 n^{2}-(2 n-\mathrm{I}): 2 n-\mathrm{I}: 2 n-\mathrm{I}: n^{2}-(2 n-\mathrm{I}) \text {. }
$$


three times, the series $8 A B+\mathrm{1} A b+\mathrm{r} a B+8 a b$ would result. We cannot, on the observed numbers, assert quite positively that the system is not $8: \mathrm{I}$, but as observations accumulate, this supposition becomes increasingly improbable, for the numbers all point rather to $7: \mathrm{I}$ than $8:$ I.

If, as many suppose, the whole process of segregation is completed in the reduction-division, it is obvious that any suggestion involving successive segregations fails. Still it is worth noting that nothing yet limits us to the conception of segregation as occurring all at once. We know very little yet as to the cytological processes antecedent to the reduction-division. Moreover it cannot yet be asserted that all the gametes, or even all the gametes of one sex (in hermaphrodite forms) are in the same cell-generation, counting from the first cleavage-plane of the zygote.

It is to be noted also that where the germ-cells are many, as in the testes of animals and the anthers of most plants, it is not difficult to imagine the formation of even very long series of couplings. The egg-cells, on the contrary, are few, and in plants they are very often definitely grouped in special organs which again are arranged on a definite geometrical plan relatively to the gross anatomy of the plant. Even if the various accessory cells of the plant ovary are reckoned as belonging to the gametic series, the number still seems insufficient to allow for the development of a coupling which demands a long series for its expression. The question may naturally be asked whether there is any organised system of differentiation connecting the several ovaries into a common plan. The differentiation among the egg-cells might conceivably be distributed on a geometrical plan like the differentiation among the somatic organs of the plant. All the available evidence is however against this suggestion, for in maize and peas, where indications of this system might be found if they existed, all the evidence is entirely negative.

There is still another direction in which we may look for an elucidation of the nature of gametic coupling. If the factors can act upon each other in such a way that certain combinations do not occur, as we have already seen actually happening in the case of Spurious Allelomorphism, it seems 
possible that such a system as the $7:$ I : I : 7 may be the result of a complex series of repulsions exerted among a number of factors. At present this suggestion is quite unfounded. It could however be tested if breeding on a really large scale could be undertaken, and supposing it to be true, the evidence for its truth would appear in the relative infrequency with which some types appeared. From that evidence the missing gametic combinations could be identified. As yet however it is quite premature to pursue such an analysis, and we must be content to note that when, as in these Sweet Peas, there is heterozygosis between a number of distinct allelomorphic pairs, the numerical proportions in which the various combinations occur may certainly be affected by the interactions exerted by allelomorphs of different pairs upon each other.

\section{The Possibility of Selective Mating between Gametes.}

It has naturally occurred to many minds that as gametes are now known to possess differentiating qualities, these differentiations may affect the readiness with which various classes of gametes may unite. We recognize that the simple Mendelian numbers are produced when every kind of female gamete has an equal probability of uniting with every kind of gamete produced by the male. Conversely, when irregular and unexpected numbers appear as the result of experiment, the question may have to be considered whether the irregularity is not due to a selective assortment taking place among the gametes, such that certain types of unions occur in fertilisation with greater readiness than others. Hitherto it is doubtful whether any instance has been discovered in which abnormal numbers can be proved to occur with such regularity as to warrant a recourse to this hypothesis. Correns observed several families of maize where $F_{2}$ from $F_{1}$ round seed $\times$ wrinkled seed, self-fertilised, contained a great excess of round seeds. The totals were 8975 round : I I I I wrinkled where the expectation is 80I4 round : 267 I wrinkled. Thirty-five $F_{1}$ plants contributed to this total and the discrepancy between observed result and expectation was fairly constant throughout.

B. $\mathrm{H}$. 
To test whether the numerical output of gametes was abnormal, reciprocal crosses were made between $F_{1}$ plants of the same breeding and recessives. In both cases the normal equality between round and wrinkled seeds was produced. Correns therefore concludes that some process of selective mating was responsible for the aberrant $F_{2}$ numbers (65).

So far as I am aware, no case altogether similar to this one has been observed, certainly none in which the numbers available are so large. The proportions for maize seeds are usually very regular in regard to the round and wrinkled characters, as the records of both Correns and Lock testify.

Pending further acquaintance with phenomena of this class there is no more to be said. The possibility of disturbance by selective attraction between particular kinds of gametes must be recognized, though without much more definite evidence its occurrence can scarcely be regarded as demonstrated.

In another instance of a different kind the same suggestion was made by Cuénot. Of this case I have already spoken* in describing the inheritance of yellow colour in animals. Experimenting with mice he found it impossible to find a yellow mouse pure to yellowness. Among mice yellow behaves as a dominant, in the sense that agoutis or blacks may be bred from two yellows. If the case were an ordinary one, some of the yellows produced by the mating of two yellows should be pure, and on breeding to blacks or agoutis they would be expected to give all yellows. Cuénot's experience is that this is never realized, and all the yellows he has ever tested, amounting to $8 \mathrm{I}$ individuals, also show, in such matings, some colours other than yellow (cp. Basset Hounds, p. I 28). Miss Durham has made similar experiments with the same result. Yellows were always found to give off either agoutis, or blacks, or chocolates.

Cuénot interprets the peculiar result as meaning that two gametes both bearing the determiner for yellow are incapable of uniting in fertilisation. The numbers were

* The discussion of this remarkable case was given in another connection at p. rrg, but in view of its special importance the facts and argument are repeated here. 
232 yellows and 86 agoutis, which is a near approach to the normal $3: \mathrm{I}\left(2380^{\circ}: 79^{\circ} 5\right)$. Cuénot comments on this as a difficulty in the way of his view, saying that he would have expected the ratio 2 : I; but as $\mathrm{Mr}$ Punnett pointed out to me, if all the ova bearing the yellow factor were fertilised by agouti spermatozoa, the number of these being indefinite, the chances of the non-yellow ova being fertilised by a spermatozoon bearing yellow or non-yellow would remain sensibly equal. Thus the ratio 3 yellow : I agouti would result.

Nevertheless the impression left on my mind by these observations, and indeed by other strange phenomena which yellows exhibit, is that the genetics of yellow mice are very imperfectly investigated and that it is premature to formulate definite views as to their behaviour*.

One of the peculiarities of yellow mice, well known to fanciers, is their frequent tendency to excessive fatness. Miss Durham, who has had considerable experience with yellows, finds that this condition is not universal among them, but shows itself in frequent individuals. She has also found the genetic investigation of yellows very difficult on account of the fact that they are often sterile, and the suggestion is perhaps worth considering that this sterility may be responsible for some of the complications.

* Miss Durham has recently proved beyond all reasonable doubt that the true account of this case is not that the two 'yellow' gametes are incapable of uniting, but that zygotes so formed perish at some stage before birth. For some physiological reason unknown, they are incapable of living. From her own and other records the result of yellow $\times$ yellow is now I 5 I I yellows and 767 non-yellow, a close approach to $2: \mathrm{I}$, which gives the expectation $1518 \cdot 6: 759^{\circ} 3$. The case, in fact, is analogous to that of Baur's golden Antirrhinum (p. 253), in which the pure albinos are missing, leaving a ratio of 2 golden : I green. (See Durham, Journ. Genetics, I. 191 1, p. 167.) 


\section{CHAPTER $X$}

HEREDITY AND SEX.

Evidence from Breeding Experiments. Bryonia-Sexlimited Heredity. The Horns of Sheep-ColourBlindness-Sex and Spurious Allelomorphism. The Currant Moth-The Cinnamon Canary-The Silky Fowl-Aglia tau-Cytological Evidence-Summary.

THE facts of sexual dimorphism have to be considered in any exposition of the laws of inheritance. There are two aspects in which the phenomena of sex concern us: (I) the nature and transmission of sex itself, (2) the influence which sex has in deciding the development or suppression of characters introduced into the zygote. The evidence relating to these two questions is so closely interwoven that they must be in practice treated together; for the facts concerning the influence of sex on the distribution of characters constitute, as will be seen, a most important means of indirectly investigating the problem of the actual nature of sex, and have provided already several clues which will probably lead to the unravelling of that baffling mystery. It need scarcely be said that in view of the great obscurity still surrounding the genetics of sex any conclusions of a positive kind can only be made tentatively. The phenomena however are among the most interesting and important with which the student of genetics is concerned, and every fragment of evidence regarding them is at present worthy of record.

Numerous essays dealing with the subject of the determination of sex* have recently appeared, and the belief is extending that sex will probably be found to be a result of gametic differentiation. This conclusion rests partly on

* This chapter naturally makes no pretence to cover the whole ground. I can treat only of those parts of the subject which come more immediately within the scope of Mendel's principles. 
analogy. Mendelian experiments have shown that in all cases which can be adequately investigated, a mixture of distinct zygotic types occurring in one family is due to gametic differentiation. Sex is a case of such mixture of zygotes and the presumption is thus created that the case is comparable in causation with those amenable to more direct analysis. This suggestion was made in 1902 (Rep. Evol. Ctee, I. p. I38), as a natural deduction from Mendelian discoveries and it is interesting to know that the same possibility occurred to Mendel himself (197, p. 24I).

The argument from analogy may perhaps be carried a step further. If the distinction between the sexes is the result of gametic differentiation, the fact that in ordinary cases the two sexes are produced in equal numbers must be taken as a strong indication that one sex is heterozygous in respect of sex-character, and the other homozygous. That, as we now well know, is the simplest way by which numerical equality in the production of two types is brought about.

In his important paper on this subject Castle argued that both sexes are to be regarded as heterozygous in sex. But in order to apply this suggestion two serious assumptions are required. First, since male and female are each regarded as heterozygous in sex, unions between ova and male cells bearing similar sex-factors must be assumed to be impossible. At the present time no fact can be adduced which negatives this assumption, and there are indeed general considerations which may be appealed to as rendering it somewhat probable.

The second assumption involved is more serious. As both sexes are regarded as heterozygotes containing the same factors, the nature of their dissimilarity is still so far unrepresented. In view of this difficulty Castle regards dominance as a matter of chance. Doncaster, in respect of a case to be discussed below, suggested that dominance may belong exclusively to the cells coming from one parent, say, for example, those of the mother. These postulates seem unsatisfactory and do not accord well with anything that we know in regard to the nature of dominance elsewhere observed ; for the applicability of all schemes hitherto discovered lies in the fact that it has been found possible to represent zygotic composition and structure as determined 
by the composition of the gametes of which the zygotes are formed.

Naturally therefore we look for a simpler solution of the problem of sex-determination. As was stated above, it would a priori seem most probable that one sex is heterozygous in sex and the other homozygous. I now propose to consider the applicability of this simpler account.

Two lines of work have been followed, that of experimental breeding, and that of cytological study of the germcells. Both have led to very positive conclusions, but these conclusions are diametrically opposed, as will be immediately seen. From experimental breeding we are on the whole led to conclude that the types used have females heterozygous in sex (female being dominant) and males homozygous recessives, while in all cases in which cytological evidence is forthcoming it appears that the females are homozygous and the males heterozygous. The meaning of this curious discrepancy will be considered when the facts have been related.

Evidence from Breeding Experiments.

\section{The Case of Bryonia.}

By the nature of the case, direct Mendelian experiment cannot be applied. There are however certain indirect lines by which the problem can be approached. The first is that followed by Correns in cross-breeding monoecious or hermaphrodite types with dioecious or bisexual types. In this connection the most striking experiment is that which he made by crossing Bryonia dioica with $B$. alba which is monoecious. The reciprocal crosses gave with much consistency a surprising difference in results. $B$. dioica, female, fertilised with the pollen of $B$. alba gave $F_{1}$ females, with or (usually) without occasional male flowers. The observed numbers from this mating were 589 females, with 2 males which must be regarded as exceptional. In the reciprocal cross, alba (monoecious) used as female $\times$ dioica $\hat{\delta}$ gave $F_{1}$ consisting of males and females in approximately equal numbers. In Cambridge we have repeated both experiments and obtained the same results. Unfortunately the hybrids, however produced, are absolutely 
sterile, forming no good pollen and failing to set seed when fertilised with pollen of the types.

Correns (8I, p. 27) interprets these facts as meaning that the germ-cells of dioica $\hat{\delta}$ are differentiated in regard to sex, and respectively bear either maleness or femaleness. The male is thus heterozygous in sex, maleness being dominant. The female dioica is consequently taken to be homozygous in femaleness. The condition of the sexual cells of the monoecious alba is not quite so readily represented. Since dioica $q \times a l b a$ o gives plants all $q$ or with only traces of maleness, the male cells of alba are regarded as all alike undifferentiated in respect of sex, bearing, that is to say, the monoecious character, and presumably the female cells of alba are in the same condition.

The facts would then be represented thus:

dioica $q$ is taken to be $q$ ㅇ.

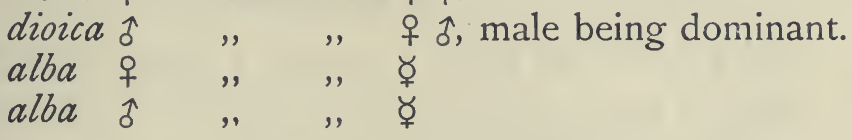

Thus as the results of breeding we have:

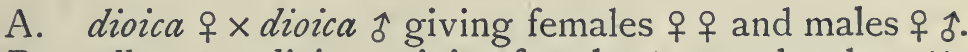

B. alba $q \times$ dioica $\hat{\sigma}$ giving females $q \not$ and males $\hat{\sigma} \not$.

C. dioica $\uparrow \times a l b a \hat{o}$ giving all females of the form $q \not{q}$.

The difficulty in this scheme is that, if maleness were dominant, it is not clear why the plants produced in case C should be almost entirely females; for it seems natural to expect that some of the dominance of maleness would attach to the gametes of monoecious character produced by $a l b a$, and hence that male flowers should be produced with frequency by the female hybrids. This nevertheless is not the case. In our experiments, out of 37 plants thus bred, 34 were purely female and only 3 showed any male flowers. These were in all cases at the lowest flowering nodes. In one plant 4 male flowers occurred in this position, and in each of the other two plants a single male flower appeared. The reciprocal cross, alba $+\times$ dioica of gave 6 pure females, I remale with a single male flower, and 9 pure males.

Correns observed a similar occurrence of male flowers in the same position on the otherwise female hybrids raised 
by him, but the occurrence was quite exceptional. One plant alone showed a distinct tendency to the monoecious condition, almost resembling alba. If maleness were dominant this prevailing absence of indications of the monoecious condition is not easily accounted for.

In view of this difficulty it is worth considering whether other schemes are not equally possible, and it seems to me that there is another method of interpreting the facts which is certainly not yet excluded. In Correns' scheme maleness is taken to be dominant, but if femaleness is taken to be dominant we can then represent the appearances thus :

dioica $q$ has egg-cells $q$ and $\hat{\delta}$.

dioica $\hat{\delta}$ has pollen all $\hat{\delta}$.

alba $\Varangle$ has egg-cells + and $\hat{\sigma}$; and pollen all $\circ$.

The matings will then stand as follows :

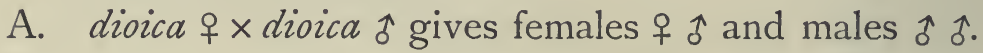

B. dioica $q \times$ alba $\hat{\delta}$ gives pure females $q+q$ and hetero. zygous females o 1 .

C. alba $+\underset{x}{\times}$ droica $\hat{\delta}$ gives females $q \hat{\delta}$ and males $\hat{\delta} \hat{\delta}$.

D. It follows that alba self-fertilised should give pure females as well as the ordinary monoecious plants. Whether this is the case or not I do not know. Only three plants were raised from seed here, and these were monoecious, but there would be of course nothing unusual in a species, often monoecious, producing female plants, for many such examples are known.

The view that it is the female and not the male which is heterozygous in sex is not improbable because, as will soon be shown, there is a remarkable group of cases among animals in which that interpretation is almost forced upon us.

Lastly, for the apparently anomalous representation of the pollen-cells of alba as of a constitution dissimilar to that of the egg-cells of the same plant, we can quote (Chap. xI) a somewhat parallel case which is well established in regard to Stocks (Matthiola), where certain strains have the eggcells of two types and pollen-cells of one type. In that case however the pollen-grains bear the recessive character, not the dominant as they must be supposed to do here. 
Admittedly there are difficulties in the way of this method of representation but they seem to be no greater than those besetting the hypothesis that maleness is dominant*.

Correns $(8 \mathrm{I}, 72,75)$ has observed another group of facts, doubtless important though equally difficult of interpretation, in regard to the results of breeding from species which have female, and hermaphrodite or gyno-monoecious individuals. His experience is that when the females are used as mothers and are fertilised with pollen from the hermaphrodites, the offspring are almost exclusively female. When however the hermaphrodites are used as mothers the offspring are mostly, though not so exclusively, hermaphrodites. From experiments of this kind it is likely that a good deal of light will be obtained when statistics on an ample scale are available. Those who may undertake such work will of course remember that as the constitution of the individuals may be dissimilar, each must be separately tested.

The relation of dioecious to hermaphrodite and monoecious forms will not in all probability be satisfactorily or rapidly elucidated until some case can be found in which the two types can be crossed together with a fertile result. No evidence from such a case as that of Bryonia is free from the suspicion that the sterility of $F_{1}$ may itself be introducing a complexity.

\section{Heredity limited by Sex: the Horns of Sheep.}

I now pass to the consideration of evidence as to the part which sex plays in determining or limiting the descent of certain characters in heredity. The manner in which these effects are shown is well illustrated by the following example investigated by Professor T. B. Wood (3 I 2, 3 I3) in the case of horned and hornless sheept. As a horned breed he chose the Dorset Horned, in which the

* A fragment of evidence bearing on these problems is that contributed by Gärtner's experiment witl Lychnis diurna $\$$ crossed with pollen from $L$. flos-cuculi $\Varangle$. This gave 4 males and 2 females; but of these, one only, a female which was totally sterile, gave any certain indication of having resulted from the cross. Pending a repetition of the experiment no conclusion can be drawn with much confidence from this account.

+ As was stated before, hornlessness is a dominant in both sexes so far 
horns are well developed in both sexes. These he crossed reciprocally with Suffolks, a breed without horns in either rams or ewes. The $F_{1}$ male lambs all developed horns of fair size, but the $F_{1}$ ewes remained hornless. The horned character may therefore be described as dominant in males and recessive in females (Fig. 20).

The $F_{2}$ generation bred from these consisted of all the four types, horned and hornless males, horned and hornless females. The experiment was not carried out on a scale sufficient to justify a statement that the numbers are simply

Males

3 horned : I hornless
Females

I horned : 3 hornless

but so far as the evidence went these simple ratios seem to be followed. (For diagram of such a descent see Fig. 33.)

Subsequent experiment has confirmed the conclusion indicated by these facts, namely that horned ewes in $F_{2}$ are pure for the presence of horns, and that hornless rams in $F_{2}$ are pure for the absence of horns.

In order therefore that the female may possess horns, she must be homozygous in that character. The factor for hornedness must come in from both sides of the parentage. Conversely, in order that the male should be hornless, he must receive the deficiency from both sides of his parentage. A case that may be compared with this has been observed in regard to the descent of wing-development in a cross between two species of moths of which the one Boarmia (Biston) hirtaria has a winged female, while the other, Boarmia (Biston) pomonaria, has a wingless female. Oberthür* crossed these two species and obtained $F_{1}$ males all with fully developed wings and four females with wings only half-formed, in a condition thus intermediate between

as horned cattle are concerned. The heterozygote is either quite hornless, or has only small loose horny lumps-"scurs" as they are called in the north.

As to the descent in goats I have no thoroughly adequate evidence. The Rev. E. P. Boys-Smith has kindly given me particulars of many matings which he has made, but the details are complex and I have not been able to extract a consistent scheme from them. There is probably some intricacy due to gametic coupling comparable with that described in the next section, or perhaps to sex-limitation.

* Bull. Soc. Ent. France, 1897, p. 256. 


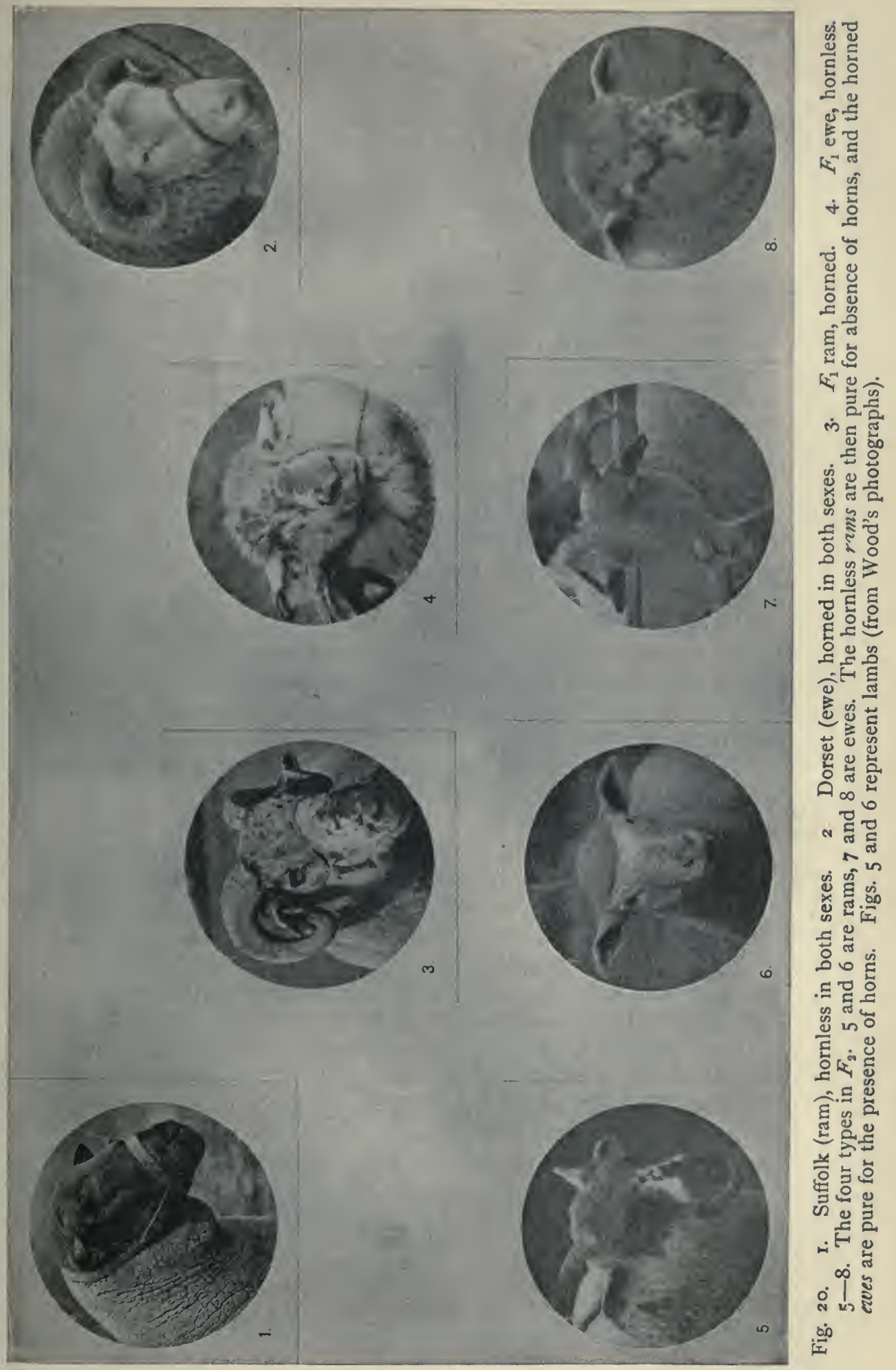


those of the two parent species. Unfortunately the hybrids were completely sterile and the experiment went no further. Here we see that the one "dose" of wingedness-as we may call it-sufficed only to bring the wings to half the full size, and two "doses" are needed to develop them properly.

Doncaster has collected evidence about the inheritance of tortoise-shell colour in cats which illustrates the same phenomenon. It has long been known that tortoise-shells are almost always females. The suggestion which Doncaster made (I09) is that this is the female form of the heterozygote between the colours orange and black. The facts, as he pointed out, show fairly clearly that the corresponding heterozygote in the male is orange. Orange colour is thus dominant in males, but in females the dominance is imperfect and the patch-work form, tortoise-shell, results. The same is true for cream and blue, which are only the dilute forms of the colours orange and black respectively*. It is true that the exceptional tortoise-shell males do occasionally exist, but they are exceedingly rare and nothing as yet is known respecting their breeding.

More complex systems of sex-limited descent are followed by certain abnormal conditions met with in Man. Of these the most familiar is colour-blindness.

The ordinary form of that affection may roughly be described as the inability to distinguish red from green. Colour-blind persons are commonly male, and in European countries it appears that at least 4 per cent. of the male population are colour-blind (of females less than 0.5 per cent.). The children of colour-blind fathers are usually not colour-blind, whether they be sons or daughters. The daughters however frequently transmit colour-blindness to their sons again. The unaffected males in these families do not transmit the condition, and their posterity are exempt unless the colour-blindness be introduced afresh. Until the facts were examined in the light of Mendelian discoveries nothing could be more puzzling. The statement however that colour-blindness is a condition dominant in males but recessive in females will express a great part of

* The idea is sometimes met with that it is only tortoise-shells without white which are almost exclusively female; but there is no truth in this supposition. 
the facts respecting the genetics of colour-blindness. The transmitting daughters of colour-blind fathers are evidently heterozygotes in whom the affection is, like the horned character in the $F_{1}$ ewes, recessive; but in the fact that both the sons and their offspring are free from the affection (unless of course it be introduced by the mother) we meet a fresh complication the meaning of which is considered later (p. I95, note). Here it must suffice to say that the most obvious test of the nature of the inheritance is provided by the families of colour-blind women. According to the scheme the simplest expectation is that all sons of such women should be colour-blind. Up to the present time we have records of seven colour-blind women only who had sons. In all they had I 7 sons who lived to be tested, and all were found to be colour-blind*. We may therefore rest assured that the scheme provides at least a substantial part of the truth, and that colour-blindness is a condition produced by the addition of a dominant factor.

In the examples just considered sex itself acts as a specific interference, stopping or inhibiting the effects of a dominant factor, and it is not a little remarkable that the inhibition occurs always, so far as we know, in the female, never in the male. When the effects of a factor fail to appear in a zygote the failure is due to one of two causes. Either some complementary element is absent which is needed to produce the effect, or some other element is present which inhibits it. 'The facts scarcely enable us to distinguish between these two possibilities but we may feel some confidence that our cases belong not to the first group but to the second. For since the condition can be developed in females, it is evident that maleness itself is not a necessary complement : and it is not easy to suppose that there is some other factor regularly coupled with maleness which has this property, though that possibility cannot be absolutely excluded. The suggestion however that the female contains something which suppresses the effect of the otherwise dominant factor is consistent with the observation that when these sex-limited conditions, as they are called, do appear in females, they are developed to a somewhat less degree than in males, just as in horned breeds of sheep the ewes have horns smaller than those of the rams. The 
inhibiting factor may be thought of as able to suppress completely the development of the character when that character is heterozygous-introduced, to use that expression, as one "dose" only-but unable to suppress it when it is homozygous, represented by a double "dose."

On the view that femaleness itself is the suppressing factor one difficulty has to be considered. Just as disease or removal of the ovaries may lead to the appearance of male characters we should expect that such disease might lead to the occasional appearance of colour-blindness in females. I do not however know of such a case. It must nevertheless be remembered that even the appearance of male characters is itself by no means a regular consequence of these lesions, and perhaps we need not regard the difficulty specified as a serious one in the case of colourblindness.

\section{Sex and Spurious Allelomorphism: the Currant Moth.}

The next case of which I shall speak is one which has been worked out in considerable detail, and I anticipate that for a long time to come it must rank as a classical experiment in all discussions as to the nature of sex. The work in question was done by Doncaster and Raynor and it relates to crosses made between the Geometrid moth Abraxas grossulariata and its variety lacticolor (I I I).

Lacticolor was originally known as an exclusively female form (see Plate I). The experimental crossings gave the following curious series of results:

I. Lacticolor $+\times$ grossulariata of produced $F_{1}$ ofs and is all grossulariata.

2. $F_{1}$ grossulariata + $\times F_{1}$ grossulariata $\hat{\delta}$ gave grossulariata ôs and is and lacticolor is; no ofs of lacticolor being formed.

3. Lacticolor $+\times F_{1}$ grossulariata $\{$ gave all four possible forms, namely grossulariata $\delta^{\mathrm{s}} \mathrm{s}$ and $\mathrm{q} \mathrm{s}$, and lacticolor to and is. The lacticolor males were the first that had ever been seen. 
4. $F_{1}$ grossulariata $+\times$ lacticolor $\delta$ gave all the $\delta \mathrm{s}$ grossulariata and all the os lacticolor.

Adopting Castle's view that both sexes are heterozygous in sex, Doncaster showed that a consistent scheme could be devised which represented the foregoing experimental results. According to this scheme the following suppositions are made

(I) Each sex is regarded as giving off $\hat{\delta}$-bearing gametes and of-bearing gametes.

(2) In females heterozygous for grossulariata and lacticolor there is gametic coupling such that each gamete bearing the grossulariata factor bears maleness, and conversely each gamete bearing lacticolor bears femaleness also.

(3) In the heterozygous male there is no coupling.

(4) There is selective mating between the gametes, such that union can take place only between gametes of opposite sex, namely such as bear maleness and femaleness respectively.

(5) Dominance attaches to the sex which is brought into the zygote by the egg.

On these assumptions the observed facts would be produced. Inspection however shows that there is a simpler solution, which avoids the need for assumption (4) that selective mating occurs. On this scheme two assumptions only are made.

(I) That the female is heterozygous in sex, femaleness being dominant, and the male a homozygous recessive.

(2) That when in $F_{1}$ the two dominant characters femaleness and the grossulariata factor co-exist, there is spurious allelomorphism or repulsion between them, such that each gamete takes one or other of these factors, not both. 
The whole series of facts is then consistently represented as follows :

I.

lact. $q \times$ gross. $t$
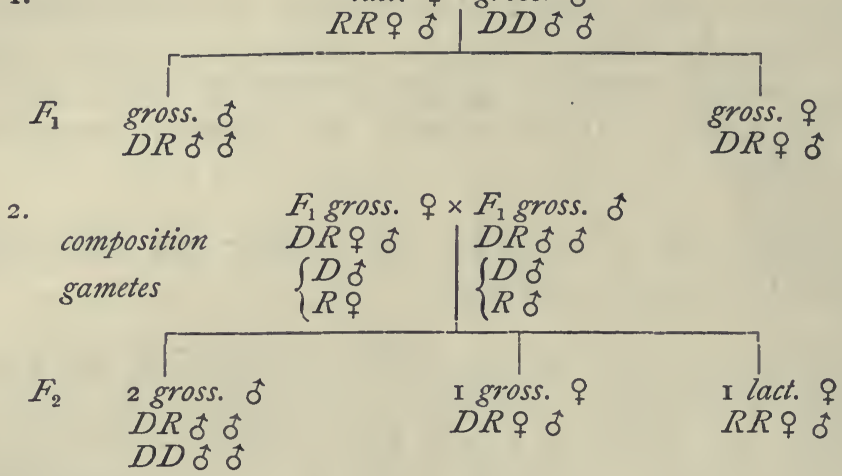

3 .

lact. $q \times F_{1}$ gross. $\hat{\delta}$

$R R$ के $\mid D R$ ठै ठै

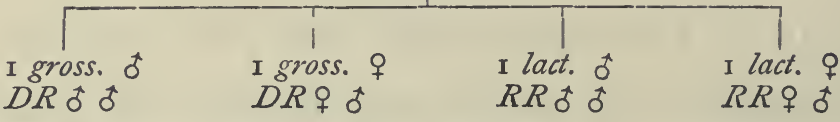

4 .

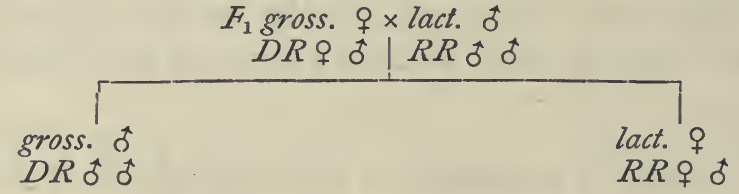

In Doncaster's first series of experiments the numbers observed were very wild and irregular, but more recently he has repeated the matings and got numbers according sufficiently well with those predicted by the scheme. The spurious allelomorphism supposed to exist between femaleness and the grossulariata factor would be a phenomenon similar to that described already (p. 1 53) in the Sweet Pea, where the factor for blueness and that for the erect standard were shown to behave as if mutually repellent, and alternative to each other in the formation of the germ-cells. The facts as to the limitations which sex introduces into the descent of a varietal character are thus consistent with the possibility that sex is a Mendelian character and that femaleness is a dominant, depending for its development on the presence of some definite factor, and that this factor has 
properties like those elsewhere proved to attach to other dominants.

One very curious observation made in the case of grossulariata is that which remains to be stated. It was communicated by Doncaster to the Dublin Meeting of the British Association (Sept. I908). The experiments enumerated were, it will be observed, incomplete in so far as the mating wild gross. $+\times$ lact. $\delta$ had not been made. The results of this mating are now known. Families thus bred consist of males grossulariata, and females lacticolor! In other words, the ordinary wild grossulariata even in districts where lacticolor is unknown, are in reality a race of which the males are pure grossulariata, though the females are in reality hybrids of lacticolor, and so continue from generation to generation. The normal female grossulariata and the $F_{1}$ o grossulariata bred from lact. $q \times$ gross. 0 are thus seen to be identical in composition. Whether a gross. o h has a gross. o for a mother, or a lact. of for a mother makes no difference to its composition and properties. This fact is one of the most striking to which genetic research has yet led. It affords strong confirmation of the interpretation of the series of phenomena given in the text, and enables us to see in the evidence both as to the grossulariata case and as to the other cases which follow, a consistent mass of testimony all pointing in the same direction. In a recent paper (I 14) Doncaster accepts the view here suggested. It must of course still be remembered that attractive though the present suggestion is, by reason of its simplicity, we have no proof that the natural scheme may not be more complex.

The two cases next to be considered resemble that of grossulariata in the fact that reciprocal crosses between pure types give dissimilar results. Though both examples to be discussed are only imperfectly explored, the facts elicited are so curious that some preliminary notice of them is called for. We may confidently anticipate that further search will discover other comparable instances. It will be seen that these phenomena point very plainly in the same direction as those previously described, to the conclusion, namely, that, in the types used, femaleness depends on the presence of a definite dominant factor.

B. $\mathrm{H}$. 


\section{The Cinnamon Canary.}

The first case of a sex-limited distinction between reciprocal crosses is provided by the evidence as to the descent of what is called "Cinnamon" in Canaries. The characteristic of these canaries when adult is the presence of the pale drab colour known as Cinnamon in the feathers, evidently replacing the black pigment characteristic of "green" canaries. The green is of course due to the black pigment showing through the yellow. According to Miss Durham's observations the cinnamon pigment is of the same nature as the "chocolate," of which we have spoken in discussing the pigmentation of mammals, and it is probable that the various aberrational forms of mammals and birds so often recorded by systematists as "isabelline" are all similar cases of the replacement of black by chocolate.

When newly hatched, Cinnamons differ very strikingly from ordinary canaries in the fact that they have pink or unpigmented eyes, like the albinos of many animals. As they grow up this distinction is scarcely, if at all, perceptible on ordinary examination. The eyes become pigmented and come to look about as dark as those of ordinary canaries. Some fanciers allege that they can distinguish the eyes of Cinnamons throughout life, but the difference is evasive and these determinations are unreliable. Microscopically, however, Miss Durham finds that there is a real difference in the fact that the pigment of the Cinnamon eye is chocolate, like that of the chocolate mouse. Apparently the cinnamon feathers are never developed in birds that have black eyes, but the pink eyes can be transferred to birds which are pure yellow and without any trace of cinnamon in their feathers.

The inheritance with which we are concerned is that of the pink eyes. It has long been declared by fanciers that when Cinnamon hens, viz. pink-eyed, are bred with green, viz. black-eyed, cocks the offspring of both sexes all come black-eyed. When however green (black-eyed) hens are bred to Cinnamon (pink-eyed) cocks, both greens and Cinnamons may be bred, but these Cinnamons are always hens. The $F_{1}$ black-eyed cocks are said to have again the power of producing pink-eyed offspring from 
black-eyed hens, but again all these pink-eyed birds are hens. Statements to this effect are to be found in many of the fanciers' books, but a particularly good and lucid account of the phenomena was given by Mr Noorduijn (2 I3), of Groningen, who has been good enough to answer many questions on the subject.

Miss Durham has begun a series of experiments designed to investigate the problem constituted by these facts, but the work has not yet gone far enough to provide a complete solution. She finds (1 I 8 ) that, 'as stated,

(I) Cinnamon $q \times$ pure green $\delta$ gives all offspring of both sexes black-eyed.

(2) Green $q \times$ Cinnamon $\hat{\delta}$ gives in general $F_{1}$ cocks black-eyed, and $F_{1}$ hens pink-eyed. Two black-eyed hens of unknown breeding mated to Cinnamon cocks have also given, in addition, black-eyed hens as well as pink-eyed hens. This result is exceptional.

(3) Cinnamon $q \times F_{1}$ black-eyed $\hat{o}$ gives all four types, black-eyed cocks and hens, and pink-eyed cocks and hens.

(4) Green $+\times F_{1}$ black-eyed $\hat{\sigma}$ gives cocks all blackeyed, and hens of both types, black-eyed and pink-eyed.

The mating of $F_{1}$ black-eyed hens (from Cinnamon $q \times$ black-eyed $\hat{\jmath}) \times$ Cinnamon $\hat{o}$ has not yet been made, but there is little doubt that such a pair of birds will give the same results that are obtained in mating (2).

As regards the descent of the pink-eye there seems to be no difference in result whether green or yellow blackeyed birds are used.

The general run of these experiments is now intelligible. The case is evidently comparable with that of Abraxas grossulariata and lacticolor. Were it not for the occasional production of black-eyed hens from green $q \times$ Cinnamon $\hat{o}$ the whole series of results could be represented in one simple scheme. That exceptional occurrence proves of course that there is some further element to be considered, but neglecting that for the present, the scheme of descent is as follows. As before, we take female as heterozygous, femaleness being dominant, and we assume that there is spurious allelomorphism between femaleness and the blackeye factor. 
The allelomorphs are

+ , $\hat{\delta}$, presence and absence of femaleness.

$B, b$, presence and absence of the factor for black eye

The "pure" green hen is thus 우 $B b$, being, as experiment proves, actually heterozygous for the black-eye-factor. The gametes are $B \hat{\delta}$ and $b$ o .

The pure black-eyed green male is pure in maleness (recessive) and in the dominant black-eye-factor, and his composition is represented thus $\hat{\delta} B B$.

The Cinnamon hen is $9 \hat{\alpha} b b$ and the Cinnamon cock is ๙ิ่bbb.

The matings are then as follows.

I.

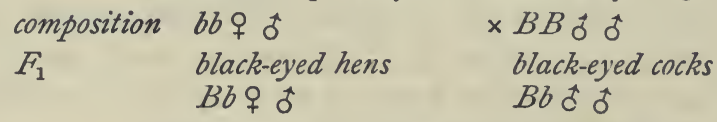

2.

Black-eyed $q \times$ Cinnamon pink-eyed $\delta$

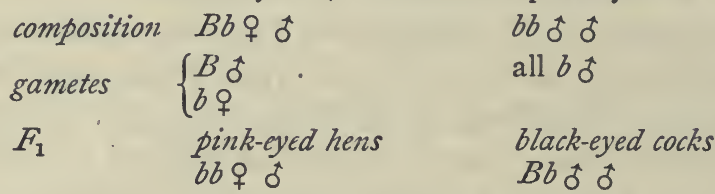

3.

Pink-eyed $q \times$ black-eyed $F_{1} \delta^{\dagger}$

composition $b 6$ 우 0

$B b$ oे ơ

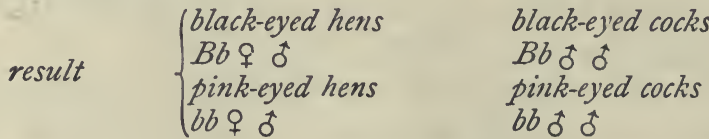

4. $\quad$ Black-eyed $q \times$ black-eyed $F_{1}$ đ

\begin{tabular}{|c|c|c|}
\hline composition & $B b$ 웅 & $B b$ ठ ฮ \\
\hline gametes & 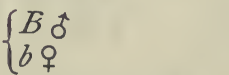 & \\
\hline result & $\left\{\begin{array}{l}\text { black-eyed hens } \\
\text { Bbo o } \\
\text { pink-eyed hens } \\
\text { bb } 9 \text { ot }\end{array}\right.$ & $\begin{array}{l}\text { black-eyed cocks } \\
B B \text { o } 0 \\
\text { and also } \\
B b \delta^{\star} \delta\end{array}$ \\
\hline
\end{tabular}

This representation is therefore complete except in so far as it takes no account of the production of black-eyed hens together with pink-eyed, which Miss Durham has twice seen in mating (2). These create a definite difficulty which as yet there is no means of overcoming. The conjecture may be hazarded that they owe their origin to the 
disturbing effects of some other dominant factor undetermined, which alters the normal distribution of the factors $B$ and $q$ in the gametogenesis of certain black-eyed hens, but that is mere conjecture.

Attention must be drawn to the paradoxical conclusion that black-eyed hen Canaries, whether green or yellow, are normally heterozygous in respect of the black-eye character*, being thus in common parlance "hybrids" of Cinnamon! The males, on the contrary, are in general homozygous or pure in the black-eye character. This discovery gives rise to many reflections, some of which will be spoken of after the facts of the next case are described.

\section{The Silky Fowl.}

The example of heredity limited by sex that we are about to consider exhibits a complication which at first sight suggests that the phenomena are the converse of those last detailed. In the Canaries when the two sexes raised from a cross differed from each other it was the females which presented the recessive feature and the males which showed the dominant. We shall here see that when the sexes differ it is the female which ostensibly shows the dominant. Nevertheless the difference is apparent, not real. In the case of the Cinnamon Canary the observed results were due to the combinations of two pairs of factors, while those about to be described are due to the combinations of three pairs. The presence of the third dominant cannot however be told by inspection. Breeding tests alone reveal its existence.

Mr Punnett and I have been engaged for some years in cxperiments with Silky fowls. The most interesting results were obtained in crosses between Silkies and certain ordinary fowls with unpigmented shanks.

Silkies are remarkable in many ways, but the peculiarity with which we are here concerned is the intense black pigmentation of the mesoblastic membranes. The periosteum, pia mater, somatopleure, parts of the splanchnopleure, the sheaths of some blood-vessels, and the connective tissue

* Miss Durham has so far tested 7 yellow hens and 6 green hens, and all of these have thrown pink-eyed hens when mated to pink-eyed cocks, thus proving their heterozygous nature. 
beneath the skin, are deeply pigmented with black*. The skin of the bird has thus a deep purple or blue colour, due to the black pigment showing through the uncoloured epithelium of the skint. Ordinary fowls, Brown Leghorns for instance, the breed chiefly used in our work, have no pigment in these parts beyond dubious traces in rare exceptions. Such fowls we may call by contrast nonpigmented, and the phenomena to be described occur as the results of crossing them with Silkiesł.

The essential facts are as follows. When the Silky hen is bred with Brown Leghorn cock all the $F_{1}$ offspring, both male and female, are either destitute of the pigmentation, or only show a small amount of it in certain parts, especially in the ribs, on the vertex of the skull, and on the iris. In the case of the iris it shows in adult birds as minute dots of black on the red ground. The skin of such $F_{1}$ birds is scarcely different from that of unpigmented breeds. Both the skin and the periosteum sometimes have well-defined patches of pigment.

When however the Brown Leghorn hens are bred with the Silky cock, the $F_{1}$ males are indistinguishable from those bred in the reciprocal mating, but the $F_{1}$ hens are almost as much pigmented as the pure Silky hens.

Of the many possible matings into which the $F_{1}$ birds, male or female, can be introduced, few have yet been made on a scale sufficient to justify very confident statements. In most of the derivative families thus produced there is also a good deal of grading, and the analysis and classification of these intermediate types have not yet been adequately carried out.

The main facts however are

(1) That when the $F_{1}$ hens, whether of the deeply pigmented sort or of the slightly pigmented kind formed in the reciprocal mating, are bred with Brown Leghorn cocks,

* The liver and the lungs are little if at all invaded by the pigmentation, and curiously enough, the allantois is entirely unpigmented.

+ When the pigmentation occurs in birds having a yellore skin the general appearance is greenish. White skin is dominant to yellow skin, but the transmission of these characters is independent of that of the peculiar pigmentation of the Silky.

t. The silkiness of the feathers is an ordinary recessive to the hard plumage of common fowls. This character seems to be distributed independently of the rest. 
their offspring, whether male or female, are never more than moderately pigmented. Just as in the making of $F_{1}$ therefore, the pigmentation must come in from the male side in order that it may appear to any full degree in the offspring.

(2) The $F_{1}$ males from both kinds of mating are identical in composition so far as pigmentation and their powers of transmitting it are concerned. When they are bred with Brown Leghorn hens they produce one bird in eight, on an average, deeply pigmented, and these are always females.

This result was exceedingly definite and regular. A long series of matings between $F_{1}$ os from Brown Leghorn hen $\times$ Silky cock as fathers and unpigmented hens gave 205 birds with various degrees of pigmentation from moderate to $n i l$, and $3 \mathrm{I}$ fully pigmented hens. Using $F_{1} \hat{\jmath} \mathrm{s}$ from Silky $q \times$ Brown Leghorn $\delta$ the numbers of these two classes were I 70 and 23 , the 23 fully pigmented birds being again all hens. The total, 375 to 54 , is 7 to $\mathrm{I}$.

(3) The $F_{1}$ cock has only been tried once with a Silky hen, and the offspring consisted of 7 males and 8 females, showing all the different degrees of pigmentation, but from so few no quantitative conclusions can be drawn.

(4) When the deeply pigmented $F_{1}$ hens are bred with the pure Silky cock, all the offspring of both sexes are deeply pigmented.

(5) From a slightly pigmented $F_{1}$ hen (offspring namely of Silky $+\times$ Brown Leghorn $\hat{\delta}$ ) bred with a pure Silky cock only a few birds have yet been bred.

As a test of the distribution of the factors among the gametes of the hen this mating is one of the most important and many critical questions can be answered by it. The numbers are as yet too small to be of much significance. They are 8 hens all deeply pigmented, and 5 cocks ranging from deeply pigmented to intermediate.

The $F_{2}$ results are so complicated that until they have been obtained on a very large scale it would be premature and useless to describe them in any detail. In general terms the $F_{2}$ families contain both males and females of the deeply-pigmented, the slightly pigmented, and the 
non-pigmented kinds, with many puzzling intergradations between them.

Leaving aside all that is obscure in this experiment for future consideration one fact stands out clearly as an almost inevitable conclusion from the data, namely that in addition to the sexual differentiation into male and female, and the racial differentiation into pigmented and non-pigmented, the operation and interference of some unknown third kind of differentiation has to be reckoned with. In no other way can the results from the reciprocal crosses between the pure types, and those from the non-pigmented hens bred to the $F_{1}$ male, be brought into harmony. It is the appearance of the one deeply-pigmented female in eight birds which gives the clue.

Since half the gametes of the $F_{1}$ male must be bearing pigmentation, and since from results of the mating Brown Leghorn $\times$ Silky male we know that a pigment-bearing gamete from the male may dominate in the female, it would be expected that half the female offspring would be deeply pigmented ; for certainly half of them contain the necessary element. But as only a quarter of the female offspring are of the deeply-pigmented class there must be some other element present which obliterates the pigmentation, or holds it in check in the missing quarter. In several cases we are well aware of the existence of such inhibiting factors, for example that which causes the flower of a Chinese Primula to be white though the factors for colour are present in it (see Chap. v, p. IO5).

This factor may be spoken of as $D$. In the Silky it is evidently not present and therefore it must come from the Leghorn. Since Silky $q \times$ Brown Leghorn $\hat{o}$ gives no deeply-pigmented offspring we must consider the Brown Leghorn cock to be homozygous in $D$. But as the Brown Leghorn $q \times$ Silky $\hat{\sigma}$ gives the female offspring deeply pigmented, the Brown Leghorn hen must be heterozygous in $D$, and there must be some system or mechanism by which this factor $D$ descends to her male offspring and not to her female offspring*.

* A priori it might be thought possible that the dominance of the pigmentation-element in the $F_{1}$ o was due to some special differentiation of half the gametes of the male. On the hypothesis that each sex is heterozygous for sex such a system might without improbability be con- 
Now if femaleness be a dominant factor and can repel $D$, forming a spurious allelomorphism with it in the way suggested for the case of Abraxas grossulariata $\times$ lacticolor and the Canaries, a system would be provided which would fulfil all the chief conditions marked out by the experimental data.

Taking then the following allelomorphs, the occurrences in the three matings which have given clear results may be represented in a tabular form.

우, $\hat{\delta}$, presence and absence of femaleness, a dominant factor without which the zygote developes into a male.

$P, p$, presence and absence of the black pigmentation.

$D, d$, presence and absence of a factor which can suppress or mask the development of $P$.

I.

Silky $q \times$ Brown Leghorn $\delta$

composition PPdd $q$ oै

gametes $\quad\left\{\begin{array}{l}P d q \\ P d \delta\end{array}\right.$

$F_{1} \quad$ females slightly males slightly

pigmented pigmented

ppDD के के

$p D$ o

2.

Brown Leghorn $q \times$ Silky $\delta$

composition $p p D d q$ oै

gametes $\left\{\begin{array}{l}p D \text { o } \\ p d \sigma\end{array}\right.$

$F_{1} \quad$ females deeply males slightly

pigmented pigmented

Ppdd के PpDd के के

3. Brown Leghorn $q \times F_{1} \delta$

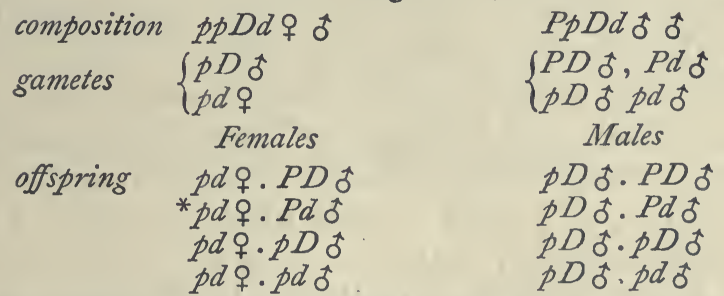

According to this analysis one bird in eight, namely the female marked *, will be of the deeply-pigmented type.

ceived. It would, however, fail to represent the I deeply-pigmented $q$ in 8 birds from Brown Leghorn $q \times F_{1} \delta^{\circ}$, and would increase the expectation to 2 in 8 birds, and this is negatived by the results of experiment. 
The above tabulation represents so well the main outline of the results of experiment that it is probably a close approximation to the truth. With regard to the somewhat meagre results of the other matings it may be said that, as far as they go, they are fairly consistent with the schematic representation, though some discrepancies occur, and unquestionably much remains still to be cleared up in this remarkable case. The distribution of the several factors among the gametes of the two types of $F_{1}$ females cannot yet be satisfactorily represented, and there is some evidence that the repulsion between femaleness and $D$ is not always so complete as the scheme demands.

In introducing the evidence as to Silkies attention was called to the fact that the difference between reciprocal matings occurred when the non-pigmented parent was of a breed which had unpigmented shanks. There are of course many breeds which though they have nothing corresponding to the general pigmentation of the Silky, are black or bluish in the shanks from a deposition of black pigment in the skin of that part*. When such hens are crossed with the Silky cock the pigmentation is developed not only in the female offspring, but also in the males. Davenport made crosses between Silkies and Spanish (black-shanked) and Frizzles (slaty shanks), and found no distinction between reciprocal matings, both sexes of the offspring being deeply pigmented. We crossed white rose-combed Bantam hens with a Silky cock with the result that the $F_{1}$ males and females were both deeply pigmented on hatching, though as they became adult the males lost much of their pigmentation. The white rose-comb is slightly bluish in down-colour and the shanks are slightly pigmented in varying degrees.

From many signs we know that there exists some complex relation between the colour of the shanks in fowls generally, and sexual differentiation. Some years ago we described (19, p. 95) a case of this kind in which Indian Game $+\times$ White Leghorn $\hat{\sigma}$ always gave $F_{1}$ yellow-shanked like both the parent breeds. But White Leghorn $q \times$ Indian Game $\hat{\sigma}$ gave cocks yellow-shanked like the parents, while

* This pigment is confined to the skin. There is none in the periosteum. When the general skin-colour is yellow these pigmented shanks have a greenish tinge, the "willow" of fanciers. 
the hens came with a good deal of pigment in the shanks ranging to nearly a full black.

Another case illustrating this relationship between sex and shank-colour is to be seen in the newly made breed called Black Leghorn. According to the fanciers' ideal both sexes should have full yellow shanks. There is no difficulty in getting this quality in the cocks, but hitherto clear yellow-shanked hens have been very rare, and the same difficulty is encountered in breeding Black Wyandottes.

Lastly, though the $D$ factor seems to be always present in Brown Leghorns, the cocks of which are homozygous and the hens heterozygous in respect of it, there is evidence that in an Egyptian breed of various nondescript brown colours, with which we have worked, the $D$ factor may be absent from both sexes, though the shanks are not dark in colour. Search among the breeds of our own country will probably lead to the discovery of other such material.

\section{The Case of the Moth Aglia tau and its Vaviety lugens.}

There is one very remarkable group of facts which cannot, so far as I see, be brought into harmony with the system proposed for the three cases already considered. These are the results recorded by Standfuss $(253, b)$ as the outcome of experiments with the Moth Aglia tau and its dark variety lugens. These experiments were brought into prominence by the discussion which Castle devoted to them in his important paper on sex (46).

The facts were briefly these. We have no explicit statement of a comprehensive kind as to the results of the cross between pure lugens and pure tau, but from subsequent results it is clear that, as usual in moths, the dark form lugens is dominant over the light form tau. With heterozygous lugens five matings were made as follows:

\begin{tabular}{|c|c|c|c|c|c|}
\hline & & & & \\
\hline & & lug. & tau & lug. & tau \\
\hline \multirow{2}{*}{\multicolumn{2}{|c|}{$\begin{array}{l}\text { tau }(R R)+\text { o lugens }(D R) \text { o } \\
\text { lugens }(D R) \text { \% } \times \text { tau }(R R)\end{array}$}} & $3^{1}$ & 14 & 13 & 28 \\
\hline 2. & & 26 & 13 & I I & 25 \\
\hline 3 . & $\begin{array}{l}\text { lugens }(D R) q \times \text { tau }(R R) \delta \\
\text { lugens }(D R) \& \times \text { lugens }(D R) \delta\end{array}$ & 34 & 10 & $2 \mathrm{I}$ & $2 \mathrm{I}$ \\
\hline 4 . & $"$ " & 49 & 3 & 42 & 8 \\
\hline 5. & $"$ & 46 & 3 & 31 & 7 \\
\hline & Total of $D R \times D R$ & 129 & 16 & 94 & 36 \\
\hline
\end{tabular}


In his discussion of these curious numbers Castle calls attention to the fact that the results of $D R \times R R$ are very nearly $2 D \hat{\delta}$ : I $R \hat{\delta}$ : I $D$ 우 : $2 R$ 우, and developing his view that each sex is heterozygous in sex, he suggests that the gametes of $D R$ females and males may bear the sexcharacters and the colour-characters coupled in this way, forming a series $2+\mathrm{I}+\mathrm{I}+2$. Assuming also that in fertilisation union can only take place between gametes of opposite sex, the $F_{2}$ numbers would be $8 D \hat{\delta}: 1 R_{\hat{\delta}}: 6 D$ 우 $: 3 R$ 웅 a series which, as he points out, fits the total of the observed numbers extraordinarily well.

Recognizing the great interest of the case I feel, nevertheless, that so long as it stands entirely alone, we are justified in treating it as of somewhat doubtful significance. We know from divers sources that the sex-ratios of the Lepidoptera are liable to astonishing fluctuations. Very large families consisting of all, or nearly all, females, or males, as the case may be, having been not rarely witnessed, and until experiments with tau and lugens are repeated with a full understanding of the importance that may attach to them, we may postpone positive conclusions.

\section{The Cytological Evidence.}

We have now to mention a group of facts which, though agreeing with the general conclusion that one sex is heterozygous and the other homozygous, suggests that in the types concerned, the rôles are reversed.

From the cytological side a remarkable advance in the problem of sex has been made in the discovery of the accessory chromosome in the spermatogenesis of certain Insects. McClung, studying this structure, originally observed by Henking, was the first to insist on its importance. He showed that in certain Insects half the sperms have it and half are without it. This fact led him to make the natural suggestion that the structure might be concerned in the differentiation of sex. This suggestion has been shown by E. B. Wilson to be correct, but the accessory. body proves to be the peculiarity of the sperms which are destined to form females, not of those which will form males, as had been previously supposed. The evidence for this is the fact that while the number of chromosomes in the male cells of the species concerned is either $n$, or $n-I$, 
the number in the female cells is $n$. The male cells therefore which have $n$ chromosomes on uniting with an ovum in fertilisation make up the zygotic number to $2 n$; but the $n$ - I spermatozoa can only make up the zygotic number to $2 n-$ I, forming thus a male. The germ-cells of the male again divide in gametogenesis to form cells with $n$, and cells with $n-$ I chromosomes as before.

In such cases it can scarcely be stated yet that the accessory chromosome is the cause of femaleness, for conceivably it may be a feature associated with that cause; but the evidence must be taken with confidence as the longexpected proof that sex is determined, in this case at least, by gametic differentiation. The female in these Insects must then be regarded as homozygous in sex, and may be represented as $D D$, while the male is heterozygous and may be represented as $D R$. Such a result accords well with the general conclusions to which breeding experiments, on the whole, point. For though great disparities between the numerical proportions of the sexes occur in certain matings, these disparities seem to be obliterated in succeeding generations. If the one sex were homozygous and the other heterozygous such impermanence of the numerical divergences is what we might naturally expect. Nevertheless the extraordinary fact remains that while the cytological evidence suggests that the male is heterozygous, equally cogent evidence-from breeding in other types-indicates the female as the heterozygous sex.

T. H. Morgan (202)* has lately carried the discussion a stage further. It is well known that in the case of animals having a series of parthenogenetic female generations, such as Aphis, Daphnia, \&c., when fertilisation does take place, the result of such fertilisation is always a female. In a Phylloxera Morgan has found that the spermatids are of two kinds, those which contain an accessory chromosome, and those which do not. The spermatids lacking the accessory body degenerate, and consequently only those provided with it take part in fertilisation. The fact that the results of fertilisation are all females, taken in connection with the degeneration of half the sperms, points evidently to the view that the accessory chromosome is here responsible for

* Since confirmed by von Baehr (7) for Aphidae. 
the femaleness. It is of especial interest to know that in this case the accessory chromosome goes through a stage in which it is partially divided into two, though after this partial division the two parts reunite and then the whole passes into one of the daughter-cells. The point that remains obscure is the nature of the cell-division by which the males are produced. For at one stage in the cycle males are of course born parthenogenetically from the females, and it would be of the greatest importance to know something of the cytological process by which the ova are destined to become males-or male-bearing females if there are two lines (as in the Aphides studied by Miss Stevens, 256, 260)*. Many other very interesting questions arise in connection with this part of the evidence, but further discussion must be postponed pending the accumulation of further evidence.

\section{Summary of Experimental Evidence as to the Heredity of Sex.}

In a full discussion of the problem of sex-inheritance many other kinds of fact would need to be considered. To these no reference can now be made. All that I have attempted is to provide a sketch of the new evidence on the problem which Mendelian experiments contribute. The main conclusion to which several quite distinct lines of inquiry unmistakably point, is that in the two Vertebrates and in the Currant moth the female is a sex-heterozygote, with femaleness dominant. The female is a hybrid, "femalemale," while the male is pure male, or "male-male." The eggs of the female are thus females and males respectively $\uparrow$, while the spermatozoa are all male. In other words, the female contains a factor which makes her female, but the male is male because he is without this factor. The phenomena can, as has been shown, with certain exceptions, be represented symbolically by a system based on this conclusion. The exceptions are real, but they are manifestly exceptional, and for the present we may be content to deal with the main course of the descent. It may conceivably

* These papers contain an account of the beginning of a very valuable experiment on the descent of colour in Aphis and its relation to sex.

$\dagger$ As those of Dinophilus apatris are. 
be through some extraordinary coincidence that the facts fall into a system which can be represented by this sym. bolism, but the convergence of the several sets of facts strongly supports the belief that the symbolical system gives a true representation of the physiological basis of sex in those forms at least to which the system applies.

The evidence from the descent of the dominant sexlimited diseases, such as colour-blindness, and of the horns in Sheep is also consistent with the same view, namely that femaleness is due to the presence of a dominant factor. For in these examples there is evidently some additional element present in the female which inhibits or suppresses the operations of the sex-limited dominant, and that additional element may not improbably be the factor for femaleness.

At the same time we should be wrong in supposing that the recognition of femaleness as a definite, allelomorphic factor is more than a first step towards an understanding of the phenomenon of sexual dimorphism. There are abundant signs that this representation expresses only a part of the truth. There seems to be no reason, a priori, why the gametic constitution of the two sexes should be the same in all types. In the various forms on which our conclusion is founded, the arrangement, we have seen, is probably for the females $D R$, and for the males $R R$. In the Insects studied by E. B. Wilson and Morgan the cytological evidence suggests $D D$ for the females and $D R$ for the males. All that can be claimed is that Mendelian analysis provides a hint of the way in which we may proceed in the attempt to unravel these intricacies.

It is, I think, on the lines indicated in the last paragraph that we must look for a reconciliation between the cytological and the experimental evidence. On the one hand the cytologists show that in most orders of Insects proof that the male is heterozygous can be obtained. From breeding experiments we find that in Vertebrates and in one Lepidopteran the female must almost certainly be regarded as heterozygous. The cytological evidence shows extraordinary differences even in nearly allied forms, some having a distinct unpaired chromosome, while in others this body is either fully or imperfectly paired. In Lepidoptera, as it happens, the accessory chromosome has not been found. 
Improbable as at first sight it may appear, the view that most commends itself to me is that in different types Sex may be differently constituted. From the results of castration-experiments we are led to a similar view ; for castration of the male Vertebrate on the whole leads merely to the non-appearance of male features, while injury or disease of the ovaries may lead to the assumption of male characters by the female. In Crustacea the evidence of Geoffrey Smith* and Potts shows that these consequences are there reversed. The great diversity of cytological features in allied Insects is also consistent with the expectation that the phenomena are specific rather than universal.

Then again we may feel fairly sure that the allelomorphism between the sex-characters must be a relation of no common order. The curious numbers that so often occur in collections of sex-ratios are evidence of this. The records given in the case Aglia tau and lugens bring us at once to difficulties with which as yet we cannot deal.

From time to time evidence has been advanced to show that the production of the sexes can be influenced by special modes of nutrition or other environmental influences. An adequate discussion of this evidence would run to great length. Some of the evidence has been found faulty in various respects $\dagger$, and I am not aware that any example has been confirmed by successive observers in such a way as to warrant definite belief in its validitył. It is not impossible,

* My attention has been called to the fact that in his monograph of Rhizocephala (Fauna u. Flora d. Golf. Neapel, xxıx. 1906, p. 89) Geoffrey Smith explicitly suggested, probably for the first time, the view here adopted, that one sex, sometimes the female, sometimes the male, is heterozygous in sex.

† See for instance Punnett (227) on sex-determination in Hydatina with a criticism of the evidence of Nussbaum on the positive effects of nutrition.

$\ddagger$ The most striking case in which positive results are said to have been attained is that lately published by Russo (236). This observer claims to have demonstrated in the rabbit the difference between the ova destined to become females and those destined to become males. His conclusion is so far in harmony with that to which genetic experiments have led us, that the female is heterozygous in sex. Heape, however, has described (Proc. Roy. Soc. vol. 76, B, 1905) in the rabbit processes by which ovarian ova frequently degenerate, apparently as a normal occurrence. $\mathrm{Mr}$ Heape very kindly gave me an opportunity of examining his preparations, and it was impossible to avoid being impressed with the general 
nevertheless, to imagine that the output of gametes representing respectively one or other of a pair of allelomorphs may be influenced by circumstances. We have not yet any proof that such a phenomenon may occur; but the long runs of unexpected numbers which we from time to time witness in the course of Mendelian experiments are suggestive of some definite disturbance of the normal equality in the output of the two kinds of gametes. Data for an adequate statistical examination of this question scarcely exist as yet, but if it be found that the long runs are too frequent to be accepted at all readily as chance aberrations, the search for environmental causes of disturbance will have to be undertaken.

The second conclusion that we have reached is one which no previous experience of nature could have led us to anticipate. Naturalists are so accustomed to considering the males and females of a true-breeding strain as of identical composition, except in so far as their sex is concerned, that those who have no practical acquaintance with genetic phenomena may find a difficulty in realizing that the females of a breed can be hybrid in some important respect though the males are not. The evidence however leaves no doubt of the reality of this conclusion. The consequences of such a discovery are not easy to foresee, but as in our very limited range of experimental study three such cases have already been encountered, we may feel fairly sure that this

resemblance which such degenerating ova bore to those which Russo regards as destined to become males. Consequently before that view of their nature is adopted, the relation of the so-called "male" ova to the degenerating ova will need very careful study; for it seems as yet not unlikely that those differences which Russo has taken to indicate maleness may prove to be due to incipient degeneration.

In addition to the detection of the distinction between male and female ova Russo states that he has, by administration of lecithin, succeeded in greatly increasing the proportion of female births. He gives figures to show that in rabbits, normally bred, male births are largely in excess of female births, and another series of figures showing a great excess of female births from females treated with lecithin. Both lists of figures are however declared to be selected from a larger number in order to illustrate the author's thesis. It is to be hoped that the full lists will soon be published. Meanwhile I may mention that Mr Hurst in his very considerable experience of breeding rabbits under normal conditions lias found the male and female births to be sensibly equal in number.

B. $\mathbf{H}$. 
special heterozygosis of females is no rarity* Bred with their own corresponding male type these females would in the ordinary course never reveal that they were heterozygous; for the spurious allelomorphism subsisting between the femaleness and the dominant factor which it repels would preserve the males homozygous and the females heterozygous, and so the impurity would be hidden in perpetuity. The real condition could only be brought to light either by a cross with a distinct race; or by a variation which through the introduction of some new factor or the omission of one previously existing, upsets the equilibrium hitherto maintained among the several elements through the cell-divisions of gametogenesis.

The recognition of these facts brings us a step, if a small one, nearer to the discovery of the nature of variation. In such a case as that of the Canary, since the ordinary green hens are "hybrids" of Cinnamon, the Cinnamon variety is perceived to have been already in existence, ready to appear if the critical event which could release the variety should occurt. What that critical event may have been we still cannot suggest.

The minute analysis of such cases as these will almost certainly disclose systems of interrelationship between the factors more complex than that we have been considering. In particular, it will be well to keep in view the obvious possibility that sexual dimorphism may be, sometimes at least, a phenomenon in which a compound character plays a part, so that for the production of femaleness, for instance, it may be necessary that two factors should coincide in the same zygote.

Finally, in a preliminary survey of the subject, the attractiveness of Castle's suggestion is not to be denied. It may well be that the unions of spermatozoa with ova

* Amongst much evidence which Professor C. O. Whitman most kindly gave me in 1907 concerning the results of his long-continued experiments in crossing the species of Pigeons, was an example which is strongly suggestive of the same heterozygosis of the female. When Turtur humilis $q$ is crossed with a white $T$. risorius $\delta$, white young may come in $F_{1}$ and are then always females.

t The same remarks apply of course also to the moth Abraxas grossulariata of which the males are pure to the type, while the females are hybrids of lacticolor. 
are not all equally possible, and that fertilisation can only take place between gametes dissimilar in respect of sexfactors*. A fact perhaps favourable to this conception is the almost universal arrangement of gametic systems in groups of four. The maturation-processes of male cells, and, with very rare exceptions, those of ova which are to undergo fertilisation, take place in such a way that a group of four, not two, reduced nuclei results. Both pollen-grains and spermatozoa are arranged in tetrads. The ovum prepared for fertilisation by the maturation-processes has ejected, except in a few special cases, three nuclei comparable with itself (though very often two of these may by an equationdivision be extruded in combination). Such differentiation by groups of four is strongly suggestive of the possibility that the four parts are not all comparable. It may be that the simple allelomorphism we so often find is really a phenomenon of simple cases only, and that the fundamental differentiation is in reality dimorphic for each sex. The gametic series for the heterozygote may not be $A, a, A, a$, but $A, a, A^{\prime}, a^{\prime}$ both on the female and male sides; and this may be the meaning of the grouping into sets of four, not sets of two. Naturally however very cogent evidence must be produced in order to establish such a proposition as this.

* There is one piece of direct evidence strongly suggestive of dimorphism among spermatozoa. It was mentioned (p. 172) that the daughters of colour-blind men can transmit the affection, while the sons of these same fathers are free from it and cannot transmit it. Until proper statistics are forthcoming it is not possible to build with complete confidence on this fact, but it is a clear indication of dimorphism among the sperms, such that those destined to take part in the production of females bear the colour-blindness factor, while those destined to fertilise the male ova are free from this factor. We do not yet know that all the daughters of colour-blind men can transmit, but the records are I think consistent with the belief that they may. While holding to the view expressed in the text that the female is heterozygous in femaleness $(F)$ we may perhaps suppose that the male is heterozygous for maleness $(M)$. We thus avoid the difficulties entailed by the theory that both sexes are heterozygous in "sex" (see p. 165), for two allelomorphic pairs are now involved, $F$ and its absence, $M$ and its absence. The eggs may be represented as (E) and $O$; the sperms as $(3)$ and $O$, so that in fertilisation the union is always between $F$ and a blank sperm or between $M$ and a blank ovum. The spurious allelomorphisms described in the canary, Abraxas grossulariata and the silky fowl are cases in which $F$ repels certain factors, while in colour-blindness there may be an exactly similiar spurious allelomorphism between $M$ and the factor for colour-blindness. 


\section{CHAPTER XI}

DOUBLE FLOWERS.

Miscellaneous Cases. Recessive and Dominant Doubling"Hose-in-Hose" Flowers-The Special Case of Double Stocks.

THE inheritance of doubling in flowers has been only studied with success in a few instances. In one of these, however, that of Stocks (Matthiola), a feature of such great physiological significance has been discovered that we may be sure the subject will before long assume considerable importance. A special chapter, though a brief one, must be devoted to it.

Doubling, the multiplication, that is to say, of the conspicuous parts of flowers, especially the petals, may occur as the result of a number of various and apparently quite distinct physiological processes. The different sorts of doubling have been often described in the treatises on plant teratology*. In the commonest kind the stamens are bodily transformed into petals, as a manifestation of that phenomenon which I have called Homoeotic + variation, viz. the transformation of a part into the likeness of another with which it stands in a series. This is the kind of doubling which occurs so conspicuously in the Rose, Ranunculus, Godetia, \&c. In other cases doubleness is

* A good general account of the phenomena will be found in Masters' Teratology, 1869. For a more minute description see K. Goebel, "Beiträge zur Kenntniss gefüllter Blüthen," Pringsheim's Fahrbiicher, xviI. I886.

+ Materials for the Study of Variation, 1894, p. 85 . 
attained by an actual multiplication or division of the petals, the stamens and other parts remaining apparently unchanged. Of this some of the double Fuchsias, Hyacinths and several Liliaceae afford very clear examples. These two processes, however, very often, in fact most usually, occur in combination with each other, and it is not generally possible to distinguish how much of the change in number is due to the one process and how much to the other.

In some flowers, especially those with gamopetalous corollas, the reduplication can occur in such a way that the corolla is simply repeated, two or more corollas standing in the place of one, but sometimes when two corollas are thus formed it may be seen that the outermost is in reality formed by a homoeotic variation of the sepals into the likeness of the petals. The most familiar examples of this "hose-in-hose" arrangement are known in Primula, Campanula and Mimulus. Another kind of doubling is due to what is termed proliferation or prolification (Masters) of the floral envelopes. The best illustration of this is the common double Arabis albida, in which the corolla and calyx are repeated tier above tier on an elongation of the axis. The different forms of increase in the number of the petals may commonly occur in varying degrees of perfection, and many grades of doubling are often to be seen on the same individual plant. There is also evidence that, in certain cases at least, high feeding and generous cultivation greatly promote the doubling. In some forms too it is known that the amount of doubling undergoes a change with the age of the plant, showing what has been called "Periodicity*," the most extensively doubled flowers appearing on the strongest stems and at the height of the flowering period.

To students of genetics the interest of the doubling of flowers arises partly from the fact that it is a character the heredity of which can be readily investigated, but especially from the obvious suggestion that the phenomenon is or at least may be associated with disturbance in the sexuality of the plants.

* See Correns, $\mathcal{F} B$. Wiss. Bot. xuI. x 905, p. 465, de Vries, Ber. Deut. bot. Ges. xviI. 1899, p. 45 . 
In many fully double flowers fertility is obviously impaired through the conversion of the reproductive organs into vegetative parts. It must not however be assumed that the sterility so often accompanying doubling is solely due to this comparatively definite circumstance. In the hose-in-hose Campanula, which has the sepals petaloid, the well-formed anthers contain plenty of pollen (some may be petalodic), but the female organs are in some way influenced by the variation of the sepals so that they are in some strains sterile. In the double Arabis albida mentioned above there are neither male nor female organs, but the tierupon-tier structure, which is here the form the doubleness assumes, plainly shows that something more than a simple homoeosis of the stamens and carpels has occurred.

In the Stock two kinds of doubling occur which are of quite distinct nature. The ordinary double Stocks much used in gardens are fully double, possessing an immense number of petals but no sexual organs, male or female*. Such doubles must therefore, as we shall see, always be bred from singles, a phenomenon which furnishes one of the most curious problems that the study of heredity has to elucidate. Besides these real doubles, plants are occasionally seen with one or two extra petals, but the experience of breeders makes it probable that these plants are not more prone than ordinary singles to produce the real doubles.

In Petunia another problem is presented. The doubles have an immense mass of petals, apparently formed at the expense of the stamens. A few anthers are nevertheless formed which contain good pollen. The female organs on the contrary are abortive and the double flowers set no seed $t$.

The case of Begonia offers some points of interest. The plants are of course monoecious and it is only the male flowers which are double as a rule. When the plants flower for their first season the doubleness is frequently extreme, no anthers being formed. But in their second season such plants bear male flowers which contain good anthers, especially, according to the experience of practical breeders,

* Goebel states that rudiments of anthers are very rarely produced.

+ See Vilmorin, Fleurs de pleine Terre, I886, p. 669, and various horticultural authorities. 
if the plants are starved*. The female flowers are generally normal, but sometimes extra petals are formed in them also. Such female flowers have singular malformations which have been often described, the most noticeable being an opening of the ovary which causes the ovules to lie freely exposed.

Of the hereditary descent of doubleness we know little, but from what is known it appears that several distinct systems are followed.

In Primula Sinensis doubleness is an ordinary recessive, singleness being completely dominant. The doubleness of Primula is of a very unusual kind. It may be primarily a petalody of the anthers, but I have never fully satisfied myself of this, nor do I know any critical observations on the subject. When a large collection of Primulas is examined, strains can usually be found, the members of which exhibit the lower degrees of petalody in some or all of their flowers. In the fully double flowers there is a complete series of petals inside the normal ones and arising from them. These are formed as images of the outer petals, so placed that their inner surfaces correspond with the outer surfaces of the normal petals, and the two adjacent surfaces are structurally both alike inner surfaces. The bizarre colour of double Primulas is due to this circumstance. Inside the corolla the stamens stand properly formed, and in their normal relations. The appearance of these fully double flowers is strongly suggestive of a delamination in the petals themselves; but as in those partially double flowers which have one or two extra petals imperfectly formed and showing their staminal origin, the petaloid tissue similarly faces outwards, it becomes impossible to distinguish any boundary between the two phenomena. The morphological problems which these facts create must be left to the expert botanist.

The segregation of the single character from the fully double is, in some families, clearly quite sharp, and doubles always breed true when fertilised inter se. The inheritance of the partial or petalodic doubles has been investigated by $\mathrm{Mr} \mathrm{R} \mathrm{P}$. Gregory, and his experiments show that when such a strain is crossed with a pure single, $F_{1}$ is single, and in $F_{2}$ singles, partial petalodics, and some doubles

* For information on this point I am obliged to Mr Leonard Sutton and to Mr Wootten. 
appear, but owing to the great fluctuation in the degree of doubleness exact counts are impossible.

In Carnation, where the doubling is of the ordinary kind, due to petalody combined with sub-division of parts, there is some reason for suspecting doubleness to be a dominant. Everyone who has grown Carnations from seed is aware that a proportion, often considerable, of the seedlings come single. It is most improbable that any large number of these can owe their singleness to cross-fertilisation with single plants, for breeders would not keep such plants wittingly. Till critical experiments are made, however, the point cannot be regarded as certain beyond question.

In Poppies, on the contrary, the dominance of the single type appears to be quite complete. In the annual Larkspur (Delphinium consolida) also, from the fact that pink doubles exposed to the pollen of blue singles produce among their seedlings some blue singles*, there is no doubt that the single type is dominant. Instances of this sort could probably be multiplied without difficulty, and from what we know of plant-breeding in general, there is no practical doubt that doubling of the ordinary type is usually recessive to singleness.

\section{The Hose-in-Hose or Calycanthemous Campanula and Mimulus.}

The inheritance of this well-known variation or monstrosity was studied by Correns (76). The calyx, as described above, is petaloid in many degrees, varying from a condition in which the sepals are still partially green up to the full development of a second corolla. In Correns' experience this variation in the calyx is accompanied by a marked diminution in the fertility of the female organs. This in Campanula persicifolia amounted to total sterility, though in $C$. media a few seeds were set.

The abnormal character proved to be a partial and somewhat irregular dominant, considerable fluctuation occurring on the individual plants. Owing to complete failure of $F_{1}$ to set seed $F_{2}$ could not be raised, but when the normal type was fertilised with pollen from $F_{1}$ a mixture of the two

* I have observed this in my own garden. 
forms resulted*. The Campanulas are plants well suited for this kind of experiment and it is to be hoped their genetic properties may be fully explored. Interesting experiments, for example, could be made with the white double form of persicifolia known in horticulture as $C$. Moerheimii. In it there is extreme doubling, apparently of the ordinary kind, due to petalody of stamens and splitting of the parts.

In the hose-in-hose Mimulus Correns found also that that variety is an irregular dominant. Here however there was no sign of sterility in the female organs.

\section{Double Stocks (Matthiola).}

Elaborate experiments on the heredity of doubleness in Stocks have been made by Miss E. R. Saunders, and though the research must still be regarded as in an incipient stage, some facts of quite unusual interest have been discovered.

Single Stocks in general breed true to singleness. As a rarity an extra petal may appear, but there is no evidence to connect such an appearance with the extreme and most definite kind of doubling characteristic of the ordinary double Stocks of horticulture. In double Stocks both carpels and stamens are wholly absent so far as our observations have gone, though, according to Goebel, rudimentary anthers are sometimes formed. From time immemorial these doubles have been bred entirely as the offspring of special strains of single Stocks which are maintained for that purpose. Since all doubles are absolutely sterile the succession is represented thus :-

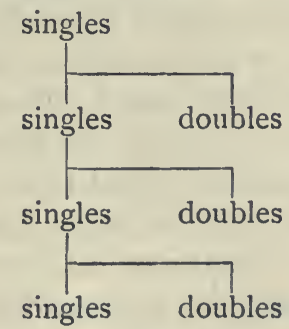

* Correns states that seed sold for "hose-in-hose " or "cup-and-saucer" Campanula gives a majority of plants showing the variation. The sterility of the ovules is perhaps an accompaniment of the petalody when strongly pronounced. Campanula media, with considerable though not complete calycanthemy, that I have examined had no obvious reduction in fertility. 
The numerical proportion in which the doubles appear cannot be confidently stated, but it is certain that this proportion differs greatly in different strains. There is some reason for supposing the ratio 9 doubles : 7 singles to be the common one in some families, but the experience of breeders points clearly to the fact that the proportion of doubles may be much higher than this in specially good strains, while in others the proportion again may be much lower. Generally speaking, however, we have the certain fact that plenty of strains consist of singles which throw a great excess of doubles.

Many writers have recommended special cultural devices for increasing the output of doubles. Starvation, drying the plants off, keeping the seeds from the lower ends of the pods, are among the expedients advised. I do not know that any of these suggestions have been properly tested, and it would be rash to deny that they may have some effect. On the other hand it is practically certain that horticultural bad treatment will not cause a double Stock to produce stamens or carpels. Even in the weakest flowers on the doubles, which often may be so reduced as to have only 4 or 5 petals, no sexual organs are formed.

The morphological nature of the doubleness of Stocks is by no means clear. When such flowers are examined it is seen that the 4 sepals are normal. Above them the floral axis is continued for some length, and on it are set the crowded petals in imbricated fashion. There is no repetition of the sepals as in the case of Arabis albida, and the variation cannot readily be described as a strobilisation.

Since the doubles are totally sterile, the problem of the hereditary transmission of the condition must be investigated by making crosses with the single-flowered parents which produce these doubles. As the doubleness appears in the offspring of these singles the condition is evidently present either in the male cells of such flowers or in the female cells, or is produced by the combination (in fertilisation) of the factors with which these cells are endowed.

Miss Saunders has made many crosses between different strains of double-throwing singles, and as was to be expected, such crosses have given a mixture of doubles and singles just as either parent would have done if self-fertilised. 
The numbers are irregular and obviously. need a further analysis.

The crosses which are instructive are those which were made between the double-throwers and the pure single strains. Whichever way the cross is made, $F_{1}$ is always single. When $F_{2}$ is raised from these plants the curious fact appears that the result differs according to the way in which the original cross was made. When the cross is in the form pure single $q \times$ double-throwing $\hat{\delta}$, all* the $F_{1}$ plants give a mixture of doubles and singles in $F_{2}$; but when the reciprocal cross is made, namely double-thrower used as $q \times$ pure single used as $\hat{\sigma}$, it is found that the $F_{1}$ plants are of two kinds, (I) those which throw doubles mixed with singles in $F_{2}$, and (2) those which throw only singles in $F_{2}$.

The conclusion to be drawn is evidently that the pollencells of the double-throwers are all (perhaps nearly all) bearing the double character, but that the egg-cells of these same plants are of two kinds, those which bear singleness and those which bear doubleness (see 22, pp. 5 and 36 ).

For the first time therefore we have a proof that, in a hermaphrodite form, there may be a substantial difference between the factors borne by the male and female cells of the same plant. It is the existence of this instance which leads me to hazard the suggestion introduced in the discussion of the facts of sex-inheritance in Bryony, that the female cells of Bryonia alba may be of two kinds and male cells of one kind (see p. I68).

This discovery, though it may prove to be the clue to the problem of the double Stocks, leaves the main difficulty still unsolved. We have to find the scheme whereby it comes to pass that the doubles, though bred from singles, are nevertheless as a rule in the majority, and the difficulty of offering a plausible suggestion is not diminished by a knowledge of the fact that the male cells may be all double, for this fact implies that a majority of the egg-cells must also be bearing doubleness, and ultimately that part of the singleness must have, as it were, disappeared in gametogenesis. We are at present quite unable to offer any

* No exception has yet been met with. There are however some reasons for anticipating that exceptions may exist. 
symmetrical or probable scheme of character-distribution by which such a phenomenon can be represented*.

The various difficulties of the case culminate in the fact which seems well established, that though the doublethrowers generally give 7 singles : 9 doubles-with certain departures from this ratio which are probably not fortuitousyet $F_{1}$ raised from single $\times$ double-thrower, and from the reciprocal cross, always on self-fertilisation gives 3 singles : I double $\uparrow$.

* For further information on this subject see the Appendix to Part I.

$\dagger$ The relation of white to cream-colour in the strain known as "Sulphur" is approximately the same as that of single to double described above. "Sulphurs" are single whites throwing singles all white, and doubles which are usually creams with whites as exceptions. The pollen-grains of "Sulphur" all bear cream, while of the ovules some are creams and some whites. 


\section{CHAPTER XII}

EVIDENCE AS TO MENDELIAN INHERITANCE IN MAN.

Normal Characters-Diseases and Malformations. Dominants-Sex-limited Dominants-Recessives-Note on Collecting Evidence.

OF Mendelian inheritance of normal characteristics in man there is as yet but little evidence. Only a single case has been established with any clearness, namely that of eye-colour. The deficiency of evidence is probably due to the special difficulties attending the study of human heredity. Human families are small compared with those of our experimental animals and plants, and the period covered by each generation is so long that no observer can examine many. Now that the critical methods of study are understood we may have every confidence that progress will be made.

In human inheritance there is however one somewhat peculiar feature, the complexity of the transmission of the various colour-characters*. In our experimental studies of animals and plants we have rarely met with examples of a descent so complex as that which the colour of hair and complexion in the mixed populations of western Europe certainly presents. If the colours that we see in our own population followed in their descent rules so simple as those traced in the mouse, or the sweet pea, or even as those which a little study would undoubtedly detect in regard to the colour of cats, the essential facts of Mendelism must have long ago been part of the common stock of human knowledge

The case of eye-colour is comparatively simple. As Hurst has shown by examining the children in a Leicester-

* See also later with regard to albinism in man. 
shire village and comparing them with their parents, the type in which pigment is present on the front of the iris is a dominant, the absence of such pigment being recessive. When the pigment is present in some quantity the eye is called brown or black, while when it is absent the eye is called blue or grey. In general terms therefore it may be said that brown eyes are dominant to blue eyes. Careful examination however shows that many eyes which might on a hasty glance be called blue really have some of the characteristic pigment. Casual descriptions therefore made by use of the popular names for the colours are quite unreliable, and the propositions based on them can only be received with great caution. Some further details and pedigrees are given in the Chapter on Eye-Colour (see p. ro8).

With respect to hair-colour in our own population nothing can yet be said with much confidence. "The segregation of red hair from black hair may be seen in many families and this red is presumably a recessive, but to work out the interrelations of hair-colours in general would be a very difficult undertaking*. Just as in the case of eyecolours, so here, the attempt to force the various colours into a continuous colour-scale and to classify the material by reference to that scale is useless; for though probably intermediates could be found existing in such gradations as to bridge the gaps between the more distinct types, there cannot be the least doubt that so soon as a strict method of analysis is instituted the various intermediates will be shown to be caused by the interactions of a limited number of definite factors. (See Intermediates, Chap. xiII.) In the analysis of such phenomena research must proceed by the detection of the pairs of factors, beginning with the more obvious, and when their behaviour and powers are thoroughly understood, a search for the remainder may be attempted.

The fact of continuous descent through many generations creates a probability that several notorious family characteristics, such as the Hapsburg lip and many more which could be cited, would prove on examination to be dominants, but the evidence for a proper analysis has not 
hitherto been compiled. Two such cases may be given. The first is that of a peculiar form of short woolly hair, resembling that of the negro (Gossage (132), on the

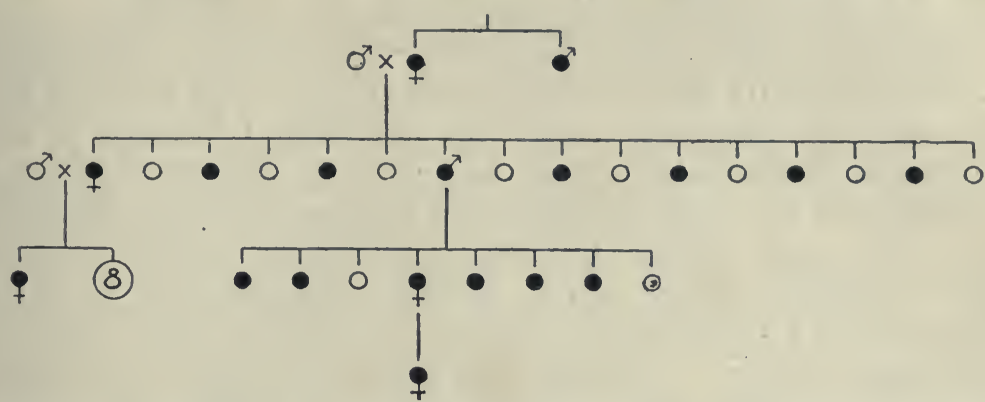

Fig. 21. Descent of a peculiar form of curly hair recorded by Dr Walter Bell. (After Gossage, 132.) The black symbols are the affected members.

authority of Dr Walter Bell). Family tradition attributed this peculiarity to a "Mexican" ancestor several generations back. The peculiarity was transmitted directly through those who exhibited it, as shown in the diagram (Fig. 2I).

The second is a similar inheritance of a lock of congenitally white hair recorded by Rizzoli, which clearly behaved as an ordinary dominant (see Fig. 22).

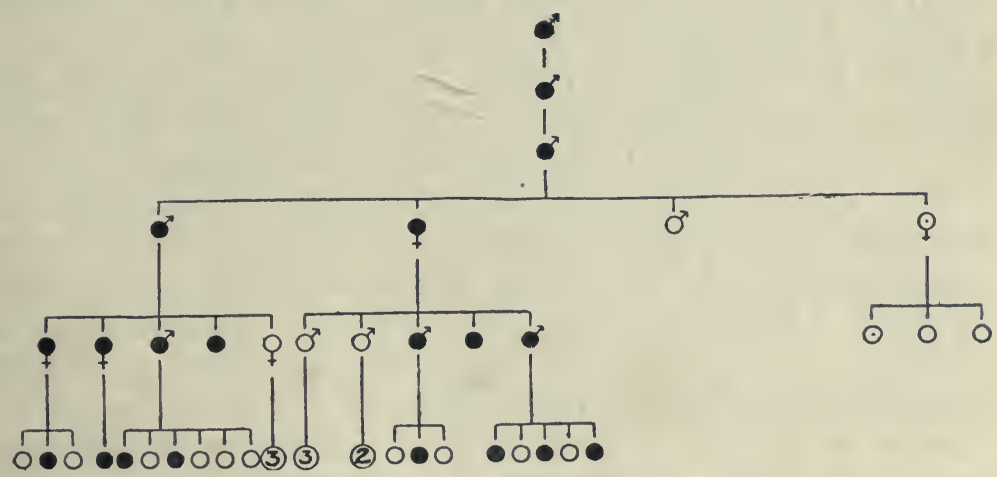

Fig. 22. Descent of a congenital lock of white hair, recorded by Rizzoli. (After Gossage, 132.)

There can be little doubt that if pains were taken to record the descents of striking features many such pedigrees could be compiled. It is of course necessary, if oral testi- 
mony and tradition have to be appealed to, that the characteristic should be a definite one about which mistakes could scarcely occur. It is necessary also that the feature should not be dependent on external influences. For example, in many families a crooked little finger is common. This peculiarity may descend both through the affected and through the unaffected, but the affection, I believe, is like that seen in chickens whose feet may be deformed through weakness. In some families such chickens occur commonly, but with much greater frequency when the incubators have been working badly, a fact which suggests that it is the disposition to the peculiarity which is transmitted, not the peculiarity itself. Probably the crooking of the little finger is an expression of a certain weakness, which if circumstances are favourable need not become apparent. Pedigrees of such peculiarities cannot be expected to give results of much positive value.

As to the results of inter-crossing between distinct races of mankind there exist, so far as I am aware, no records of that critical and minute sort which are alone of value for the adequate study of Mendelian inheritance. For example, the statement has been repeatedly made that the mulatto formed between the European and the Negro breeds true to an intermediate type, but we still await precise data in support of this statement. It may well be that there is no sensible segregation in regard to colour in that case, and certainly many intergrading colours exist, but there is no material yet upon which a definite pronouncement can be made. As regards length and curliness of hair, indeed, I anticipate from casual observation that the consequences of segregation could be made evident without very much difficulty. With regard to skin-colour the general trend of evidence is in favour of the conclusion that if definite determining factors are responsible for the colour seen, the number of such factors or of their subtraction-stages must be considerable. A point not to be forgotten is the diversity not only of the negro races, but of the white population, from which the mulattos arise.

From such information as I have been able to glean from travellers I am disposed to think that in the crosses between white races and the inhabitants of India there are 
signs of a segregation more complete than exists among mulattos*.

Mr Mudge has published also reports of the existence of segregation in regard to several characteristics in the crossbred offspring of Canadian Red Indians and Europeans (Nature, Nov. 7, 1907).

Pending further research we must take it that the mulatto is probably a genuine exception, in so far at least as no obvious segregation normally occurs.

Respecting the genetics of other normal characteristics in man, such as stature, little progress has been made. The case of human stature is interesting as it furnished material for Galton's original determination $\dagger$ of the Law of Ancestral Heredity spoken of above (p. 6). We may feel assured that this deduction is to be interpreted as meaning that the number of factors involved in deciding human stature is large-which indeed is evident, if it be remembered how many kinds of physiological difference must contribute to the determination of the length of the body. Something must be decided by the number of cells in the leg-bones, vertebrae and various cartilages, by the size of these cells, by the amount and density of the bony substance secreted, by the shape of the skull, by the obliquity of the neck of the femur, by the curvature of the spine, and by many elements of bodily proportions, some easily influenced by conditions, which interact with each other to produce the single "character" we call height. Such a character could only show segregation if it were studied in families made by the inter-crossing of extreme forms each breeding sensibly true to type. It is perhaps a little remarkable that in the pea and the sweet pea segregation in height should be so marked, but the reason is evidently to be found partly in the immense differences there available for study, and in the existence of one predominant contributing factor, the internodal length.

* There is a good deal of scattered information which tends to show that even among negro-mulattos segregation sometimes occurs. See, for instance, W. Lawrence, Lectures on Physiol., 1823, p. 259.

$\dagger$ Natural Inheritance, 1889. 


\section{Dominant Hereditary Diseases and Malformations}

Though knowledge of genetics of normal characters in man has advanced so little, we have now clear evidence as to the laws of descent followed by many striking peculiarities which are of the nature of deformity or disease. It is somewhat singular that nearly all the abnormal features (except those which are sex-limited) that have been yet positively shown to follow Mendelian rules in man are dominant to the normal. There are indications that certain abnormal conditions are recessive, but in two of these only is there much evidence. Dominants are of course much easier to trace, as the peculiarity then descends directly from parent to offspring, and so a continuous history is provided. Probably it is to this circumstance that the comparative plenty of evidence respecting the dominants is due.

\section{Brachydactyly.}

A good example of these dominants, which indeed was the first Mendelian case to be demonstrated in man, is that described by Farabee. The peculiarity consists in a shortening of the fingers and toes, which had only one phalangeal articulation like the thumbs instead of two (Figs. 23 and 25). The condition is said to have been the same in all the fingers and toes of the affected members of the family. The descent is represented in the diagram. As there indicated, the peculiarity descended solely through the afiected, and the children of the unaffected did not in any single instance reproduce it, for they were evidently pure recessives. The offspring of the affected, on the contrary, consist of affected and unaffected, in approximately equal numbers $(36: 33)$. The affected parents in each case married with normal persons, so these unions are all of the form $D R \times R R$, and the equality of affected and unaffected is in accordance with Mendelian expectation.

Farabee's families are American, being centred in Pennsylvania, and the diagram shows records referring to five generations. Recently Drinkwater has published a full and 
excellent account of the same peculiarity as manifested in an English family extending over seven generations. In his cases also the same law of inheritance is strictly followed, the normal offspring of abnormals being always free of the abnormality; while the abnormals marrying with normal persons produce on an average equal numbers of affected and unaffected children ( $39: 32$, counting those only whose condition is definitely known).

There are several physiological questions of importance arising out of this case. First, as to the exact nature of

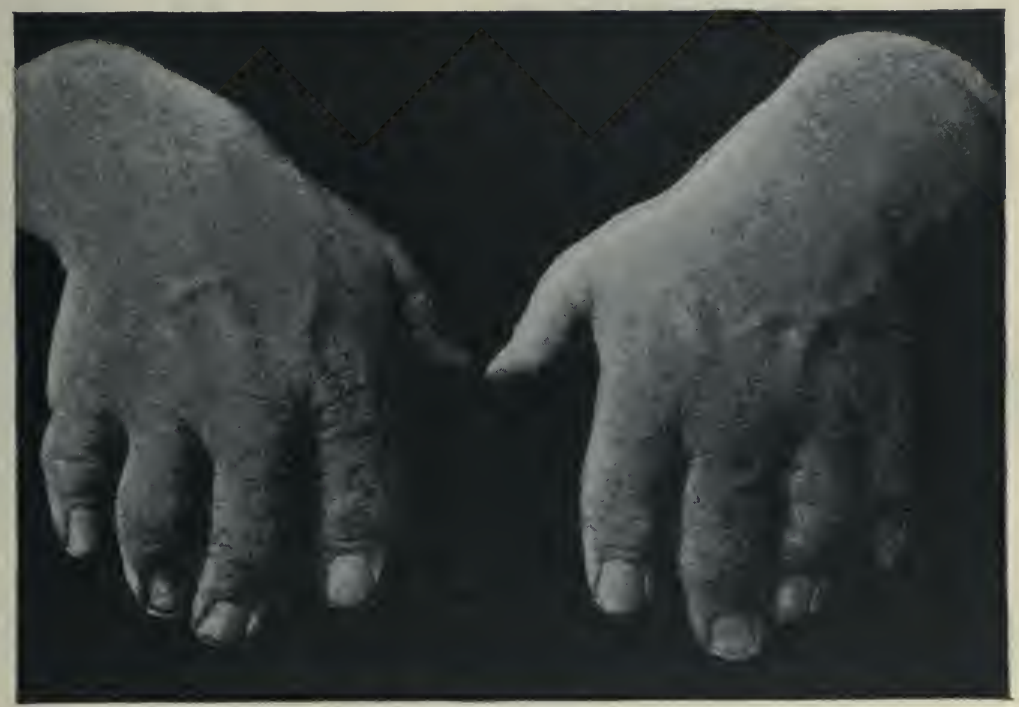

Fig. 23. Brachydactylous hands. (After Farabee, I22.)

the malformation, it seems to be clear that in all abnormal individuals examined the fingers and toes are all alike in being short and in having only one phalangeal articulation. Farabee from his radiographs concluded that a phalanx was definitely absent in all cases, but Drinkwater in the extensive series studied by him proved that in some individuals there is in digits III and IV a distinct representation of the middle phalanx as well as the proximal and terminal. His view is that the basal epiphysis of phalanx II is absent, and that this absence constitutes the essential malformation. 
Perhaps the epiphysis of phalanx III is sometimes also unrepresented and it may be that phalanges II and III are sometimes originally one cartilage, but there is not always complete union between these phalanges even in adult life (compare Figs. 24 and 25).

Another point of exceptional interest is the fact that all the abnormal persons were unusually short in stature as well as short in the fingers and toes. This association of characters was equally pronounced in Farabee's and in

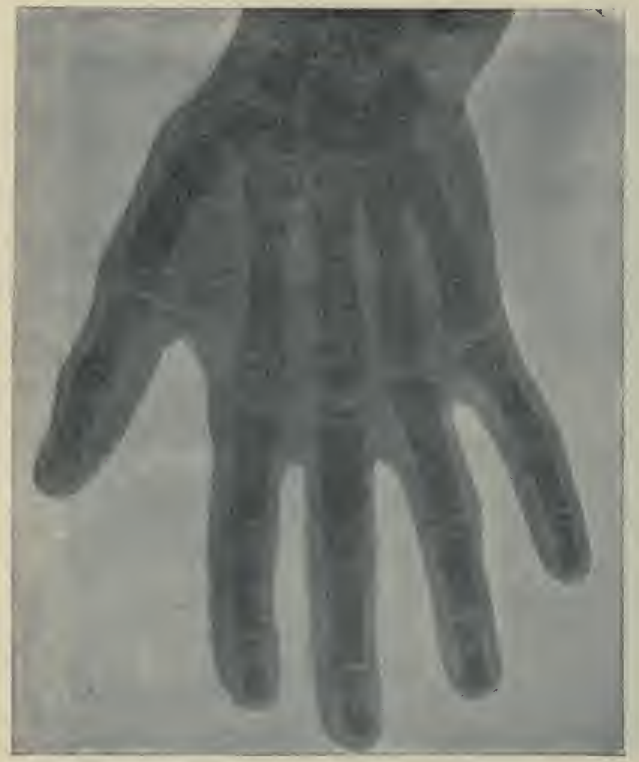

A

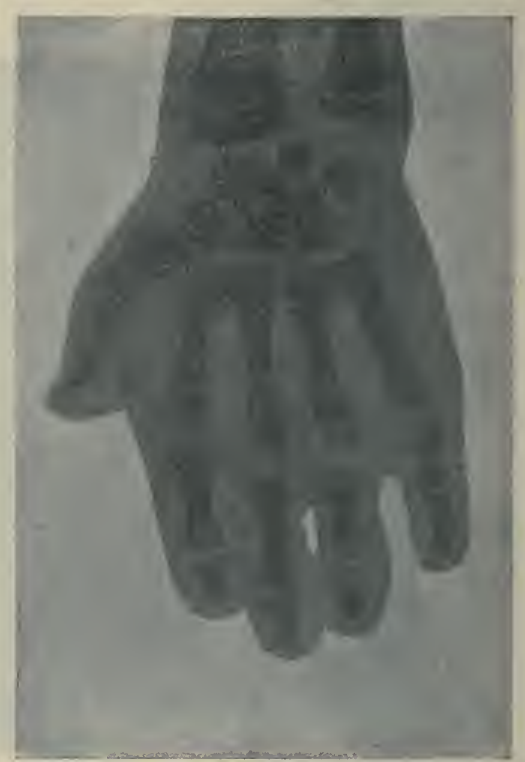

B

Fig. 24. Skiagrams of normal $(A)$ and brachydactylous $(B)$ members of Drinkwater's family. Both are adult. (From his photographs.)

Drinkwater's families. The latter author gives many details of measurement from which it appears that the average height of the normals exceeds that of the abnormals in males by $8 \frac{1}{2}$ inches and in females by $4 \frac{3}{4}$ inches. The disproportion between the head-lengths was relatively much larger. Upon what structural peculiarity the reduction in stature depended was not discovered. There was no indication that the length of the limb-bones was out of proportion to that of the trunk. The whole series of facts has 
obviously a close bearing on the nature of meristic variation, but a discussion of that problem is beyond my present scope*.

If we were dealing with natural species or varieties a debate might arise on the question whether it is readily to be imagined that so definite a variation could have arisen independently in the two sets of families, in America and England respectively. To those who have experience of variation and who know how large and well defined discontinuous variations may frequently be, it will not seem much more difficult to conceive of the repetition of the variation

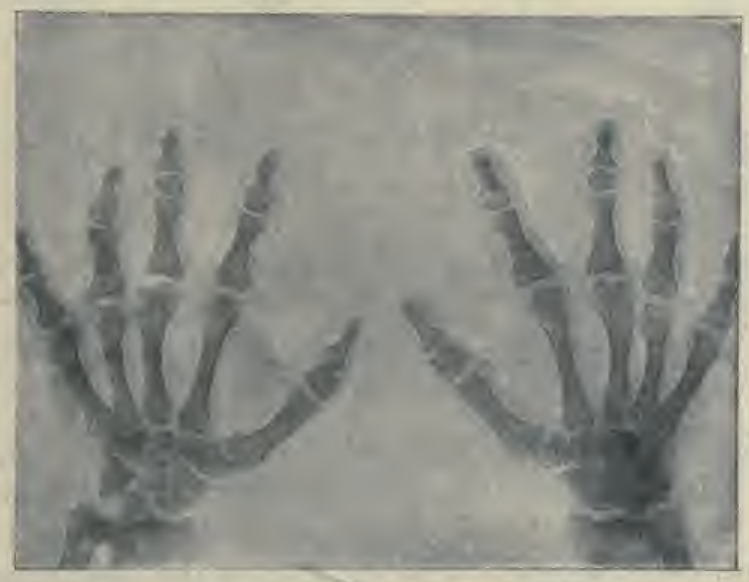

Fig. 25. Hands of brachydactylous woman, age 32. A separate ossification can be seen in digit III between the 1st and 3 rd phalanges. In the other digits union of this element with the 3 rd phalanx has already taken place. (From Drinkwater's unpublished photograph.)

than of its first occurrence. There is however some plausibility in the suggestion that these two families may in reality be one in origin, for it is known that a male abnormal of the 4th generation in Drinkwater's strain did emigrate to America. On the other hand the abnormal first recorded in Farabee's strain was a female in the $5^{\text {th }}$ generation from

* It must be observed that in view of Drinkwater's facts the variation is not simply meristic in the sense that the digit divides into two joints instead of three. As he himself is inclined to suppose, the case is more probably to be regarded as a homoeotic variation of the digits into the likeness of the hallux and pollex. 
the youngest. There is consequently no very probable correspondence between the two, but their connection is not impossible on our present evidence.

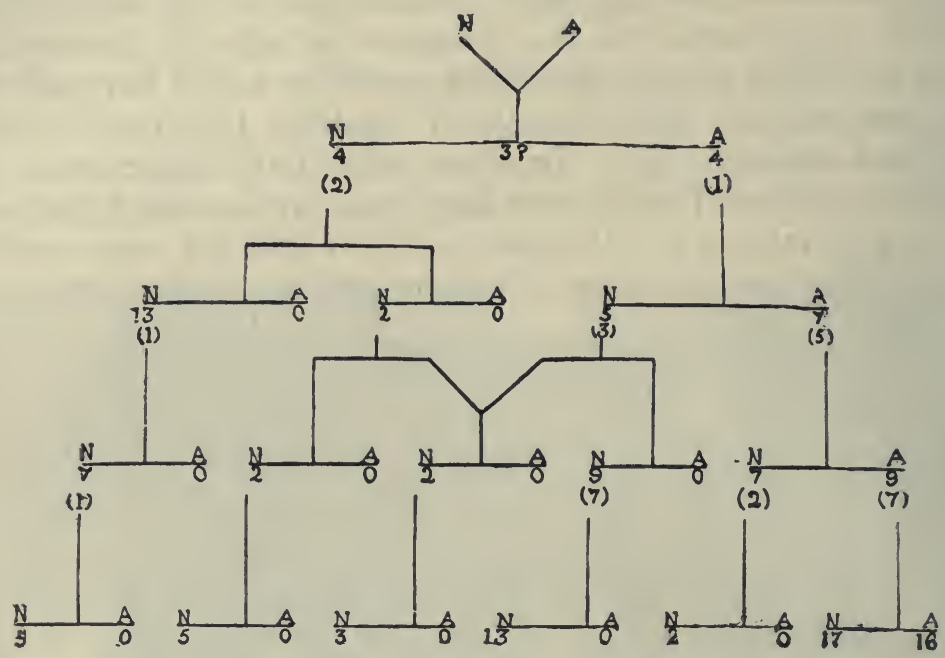

Fig. 26. The pedigree of Farabee's brachydactylous family. $N$, normal. $A$, abnormal.

Another type of brachydactyly has also been recorded by Walker*. In it there was an imperfect union of the phalanges affecting especially the first and second phalanges of the middle and ring fingers. The degree of the affection varied

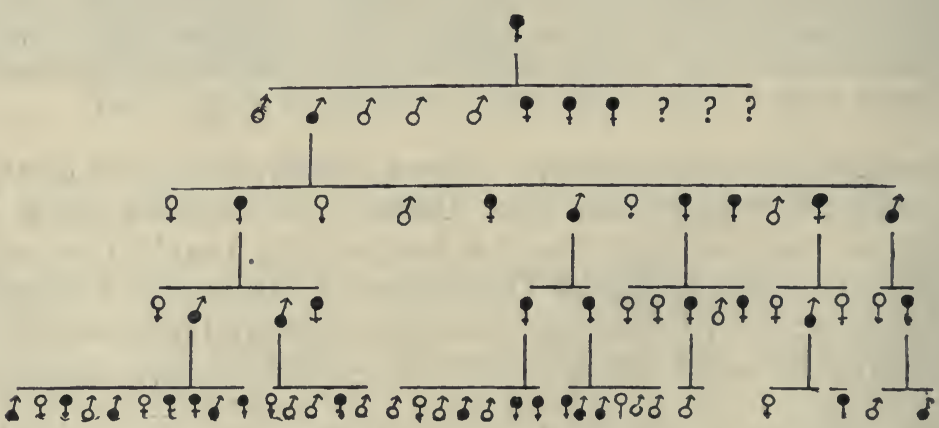

Fig. 27. Pedigree of affected members of Farabee's brachydactylous family. Black symbols are affected persons.

* Walker, G., Johns Hopkins Hosp. Bull., xII. r9or. In copy sent by author 2 unaffected in family of John B. are deleted. 


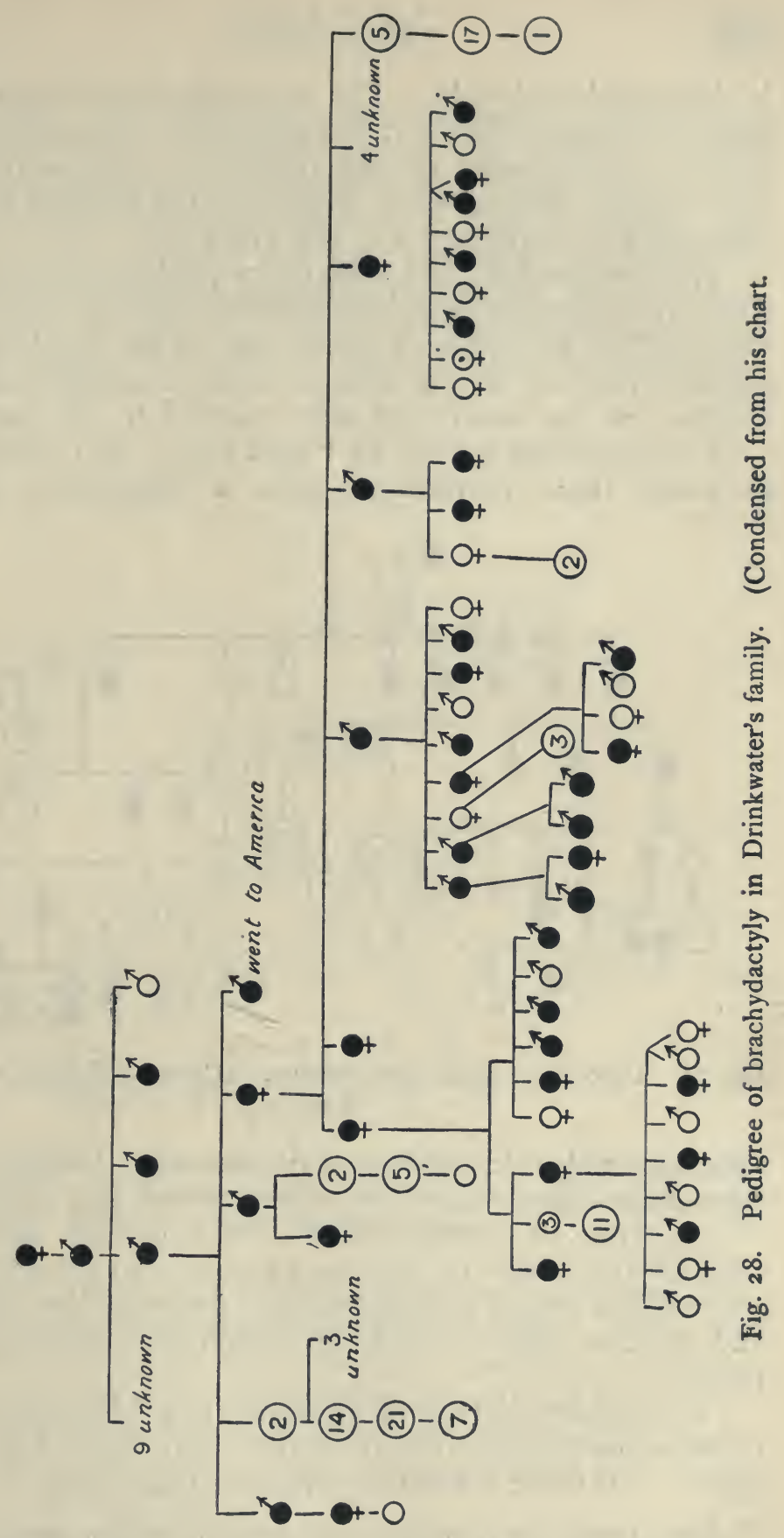


in different individuals. The pedigree shows that this condition descended through the affected in four generations, but the descendants of the unaffected are not recorded. According to this table the families of the affected add up to Affected 8; Unaffected I 5 ; not known 2.

A correspondent has sent me a pedigree of a family in which the fingers were almost entirely aborted. The descent was that of an ordinary dominant. The families of the affected add up to 19 affected and 16 unaffected. The children of the unaffected were normal in all cases.

The condition known as "split hand" and "split foot," in which these extremities have a monstrous, claw-like

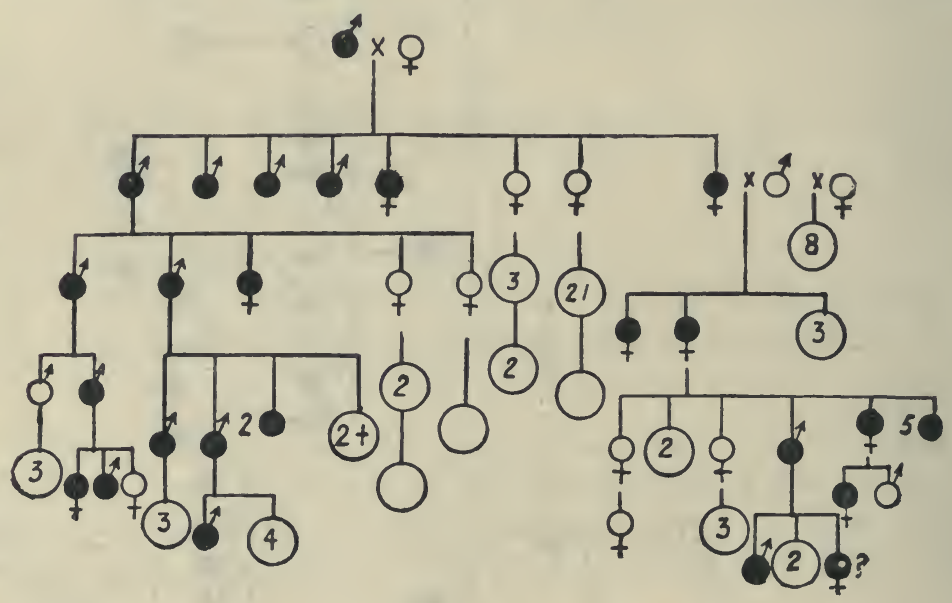

Fig. 29. Descent of prae-senile cataract. (Condensed from Nettleship's chart.)

appearance also descends as a dominant*. Partial suppression of the digits is one of the features of this abnormality. The degree of malformation varies greatly among the affected individuals of the same family, and though there is generally a rough correspondence between the two hands and the two feet of the same person the symmetry is only partial.

A considerable list of abnormalities following a course of heredity similar to that of the short digits can now be given. Of these a few only are mentioned here.

* For full collection of evidence see Lewis, T., and Embleton, D. (I 70). 
Cataract. A study of the extensive collection of evidence made by Nettleship shows that several forms of cataract are usually transmitted as dominants, the descent passing through the affected persons. This is shown most clearly in regard to the congenital varieties. In these the cataract is present and generally recognized very early in life. Some of the family records exemplifying descents of this kind are very extensive. Occasionally there is descent

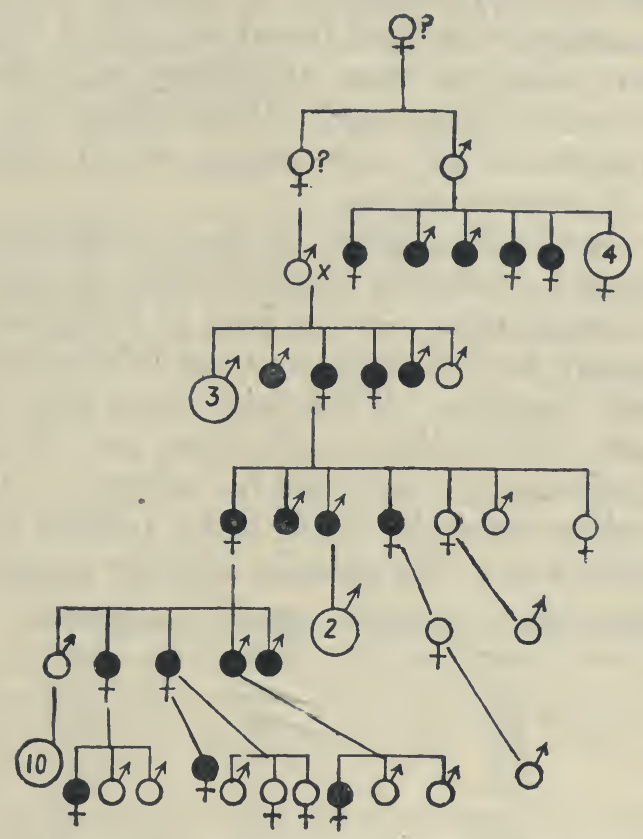

Fig. 30. Descent of prae-senile cataract. The condition was here transmitted, exceptionally, through one individual who was probably unaffected. (Condensed from Nettleship's chart.)

of the cataract through a parent recorded as unaffected, but the course of the transmission is usually normal. Whether in the exceptional cases the apparently normal persons who transmitted were in reality slightly affected cannot yet be said, but it is noticeable that several of these alleged exceptions occur in the case of progenitors whose state is only known by tradition (Fig. 30).

Cataracts acquired later in life, and even the senile forms, seem, so far as the evidence goes, to follow the same rule in 
their descent. But in dealing with this evidence difficulty arises from the fact that the age at which the cataract appears is liable to great variation and consequently the negative record, that a person was not affected, is of doubtful value, for the affection might have been due to appear later in life. As we should expect, however, it is in respect of cataracts of this kind that transmission through the unaffected is recorded with considerable frequency*.

There are several examples of families showing even congenital cataract in several members where the affection seems to have arisen de novo, no history of cataract being discoverable in the parentage. With regard to the subsequent transmission of such cataracts no very definite information at present exists.

As there is obvious doubt of the completeness of the records in many instances it is not possible to give numerical data with exactitude. A fair idea of the general run of the numbers may be gathered from the following list of the more complete families. These are somewhat arbitrarily selected from Nettleship's collection, but all the more extensive families are included $t$. Some of the more aberrant numbers even in these more perfect records are almost certainly due to the sources of error named above.

\begin{tabular}{lcccc} 
Recorder & Nettleship's case-number & Affected & Unaffected & Not knozen \\
Berry & 27 & 20 & 20 & \\
Fukala & 46 & 19 & 22 & \\
Nettleship & 58 & 29 & $25+$ & \\
Bandon & 83 & 11 & 5 & \\
Froebelius & 90 & 13 & 26 & \\
Gjersing & 97 & 17 & 4 & \\
Fisher & 108 & 8 & 13 & \\
Zirm & 112 & 14 & 15 & \\
Nettleship and & & & & \\
$\quad$ Ogilvie [21 2] & & 17 & 25 & 17 \\
& & 148 & 155+ & 17 \\
\hline
\end{tabular}

* It should nevertheless be remarked that the appearance of the cataract at earlier ages in successive generations- "anticipation," as Nettleship calls it-has been observed with some frequency.

$\dagger$ Except No. 98, Green's family, where the cataract appeared at various ages, sometimes not till advanced life. The numbers in it are I 8 affected, and 37 unaffected. Transmission here occurred twice through the unaffected. Since this table was made Nettleship has published some new and important pedigrees (2 I0). One of these gives a family from two $D R$ parents with senile cataract. Up to time of writing their children were 7 affected, 2 unaffected, and I died at 45 with sight good. 
A valuable collection of pedigrees illustrating Mendelian descent chiefly of several affections of the skin and hair has been published by Gossage. All the conditions there considered behave as dominants, and respecting several of these the evidence is fairly adequate (1 32 ).

Respecting tylosis palmaris et plantaris the records are especially clear. There is a condition in which the skin of the palms and soles is abnormally thick in varying degrees*. From a study of over thirty family groups affected with this peculiar thickening of the skin it appears that transmission was always through affected members except in the case of one family historyt. Adding together all the available recorded numbers of children born to affected parents there were 220 affected and I 84 unaffected. Since from the nature of such records it is practically certain that affected members of families would be less likely to be missed or forgotten than the unaffected, we may regard these numbers as not an impossible representation of an actual equality. In view of the complete escape of the offspring of the unaffected except in one family tree the fact that tylosis (called also keratosis) almost always behaves as a simple dominant may be regarded as established.

One family group was unique in the fact that the ancestor first showing the disease came from parents believed not to have had it. This person had a family of I 2, all affected In general we know nothing definite as to the origin of such variations, and whether the uniform

* Compare the famous family of "porcupine-men" often alluded to by the earlier anthropologists. A good account of this family is given by Sir W. Lawrence (Lectures, I823, p. 385), who remarks:- "Let us suppose that the porcupine family had been exiled from human society, and had been obliged to take up their residence in some solitary spot or desert island. By matching with each other, a race would have been produced, more widely different from us in external appearance than the Negro. If they had been discovered at some remote period, our philosophers would have explained to us how the soil, air, or climate, had produced so strange an organization; or would have demonstrated that they must have sprung from an originally different race ; for who would acknowledge such bristly beings for brothers?"

+ This is the family recorded by Ballantyne, Pediatrics, 1896, I. p. 337. This family was peculiar not only inasmuch as transmission occurred through the unaffected, but also in the fact that the condition appeared in females only. This sex-limitation suggests that the etiology of the condition was in this family distinct from that of the ordinary tylosis. 
affection of all the family of the first case is in any way connected with the sudden appearance of the condition cannot of course be said.

The other conditions which Gossage finds to be dominants as a rule are:

Epidermolysis bullosa: in which the skin is liable to blister for trifling causes.

Xanthoma: the presence of yellow patches on the skin. Multiple Teleangiectasis: small naevus-like spots.

Hypotrichosis congenita familiaris: the loss of hair at or soon after birth.

Monilithrix: a nodose condition of the hair.

Porokevatosis : a curious disease in which a raised horny ridge appears on the skin, spreading centrifugally, leaving behind it a patch in. which the constituents of the skin undergo partial atrophy.

The spontaneous origin of porokeratosis has several times been observed. In epidermolysis transmission through unaffected persons occurred in some of the strains but was exceptional.

Besides these skin-diseases Gossage suggests that enlarged spleen is generally transmitted as a dominant. There are also four pedigrees showing the same phenomenon in the case of the condition called diabetes insipidus or polyuria.

To these may be added with some probability certain forms of inherited oedema and some of the kinds of harelip.

The most extensive pedigree that has yet been compiled for any disease in man is that for congenital stationary night-blindness, of which the first part was published by Cunier in $1838 *$. Working on this foundation Nettleship succeeded in obtaining material for a most elaborate genealogy including 2116 persons (Fig. $3 \mathrm{I}$ ). The aftection consists in a marked inability to see in a dim light. In this family the transmission is through the affected, and no

* Annales Soc. Méd. de Gand, 1838, p. 383. [Not seen: quoted from Nettleship.] 


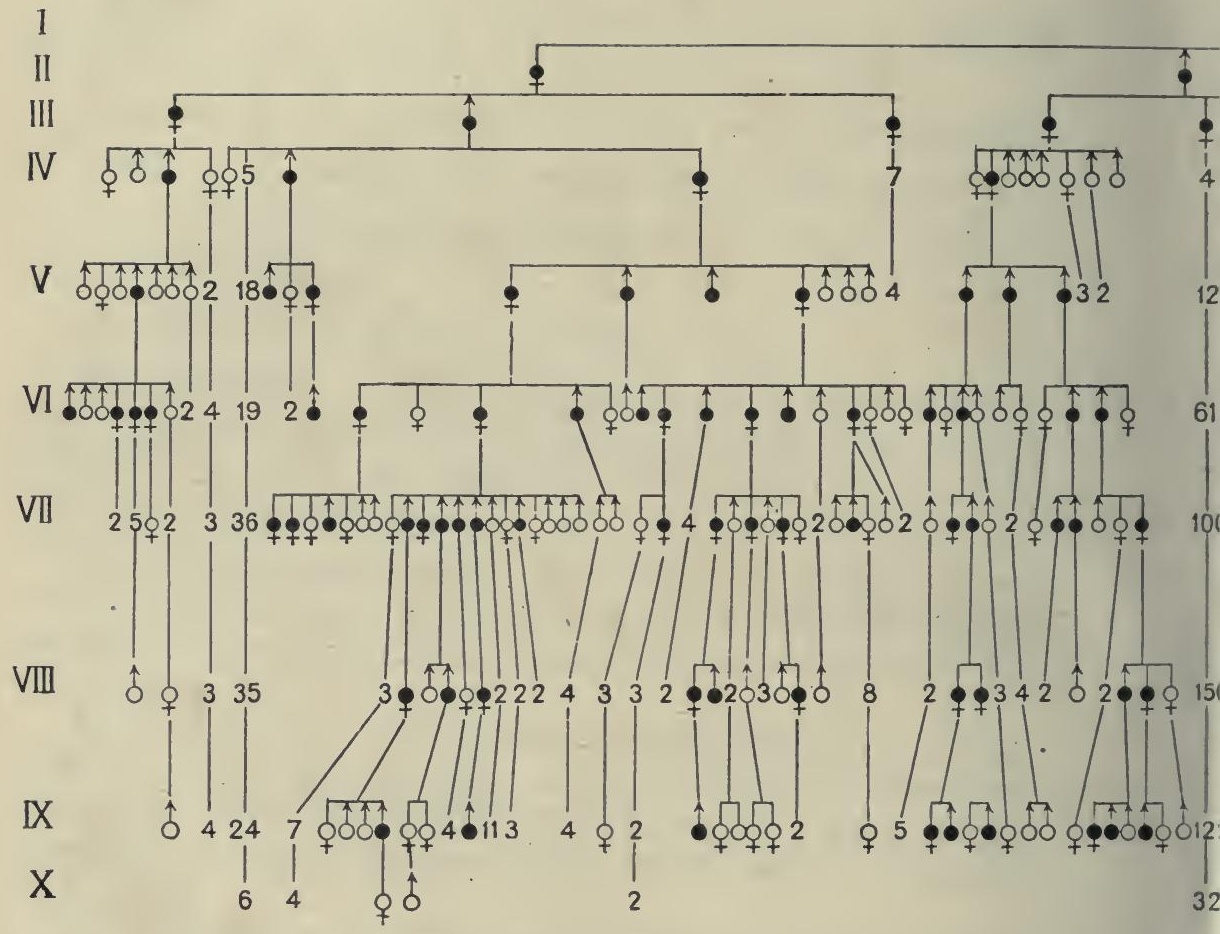

Fig. 3 r. Descent of a form of stationary night-blindness. (Condensed fr Only those families which contain affected members are here set out in by the $16^{\text {th }}$ black symbol from the left in generation viI.

Black symbols show the night-blind individuals. The descent $\mathrm{i}$ According to the records there is a great excess of normals over the aff theless the table gives a remarkable illustration of the permanence and 

departure from this rule has occurred in the ten generations that have elapsed since the birth of the earliest known case (born 1637). The people are peasants living in a group of somewhat isolated villages in the south of France. Adding together all the families which can be regarded as the offspring of affected and presumably heterozygous $(D R)$ individuals mated with normals the numbers are I 30 affected and 242 unaffected*. These figures depart very widely from the expected equality. Nettleship thinks there are reasons for supposing the number of affecteds somewhat understated, through a disposition to conceal the infirmity This cannot however account for the whole discrepancy, since error introduced from this cause would lead to the production of affecteds from alleged normals which has never occurred. It is of course possible that several who died before their condition was ascertained may have been counted as normals in past generations.

Qualitatively the descent is evidently that of a dominant, and though perhaps these aberrant numbers point to a genuine complication such a conclusion can scarcely be drawn positively from materials of this kind.

It is somewhat remarkable that stationary night-blindness usually follows a sex-limited descent, and the absence of any sex-limitation in the genealogy just spoken of, suggests that the physiological nature of the affection in this family may be distinct. There is, I understand, nothing which yet differentiates the one condition from the other unless it be that the sex-limited kind is usually associated with myopia which was not conspicuous here.

In the recorded pedigrees there are indications that, in addition to those already mentioned, the following ophthalmic diseases or malformations may be transmitted as dominants sometimes, though the evidence does not justify a comprehensive general statement and many exceptional cases are known.

Distichiasis: development of eye-lashes in place of glands on inside of eye-lids (perhaps a homoeotic variation).

* In one case an affected pair married and their two daughters were affected. Of these one (who may have been $D D$ ) had two affected children. The totals given above are arrived at after deduction for this fanily. 
Ptosis: droop of the upper lid.

Coloboma or Irideremia: a congenital defect in or absence of the iris.

Ectopia lentis: dislocation of the lens.

Glaucoma is a condition arising from various distinct pathological causes. From the pedigrees I have examined it is likely that in some of its forms the dominance rule is followed*.

The heredity of these various conditions could only be discussed adequately at great length. To do this the time is scarcely ripe. Owing to the interest now taken in the problems of genetics evidence is being rapidly accumulated and detailed analysis is best postponed till this new information becomes available.

\section{Dominant Diseases or Variations in Man following a} Sex-limited Descent.

In discussing the phenomenon of Sex allusion was made to the sex-limited diseases (p. I 72). The best known of these are :

Haemophilia: the liability to bleed profusely from trifling cuts or abrasions.

Colour-blindness: in its commonest form an inability to distinguish certain reds and greens.

Pseudo-hypertrophic muscular paralysis, or Gowers' disease.

Night-blindness of certain kinds.

The peculiarities in the descent of these conditions are that :

(I) They affect males much more commonly than females.

(2) They may be transmitted by the affected malest, but are not-save in very rare exceptions (haemophilia)transmitted by unaffected males.

* See for instance Howe, Arch. of Ophth., xvi. p. 72.

$\dagger$ Patients suffering from Gowers' disease rarely survive to adult life and practically nothing is known of their powers of transmission. 
(3) They are nevertheless transmitted by the unaffected females. Apparently normal women, daughters or sisters of the affected males, thus may transmit the condition to some of their sons (Fig. 32).

Such a system of heredity has long been a physiological paradox, and one of the most curious and interesting deductions from Mendelian research is the clue which it has provided to the solution of this problem of sex-limited descents (cp. p. I 73).

The experiment dealing with the inheritance of horns in sheep showed very clearly the probable lines on which an explanation was to be found. Just as in the sheep the horned character is dominant in males and recessive in females, so with these sex-limited conditions. If the males

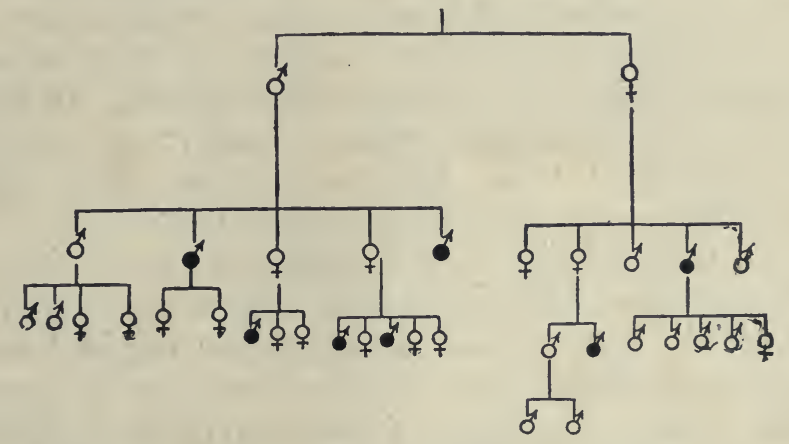

Fig. 32. Pedigree illustrating descent of colour-blindness. This family was found by $\mathrm{Dr}$ W. H. R. Rivers among the Todas, a hill-tribe of Southern India.

contain the factor for the condition, they exhibit it ; consequently the affected males can transmit, while the unaffected males cannot. In the females, on the contrary, somethingalmost certainly the presence of some other factor-prevents or inhibits the development of the condition, and then they may possess the factor without its making itself apparent. Such females may then transmit it to their offspring, but it will only be visible in the males, except in the rare case of a union between a heterozygous female and an affected male. Then the female children also may be affected, because they may be homozygous to the factor, receiving two "doses" of it, one from their father and one from their mother. 
According to the simple plan thus sketched, all the sons of the affected females will have the affection and exhibit it.

The only one of the notoriously sex-limited conditions which is available for testing this rule is Colour-blindness. Haemophilia and Gowers' disease are too fatal, and nightblindness is too rare and too little known. Regarding Colourblindness even, the evidence is not enough yet to provide a statement as to the quantitative results. A search undertaken by $\mathrm{Mr}$ Nettleship has however been successful in so far that he has obtained evidence of five colour-blind women with sons, in all eleven, who are all colour-blind. In three cases also there is, in addition, evidence that the women's fathers were colour-blind, as required by the scheme; and in one case also there is a record that the woman came from a strain having colour-blind members. Besides these there are two published accounts of families of two colour-blind women, each with three sons, all.colour-blind. In all therefore the seven colour-blind women had I 7 sons, all colourblind. We can I think feel no further doubt that the scheme must so far present an approximation to the actual facts. In dealing with such phenomena exceptional cases must be expected, but hitherto they have not been found*

Colour-blindness is not, therefore, as might have been imagined, a condition due to the omission of something from the total ingredients of the body, but is plainly the consequence of the addition of some factor absent from the normal. We can scarcely avoid the surmise that this added element has the power of paralysing the colour-sense, somewhat as nicotin-poisoning may do.

The other sex-limited diseases in all probability follow similar rules, but in regard to haemophilia there are various difficulties. The records are too heterogeneous for satisfactory tabulation as yet, and it is to be suspected that more than one condition may pass by the same name. One remarkable feature must be mentioned, namely that the records show with great constancy that too many males are affected and too many females transmit, the excess being far greater than any which could readily be ascribed to recorders' errors. This excess is also strongly exemplified

* By the kindness of Miss J. E. Downey of Wyoming University I have since been informed of a case which is probably a real exception-a woman with defective colour-sense having a child with normal colour-vision. 
in the case of another sex-limited disease-a peculiar paralysis of a "peroneal" type, studied by Herringham*. Some definite disturbing complication must be looked for in these phenomena, and the statement that the factor is dominant in males, and recessive in females, can only be taken as giving a qualitative description of its behaviour.

In Gowers' disease we have perhaps also to deal with more than one condition, and the evidence suggests that the recessiveness in females is not universal.

Tabular representations of the most probable expectations in the case of a sex-limited condition are given in Figs. 33 and 34 .

\section{Recessive Variations in Man.}

As so many abnormalities are known to behave as dominants with some consistency it is perhaps surprising that we have no quite positive case of pathological conditions behaving as recessives in man. The evidence regarding the normal light eye-colour has already been given, but this as yet stands practically alone. Hurst ( 162 ) has given some further facts suggesting that the musical sense is a recessive, but great and obvious difficulties make it very hard to obtain convincing proofs in that case.

In regard to diseases that may be recessive, there are several records which are suggestive, but little that amounts to proof. Naturally, as evidence of direct transmission is not to be expected, the likeliest place to look for recessives will be among those conditions which have been noticed as coming with special frequency in families resulting from consanguineous matings. In such matings, and particularly in those of first cousins, bearers of similar recessive characters may come together, and thus by the meeting of two similar germs in fertilisation offspring exhibiting the recessive character may be formed. The comparatively frequent appearance of a variation among the children of such unions is thus prima facie a suggestion that it is a recessive to the normal. This has been observed very noticeably in regard to retinitis pigmentosa, a degenerative disease of the retina. In Herrlinger's $\dagger$ collection of

* Herringham, W. P., Brain, xı. 1889, p 230.

+ Herrlinger, "Ueb. d. Aetiologie d. Retinitis Pigmentosa," Inaug. Diss., Tübingen, 1899 .

B. $\mathrm{H}$. 
records among $76 \mathrm{I}$ cases, 228 are said to have been offspring of consanguineous marriages. It is not in dispute that the condition may be produced by various specific causes also, but the heredity through consanguineous marriages creates a presumption that a group of cases may be of a recessive nature.

It should perhaps be pointed out categorically that nothing in our present knowledge can be taken with any confidence as a reason for regarding consanguineous marriages as improper or specially dangerous. All that can be said is that such marriages give extra chances of the appearance of recessive characteristics among the offspring. Some of these are doubtless bad qualities, but we do not yet know that among the recessives there may not be valuable qualities also.

From. what is known of the genetics of albinism in other types we should on analogy expect it to be recessive in man. There is no reasonable doubt that this description is true so far as it goes, but obvious complications are met with. No union of two albinos is on record so far as I know, but the frequency with which the albinos have proved to be offspring of related parents, especially of first cousins, justifies us in definitely inclining to the view that albinism is in man a recessive character. The families available for numerical comparison are scarcely well enough reported for a proper analysis to be made, but it is clear that they exhibit again the difficulty met with before in human pedigrees, namely, that the affected are far in excess of expectation (on the hypothesis that they are ordinary recessives). For example, from a set of figures derived from various sources* I get the numbers I I 5 albinos and I 74 normals, where the expectation is only 72 to 216. There are reasons for thinking these records of albinos too high, and those of normals too low, but the discrepancy is too large to be accounted for thus.

Human albinism differs from that of our domestic animals in the fact that it shows many gradational forms which connect it with the normal. There are also several

* I am especially grateful to Dr V. Magnus of Christiania for a series of ro albino pedigrees. One of these contains an extraordinary family of which seven are stated to be albinos and only one pigmented. 
records of congenital albinos acquiring pigmentation more or less complete. Lastly, albinism in man differs greatly from that in other forms in the fact that it is very often associated with disease, especially of the nervous system. Even nystagmus, the oscillating movement of the eyes so generally associated with human albinism, is not, so far as I know, met with in the pink-eyed rabbits, guinea-pigs, rats or mice. In the cat however something more like human albinism is to be seen, for in that animal we find the association of certain types of albinism with deafness, and in it also several degrees of pigmentation in the iris occur. Careful pedigrees of crosses with albino cats might help to a solution of this problem. But in studying the subject of human albinism and also that of retinitis pigmentosa one meets not infrequently features indicative of a widespread and multiform degeneration in the affected family not at all resembling the simple course of Mendelian inheritance where natural variations are concerned. It is at least doubtful whether there may not be some distinction between albinism thus appearing and that less definitely associated with disease.

An interesting observation is recorded by Stedman (Surinam, I806, Ir. p. 260) to the effect that an albino negress married to a European had children all mulattos. Hence we may infer that the factor determining the blackness of the negro may be carried by the albino. The great frequency of albinos among several coloured races of men has often been remarked on by anthropologists.

A rare condition known as Alkaptonuria in which the urine is red from the presence of the substance alkapton must surely be a recessive. The facts published by Garrod* make it likely that the disease follows recessive lines, for of 17 families in which cases have been seen, 8 were offspring of first cousins. On the other hand Garrod gives

* Garrod, A. E., Lancet, 1902, Dec. 13, and Arch. f. Ges. Physiologie, Bd. 97,1903 , p. 410. The inheritance of cystinuria would be equally interesting; see Garrod, ibid. and Abderhalden, Ztsch. f. Physiol. Chemie, 1903, xxxviII. p. 557. For a valuable discussion of the evidence see also Garrod (127). Since that paper was published he has called my attention to a fresh tamily discovered by Fromherz, Inaug. Diss., Strassburg, 1908, containing 3 alkaptonurics and 8 or 9 normals. 
one case of direct transmission from an alkaptonuric parent, and it is of course improbable that this extremely rare condition should have existed on the other side of the parentage. Though the evidence is scarce, it is very important, since the question of the actual mode of descent followed by such a "chemical sport," as Garrod has called it, is of absorbing interest. Anyone who has an opportunity should not fail to use every effort to trace the descent of alkaptonuria.

\section{Miscellaneous irregular Phenomena of Inheritance in Man.}

There remain great numbers of pedigrees of malformations and diseases which in our present ignorance seem altogether irregular. Of these some, for example, polydactylism, are perhaps to be regarded as due to dominant factors which can be inhibited or suppressed as a result of the presence of other factors. In poultry we know by experiment that the presence of extra toe may behave as a dominant, following the simple rule with fair regularity, but in other families the number of dominants produced is too small and transmission may occur through normals destitute of extra toes Such facts point to the existence of some unknown complication in those families.

Several pedigrees of ectrodactylism, the deformity of hands or feet by the absence of digits, are recorded in medical literature. Transmission in these cases usually occurs through the affected members, but the degree of malformation is exceedingly irregular, and the number of affected persons is higher than expectation. On the evidence it is improbable that any simple Mendelian scheme will express all these descents*. In Fotherby's "split-hand" family a case of polydactylism also occurred, and the condition is not very rarely recorded as associated with the more extreme forms of human monstrosity.

A vast literature exists relating to the heredity of various

* See for instance Fotherby, Brit. Med. Jour., r 886 (I), p. 975; Tubby: Lancet, 1894 (I), p. 396 ; for a full and more recent collection, also Lewis and Embleton ( 7 I 1 ). 
forms of paralysis, of deaf-mutism, and of mental disease. From the analysis of considerable collections of such evidence it is clear that in the present state of knowledge any reduction of the phenomena into a simple scheme is impossible. The first difficulty is that the pathology of these diseases is obscure and their diagnosis often imperfect, various dissimilar affections passing by one name.

Deaf-mutism, for instance, though at first sight a very definite phenomenon, may obviously be due to congenital deafness arising from many distinct causes, and hence the genealogies of deaf-mutism cannot be treated as though they all related to a single physiological condition*.

Next in dealing with diseases of the nervous system it must be remembered that many of them depend for their appearance on the presence of external stimuli. Forms of insanity which appear when the individual is subjected to various strains and excitements may not appear at all if these causes be absent. The element transmitted is evidently the liability, not necessarily the developed condition. The descent of such peculiarities is therefore beyond the range of our analysis.

The peculiar form of insanity known as Hereditary Chorea is exceptional, in that it very clearly follows the course of an ordinary dominant with few complications. Adding all the families apparently from heterozygous $(D R)$ parents recorded as mating with unaffected persons I get the totals I I 7 affected, 99 unaffected, as nearly approaching the normal equality as we can expect when the nature of the evidence is remembered.

\section{Note on Collecting Evidence as to Human Descent.}

As some persons may read this chapter who have not leisure for study of the more elaborate phenomena of Mendelism a few words as to the collection of evidence may usefully be introduced here. The one essential point in such collections is that the normal members of families

* Dr C. J. Bond, Brit. Med. Jour., Oct. 28, 1905, calls attention to evidence suggesting sex-limitation in certain deaf-mute families, sometimes the male, sometimes the female being the affected sex. 


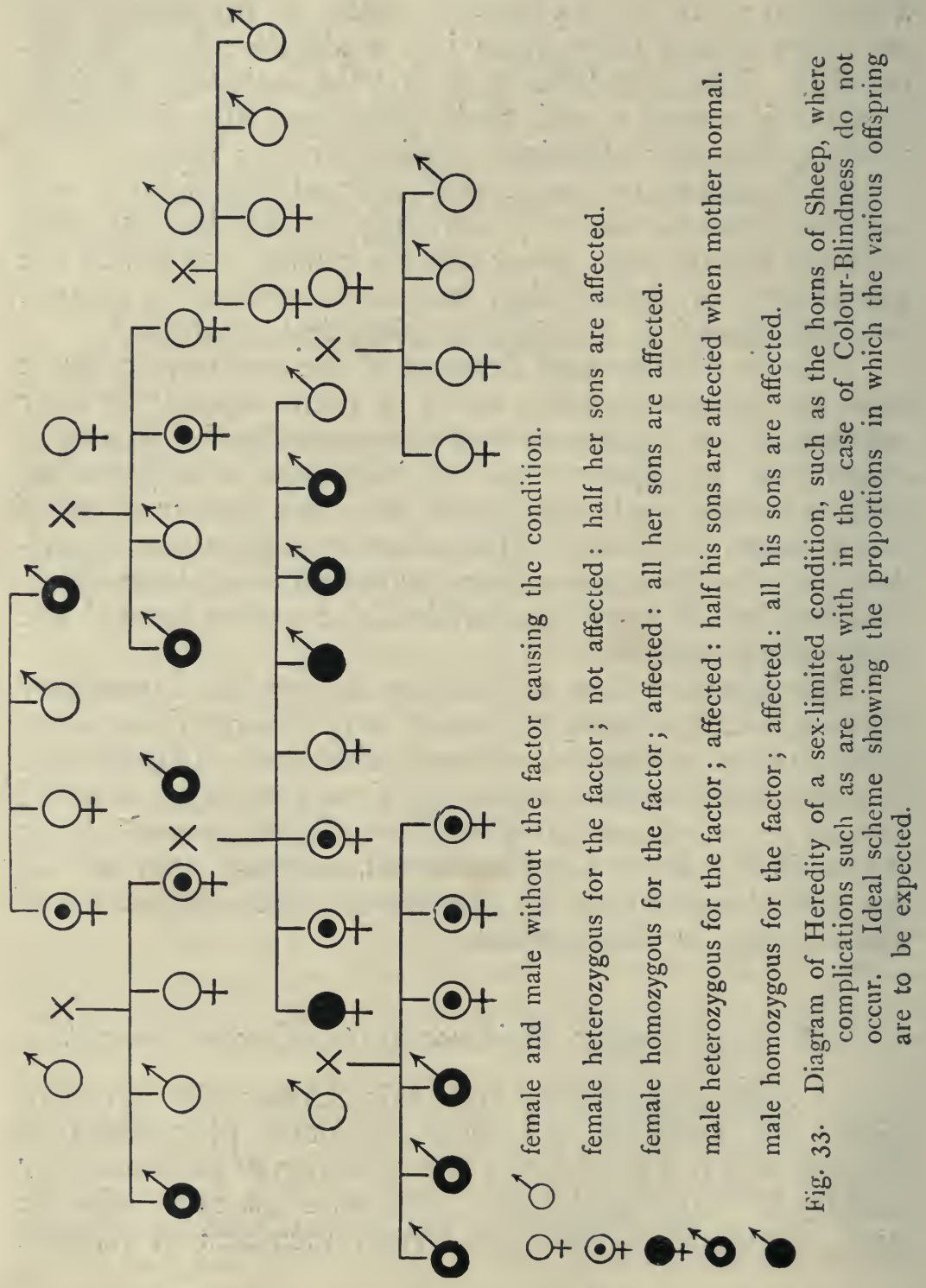


should be recorded with as much care as the abnormals. In all cases, where possible, inquiry should be made, respecting the children of the normals. The sex and age as far as possible of each individual should be noted. If the condition studied be not a congenital one, the age at which it appeared in each individual should be entered.

Dominant characters will in general be recognized as such from the fact that they are transmitted through affected persons only. The dominants will as a rule have had one parent affected with the peculiarity and one parent free from it. It is then to be expected that the children of such dominants, resulting from their marriages with unaffected persons, will be in equal numbers affected and normal.

Sex-limited dominant characters such as colour-blindness and haemophilia affect one sex, generally the male, most often.

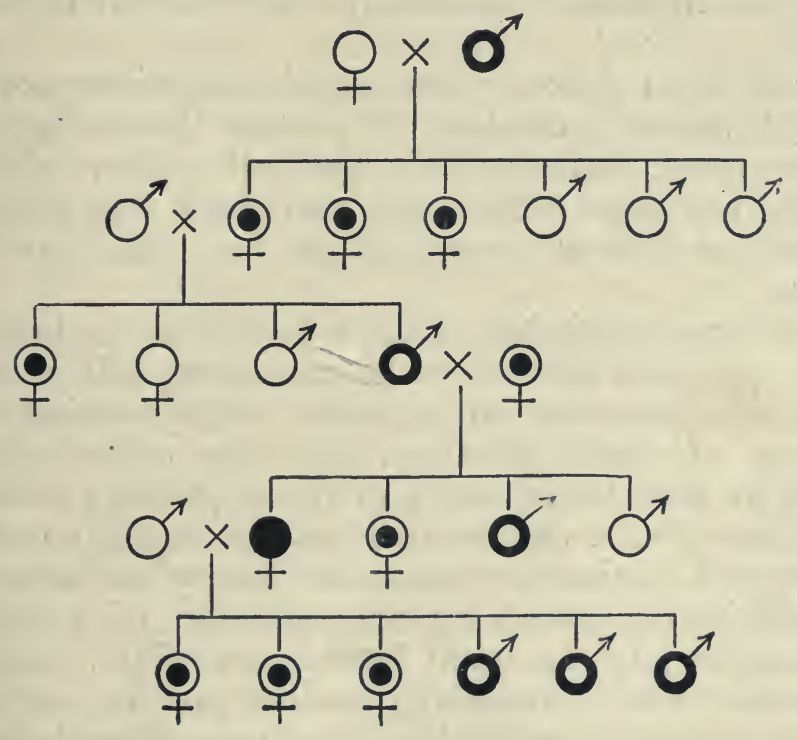

Fig. 34. Tentative representation of the descent of colour-blindness, drawn up on the hypothesis stated p. 195, note. Symbols as in Fig. 33. On this scheme a homozygous colour-blind male cannot be produced even by the union of two colour-blind parents. 
The simplest kind of sex-limitation is illustrated in Fig. 33. No example is yet known in man which exactly follows this system, though perhaps haemophilia and nightblindness may approximate to it. Colour-Blindness, as stated above (pp. 172 and 195), shows remarkable complications in the fact that not merely the development of the peculiarity but also the actual transmission of the responsible factor is affected by sex. Fig. 34 gives a tentative representation of the system which is perhaps followed by such a condition, but it is given rather as a hint to collectors of evidence than as a positive statement of ascertained fact.

Recessive characters will be recognized by the fact that they may appear in the children of parents not exhibiting such characters, and especially among children born of consanguineous marriages. Complete proof of the recessive nature of a characteristic will only be obtained by evidence that all the children of affected parents exhibit the characteristic.

In all these descents occasional exceptions are to be expected, which in the light of present knowledge cannot be interpreted; but when the habitual course of the inheritance has been determined, an inquiry into the nature of these exceptions may perhaps be undertaken with success.

A correct knowledge of the system which a hereditary disease follows in the course of its descent will, it may be anticipated, contribute to a proper understanding of the pathology of these affections, and thus make a definite advance in the general study of the physiology of disease. If, for example, a disease descends through the affected persons, as a dominant, we may feel every confidence that the condition is caused by the operation of a factor or element added to the usual ingredients of the body. In such cases there is something present, probably a definite chemical substance, which has the power of producing the affection. Thus the abortion of the digits, or the inability to distinguish certain colours, must be due to such added factors. On the contrary, when the disease is recessive we recognize that its appearance is due to the absence of some ingredient which is present in the normal body. So, for 
example, albinism is almost certainly due to the absence of at least one of the factors, probably a ferment, which is needed to cause the excretion of pigment; and, as Garrod has shown, alkaptonuria must be regarded as due to the absence of a certain ferment which has the power of decomposing the substance alkapton. In the normal body that substance is not present in the urine, because it has been broken up by the responsible ferment; but when the organism is deficient in the power to produce that ferment, then the alkapton is excreted undecomposed and the urine is coloured by it.

Similarly when we find that a condition such as retinitis pigmentosa sometimes descends in one way and sometimes in another, we may perhaps expect that a fuller knowledge of the facts would show that more than one pathological state may be included under the same name.

It need scarcely be remarked that when a disease, such as tuberculosis, which is due to a pathogenic organism, affects certain families or strains with special frequency, the hereditary or transmitted property is either the presence of something which renders the organism specially liable, or the absence of something which confers a higher degree of resistance. From the nature of the case pedigrees are not of much service in the analysis of these examples, for it cannot be asserted that an individual who escapes underwent the same risk of infection as those who took the disease. Though it cannot be doubted that the study of the descent of disease-resistance is of the highest importance, both theoretical and practical, progress must here be made by careful breeding experiments with animals and plants which can be tested by uniform methods of infection. As yet we know only one clear example in which such a rule of descent has been ascertained, namely that discovered by Biffen (29), who proved that, in wheat, resistance to Rust-disease is a recessive. In that classical example therefore we may suppose that this resistance is due to the absence of some ingredient which is present in common wheats and enables the rust-fungus to attack them successfully. Varieties in several degrees possessing the property of resisting disease are known in many orders of plants, and though regarding animals less is known, there are some 
indications of a similar character. An attractive field of research is thus provided. We may surmise that various types of resistance will be found, some dominant as well as others like that of wheat, which are recessive. Whatever answers are obtained in regard to these critical distinctions and their physiological nature, the evidence must in no ordinary measure contribute to the advance of general pathology. 


\section{CHAPTER XIII}

\section{INTERMEDIATES BETWEEN VARIETIES AND THE "PURE LINES" OF JOHANNSEN.}

Misunderstanding in regard to the physiological significance of intermediates has been a fertile source of error, and more than any other perhaps has tended to obscure the true interpretation of genetic phenomena. The existence of intermediates has been often alleged as a proof that segregation does not occur in regard to the particular variations towards which the gradational forms seem to lead, and the misuse of statistical method so frequent in biometrical attempts to investigate heredity has come about chiefly through misinterpretation of the nature of such gradational forms.

These errors are of course a legacy from the period of the essayists, when evolution at large was the chief object of study, and an examination of variation in detail as occurring in specific instances had not been undertaken.

Biologists committed to the proposition that varieties arise through the transformation of masses of individuals by the selective accumulation of minute differences saw that with each new case in which discontinuous variation could be proved to occur, the scope for their views was reduced, and the existence of intermediates constituted the most promising line of defence. When intermediate and gradational forms could be found, the contention might always be hazarded that the definite and extreme forms had been reached by transition through them.

Now the application of analytical methods to those 
cases where much gradation occurs is usually troublesome, but it is becoming clear that the difficulty is often a practical one only. To analyse the intermediates and to determine the various elements that have taken part in their production may be a very laborious process, still it can be shown in many examples that the intermediate character is only a superficial or nett result of the interaction of factors which are transmitted as units. The several classes under which such intermediate types present themselves are worth enumerating.

I. Intermediates as heterozygous forms. Sometimes, as is the case of the blue Andalusian fowl, the whole group of heterozygotes forms a recognizable, distinct class which might nevertheless be regarded as intermediate between the two pure types black and white, with splashes of grey and black. Similar intermediates occurring as heterozygous forms have now been often seen and described. In the case of Primula Sinensis the heterozygote between the star-shaped flower of the modern stellata varieties and the common type with imbricated petals is always an intermediate*.

In fowls though no perfectly distinct heterozygous type of comb exists, yet with experience it is usually possible to distinguish the pure $(D D)$ pea combs from the $D R$ group, and, though with much less confidence, the same distinction can sometimes be made among the rose-combs.

In Lychnis the crosses between white vespertina and red dioica are, so far as I know, always of an intermediate shade of pink. In Pisum the crosses between the races I have called "half-dwarf," standing about 3 to 4 feet high, and actual dwarfs, about I foot high, are usually intermediate in height. The researches of Miss Wheldale on Antirrhinum have shown that in that species numerous intergrading forms are definite heterozygotes, and Miss Marryat has proved that the same is true for Mirabilis.

Such instances may be multiplied indefinitely. Dominance indeed is not often so pronounced that a practised

* Whether any such intermediate flower shape exists as a gametic form which can be bred true I do not know for certain. Mr Gregory and I have not met one among our derivatives from various crosses between stellata and Sinensis. 
observer cannot distinguish the $D D$ types from the $D R$ 's with fair certainty by a thorough and minute examination. As examples in which heterozygotes are indistinguishable from pure dominants, may be mentioned tall and dwarf in Peas and Sweet Peas, coloured flowers and white flowers in Sweet Peas and Stocks, hoary and glabrous in Stocks. In all these the one "dose" of a dominant factor is sufficient to produce the full effect.

2. Intermediates due to subtraction-stages of dominant factors. This is also a large and important class, one, too, which has been perhaps more especially a source of the confusion alluded to in the introduction to this section. Of such intermediates the colour of the Dutch rabbit may be taken as a type. The Dutch rabbit has the posterior half of the body coloured, while the anterior half is white except for patches including the eyes and ears. Such a rabbit may be described as in a sense intermediate between the self-coloured rabbit and the albino. Its particoloured nature is due to the restriction of one (or conceivably more) of its pigment-factors to certain areas. In the self-coloured animal this factor has a more extended distribution. But in speaking of the Dutch pattern as an intermediate, an essential point, the definiteness of the Dutch pattern, is missed. It is intermediate only in a strictly limited sense. It is not a transition-stage between a self-colour and an albino, nor would there be any better chance of breeding albinos from genetically pure Dutch than there would be from genetically pure self-coloured rabbits. The Dutch pattern in fact constitutes a definite type and depends for its appearance on the presence of a certain factor in a fairly definite stage, and it does not appear when selfcolours are crossed with albinos unless the particular necessary factor in its appropriate stage is introduced in one of the parents.

Within the Dutch class there are subordinate types again, the interrelations of which have not yet been worked out. 'Some of these are very probably gametic types which could be bred pure, while others are probably caused by fluctuations of an irregular kind or by the interactions between the factors. It will need very elaborate research 
to determine the interrelations of these finer differences. As one of the few points that are clear may be mentioned the impossibility of fixing a form with the sharply-cut division between the white and the colour which is the fancier's ideal for Dutch. From this negative evidence it may be inferred that that particular distribution is not one of those represented as a gametic type.

Intermediates of this sort are commonly met with in breeding. The "half-dwarf" peas are an instance. In Stocks Miss Saunders has described a "half-hoary" race ( 19, p. 33) in which the lower surfaces of the leaves were hoary while the upper surfaces were glabrous or nearly so. No such "intermediates" have occurred among the thousands of plants raised by Miss Saunders except when the definite "half-hoary" type was originally introduced as a parent. In the Sweet Pea one of the most definite and unchanging types is the nearly white flower with a pink streak, that comes next to the pure white if judged by the criterion of amount of deficiency of colour. In the absence of breeding experiments an ignorant person having raised such a plant as offspring of a purple or a red might consider that he had made an advance towards pure white. Nevertheless he would be no more likely to raise true whites from such a tinged white than if he began with a wild Sicilian purple pea. Indeed if I required to raise a white Sweet Pea de novo, I should think the chance of getting it from the wild pea much better than from the tinged white, for again and again wild types have been found to throw off albinos soon after their introduction into cultivation-by the occurrence, of course, of a new genetic variation.

3. Intermediates produced by the interference of other factors. There are many intermediates of this kind also. In both animals (Fowl, Rabbit, Mouse) and plants (Primula) we have seen that a dominant factor may exist which has the power, for example, of suppressing the development of colour, leaving those parts white which in the absence of that factor would otherwise be coloured. This suppressing or inhibiting effect may be total, but when it is not total some puzzling intermediate types may be constituted. Only most careful breeding experiments can reveal the true 
nature of these cases. To the ordinary observer the "Painted Lady" Sweet Pea with its whitish wings and red standard might appear intermediate between a white and a totally red type. But as experiment shows, the bicolour type with its whitish wings is dominant to the self-coloured forms, and its wings are white, not as the Dutch rabbit is, owing to the omission of something, but on account of the addition of another distinct element which suppresses the pink colour in that particular part more or less completely. The real white is of course due to the absence of one or more of the colour-factors, an altogether different cause, and it is therefore evident that to speak of the bicolour form as in any way intermediate between self-colour and white would be a complete misrepresentation of the facts.

Similarly in the Rabbit, Hurst has shown that the curious type known as "English" pattern-something like a Dalmatian dog but for the more or less regular disposition of the spots-is a dominant to self-colour, though a careless observer might guess it to be a transition-stage towards albinism.

In such cases again there is every likelihood that careful selection might succeed in isolating subordinate types in which the suppression attains particular degrees of completeness ranging within well defined limits, but the evidence distinctly negatives any attempt to treat these several forms as a continuous series in which any member is capable of reproducing any other among its offspring.

4. Intermediates as fuctuational forms. Lastly there are intermediates due to the disturbing effects of many small causes not of genetic but presumably of environmental origin. Such are the fluctuations in the weight of individual seeds which Johannsen has studied with success in beans (Phaseolus).

When the weights of the seeds of a single variety of bean are marshalled statistically they arrange themselves in a normal curve round the weight of greatest frequency. Similarly when the seeds are sown and the seeds from the self-fertilised individual plants which grow from them are harvested separately, the crops from each individual again can be grouped according to their weights in normal curves 
round the most frequent or modal weight characteristic of each individual. There will then be seen that some rough correspondence exists between the modes for the individual plants and the weight of the individual seeds from which they sprang. The heavier families on the whole come from the heavier seeds, and the lighter from the lighter seeds. Such correspondence is nevertheless very imperfect, and the weight of the seed chosen from the original mass gives only a vague indication of what the modal weight of that plant will be.

But when any one family raised from a single seed was taken, and the heavier and the lighter of its self-fertilised seeds chosen and separately sown, Johannsen found that the modal weights were approximately the same for the produce of both the heavier and the lighter seeds. Selection inside the family raised from a single seed did not alter the modal weight, which went back or regressed to that of the individual common parent. Such a family thus raised from the single seed constitutes what Johannsen has called a pure line. Within the genetically pure line there are fluctuations in weight, but these fluctuations are due to interference which is external, or environmental in the wide sense, and selection of those extremes which are due to such interference produces no effect on the result, for the differences in the weights of the seeds of a pure plant do not indicate differences between the germ-cells which produced them (164).

It is to variation of this type that the name fuctuation is given.

Johannsen has made a similar series of experiments in regard to the proportion of defective grains present in certain barleys and has reached a similar conclusion.

Whether the distinctions characteristic of the pure lines segregate or not cannot yet be said. By the nature of such cases the distinctions themselves are fine, and even if segregation occurred the proof of its existence would involve many and serious practical difficulties.

When the complex diversity of intermediates is thus appreciated it will be understood that extreme caution is needed before the statement is made that in any specific 
case segregation does not occur. Even a sensible continuity between varieties is no proof that there is not segregation which with careful work could be demonstrated. To take for instance the case of piebald mice, it would be possible with industry to collect from the results of miscellaneous breeding specimens ranging from a self-colour to a black-eyed white. From such series any number of false conclusions could be drawn respecting the continuous variation of mice. By appealing to the "ruby" eyes of certain chocolates an uncritical observer might even argue that a series of intermediates connected the black and the pink eyes.

Analytical breeding immediately shows the fallacy of such deductions. For it would be found that the so-called intermediates consisted of numbers of genetically distinct types with distinct genetic properties depending on the factors which constituted them. Some would carry the colour-factors for the Dutch pattern, others those for more complete or less complete pigmentation, while others would owe their partial whiteness to the presence of the dominant factor which can suppress pigmentation in several stages of completeness.

In general it is to be concluded that when the interbreeding of recognizably distinct types produces only those types again without intermediates or with only few individuals that can be so regarded, the fact points strongly to the existence of gametic segregation; but on the other hand the appearance of even a complete series of intergrading forms is not to be accepted as proof that gametic segregation does not occur.

Misconception of the nature and significance of intermediates has deprived the work of the biometrical school of scientific value as a contribution to the study of heredity. This is well seen in the case of the colours of Shirley Poppies, one of the subjects with reference to which copious statistics have been amassed and published*. The colours are red, pink, and white, of many shades, and mixtures of these tints variously disposed and graded. The problem was to discover the laws of inheritance of the colours. The

\section{* Biometrika, II. I, 1902, and IV. 4, 1906.}


Mendelian method would be to institute breeding experiments with individual plants, self-fertilising and crossing these individuals together, and recording the offspring produced by each plant and by each combination. True that by sufficient search flowers can be found which when arranged in order provide a series of intergrades from the darkest red to the whites so nearly complete as to persuade an observer of moderate experience that there is full continuity. Nevertheless, from what we know of every such case to which Mendelian analysis has been extensively applied, there cannot be the smallest doubt that, as in Antirrhinum, the Mouse, and many other examples, the apparent continuity is misleading. It will, when analysis is undertaken, unquestionably. be shown that there are definite, pure homozygous types, containing factors which display the usual phenomena of dominance, and segregate in an orderly fashion; and that though, between these, intermediates may occur, yet they again will be found for the most part to be various consequences of heterozygosis. There may, not improbably, be a residuum of intermediates which cannot be thus represented, but it will be surprising if these are more than insignificant fractions of the whole, owing their peculiarities to various fluctuating influences. The ground will then be approximately mapped, and the laws of colour-inheritance approximately established for the Shirley Poppy.

The method of Professor Pearson and his assistants dispenses with all analysis. No attempt is made to discover the factors concerned, to distinguish the pure types, or the properties of dominance which they possess. No artificial crosses are undertaken*. The flowers are left uncovered, open to the insects which may introduce the pollen of any other flower which they happen to be carrying. The records show-probably with much accuracy-the colours of the offspring derived from mothers of recorded colours, and no more. In this attempt to study the laws of heredity of colour, the fathers of the progeny examined are in every case unknown.

* Self-fertilisation was subsequently tried, but, owing to self-sterility, gave no results. 
According to the biometrical system, if the characters of one parent and of the offspring are known, the material can, after mathematical treatment by means of the correlationtable, be used to deduce a law of inheritance. The theoretical conceptions upon which this method is based were formed and elaborated first in ignorance and subsequently in disregard of Mendelian facts; but improbable as their validity was in pre-Mendelian times, it must now be obvious that these methods can merely obscure the essential phenomena they were devised to discover.

There are moreover abundant examples of other fluctuations more obviously depending on environmental influences. In studying the inheritance of the cotyledon-colours in the seeds of peas such cases are met with. The cotyledons of many varieties are in all ripe seeds a full yellow, while those of many others are a full green. The contrast between the yellow and the green seeds in $F_{2}$ after segregation may be perfectly sharp and clear. Some varieties, especially the more modern sorts, have many seeds which are in various degrees partly yellow and partly green, and occasionally such particoloured seeds are the commonest type of the variety. In his criticism of Mendel's work the late Professor Weldon called attention to these particoloured seeds, contending that they showed failure of segregation. Before looking into the facts ${ }^{\circ}$ was disposed to admit the justice of this contention. After experimenting with such kinds however I found that the particoloured appearance was not caused by any admixture of the yellow character, but by exposure to sun and weather. All those seeds which tinge are by nature green and breed true to greenness. If in a pod several are found yellowed, this bleaching affects those sides of the seeds which face the same way and is obviously due to effects of light just like the reddening of the exposed sides of many apples.

The hereditary property characteristic of these tinging sorts is the power of bleaching readily if exposed, but the bleaching is checked and can generally be prevented if the pods are gathered as soon as they are ripe. It may be thought that the power of bleaching to which the ordinary yellow varieties owe their colour is the same faculty intensified. It may be so, but there are very marked distinctions ; 
for seeds of yellow varieties turn yellow before they harden, while the particoloured sorts bleach after they have become ripe.

In some yellow varieties greenish seeds occur. This phenomenon, the converse of the last, occurs in wet years with many kinds, some of course being specially liable.

The fact that such differences are fluctuational and not genetic appears at once when breeding is begun; for if in a green which bleaches (e.g American Wonder) the yellowest and the greenest are taken from the same plant and sown, there will be no corresponding difference between their respective produce. By selecting the greenest and yellowest from a large mass of commercial seeds of a variety, not harvested plant by plant, some correspondence may be obtained occasionally (e.g. Telephone)-but the occurrence merely means that the mass, though called one variety, had not really been selected down to a "pure line," but was in fact a mixture of sorts. In such a type as "Nonpareil," for instance, where the difference between yellows and greens is actually genetic, the yellows and the greens will give distinct results. "Nonpareil," in fact, is a form which consists of true yellows and true greens in about equal numbers, and it is simply a mixture of two varieties not sensibly different except in seed-colour. No doubt it arose from a plant saved in one of the later generations derived from a cross, which plant was homozygous in other respects but heterozygous in seed-colour. 


\section{CHAPTER XIV}

\section{MISCELLANEOUS EXCEPTIONAL AND UNCONFORMABLE PHENOMENA.}

Crosses breeding true without Segregation. Parthenogenetic or Apogamic Forms. Hieracium-Sexual FormsNumerical Aberrations-Irregularities of Dominance - Alternation of Generations-Maternal Characters in certain Seeds.

OF the various cases alleged as exceptional, or declared to be incompatible with Mendelian principles, few have any authenticity. Several rest on errors of observation or of interpretation and some have even been created by a mistranslation or a misprint. With this class of exception I have dealt on previous occasions. At a time when the Mendelian idea was novel it was perhaps natural that a doctrine so utterly at variance with received opinions should have been regarded as suspect, and that from the conventional standpoint the mere fact that a record was incompatible with Mendelism should have raised a presumption in favour of its genuineness. That stage of the debate is over, and we have now learnt that phenomena consistently departing from Mendelian rules are so rare that announcements of discoveries irreconcileable with the principle of factorial composition may be safely disregarded, unless they are made by observers experienced in this class of investigation, or supported by evidence of exceptional strength.

The progress of research has gone steadily to show that facts of heredity which at first seemed hopelessly complicated can be represented in terms of a strict Mendelian system. This simplification of the problem has far exceeded our earlier anticipations, and I have to regret that in dealing with several sets of phenomena I countenanced 
non-Mendelian interpretations which in almost every case it has been found possible to replace by simple Mendelian formulae. Where this reduction to a common plan has not been yet effected, the difficulty, we feel fairly confident, is genevally created rather by the disturbance of environmental causes or by the influence of undetermined factors than by any more profound aberration in physiology.

It is the object of the present chapter to discuss some of the more prominent of the phenomena which are really or apparently unconformable. As.will be seen, they are of a miscellaneous nature.

\section{Crosses breeding true without Segregation.}

\section{A. Parthenogenetic Cases.}

Of crosses breeding true there are two types quite distinct in nature and significance. In the first we now know that the absence of segregation is due to the fact that the reproduction is parthenogenetic, or apogamic, as it is more often called by botanists. The famous example of this kind of reproduction is provided by Hieracium (Hawkweeds). Mendel himself investigated the inheritance of Hieracium experimentally, and his paper is given in translation at the end of this book. This genus being one of the most strikingly polymorphic, he chose it after his discovery regarding the inheritance of peas, as the subject of further research. We may surmise that he expected to find in it illustrations of the new principles. The technical difficulties were extreme. The minuteness and the delicacy of the flowers made the operation of castration almost impossible to carry out on a large scale. It is recorded that in the course of this work he injured his eyesight; and after all precautions were taken he was mortified by what seemed to be frequent failures. Seeds that were supposed to be hybrid gave plants of pure maternal type, which he could, in those days, only attribute to accidental pollination from anthers of the mother-plant, caused by imperfect emasculation. In a few cases however he did succeed in raising genuine and obvious hybrids. These hybrids were partially sterile, but the seeds which they did give reproduced the hybrid form again. 
In the earlier discussions which followed the rediscovery of these papers we all were inclined to follow Mendel in supposing that Hieracium illustrated a distinct kind of sexual inheritance in which segregation was absent, and it seemed natural to suspect that the association of this phenomenon with partial sterility was not accidental. As we now know, there is a distinction between the inheritance exemplified by these true breeding hybrids, but it is not a speciality of Hieracium, or in any sense to be regarded as an exception to the general rule that in sexual heredity segregation of characters occurs. The meaning of the facts was first discovered by Ostenfeld (2I9) following up an experiment of Raunkiaer (228). These two observers found that both Taraxacum and many Hieracia have the power of setting seeds parthenogenetically without any fertilisation. Such plants may set seed profusely when all the anthers and stigmas are cut off in an unopened bud. The cytology has been investigated by Rosenberg (233), and he has shown that in the ovules which are capable of parthenogenesis no reduction-process occurs. In the strict sense therefore this method of reproduction is not sexual, but asexual, being comparable with the reproduction from buds or cuttings in which, as is well known, the parent type almost always reappears without modification.

This fact explains not only why Mendel's hybrid Hieracia bred true, but also why he so often failed to produce a hybrid when all precautions were taken to avoid self-fertilisation. The plants which were supposed to be due to accidental self-fertilisation were in reality parthenogenetic. From Ostenfeld's experiments it was shown-that some only of the ovules are capable of fertilisation; and it is practically certain that the ovules with unreduced nuclei, though they may give rise to plants by parthenogenesis or apogamy, are incapable of being fertilised. The evidence that the unreduced egg-cells merely reproduce the maternal type is of course a strong support to the view that it is in the reduction-division that the segregation of factors is effected.

It is by no means alone in the Compositae that parthenogenesis has been observed. Fresh cases are continually being discovered among flowering plants, and in any 
discussion of unexpected genetic phenomena this possibility must be taken into account*.

There is a further difficulty to be considered in this connection. The fact that no seed is set when fertilisation is excluded is in itself no proof that the inheritance is not of a parthenogenetic type. A small but very definite and well-ascertained group of cases occurs among Orchids in which, though no seed is set unless the flowers are pollinated, yet the offspring exhibit no trace of the paternal characters. The facts are well known to orchid-growers, but they were first made known to students of genetics by Hurst ( 152 , p. 104). The most notorious example is that of Zygopetalum Mackayi, which when fertilised with pollen of various other orchids has given nothing but Zygopetalum Mackayi. Four species of Odontoglossum, a Lycasta, and an Oncidium, used as father all gave this curious result. Hurst quotes other records showing that several other orchids may behave in the same way as $Z$. Mackayi, giving purely maternal plants when fertilised with pollen of other types. In some of these cases it has also been shown that self-fertilisation of the supposed cross-bred again gives nothing but the same type.

Pending cytological investigation it is not possible to form a satisfactory rationale of these cases, but we cannot avoid the conclusion that fertilisation has acted as a stimulus to development without any admixture of paternal character. The result is, as Hurst has pointed out, exactly as if parthenogenesis had in reality occurred, although pollination had taken place and is indeed necessary to effect production of seed. I have proposed to distinguish this phenomenon as Monolepsis, in which characters are taken from one parent only, in contrast to Amphilepsis, in which they are brought in from both sides.

In the earlier stages of these discussions we were disposed to attach much importance to an observation of Millardet (I98), to the effect that in certain crosses among strawberries the supposed hybrid was of purely paternal type, showing no maternal features, and breeding true. The term "false hybrids" was suggested by him as descriptive of such plants. No exactly similar example has been

* For important summary of new evidence see Rosenberg, Svensk Bot. Tidsk., 1909, III. p. 159 . 
found in the course of contemporary experiments. It is to be hoped that the original experiment may be repeated, for Millardet's note is very brief, and we have no means of judging whether the various possibilities of error were excluded (e.g. heterozygosis of the mother-plant).

Whatever the future may reveal as to the significance of these ambiguous occurrences it is certain that.in all cases where irregular results are observed we may have to reckon with two possibilities: (I) actual parthenogenesis, or the development of unfertilised ova without fertilisation; (2) a phenomenon tantamount-as regards heredity - to parthenogenesis, occurring after fertilisation*. In both events the offspring are purely maternal. The latter exemplifies the conception of Strasburger and Boveri, that fertilisation may consist of two distinct processes, the stimulus to development, and the union of characters in the zygote.

B. Sexual Types.

The literature of hybridisation and heredity abounds with examples of hybrids or cross-breds which are said to have bred true without splitting up, or as we should now say, without segregation. Some of these, so far as they refer to plants at least, are open to the criticism made in the last section, that either actual parthenogenesis or monolepsis may be occurring. But an even simpler and more probable account is hardly ever excluded, for we have rarely if ever any means of knowing that the plants studied were in reality first crosses and not members of derivative generations. Moreover even in cases such as the famous series of Salix hybrids made by Wichura (304), where this objection does not apply, we know almost nothing as to the numbers of individuals raised. These remarks probably apply to all the illustrations given by Focke (123) so far as I have been able to ascertain. In Mutationstheorie, II. p. 66,

* On a former occasion (18, p. 156) I suggested that in mosaic forms the presence of the recessive character in patches might be thought of as due to failure of fertilisation in those areas. More accurate knowledge of the facts has practically disposed of this suggestion. It was chiefly based on evidence relating to the mosaic seeds of Peas, patched with green and yellow in the cotyledons, but we may now feel fairly sure that the abnormalities seen in their behaviour are due to disturbance caused by external influences. 
de Vries mentions some cases which he accepts as satisfactory, the most definite being that of Oenothera muricata $\times$ biennis raised by himself. The hybrids were partially sterile in a high degree, but the subsequent generations raised from them showed no definite departure from the $F_{2}$ type. The evidence, as it stands, must be taken as constituting a definite exception. Nevertheless in view of the great sterility exhibited by the hybrids, and the fact that all that we know of the Oenothera crosses points to the existence of very unusual features in their genetic physiology, the significance of this curious observation is still somewhat problematical*

Another case given by de Vries is that of Anemone silvestris $\times$ magellanica on the authority of Janczewskit. As this case well illustrates the anomalous and uncertain behaviour of partially sterile hybrids, the facts are worth giving in some detail. In the first account the hybrids are said to have been either totally sterile, or fertile in a very slight degree. The pollen is said to have been all bad, and in flowers containing about Ioo pistils, one or rarely two good akenes formed. Janczewski thinks that these were fertilised from one of the parents. But the hybrid sometimes gave rise by an adventitious bud, from the root, to a plant having the female parts perfectly fertile, producing akenes of intermediate shape. The pollen of these flowers was not examined, and it was supposed that fertilisation was effected by pollen of $A$. sylvestris growing near by

In the later account it is recalled that the hybrids sometimes produced good akenes among a multitude of abortive ones, and it is said that the pollen, though very bad, contained occasional good grains which could effect fertilisation without foreign pollen. Some stems of this hybrid-not the whole plant-gave rise suddenly to flowers perfectly fertile, with all the akenes good. These flowers had mixed pollen-grains, of which about three-fourths were good and effected selffertilisation. The second and also the third generations raised from the hybrid, whether from the sterile or from the fertile flowers, reproduced precisely the characters of the original hybrid, exhibiting the complete fertility of the female

* See Appendix to Part I.

$\dagger$ Bull. Ac. Cracovie, 1889, June, No. 6, p. xxiv, and ibid. 1892, p. 228. 
organs of the fertile stems, and the mixed pollen of those stems. Janczewski concludes that in this way a new specific type could arise which, but for its mixed pollen-grains, would be indistinguishable from a natural and original species.

This extreme irregularity and sportiveness in the degree of fertility manifested is a frequent concomitant of partial sterility in hybrids. The fact that the sterility once lost does not return, obviously suggests that it is due to the presence of some dominant factor or factors, and that they, or the compound they make, can be eliminated by budvariation. Other reflections will occur to the reader which we cannot now consider, but I feel it difficult to accept such an example without further comment as a proof that in ordinary sexual reproduction segregation may fail as a normal event.

It is most desirable that search should be made for genuine cases of failure of segregation amongst animals. The example of the human mulatto is as yet one of the best which ordinary experience suggests*. In experimental breeding perhaps the single clear instance is provided by Castle's experiment (48) on the lengths of ears in rabbits. $\mathrm{He}$ crossed the long-eared lop rabbit with ordinary shorteared individuals. $F_{1}$ had ears of intermediate length, and the subsequent generations retained this character very definitely. The later details have not yet been published, but Professor Castle very kindly showed me many of his records which plainly established the fact that no return to the parental types took place.

In some experiments made with the butterfly Pararge egeria I obtained a comparable result. In Spain and the plains of Southern France egeria has a bright fulvous brown colour (intersected with darker brown), while in England our representative form egeriades has the bright brown replaced by primrose yellow. In the Loire valley and in Brittany a race almost exactly intermediate between egeria and egeriades is found. By crossing the two extremes the intermediate type was produced with great exactitude. $F_{2}$ was not raised in any adequate numbers, but those that 
were bred showed only dubious traces of segregation. On the other hand $F_{1}$ was crossed with both parent types and some large families raised. The general aspect of these families might be described by the statement that they came on the whole intermediate between $F_{1}$ and the pure parents respectively. Pending a repetition of the experiment, and especially before $F_{2}$ has been properly investigated, the negative cannot be asserted with complete confidence, but I am disposed to regard the case as one in which segregation is really absent.

Experiments made by Bacot and Prout with Acidalia virgularia (Geometrid moth) and a light variety from Hyères called canteneneiraria showed that $F_{1}$ was always intermediate. In subsequent generations no segregation could be detected. A good deal of fluctuation occurred, but the evidence went to show that either extreme could produce the other. The facts were described by $\mathrm{Mr}$ Bacot in a lecture at the London Hospital in 1908 , and I am indebted to him for further information. A full account is shortly to appear.

\section{Departures from Numerical Expectation.}

The material for a proper investigation of this important question is still quite insufficient. It is the experience of all who have made breeding experiments that from time to time individual families show great aberration from the numbers which are predicable by the simple rules. "Not till a large mass of otherwise homogeneous statistics are available will it be possible to ascertain whether any of these aberrations are really indicative of the effects of special causes of disturbance or are to be accepted as random departures from normality. From the point of view of the statistician it may seem a comparatively simple matter to procure such material, but in practice there are many difficulties to be overcome. The impression formed on my own mind is that the output of allelomorphic gametes, where equality is expected, does in certain types show individual departures which are not purely fortuitous, but in any collection of statistics large enough to give reliable conclusions these individual aberrations would be completely 
masked; for though they may be really indicative of physiological disturbance, these disturbances must be rather of the nature of sporadic events than the consequence of predominating large causes affecting long series of records.

Occasionally however the effects of such definite causes can be traced in our numbers. One excellent example is provided by the work of E. Baur (24), who investigated the inheritance of the colour of the leaves in Antirrhinum. He found that the green type breeds true, but that the golden-leaved or "aurea" form gives, on self-fertilisation, a mixture of goldens and greens. The resulting numbers were 573 golden and 286 green, showing very clearly the proportion $2: \mathrm{I}$, the golden being a majority. The question why are the goldens not 3 to I of the greens is, as he points out, readily answered. The missing group of plants are those which would have been pure to the yellow character. Such plants would contain no chlorophyll and consequently would perish. A study of the germinating seeds subsequently proved that this was the true account, for such an examination gave 77 green, 160 golden, 5I almost or quite without green. The last group all died. Such an observation throws a clear light on the meaning of some at least of those "ever-sporting" types to which de Vries drew attention*. As stated in a note on p. 163 Miss Durham has now proved the condition of yellow mice to be exactly comparable with that of these varietates aureae. It would be a matter of very great interest to determine what is the exact cause of the non-viability of the pure yellows, and what the physiological action of the "yellow factor" may be.

In the light of this experiment, and indeed from a priori consideration, it is clear that the non-viability of zygotes or even of gametes bearing special combinations of factors may play a large part in genetic phenomena. Nevertheless with the exception of some cases in Primula and the peculiar phenomenon recorded in regard to maize (p. 16I) I know no large series of numbers which show a consistent departure from expectation. There are however suggestions 
that in some types of animals and plants the amplitude of the fluctuations about normality is greater than in others. This is of course a point well adapted to statistical study which sooner or later must be undertaken. Similarly there are indications that in cases where the point can be tested, the seeds of individual pods may give strangely aberrant numbers though the plants as a whole may give regular results. Miss Saunders has often been struck with this peculiarity in studying the genetics of Stocks, but as yet the figures have not been examined by statistical methods. Knowing what we do as to the capacity of individual flowers to sport, the suggestion obviously arises that such individuality may be manifested by the collective offspring of one ovary even though the flower in which it was formed may show no peculiarity.

The very wide departures from expectation shown in many pedigrees of human diseases and defects are certainly in part attributable to the imperfections of the records, but I cannot doubt that the discrepancies are in part due to genuine physiological causes. In regard to some of these it is I think still open to question whether the transmission is a process comparable with that which we ordinarily designate as Heredity. Some element is obviously handed on from individual to individual, but it seems to me possible that this element or poison is distributed irregularly among the germ-cells, spreading among them by a process which is mechanical, like the spread of an oil-stain in a heap of paper, or of a fungus in a heap of seeds. In the present state of pathological knowledge it is premature to make any suggestion as to the possible nature of such poisons. I am told by competent authorities that in the cases, for example, of the various polymorphic hereditary paralyses it is very improbable that pathogenic organisms can be the exciting cause; nevertheless, from a study of the inheritance in an ample series of families, I am inclined to suppose that the element transmitted is something apart from the normal organism, and that it is handed on by a process independent of the gametic cell-divisions. In such cases I do not anticipate that any "law" of inheritance can be discovered, for if my view is correct, the process is not heredity in the naturalist's sense at all. 


\section{Irregularities of Dominance.}

It must be anticipated that irregularity or fluctuation in dominance will prove a frequent source of exceptions. Neither among animals nor plants is material often to be found so homogeneous that the heterozygotes formed from various individual pairs are quite identical, and the dominant factors thus produce their effects in varying degrees. Reference has already been made (p. 236) to the fact that dominance may sometimes be so complete that the heterozygote may pass for the pure homozygous type, while in other individuals the distinction can be recognized. Such cases present no real difficulty in analysis, for the two classes can be taken as one in the counts of $F_{2}$. Serious difficulty is caused however in a few examples by the fact that a dominant factor may be present without producing any effect by which its presence can be perceived. Such dominant individuals are indistinguishable from recessives, and analysis thus becomes difficult or impossible. Conspicuous examples of this ambiguous phenomenon have occurred in two of the subjects investigated on a considerable scale. The first is the heredity of the extra toe in fowls. In our work we have found the extra toe usually to be a dominant. Such a 5-toed breed as Dorking or Houdan crossed with a normal 4 -toed breed commonly gives $F_{1}$ birds with extra toes developed to an extent somewhat less than that found in the normal 5 -toed type, and $F_{2}$ from such birds is of the usual composition, averaging 3 birds with extra toes more or less developed, to I normally 4-toed. But it is by no means rare to meet with birds of the 4-toed type which when crossed with an extra-toed breed will throw many $F_{1}$ with 4 -toed feet, or with traces only of the extra digit. The two feet of the same bird may also differ, presenting every combination. Such birds have in several instances been proved by their progeny to be ordinary heterozygotes. At first one might be disposed to attribute the peculiarity to interference caused by some epistatic factor repressing the development of the dominant characteristic; but this account cannot very readily be reconciled with the actual numbers, for there is no consistent diminution in the number of the dominant offspring of such birds. The 
nature of these unconformable families must be regarded at present as altogether obscure. The records of human polydactylism indicate that very similar disturbances of the usual course of descent operate in regard to man.

Another example almost exactly similar has been encountered both by Correns and by Lock in the heredity of the blue or black colour of the aleurone layer of the seeds of maize. The blue is generally a dominant appearing in various degree, but some white $F_{1}$ seeds proved able to carry on the blue character. The problem raised by this defect of dominance is discussed at length by Lock (I 74), but no altogether satisfactory elucidation has been found.

It may be pointed out that when, as in these examples, the abnormal result is clearly perceptible in $F_{1}$, no question arises as to the occurrence of an imperfect segregation. The peculiarity is evidently zygotic, and is caused either by some feature of zygotic organisation, or by the influence of external circumstances. This conclusion, so plainly deducible from results obtained in experimental crosses between pure races, may be appealed to when, as in the descent of some human characteristics, we witness as an exception the handing down of a normally dominant factor through an unaffected member of the family. In view of such occurrences the reality of the segregation may be doubted. No experiment with pure types is possible in dealing with those phenomena but the analogy of the genetics of extra toe in fowls supports the view that the peculiarity of these cases is zygotic and not due to failure of segregation.

\section{Alternation of Generations : an outstanding difficulty.}

The question may be asked whether, on a general survey of the facts of Natural History, any large classes of phenomena are encountered which are altogether inconsistent with the Mendelian system to such a degree as to suggest that they are quite incompatible with any scheme of factorial analysis. Various exceptional cases have been enumerated and considered. It is not in dispute that, for example, in the phenomena of hybridisation between the races of mankind, the facts (fairly well authenticated) must 
be taken as pointing at all events to the existence of special features in them. In most of these, however, there is nothing that can be construed as indicative of some wholly different system of genetic physiology. There is nevertheless one phenomenon, alternation of generations, which stands out as at present incapable of factorial representation.' This is best exemplified by the case of seasonal dimorphism so frequently met with in insects, especially Lepidoptera. For example many species exist in two types of which one emerges after the winter as a spring form. This breeds and lays its eggs. The larvae feed up rapidly and emerge in summer as the second or summer form. In their turn individuals of the summer type give rise to the larvae which are destined to become the spring type again. A similar sequence of distinct types is a common feature of many tropical species in which the wet and dry season forms correspond to the winter and summer forms of temperate countries. The cycle may be complicated in a variety of ways, by the intercalation of additional generations and otherwise. Of these more complex cycles the Aphidae and Coccidae afford many striking illustrations. In none of these examples can we conceive that the distinctions between the recurring types are due to addition or removal of factors. The only other suggestion which can be made is that these distinctions are ultimately referable to the effects of external conditions. As yet very little if any evidence can be adduced in support of this suggestion. The experiments of Standfuss, Fischer, Merrifield, and others have shown that the form which the individual assumes can within rather narrow limits be determined by altering the temperature to which it is exposed, but in order to obtain a satisfactory elucidation of the phenomena I am here considering this evidence is insufficient. In the known examples the experimental influences were brought to bear on the individuals themselves. It is not impossible that something might be accomplished by changing the conditions to which the parents are exposed. It is, for example, a familiar experience of lepidopterists that when a one-brooded type is bred in captivity a second brood is very commonly produced in the autumn, and it is natural to refer this variation to some influence of the abnormal 
conditions. But we here meet the old difficulty that the distinction between the living types is often sharp, whereas that between the environments is one of degree. We know also that from the same parents a brood of larvae may be raised some of which will feed up fast and emerge as the autumn form while others will feed up slowly and emerge with all the characteristics of the spring form after the winter, and we can form no idea as to the circumstance which determines the distinction. Though I can give no hint as to the rationale of these occurrences which in the cases of the sex of Aphidae \&c. have an extreme importance to the progress of genetics, I call attention to them as constituting what must be regarded as the group of phenomena least in accord with the conception that the characteristics of living things are an expression of their factorial composition*.

\section{Maternal Characters in Embryos.}

\section{A. Seeds of Wheat.}

In certain plants a curious and at present quite unintelligible phenomenon has been met with. Of this four examples are known. The simplest is that seen when a wheat having long glumes and seeds is crossed with a variety having short seeds in short glumes. This case has been studied by Biffen. The $F_{1}$ seed, resulting from the cross-fertilisation, is unchanged, and resembles the normal seeds of the mother-plant. When this $F_{1}$ seed is sown the plant in due course flowers. Its glumes are of intermediate length (Fig. 35) and the seeds they contain $\left(F_{2}\right)$ are all alike of intermediate length (Fig. 36). Apparently therefore segregation has not taken place. But when these $F_{2}$ seeds are sown, the plants which they become are respectively longs, intermediates (like $F_{1}$ ), and shorts, as regards their glumes and seeds. Hence it is clear that segregation had occurred among the gametes produced by $F_{1}$, but owing to some control exercised by the motherplant, all the seeds have a similar length and appearance.

* It is not impossible that the difficulties which have been met with in the attempt to pursue Mendelian analysis in the double-brooded Lepidoptera may be connected with the phenomena spoken of above. 
This case at first sight does not seem altogether paradoxical, for it may appear natural that the size of the seeds should - at all events as regards the upper limit-be governed by the size of the maternal envelopes. The next group of cases however, though showing that the peculiarity is in some way caused by the maternal tissues, suggests that the influence may be of a more recondite nature.

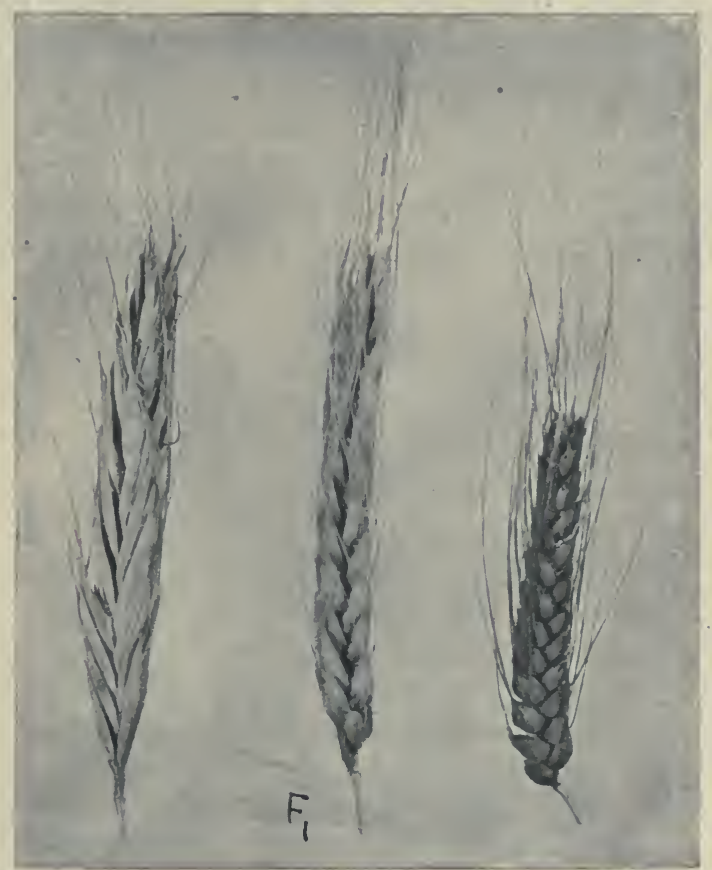

Fig. 35. Polish wheat, with long glumes, crossed with a short-glumed Rivet wheat. $F_{1}$ has glumes intermediate in length. (Biffen's specimens.)

\section{B. The Indent Peas.}

This example is that of the Peas (Pisum) known as "Indent." The crosses of this type of pea give rise to an intricate series of results. For our knowledge of these facts we are indebted in the first place to Tschermak* : and

* Tschermak's account is sometimes difficult to follow, because his terminology does not always show whether he is referring to indent or to wrinkled peas. 
several experiments have also been carried out both by Lock, and by myself assisted by Miss Killby. In the following account only the essential features are related.

As the type of an "indent" pea I take the French Purple Sugar pea* (Grane Riesen of German seedsmen). Its flowers are purple. The seeds have deep, irregular indentations, but with experience of peas it is fairly easy to distinguish these "indents" from all wrinkled types. As always on plants with coloured flowers, the seed-coats are coloured, being here a grey brown (with some purple spots). In connection with the colour of the seed-coats we meet the first complication. So far as is known, the thoroughly indent type of seed never appears except in coloured coats, but round or wrinkled seeds may exist in coats of all sorts,

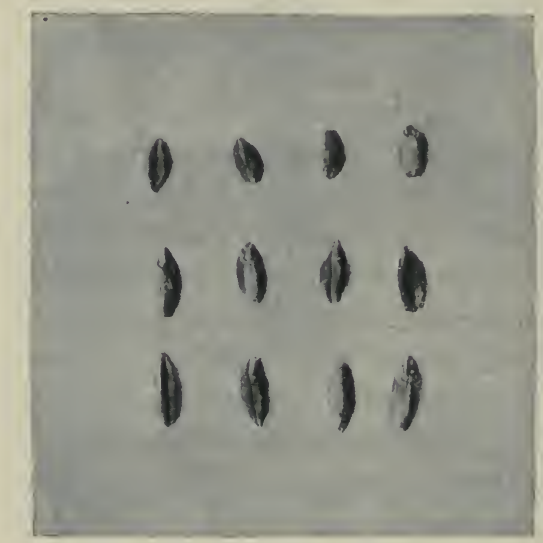

Fig. 36. Upper row: Seeds of Rivet. Lower row : Seeds of Polish wheat. Middle row: $F_{2}$ seeds borne by $F_{1}$ plant, all similar and of intermediate length, though segregation has occurred. (Biffen's specimens.)

whether coloured or uncoloured. As regards the nature of the reserve-materials in these peas Gregory found that the starch grains of indents are like those of round peas (see Fig. 9), being large and simple.

For simplicity's sake I take first the results of crossing

* This pea has soft "sans parchemin" pods, but the characters of the pods are not involved in the problem discussed in the text. 
indent with rornkled (e.g. Satisfaction, Laxton's Alpha), for the consequences are there comparatively regular. Indent fertilised by wrinkled gives the $F_{1}$ seed indent and unchanged. Wrinkled fertilised by indent gives the $F_{1}$ seed round. This is evidently in consequence of the fact that the wrinkled variety used was a white-flowered plant, with of course uncoloured seed-coats, in which the indentation cannot develop. Such an $F_{1}$ seed becomes an $F_{1}$ plant with coloured flowers and coats, and the $F_{2}$ seeds it bears show normal segregation, being indents and wrinkleds* in the usual proportion $3: \mathrm{I}$. The wrinkled seeds if sown give normal results, viz. 3 wrinkled with coloured flowers : I wrinkled with white flowers. The indents if sown also give 3 coloured-flowered : I white-flowered, but all seeds of "round" nature on the coloured plants are of course indents (whether all indents, or 3 indents : I wrinkled as before), while all the corresponding seeds on the white-flowered plants are true rounds (being either all rounds, or 3 rounds : I wrinkled).

Thus far all is intelligible, but when we proceed to the next combination, indent crossed with round, the real difficulties are reached. Indent fertilised by a round gives of course the $F_{1}$ seed indent; and reciprocally, the round fertilised by indent gives the $F_{1}$ seed round. When these seeds are sown, the $F_{1}$ plants grow up (with coloured flowers), but the condition of their $F_{2}$ seeds differs according to the round variety used as original parent. I take first the result which has been most commonly observed. Tschermak observed it in many crosses made with "Victoria," a whiteflowered variety, of which more will be said later. Lock saw the same thing with a round-seeded native Ceylon pea having coloured flowers. I have similar results from Fillbasket, Express and Blue Peter, all white-flowered types. In all these cases the $F_{2}$ seeds, borne by $F_{1}$ plants, are all alike indent, none being round.

On inspection of such seeds it seems impossible to suppose that segregation has occurred. On sowing it is nevertheless found that the resulting $F_{2}$ plants are respectively

* In such a case the sorting of living seeds can only be approximate, for to the eye the distinction is not quite sharp. They can be sorted immediately by microscopical examination of the starch. 
coloured and uncoloured in the proportion $3: \mathrm{I}$, the coloured all have indent seeds and the white-flowered all have round seeds. Segregation is therefore normal, and the fact that all the $F_{2}$ seeds are indent is in some way brought about by the nature of the maternal envelopes in which the seeds develop. How this influence is exerted we cannot suggest, but perhaps there is some quality in these seed-coats which causes the loss of water on ripening to take place irregularly and so induces an irregular shrinking of the cotyledons. Such an account is difficult to apply, for the seed-coats seem uniform and homogeneous, and as the next case proves, the influence of the seed-coat, whatever it be, operates in an extraordinarily capricious and specific manner.

Until recently the account given above was supposed to apply to all crosses of round with indent. Going over seeds which were harvested in 1904 and I905 without examination at the time I find that one case is altogether different from the rest. In this the round parent was Nain de Bretagne, a small, very round, white-flowered variety: Indent fertilised by this variety gave $F_{1}$ seeds indent. These when sown became coloured $F_{1}$ plants, but their $F_{2}$ seeds, instead of being all indent as in the examples described, are quite definitely indents and rounds, in the usual 3: I ratio*. Moreover among the few $F_{2}$ plants of which the seeds were harvested, was one which had only round seeds (in coloured coats) and it is evident that this plant came from a round $F_{2}$ seed which was sown without note being taken of its character.

We are thus presented with the exceedingly definite and specific fact that one round-seeded variety, by virtue of its intrinsic nature, behaves quite differently from the others that have been tried. Presumably its reserve-materials (and by inference the ferments which lead to their formation) have some distinctive property such that though ripening in a seed-coat which would make the other round peas shrink as indents, they are still able to retain their own characteristics. Such a fact may well be remembered in any discussion of the nature of specificity.

* Several were intermediate in appearance, as in such cases must be expected. Three plants, for instance, had 339 indent, I 9 round, and 39 uncertain. 
One further phenomenon of importance has been discovered respecting indent peas. It was said above that the true indents are in coloured coats, but it is obvious that a coloured coat, as such, is not sufficient to make peas indent, for there are many round varieties with coloured coats. It is clear from the experiments of Tschermak (273) and Lock ( $173, \mathrm{I} 75)$, as they point out, and from results of my own, that the properties of the indenting coats depend on two factors, viz. (I) the indenting factor proper, (2) a requisite pigmentation. For crossing coloured, round varieties with "Victoria," a white variety commonly round, $F_{1}$ bore $F_{2}$ seeds indent. Lock had a similar result from a round, coloured, native Ceylon pea crossed with Satisfaction (white, wrinkled), but as wrinkledness was introduced, $F_{3}$ seeds were indent and wrinkled. I also by crossing Maple (round, purple flowers) with Victoria Marrow, found all $F_{2}$ seeds indent.

In these cases it is evident that the indenting factor was introduced in the white-flowered plant, and this, meeting its complementary pigmentation-factor from the coloured side, was able to indent the $F_{2}$ seeds. It is worth noting that "Victoria," which I take to be our "Victoria Marrow," though generally a round type, produces sporadically a good many seeds with some degree of indentation or pitting*. These (and William I) come nearer to the true indent than those of any white-flowered variety known to me. Probably this is due to an imperfect and occasional manifestation of the powers of the indenting factor, which cannot produce its full effect as the pigment-element is missing; but when that element is brought in by the cross the compound factor is complete and the seeds are indented.

An experiment of Tschermak's (273, p. 30, Case 9) contributes one more important and instructive fact, which gives a clear indication as to the identity of this pigmentation-factor. He crossed two round-seeded varieties both having coloured flowers, and the $F_{2}$ seeds were indented. Now one of these was an ordinary purple type, but the other was of the pink (salmon-pink) colour well known for

* I found by sowing that the most indented and the roundest give identical results in the next generation. The distinction is fluctuational, and is unaffected by selection. 
instance in the "Mummy-pea" of our gardens. The difference between these two is that the purple has the blueing factor which in discussing Sweet Peas \&c. we have called $B$, and there can thus scarcely be a doubt that this $B$ factor is the complement of the indenting factor which here came in from the pink side.

Analogous observations have been made in regard to Maize, especially by Lock. According as the seeds are opaque or semi-transparent, the varieties are distinguished as "Dent" or "Flint." These distinctions are also maternal characters, and though segregation is normal, its effects cannot be seen by examining the cob of $F_{2}$ seeds. Conditions however, in particular the degree of ripeness, cause a complication in this case, for the seeds, at the top and bottom especially, may remain comparatively flinty after the rest have assumed the dent character.

In Wheat the relation between hard and soft endosperms is probably similar, but Professor Biffen tells me that in that case also complications occur.

This group of cases introduces us to several points of interest. We have first the remarkable fact that the motherplant can impress varietal characters on her offspring by influences which are not heredity in the ordinary sense. Seeds are in botany what larvae are in zoology, and no example is yet known in which the maternal impress extends beyond the seed-stage. But without any serious stretch of imagination we may suppose that a maternal impress may be such as to produce an effect lasting at least for the lifetime of the immediate offspring; and it would not be altogether surprising if such results were actually detected in the cases enumerated; for the difference in food-materials between those provided by a dent seed and a flint, a glutenous and a starchy, may, for aught we know, influence the later life of the plant, just as the nature of the milk supplied to the human infant is believed to do. Such influences may probably enough be limited and perhaps trifling in comparison with those that are in the strict sense genetic, but we do not yet know that they are negligible. 
The next feature of interest is the specific behaviour of the round pea Nain de Bretagne, which distinguishes it from the other round peas, creating a problem in the chemistry of the reserve-materials well worth investigating.

Lastly we have one more illustration of the special properties of the " $B$ factor." This factor, it will be remembered, was concerned both in producing some of the phenomena of coupling, and spurious allelomorphism in the Sweet Pea, and we may anticipate that its presence will be shown to have profound effects on the constitution of plants*.

* The seed-shapes of Sweet Peas have not been studied. There is a great diversity in size. Some also are quite spherical, others being somewhat elongated. The self-coloured violet-flowered kinds alone (Countess Radnor, \&c.) have shrivelled seeds. In $F_{2}$ seeds from these crossed with common round sorts no mixture of shapes occurs, and the shape is evidently a plant-character. 


\section{CHAPTER XV}

BIOLOGICAL CONCEPTIONS IN THE LIGHT OF MENDELIAN DISCOVERIES.

Nature of Units-Nature of Segregation-Moment of Segregation-Differentiation of Parts compared with Segregation-Reversion and Variation. "Bush" and "Cupid" Sweet Peas-"Mendelian Segregation and Species-Discontinuity in Variation-Mendelism and Natural Selection.

THE purpose of the preceding chapters has been to show the method of Mendelian analysis in application to a variety of problems, and to describe the concrete discoveries to which that method has already led. $\bar{T}$ o discuss the bearing of the new facts on biological science is now a considerable undertaking and all that will be attempted within the limits of this volume is a slight sketch of the possibilities which these facts suggest.

\section{Nature of the Units.}

With the recognition of unit-characters our general conceptions of the structure and properties of living things inevitably undergo a change. We begin to perceive outlines where previously all was vague, nor can we doubt that those outlines will very soon become clearer. What the physical nature of the units may be we cannot yet tell, but the consequences of their presence is in so many instances comparable with the effects produced by ferments, that with some confidence we suspect that the operations of some units are in an essential way carried out by the formation of definite substances acting as ferments. 
The conception of dominant characters as each due to the presence of something which is absent from the corresponding recessive may prove of use as assisting towards the identification of these problematical bodies. To most of the cases of allelomorphism between characters yet detected, this method of representation may be very readily applied. The round seed of peas, or of maize, is one which contains something possessing the power of turning most of the reserve-materials into starch. If the dominant factor endowed with this power is absent, much of the sugar remains sugar, and the seed wrinkles on ripening. The actual physiological processes involved are doubtless more complex than this, but there is no mistaking the essential nature of the distinction between the round and the wrinkled seed. So also it is easy to understand that an albino is an organism from which a ferment responsible for the production of colour has been omitted. No great strain is put on this hypothesis of dominance even by the suggestion that the production of hairs on the leaves of a Stock, or of the disposition to go broody in a hen, may be directly caused by specific substances, for it would not be difficult to adduce pathological parallels for the production of bodily and mental changes such as these in cases where the change is plainly the work of a specific poison. Again it is easy to imagine that the presence or absence of a ferment can confer a greater power of resistance to the attack of a fungus. I suppose also that the dominant whites met with in some animals and plants may reasonably be represented as organisms possessing a substance which has the power of suppressing the development of pigment, whether by preventing its excretion or by destroying it when formed.

On the other hand it must be admitted that in application to those examples in which the dominant factor operates by inducing or suppressing the division of a certain organ, the analogy with ferment-action is more difficult to maintain. The double comb of fowls is nevertheless a single comb made bifid by the addition of a dominant factor. To suppose also that the suppression of a phalanx in the digits of a brachydactylous man, or the development of a rabbit's fur in the normal and not in the Angora manner is thus decided by a specific substance, is to contemplate a 
serious extension of the powers of specific substances as usually imagined.

But disregarding for the present these more obscure cases in which meristic phenomena play the chief part, we * may draw from Mendelian observations the conclusion that in at least a large group of cases the heredity of characters consists in the transmission of the power to produce something with properties resembling those of ferments. It is scarcely necessary to emphasise the fact that the ferment itself must not be declared to be the factor or thing transmitted, but rather, the power to produce that ferment, or ferment-like body.

\section{Nature of Segregation.}

Next we have to recognize that this antecedent power must be of such a nature that in the cell-divisions of gametogenesis it can be treated as a unit, being included in one daughter-cell and excluded from. the other at some definite cell-division. As we have no knowledge as to the actual nature of the factor-and only a conjecture as to whether it is a material substance, or a phenomenon of arrangement-. we are not in - a position to hazard so much as a guess respecting the physical process of segregation. We may be fairly conifdent that segregation is not a process - of chemical separation. I ts features point rather to mechanical analogies. If some mental picture be demanded, I would for the purpose of illustration suggest a comparison with the separation of a fairly heavy precipitate from a filtrate by decanting. The precipitate is to represent the factor, and the filtrate the recessive product of division, lacking the factor. The analogy is probably quite erroneous, and small purpose would be served by developing it further.

Whatever that process may be, it must be one which is applicable to an extraordinary variety of factors. No conclusion to which genetic research has led is so surprising as the fact that the same system of transmission should be followed by characters which, by whatever test they are judged, must be supposed to be most diverse in physiological causation. Even if it were fairly easy to conceive of all the dominant characters of animals and plants as due 
to the operation of ferments their diversity must be anyhow very great, and it seems strange that all these multifarious potentialities should exhibit gametic allelomorphism. Let us take an illustration. Colour, as we can prove in regard to several plants and as we suspect in the case of animals, is due to the meeting of two complementary factors. One is presumably a ferment. Recent research strongly suggests that it may be a tyrosinase*. The other is referred to sometimes as "chromogen." But whatever they are, the two bodies-and surely the factors which produce themmust be of utterly different nature, and yet genetically the two potentialities are treated similarly. Each is allelomorphic to the absence of such a power.

Similarly in regard to the anthocyanin colours of Antirrhinum Miss Wheldale has given reasons for the belief that they are formed by the meeting of a tanninlike body with a ferment, perhaps an oxydase. Both these factors, whatever be their nature, are allelomorphic to their absences.

How much more astounding is it, that when we pass to qualities such as length of stalk, and shape of flower, the shape of a cock's comb, or the development of interlocking barbules on the webs of its featherst, we still find the same rules in undeviating operation!

\section{Moment of Segregation.}

At what particular cell-division of the many by which the germinal cells are brought to their maturity does this process of segregation happen? The question is of extreme interest, but no positive answer can yet be made. Naturally the common expectation we all share is that the reduction-division is the critical moment. At that division the number of chromosome-elements, of which the nucleus is formed, can be seen to be halved. Up to this point the nucleus of each daughter-cell seems to be simply a repetition of the nuclei of the body and may be supposed to contain all the elements which they contain. But when the number of these elements is halved, the germ-cell.

* See F. M. Durham, Proc. Roy. Soc. I 904, vol. 7.4, p. 3 I I.

$\uparrow$ Distinguishing them from the loose, non-cohering webs of the feathers in the Silky breed. 
begins to acquire its own special features, and we may without much difficulty imagine that if two daughter-cells are to be differentiated allelomorphically from each other, the differentiation will come about at this reduction, or meiotic division, as it is called. When however we seek for proof, there is as yet none which is quite convincing. The one observation bearing immediately on the problem is that to which Correns has appealed*. In the maize we know that those $F_{2}$ seeds which have wrinkled or sugary endosperms will give rise to plants with similar sugary seeds. But the seed of maize is formed by a double fertilisation. It consists of two parts, an embryo, and an endosperm, and it is the sugary condition of the reserve-material in the endosperm which causes the wrinkled appearance of the seed. From the fact that wrinkled seeds always give rise to plants bearing wrinkled seeds, it is clear that the same elements have entered into the composition of both the embryo and the endosperm of the same seed. But the embryo is formed by the union of one nucleus of the eggcell with one from the pollen-tube, and the endosperm is similarly, formed by the union of the united polar nuclei with another from the pollen-tube. Hence we may infer that the two nuclei brought in from the pollen-grain bear similar allelomorphs, and that all the nuclei of the eggcell must also be similar in composition. Therefore the segregation of characters cannot in the maize take place after the division by which the pollen-grain was formed, for both nuclei to which it gives rise are of the same composition ; and the same argument applies to the egg-cell.

\section{Chromosomes as the possible Bearers of Factors.}

These are, I believe, at present, the only facts which positively limit the inquiry. It has been pointed out by the cytologists that the details of the processes by which nuclear reduction is accomplished may be readily construed as effecting the operation of segregation, but while admitting this as a fair and even probable interpretation, nothing in my judgment yet compels us to accept it as proved.

* The same conclusion is strongly supported by the evidence from Hieracium (see p. 247). 
Much that is known of chromosomes seems inconsistent with the view that they are the sole effective instruments in heredity.

Without presuming to a definite opinion on this question, I venture to state what seem to me formidable difficulties in the way of this expectation. If the chromosomes were directly responsible as chief agents in the production of the physical characteristics, surely we should expect to find some degree of correspondence between the differences distinguishing the types, and the visible differences of number or shape distinguishing the chromosomes. So far as I can learn, no indication whatever of such a correspondence has ever been found. Besides this, although no very thorough investigation of the chromosomes of somatic structures has yet been made on an extensive scale, I believe that consistent cytological distinctions between the nuclei of the various tissues of the same body have not been detected. If chromosomes were the chief governors of structure, surely we should find-great differences between the chromosomes of the various epithelia, which differ greatly in their structure and properties. As these cytological differences have not been found consistently there, the prospect of successfully tracing them among the specific types does not look very hopeful.

Again, no correspondence between the chromosome numbers and complexity of structure has ever been asserted to exist. Low forms may have many; highly complex types may have few.

Then, on the contrary, very closely allied types may show great differences in these respects. As is well known, Rosenberg has shown that one species of Drosera has 20, while another has Io. Again, Miss Lutz, and, independently, Gates, have found remarkable diversities in Oenothera, especially that gigas has 28 , while lata has $14 *$. Obviously this doubling means something definite, but it is not suggestive of the determination of specific difference.

In Aphis Miss Stevens, on the other hand, has shown how wide a diversity may be presented by the chromosomes of forms so alike as to have passed for one species. These

* Important evidence as to variations in chromosone numbers has been published by R. R. Gates, Botanical Gazette, July, 1908. 
differences prove both too little and too much. I cannot but believe that all this evidence points to the conclusion that we are about to find among the chromosomes one more illustration of the paradoxical incidence of specific difference, not the fundamental phenomena on which that difference depends. Among coleopterists punctulation is sometimes a feature of great systematic importance. To dipterists neuration and chaetotaxy sometimes give useful critical data. In certain orders of Lepidoptera, the Hesperidae, for example, the structure of the gonapophyses sharply distinguishes the species where all outward tests fail. But proceeding farther with each of these criteria, we are sure to come upon other groups where for a long series of diverse types the critical feature, so important elsewhere, may show no differences, or, on the contrary, may show marked instability.

There remains the suggestive fact that all that has been witnessed regarding the behaviour of the chromosomes is in fair harmony with the expectations which our Mendelian experience would lead us to form respecting the hypothetical "bearers" of varietal differences*. On the other hand,-with one striking exception, nobody has been able to connect a cytological difference with a character-difference in any instance. The exception, of course, is the case of the accessory chromosome which the researches, especially of Wilson and Morgan, have proved to be definitely connected with the development of femaleness (see p. I88).

\section{The Case of Bud-sports.}

There is another circumstance which must be taken into account in any attempt to see the facts of segregation in proper relation to other biological phenomena. This is the obvious fact that when a bud-sport occurs on a plant, the difference between the sport and the plant which produced it may be exactly that which in the case of a seminal variety is proved to depend on allelomorphism. This subject may best be discussed in reference to a practical illustration. All naturalists remember the passage in Animals and Plants

* The recent work of Godlewski gives however strong reason to believe that heredity in Echini may be governed by the cytoplasm of the egg. 
under Domestication* in which Darwin collected the evidence about the relation between peaches and nectarines. The records proved abundantly, (I) that the seeds of peaches may come up nectarines; (2) that conversely the seeds of nectarines may give rise to peaches; (3) that peach-trees may by bud-sports produce nectarines, the sport either involving a part so large that a definite nectarinebearing branch is formed, or so small that only a segment of a fruit is affected, part of the fruit being peach and part nectarine; (4) in one instance, the (Carclew) nectarine is said to have produced a branch which bore peaches.

With the exception of the last fact (4) the significance of this series of observations is now clear. The nectarine is essentially a glabrous variety of the peach, and on the analogy of other cases, the hoariness of the peach is presumably a dominant character. Thus, when from the seeds of peaches, nectarines spring, we perceive that this is the ordinary phenomenon of a recessive variety arising from a dominant hybrid. When, on the contrary, peaches are produced from the seeds of nectarines, the fact plainly suggests that the nectarine has been pollinated from a peach. To come next to the cases of the bud-sports, that in which nectarines appear on peaches must be interpreted as meaning that in the formation of that bud or cell from which the branch, or fruit, or part of a fruit, derived its separate existence, the element or factor for the peachcharacter was omitted. Therefore at some cell-division, evidently a somatic division, segregation of the allelomorph for hoariness must have taken place, and we are thus obliged to admit that it is not solely the reduction-divisions which have the power of effecting segregation.

Case (4) remains unelucidated. The record is most circumstantial, and its truth can scarcely be called in question. Nevertheless it stands, so far as I know, as an isolated instance. Whether the case is, as we might perhaps be tempted to suppose, one of actual, de novo origin of a dominant feature; or whether, as seems more probable, this particular tree was in reality a monstrosity due to imperfect segregation of that character in the germ of a

* Vol. I. p. 362.

B. II. 
heterozygous parent*, we cannot say with any confidence; but in considering the significance of the phenomenon of bud-sporting that special problem is of subordinate consequence, for in either event there must have been a process of allelomorphic segregation at some somatic division.

In our Sweet Pea cultivations a phenomenon precisely comparable occurred in two individuals of similar breeding. The plants were purples of the dark type with purple wings (Plate III, fig. 7), and were heterozygous for the blue factor, $B$. After a few hot days they stopped flowering. Then wet weather succeeded and much secondary growth was made, young flowering shoots springing in the axils of the older stems. On two individuals one of these young shoots bore a flower of the red, or Miss Hunt type (Plate III, fig. 8), showing that the factor $B$ had been omitted in one of the cell-divisions by which they were formed.

\section{The Differentiation of Repeated Parts compared with Segregation.}

Such facts raise a theoretical question of fundamental importance. If upon the same individual, parts may as an abnormal occurrence present the same differentiation which is known to be characteristic of dominant and recessive, may not the differentiation normally existing between repeated parts of the same individual be a phenomenon of segregation? Why, for instance, may not the differentiation normally existing between petal and leaf, or between the appendages of arthropods, or any other meristically repeated parts, be due to a segregation acting amongst somatic parts as amongst gametes? Evidently, as morphologists have often argued, the relationship between individuals is comparable to that existing between repeated parts. By tracing the comparison in one direction we reach the fact that hereditary resemblance is the same phenomenon as that which we elsewhere know as symmetry: for if a cell divides into two similar halves, and each half undergoes similar

* Comparable, for instance, with gynandromorphous insects, half male, half female; or with white flowers showing a well-defined patch of some coloured variety. 
changes and developments, while remaining attached to the other half, we call the resemblance between the two halves Symmetry; but if the division is one by which two new individuals are formed, and the two halves separate and lead independent lives, then we ascribe the resemblance between the two individuals to heredity. On a previous occasion (I I) I pointed out that when the comparison is followed in the other direction it appears that if

I. Symmetrical Repetition of Parts is comparable $v$ with Heredity,

then 2. Differentiation between Parts is comparable with Variation.

That there must be limits beyond which the comparison fails, is clear enough, but I do not think they have been yet satisfactorily defined. The discovery of a true delimitation of the properties and attributes of individuals, which distinguish them from parts, would constitute a great advance in biological theory. Perhaps the nearest we can get to such a distinction is a recognition of the fact that ordinary somatic differentiation is usually distributed in a symmetrical manner about one or more axes, while among gametic tissues such axes are not usually perceptible; but to both statements there are some notable exceptions. Bud-sports, however, never, so far as I know, are distributed symmetrically about the axis of the individual producing them, and thus are distinguishable from ordinary somatic differentiations. Dr S. F. Harmer in his Presidential Address to Sect. D of the British Association (Dublin, I 908), describing the various forms of avicularia* found on the same colony of certain Polyzoa, made the interesting suggestion that there may be an allelomorphic relationship between these parts. Sometimes one special type of avicularia characterises a species, sometimes another type; while again both types may occur together on the same colony. Now this is a case to which such a suggestion is especially applicable; for the different types of avicularia are not distributed in a symmetrical pattern, but apparently at random on the

* These structures are prehensile organs of various patterns, somewhat resembling minute crab's claws or the heads of birds. Morphologically they are regarded as much modified "individuals." 
colony just as bud-sports are on plants, and hence it is by no means unlikely that the differences between the avicularia may be due to allelomorphic segregation.

\section{Asymmetry and Variation.}

Stripped of all that is superfluous and of all that is special to particular cases, genetics stand out as the study of the process of cell-division. If we had any real knowledge of the actual nature of the processes by which a cell divides, the rest would be largely application and extension. It is in cell-division that almost all the phenomena of heredity and variation are accomplished.

Heredity being a special case of symmetrical division, genetic variation is the consequence of asymmetrical division. The cause of the asymmetry may lie far back in the history of the tissue or of the germs. The germ-series may for instance be represented as

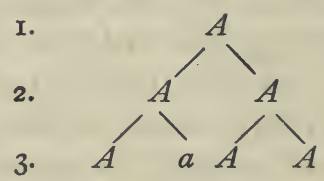

where the division in which the variant, $a$, first appears is actually an asymmetrical one; or we may imagine the process occurring in such a way that the first appearance of $a$ is produced by the division of an $A$ cell into $a$ and $a$ thus :

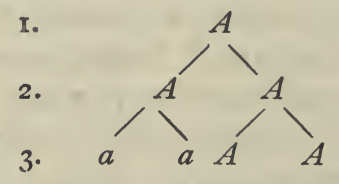

In the latter case the asymmetry was introduced into the series at some division antecedent to that at which the $a$ form actually appeared. The question whether one of these schemes is correct as a representation of the natural processes, to the exclusion of the other, is a not unimportant one, but there is no adequate ground for a positive answer as yet.

In comparing the somatic differentiations with genetic 
variations it may perhaps be found hereafter of use to bear in mind that just as the divisions of germ-cells are of two $r$ kinds,

(I) Symmetrical, producing the resemblance called Heredity,

(2) Asymmetrical, producing the difference called Variation,

so are somatic cell-divisions recognizably of two kinds. For there also we meet divisions by which similar parts are divided from each other, and differentiating divisions by which parts with distinct characters and properties are separated. It is evident that when the conception of symmetry is applied to such phenomena it must be understood to include the case of production of like parts by division which result in the formation of a successive series.

In this connection reference should be made to a work of more than ordinary suggestiveness lately published by Jennings $\left(\mathrm{I}_{3}\right)$. Speaking of the fission of Paramoecium which results in the formation of two individuals by a transverse division, he truly says that "it is evident that even in Protozoa heredity is not a mere result of subdivision," for in the new individuals the head-end produces -regenerates, perhaps we might say-a tail, and the new tail-end grows a head.

That there must be a real difference between the mechanical processes by which this repetition comes about, and that in which two halves are formed as optical images is clear. The latter is the obvious case of real geometrical symmetry. But when two similar individuals are formed, so that one is placed in succession to the other, this result may be described as symmetrical in so far as the two products are similar, but the homologous parts instead of being adjacent to each other, are arranged in alternating series.

Jennings' observations relate to a remarkable case. He found a Paramoecium with a monstrous outgrowth, or spine of protoplasm, and as the animal successively divided, this spine was handed on to one not both of the products of division. Obviously there is here something which may be interpreted as providing a rough model of the process of 
segregation. The spine may be taken as a thing added and "present," or in our terms, a dominant character. In each division there is found a dominant half which has the spine, and a recessive half without it. If only we could know what would happen as the result of conjugation between two Paramoecia possessing similar "spines," it is possible that the manner in which segregation occurs in the gametogenesis of the Metazoa would be elucidated. In them, as mentioned above (p. I95), the gametes are almost always formed in sets of four, and a presumption is thus created that the members of each set of four are not all equivalent to each other. If they were equivalent we could represent the set as $A A A A$, supposing the zygote which produced them were homozygous. If the organism were heterozygous we should then imagine the series to be $A a A a$. But if the differentiation is not by pairs but by fours, the series must be represented by $A a A^{\prime} a^{\prime}$ (or $\left.A A^{\prime} a a^{\prime}\right)$ for the heterozygote, and as $A A^{\prime} A A^{\prime}$ for the homozygote.

\section{Reversion and Variation.}

It follows from what has been said of allelomorphism that variation must now be regarded in the main as a phenomenon due to the addition or omission of one or more definite elements. When, as in the case of the combs of fowls, the types which have obviously arisen later in the evolution of the species, for example, rose, pea, and walnut, dominate over the primitive type, in this case the single, the variation by which those dominant varieties came into existence must have consisted in the addition of an element (or in the case of the walnut-comb, two elements) to the original stock of the species. When, on the contrary, the new variety is recessive, it is clear that the variety occurs in consequence of the omission of an element. (The suggestion made by de Vries, that the dommant is always the phylogenetically older form, has not been substantiated by further investigation.) The problem of the causation of variation is thus to some extent narrowed down. The "cause" of a variation is the event which brings about the addition or omission of a factor. 
In reply to the question so often asked, Has modern investigation given evidence as to the nature of these causes? the answer must still be, Almost nothing. Signs there are however that the search for such evidence is beginning at last to be not altogether unfruitful, but the detailed consideration of this part of the subject must be postponed to another occasion.

Reversion occurs when the sum total of the factors returns to that which it has been in some original type. Such a return may be brought about by the omission of an. element or elements, as when the rose-comb fowl for any reason has a single-combed offspring. Conversely, the return may occur by the addition of some missing element needed to complete the original type. As yet no means are known by which the omission or addition of elements can be made at will, except by crossing. Reversion on crossing is thus the particular case in which one or more missing factors are brought in by the parents of the cross-bred. The most striking cases of such reversion on crossing are those in which neither parent seems to the observer to contain anything specially reminiscent of the original type, and yet the offspring of the cross are all of that type. Such cases are those of the two white Sweet Peas which, though each severally breeding quite true to whiteness, when crossed together have a reversionary offspring; or of the two breeds of Pigeon, which though neither has the bluebarred plumage of the Rock Pigeon, yet contain materials from which blue-barred birds may be compounded.

Not often can we hope to be able to specify the complementary elements which must meet each other in order that a certain compound character may be produced. Nevertheless, by the co-operation of physiological chemistry with genetics there is every hope that in favourable cases of a simple order actual demonstrations of these elements may be carried out. Perhaps the nearest approach to such an achievement is that made by Miss Wheldale in her experiments on Antirrhinum (Snapdragon). Crossing the very pale yellow, known as "Ivory," with a white variety, she obtained the $F_{1}$ generation all of the dull red of common Snapdragons $(303)^{*}$. The work of Overton and others had * $F_{2}$ was of the normal kind expected in such cases. 
indicated, as she points out, that the red anthocyan is probably a glucoside formed by a combination of tannic acid with a sugar. Now in this case Miss Wheldale found that the "ivory" flowers do contain a glucoside and that this body is absent from the white flowers. The breeding experiment proves that the white variety introduces a reddening factor which is absent from the "ivories." According to her view the contribution which the "ivory" makes is the glucoside, and the complementary contribution made by the white is an oxidase which acts upon the glucoside to make anthocyan.

With the development of the inquiry it has become clear that variation, in so far as it consists in the omission of elementary factors, is the consequence of a process of "unpacking." The white Sweet Pea was created in the variation by which one of the colour-factors was dropped out. Such variation is not, as it was formerly supposed that all variation must be, a progress from a lower degree of complexity to a higher, but the converse. When from a single wild type, man succeeds in producing a multitude of new varieties, we may speak of the result as a progress in differentiation: but we must recognize that the term is only applicable loosely, and that the obvious appearance of increased complexity may in reality be the outcome of a process of simplification. The facts nevertheless preclude the suggestion that all variation even under domestication is of this nature, nor till experimental research has developed far beyond its present limits, can we make any confident estimate whether it is the one process or the other which has played the larger part in the formation of the diversity of living forms.

It is legitimate to conclude from what is known of reversionary cases that when reversion in characteristics other than colour results from crossing, similar processes are operating. One such instance was described in connection with the peculiar properties of colour-factors (p. I 33), a reversion to the hoary leaf resulting from the cross of two glabrous types of Stock (Matthiola). As yet however the only other example of reversion in a structural character is that furnished by the crosses between two varieties of Sweet Peas known as Bush and Cupid. The Cupids are the 
little prostrate plants shown in Fig. I. Crossed with ordinary tall plants they give a normal Mendelian result, the tall being dominant. The Bush is a distinct type. It is only half or two-thirds the height of the taller, and its habit of growth is peculiar. The stems are erect, thin and wiry, branching profusely, whence the description Bush is derived. Bush again is an ordinary recessive to tall. But Mr Punnett and I found, a good deal to our surprise, that the cross Bush $\times$ Cupid gives $F_{1}$ tali, i.e. reversionary (Fig. 37). Though we cannot venture on any surmise as to the chemical causation of this phenomenon it is obvious that once again the reversionary character, here the tallness, is a compound character due to the meeting of complementary elements. The allelomorphs are

\section{Dominant.}

I. Tallness $(T)$.

2. Prostrate habit: non-branching $(P)$.
Recessive.

Dwarfness $(t)$.

Erect: branching $(p)$.

In $F_{2}$ therefore a new type occurs, namely an erect or Bush-like Cupid (Fig. 38 ), and the $F_{2}$ series is

9 Tall $(T P): 3$ Bush $(T p): 3$ prostrate Cupid $(t P):$ I erect
Cupid $(t p)$.

The "height" of Sweet Peas is evidently therefore a compound character and in part depends on the existence of a factor which suppresses or inhibits that stimulus which otherwise would compel it to branch. A successful analysis of the physiological processes concerned in this series of phenomena would be a significant addition to plant physiology.

The analysis of compound structural characters may confidently be expected to lead to the recognition of numerous examples comparable with these two.

With the progress of such analysis other examples will certainly be encountered illustrating those curious interrelations between the factors spoken of under the names Coupling and Spurious Allelomorphism. In the existence of such phenomena we meet evidence that the central problem of genetics is in part a geometrical one. Perhaps 


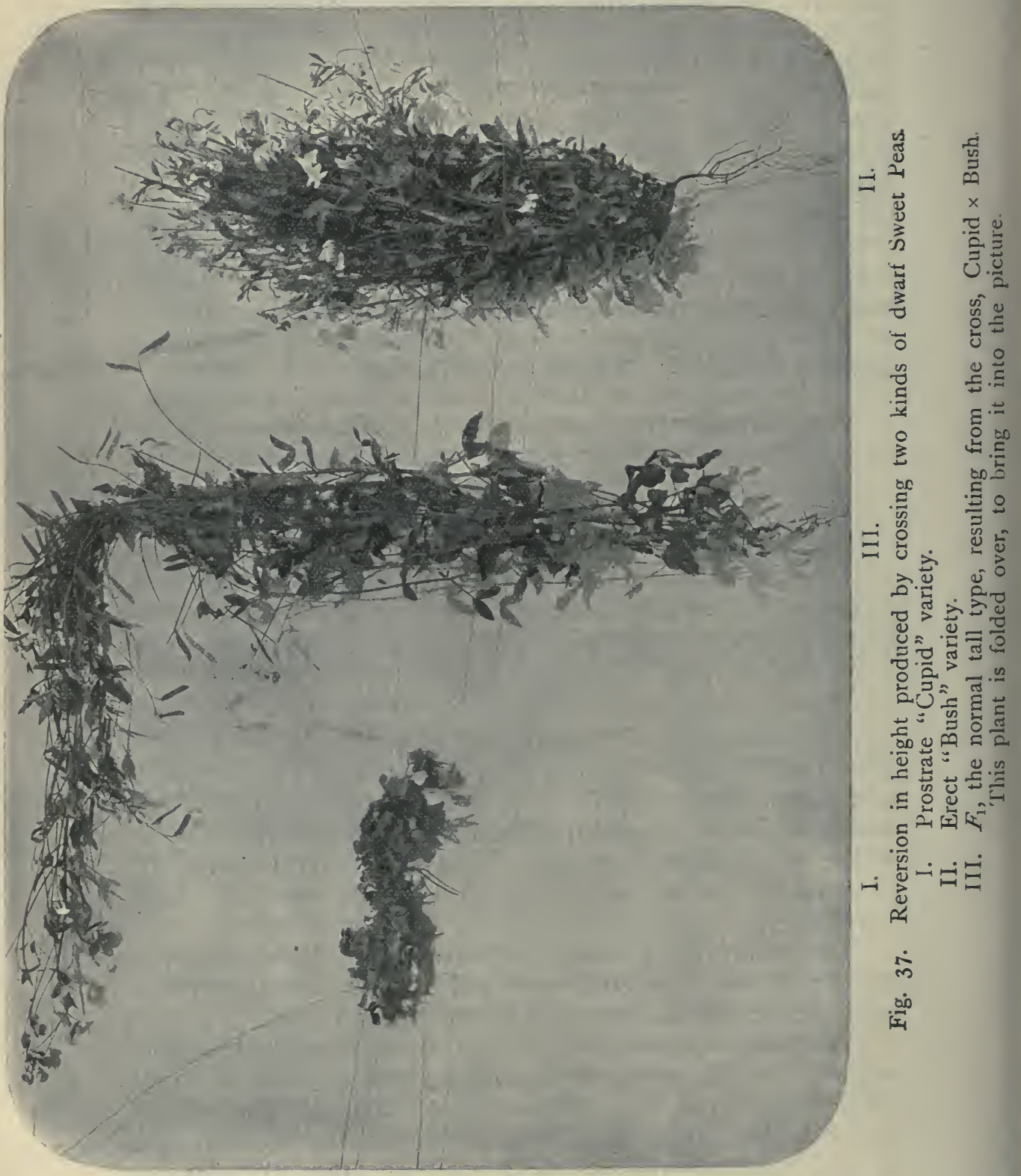


the greatest advance that can be foreseen in this department of physiology will be made when the nature of the interaction between the chemical and the geometrical phenomena of heredity is ascertained.

\section{Bearing on the Theory of Evolution.}

The consequences of these discoveries to the general theory of Evolution must be examined in reference to concrete examples in order that the extent to which they reach, and the limitations by which they are beset, may rightly be apprehended. It is only by the detailed study of actual cases of interrelation between kindred species that the scope of Mendelism in this region of inquiry can be measured and illustrated. I hope subsequently to publish a separate volume* in which this part of the subject will be considered. Two features which emerge salient on such a survey may be named at once.

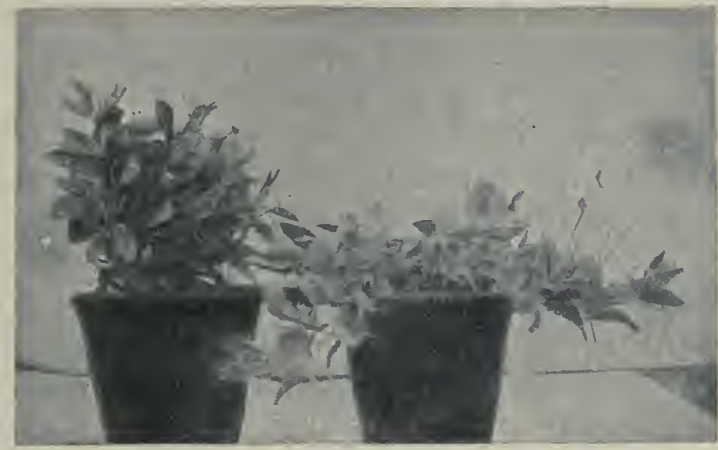

II.

I.

Fig. 38. The two "Cupid" types in $F_{2}$ from Cupid $\times$ Bush (Sweet Peas). I. The ordinary, prostrate Cupid. II. The erect Cupid.

\section{Mendelian Segregation and Species.}

First it is certain that segregation in countless instances plays a part in the constitution and maintenance of characteristics held by systematists to be diagnostic of species. One has only to glance over trays of birds' skins, the

* This volume which is designed to be in some respects a continuation of the present book will be based on lectures given as Silliman Lecturer to Yale University in 1907 , under the title "The Problems of Genetics." 
portfolios of a herbarium, or drawers of butterflies and moths to discover abundant "species" which are analytical varieties of others. The principles of heredity we trace in our experimental breeding are operating throughout the natural world of species. They may apply to the interrelations of allied forms which are species in the strictest acceptance of that term. Remarkably clear and uncontrovertible evidence of this fact has been obtained by Dr Ezra Brainerd in his studies of the American Violets. The whole series of observations is full of significant details which I must be content to omit from this summary. The essential facts are as follows. Finding wild in nature plants which from his knowledge of the genus Viola he determined as accidental hybrids between certain species, he removed these plants to a garden and kept them under observation. They proved to be very nearly, but not quite completely, sterile, thus manifesting one of the attributes of crosses between genuine species in the strictest sense. Some small quantity of seed was however produced in the cleistogamic capsules, and therefore may be taken as certainly the result of self-fertilisation. This seed gave rise to plants showing obvious segregation in regard to many features-the colour of the stems, the fruits, and the seeds, and also, though not quite so palpably, in the shapes of the leaves. In some at least of these derivative plants there was-as Herbert and others have found in similar studies of the offspring of sterile hybrids-some return of fertility. Such an experiment raises the hope that successful investigation of the nature even of the sterility consequent on crossing, the most obscure of all genetic phenomena, may become one of the possibilities of Mendelian research.

It is scarcely necessary to insist that plenty of the characters which are now known to segregate would be far more than sufficient to constitute specific differences in the eyes of most systematists, were the plants or animals in question brought home by collectors. We may even be certain that numbers of excellent species universally recognized by entomologists or ornithologists, for example, would if subjected to breeding tests be immediately proved to be analytical varieties, differing from each other merely in the presence or absence of definite factors. 
But this is not enough. We must eventually go further; and, supposing such tests to be applicable on a comprehensive scale to great numbers of natural forms, we must ask whether the result of such an investigation will show first that certain kinds of differences segregate and that certain other kinds do not segregate; and secondly whether we shall then recognize that it is to the non-segregating that the conception of species attaches with the greater propriety. Since of the non-segregating characters we know as yet almost nothing, I am loth to attempt an answer, but I cannot imagine upon what evidence anyone would rely who should maintain that the answer must be affirmative. De Vries has, as it seems to me, incautiously, defined with some strictness the differences between varietal and specific distinctions, declaring that it is the property of varietal characters alone to exhibit Mendelian heredity (295, \&c.). Though I agree with him in perceiving that genetic research may ultimately provide some approach to a valid distinction between species and variety, I am reluctant to accept any evidence yet attained as an adequate basis for so vast a generalisation. Of the consequences of specific crossesin the stricter sense-little is known, and no case has been fully explored. Even as to the results of crosses between petites espèces differing in several characters, present information is most imperfect. Such cases as that of the Violets, mentioned above, must be thoroughly investigated by critical methods; and when a good number of these examples taken from an ample range of types have been submitted to factorial analysis it will perhaps be possible to come to a more confident decision. But before any decision at all is pronounced or even contemplated, the laws which govern the incidence of sterility must be most carefully determined.

Feeling thus the impossibility of now defining the segregating from the non-segregating, I am unable to follow de Vries in the further step which he has taken (299) in assigning a definite physiological reason for the difference between these classes. His suggestion is that in Mendelian heredity there is a process, spoken of as "bisexual," in which each determining factor (Anlage) meets a corresponding opponent; while in the other, or "unisexual" case, 
the factor in question remains unpaired in the hybrid. The latter description, "unisexual," is applied to characters which breed true in the cross-bred. This suggestion is the outcome of an attempt to incorporate the facts of Mendelian inheritance with the conclusions previously drawn from the mutations studied in Oenothera. But as knowledge of Mendelian cases has increased, the applicability of what is here spoken of as the "presence and absence" hypothesis becomes more and more clear. We now, in fact, feel fairly sure that a heterozygote is properly represented as one which contains an unpaired factor. Hence the doubt may be expressed whether if de Vries' terminology is to be maintained, its application should not be reversed.

\section{Discontinuity in Variation.}

When some years ago I published a collection of facts illustrating the phenomenon of Variation in animals*, I pointed out that variation is frequently definite, or Discontinuous. That conclusion is one which cannot fail to strike an observer who makes a study of this part of physiology. Inasmuch as the discontinuity of variation is manifested again and again in respect of exactly those differences which we are accustomed to recognize as distinguishing specific forms from each other, the further conclusion followed that the diversity of species may be regarded as having come about very largely by the occurrence of these discontinuous variations.

The materials then put forward related almost entirely to a restricted group of phenomena in animals, those which are known as meristic, exemplifying the processes of change in the number of parts and the relation of repeated parts to each other. Had the field of inquiry been widened by the inclusion of variations in other characteristics of animals, or in those of plantst, a body of evidence more clearly demonstrating the truth of this thesis could have been presented. There were, however, reasons which led to the

* Materials for the Study of Variation, I 894 .

+ A useful collection of facts of this nature in plants has been published by Korschinsky, under the title "Heterogenesis und Evolution," Flora, 89, I90r. 
preference of the one special group of facts-the meristicas being in their nature more fundamental and homogeneous, and my object was rather to map out the ground than to erect a definite proposition upon it. The book was to have been followed by similar collections dealing with the other manifestations of variation; but with the development which genetics almost immediately underwent, it became clear that the method of miscellaneous collection was no longer the most direct, and that by experimental investigation of special cases progress of a far more valuable order was possible.

Views somewhat similar to those that I had formed from a general survey of the facts of variation were shortly afterwards published by de Vries in his famous book Die Mutationstheorie, I9OI-3. Having at command a mass of evidence far larger and more coherent than mine he was at last successful in bringing workers of many schools to give these suggestions a serious consideration. For the first time he pointed out the clear distinction between the impermanent and non-transmissible variations which he speaks of as fluctuations, and the permanent and transmissible variations which he calls mutations. Of his proofs, the most striking, to many the most convincing, is that provided by his study of Oenothera, in which he witnessed the actual occurrence of sudden departures from type-not one but several-by which at one step "in descent distinct and frequently pure-breeding types were produced. Whatever be the true interpretation of these particular observations, they manifestly provide examples of something so like the generation of new species that in any future discussion of Evolution they cannot possibly be passed over.

We may be doubtful of the validity of the superstructure which de Vries has created, and yet in full agreement with him in recognizing the fundamental truth, that there is a natural distinction between fluctuational variations and actual genetic variations; that the latter are those alone by which permanent evolutionary change of type can be effected; and that commonly, though, as it seems to me, not always, the steps by which such changes occur are so discontinuous as to merit the name Mutations.

It is at this point that Mendelian discovery aids. Whereas formerly, though the fact of Discontinuity was not 
doubtful, there was nothing to indicate how or when it was determined. We now see that the discontinuous variations are in the main the outward manifestations of the presence or absence of corresponding Mendelian factors, and we recognize that the unity of those factors is a consequence of the mode in which they are treated by the cell-divisions of gametogenesis. With the discovery of these factors precise analytical treatment can at length be applied to the problem of Evolution.

\section{Mendelism and Natural Selection.}

Knowledge of the physiology of heredity thus abolishes an old difficulty often admitted to be an obstacle in the way of any affirmation of Evolution by the process of natural variation. The notion that a character once appearing in an individual is in danger of obliteration by the intercrossing of that individual with others lacking that character proves to be unreal; because in so far as the character depends on factors which segregate, no. obliteration takes place. The factors are permanent by virtue of their own properties, and their permanence is not affected by crossing.

If the acquisition of a new factor or the loss of an old one is so damaging as sensibly to impair the chances of life of the variety thus constituted, that variety must surely be extinguished. On the contrary, the addition of a new factor contributing sensibly to the success of its possessor, or the omission of a detrimental element, will aid in the preservation of those which exhibit that variation.

Mendelism, though aiding us greatly in showing how the diversity of species and varieties may arise and be $\checkmark$ maintained, provides no fresh clue to the problem of Adaptation; except in so far as it is easier to believe that a definite, integral change in attributes can make a perceptible difference to the prospect of success than that an indefinite and impalpable change should entail such consequences. Definite variational changes are being continually offered, each giving an opportunity to natural or to artificial selection, and we need not hesitate to declare that of such materials the diversity of nature has been compiled. If anywhere in such a province as evolutionary science certainty 
may be reached, it is here. The conception of Evolution as proceeding through the gradual transformation of masses of individuals by the accumulation of impalpable changes is one that the study of genetics shows immediately to be false. Once for all, that burden so gratuitously undertaken in ignorance of genetic physiology by the evolutionists of the last century may be cast into oblivion. For the facts of heredity and variation unite to prove that genetic variation is a phenomenon of individuals. Each new character is formed in some germ-cell of some particular individual, at some point of time. More we cannot assert. That the variations are controlled by physiological law, we have now experimental proof; but that this control is guided ever so little in response to the needs of Adaptation there is not the smallest sign. If chance variation was an improbable source of the adapted diversity which living things exhibit, the improbability remains, undiminished perhaps, but certainly not increased, by the recognition of that control.

There is also nothing in Mendelian discovery which runs counter to the cardinal doctrine that species have arisen "by means of Natural Selection, or the preservation of favoured races in the struggle for life," to use the definition of that doctrine inscribed on the title of the Origin. By the arbitrament of Natural Selection all must succeed or fail. Nevertheless the result of modern inquiry has unquestionably been to deprive that principle of those supernatural attributes with which it has sometimes been invested. The scope of Natural Selection is closely limited $\nu$ by the laws of variation. How precise and specific are those laws we are only beginning to perceive. In the light of the new knowledge various plausible, but frequently unsatisfying, suggestions put forward, especially by Wallace, Weismann, and their followers, as probable accounts of evolutionary progress, must be finally abandoned. It cannot in candour be denied that there are passages in the works of Darwin which in some measure give countenance to these abuses of the principle of Natural Selection, but I rest easy in the certainty that had Mendel's paper come into his hands, those passages would have been immediately revised.

For Darwin, indeed, Mendelism would have provided B. H. 
sound reasons for a return to his own earlier views. In abandoning his belief in the importance of individual variations, which previously he had held in a form not incompatible with that now demonstrated to be right, he took a step in the wrong direction. The criticism before which he then gave way-has proved invalid*. To him, most of all men, would the knowledge have come as a delight, that progress, even if in a direction unexpected by himself, had been made with that problem the solubility of which he was the first to make apparent to the world.

* As to this change of opinion see Darwin's letters to A. R. Wallace (Life and Letters, 1888, ज1. p. 108). 


\section{CHAPTER XVI}

PRACTICAL APPLICATION OF MENDELIAN PRINCIPLES.

\section{Meaning of Pure-bred-Rogueing-Raising Novelties- A Practical Example-Unfixable Types-Technical Methods-Sociological Application.}

No one who is acquainted with Mendelian method will doubt that by its use practical breeders of animals and plants may benefit. In so far as they are concerned with the fixation of desirable varieties, or with the creation of new types by re-combination of pre-existing characters, their operations may now be greatly accelerated.

\section{"Pure-bred" and "Cross-bred."}

But apart from these obvious advantages which it may confer, the new knowledge of heredity will react most profoundly on the art and practice of the breeder by introducing a new standard of precision. We at length understand the physiological meaning of "pure-bred" and "cross-bred." We know that these ideas must be applied to the several characters of the animal or the plant, rather than to the individual as a whole. For the individual to be altogether pure-bred it must be homozygous in all respects. In current parlance, dogs, for example, derived from a cross a few generations back have been spoken of as $\frac{1}{8}$ Bulldog, or $\frac{1}{3.2}$ Pointer blood, and so forth. Such expressions are quite uncritical, for they neglect the fact that the characters may be transmitted separately, and that an animal may have only $\frac{1}{32}$ of the "blood" of some progenitor, and yet be pure in one or more of his traits. 


\section{Rogueing.}

The introduction of these ideas will help much by showing what is to be expected of a pure variety. I may give an example. Hitherto, when in growing seed-crops, undesirable "rogues" recur continually through long periods of years, the fact has been accepted as part of the natural perversity of the variety. The grower devotes much time and expense in keeping the rogues down, but the idea that they can be got rid of altogether does not generally occur to his mind. Nevertheless in many such cases Mendelian observation at once provides the means of carrying out this radical treatment with success. I cannot here discuss the intricate question of the reality and signification of that degeneration of cultivated varieties which is believed to occur generally and certainly occurs sometimes when selection is suspended. All that we can insist on at this stage of the inquiry is the fact that much of the irregularity of crops which passes for such natural degeneration is readily preventible.

The rogue-plants may be of various kinds, and their nature must be separately determined in each case. For example, they may be recessives merely, and if so they can be eliminated by breeding from pure dominant individuals, according to the system now well understood. It is possible also that they may owe their existence-if the plants are fertilised by insects-to special combinations of complementary characters. In that case, to exclude the possibility of their production must be a more difficult, though not necessarily a hopeless task. But in the light of present knowledge one definite and conspicuous conclusion has been attained, that for the appearance of each type in a crop there must be some specific and usually ascertainable cause ; and an aim of practical seed-growers should in future be to search carefully for such causes. When this search is made, the guess may even be hazarded with some confidence that in numerous examples the cause of impurity in seed-crops will often be found to be nothing more recondite than an unsuspected admixture of another variety, the seeds of which are overlooked as apparently belonging to the selected type. In a certain strain of eating peas I have 
seen a case of this kind. The true variety has a wrinkled seed. The "rogues" had round seed. Every year the variety was picked over by hand and the round seed rejected. Nevertheless a pretty constant proportion of the rogues persisted. Knowing that it was in the highest degree unlikely that a wrinkled pea, being recessive in that respect, could give off a round-seeded form, I examined the seed with care and found that the seeds at the ends of the pods of the rogues were liable to be shrivelled so much as to pass for the true wrinkled type, and being thus admitted into the selected seed, perpetuated the "rogues." This is only one of many sources of error. Hitherto, through the prevalence of incorrect views of the nature of variation and heredity it has been thought more natural and likely that plants should throw rogues than that they should not. With the attainment of exact knowledge we see that the opposite expectation is more probable, and in that hope all operations of this sort should now be guided.

\section{Raising Novelties.}

When crossings between varieties are made, either with the definite purpose of producing a combination of two desirable qualities, or in the general expectation that some novelty will turn up, it is scarcely necessary nowadays to insist that the appearance and attributes of $F_{1}$, the first cross, give no indication as to the failure or success of the attempt. That outstanding fact is at length very generally known and appreciated, with the result that first crosses are now preserved which a few years ago would have been rejected. More important is it to lay stress on the necessity for sowing a really large sample of the seed from which $F_{2}$ is to be raised, for there must be enough to give a chance of seeing the rarer combinations. Since by the nature of the case most of the obvious crosses between the familiar varieties of cultivated plants have been tried in horticultural practice, the novelties are likely to be found more often among these less frequent combinations.

\section{A Practical Example.}

As a good example of an $F_{2}$ family consisting of a long series of types, that derived from one of the crosses 
between a white and a coloured Primula Sinensis is figured in Plate VI. The results of this mating have for several years been studied by $\mathrm{Mr} \mathrm{R}$. P. Gregory in collaboration with me. Though the quantitative relations between the types, and their factorial composition are still not completely worked out, the case forms a good illustrative example of the operation of Mendelian processes. The coloured parent is Sutton's Crimson King, a well-known very dark red sort, with red stigma, red spots round the eye and full red stalks. The white parent used was Primrose Queen, a stellate or Star Primula, with a white flower and reddish stem. This white type has the large yellow eye spoken of in connection with the inheritance of heterostylism (p. 7o), and as there described, the style does not reach above the level of the anthers, this being the condition known as homostyle. Both types breed absolutely true to their respective characters.

The plants, $F_{1}$, produced by crossing these two types are quite uniform. In habit they are intermediate between the star type and the compacter shape of Crimson King. The flower shape is also intermediate. The petals are whitish, with a slight tinge of magenta, especially on the lateral edges. When kept warm they lose the colour almost entirely, but when they are kept cool, the colour increases somewhat in amount.

By the self-fertilisation of such $F_{1}$ plants a very complex $F_{2}$ series is produced. Plate VI gives a fair idea of some of the more conspicuous types which appear, though it will be understood that only the colour and the shapes of the individual flowers can be represented there. In the mode of growth, the shapes of the inflorescence and many other features there is an equal diversity, so that an observer not accustomed to the results of crossing may well find it difficult to believe that this heterogeneous assemblage of plants can all be the offspring of a single individual. A systematist might make ten or even twenty species out of such a family, were the several types found isolated in nature, and no one could accuse him of excessive "splitting."

The magenta colours are evidently due to a factor epistatic to the crimson or claret-coloured reds, and this factor was obviously introduced by the white parent. In 
$x=$

$+5$ 
5

$\frac{0}{\frac{\pi}{2}}$

c

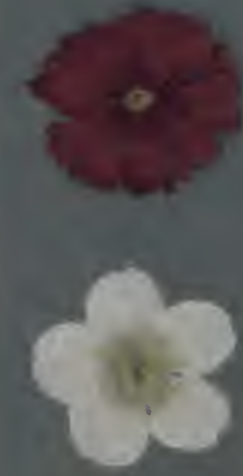

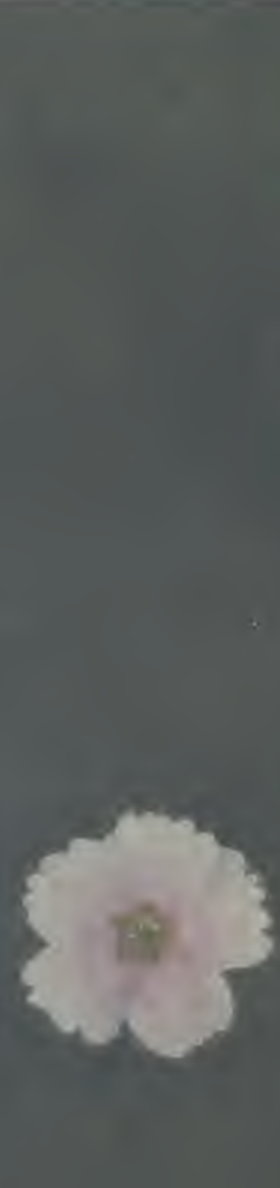

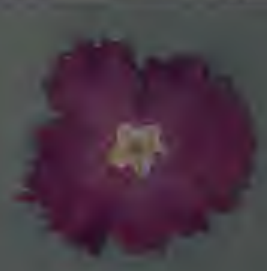

๑

0

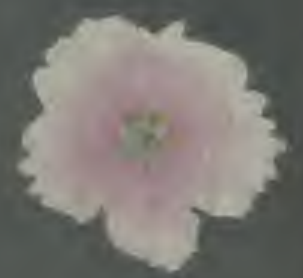

เ)

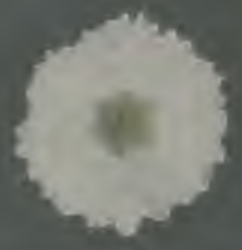




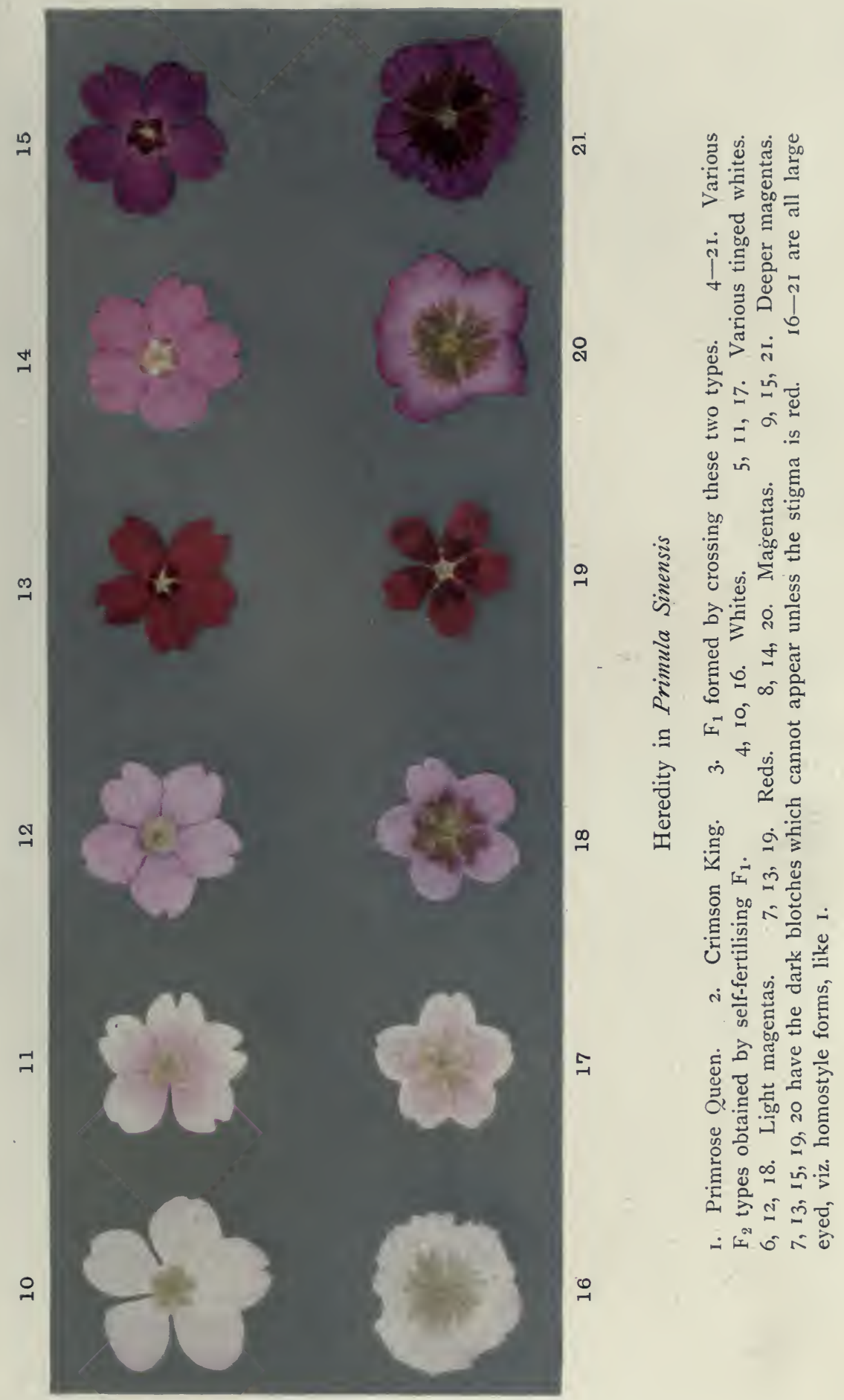



$F_{2}$ those whites which are quite devoid of colour, are pure to whiteness. The crimson-reds are pure to redness. The magenta types may be pure to magenta, or may throw the hypostatic reds. The darker colours are recessive to the lighter, in the magenta classes, though the mutual relations of the light to the dark among the reds are not so certain.

The large eye, combined with a homostyle structure, is recessive to the small or long-styled type. As mentioned (p. I39) the dark blotches round the eye can appear only in those plants which have coloured stigmas. Another curious point is to be noticed in this respect : that when the dark blotches are developed in a large-eyed type, the blotch extends over the whole area included in the "eye." If the stigma be green the eye is therefore yellow (Plate VI, fig. 20). The stigma may be coloured, though the eye is yellow; but if the stigma be coloured and the factor for the blotches is present, then the appearance of the deep red and deep magenta flowers figured in the bottom row (Plate VI, figs. I 9 and $2 \mathrm{I}$ ) is produced. Such types are exceedingly distinct, and might most naturally pass for different species. Moreover, of these singular varieties the red would always breed true in both shape and colour, so that the illusion in that case would be complete. The deep magenta type might also be homozygous and breed true, but some individuals would throw the red variety also. All the offspring of these dark-eyed types would of course have the large dark eye.

As regards the relations of the various types all that can be positively asserted is that the chief classes are (I) pure white, (2) tinged white, (3) crimson-reds, and (4) magenta-reds. There is some grading between the two red classes, and between the pure and the tinged whites. The distinction between the two kinds of red also cannot be followed among the tinged whites. The numerical proportions are therefore not quite certain, but presumably the various whites collectively are i 2 , magenta-reds 3 , and the crimson-reds $\mathrm{I}$.

In the plate no attempt is made to indicate these numerical proportions and the flowers there shown are simply chosen as representing the most distinct types which occur. The polymorphism of such an $F_{2}$ tamily is greatly increased by the existence of extreme diversity in the shapes of the 
umbels, the size of the calyx, of the involucre of bracts, and many other structural features the inheritance of which has not been studied in detail.

Such a series will illustrate the ordinary practice of the horticulturist who is engaged in the production of novelties. $\mathrm{He}$ crosses together two types and picks out those novelties which are produced in $F_{2}$ by the re-combination of pre-existing factors. This has been the method which has led to the creation of nearly all our modern varieties of vegetables and flowers. From Mendelian discovery the practical breeder learns two lessons, both of importance. The first is that he must not discard the $F_{1}$ generation merely because it does not give him anything he wants. This generation may be uniform, and indeed must always be uniform if both parental types were homozygous in all their several factors. It may also be quite uninteresting from the horticultural standpoint, exhibiting some old-fashioned or reversionary type which is reproduced because all the factors which constitute it happen to have been brought together into one individual. But from the appearance and properties of $F_{1}$ no guess can be made as to the possibilities of $F_{2}$. A vast amount of valuable material has again and again been discarded by practical horticulturists through ignorance of Mendelian principles. The uninteresting types produced by crossing, though no improvement on the old and familiar varieties would, if their seed had been saved, have given abundant novelties in the next generation.

\section{Fixing the Type.}

The second practical lesson is more important. If the plant is of a kind which is habitually propagated by budding grafting, cuttings, or other asexual mode of division, no doubt the object of the breeder is attained at once with the appearance of his novelty. All that he has to do is to multiply it. But if, as is generally the case with vegetables, and very often nowadays with flowers also, he requires to work up a strain true from seed, Mendelian analysis shows him how to accomplish this in the shortest time. He must breed from each individual separately. Take this case of Primula. Suppose he wishes to get a pure strain of the dark magenta (e.g. Plate VI, figs. 9, I 5, 2I). If he saves all 
these together, some will be impure and throw the reds (Plate VI, figs. 7, 13, 19), and he may go on for some years saving collectively from the dark magentas but still find a proportion of reds produced. The first time he saves from each individual separately he will find that some only are impure and others pure. Then by saving from the pure plants, his pure strain is immediately established.

When the desired combination has appeared, the readiest way to perpetuate it is by self-fertilisation. But if, as happens more often than is commonly supposed, there is any considerable degree of self-sterility, the plant must be fertilised by some other individual of the same type if that can be obtained, or by one which is suspected of being a heterozygous form containing the selected variety.

The advice to attend very carefully to this matter of self-fertilisation may seem superfluous, but I know actual cases where practical men have attempted for several years to fix a new variety of a plant growing in the open ground by merely leaving the individuals uncovered, exposed to the visits of insects, though in an adjacent bed were plants of the original type from which the novelty had been derived. Year by year the proportion of plants which came true to the new variety continued very small, and the fact was accepted as a symptom of especial difficulty in fixing that particular variety. A few yards of muslin arranged as a cage over the plants and a few minutes spent in pollinating the covered flowers would have saved much further trouble, and the variety could have been raised true or "fixed" in one season. In reality, of course, the supposed "tendency to throw back" to the parent type was due simply to the pollination of the variety by insects which had visited the adjacent dominants.

The animal breeder, as he cannot self-fertilise his productions, must follow a rather more complicated procedure, but by the use of Mendelian methods he also can work with certainty. He must take his birds or other animals and test them for purity individually, usually by breeding each first with a recessive, and then having found a pure individual of each sex, he can by breeding these two together create material for building up a pure strain. 
Mendelian discovery at once abolishes the old delusion that time and continued selection are needed in order to make a variety breed true; for the homozygous individuals, which are the only ones that will breed true, may appear in $F_{2}$. It is the business of the breeder to find such individuals. By continued selection he may perhaps succeed ultimately, for at each selection he somewhat increases his chance of finding them, but by following Mendelian method he can go straight to the desired end, obviating many years' work.

\section{Unfixable Types.}

There are of course certain types which cannot be fixed at all, for the reason that their special character is not represented in their gametes, but is a special consequence of the meeting of dissimilar gametes. In animals the Andalusian fowl is a case in point.

The colours of Canaries are mostly of this class, and in order to obtain the requisite shades of yellow various crosses between pure coloured varieties are made, scarcely any being bred pure for exhibition. The "Golden Duckwing" of Game fowls is another heterozygous colour, and can be produced by crossing Silver Duckwing with Blackred. The only structural feature of this kind that I can name is the crest of the crested Canaries, which is always bred for shows by mating cresteds with plain-headed birds (R.E.C., 19; Davenport, IO5). The admired crest consists of long, drooping feathers radiating symmetrically from the top of the head in one of several approved patterns. This neatlylaid appearance is only produced when the bird is heterozygous for the crest-factor.

Among plants there are doubtless many examples*. For instance, among their pedigree strains of Primula Sinensis Messrs Sutton have met with two which are clearly of this nature. One is a large-flowered type known as "Giant Lavender," having a pale magenta flower. This never comes true from seed, throwing always a number of bright magenta-reds, and a corresponding number of whites more

* Compare Baur's case of Antirrhinum (p. 253), which like many other variegated plants proved unfixable because it is a heterozygous type. 
or less tinged with magenta, which evidently exhibit the two gametic elements that must be combined in one zygote in order to produce the Lavender.

In this case the bright magenta-reds immediately breed true when self-fertilised. In regard to the tinged whites there is a complication which might repay further study*.

There is another colour in Primula Sinensis which apparently cannot exist in a pure form. This is a peculiar shade of "crushed strawberry," and the two pure forms by the union of which it is formed are the deep crimson of "Crimson King," and the white with a bright pink eye brought out by Messrs Sutton under the name "I)uchess." By crossing these two together, the peculiar heterozygous colour can at once be produced.

But though such examples are not rare, they are in a minority, and speaking generally we may feel fairly confident that a given type can be made to breed true, and to perpetuate its good qualities indefinitely. A doubt should perhaps be expressed as to the possibility of fixing permanently such a property, as a high degree of fertility. Respecting the transmission of that character and the conditions governing its existence little is positively known, and to discuss the various indications properly is not possible within present limits; the remark must suffice that the evidence on the whole suggests that hopes of fixing permanently such a quality as excessive egg-production of

* When such tinged whites are self-fertilised, most of them are found to breed true. Some, however, in addition to their own type throw some lavenders, and occasionally even a magenta-red. This result has occurred both at Messrs Sutton's and also in the sowings which Mr R. P. Gregory and I have made. The fact is probably due to imperfect classification of the lavenders and tinged whites. These shades of colour are liable to vary somewhat, especially with temperature, colder temperatures causing more, higher temperatures less colour in the flowers. From our general experience of Primula colours we may feel assured that the white of the tinged whites in this case is due to the presence of some dominant inhibiting factor, which we may call $D$. The factor for colour, $C$, is common to all these plants. The bright magentas are $C C d d$, the lavenders are $C C D d$, and the tinged whites are $C C D D$. In all probability there is an irregularity in the dominance of $D$, for which we cannot at present account, such that a lavender may occasionally be so pale in tint as to pass for a tinged white. Further analysis would doubtless show that the number of these dubious plants is in fact definite, and possibly that their abnormal behaviour is due to the possession of some distinct element. 
fowls or excessive fertility in pigs should not be entertained with great confidence.

To avoid raising false expectations it should also be said that many of the small fancy points which distinguish individuals of the same breed from each other are rather of the nature of fluctuations than definite transmissible attributes. In the regulation of these finer details it is improbable that heredity plays any very prominent or at least assignable part. A Dutch rabbit, for instance, having the transverse division between the coloured and the white parts of the trunk exactly disposed as the fancier desires is scarcely if at all more likely to have offspring correctly marked than an average specimen of the same strain. In such cases all the fancier can do is to use strains otherwise good. A knowledge of genetic physiology will only help him here in so far as it may warn him not to pay extravagant prices for animals whose qualities are not genetic or transmissible.

The domesticated animals and plants of European countries have already been "brought to such perfection that in them the scope for new combination is reduced. Nevertheless the line of progress which Professor Biffen has inaugurated, by combining the resistance to rust-disease of one variety of wheat, with productiveness and other qualities derived from another variety, is one which should be capable of indefinite extension. It may be that resistance to disease is incompatible with some of the valuable qualities of animals and plants, but the attempt to produce these combinations should be made on the largest possible scale. The search for disease-resisting strains of animals and plants is hardly begun.

In its application to the improvement of the domesticated animals and plants of tropical regions the aid of Mendelian method may be expected to be more immediate and direct. A part from the actual creation of new types by re-combination much can undoubtedly be accomplished, as $\mathrm{Mr}$ W. L. Balls has indicated in the case of the Egyptian Cotton crop, by purification of the cultivated sorts. According to the traditional practice the "variety." used consists in reality of an immense number of distinct strains which under ordinary 
conditions are continually being crossed together by insect agency. To purify such crops by "rogueing" is an interminable and hopeless task. The proper course is to identify the factors governing the various characters, and having ascertained their relations to each other, to build up a desirable strain by individual selection. In former times the confusion of types in the crop would have passed for "variability." Mendelian analysis shows that representation of the facts to be entirely mistaken.

\section{Technical Methods.}

The technique of Mendelian experimentation is usually very simple. In crossing plants together the anthers of the plant to be used as female must of course be picked out with forceps before they dehisce. The other parts should be injured as little as possible. The flower is then covered to exclude insects. Muslin bags may be used for this purpose, but they are neither so convenient nor so safe as rain-proof bags made of parchment-paper, which most manufacturing stationers can now supply in any required size. The bag is put over the flower, the mouth being crushed up so as to fit to the stem, and it is fixed in place with a small bent piece of copper wire. If the stem be delicate a thin bamboo stuck in the ground must be also held in the copper clip. The use of the clip obviates all tying and untying. Muslin bags are objectionable for various reasons, especially because unless they are continually readjusted, flowers are sure to touch the sides and there is the risk of fertilisation by insects. Bees will often visit flowers covered by muslin, and I suspect that nocturnal moths may do the same. If it is desired to give ventilation, holes may be punched in the top of the paper bags, above the flowers. The flower from which pollen is to be taken must also be covered before it opens, in order to keep its pollen from pollution. There are indications that some of the results obtained by the older hybridists were confused by neglect of this precaution.

In transferring pollen the use of brushes is to be deprecated, as tending to the introduction of errors. The best plan is to pick out with fine forceps an anther from 
the flower to be used as male, and with it to touch the stigma of the female flower. The anther is then thrown away, unless the supply of pollen is very limited. Absolute cleanliness is of course most essential. The fingers and forceps should be continually cleaned with spirit in order to kill any pollen-grains adhering to them before proceeding to the next fertilisation. In operating on plants with much pollen like Oenothera, Poppies, \&c., care should be taken to avoid including in the bag leaves on which pollen has already fallen, and many other small precautions of this kind will occur to the experimenter in practice. For example, if muslin bags are used, they should not be laid on the greenhouse stage where the plants have been standing, for obviously the fallen pollen may adhere to them and be passed on to the stigmas.

In sowing fine or light seed in pans the presser with which the soil is patted down must be cleaned as each pan is finished. Otherwise it is liable to carry on seed to the next pan sown.

In recording results the capsule of seed resulting from a cross should have a register-number under which it is sown. Each plant which comes up must then be numbered separately, and its number is written as an index-number to the original number of the capsule. Thus, a pod of Emily Henderson Sweet Pea $q \times$ Blanche Burpee $\hat{\sigma}$ may, when sown in 1904 , have the number 20 . If seven plants come up, they are numbered $20^{1 \rightarrow}$. In 1905 each of these is sown under a fresh number. $20^{1}$ may for instance become I $5,20^{2}$ becomes I 6, \&c. The families I I 5, I I6, \&c., will each contain a great number of individual plants, and each of these from which the breeder intends to save seed must then receive an index-number $115^{1}$, I $15^{2}$, \&c., under which its characters are recorded in the register. In 1906 the offspring of each of these plants receives a fresh number for the year. The family of I I $5^{2}$ may become 305 , and so on. Reference is thus made easy, and the history of any individual plant can be rapidly traced. The same system can of course be easily modified so as to adapt it to the case of animals where the mating must be bi-parental. 


\section{Sociological Application.}

It may be anticipated that a general recognition of the chief results of Mendelian analysis will bring about a profound change in man's conceptions of his own nature and in his outlook on the world. Many have in all ages held the belief that our powers and characteristics are directly dependent on physical composition; but when it becomes known that the dependence is so close that the hereditary descent of certain attributes can be proved to follow definite predicable formulae, these ideas acquire a solidity they never possessed before, and it is likely that the science of sociology will pass into a new phase. The evidence at our disposal already proves that in many simple cases of defects and abnormalities the descent is of this definite order, and it is scarcely doubtful that future search will reveal comparable examples in abundance. As regards more complex phenomena of human inheritance, the descent of characters involving the coincidence of several factors, and effects due to interference between factors, a complete analysis may be unattainable; but even in some of these more obscure examples a close scrutiny will probably discover positive traces of regularity in descent of such a kind as to indicate in them also that the bodily or mental characteristic considered is a consequence of definite factorial composition. It is not in dispute that the appearance or non-appearance of a characteristic may be in part decided by environmental influences. Opportunity given may decide that a character manifests itself which without opportunity must have lain dormant. The question of opportunity and of the degree to which the conditions of life are operative in controlling or developing characters will some day demand attention, but in order to answer such questions successfully it is the first necessity that a knowledge of the genetic behaviour of the factors should be obtained. They are the fundamental elements, and the consequences of environmental interferences are subordinate to them. The previous attempts, experimental and statistical, to determine the results of changed conditions have led to quite inconclusive results because no pains were taken to ascertain that the material subjected to these various influences was genetically similar. 
In the pre-Mendelian period, indeed, such an expression had no definite meaning. From a knowledge of the physiology of descent under uniform conditions it may be possible to proceed to a determination of the consequences following a change in those conditions. We cannot investigate both unknowns at once. In the analysis of these wider problems we must begin with the more tractable of the two, and it has become obvious that this is to be found in the genetic aspect of the phenomena.

The outcome of genetic research is to show that human society can, if it so please, control its composition more easily than was previously supposed possible*. Whether such control should be exercised, or the form which it is to take, scarcely falls within the province of this text-book to discuss. Nevertheless, as many are already becoming urgent in advocating the practical application of genetic science to human affairs, some few words on that subject may be appropriate. Whatever course civilisations like those of Western Europe may be disposed to pursue, there can be little doubt that before long we shall find that communities more fully emancipated from tradition will make a practical application of genetic principles to their own population.

The power is in their hand and they will use that power like any other with which science can endow them. The consequence of such action will be immediate and decisive. For this revolution we do well to prepare.

Interference may take one or both of two courses.

* Mr F. Galton's long-continued efforts have at length been successful in directing public attention in some degree to the overwhelming importance of Eugenics. Some of the earlier attempts in the same direction are worth remembering. For example, Sir W. Lawrence frequently adverts to the subject in language almost identical with that now current. "The hereditary transmission of physical and moral qualities, so well understood and familiarly acted on in the domestic animals, is equally true of man. A superior breed of human beings could only be produced by selections and exclusions similar to those so successfully employed in rearing our more valuable animals. Yet in the human species, where the object is of such consequence, the principle is almost entirely overlooked. Hence all the native deformities of mind and body, which spring up so plentifully in our artificial mode of life, are handed down to posterity, and tend, by their multiplication and extension, to degrade the race." (W. Lawrence, Lectures on Physiology, Zoology, and the Natural History of Man, London, 3rd Ed., I823, p. 393. See also ibid. pp. 260 and 389.) 
Measures may be taken to eliminate strains regarded as unfit and undesirable elements in the population, or to encourage the persistence of elements regarded as desirable. From the standpoint of the sociologist these two kinds of interference may seem merely complementary to each other, but in the light of genetic physiology they are entirely different.

To the naturalist it is evident that while the elimination of the hopelessly unfit is a reasonable and prudent policy for society to adopt, any attempt to distinguish certain strains as superior, and to give special encouragement to them would probably fail to accomplish the object proposed, and must certainly be unsafe.

Comprehensive discussion of these questions would be quite out of place here. It must suffice to point out that the distinction is created partly by the fact that, whereas our experience of what constitutes the extremes of unfitness is fairly reliable and definite, we have little to guide us in estimating the qualities for which society has or may have a use, or the numerical proportions in which they may be required. But specially important are the indications that in the extreme cases, unfitness is comparatively definite in its genetic causation, and can, not unfrequently, be recognized as due to the presence of a simple genetic factor. There is as yet nothing in the descent of the higher mental qualities to suggest that they follow any simple system of transmission. It is likely that both they, and the more marked developments of physical powers, result rather from the coincidence of numerous factors than from the possession of any one genetic element.

Some serious physical and mental defects, almost certainly also some morbid diatheses, and some of the forms of vice and criminality could be eradicated if society so determined. That however is the utmost length to which the authority of physiological science can in the present state of knowledge be claimed for interference. More extensive schemes are already being advocated by writers who are neither utopians nor visionaries. Their proposals are directed in the belief that society is more likely to accept a positive plan for the encouragement of the fit than negative interference for the restraint of the unfit. Genetic science, as I

B. $\mathrm{H}$. 
have said, gives no clear sanction to these proposals. It may also be doubted whether the guiding estimate of popular sentiment is well-founded. Society has never shown itself averse to adopt measures of the most stringent and even brutal kind for the control of those whom it regards as its enemies.

Genetic knowledge must certainly lead to new conceptions of justice, and it is by no means impossible that in the light of such knowledge public opinion will welcome measures likely to do more for the extinction of the criminal and degenerate than has been accomplished by ages of penal enactment. 


\section{APPENDIXES}

Since this book first appeared advances have been made which extend and in some cases modify several statements contained in the previous chapters. Pending an opportunity of re-writing the whole, some of these researches are mentioned very briefly in the following paragraphs. I have arranged them in the order followed in the book, so that they may be read as annotations to the several chapters.

The work done in the past three years with a bearing on Mendelian Principles has been of vast extent, and the contributions here enumerated are those only which either for extent or for novelty seem especially important.

Appendix to Chapter II. Structural Characters.

Peas. As to inheritance of Stature and the Time of flowering see Keeble, F. and Pellew, C., Jour. Gen., I. I 9 ro, p. 47. The following characters were found to be dominants, Thick stem, Long internode, Late flowering.

Potato. Elaborate investigation of numerous characters. Salaman, R. N., Jour. Gen., I. I910, p. 7.

Capsella. Several leaf-characters analysed by Shull, G. H., "Bursa bursa-pastoris and Bursa Heegeri biotypes and hybrids," Publication No. I I 2 of Carnegie Institution, I 909 .

Beta. Kajanus (Zts. f. indukt. Abstam. u. Vererbungslehre, I9I I, vI. p. I37) describes experiments on the inheritance of sugar-beets and mangels. Besides colours, which were found to be controlled by very numerous factors, some structural chararters were-investigated, especially the shape of the bulb and the number of leaves. The results were complex and some apparent inconșistencies were met with. 
Brassica. Kajanus has investigated the genetics of some species of this genus, particularly napus and rapa and their hybrids. He confirms older observations as to the production of lumpy outgrowths (resembling "finger and toe") as the result of certain such crosses, and in this respect he found remarkable differences between reciprocals. Zts. f. ind. Abstam., I 9 I 2, vi. p. 2 I 7.

Cotton. Leake found the sympodial habit partially dominant over the monopodial, with many forms of blending between the two types. The inheritance of the leaf-shapes was determined by a special method of measurement and it was found that certain shapes were always heterozygous. Leake, H. M., Jour. Gen., I 9 I I, p. 205.

Tomato. Genetic properties of several characters, especially leaf-shape, investigated by Groth, A., Bulletins 238 and 239. New Jersey Agricultural Station, I 9 I I.

Cattle. Evidence as to inheritance of long and short legs, and red or black colour in Dexter Kerry. Wilson, J., Sci. Proc. R. Dublin Soc., xir. I 909, p. I.

Dogs. Genetics of several characters investigated by A. Lang, Zts. f. ind. Abstammungslehre, III. I9IO, p. I.

\section{Colour-Characters. Plants.}

Antirrhinum. Full and minute analysis of colours, for the most part confirming Miss Wheldale's results, and adding a remarkable case of coupling (see later, p. 318 ). Baur, E., Zts. f. ind. Abst., I9IO, III. p. 34. An account of Mendelian heredity in the case of a cross between distinct species of this genus was given by Baur at the Genetics Conference, Paris, I9II, but publication has not yet been made.

Cotton. Curious case of complete correlation between small size of petals and white colour, and large size and yellow colour. Leake, H. M., Jour. Gen., I. I9I I, p. 24 I.

Digitalis. Analysis of flower-colour, with evidence that white may be a dominant. Keeble, Pellew, and Jones, W. N., New Phytologist, Ix. I910, p. 68.

Helianthus. Discovery of a new red-flowered variety. The heterozygote with the yellow type is intermediate in colour. Cockerell, T. D. A., Pop. Sci. Monthly, I9I2, p. 373 . 


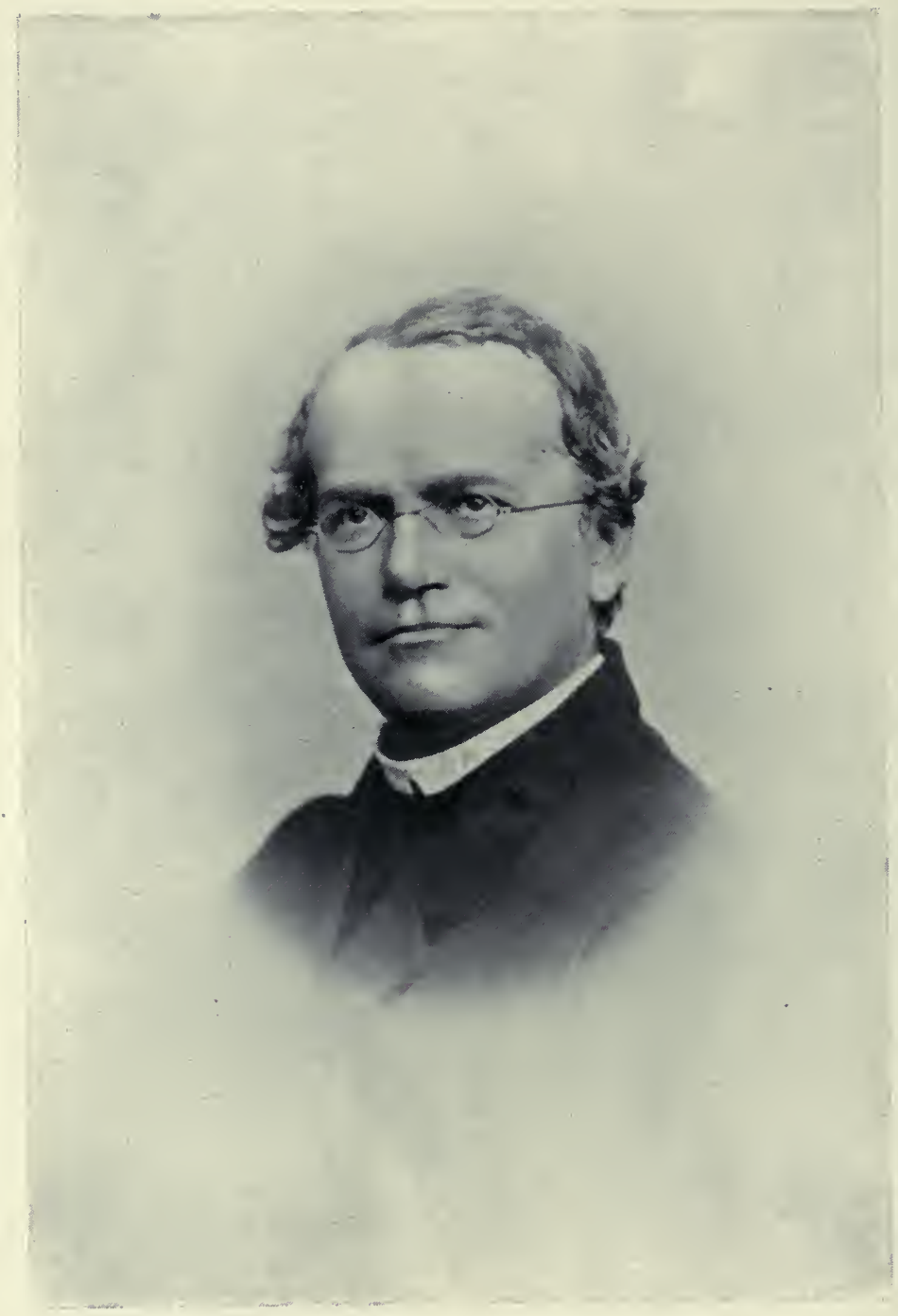

GREGOR MENDEL

About the year 1862 
Mirabilis. Full analysis of the self-coloured types, correcting the account previously given by Correns. Marryat, D. C. E., Rep. Evol. Ctee. Roy. Soc., 1909, p. 32.

Primula Sinensis. Genetics of colour-characters in flowers and stems; also of several structural characters. Gregory, R. P., Jour. Gen. I. I9I I, p. 73.

Colour-Characters. Animals.

Cattle. Wilson, J., "The colours of Highland Cattle," Sci. Proc. R. Dublin Soc., I 909, xII. (N. S.) p. 66, contains evidence as to the relations between dun, brindled, and other colours hitherto little studied.

Mice. Analysis of the coat-colours of mice having pink eyes. Durham, F. M., Jour. Gen., I. I91 I, p. I59.

Analysis of coat-colours of mice in general with discussion of the relation of the phenomena to those seen in other rodents. Incidentally (p. I 26) evidence is given as to a case in which the factor for colour seemed to repel the agouti factor. Hagedoorn, A., Zts. f. ind. Abstami., I9I I, vi. p. 99.

Horse. Factors for the various colours provisionally worked out. Sturtevant, A. H., Jour. Gen. II. I9I2, p. 4I. Walther, A. R., Beitr. zur Kenntniss d. Vererbung $d$. Pferdefarben. Hannover, M. and R. Schaper, I I I I 2. Wilson, J., Sci. Proc. R. Dublin Soc., igro, xiIr. (N.S.) p. 33I and ibid. xiir. (N.S.) I9I2, p. I84 (especially regarding dun colour).

From these researches it seems to be established that in the horse chestnut is the lowest colour in the series, and that white is epistatic to all. As to the order of the other factors there is not complete agreement. Sturtevant gives reasons for the view that black stands next above chestnut, and bay-or-brown taken as one indivisible type next above black. Grey and roan come above these and below white. Walther has much useful information as to the behaviour of the peculiar piebald and spotted types.

Pigeons. Relations of black, blue, dun and silver. R. Staples-Browne, Jour. Gen. II. I9I 2, p. I3I. Further notes on colours of Pigeons; Doncaster, ibid. p. 89. Interrelations of various colours, especially concerning Grizzles and Mealies; Bonhote, J. S. and Snalley, F. W., P.Z.S., 
I9II, p. 60I. The authors disagree with some of StaplesBrowne's conclusions. He had held that blue was a dilute form of black, and that silver is similarly a dilute form of dun; but Bonhote and Smalley came to the conclusion that silver is dilute blue. It is not in dispute that blues do not throw blacks, and that silvers do not throw duns, and that both blue and dun are recessive to black. As however there is no microscopical or chemical test by which the nature of the pigments can be certainly distinguished the point in question cannot be positively decided at present.

\section{LEPIDOPTERA.}

[For sex-limited cases in Colias, \&c. see Appendix to Chap. X.]

Aglia tau and the dark vars. lugens, \&c. have been investigated again by Standfuss, see especially Iris, 1910, xxiv. p. I55, and Deut. Entom. National-Bibliothek, I9I0, I. In these experiments the peculiar sex-distribution before recorded was not seen again. For an interpretation of Standfuss's results, see Plate L, Arch. Rass. u. Gesel., I9Io, Hft. 6, p. 678.

Pygaera. Important paper on species-hybrids. Federley, H., Arch. Rassen u. Gesel.-Biol., I 9 I 1.

Bombyx mori. Extensive series of experiments on genetics of Silkworms. Kellogg, V. L., Leland Stanford Univ. Publ., No. I, I908. As regards the colours of cocoons many special idiosyncrasies of strains and of individuals were found, which have not yet been analysed successfully. The larval colours were more regular in their behaviour.

\section{Diptera.}

Drosophila ampelophila has been the subject of elaborate investigation by Castle and his assistants (see especially Proc. Amer. Acad. Arts and Sci., XLI. I 906) with special regard to the genetics of fertility; by T. H. Morgan (chief paper in Jour. Exp. Zoology, I 9 I I, Vol. XI. p. 365), who has discovered many recessive forms, and shown that remarkable cases of sex-limited inheritance occur (see Appendix to Chap. X.) ; and by Lutz (Carnegie Institution, 
Publication No. I43, I9II), who has investigated the inheritance of abnormal venation on a very large scale, chiefly with negative results which suggest that these features are largely fluctuational.

\section{Mollusca.}

Lang, Zts. indukt. Abstam., I9 I I, v. p. 97, has continued and extended his researches on the genetics of $H$. nemoralis and hortensis, and has found that in some cases, as previously recorded, when these two species are crossed, genuine hybrids are produced; but in other instances the results of the cross are purely maternal. This evidence naturally suggests a comparison with the observations made by those who have experimented with Echinoderm hybrids. The literature of this latter subject is too extensive for consideration on this occasion, and as no one has yet succeeded in rearing the products to maturity the genetics of the various species are still unknown.

\section{Appendix to Chapter IV.}

The analyses made by Nilsson-Ehle of the genetic properties of Cereals have led him to the conclusion that several factors all possessing the same property may coexist in one individual. For example, he gives evidence that a certain wheat has in it three distinct factors having the power of producing the red colour. Obviously such possibilities must be remembered in future analyses, and to them may perhaps be due various numerical aberrations hitherto unelucidated. See especially, H. Nilsson-Ehle, Kreuzungsuntersuchungen an Hafer und Weizen, Lund, I909, p. 69.

Some new types of ratio, brought about by the interaction of various factors leading to the masking of some of the terms in the series, are discussed and illustrated by Shull, G. H., Amer. Nat., 1908, p. 433.

\section{Appendix to Chapter VII.}

As stated above (p. 309) the analysis of the colours of Horses has now been carried somewhat beyond the point reached in this discussion. 
Appendix to Chapter VIII.

In this chapter an attempt is made to discuss the relation of self-coloured to pied types in the simplest possible cases, such as that of the self-coloured and pied rabbits. All further researches on this subject tend to show that it is one of great complexity. Even in animals the interrelations of the various degrees of piedness are not established satisfactorily for any one example, and in plants there are evidently phenomena to be explored which as yet evade our analysis. In the case of the flaked flowers of Mirabilis for example the experiments of Miss Marryat showed that though the numbers suggest orderly behaviour, it was not possible yet to express them in factorial terms, and the same is true for Primula Sinensis. In regard to variegation of the leaves of Mirabilis Correns has published some very remarkable evidence. In an earlier paper, $Z$ ts. $f$. indukt. Abstam., I 909, I. p. 29I*, he showed that a peculiar variegated type which he called variegata usually behaved as a recessive to the normal green. Sometimes however this variegated form bears a branch entirely green. The self-fertilised offspring of the variegated and of the green branches were separately studied; and it was found that the variegated branches gave families consisting almost entirely of variegated $+a$ small and irregular percentage of greens; but the green branches gave normal Mendelian families, of 3 greens : I variegated, 25 per cent. of the greens being pure greens, the rest heterozygous. The variegateds and the greens, of whatever origin, behaved in the same way.

As regards the striping of the flowers in Mirabilis Correns has also provided some new evidence. When a plant bears both striped branches and unstriped branches, each type produces offspring which in the great majority resemble itself. The striped offspring then throw a small and irregular proportion of self-coloured, but the self-coloured consist of heterozygotes and homozygotes approximately in the ratio $2: \mathrm{I}$. The facts in Correns's opinion indicate that a homozygous branch can by some change pass into a

* For further discussion of cognate phenomena see Correns, ibid. II. I909, p. 33 I. 
heterozygous condition, and he argues that observations of de Vries on Veronica, and of MacDougal on Oenothera indicate the possibility that this process may be reversed, a heterozygous branch becoming a homozygous dominant. (Correns, Ber. Deut. Bot. Ges., xxviII. I9Io, p. 433.)

The whole problem is evidently one of great obscurity, but the facts suggest that a clear understanding of the genetics of these variegated forms may give light on the nature of segregation in general.

This paper of Correns should be read in connexion with that of Erwin Baur on the periclinal variegation of Pelargonium (Zts. ind. Abstam., I. 1909, p. 330)*, in which one of the most notable of recent advances in genetic physiology is made. Plants with green inside and white epidermis and sub-epidermal layers have exclusively albino offspring, while plants in which the inside is white and external layers green have exclusively green offspring. Similarly when the external layers are golden the offspring are greens and goldens, just as when derived from wholly golden plants (see p. 253). [The last two statements I have myself confirmed experimentally, but with the albo-marginatae I have hitherto failed to obtain offspring.] The characters of the cells of the sub-epidermal layer thus indicate the characters of the germ-cells. In view of this discovery of Baur's we are led to speculate whether the sub-epidermal layer of any heterozygote is not in reality a patchwork of cells, bearing or not bearing a given factor, and that according as this patchwork is coarse, or fine (as in an emulsion) we obtain aberrant or expected numbers in our ratios. It must be remembered that external appearance is only an imperfect guide to the genetic properties. For instance Tropaeolum with variegated leaves breeds nearly if not quite true in this respect, though we might expect its germ-cells to be green, yellow, or mosaic in nature. If in the mixtures of substances capable of forming coarse or fine emulsions we have a true picture of the heterozygous state, such phenomena as those observed by Correns for the green and variegated branches become capable of representation without any great straining of the facts. Segregation on this view is regarded as a phenomenon

* See also ibid. iv. 1910, p. 81 . 
capable of occurring at any cell-division, and not merely in gameto-genesis. The moment of segregation will thus probably be found to vary with different types and with different factors, and for some it is likely that great irregularity in this respect will be found as a common occurrence. In this way can we best hope to reconcile the facts that with certain types such as wheat and barley the expected ratios are almost always approximately realised, whereas in other types, such as Matthiola there is great fluctuation in the numbers from individual pods, as Miss Saunders assures me, though when large series are taken the normal ratios are usually approached.

\section{Appendix to Chapter IX.}

The result of further experiment has led to a great simplification of the views as to coupling and repulsion given in the text. We have now proof that coupling and repulsion are consequences which may affect the same pair of factors. If $A B \times a b$ gives an $F_{1}$ in which $A$ and $B$ are coupled, then $A b \times a B$ will give $F_{1}$ in which $A$ and $B$ are "repelled." It is moreover established in three cases that the appearance of "repulsion" is in reality a consequence of the fact that the gametes $A b$ and $a B$ are represented in numbers larger than those in which $A B$ and $a b$ occur. In other words, if $A B \times a b$ gives $F_{1} A a B b$ having gametes

$$
7 A B: \text { i } A b: \text { г } a B:: 7 a b \text {; }
$$

then $A b \times a B$ will give $F_{1}, A a B b$ having gametes

$$
\text { I } A B: 7 A b: 7 a B: 1 a b \text {, }
$$

or at least displaying some similar coupling. We have as yet no sufficient data for asserting that the actual numbers will be the same in the two cases, though this is perhaps the simplest expectation.

The essential phenomenon in each case is the formation by the heterozygote of a greater number of germ-cells representing those combinations of factors which were possessed by the original parents, and fewer germ-cells representing the new combinations*.

* I have only lately seen Emerson, R. A., "Genetic Correlation and Spurious Allelomorphism in Maize," 24th Ann. Rep. Nebraska Agric. Exp. Sta. I III, in which this possibility is pointed out already. 
To distinguish the two kinds of $F_{1}$ we ought to write $A B \times a b$ as $A B, a b$; and $a B \times A b$ ought to be written $a B . A b$. We must in fact recognize that in the dividing zygotic cell, at some time before segregation is effectedprobably from the moment of fertilisation-a polarity is established; and that the constituents derived from the two parents are not, in these cases at least, placed indifferently, but are grouped according to their parental origin.

Hence we may construct the following table:

\begin{tabular}{|c|c|c|c|c|c|c|c|c|c|c|c|}
\hline \multirow{4}{*}{$\begin{array}{l}\text { Partial } \\
\text { repulsion }\end{array}$} & \multicolumn{4}{|c|}{ Gametic series } & \multirow{2}{*}{$\begin{array}{l}\text { Number } \\
\text { of gametes } \\
\text { in series }\end{array}$} & \multirow{2}{*}{$\begin{array}{l}\text { Number of } \\
\text { zygotes } \\
\text { formed }\end{array}$} & \multicolumn{4}{|c|}{ Nature of zygotic series } & \multirow[b]{2}{*}{$a b$} \\
\hline & $A B$ & $A b$ & $a B$ & $a b$ & & & $A B$ & $A b$ & $a b$ & & \\
\hline & $\mathbf{I}$ & $(n-\mathrm{I})$ & $(n-1)$ & $\mathbf{I}$ & $2 n$ & $4^{n^{2}}$ & $2 n^{2}+\mathrm{I}$ & $n^{2}-1$ & $n^{2}-\mathrm{I}$ & & I \\
\hline & $\mathbf{I}$ & $3 I$ & 31 & I & 64 & 4096 & 2049 & 1023 & 1023 & & $\mathbf{I}$ \\
\hline from zygote & $\mathbf{I}$ & I5 & $I_{5}$ & $\mathbf{I}$ & 32 & 1024 & 513 & 255 & 255 & & $\mathbf{I}$ \\
\hline of form & $\mathbf{I}$ & 7 & 7 & $\mathbf{I}$ & 16 & 256 & 129 & 63 & 63 & & $\mathbf{I}$ \\
\hline$A b \times a B$ & $\mathbf{I}$ & 3 & 3 & I & 8 & 64 & 33 & I 5 & I5 & & $\mathbf{I}$ \\
\hline & $\mathbf{I}$ & I & I & I & 4 & 16 & 9 & 3 & 3 & & I \\
\hline & 3 & I & I & 3 & 8 & 64 & $4 I$ & 7 & 7 & & 9 \\
\hline couplino & 7 & I & I & 7 & I6 & 258 & 177 & 15 & I5 & & 49 \\
\hline from zygote & 15 & I & I & 15 & 32 & 1024 & 737 & 31 & 31 & & 225 \\
\hline $\begin{array}{l}\text { Hom zygote } \\
\text { of form }\end{array}$ & $3 I$ & I & I & 31 & 64 & 4096 & 3009 & 63 & $6_{3}$ & & $96 I$ \\
\hline$A B \times a b$ & 63 & I & I & 63 & I 28 & I6 $38_{4}$ & 12161 & 127 & 127 & & 3969 \\
\hline$A B \times a b$ & $(n-1)$ & I & $\mathbf{I}$ & $(n-1)$ & $2 n$ & $4 n^{2} \quad 3 n$ & $2-(2 n-I)$ & $2 n-1$ & $2 n-\mathrm{I}$ & $n^{2}-$ & $(2 n-1)$ \\
\hline
\end{tabular}

For details as to these phenomena see Bateson and Punnett, Proc. Roy. Soc. I9 I I, vol. 84 B, p. 3; de Vilmorin and Bateson, ibid.; Gregory, ibid.; Bateson and Punnett, Jour. Gen., I. I9I I, p. 293.

The evidence has been obtained from observation of various characters in Sweet Pea; the relations of tendrilled leaves to the "Acacia" form in Pisum, and of Magenta to Short-style in Primula Sinensis (Gregory).

Now that we may regard the formation of four cells of composition $A B, A b, a B, a b$, as the foundation both of the coupling- and of the repulsion-series the problem is manifestly somewhat simplified. The time, excluding gametogenesis, at which we can most readily imagine four such definite quadrants to be formed is during the delimitation of the embryonic tissues. It is then that the plant is most clearly a single geometrical system. Moreover the excess of gametes of parental composition characterising the coupling- and repulsion-series must certainly mean that the position of the planes of division by which the four quadrants are constituted is determined with regard to the gametes taking part in fertilisation. Though the relative 
positions of the constituents of the cells may perhaps be maintained throughout the history of the tissues, it is easier to suppose that the original planes of embryonic division are determined according to these positions than that their influence can operate after complex somatic differentiation has been brought about.

At some early stage in the embryonic development or perhaps in later apical divisions we can suppose that the $n-I$ cells of the parental constitution are formed by successive periclinal and anticlinal divisions of the original quadrants which occupy corresponding positions. The accompanying diagram gives a schematic representation of the process as we imagine it. Obviously it does not pretend to give more than a logical or symbolic presentation
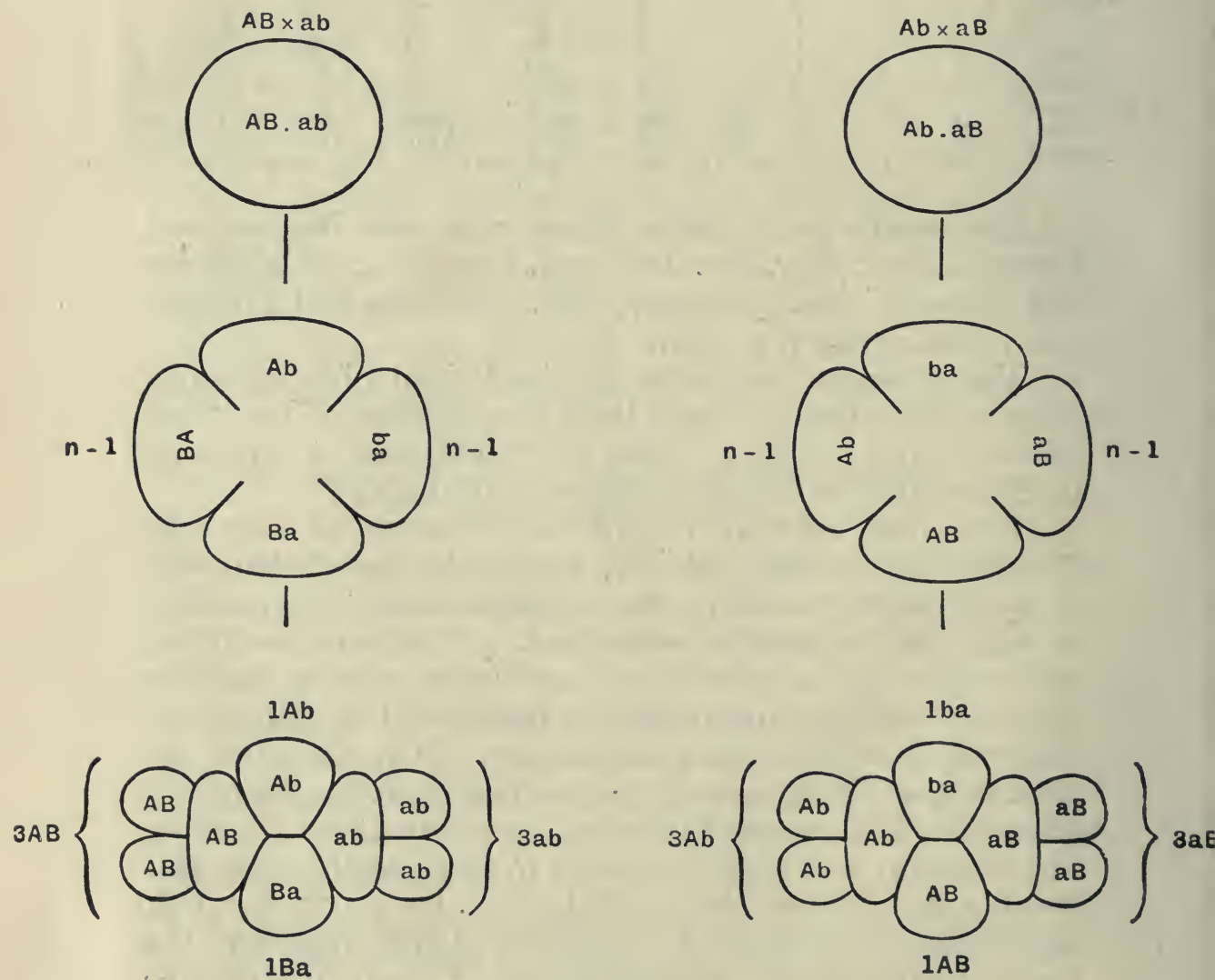

Fig. 39 . 



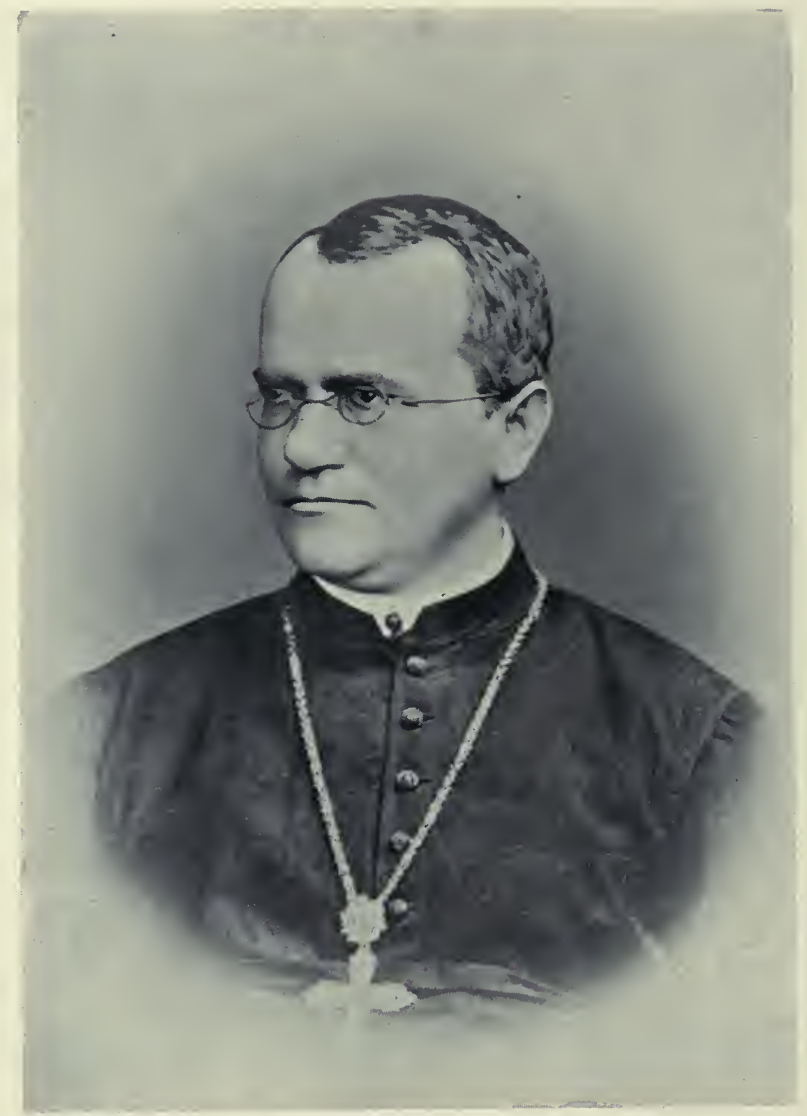

GREGOR MENDEL AS PRÄLAT

Taken about the year 1880 
of the phenomena. If such a system of segregation is actually formed at the apex, it must be supposed that the axes of the system revolve with the generating spiral.

Whatever hypothesis be assumed, the following points remain for consideration.

I. We are as yet unable to imagine any simple system by which the four original quadrants can be formed by two similar divisions. Evidently there must be two celldivisions, and if in one of them we suppose $A B$ to separate from $a b$, we cannot then represent the formation of $A b$ and $a B$. Therefore we are almost compelled to suppose that the original zygotic cell forms two similar halves, each $A a B b$, and that the next division passes differently through each of these two halves, in the one half separating $A B$ from $a b$, and in the other half separating $A b$ from $a B$. The formation of these four quadrants must take place in every case in which there is segregation in respect of two pairs of factors. (For three pairs there must similarly be eight segments, and so on.) The axes of this system may well be determined by the position of the constituent parental gametes. Reduplication or proliferation resulting in $n-I$ gametes may then take place in either of the opposite pairs of quadrants according to the parental composition.

2. If in the gametes of any plant some factors are distributed according to one of the reduplicated series and other factors according to the normal Mendelian systemas we know they may be-the segregations by which such a system is brought about cannot have happened simultaneously. Moreover if various reduplications can take place very early in some individuals and not in others, we cannot imagine how the normal form of the plant remains unchanged, unless these reduplications affect tissues originally set apart as germinal.

As possibly significant we note here the fact that in the embryonic development of plants the order of the various divisions is known to be subject to great variation and it is not inconceivable that such disturbances of the order in which the planes of division occur may indicate variations in the process of segregation*. p. 187 .

* See Coulter and Chamberlain, Morphology of Angiosperms, I903, 
3. We do not yet know whether independent reduplicated systems can be formed in the same individual. In the sweet pea for instance we have not yet seen the consequences of combining blue, erect standard, and long pollen with the fertile-sterile, dark-light axil series, and much may be discovered when such families come to be examined.

The phenomena seen in animals may well be produced by the segmentations in which the parts of the ovary or testis are determined. Hitherto no case of coupling has been found in animals. Among the phenomena of repulsion, however, of which many examples exist, certain suspicious cases have been observed which may mean that in animals reduplicated systems exist like those of the plants. Nevertheless at present it seems not impossible that the two forms of life are really distinguished from each other in these respects.

Lastly, in view of what we now know, it is obvious that the terms "coupling" and "repulsion" are misnomers. "Coupling" was first introduced to denote the association of special factors, while "repulsion" was used to describe dissociation of special factors. Now that both phenomena are seen to be caused not by any association or dissociation, but by the development of certain cells in excess, those expressions must lapse. It is likely that terms indicative of differential multiplication or proliferation will be most appropriate. At the present stage of the inquiry we hesitate to suggest such terms, but the various systems may conveniently be referred to as examples of reduplication, by whatever means the numerical composition of the gametic series may be produced.

It remains to be seen whether systems of reduplication not contemplated by this scheme will be proved to exist, but it should be mentioned that Baur has already shown the probability that other ratios, especially $6: 1: 1.6$, occur in Antirrhinum (see Zts. f. ind. Abstam., I912, vi. p. 20I); and once it is realized that the phenomenon is probably one of reduplication of certain terms, there seems no reason for supposing that various less simple series will not be found. 
Appendix to Chapter $X$.

With respect to sex-limitation a great deal of recent evidence has been discovered. Several characters in Poultry have been shown to follow the same system of descent as the black colour of Canaries. Especially may be mentioned the barred markings of Plymouth Rock (Pearl and Surface, Arch. Entrum. xxx. 1910). This barring is a dominant, males being homozygous and females heterozygous. The females crossed with black males give black females and barred males.

Staples-Browne (Jour. Gen., I9I 2, II. p. I 58) gives additional evidence that the brown colour of the Barbary Dove is similarly sex-limited, crosses with a white male giving usually dark males and white females.

Gerould (Amer. Nat., I9I I, p. 257) has investigated the case of the white females of Colias, showing that white is probably dominant in the female, recessive in the male; and much work has been done in reference to the polymorphic females of tropical butterflies, several of which have been shown with great probability to follow a simple Mendelian scheme in their descent (see especially Punnett, Mendelism, ed. 3, I9I 1, p. I34; Spolia Zeylanica, 1910, viI. in which the case of Papilio polytes, aristolochiae, and hector is elucidated).

The subject upon which the most extensive work has been done is Drosophila ampelophila, the pomace-fly, especially by T. H. Morgan (chief paper in Jour. Exp. Morph., I9I I, vol. xI). The eyes are of several colours, dark red, vermilion, orange, white, \&c., and complex rules of sex-limitation have been found governing their descent. The simplest case is that of red eye and white eye, which follow the same rules as colour-blindness in man, white eye being the equivalent of colour-blindness. The whole subject is full of interest, but could only be made clear at great length.

In regard to colour-blindness and analogous phenomena Doncaster has made a suggestion of great importance (Jour. Gen., I9I I, I. p. 377). Hitherto we have supposed, following the analogy of horns in sheep, that the colour-blindness must be the dominant, for the reason that the normal male. 
cannot transmit it. Doncaster shows that by assuming both sexes to be heterozygous for sex-determiners it is possible to construct a scheme on which normal vision is the dominant, and colour-blindness the recessive, which will express all the facts, and is in my judgment on the whole the most probable account of these phenomena yet suggested. Substituting white eye for colour-blindness, the same scheme expresses Morgan's observations for red and white eye in Drosophila.

Doncaster's scheme is thus expressed: "Since the male transmits the factor for colour-blindness only to his daughters, it must be assumed that the male in this case is heterozygous for the sex-determiner. In former papers I have suggested that if maleness is determined by a factor $\hat{\delta}$, femaleness by a factor $q$ epistatic to $\hat{\delta}$ when both are present, then a male individual may be represented $\hat{0} 0$, a female $\hat{\delta} q$; i.e. that both sexes are heterozygous for sexdeterminers, with selective fertilisation between $\hat{\delta}$-bearing eggs and $O$-bearing spermatozoa, and between + -bearing eggs and $\delta$-bearing spermatozoa. If we adopt this scheme

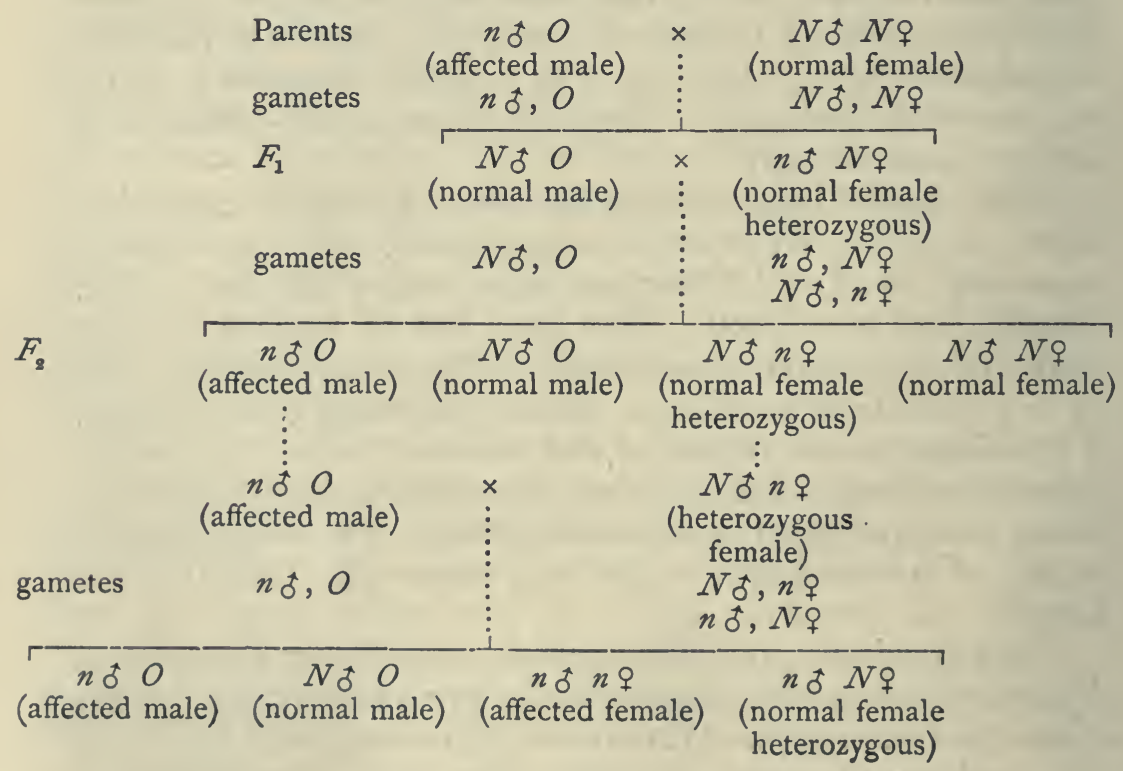

* Doncaster, ibid. p. 378 . 
as a working hypothesis, and then represent normal sight by $N$, colour-blindness by absence or modification of $N(=n)$, and further suppose that $N$ can only be borne by gametes containing a sex-determiner ( $\hat{0}$ or $q$, not $O$ ), we obtain the observed results."

Facts bearing on the general theory of sex-determination have accumulated so fast that they would require a separate treatise for adequate enumeration and discussion. Reference can here be made to one group of experiments alone, those of Goldschmidt (Zts. indukt. Abstam., I9I 2, viI. p. I), in which phenomena of extraordinary novelty and significance are recorded. The most striking experiment was that in which the moths Lymantria dispar and japonica were reciprocally crossed.

L. japonica o $\times$ dispar $\hat{\alpha}$ gives constantly both sexes hybrid, showing mixture of the parental characters.

The reciprocal, $L$. dispar $+\times$ japonica $\hat{\delta}$, gives families which at first sight seem to be all males, but on closer examination it is found that in reality they consist of normal males and gynandromorphs having the characters of the sexes mixed in various degrees. From these gynandromorphs it was however possible to breed, and subsequent generations were obtained from both types of crosses. The results were complex and difficult to interpret, but an analysis of great interest is suggested by Goldschmidt.

In connexion with this paper reference should be made to another curious series of results obtained by Kuttner (Intern. Rev. Gesamt. Hydrobiol., II. 1909) in an investigation of the descent of sex in Daphnia. In this case also the descent from certain peculiar forms called "pseudohermaphrodites" was studied. Actual hermaphrodites regarded as originally females and others regarded as originally. males occurred in these series. The numbers obtained were considerable, but as yet no satisfactory analysis has been made.

\section{Appendix to Chapter XI.}

Two important contributions to the investigation of the genetics of doubleness have been made by Miss Saunders. A factorial analysis is provided by which the descent of

B. H. 
doubleness in Stocks is approximately represented. Taking $X$ and $Y$ as two factors, either of which is sufficient to make the flower single, and representing the ever-sporting type of single as $X x Y y$, it is shown that the inheritance from such a plant is given by a scheme in which the ovules are arranged in a system

$$
\text { I } 5 X Y: \text { I } X y: \text { I } x Y: \text { I } 5 x y
$$

and the pollen is all $x y$.

To represent the descent from such a plant, if it be also heterozygous for white and cream plastids, much more complex expressions are provided. In these it is suggested that the factors $X, Y$, and $W$ (the factor for white plastids) or any two of them may behave as inseparable in certain ovules, and as separable in others. In the light of the new facts as to coupling and repulsion this representation should be regarded as provisional only. (For the evidence see Saunders, E. R., Jour. Gen., I 9 I I, I. p. 303.)

In the same Journal, I. I9IO, p. 57, Miss Saunders describes experiments with Petunia, which show that the pollen of singles is always single so far as has been determined, but the ovules of singles, of whatever source, consist of a mixture of singles and doubles. Thus, any mixture fertilised by a double, gives a mixture of singles and doubles, but the singles thus produced will not give doubles, whether self-fertilised or crossed inter se. It is not as yet clear why the interbreeding of singles should give no pure singles, but that nevertheless seems to be the fact.

Appendix to Chapter XII.

In regard to inheritance of sex-limited conditions, see Appendix to Chap. $\mathrm{X}$.

The whole subject of the inheritance of human diseases and malformations is now being rapidly explored, and for extensions of knowledge on this branch of inquiry the reader should consult the Treasury of Human Inheritance, and especially the Transactions. of the Ophthalmological Society, in which the papers of E. Nettleship appear. Among the latter is a collection of evidence as to hereditary nystagmus (ibid., xxxi. I91 I), a condition following lines of descent similar to those of colour-blindness. 
Appendix to Chapter XIV.

Parthenogenetic cases. To the cases in the text must be added the evidence of Przibram as to Mantis, Arch. Entrom., 1909, xxviII. pp. 602 and 61 2, and of Lang, as to Helix nemoralis and hortensis (Zts. indukt. Abstam., I9I I, v. p. I 35 ).

Sexual cases. Reference was made in the text to de Vries's observation that the hybrid Oenothera muricata $\times$ biennis breeds true. In a recent paper (Biol. Cbltt. I9I I, xxxi. p. 97) he has briefly published a statement of the very remarkable results which will probably provide an elucidation of this and other paradoxical cases. It appears that reciprocal crosses between these two species give different hybrid types, and that both breed true. Next, calling the reciprocal hybrids $M B$ and $B M$, it was found that $M B \times B M$ gives only muricata, and that $B M \times M B$ gives only biemnis! De Vries conjectures that this behaviour may be due to abortion of a class of gametes on each side ; but I am inclined to suspect that we should rather compare the phenomenon with that seen in those Stocks and Petunias in which the male and female parts of the same plant have a different genetic constitution. However that may be, we must anticipate that the study of the results of reciprocal crosses will lead to a great extension of genetic science.

As to lop-eared Rabbits, and the alleged absence of segregation in crosses between long and short-eared races, see A. Lang, ibid., I910, IV. p. I, who argues that the observed data are not inconsistent with the possibility that there is segregation of numerous factors.

Much information additional to that contained in this volume will be found in the three text-books which have appeared since :

Johannsen, W. Elemente der exakten Erblichkeitslehre. Fischer, Jena, 1909.

Baur, E. Einfiihrung in die Experimentelle Vererbungslehre. Berlin, I9I I.

Punnett, R. C. Mendelism, ed. 3, I9Ir. 



\section{PART II}

เ. BIOGRAPHICAL NOTICE OF MENDEL

2. TRANSLATION OF THE PAPER ON HYBRIDISATION

3. TRANSLATION OF THE PAPER ON HIERACIUM 



\section{BIOGRAPHICAL NOTICE OF MENDEL*.}

Gregor Johann Mendel was born on July 22, i 822 , at Heinzendorf bei Odrau, in the "Kuhland" district of Austrian Silesia. His father was a small peasant proprietor, being the first of the family to raise himself to that degree, and he held his land by a kind of socage, performing "Robot" (agricultural labour) for the lord.

The name Mendel suggests a Jewish origin, but it is practically certain that the suggestion is incorrect. The family appears in the Church Register of the seventeenth century - the earlier ones were burnt by the Hussitesusually under the name Mandel, whereas it was not till the reign of Joseph II (I765-I790) that the Jews in Austria assumed definite surnames. At the time of the Thirty Years' War Kuhland was a protestant district, and several of Mendel's ancestors were of that persuasion. His four grandparents were all of the local Heinzendorf stock, which may be described as a German colony surrounded by a

* I regret that the short paragraph which I published in 1902 respecting Mendel's career contained several inaccuracies. The materials supplied to me were meagre and in many respects incorrect. Somewhat fuller sources of information are before me now. Of these the chief is an annotated report of the oration delivered by Mendel's nephew, Dr Alois Schindler, in July, I902, at the unveiling of a memorial tablet at Heinzendorf. Other facts are to be gathered from Mendel's letters to Nägeli dated I 866-I873 (Bibliography, 197); an article by Dr E. von Proskowetz in Neue Fireie Presse, 24 July, I902; a similar notice by Dr Wiesner in the Wiener Abendpost, November, $190 \mathrm{r}$; and from a somewhat fuller account published by Dr H. von Iltis in Tagesbote aus Mähren, 1906. I have to thank Dr Janetschek and Dr von Niessl for assistance given in the course of inquiries which I made in Brünn, and both Dr Ferdinand Schindler and his brother, Dr Alois Schindler, for letters giving many interesting particulars respecting their uncle. I understand that Dr von Iltis has a fuller biography in hand. My most serious misstatement was to the efiect that in his later years Mendel devoted himself to the Ultramontane Controversy. This was a complete mistake. The dispute in which he engaged was, as is described above, of a totally different nature. 
Slavonic population. It is recorded of his father that he took special interest in fruit-culture, initiating his son at an early age into the methods of grafting. Mendel's maternal uncle, Anton Schwirtlich, was evidently a man of intellectual tastes, which is shown by the fact that he started private classes for the children of Heinzendorf who could not walk so far as the neighbouring village, for in Heinzendorf itself there was at that time no regular school. Mendel was thus able to say with some pride that he came from an educational family.

On the death of Schwirtlich a government-school was established which Mendel attended as a young boy. His talent was noticed and encouraged by the master. At this time also two older boys who had gone away to the school at Leipnik fell in with Mendel during their holidays, and excited his ambition, with the result that he asked his parents to let him study, and eventually he too was sent to Leipnik at I I years old, though this involved considerable sacrifice on the part of the family. Here he distinguished himself so much that it was decided to continue his education at the gymnasium at Troppau, a course which finished with a year at Olmütz. The parental resources were severely taxed by such expenses, and Mendel was only enabled to complete his course through the generosity of a younger sister, who voluntarily contributed a part of her dowry for this purpose. In after years he repaid her advance many times over, himself providing the education of her three sons, his nephews.

At Troppau one of the teachers was an Augustinian, and it is surmised that perhaps his description of the scholarly tranquillity of the cloister may have turned Mendel's thoughts towards a monastic life. However that may have been, when his time at the gymnasiun was ended he became a candidate for admission to the Augustinian house of St Thomas in Brünn, an institution generally spoken of as the Königskloster. His application was successful, and he was elected with a view to his taking part in the educational work which then devolved on the institution. On admission he took the name of Gregor "in religion," Johann being his baptismal name. In I 847 he was ordained a priest. 
At the expense of the cloister he was sent in $185 \mathrm{I}$ to the University of Vienna, where he remained till I 853 , studying mathematics, physics, and natural sciences*. Returning to Brünn he became a teacher, especially of physics, in the Realschule. He appears to have taken great pleasure in teaching and to have been extraordinarily successful in interesting his pupils in their work. He continued this occupation till i 868 , when he was elected Abbot, or more strictly, Prälat of the Königskloster.

The experiments which have made his name famous throughout the world were carried on in the large garden of the cloister. From the time of his novitiate he began experimental work, introducing various plants into the garden and watching their behaviour under treatment. $\mathrm{He}$ was fond of showing these cultures to his friends. Dr von Niessl relates how on one occasion he was taken to see Ficaria calthaefolia and Ficaria ramunculoides (two forms now regarded as varieties of Ranunculus Ficaria) which had for some years been cultivated side by side without manifesting any noticeable change. Mendel jokingly said: "This much I do see, that nature cannot get on further with species-making in this way. There must be something more behind."

With the views of Darwin which at that time were coming into prominence Mendel did not find himself in full agreement, and he embarked on his experiments with peas, which as we know he continued for eight years. The results were communicated to the Brünn Society in 1865 and published in 1866, but they passed unheeded. The subsequent paper on Hieracium appeared in 1869, meeting a similar fate.

During his period of scientific work Mendel, as we now know, was engaged on a great variety of cognate researches. In his letters to Nägeli (I97) there are allusions to some of these subjects, but unhappily few statements of results. His largest undertaking besides the work on Pisum was an investigation of the heredity of bees. $\mathrm{He}$ had 50 hives

* To this period belong two notes which he published in the Verh. zool. bot. Verein, Wien, on Scopolia margaritalis (1853, III. p. I I6) and Bruchus pisi (ibid. I854, IV. p. 27). In these papers he speaks of himself as a pupil of Kollar. 
under observation. He collected queens of all attainable races, European, Egyptian, and American, and effected numerous crosses between these races, though it is known that he had many failures. Attempts were made to induce the queens to mate in his room, which he netted in with gauze for the purpose, but it was too small or too dark, and these efforts were unsuccessful. We would give much to know what results he obtained. In view of their genetic peculiarities a knowledge of heredity in bees would manifestly be of great value. The notes which he is known to have made on these experiments cannot be found, and it is supposed by some that in the depression which he suffered before his death they were destroyed.

In 1905 I had the pleasure of visiting the Königskloster, hoping that some trace of the missing books might be discovered. I was most courteously received by the present Prälat and the brethren of the cloister. My thanks are due in particular to Dr Janetschek for the assistance he gave me. It is to him that I owe the photographs of Mendel given in this volume. I saw the hives which had been used standing in their places, but the note books are gone*. A rich harvest of discovery awaits those who may successfully repeat the work.

With his appointment as Prälat his researches may be said to have ended. To Nägeli he wrote that he hoped that after an interval his elevation might enable him to find better opportunities for study, but it was not to be. In I 872 the Government passed a law imposing special taxes on the property of religious houses. This enactment Mendel conceived to be unjust and he decided to resist, claiming that all citizens should be equal in law, and that if these taxes were imposed on one class of institution they should be imposed on all. He thus took up a position which in England we should call that of a "Passive Resister." At first several monasteries stood out with the Königskloster, but gradually they conformed, Mendel alone remaining firm. The quarrel involved him in protracted trouble and litigation. High emissaries are said to have visited him pro-

* On chance of finding something I obtained a file of the local beejournal of Brünn, but beyond the fact that Mendel was a Vice-President of the Verein, whose organ it is, I could discover in it nothing relating to him. 
posing a compromise, and even offering honours in case of submission. Old friends and acquaintances tried to influence him, but it was all in vain. He attended neither to cajolement nor menace. The property of the house was eventually distrained upon, but he did not give in. $\mathrm{He}$ became also involved in the racial controversies which are often rife in this part of Austria, and it is only too certain that the last ten years of his life were passed in disappointment and bitterness. From being a cheerful, friendly man he became suspicious and misanthropic. During this period he fell into ill-heath, contracting a chronic nephritis, of which he died January 6, I 884 .

As to the propriety of his action in the great quarrel with the Government I have no means of forming an opinion. It is nevertheless interesting to know that a few years after his death the tax was removed without debate or dispute.

For many years he attended closely to meteorology and published his records annually in the Brünn Abhandhungen. He also took a great interest in sunspots, making such observations on them as he could by simple means*, drawing them and recording the frequency of their occurrence. He was among those who incline to the view that there is a connection between the appearance of spots on the sun and meteorological events on the earth. His notes on this subject are also lost. He served a term as President of the Naturforscher Verein in Brünn. That he was credited with good faculties for business is shown by the fact that he was chosen to be Chairman of the Moravian Hypotheken-Bank in that city. $\mathrm{He}$ is said also to have attained considerable skill as a chess-player, and he composed a good many problems which however were not published. This faculty reappears in one of his nephews.

His handwriting is remarkable for its extreme neatness, every letter being formed with meticulous precision.

In Heinzendorf, his native village, he is remembered as having been the organiser of a fire-brigade. When he eventually became famous, the erection of a new fire-station was used as an opportunity of commemorating him, and a memorial tablet was placed over the building in his honour.

* He used one of Fritsch's "brachytelescopes." 
The types of the great discoverers are most various. To the naturalist the fact is full of meaning. The wild, uncertain, rapid flash of genius, the scattered, half-focussed daylight of generalisation, the steady, slowly-perfected ray of penetrative analysis, are all lights in which truth may be seen. Mendel's faculty was of the latter order. From the fragmentary evidence before us we can in all probability form a fairly true notion of the man, with his clear head, strong interest in practical affairs, obstinate determination, and power of pursuing an abstract idea.

The total neglect of his work is known to have been a serious disappointment to him, as well it might. $\mathrm{He}$ is reported to have had confidence that sooner or later it would be noticed, and to have been in the habit of saying "Meine Zeit wird schon kommen!" This episode in the history of science is not a very pleasant one to contemplate. There are of course many similar examples, but there must be few in which the discovery so long neglected was at once so significant, so simple, and withal so easy to verify. The scientific world may comfort itself with the thought that in this case it sinned through inadvertence. With the exception of Nägeli* perhaps none of the leading naturalists ever saw the paper on peas. We would like to know whether Mendel made any other attempt to interest his contemporaries in his discovery. Probably having tried Nägeli and failed, he gave up further efforts.

So far as I have discovered there was, up to I 900 , only one reference to Mendel's observations in scientific literaturet, namely that of Focke, Pfanzenmischlinge, I88 I, p. IO9, where it is simply stated that Mendel's numerous experiments on Pisum gave results similar to those obtained by 'Knight, but that he believed he had found constant numerical ratios among the types produced by hybridisation. In the same work a similar brief reference is made to the paper on Hievacium. For these references we may now be grateful since it was through them that the papers were rediscovered.

The fact that the Brün journal is rather scarce does

* See p. 54 .

†. The Hieracium paper is referred to by Peter, Engler's bot. Jahrb. Bde. v and vi, I884, but only in its systematic bearings. 
not in itself explain why the work was not noticed. Such a circumstance has seldom long delayed general recognition. The cause is unquestionably to be found in that neglect of the experimental study of the problem of Species which supervened on the general acceptance of the Darwinian doctrines. The problem of Species, as Kölreuter, Gärtner, Naudin, Wichura, and the other hybridists conceived it, attracted thenceforth no workers. The question, it was imagined, had been answered and the debate ended. No one felt much interest in the matter. A host of other lines of work were suddenly opened up, and in 1865 the more original investigators naturally found those new methods of research more attractive than the tedious observations of the hybridisers, whose inquiries were supposed, moreover, to have led to no definite result.

Nevertheless the total neglect of such a discovery is not easy to account for. Those who are acquainted with the literature of this branch of inquiry will know that the French Academy offered a prize in I861 to be awarded in 1862 on the subject "Étudier les Hybrides végétaux au point de vue de leur fécondité et de la perpétuité de leurs caractères." This subject was doubtless chosen with reference to the experiments of Godron of Nancy and Naudin, then of Paris. Both these naturalists competed, and the accounts of the work of Godron on Datura and of Naudin on a number of species were published in the years 1864 and 1865 respectively. Both, especially the latter, are works of high consequence in the history of the science of heredity. In the latter paper Naudin clearly enunciated what we shall henceforth know as the Mendelian conception of the dissociation of characters of cross-breds in the formation of the germ-cells, though apparently he never developed this conception.

In the year 1864, George Bentham, then President of the Linnean Society, took these treatises as the subject of his address to the Anniversary meeting on the 24th May, Naudin's work being known to him from an abstract, the full paper having not yet appeared. Referring to the hypothesis of dissociation which he fully described, he said that it appeared to be new and well supported, but required much more confirmation before it could be held as proven. (J. Linn. Soc. Bot. virI. Proc. p. xiv.) 
In 1865, the year of Mendel's communication to the Brün Society, appeared Wichura's famous treatise on his experiments with Salix to which Mendel refers. There are passages in this memoir which come very near Mendel's principles, but it is evident from the plan of his experiments that Mendel had conceived the whole of his ideas before that date.

In I 868 appeared the first edition of Darwin's Animals and Plants, marking the very zenith of these studies, and thenceforth the decline in the experimental investigation of Evolution and the problem of Species has been steady. With the rediscovery and confirmation of Mendel's work by de Vries, Correns and Tschermak in I900 a new era begins.

That Mendel's work, appearing as it did at a moment when several naturalists of the first rank were still occupied with these problems, should have passed wholly unnoticed, will always remain inexplicable, the more so as the Brün Society exchanged its publications with most of the Academies of Europe, including both the Royal and Linnean Societies.

Naudin's views were well known to Darwin and are discussed in Animals and Plants (ed. 1885, II. p. 23); but, put forward as they were without full proof, they could not command universal credence. Darwin took the objection that Naudin's ideas were not compatible with cases of reversion, though as we now know, such cases are perfectly consistent with the phenomenon of segregation. Gärtner, too, had adopted opposite views; and Wichura, working with cases of another order, had proved the fact that some hybrids breed true. Consequently it is not to be wondered at that Darwin was sceptical. Nioreover, the Mendelian idea of the "hybrid-character," or heterozygous form, was unknown to him, a conception without which the hypothesis of dissociation of characters is quite imperfect.

Had Mendel's work come into the hands of Darwin, it is not too much to say that the history of the development of evolutionary philosophy would have been very different from that which we have witnessed. 


\title{
EXPERIMENTS IN PLANT- HYBRIDISATION*:
}

\author{
By Gregor Mendel.
}

(Read at the Meetings of the 8th February and 8th March, 1865.)

\section{INTRODUCTORY REMARKS.}

EXPERIENCE of artificial fertilisation, such as is effected with ornamental plants in order to obtain new variations in colour, has led to the experiments which will here be discussed. The striking regularity with which the same hybrid forms always reappeared whenever fertilisation took place between the same species induced further experiments to be undertaken, the object of which was to follow up the developments of the hybrids in their progeny.

To this object numerous careful observers, such as Kölreuter, Gärtner, Herbert, Lecoq, Wichura and others, have devoted a part of their lives with inexhaustible perseverance. Gärtner especially, in his work "Die Bastarderzeugung im Pflanzenreiche" (The Production of Hybrids in the Vegetable Kingdom), has recorded very valuable observations; and quite recently Wichura published the results of some profound investigations into the hybrids of the Willow. That, so far, no generally applicable law governing the formation and development of hybrids has been successfully formulated can hardly be wondered at by anyone who is acquainted with the extent of the task, and can appreciate the difficulties with which experiments of

* [This translation was made by the Royal Horticultural Society, and is reprinted with modifications and corrections, by permission. The original paper was published in the Verh. naturf. Ver. in Briinn, Abhandlungen, Iv. 1865, which appeared in I866.] 
this class have to contend. A final decision can only be arrived at when we shall have before us the results of detailed experiments made on plants belonging to the most diverse orders.

Those who survey the work done in this department will arrive at the conviction that among all the numerous experiments made, not one has been carried out to such an extent and in such a way as to make it possible to determine the number of different forms under which the offspring of hybrids appear, or to arrange these forms with certainty according to their separate generations, or definitely to ascertain their statistical relations*.

It requires indeed some courage to undertake a labour of such far-reaching extent; this appears, however, to be the only right way by which we can finally reach the solution of a question the importance of which cannot be overestimated in connection with the history of the evolution of organic forms.

The paper now presented records the results of such a detailed experiment. This experiment was practically confined to a small plant group, and is now, after eight years' pursuit, concluded in all essentials. Whether the plan upon which the separate experiments were conducted and carried out was the best suited to attain the desired end is left to the friendly decision of the reader.

\section{Selection of the Experimental Plants.}

The value and utility of any experiment are determined by the fitness of the material to the purpose for which it is used, and thus in the case before us it cannot be immaterial what plants are subjected to experiment and in what manner such experiments are conducted.

The selection of the plant group which shall serve for experiments of this kind must be made with all possible care if it be desired to avoid from the outset every risk of questionable results.

* [It is to the clear conception of these three primary necessities that the whole success of Mendel's work is due. So far as I know this conception was absolutely new in his day.] 
The experimental plants must necessarily-

I. Possess constant differentiating characters.

2. The hybrids of such plants must, during the flowering period, be protected from the influence of all foreign pollen, or be easily capable of such protection.

3. The hybrids and their offspring should suffer no marked disturbance in their fertility in the successive generations.

Accidental impregnation by foreign pollen, if it occurred during the experiments and were not recognized, would lead to entirely erroneous conclusions. Reduced fertility or entire-sterility of certain forms, such as occurs in the offspring of many hybrids, would render the experiments. very difficult or entirely frustrate them. In order to discover the relations in which the hybrid forms stand towards each other and also towards their progenitors it appears to be necessary that all members of the series developed in each successive generation should be, without exception, subjected to observation.

At the very outset special attention was devoted to the Leguminosae on account of their peculiar foral structure. Experiments which were made with several members of this family led to the result that the genus Pisum was found to possess the necessary qualifications.

Some thoroughly distinct forms of this genus possess characters which are constant, and easily and certainly recognizable, and when their hybrids are mutually crossed they yield perfectly fertile progeny. Furthermore, a disturbance through foreign pollen cannot easily occur, since the fertilising organs are closely packed inside the keel and the anther bursts within the bud, so that the stigma. becomes covered with pollen even before the flower opens. This circumstance is of especial importance. As additional advantages worth mentioning, there may be cited the easy culture of these plants in the open ground and in pats, and also their relatively short period of growth. Artificial fertilisation is certainly a somewhat elaborate process, but nearly always succeeds. For this purpose the bud is opened before it is perfectly developed, the keel is removed, and each stamen carefully extracted means of forceps, after which the stigma can at once be dusted over with the foreign pollen.

B. H. 
In all, thirty-four more or less distinct varieties of Peas were obtained from several seedsmen and subjected to a two years' trial. In the case of one variety there were noticed, among a larger number of plants all alike, a few forms which were markedly different. These, however, did not vary in the following year, and agreed entirely with another variety obtained from the same seedsman; the seeds were therefore doubtless merely accidentally mixed. All the other varieties yielded perfectly constant and similar offspring; at any rate, no essential difference was observed during two trial years. For fertilisation twenty-two of these were selected and cultivated during the whole period of the experiments. They remained constant without any exception.

Their systematic classification is difficult and uncertain. If we adopt the strictest definition of a species, according to which only those individuals belong to a species which under precisely the same circumstances display precisely similar characters, no two of these varieties could be referred to one species. According to the opinion of experts, however, the majority belong to the species Pisum sativum; while the rest are regarded and classed, some as sub-species of $P$. sativum, and some as independent species, such as $P$. quadratum, $P$. saccharatum, and $P$. umbellatum. The positions, however, which may be assigned to them in a classificatory system are quite immaterial for the purposes of the experiments in question. It has so far been found to be just as impossible to draw a sharp line between the hybrids of species and varieties as between species and varieties themselves.

\section{Division and Arrangement of the Experiments.}

If two plants which differ constantly in one or several characters be crossed, numerous experiments have demonstrated that the common characters arè transmitted unchanged to the hybrids and their progeny ; but each pair of differentiating characters, on the other hand, unite in the hybrid to form a new character, which in the progeny of the hybrid is usually variable. The object of the experiment was to observe these variations in the case of each pair of 
differentiating characters, and to deduce the law according to which they appear in the successive generations. The experiment resolves itself therefore into just as many separate experiments as there are constantly differentiating characters presented in the experimental plants.

The various forms of Peas selected for crossing showed differences in the length and colour of the stem; in the size and form of the leaves; in the position, colour, and size of the flowers; in the length of the flower stalk; in the colour, form, and size of the pods; in the form and size of the seeds; and in the colour of the seed-coats and of the albumen [cotyledons]. Some of the characters noted do not permit of a sharp and certain separation, since the difference is of a "more or less" nature, which is often difficult to define. Such characters could not be utilised for the separate experiments; these could only be applied to characters which stand out clearly and definitely in the plants. Lastly, the result must show whether they, in their entirety, observe a regular behaviour in their hybrid unions, and whether from these facts any conclusion can be come to regarding those characters which possess a subordinate significance in the type.

The characters which were selected for experiment relate:

I. To the difference in the form of the ripe seeds. These are either round or roundish, the depressions, if any, occur on the surface, being always only shallow ; or they are irregularly angular and deeply wrinkled ( $P$. quadratum).

2. To the difference in the colour of the seed albumen (endosperm)*. The albumen of the ripe seeds is either pale yellow, bright yellow and orange coloured, or it possesses a more or less intense green tint. This difference of colour is easily seen in the seeds as [ = if] their coats are transparent.

3. To the difference in the colour of the seed-coat. This is either white, with which character white flowers are constantly correlated ; or it is grey, grey-brown, leatherbrown, with or without violet spotting, in which case the colour of the standards is violet, that of the wings purple,

" [Mendel uses the terms "albumen" and "endosperm" somewhat loosely to denote the cotyledons, containing food-material, within the seed.] 
and the stem in the axils of the leaves is of a reddish tint. The grey seed-coats become dark brown in boiling water.

4. To the difference in the form of the ripe pods. These are either simply inflated, not contracted in places; or they are deeply constricted between the seeds and more or less wrinkled ( $P$. saccharatum).

5. To the difference in the colour of the unripe pods. They are either light to dark green, or vividly yellow, in which colouring the stalks, leaf-veins, and calyx participate*.

6. To the difference in the position of the flowers. They are either axial, that is, distributed along the main stem; or they are terminal, that is, bunched at the top of the stem and arranged almost in a false umbel; in this case the upper part of the stem is more or less widened in section $(P$. umbellatum) + .

7. To the difference in the length of the stem. The length of the stem $\ddagger$ is very various in some forms; it is, however, a constant character for each, in so far that healthy plants, grown in the same soil, are only subject to unimportant variations in this character.

In experiments with this character, in order to be able to discriminate with certainty, the long axis of 6 to $7 \mathrm{ft}$. was always crossed with the short one of $\frac{3}{4} \mathrm{ft}$. to $\mathrm{I} \frac{1}{2} \mathrm{ft}$.

Each two of the differentiating characters enumerated above were united by cross-fertilisation. There were made for the

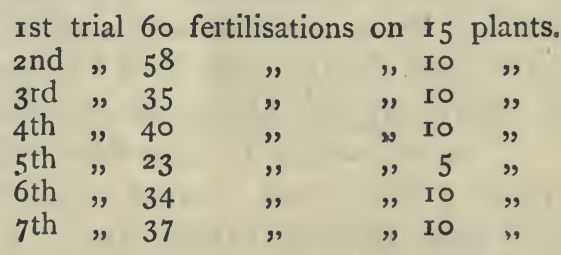

* One species possesses a beautifully brownish-red coloured pod, which when ripening turns to violet and blue. Trials with this character were only begun last year. [Of these further experiments it seems no account was published. Correns has since worked with such a variety.]

$\dagger[$ This is often called the Mummy Pea. It shows slight fasciation. The form I know has white standard and salmon-red wings.]

$\ddagger$ [In my account of these experiments (R.H.S. Journal, vol. xxv. p. 54) I misunderstood this paragraph and took "axis" to mean the floral axis, instead of the main axis of the plant. The unit of measurement, being indicated in the original by a dash ('), I carelessly took to have been an inch, but the translation here given is evidently correct.] 
From a larger number of plants of the same variety only the most vigorous were chosen for fertilisation. Weakly plants always afford uncertain results, because even in the first generation of hybrids, and still more so in the subsequent ones, many of the offspring either entirely fail to flower or only form a few and inferior seeds.

Furthermore, in all the experiments reciprocal crossings were effected in such a way that each of the two varieties which in one set of fertilisations served as seed-bearer in the other set was used as the pollen plant.

The plants were grown in garden beds, a few also in pots, and were maintained in their naturally upright position by means of sticks, branches of trees, and strings stretched between. For each experiment a number of pot plants were placed during the blooming period in a greenhouse, to serve as control plants for the main experiment in the open as regards possible disturbance by insects. Among the insects* which visit Peas the beetle Bruchus. pisi might be detrimental to the experiments should it appear in numbers. The female of this species is known to lay the eggs in the flower, and in so doing opens the keel; upon the tarsi of one specimen, which was caught in a flower, some pollen grains could clearly be seen under a lens. Mention must also be made of a circumstance which possibly might lead to the introduction of foreign pollen. It occurs, for instance, in some rare cases that certain parts of an otherwise quite normally developed flower wither, resulting in a partial exposure of the fertilising organs. A defective development of the keel has also been observed, owing to which the stigma and anthers remained partially uncovered $t$. It also sometimes happens that the pollen does not reach full perfection. In this event there occurs a gradual lengthening of the pistil during the blooming period, until the stigmatic tip protrudes at the point of the keel. This remarkable appearance has also been observed in hybrids of Phaseolus and Lathyrus.

The risk of false impregnation by foreign pollen is,

* [It is somewhat surprising that no mention is made of Thrips, which swarm in Pea flowers. I had come to the conclusion that this is a real source of error and I see Laxton held the same opinion.]

+ [This also happens in Sweet Peas.] 
however, a very slight one with Pisum, and is quite incapable of disturbing the general result. Among more than I0,000 plants which were carefully examined there were only a very few cases where an indubitable false impregnation had occurred. Since in the greenhouse such a case was never remarked, it may well be supposed that Bruchus pisi, and possibly also the described abnormalities in the floral structure, were to blame.

\section{$\left[F_{1}\right]$ The Forms of the Hybrids*.}

Experiments which in previous years were made with ornamental plants have already afforded evidence that the hybrids, as a rule, are not exactly intermediate between the parental species. With some of the more striking characters, those, for instance, which relate to the form and size of the leaves, the pubescence of the several parts, \&c., the intermediate, indeed, is nearly always to be seen; in other cases, however, one of the two parental characters is so preponderant that it is difficult, or quite impossible, to detect the other in the hybrid.

This is precisely the case with the Pea hybrids. In the case of each of the seven crosses the hybrid-character resembles t that of one of the parental forms so closely that the other either escapes observation completely or cannot be detected with certainty. This circumstance is of great importance in the determination and classification of the forms under which the offspring of the hybrids appear. Henceforth in this paper those characters which are transmitted quite, or almost unchanged in the hybridisation, and therefore in themselves constitute the characters of the hybrid, are termed the dominant, and those which become latent in the process recessive. The expression "recessive" has been chosen because the characters thereby designated withdraw or entirely disappear in the hybrids,

* [Mendel throughout speaks of his cross-bred Peas as "hybrids," a term which many restrict to the offspring of two distinct species. $\mathrm{He}$, as he explains, held this to be only a question of degree.].

$\dagger[$ Note that Mendel, with true penetration, avoids speaking of the hybrid-character as "transmitted" by either parent, thus escaping the error pervading the older views of heredity.] 
but nevertheless reappear unchanged in their progeny, as will be demonstrated later on.

It was furthermore shown by the whole of the experiments that it is perfectly immaterial whether the dominant character belong to the seed-bearer or to the pollen-parent; the form of the hybrid remains identical in both cases. This interesting fact was also emphasised by Gärtner, with the remark that even the most practised expert is not in a position to determine in a hybrid which of the two parental species was the seed or the pollen plant*.

Of the differentiating characters which were used in the experiments the following are dominant:

I. The round or roundish form of the seed with or without shallow depressions. ledons].

2. The yellow colouring of the seed albumen [coty-

3. The grey, grey-brown, or leather-brown colour of the seed-coat, in association with violet-red blossoms and reddish spots in the leaf axils.

4. The simply inflated form of the pod.

5. The green colouring of the unripe pod in association with the same colour in the stems, the leaf-veins and the calyx.

6. The distribution of the flowers along the stem.

7. The greater length of stem.

With regard to this last character it must be stated that the longer of the two parental stems is usually exceeded by the hybrid, a fact which is possibly only attributable to the greater luxuriance which appears in all parts of plants when stems of very different length are crossed. Thus, for instance, in repeated experiments, stems of I ft. and $6 \mathrm{ft}$. in length yielded without exception hybrids which varied in length between $6 \mathrm{ft}$. and $7 \frac{1}{2} \mathrm{ft}$.

The hybrid seeds in the experiments with seed-coat are often more spotted, and the spots sometimes coalesce into small bluish-violet patches. The spotting also frequently appears even when it is absent as a parental character $†$.

The hybrid forms of the seed-shape and of the albumen [colour] are developed immediately after the artificial ferti-

* [Gärtner, p. 223.]

$\dagger$ [This refers to the coats of the seeds borne by $F_{1}$ plants.] 
lisation by the mere influence of the foreign pollen. They can, therefore, be observed even in the first year of experiment, whilst all the other characters naturally only appear in the following year in such plants as have been raised from the crossed seed.

\section{$\left[F_{2}\right.$ ] The First Generation [Bred] from the Hybrids.}

In this generation there reappear, together with the dominant characters, also the recessive ones with their peculiarities fully developed, and this occurs in the definitely expressed average proportion of three to one, so that among each four plants of this generation three display the dominant character and one the recessive. This relates without exception to all the characters which were investigated in the experiments. The angular wrinkled form of the seed, the green colour of the albumen, the white colour of the seed-coats and the flowers, the constrictions of the pods, the yellow colour of the unripe pod, of the stalk, of the calyx, and of the leaf venation, the umbel-like form of the inflorescence, and the dwarfed stem, all reappear in the numerical proportion given, without any essential alteration. Transitional forms were not observed in any experiment.

Since the hybrids resulting from reciprocal crosses are formed alike and present no appreciable difference in their subsequent development, consequently the results [of the reciprocal crosses] can be reckoned together in each experiment. The relative numbers which were obtained for each pair of differentiating characters are as follows:

Expt. I. Form of seed.-From 253 hybrids 7,324 seeds were obtained in the second trial year. Among them were 5,474 round or roundish ones and 1,850 angular wrinkled ones. Therefrom the ratio 2.96 to $\mathrm{I}$ is deduced.

Expt. 2. Colour of albumen. $-25^{8}$ plants yielded 8,023 seeds, 6,022 yellow, and 2,00 I green; their ratio, therefore, is as $3^{\circ} \mathrm{OI}$ to $\mathrm{I}$.

In these two experiments each pod yielded usually both kinds of seed. In well-developed pods which contained on the average six to nine seeds, it often happened that all the 
seeds were round (Expt. I) or all yellow (Expt. 2); on the other hand there were never observed more than five wrinkled or five green ones in one pod. It appears to make no difference whether the pods are developed early or later in the hybrid or whether they spring from the main axis or from a lateral one. In some few plants only a few seeds developed in the first formed pods, and these possessed exclusively one of the two characters, but in the subsequently developed pods the normal proportions were maintained nevertheless.

As in separate pods, so did the distribution of the characters vary in separate plants. By way of illustration the first ten individuals from both series of experiments may serve.

Experiment I.

Form of Seed.

\begin{tabular}{|c|c|c|c|}
\hline Plants & Round & Angular & \\
\hline I & 45 & I2 & 3.75 \\
\hline 2 & 27 & 8 & 337 \\
\hline 3 & 24 & 7 & $31 \div 2$ \\
\hline 4 & I9 & Io & 1.9 \\
\hline 5 & 32 & I I & 29 \\
\hline 6 & 26 & 6 & 6,3 \\
\hline 7 & 88 & 24 & 3.66 \\
\hline 8 & 22 & I0 & 2.2 \\
\hline 9 & 28 & 6 & 4.46. \\
\hline I0 & 25 & 7 & 2.57. \\
\hline
\end{tabular}

Experiment 2.

Colour of Albumen.

$\begin{array}{cr}\text { Yellow } & \text { Green } \\ 25 & \text { II } \\ 32 & 7 \\ \text { 14 } & 5 \\ 70 & 27 \\ 24 & \text { I3 } \\ 20 & 6 \\ 32 & 13 \\ 44 & 9 \\ 50 & \text { I } 4 \\ 44 & \text { I8 }\end{array}$

As extremes in the distribution of the two seed characters in one plant, there were observed in Expt. I an instance of 43 round and only 2 angular, and another of 14 round and I 5 angular seeds. In Expt. 2 there was a case of 32 yellow and only I green seed, but also one of 20 yellow and 19 green.

These two experiments are important for the determination of the average ratios, because with a smaller number of experimental plants they show that very considerable fluctuations may occur. In counting the seeds, also, especially in Expt. 2, some care is requisite, since in some of the seeds of many plants the green colour of the albumen is less developed, and at first may be easily overlooked. The cause of this partial disappearance of the green colouring has no connection with the hybrid-character of the 
plants, as it likewise occurs in the parental variety. This peculiarity [bleaching] is also confined to the individual and is not inherited by the offspring. In luxuriant plants this appearance was frequently noted. Seeds which are damaged by insects during their development often vary in colour and form, but, with a little practice in sorting, errors are easily avoided. It is almost superfluous to mention that the pods must remain on the plants until they are thoroughly ripened and have become dried, since it is only then that the shape and colour of the seed are fully developed.

Expt. 3. Colour of the seed-coats.-Among 929 plants 705 bore violet-red flowers and grey-brown seed-coats; 224 had white flowers and white seed-coats, giving the proportion 3 . I 5 to $\mathrm{I}$.

Expt. 4. Form of pods.-Of I, I 8 I plants 882 had them simply inflated, and in 299 they were constricted. Resulting ratio, $2^{\circ} 95$ to $\mathrm{I}$.

Expt. 5. Colour of the unripe pods.-The number of trial plants was 580 , of which 428 had green pods and I 52 yellow ones. Consequently these stand in the ratio $2 \cdot 82$ to I:

Expt. 6. Position of flowers.-Among 858 cases $65 \mathrm{I}$ had inflorescences axial and 207 terminal. Ratio, $3^{\circ}{ }^{\circ} 4$ to $I$.

Expt. 7. Length of stem.-Out of 1,064 plants, in 787 cases the stem was long, and in 277 short. Hence a mutual ratio of 2.84 to I. In this experiment the dwarfed plants were carefully lifted and transferred to a special bed. This precaution was necessary, as otherwise they would have perished through being overgrown by their tall relatives. Even in their quite young state they can be easily picked out by their compact growth and thick dark-green foliage*.

If now the results of the whole of the experiments be brought together, there is found, as between the number of forms with the dominant and recessive characters, an average ratio of 2.98 to $I$, or 3 to $I$.

* [This is true also of the dwarf or "Cupıd" Sweet Peas.] 
The dominant character can have here a double signification-viz. that of a parental character, or a hybridcharacter*. In which of the two significations it appears in each separate case can only be determined by the following generation. As a parental character it must pass over unchanged to the whole of the offspring; as a hybridcharacter, on the other hand, it must maintain the same behaviour as in the first generation $\left[F_{2}\right]$.

\section{$\left[F_{3}\right]$ The Second Generation [bred] from THE Hybrids.}

Those forms which in the first generation $\left[F_{2}\right]$ exhibit the recessive character do not further vary in the second generation $\left[F_{3}\right]$ as regards this character; they remain constant in their offspring.

It is otherwise with those which possess the dominant character in the first generation [bred from the hybrids]. Of these two-thirds yield offspring which display the dominant and recessive characters in the proportion of 3 to $I$, and thereby show exactly the same ratio as the hybrid forms, while only one-third remains with the dominant character constant.

The separate experiments yielded the following results:

Expt. I. Among 565 plants which were raised from round seeds of the first generation, 193 yielded round seeds only, and remained therefore constant in this character; 372 , however, gave both round and wrinkled seeds, in the proportion of 3 to $\mathrm{I}$. The number of the hybrids, therefore, as compared with the constants is $\mathbf{r}^{\circ} 93$ to $\mathrm{I}$.

Expt. 2. Of $5^{19}$ plants which were raised from seeds whose albumen was of yellow colour in the first generation, I 66 yielded exclusively yellow, while 353 yielded yellow and green seeds in the proportion of 3 to I. There resulted, therefore, a division into hybrid and constant forms in the proportion of $2 \cdot \mathrm{I} 3$ to $\mathrm{I}$.

* [This paragraph presents the view of the hybrid-character as something incidental to the hybrid, and not "transmitted" to it-a true and fundamental conception here expressed probably for the first time.] 
For each separate trial in the following experiments 100 plants were selected which displayed the dominant character in the first generation, and in order to ascertain the significance of this, ten seeds of each were cultivated.

Expt. 3. The offspring of 36 plants yielded exclusively grey-brown seed-coats, while of the offspring of 64 plants some had grey-brown and some had white.

Expt. 4. The offspring of 29 plants had only simply inflated pods; of the offspring of $7 \mathrm{I}$, on the other hand, some had inflated and some constricted.

Expt. 5. The offspring of 40 plants had only green pods; of the offspring of 60 plants some had green, some yellow ones.

Expt. 6. The offspring of 33 plants had only axial flowers; of the offspring of 67 , on the other hand, some had axial and some terminal flowers.

Expt. 7. The offspring of 28 plants inherited the long axis, and those of 72 plants some the long and some the short axis.

In each of these experiments a certain number of the plants came constant with the dominant character. For the determination of the proportion in which the separation of the forms with the constantly persistent character results, the two first experiments are of especial importance, since in these a larger number of plants can be compared. The ratios $I^{\circ} 93$ to $I$ and $2^{\circ} I 3$ to I gave together almost exactly the average ratio of 2 to $\mathrm{I}$. The sixth experiment gave a quite concordant result; in the others the ratio varies more or less, as was only to be expected in view of the smaller number of 100 trial plants. Experiment 5, which shows the greatest departure, was repeated, and then, in lieu of the ratio of 60 and 40 , that of 65 and 35 resulted. The average vatio of 2 to 1 appears, therefore, as fixed with certainty. It is therefore demonstrated that, of those forms which possess the dominant character in the first generation, two-thirds have the hybrid-character, while one-third remains constant with the dominant character

The ratio of 3 to $I$, in accordance with which the 
distribution of the dominant and recessive characters results in the first generation, resolves itself therefore in all experiments into the ratio of $2: \mathrm{I}: \mathrm{I}$ if the dominant character be differentiated according to its significance as a hybridcharacter or as a parental one. Since the members of the first generation $\left[F_{2}\right]$ spring directly from the seed of the hybrids $\left[F_{1}\right]$, it is now clear that the hybrids form seeds having one or other of the two differentiating characters, and of these one-half develop again the hybrid form, while the other half yield plants which remain constant and receive the dominant or the recessive characters [respectively] in equal numbers.

The Subsequent Generations [bred] from the Hybrids.

The proportions in which the descendants of the hybrids develop and split up in the first and second generations presumably hold good for all subsequent progeny. Experiments I and 2 have already been carried through six generations, 3 and 7 through five, and 4, 5, and 6 through four, these experiments being continued from the third generation with a small number of plants, and no departure from the rule has been perceptible. The offspring of the hybrids separated in each generation in the ratio of $2: \mathbf{I}: \mathbf{I}$ into hybrids and constant forms.

If $A$ be taken as denoting one of the two constant characters, for instance the dominant, $\boldsymbol{a}$, the recessive, and $A a$ the hybrid form in which both are conjoined, the expression

$$
A+2 A a+a
$$

shows the terms in the series for the progeny of the hybrids of two differentiating characters.

The observation made by Gärtner, Kölreuter, and others, that hybrids are inclined to revert to the parental forms, is also confirmed by the experiments described. It is seen that the number of the hybrids which arise from one fertilisation, as compared with the number of forms which become constant, and their progeny from generation to generation, is continually diminishing, but that nevertheless they could not entirely disappear. If an average 
equality of fertility in all plants in all generations be assumed, and if, furthermore, each hybrid forms seed of which one-half yields hybrids again, while the other half is constant to both characters in equal proportions, the ratio of numbers for the offspring in each generation is seen by the following summary, in which $A$ and $a$ denote again the two parental characters, and $A a$ the hybrid forms. For brevity's sake it may be assumed that each plant in each generation furnishes only 4 seeds.

$\begin{array}{cr}\text { Generation } & A \\ \text { I } & \text { I } \\ 2 & 6 \\ 3 & 28 \\ 4 & \mathbf{1} 20 \\ 5 & 496 \\ n & \end{array}$

$\begin{array}{rr}A a & a \\ 2 & 1 \\ 4 & 6 \\ 8 & 28 \\ 16 & 120 \\ 32 & 496\end{array}$

\begin{tabular}{|c|c|c|c|}
\hline & Ratio & & \\
\hline$A$ & : $A a$ & : & $a$ \\
\hline I & $: \quad 2$ & : & $\mathbf{I}$ \\
\hline 3 & : & : & 3 \\
\hline 7 & : & : & 7 \\
\hline I 5 & : & : $]$ & I 5 \\
\hline $3 I$ & 2 & & 31 \\
\hline $2^{n}-I$ & 2 & : & $2^{n}$ \\
\hline
\end{tabular}

In the tenth generation, for instance, $2^{n}-\mathrm{I}=\mathrm{IO} 23$. There result, therefore, in each 2,048 plants which arise in this generation $\mathrm{I}, \mathrm{O} 23$ with the constant dominant character, $\mathrm{I}, 023$ with the recessive character, and only two hybrids.

\section{Tile Offspring of Hybrids in wiitch several Differentiating Characters are associated.}

In the experiments above described plants were used which differed only in one essential character*. The next task consisted in ascertaining whether the law of development discovered in these applied to each pair of differentiating characters when several diverse characters are united in the hybrid by crossing. As regards the form of the hybrids in these cases, the experiments showed throughout that this invariably more nearly approaches to that one of the two parental plants which possesses the greater number of dominant characters. If, for instance, the seed plant has

* [This statement of Mendel's in the light of present knowledge is open to some misconception. Though his work niakes it evident that such varieties may exist, it is very unlikely that Mendel could have had seven pairs of varieties such that the members of each pair differed from each other in only one considerable character (zesentliches Merkmal). The point is probably of little theoretical or practical consequence, but a rather heavy stress is thrown on "zesentlich."] 
a short stem, terminal white flowers, and simply inflated pods; the pollen plant, on the other hand, a long stem, violet-red flowers distributed along the stem, and constricted pods; the hybrid resembles the seed parent only in the form of the pod; in the other characters it agrees with the pollen parent. Should one of the two parental types possess only dominant characters, then the hybrid is scarcely or not at all distinguishable from it.

Two experiments were made with a considerable number of plants. In the first experiment the parental plants differed in the form of the seed and in the colour of the albumen; in the second in the form of the seed, in the colour of the albumen, and in the colour of the seed-coats. Experiments with seed characters give the result in the simplest and most certain way.

In order to facilitate study of the data in these experiments, the different characters of the seed plant will be indicated by $A, B, C$, those of the pollen plant by $a, b, c$, and the hybrid forms of the characters by $A a, B b$, and $C c$.

$\begin{array}{cc}\text { Expt. I. }-A B \text {, seed parents; } & a b \text {, pollen parents ; } \\ A \text {, form round; } & a \text {, form wrinkled; } \\ B \text {, albumen yellow. } & b \text {, albumen green. }\end{array}$

The fertilised seeds appeared round and yellow like those of the seed parents. The plants raised therefrom yielded seeds of four sorts, which frequently presented themselves in one pod. In all, 556 seeds were yielded by 15 plants, and of these there were:
315 round and yellow,
Ior wrinkled and yellow,
I08 round and green,
32 wrinkled and green.

All were sown the following year. Eleven of the round yellow seeds did not yield plants, and three plants did not form seeds. Among the rest:

38 had round yellow seeds

65 round yellow and green seeds $: \therefore A B b$

60 round yellow and wrinkled yellow seeds $A a B$

I 38 round yellow and green, wrinkled yellow and green seeds

$A a B b$. 
From the wrinkled yellow seeds 96 resulting plants bore seed, of which :

28 had only wrinkled yellow seeds $a B$

68 wrinkled yellow and green seeds $a B b$.

From 108 round green seeds IO2 resulting plants fruited, of which:

35 had only round green seeds $A b$

67 round and wrinkled green seeds $A a b$.

The wrinkled green seeds yielded 30 plants which bore seeds all of like character; they remained constant $a b$.

The offspring of the hybrids appeared therefore under nine different forms, some of them in very unequal numbers. When these are collected and co-ordinated we find :

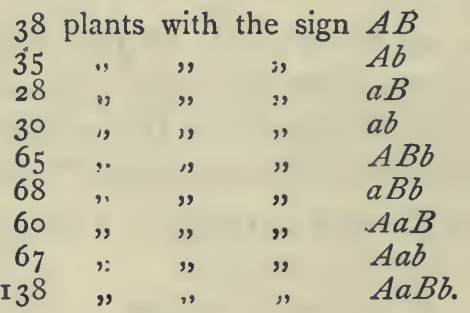

The whole of the forms may be classed into three essentially different groups. The first includes those with the signs $A B, A b, a B$, and $a b$ : they possess only constant characters and do not vary again in the next generation. Each of these forms is represented on the average thirtythree times. The second group includes the signs $A B b$, $a B b, A a B, A a b$ : these are constant in one character and hybrid in another, and vary in the next generation only as regards the hybrid-character. Each of these appears on an average sixty-five times. The form $A a B b$ occurs 138 times: it is hybrid in both characters, and behaves exactly as do the hybrids from which it is derived.

If the numbers in which the forms belonging to these classes appear be compared; the ratios of $1,2,4$ are unmistakably evident. The numbers $32,65,138$ present very fair approximations to the ratio numbers of $33,66,132$.

The developmental series consists, therefore, of nine classes, of which four appear therein always once and are constant in both characters; the forms $A B, a b$, resemble 
the parental forms, the two others present combinations between the conjoined characters $A, a, B, b$, which combinations are likewise possibly constant. Four classes appear always twice, and are constant in one character and hybrid in the other. One class appears four times, and is hybrid in both characters. Consequently the offspring of the hybrids, if two kinds of differentiating characters are combined therein, are represented by the expression $A B+A b+a B+a b+2 A B b+2 a B b+2 A a B$

$$
+2 A a b+4 A a B b \text {. }
$$

This expression is indisputably a combination series in which the two expressions for the characters $A$ and $a, B$ and $b$ are combined. We arrive at the full number of the classes of the series by the combination of the expressions:

Expt. 2.

$$
\begin{aligned}
& A+2 A a+a \\
& B+2 B b+b .
\end{aligned}
$$

$$
\begin{array}{cc}
A B C \text {, seed parents; } & a b c \text {, pollen parents } ; \\
A \text {, form round; } & a \text {, form wrinkled; } \\
B \text {, albumen yellow ; } & b \text {, albumen green } ; \\
C \text {, seed-coat grey-brown. } & c \text {, seed-coat white. }
\end{array}
$$

This experiment was made in precisely the same way as the previous one. Among all the experiments it demanded the most time and trouble. From 24 hybrids 687 seeds were obtained in all: these were all either spotted, greybrown or grey-green, round or wrinkled*. From these in the following year 639 plants fruited, and, as further investigation showed, there were among them:

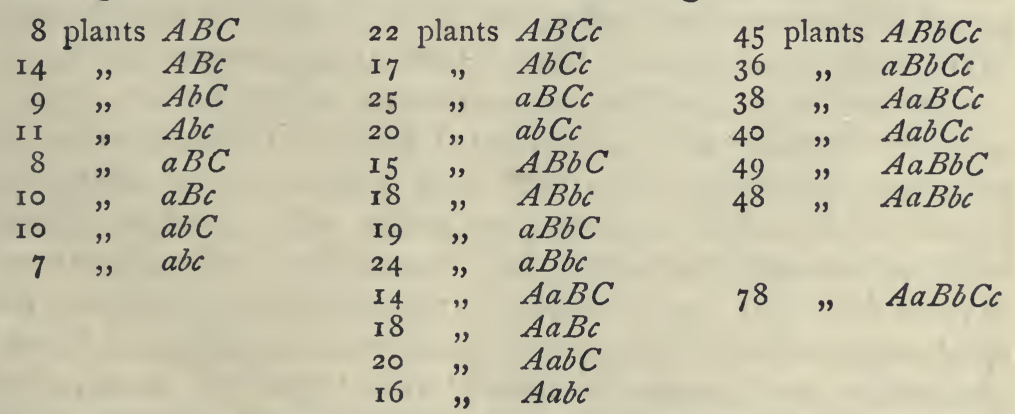

* [Note that Mendel does not state the cotyledon-colour of the first crosses in this case; for as the coats were thick, it could not have been seen without opening or peeling the seeds.]

I. $\mathrm{H}$. 
The whole expression contains 27 terms. Of these 8 are constant in all characters, and each appears on the average Io times; 12 are constant in two characters, and hybrid in the third; each appears on the average I 9 times ; 6 are constant in one character and hybrid in the other two; each appears on the average 43 times. One form appears 78 times and is hybrid in all of the characters. The ratios IO, I $9,43,78$ agree so closely with the ratios Io, $20,40,80$, or $\mathrm{I}, 2,4,8$, that this last undoubtedly represents the true value.

The development of the hybrids when the original parents differ in three characters results therefore according to the following expression:

$A B C+A B c+A b C+A b c+a B C+a B c+a b C+a b c+$ $2 A B C c+2 A b C c+2 a B C c+2 a b C c+2 A B b C+2 A B b c+$ $2 a B b C+2 a B b c+2 A a B C+2 A a B c+2 A a b C+2 A a b c+$ $4 A B b C c+4 a B b C c+4 A a B C c+4 A a b C c+4 A a B b C+$ $4 A a B b c+8 A a B b C c$.

Here also is involved a combination series in which the expressions for the characters $A$ and $a, B$ and $b, C$ and $c$, are united. The expressions

$$
\begin{aligned}
& A+2 A a+a \\
& B+2 B b+b \\
& C+2 C c+c
\end{aligned}
$$

give all the classes of the series. The constant combinations which occur therein agree with all combinations which are possible between the characters $A, B, C, a, b, c$; two thereof, $A B C$ and $a b c$, resemble the two original parental stocks.

In addition, further experiments were made with a smaller number of experimental plants in which the remaining characters by twos and threes were united as hybrids: all yielded approximately the same results. There is therefore no doubt that for the whole of the characters involved in the experiments the principle applies that the offspring of the hybrids in which several essentially different characters are combined exhibit the terms of a series of combinations, in which the developmental series for each pair of differentiating characters are united. It is demonstrated at the same time that the relation of each pair of 
different churacters in hybrid union is independent of the other differences in the two original parental stocks.

If $n$ represent the number of the differentiating characters in the two original stocks, $3^{n}$ gives the number of terms of the combination series, $4^{n}$ the number of individuals which belong to the series, and $2^{n}$ the number of unions which remain constant. The series therefore contains, if the original stocks differ in four characters, $3^{4}=8 \mathrm{I}$ classes, $4^{4}=25^{6}$ individuals, and $2^{4}=16$ constant forms ; or, which is the same, among each 256 offspring of the hybrids there are 8 I different combinations, I 6 of which are constant.

All constant combinations which in Peas are possible by the combination of the said seven differentiating characters were actually obtained by repeated crossing. Their number is given by $2^{7}=128$. Thereby is simultaneously given the practical proof that the constant characters which appear in the several varieties of a group of plants may be obtained in all the associations which are possible according to the [mathematical] laws of combination, by means of repeated artificial fertilisation.

As regards the flowering time of the hybrids, the experiments are not yet concluded. It can, however, already be stated that the time stands almost exactly between those of the seed and pollen parents, and that the constitution of the hybrids with respect to this character probably follows the rule ascertained in the case of the other characters. The forms which are selected for experiments of this class must have a difference of at least twenty days from the middle flowering period of one to that of the other; furthermore, the seeds when sown must all be placed at the same depth in the earth, so that they may germinate simultaneously. Also, during the whole flowering period, the more important variations in temperature must be taken into account, and the partial hastening or delaying of the flowering which may result therefrom. It is clear that this experiment presents many difficulties to be overcome and necessitates great attention.

If we endeavour to collate in a brief form the results arrived at, we find that those differentiating characters, which admit of easy and certain recognition in the experimental plants, all behave exactly alike in their hybrid 
associations. The offspring of the hybrids of each pair of differentiating characters are, one-half, hybrid again, while the other half are constant in equal proportions having the characters of the seed and pollen parents respectively. If several differentiating characters are combined by crossfertilisation in a hybrid, the resulting offspring form the terms of a combination series in which the combination series for each pair of differentiating characters are united.

The uniformity of behaviour shown by the whole of the characters submitted to experiment permits, and fully justifies, the acceptance of the principle that a similar relation exists in the other characters which appear less sharply defined in plants, and therefore could not be included in the separate experiments. An experiment with peduncles of different lengths gave on the whole a fairly satisfactory result, although the differentiation and serial arrangement of the forms could not be effected with that certainty which is indispensable for correct experiment.

\section{The Reproductive Cells of the Hybrids.}

The results of the previously described experiments led to further experiments, the results of which appear fitted to afford some conclusions as regards the composition of the egg and pollen cells of hybrids. An important clue is afforded in Pisum by the circumstance that among the progeny of the hybrids constant forms appear, and that this occurs, too, in respect of all combinations of the associated characters. So far as experience goes, we find it in every case confirmed that constant progeny can only be formed when the egg cells and the fertilising pollen are of like character, so that both are provided with the material for creating quite similar individuals, as is the case with the normal fertilisation of pure species. We must therefore regard it as certain that exactly similar factors must be at work also in the production of the constant forms in the hybrid plants. Since the various constant forms are produced in one plant, or even in one flower of a plant, the conclusion appears logical that in the ovaries of the hybrids there are formed as many sorts of egg cells, and in the 
anthers as many sorts of pollen cells, as there are possible constant combination forms, and that these egg and pollen cells agree in their internal composition with those of the separate forms.

In point of fact it is possible to demonstrate theoretically that this hypothesis would fully suffice to account for the development of the hybrids in the separate generations, if we might at the same time assume that the various kinds of egg and pollen cells were formed in the hybrids on the average in equal numbers*.

In order to bring these assumptions to an experimental proof, the following experiments were designed. Two forms which were constantly different in the form of the seed and the colour of the albumen were united by fertilisation.

If the differentiating characters are again indicated as $A, B, a, b$, we have:
$A B$, seed parent ;
$A$, form round;
$B$, albumen yellow.
$a b$, pollen parent ;
$a$, form wrinkled;
$b$, albumen green.

The artificially fertilised seeds were sown together with several seeds of both original stocks, and the most vigorous examples were chosen for the reciprocal crossing. There were fertilised :

I. The hybrids with the pollen of $A B$.
2. The hybrids
$a b$.
3. $A B$
" the hybrids.
4. $a b$

For each of these four experiments the whole of the flowers on three plants were fertilised. If the above theory be correct, there must be developed on the hybrids egg and pollen cells of the forms $A B, A b, a B, a b$, and there would be combined : cells $A B$.

The egg cells $A B, A b, a B, a b$ with the pollen

2. The egg cells $A B, A b, a B, a b$ with the pollen cells $a b$.

* [This and the preceding paragraph contain the essence of the Mendelian principles of heredity.] 

$a B, a b$.

3. The egg cells $A B$ with the pollen cells $A B, A b$,

4. The egg cells $a b$ with the pollen cells $A B, A b$, $a B, a b$.

From each of these experiments there could then result only the following forms:
I. $A B, A B b, A a B, A a B b$.
2. $A a B b, A a b, a B b, a b$.
3. $A B, A B b, A a B, A a B b$.
4. $A a B b, A a b, a B b, a b$.

If, furthermore, the several forms of the egg and pollen cells of the hybrids were produced on an average in equal numbers, then in each experiment the said four combinations should stand in the same ratio to each other. A perfect agreement in the numerical relations was, however, not to be expected, since in each fertilisation, even in normal cases, some egg cells remain undeveloped or subsequently die, and many even of the well-formed seeds fail to germinate when sown. The above assumption is also limited in so far that, while it demands the formation of an equal number of the various sorts of egg and pollen cells, it does not require that this should apply to each separate hybrid with mathematical exactness.

The first and second experiments had primarily the object of proving the composition of the hybrid egg cells, while the third and fourth experiments were to decide that of the pollen cells*. As is shown by the above demonstration the first and third experiments and the second and fourth experiments should produce precisely the same combinations, and even in the second year the result should be partially visible in the form and colour of the artificially fertilised seed. In the first and third experiments the dominant characters of form and colour, $A$ and $B$, appear in each union, and are also partly constant and partly in hybrid union with the recessive characters $a$ and $b$, for which reason they must impress their peculiarity upon the whole

\footnotetext{
* [To prove, namely, that both were similarly differentiated, and not one or other only.]
} 
of the seeds. All seeds should therefore appear round and yellow, if the theory be justified. In the second and fourth experiments, on the other hand, one union is hybrid in form and in colour, and consequently the seeds are round and yellow ; another is hybrid in form, but constant in the recessive character of colour, whence the seeds are round and green; the third is constant in the recessive character of form but hybrid in colour, consequently the seeds are wrinkled and yellow ; the fourth is constant in both recessive characters, so that the seeds are wrinkled and green. In both these experiments there were consequently four sorts of seed to be expected-viz. round and yellow, round and green, wrinkled and yellow, wrinkled and green.

The crop fulfilled these expectations perfectly. There were obtained in the

Ist Experiment, 98 exclusively round yellow seeds ;

3rd " 94 " " "

In the 2 nd Experiment, 3I round and yellow, 26 round and green, 27 wrinkled and yellow, 26 wrinkled and green seeds.

In the 4th Experiment, 24 round and yellow, 25 round and green, 22 wrinkled and yellow, 27 wrinkled and green seeds.

There could scarcely be now any doubt of the success of the experiment; the next generation must afford the final proof. From the seed sown there resulted for the first experiment 90 plants, and for the third 87 plants which fruited: these yielded for the

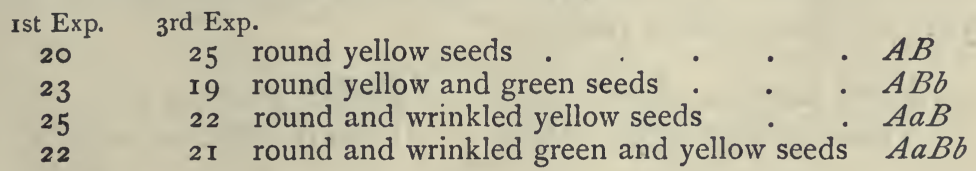

In the second and fourth experiments the round and yellow seeds yielded plants with round and wrinkled yellow and green seeds, $A a B b$.

From the round green seeds plants resulted with round and wrinkled green seeds, $A a b$.

The wrinkled yellow seeds gave plants with wrinkled yellow and green seeds, $a B b$. 
From the wrinkled green seeds plants were raised which yielded again only wrinkled and green seeds, $a b$.

Although in these two experiments likewise some seeds did not germinate, the figures arrived at already in the previous year were not affected thereby, since each kind of seed gave plants which, as regards their seed, were like. each other and different from the others. There resulted therefore from the

$\begin{array}{ccccc}\text { 2nd Exp. } & 4^{\text {th Exp. }} \text {. } & & & \\ 31 & 24 & \text { plants of the form } A a B b \\ 26 & 25 & " & " & A a b \\ 27 & 22 & " & " & a B b \\ 26 & 27 & " & \text { ", } & a b\end{array}$

In all the experiments, therefore, there appeared all the forms which the proposed theory demands, and they came in nearly equal numbers.

In a further experiment the characters of flower-colour and length of stem were experimented upon, and selection was so made that in the third year of the experiment each character ought to appear in half of all the plants if the above theory were correct. $A, B, a, b$ serve again as indicating the various characters.

$$
\begin{aligned}
& A \text {, violet-red flowers. } \quad a \text {, white flowers. } \\
& B \text {, axis long. }
\end{aligned}
$$

The form $A b$ was fertilised with $a b$, which produced the hybrid $A a b$. Furthermore, $a B$ was also fertilised with $a b$, whence the hybrid $a B b$. In the second year, for further fertilisation, the hybrid $A a b$ was used as seed parent, and hybrid $a B b$ as pollen parent.

Seed parent, $A a b$.

Possible egg cells, Abab.
Pollen parent, $a B b$.

Pollen cells, $a B a b$.

From the fertilisation between the possible egg and pollen cells four combinations should result, viz. :

$$
A a B b+a B b+A a b+a b \text {. }
$$

From this it is perceived that, according to the above theory, in the third year of the experiment out of all the plants 
Half should have violet red flowers $(A a)$, Classes I, 3

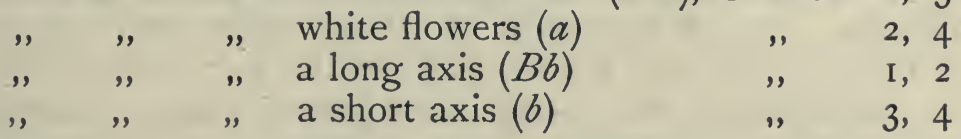

From 45 fertilisations of the second year 187 seeds resulted, of which only 166 reached the flowering stage in the third year. Among these the separate classes appeared in the numbers following:

$\begin{array}{clll}\text { Class } & \text { Colour of flower } & \text { Stem } & \\ \text { I } & \text { violet-red } & \text { long } & 47 \text { times } \\ 2 & \text { white } & \text { long } & 40 ", \\ 3 & \text { violet-red } & \text { short } & 38 \text { " } \\ 4 & \text { white } & \text { short } & 4 I ",\end{array}$

There subsequently appeared

The violet-red flower-colour $(A a)$ in 85 plants.

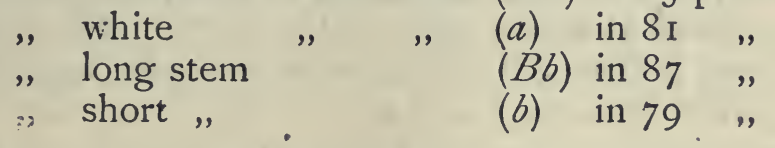

The theory adduced is therefore satisfactorily confirmed in this experiment also.

For the characters of form of pod, colour of pod, and position of flowers experiments were also made on a small scale, and results obtained in perfect agreement. All combinations which were possible through the union of the differentiating characters duly appeared, and in nearly equal numbers.

Experimentally, therefore, the theory is confirmed that the pea hybrids form egg and pollen cells which, in their constitution, represent in equal numbers all constant forms which result from the combination of the characters united in fertilisation.

The difference of the forms among the progeny of the hybrids, as well as the respective ratios of the numbers in which they are observed, find a sufficient explanation in the principle above deduced. The simplest case is afforded by the developmental series of each pair of differentiating characters. This series is represented by the expression $A+2 A a+a$, in which $A$ and $a$ signify the forms with constant differentiating characters, and $A a$ the hybrid form 
of both. It includes in three different classes four individuals. In the formation of these, pollen and egg cells of the form $A$ and $a$ take part on the average equally in the fertilisation; hence each form [occurs] twice, since four individuals are formed. There participate consequently in the fertilisation

\section{The pollen cells $A+A+a+a$ \\ The egg cells $A+A+a+a$.}

It remains, therefore, purely a matter of chance which of the two sorts of pollen will become united with each separate egg cell. According, however, to the law of probability, it will always happen, on the average of many cases, that each pollen form $A$ and $a$ will unite equally often with each egg cell form $A$ and $a$, consequently one of the two pollen cells $A$ in the fertilisation will meet with the egg cell $A$ and the other with an egg cell $a$, and so likewise one pollen cell $a$ will unite with an egg cell $A$, and the other with egg cell $a$.

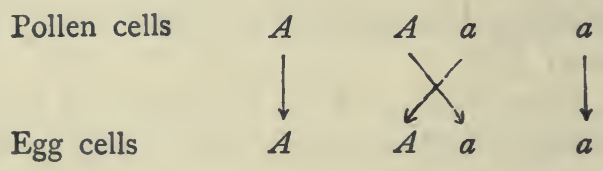

The result of the fertilisation may be made clear by putting the signs for the conjoined egg and pollen cells in the form of fractions, those for the pollen cells above and those for the egg cells below the line. We then have

$$
\frac{A}{A}+\frac{A}{a}+\frac{a}{A}+\frac{a}{a} \text {. }
$$

In the first and fourth term the egg and pollen cells are of like kind, consequently the product of their union must be constant, viz. $A$ and $a$; in the second and third, on the other hand, there again results a union of the two differentiating characters of the stocks, consequently the forms resulting from these fertilisations are identical with those of the hybrid from which they sprang. There occurs accordingly a repeated hybridisation. This explains the striking fact that the hybrids are able to produce, besides 
the two parental forms, offspring which are like themselves; $\frac{A}{a}$ and $\frac{a}{A}$ both give the same union $A a$, since, as already remarked above, it makes no difference in the result of fertilisation to which of the two characters the pollen or egg cells belong. We may write then

$$
\frac{A}{A}+\frac{A}{a}+\frac{a}{A}+\frac{a}{a}=A+2 A a+a \text {. }
$$

This represents the average result of the self-fertilisation of the hybrids when two differentiating characters are united in them. In individual flowers and in individual plants, however, the ratios in which the forms of the series are produced may suffer not inconsiderable fluctuations*. Apart from the fact that the numbers in which both sorts of egg cells occur in the seed vessels can only be regarded as equal on the average, it remains purely a matter of chance which of the two sorts of pollen may fertilise each separate egg cell. For this reason the separate values must necessarily be subject to fluctuations, and there are even extreme cases possible, as were described earlier in connection with the experiments on the form of the seed and the colour of the albumen. The true ratios of the numbers can only be ascertained by an average deduced from the sum of as many single values as possible; the greater the number the more are merely chance effects eliminated.

The developmental series for hybrids in which two kinds of differentiating characters are united contains among sixteen individuals nine different forms, viz.:

$$
\begin{array}{r}
A B+A b+a B+a b+2 A B b+2 a B b+2 A a B+2 A a b+ \\
4 A a B b .
\end{array}
$$

Between the differentiating characters of the original stocks $A a$ and $B b$ four constant combinations are possible, and consequently the hybrids produce the corresponding four forms of egg and pollen cells $A B, A b, a B, a b$, and each of these will on the average figure four times in the

* [Whether segregation by such units is more than purely fortuitous may perhaps be deternined by seriation.] 
fertilisation, since sixteen individuals are included in the series. Therefore the participators in the fertilisation are

Pollen cells $A B+A B+A B+A B+A b+A b+A b+A b+$ $a B+a B+a B+a B+a b+a b+a b+a b$.

Egg cells $A B+A B+A B+A B+A b+A b+A b+A b+$ $a B+a B+a B+a B+a b+a b+a b+a b$.

In the process of fertilisation each pollen form unites on an average equally often with each egg cell form, so that each of the four pollen cells $A B$ unites once with one of the forms of egg cell $A B, A b, a B, a b$. In precisely the same way the rest of the pollen cells of the forms $A b, a B, a b$ unite with all the other egg cells. We obtain therefore

$$
\begin{aligned}
& \frac{A B}{A B}+\frac{A B}{A b}+\frac{A B}{a B}+\frac{A B}{a b}+\frac{A b}{A B}+\frac{A b}{A b}+\frac{A b}{a B}+\frac{A b}{a b}+ \\
& \frac{a B}{A B}+\frac{a B}{A b}+\frac{a B}{a B}+\frac{a B}{a b}+\frac{a b}{A B}+\frac{a b}{A b}+\frac{a b}{a B}+\frac{a b}{a b}
\end{aligned}
$$

or

$A B+A B b+A a B+A a B b+A B b+A b+A a B b+A a b+$ $A a B+A a B b+a B+a B b+A a B b+A a b+a B b+a b=A B+$ $A b+a B+a b+2 A B b+2 a B b+2 A a B+2 A a b+4 A a B b *$.

In precisely similar fashion is the developmental series of hybrids exhibited when three kinds of differentiating characters are conjoined in them. The hybrids form eight various kinds of egg and pollen cells- $A B C, A B c, A b C$, $A b c, a B C, a B c, a b C, a b c$-and each pollen form unites itself again on the average once with each form of egg cell.

The law of combination of different characters which governs the development of the hybrids finds therefore its foundation and explanation in the principle enunciated, that the hybrids produce egg cells and pollen cells which in equal numbers represent all constant forms which result from the combinations of the characters brought together in fertilisation.

* [In the original the sign of equality $(\Rightarrow)$ is here represented by + , evidently a misprint.] 
Experiments with Hybrids of other Species of Plants.

It must be the object of further experiments to ascertain whether the law of development discovered for Pisum applies also to the hybrids of other plants. To this end several experiments were recently commenced. Two minor experiments with species of Phaseolus have been completed, and may be here mentioned.

An experiment with Phaseolus vulgaris and Phaseolus nanus gave results in perfect agreement. $P h$. nanus had together with the dwarf axis, simply inflated, green pods. Ph. vulgaris had, on the other hand, an axis io feet to I 2 feet high, and yellow-coloured pods, constricted when ripe. The ratios of the numbers in which the different forms appeared in the separate generations were the same as with Pisum. Also the development of the constant combinations resulted according to the law of simple combination of characters, exactly as in the case of Pisum. There were obtained

\begin{tabular}{|c|c|c|c|}
\hline $\begin{array}{l}\text { Constant } \\
\text { combinations }\end{array}$ & Axis & $\begin{array}{l}\text { Colour of } \\
\text { the unripe pods }\end{array}$ & $\begin{array}{l}\text { Form of } \\
\text { the ripe pods }\end{array}$ \\
\hline I & long & green & inflated \\
\hline 2 & $"$ & & constricted \\
\hline 3 & " & yellow & inflated \\
\hline 4 & $"$ & $"$ & constricted \\
\hline 5 & short & green & inflated \\
\hline 6 & " & "̈ & constricted \\
\hline $\begin{array}{l}7 \\
8\end{array}$ & $"$ & $\begin{array}{c}\text { yellow } \\
, "\end{array}$ & $\begin{array}{c}\text { inflated } \\
\text { constricted }\end{array}$ \\
\hline
\end{tabular}

The green colour of the pod, the inflated forms, and the long axis were, as in Pisum, dominant characters.

Another experiment with two very different species of Phaseolus had only a partial result. Phaseolus nanus, L., served as seed parent, a perfectly constant species, with white flowers in short racemes and small white seeds in straight, inflated, smooth pods; as pollen parent was used Ph. multiflorus, W., with tall winding stem, purple-red flowers in very long racemes, rough, sickle-shaped crooked pods, and large seeds which bore black flecks and splashes on a peach-blossom-red ground. 
The hybrids had the greatest similarity to the pollen parent, but the flowers appeared less intensely coloured. Their fertility was very limited; from seventeen plants, which together developed many hundreds of flowers, only forty-nine seeds in all were obtained. These were of medium size, and were flecked and splashed similarly to those of Ph. multiflorus, while the ground colour was not materially different. The next year forty-four plants were raised from these seeds, of which only thirty-one reached the flowering stage. The characters of $P h$. nanus, which had been altogether latent in the hybrids, reappeared in various combinations; their ratio, however, with relation to the dominant plants was necessarily very fluctuating owing to the small number of trial plants. With certain characters, as in those of the axis and the form of pod, it was, however, as in the case of $P$ isum, almost exactly $1: 3$.

Insignificant as the results of this experiment may be as regards the determination of the relative numbers in which the various forms appeared, it presents, on the other hand, the phenomenon of a remarkable change of colour in the flowers and seed of the hybrids. In Pisum it is known that the characters of the flower- and seed-colour present themselves unchanged in the first and further generations, and that the offspring of the hybrids display exclusively the one or the other of the characters of the original stocks. It is otherwise in the experiment we are considering. The white flowers and the seed-colour of Ph. nanus appeared, it is true, at once in the first generation [from the hybrids] in one fairly fertile example, but the remaining thirty plants developed flower-colours which were of various grades of purple-red to pale violet. The colouring of the seed-coat was no less varied than that of the flowers. No plant could rank as fully fertile; many produced no fruit at all ; others only yielded fruits from the flowers last produced, which did not ripen. From fifteen plants only were well-developed seeds obtained. The greatest disposition to infertility was seen in the forms with preponderantly red flowers, since out of sixteen of these only four yielded ripe seed. Three of these had a similar seed pattern to Ph. multiflorus, but with a more or less pale ground colour; the fourth plant yielded only one seed of plain brown tint. 
The forms with preponderantly violet-coloured flowers had dark brown, black-brown, and quite black seeds.

The experiment was continued through two more generations under similar unfavourable circumstances, since even among the offspring of fairly fertile plants there came again some which were less fertile or even quite sterile. Other flower- and seed-colours than those cited did not subsequently present themselves. The forms which in the first generation [bred from the hybrids] contained one or more of the recessive characters remained, as regards these, constant without exception. Also of those plants which possessed violet flowers and brown or black seed, some did not vary again in these respects in the next generation; the majority, however, yielded, together with offspring exactly like themselves, some which displayed white flowers and white seed-coats. The red flowering plants remained so slightly fertile that nothing can be said with certainty as regards their further development.

Despite the many disturbing factors with which the observations had to contend, it is nevertheless seen by this experiment that the development of the hybrids, with regard to those characters which concern the form of the plants, follows the same laws as in Pisum. With regard to the colour characters, it certainly appears difficult to perceive a substantial agreement. Apart from the fact that from the union of a white and a purple-red colouring a whole series of colours results [in $F_{2}$ ], from purple to pale violet and white, the circumstance is a striking one that among thirty-one flowering plants only one received the recessive character of the white colour, while in Pisum this occurs on the average in every fourth plant.

Even these enigmatical results, however, might probably be explained by the law governing Pisum if we might assume that the colour of the flowers and seeds of $P h$. multiflorus is a combination of two or more entirely independent colours, which individually act like any other constant character in the plant. If the flower-colour $A$ were a combination of the individual characters $A_{1}+A_{2}+\ldots$ which produce the total impression of a purple coloration, then by fertilisation with the differentiating character, white colour, $a$, there would be produced the hybrid unions 
$A_{1} a+A_{2} a+\ldots$ and so would it be with the corresponding colouring of the seed-coats*. According to the above assumption, each of these hybrid colour unions would be independent, and would consequently develop quite independently from the others. It is then easily seen that from the combination of the separate developmental series a complete colour-series must result. If, for instance, $A=A_{1}+A_{2}$, then the hybrids $A_{1} a$ and $A_{2} a$ form the developmental series-

$$
\begin{aligned}
& A_{1}+2 A_{1} a+a \\
& A_{2}+2 A_{2} a+a .
\end{aligned}
$$

The members of this series can enter into nine different combinations, and each of these denotes another colour-
I $A_{1} A_{2}$
$2 A_{1} a A_{2}$
I $A_{2} a$
$2 A_{1} A_{2} a$
$4 A_{1} a A_{2} a$
$2 \mathrm{~A}_{2} a a$
I $A_{1} a$
$2 A_{1} a a$
I $a \alpha$.

The figures prescribed for the separate combinations also indicate how many plants with the corresponding colouring belong to the series. Since the total is sixteen, the whole of the colours are on the average distributed over each sixteen plants, but, as the series itself indicates, in unequal proportions.

Should the colour development really happen in this way, we could offer an explanation of the case above described, viz. that the white flowers and seed-coat colour only appeared once among thirty-one plants of the first generation. This colouring appears only once in the series, and could therefore also only be developed once in the average in each sixteen, and with three colour characters only once even in sixty-four plants.

It must, nevertheless, not be forgotten that the explanation here attempted is based on a mere hypothesis, only supported by the very imperfect result of the experiment just described. It would, however, be well worth while to follow up the development of colour in hybrids by similar

* [As it fails to take account of factors introduced by the albino this representation is imperfect. It is however interesting to know that Mendel realized the fact of the existence of compound characters, and that the rarity of the white recessives was a consequence of this resolution.] 
experiments, since it is probable that in this way we might learn the significance of the extraordinary variety in the colouring of our ornamental flowers.

So far, little at present is known with certainty beyond the fact that the colour of the flowers in most ornamental plants is an extremely variable character. The opinion has often been expressed that the stability of the species is greatly disturbed or entirely upset by cultivation, and consequently there is an inclination to regard the development of cultivated forms as a matter of chance devoid of rules; the colouring of ornamental plants is indeed usually cited as an example of great instability. It is, however, not clear why the simple transference into garden soil should result in such a thorough and persistent revolution in the plant organism. No one will seriously maintain that in the open country the development of plants is ruled by other laws than in the garden bed. Here, as there, changes of type must take place if the conditions of life be altered, and the species possesses the capacity of fitting itself to its new environment. It is willingly granted that by cultivation the origination of new varieties is favoured, and that by man's labour many varieties are acquired which, under natural conditions, would be lost; but nothing justifies the assumption that the tendency to the formation of varieties is so extraordinarily increased that the species speedily lose all stability, and their offspring diverge into an endless series of extremely variable forms. Were the change in the conditions the sole cause of variability we might expect that those cultivated plants which are grown for centuries under almost identical conditions would again attain constancy. That, as is well known, is not the case, since it is precisely under such circumstances that not only the most varied but also the most variable forms are found. It is only the Leguminosae, like Pisum, Phaseolus*, Lens, whose organs of fertilisation are protected by the keel, which constitute a noteworthy exception. "Even here there have arisen numerous varieties during a cultural period of more than 1000 years under most various conditions; these maintain, however, under unchanging environments a stability as great as that of species growing wild.

* [Phaseolus nevertheless is insect-fertilised:]

B. H. 
It is more than probable that as regards the variability of cultivated plants there exists a factor which so far has received little attention. Various experiments force us to the conclusion that our cultivated plants, with few exceptions, are members of various hybrid series, whose further development in conformity with law is varied and interrupted by frequent crossings inter se. The circumstance must not be overlooked that cultivated plants are mostly grown in great numbers and close together, affording the most favourable conditions for reciprocal fertilisation between the varieties present and the species itself. The probability of this is supported by the fact that among the great array of variable forms solitary examples are always found, which in one character or another remain constant, if only foreign influence be carefully excluded. These forms behave precisely as do those which are known to be members of the compound hybrid series. Also with the most susceptible of all characters, that of colour, it cannot escape the careful observer that in the separate forms the inclination to vary is displayed in very different degrees. Among plants which arise from one spontaneous fertilisation there are often some whose offspring vary widely in the constitution and arrangement of the colours, while that of others shows little deviation, and among a greater number solitary examples occur which transmit the colour of the flowers unchanged to their offspring. The cultivated species of Dianthus afford an instructive example of this. A white-flowered example of Dianthus caryophyllus, which itself was derived from a white-flowered variety, was shut up during its blooming period in a greenhouse; the numerous seeds obtained therefrom yielded plants entirely white-flowered like itself. A similar result was obtained from a sub-species, with red flowers somewhat flushed with violet, and one with flowers white, striped with red. Many others, on the other hand, which were similarly protected, yielded progeny which were more or less variously coloured and marked.

Whoever studies the coloration which results in ornamental plants from similar fertilisation can hardly escape the conviction that here also the development follows a definite law which possibly finds its expression in the combination of several independent colour characters. 


\section{Concluding Remarks.}

It can hardly fail to be of interest to compare the observations made regarding Pisum with the results arrived at by the two authorities in this branch of knowledge, Kölreuter and Gärtner, in their investigations. According to the opinion of both, the hybrids in outward appearance present either a form intermediate between the original species, or they closely resemble either the one or the other type, and sometimes can hardly be discriminated from it. From their seeds usually arise, if the fertilisation was effected by their own pollen, various forms which differ from the normal type. As a rule, the majority of individuals obtained by one fertilisation maintain the hybrid form, while some few others come more like the seed parent, and one or other individual approaches the pollen parent. This, however, is not the case with all hybrids without exception. Sometimes the offspring have more nearly approached, some the one and some the other of the two original stocks, or they all incline more to one or the other side; while in other cases they remain perfectly like the hybrid and continue constant in their offspring. The hybrids of varieties behave like hybrids of species, but they possess greater variability of form and a more pronounced tendency to revert to the original types.

With regard to the form of the hybrids and their development, as a rule an agreement with the observations made in Pisum is unmistakable. It is otherwise with the exceptional cases cited. Gärtner confesses even that the exact determination whether a form bears a greater resemblance to one or to the other of the two original species often involved great difficulty, so much depending upon the subjective point of view of the observer. Another circumstance could, however, contribute to render the results fluctuating and uncertain, despite the most careful observation and differentiation. For the experiments plants were mostly used which rank as good species and are differentiated by a large number of characters. In addition to the sharply defined characters, where it is a question of greater or less similarity, those characters must also be

$$
24-2
$$


taken into account which are often difficult to define in words, but yet suffice, as every plant specialist knows, to give the forms a peculiar appearance. If it be accepted that the development of hybrids follows the law which is valid for Pisum, the series in each separate experiment must contain very many forms, since the number of the terms, as is known, increases with the number of the differentiating characters as the powers of three. With a relatively small number of experimental plants the result therefore could only be approximately right, and in single cases might fluctuate considerably. If, for instance, the two original stocks differ in seven characters, and 100 and 200 plants were raised from the seeds of their hybrids to determine the grade of relationship of the offspring, we can easily see how uncertain the decision must become, since for seven differentiating characters the combination series contains 16,384 individuals under 2187 various forms; now one and then another relationship could assert its predominance, just according as chance presented this or that form to the observer in a majority of cases.

If, furthermore, there appear among the differentiating characters at the same time dominant characters, which are transmitted entire or nearly unchanged to the hybrids, then in the terms of the developmental series that one of the.two original parents which possesses the majority of dominant characters must always be predominant. In the experiment described relative to Pisum, in which three kinds of differentiating characters were concerned, all the dominant characters belonged to the seed parent. Although the terms of the series in their internal composition approach both original parents equally, yet in this experiment the type of the seed parent obtained so great a preponderance that out of each sixty-four plants of the first generation fifty-four exactly resembled it, or only differed in one character. It is seen how rash it must be under such circumstances to draw from the external resemblances of hybrids conclusions as to their internal nature.

Gärtner mentions that in those cases where the development was regular, among the offspring of the hybrids the two original species were not reproduced, but only a few individuals which approached them. With very extended 
developmental series it could not in fact be otherwise. For seven differentiating characters, for instance, among more than I6,000 individuals-offspring of the hybridseach of the two original species would occur only once. It is therefore hardly possible that these should appear at all among a small number of experimental plants; with some probability, however, we might reckon upon the appearance in the series of a few forms which approach them.

We meet with an essential difference in those hybrids which remain constant in their progeny and propagate themselves as truly as the pure species. According to Gärtner, to this class belong the remarkably fertile hybrids Aquilegia atropurpurea canadensis, Lavatera psendolbia thuringiaca, Geum urbano-rivale, and some Dianthus hybrids; and, according to Wichura, the hybrids of the Willow family. For the history of the evolution of plants this circumstance is of special importance, since constant hybrids acquire the status of new species. The correctness of the facts is guaranteed by eminent observers, and cannot be doubted. Gärtner had an opportunity of following up Dianthus Armeria deltoides to the tenth generation, since it regularly propagated itself in the garden.

With Pisum it was shown by experiment that the hybrids form egg and pollen cells of different kinds, and that herein lies the reason of the variability of their offspring. In other hybrids, likewise, whose offspring behave similarly we may assume a like cause ; for those, on the other hand, which remain constant the assumption appears justifiable that their reproductive cells are all alike and agree with the foundation-cell [fertilised ovum] of the hybrid. In the opinion of renowned physiologists, for the purpose of propagation one pollen cell and one egg cell unite in Phanerogams* into a single cell, which is capable by

* In Pisum it is placed beyond doubt that for the formation of the new embryo a perfect union of the elements of both reproductive cells must take place. How could we otherwise explain that among the offspring of the hybrids both original types reappear in equal numbers and with all their peculiarities? If the influence of the egg cell upon the pollen cell were only external, if it fulfilled the rôle of a nurse only, then the result of each artificial fertilisation could be no other than that the developed hybrid should exactly resemble the pollen parent, or at any rate do so very closely. This the experiments so far have in no wise confirmed. An 
assimilation and formation of new cells to become an independent organism. This development follows a constant law, which is founded on the material composition and arrangement of the elements which meet in the cell in a vivifying union. If the reproductive cells be of the same kind and agree with the foundation cell [fertilised ovum] of the mother plant, then the development of the new individual will follow the same law which rules the mother plant. If it chance that an egg cell unites with a dissimilar pollen cell, we must then assume that between those elements of both cells, which determine opposite characters some sort of compromise is effected. The resulting compound cell becomes the foundation of the hybrid organism, the development of which necessarily follows a different scheme from that obtaining in each of the two original species. If the compromise be taken to be a complete one, in the sense, namely, that the hybrid embryo is formed from two similar cells, in which the differences are entirely and permanently accommodated together, the further result follows that the hybrids, like any other stable plant species, reproduce themselves truly in their offspring. The reproductive cells which are formed in their seed vessels and anthers are of one kind, and agree with the fundamental compound cell [fertilised ovum].

With regard to those hybrids whose progeny is variable we may perhaps assume that between the differentiating elements of the egg and pollen cells there also occurs a compromise, in so far that the formation of a cell as foundation of the hybrid becomes possible; but, nevertheless, the arrangement between the conflicting elements is only temporary and does not endure throughout the life of the hybrid plant. Since in the habit of the plant no changes are perceptible during the whole period of vegetation, we must further assume that it is only possible for the differentiating elements to liberate themselves from the enforced union when the fertilising cells are developed. In the formation of these cells all existing elements participate

evident proof of the complete union of the contents of both cells is afforded by the experience gained on all sides that it is immaterial, as regards the form of the hybrid, which of the original species is the seed parent or which the pollen parent. 
in an entirely free and equal arrangement, by which it is only the differentiating ones which mutually separate themselves. In this way the production would be rendered possible of as many sorts of egg and pollen cells as there are combinations possible of the formative elements.

The attribution attempted here of the essential difference in the development of hybrids to a permanent or temporary union of the differing cell elements can, of course, only claim the value of an hypothesis for which the lack of definite data offers a wide scope. Some justification of the opinion expressed lies in the evidence afforded by $P$ isum that the behaviour of each pair of differentiating characters in hybrid union is independent of the other differences between the two original plants, and, further, that the hybrid produces just so many kinds of egg and pollen cells as there are possible constant combination forms. The differentiating characters of two plants can finally, however, only depend upon differences in the composition and grouping of the elements which exist in the foundationcells [fertilised ova] of the same in vital interaction*.

Even the validity of the law formulated for Pisum requires still to be confirmed, and a repetition of the more important experiments is consequently much to be desired, that, for instance, relating to the composition of the hybrid fertilising cells. A differential [element] may easily escape the single observert, which although at the outset may appear to be unimportant, may yet accumulate to such an extent that it must not be ignored in the total result. Whether the variable hybrids of other plant species observe an entire agreement must also be first decided experimentally. In the meantime we may assume that in material points an essential difference can scarcely occur, since the unity in the developmental plan of organic life is beyond question.

In conclusion, the experiments carried out by Kölreuter, Gärtner, and others with respect to the transformation of one species into another by artificial fertilisation merit special mention. Particular importance has been attached siehen."

* "Welche in den Grundzellen derselben in lebendiger Wechselwirkung + "Dem einzelnen Beobachter kann leicht ein Differenziale entgehen." 
to these experiments, and Gärtner reckons them among "the most difficult of all in hybridisation."

If a species $A$ is to be transformed into a species $B$, both must be united by fertilisation and the resulting hybrids then be fertilised with the pollen of $B$; then, out of the various offspring resulting, that form would be selected which stood in nearest relation to $B$ and once more be fertilised with $B$ pollen, and so continuously until finally a form is arrived at which is like $B$ and constant in its progeny. By this process the species $A$ would change into the species $B$. Gärtner alone has effected thirty such experiments with plants of genera Aquilegia, Dianthus, Geum, Lavatera, Lychnis, Malva, Nicotiana, and Oenothera. The period of transformation was not alike for all species. While with some a triple fertilisation sufficed, with others this had to be repeated five or six times, and even in the same species fluctuations were observed in various experiments. Gärtner ascribes this difference to the circumstance that "the specific [typische] power by which a species, during reproduction, effects the change and transformation of the maternal type varies considerably in different plants, and that, consequently, the periods within which the one species is changed into the other must also vary, as also the number of generations, so that the transformation in some species is perfected in more, and in others in fewer generations." Further, the same observer remarks "that in these transformation experiments a good deal depends upon which type and which individual be chosen for further transformation."

If it may be assumed that in these experiments the constitution of the forms resulted in a similar way to that of Pisum, the entire process of transformation would find a fairly simple explanation. The hybrid forms as many kinds of egg cells as there are constant combinations possible of the characters conjoined therein, and one of these is always of the same kind as that of the fertilising pollen cells. Consequently there always exists the possibility with all such experiments that even from the second fertilisation there may result a constant form identical with that of the pollen parent. Whether this really be obtained depends in each separate case upon the number of the experimental plants, as well as upon the number of differentiating 
characters which are united by the fertilisation. Let us, for instance, assume that the plants selected for experiment differed in three characters, and the species $A B C$ is to be transformed into the other species $a b c$ by repeated fertilisation with the pollen of the latter; the hybrids resulting from the first cross form eight different kinds of egg cells, viz. :

$$
A B C, A B c, A b C, a B C, A b c, a B c, a b C, a b c \text {. }
$$

These in the second year of experiment are united again with the pollen cells $a b c$, and we obtain the series

$$
\begin{aligned}
A a B b C c & +A a B b c+A a b C c+a B b C c \\
& +A a b c+a B b c+a b C c+a b c .
\end{aligned}
$$

Since the form $a b c$ occurs once in the series of eight terms, it is consequently little likely that it would be missing among the experimental plants, even were these raised in a smaller number, and the transformation would be perfected already by a second fertilisation. If by chance it did not appear, then the fertilisation must be repeated with one of those forms nearest akin, $A a b c, a B b c, a b C c$. It is perceived that such an experiment must extend the farther the smaller the number of experimental plants and the larger the number of differentiating characters in the two original species; and that, furthermore, in the same species there can easily occur a delay of one or even of two generations such as Gärtner observed. The transformation of widely divergent species could generally only be completed in five or six years of experiment, since the number of different egg cells which are formed in the hybrid increases as the powers of two with the number of differentiating characters.

Gärtner found by repeated experiments that the respective period of transformation varies in many species, so that frequently a species $A$ can be transformed into a species $B$ a generation sooner than can species $B$ into species $A$. He deduces therefrom that Kölreuter's opinion can hardly be maintained that "the two natures in hybrids are perfectly in equilibrium." It appears, however, that Kölreuter does not merit this criticism, but that Gärtner rather has overlooked a material point, to which he himself elsewhere 
draws attention, viz. that "it depends which individual is chosen for further transformation." Experiments which in this connection were carried out with two species of Pisum demonstrated that as regards the choice of the fittest individuals for the purpose of further fertilisation it may make a great difference which of two species is transformed into the other. The two experimental plants differed in five characters, while at the same time those of species $A$ were all dominant and those of species $B$ all recessive. For mutual transformation $A$ was fertilised with pollen of $B$, and $B$ with pollen of $A$, and this was repeated with both hybrids the following year. With the first experiment $\frac{B}{A}$ there were eighty-seven plants available in the third year of experiment for selection of the individuals for further crossing, and these were of the possible thirty-two forms ; with the second experiment $\frac{A}{B}$ seventy-three plants resulted, which agreed throughout perfectly in habit with the pollen parent; in their internal composition, however, they must have been just as varied as the forms in the other experiment. A definite selection was consequently only possible with the first experiment; with the second the selection had to be made at random, merely. Of the latter only a portion of the flowers were crossed with the $A$ pollen, the others were left to fertilise themselves. Among each five plants which were selected in both experiments for fertilisation there agreed, as the following year's culture showed, with the pollen parent:

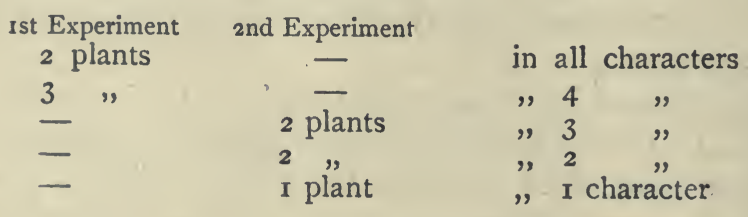

In the first experiment, therefore, the transformation was completed; in the second, which was not continued further, two more fertilisations would probably have been required.

Although the case may not frequently occur in which 
the dominant characters belong exclusively to one or the other of the original parent plants, it will always make a difference which of the two possesses the majority of dominants. If the pollen parent has the majority, then the selection of forms for further crossing will afford a less degree of certainty than in the reverse case, which must imply a delay in the period of transformation, provided that the experiment is only considered as completed when a form is arrived at which not only exactly resembles the pollen plant in form, but also remains as constant in its progeny.

Gärtner, by the results of these transformation experiments, was led to oppose the opinion of those naturalists who dispute the stability of plant species and believe in a continuous evolution of vegetation. He perceives* in the complete transformation of one species into another an indubitable proof that species are fixed within limits beyond which they cannot change. Although this opinion cannot be unconditionally accepted we find on the other hand in Gärtner's experiments a noteworthy confirmation of that supposition regarding variability of cultivated plants which has already been expressed.

Among the experimental species there were cultivated plants, such as Aquilegia atropurpurea and canadensis, Dianthus caryophyllus, chinensis, and japonicus, Nicotiana rustica and paniculata, and hybrids between these species lost none of their stability after four or five generations.

* ["Es sieht" in the original is clearly a misprint for "Er sieht."] 


\title{
ON HIERACIUM-HYBRIDS OBTAINED BY ARTIFICIAL FERTILISATION
}

\author{
By G. Mendel.
}

(Communicated to the Meeting 9 June, 1859*.)

ALthough I have already undertaken many experiments in fertilisation between species of Hieracium, I have only succeeded in obtaining the following 6 hybrids, and only from one to three specimens of them:
H. Auricula o $\times H$. aurantiacum $\hat{\delta}$
H. Auricula + $\times H$. Pilosella ô
$H$. Auricula $q \times H$. pratense $\hat{o}$
H. echioides $\uparrow+\uparrow \times H$. aurantiacum $\hat{\delta}$
H. praealtum $+\times H$. flagellare Rchb. $\delta$
$H$. praealtum o $\times H$. aurantiacum $\hat{\sigma}$

The difficulty of obtaining a larger number of hybrids is due to the minuteness of the flowers and their peculiar structure. On account of this circumstance it was seldom possible to remove the anthers from the flowers chosen for fertilisation without either letting pollen get on to the stigma or injuring the pistil so that it withered away. As is well known, the anthers are united to form a tube,

* [Published in Verh. naturf. Ver. Briinn, Abhandlungen, vill. I869, p. 26, which appeared in 1870.$]$

$\dagger$ The plant used in this experiment is not exactly the typical $H$. echioides. It appears to belong to the series transitional to $H$. praealtum, but approaches more nearly to $H$. echioides and for this reason was reckoned as belonging to the latter. 
which closely embraces the pistil. As soon as the flower opens, the stigma, already covered with pollen, protrudes. In order to prevent self-fertilisation the anther-tube must be taken out before the flower opens, and for this purpose the bud must be slit up with a fine needle. If this operation is attempted at a time when the pollen is mature, which is the case two or three days before the flower opens, it is seldom possible to prevent self-fertilisation; for with every care it is not easily possible to prevent a few pollen grains getting scattered and communicated to the stigma. No better result has been obtained hitherto by removing the anthers at an earlier stage of development. Before the approach of maturity the tender pistil and stigma are exceedingly sensitive to injury, and even if they are not actually injured, they generally wither and dry up after a little time if deprived of their protecting investments. I hope to obviate this last misfortune by placing the plants after the operation for two or three days in the damp atmosphere of a greenhouse. An experiment lately made with $H$. Auricula treated in this way gave a good result.

To indicate the object with which these fertilisation experiments were undertaken, I venture to make some preliminary remarks respecting the genus Hieracium. This genus possesses such an extraordinary profusion of distinct forms that no other genus of plants can compare with it. Some of these forms are distinguished by special peculiarities and may be taken as type-forms of species, while all the rest represent intermediate and transitional forms by which the type-forms are connected together. The difficulty in the separation and delimitation of these forms has demanded the close attention of the experts. Regarding no other genus has so much been written or have so many and such fierce controversies arisen, without as yet coming to a definite conclusion. It is obvious that no general understanding can be arrived at, so long as the value and significance of the intermediate and transitional forms are unknown.

Regarding the question whether and to what extent hybridisation plays a part in the production of this wealth of forms, we find very various and conflicting views held by leading botanists. While some of them maintain that 
this phenomenon has a far-reaching influence, others, for example, Fries, will have nothing to do with hybrids in Hieracia. Others take up an intermediate position; and while granting that hybrids are not rarely formed between the species in a wild state, still maintain that no great importance is to be attached to the fact, on the ground that they are only of short duration. The [suggested] causes of this are partly their restricted fertility or complete sterility; partly also the knowledge, obtained by experiment, that in hybrids self-fertilisation is always prevented if pollen of one of the parent-forms reaches the stigma. On these grounds it is regarded as inconceivable that Hieracium hybrids can constitute and maintain themselves as fully fertile and constant forms when growing near their progenitors.

The question of the origin of the numerous and constant intermediate forms has recently acquired no small interest since a famous Hieracium specialist has, in the spirit of the Darwinian teaching, defended the view that these forms are to be regarded as [arising] from the transmutation of lost or still existing species.

From the nature of the subject it is clear that without an exact knowledge of the structure and fertility of the hybrids and the condition of their offspring through several generations no one can undertake to determine the possible influence exercised by hybridisation over the multiplicity of intermediate forms in Hzeracium. The condition of the Hieracium hybrids in the range we are concerned with must necessarily be determined by experiments; for we do not possess a complete theory of hybridisation, and we may be led into erroneous conclusions if we take rules deduced from observation of certain other hybrids to be Laws of hybridisation, and try to apply them to Hieracium without further consideration. If by the experimental method we can obtain a sufficient insight into the phenomenon of hybridisation in Hieracium, then by the help of the experience which has been collected respecting the structural relations of the wild forms, a satisfactory judgment in regard to this question may become possible.

Thus we may express the object which was sought after in these experiments. I venture now to relate the very 
slight results which I have as yet obtained with reference to this object.

I. Respecting the structure of the hybrids, we have to record the striking phenomenon that the forms hitherto obtained by similar fertilisation are not identical. The hybrids $H$. praealtum $+\times H$. aurantiacum ô and $H$. Auricula $+\times H$. aurantiacum $\hat{f}$ are each represented by two, and $H$. Auricula + $\times H$. pratense $\hat{\sigma}$ by three individuals, while as to the remainder only one of each has been obtained.

If we compare the individual characters of the hybrids with the corresponding characters of the two parent types, we find that they sometimes present an intermediate structure, but are sometimes so near to one of the parent characters that the [corresponding] character of the other has receded considerably or almost evades observation. So, for instance, we see in one of the two forms of $H$. Auricula $+\times H$. aurantiacum is pure yellow disc-florets; only the petals of the marginal florets are on the outside tinged with red to a scarcely noticeable degree : in the other on the contrary the colour of these florets comes very near to $H$. aurantiacum, only in the centre of the disc the orange red passes into a deep golden-yellow. This difference is noteworthy, for the flower-colour in Hieracium has the value of a constant character. Other similar cases are to be found in the leaves, the peduncles, \&c.

If the hybrids are compared with the parent types as regards the sum total of their characters, then the two forms of $H$. praealtum $q \times H$. aurantiacum $\hat{\delta}$ constitute approximately intermediate forms which do not agree in certain characters. On the contrary in $H$. Auricula $q \times H$. aurantiacum $\hat{\delta}$ and in $H$. Auricula $q \times H$. pratense $\hat{\delta}$ we see the forms widely divergent, so that one of them is nearer to the one and the other to the other parental type, while in the case of the last-named hybrid there is still a third which is almost precisely intermediate between them.

The conviction is then forced on us that we have here only single terms in an unknown series which may be formed by the direct action of the pollen of one species on the egg-cells of another. 
2. With a single exception the hybrids in question form seeds capable of germination. $H$. echioides $q \times H$. aurantiacum $\hat{\alpha}$ may be described as fully fertile; $H$. praealtum $+\times H$. flagellare $\hat{\sigma}$ as fertile; $H$. praealtum $q \times H$. aurantiacum $\hat{\delta}$ and $H$. Auricula $+\times H$. pratense $\hat{\delta}$ as partially fertile; $H$. Auvicula $+\times H$. Pilosella $\hat{\delta}$ as slightly fertile, and $H$. Auricula $+\times H$. aurantiacum $\hat{\delta}$ as infertile. Of the two forms of the last-named hybrid, the red-flowered one was completely sterile, but from the yellow-flowered one a single well-formed seed was obtained. Moreover it must not pass unmentioned that among the seedlings of the partially fertile hybrid $H$. praealtum $\uparrow \times H$. aurantiacum $\hat{\delta}$ there was one plant which possessed full fertility.

[3.] As yet the offspring produced by self-fertilisation of the hybrids have not varied, but agree in their characters both with each other and with the hybrid plant from which they were derived.

From $H$. praealtum $+\times H$. flagellare of two generations have flowered; from $H$. echioides $+\times H$. aurantiacum $\hat{\delta}$, $H$. praealtum $+\times H$. aurantiacum $\hat{\delta}, H$. Auricula $q \times H$. Pilosella $\hat{\delta}$ one generation in each case has flowered.

4. The fact must be declared that in the case of the fully fertile hybrid $H$. echioides $+\times H$. aurantiacum $\delta$ the pollen of the parent types was not able to prevent selffertilisation, though it was applied in great quantity to the stigmas protruding through the anther-tubes when the flowers opened.

From two flower-heads treated in this way seedlings were produced resembling this hybrid plant. A very similar experiment, carried out this summer with the partially fertile $H$. praealtum $+\times H$. aurantiacum $\delta$ led to the conclusion that those flower-heads in which pollen of the parent type or of some other species had been applied to the stigmas, developed a notably larger number of seeds than those which had been left to self-fertilisation alone. The explanation of this result must only be sought in the circumstance that as a large part of the pollen-grains of the hybrid, examined microscopically, show a defective structure, a number of egg-cells capable of fertilisation do 
not become fertilised by their own pollen in the ordinary course of self-fertilisation.

It not rarely happens that in fully fertile species in the wild state the formation of the pollen fails, and in many anthers not a single good grain is developed. If in these cases seeds are nevertheless formed, such fertilisation must have been effected by foreign pollen. In this way hybrids may easily arise by reason of the fact that many forms of insects, notably the industrial Hymenoptera, visit the flowers of Hieracia with great zeal and are responsible for the pollen which easily sticks to their hairy bodies reaching the stigmas of neighbouring plants.

From the few facts that I am able to contribute it will be evident the work scarcely extends beyond its first inception. I must express some scruple in describing in this place an account of experiments just begun. But the conviction that the prosecution of the proposed experiments will demand a whole series of years, and the uncertainty whether it will be granted to me to bring the same to a conclusion have determined me to make the present communication. By the kindness of $\mathrm{Dr}$ 'Nägeli, the Munich Director, who was good enough to send me species which were wanting, especially from the Alps, I am in a position to include a larger number of forms in my experiments. I venture to hope even next year to be able to contribute something more by way of extension and confirmation of the present account.

If finally we compare the described result, still very uncertain, with those obtained by crosses made between forms of Pisum, which I had the honour of communicating in the year 1865, we find a very real distinction. In Pisum the hybrids, obtained from the immediate crossing of two forms, have in all cases the same type, but their posterity, on the contrary, are variable and follow a definite law in their variations. In Hieracium according to the present experiments the exactly opposite phenomenon seems to be exhibited. Already in describing the Pisum experiments it was remarked that there are also hybrids whose posterity do not vary, and that, for example, according to Wichura the hybrids of Salix reproduce themselves like pure species. In Hieracium we may take it we have a similar case. 
Whether from this circumstance we may venture to draw the conclusion that the polymorphism of the genera Salix and Hieracium is connected with the special condition of their hybrids is still an open question, which may well be raised but not as yet answered.

[The discovery of Ostenfeld and Raunkiaer that Hieracium is frequently parthenogenetic, or apogamous, of course puts an entirely new construction on the results of these experiments. See p. 247.] 


\section{BIBLIOGRAPHY}

[This list does not include works published since the appearance of the first edition. References to the more important of these will be found in the Appendixes]

I. Allen, G. M. The heredity of coat colour in mice. Proc. Am. Acad. Arts and Sci., Vol. XL. No. 2. r 904. [See also Castle.]

2. Anthony, R. Bull. Soc. Anthr., I899, p. 303. [Heredity in Manx Cat.]

3. Bacot, A. Experiments with Triphaena comes and melanic form. Proc. Ent. Soc. Lond. 1905, p. Ixvii.

4. - Larvae of Lasiocampa quercus and its vars. and of crosspairings between these races. Ent. Rec. XIII. p. I I4.

5. Balls, W. L. The wheat problem in Egypt. Year-book of Khedivial Agric. Soc. Cairo, r907.

6. - Studies in Egyptian Cotton. Ibid. I907.

7. von Baehr. Üb. d. Bildung der Sexualzellen bei Aphididae. Zool. Anz. xxxill. 1908, p. 507.

8. Bateson, W. Mendel's Principles of Heredity. Cambridge, I 902.

9. N $\quad$ Note on the Resolution of Compound Characters by Crossbreeding. Proc. Camb. Phil. Soc., Vol. xil. Nr. vi. p. 50. I902.

ro. - The present state of knowledge of colour-heredity in mice and rats. Proc. Zool. Soc. I903, Vol. II. p. 7 1. [On p. 73 the use of the terms "Cinnamon Agouti" and Golden Agouti is accidentally reversed.]

Ir. - Variation and Differentiation in Parts and Brethren. Printed for the author. Cambridge r9o3.

I 2. Presidential address to Section D. Brit. Ass. Report Cambridge. Ig04.

13. - An address on Mendelian Heredity and its application to Man. Brain, Xxix. 1906, p. I 57.

i 4. The Progress of Genetics since the rediscovery of Mendel's papers. Progr. Rei Bot. I. 1906, p. 368.

I5. Facts limiting the Theory of Heredity. Science, N. S., xxvi. 1907, p. 649.

16. Trotting and Pacing: Dominant and Recessive? Ibid. p. 908 .

I 7. and Gregory, R. P. On the inheritance of Heterostylism in Primula. Proc. Roy. Soc. B., Vol. 76, r905, p. $5^{81}$. 
18. Bateson. W. and Punnett, R. C. A suggestion as to the nature of the walnut Comb in Fowls. Proc. Camb. Phil. Soc. XIII. 1905 , p. 165 .

19. of the Royal Society. I. IgO2.

20. —— and Punnett, R. C. Ibid. II. I9ग5.

21. ——. Ibid. III. 1906.

22. — - Ibid. IV. 1908.

23. - and Punnett, R. C. The Heredity of Sex. Science, N.S. xxvil. I908, p. 785 .

24. Baur, E. Unters. üb. d. Erblichkeitsverhältnisse einer nur in Bastardform lebensfähigen Sippe von Antirrhinum majus. Ber. Deut. Bot. Ges., xxv. I 907 , p. 442. See also Zt. f. indukt. Abstammungsund Vererbungslehre, I. 1908, p. 124.

25. - Einige Ergebnisse d. experimentellen Vererbungslehre. Beihefte z. Med. Klinik. 1908, Iv. p. 265 .

26. Bell, A. Graham. On the development by Selection of Supernumerary Mammae in Sheep. Science, IX. 1899, p. 637; ibid. XIx. 1904, p. 767; also Multi-nippled Sheep, privately printed. Washington, 1904 .

27. Biffen, R. H. Mendel's laws of Inheritance and Wheat breeding. Journ. Agric. Sci. I. I. Cambridge, 1905.

28. The Inheritance of Sterility in the Barleys. Ibid. Vol. I., pt. 2, 1905, p. 250.

29. - Studies in the Inheritance of Disease-resistance. Ibid. II. I 907 , p. I09.

30. - The Hybridisation of Barleys. Ibid. II. I907, p. I83.

31. Blakeslee, A. F. Zygospore Germinations in the Mucorineae. Ann. Mycol. IV. 1906.

32. Differentiation of Sex in Thallus Gametophyte and Sporophyte. Bot. Gaz. 42, I906, p. I6I.

33. - The Biological Significance and Control of Sex. Sci., N. S., xxv. 1907 , p. 366 .

34. Boveri, T. Zellenstudien. Über die Abhängigkeit der Kerngrösse und Zellenzahl der Seeigellarven von der Chromosomenzahl der Ausgangszellen. Jena 1905 .

35. - Zellen-Studien. 6. Jena (Fischer), r9o7.

36. Brainerd, E. The behaviour of the seedlings of certain violet hybrids. Science, N. S., xxv. I907, p. $94^{\circ}$.

37. Hybridism of the genus Viola. Rhodora, Vol. vi. Igo4, p. 213.

38. —. Pt. 11. Ibid. Vol. viı. I906, p. 6.

39. —. Pt. III. Ibid. Vol. vill. I906, p. 49. 
40. Brainerd, E. Older types of N. American Violets. Ibid. Vol. IX. 1907, p. 93.

41. - Mendel's Law of Dominance in Viola. Ibid. Vol. Ix. I907, p. 2 I I.

42. Braun, $\mathbf{H}$. Über die specifischen Chromosomenzahlen in der Gattung Cyclops. Zool. Anz. xxxir. I907, p. 407 .

43. Burckhardt, F. Über Farbenblindheit. Verh. d. Naturf. Ges. Basel, v. 1873 , p. 566.

44. Cannon, W. A. Studies in Plant-Hybrids: The Spermatogenesis of Hybrid Cotton. Bull. Torrey Bot. Club, xxx. 1903, p. 133.

45. Castle, W. E. The heredity of "Angora" Coat in Mammals. Science, N. S., Vol. xviri. No. 467 , p. $760 . \quad$ r9o3.

46. The Heredity of Sex. Bull. Mus. Comp. Zool. Harvard, r 903, Vol. XL. No. 4 .

47. The Laws of Heredity of Galton and Mendel and some laws governing race-improvement by Selection. Proc. Amer. Acad. Arts and Sci., Xxxix. 1903, p. 223.

48. Heredity of Coat Characters in Guinea-pigs and Rabbits. Carnegie Instit. of Washington. 1905.

49. The Origin of a Polydactylous Race of Guinea-pigs. Carnegie Instit. of Washington. rgo6.

50. Mendel's Law of Heredity. Science, N. S., xvill. I903, p. 396.

51. Yellow Mice and Gametic Purity. Science, N. S., xxiv. r906, p. 275 .

52. - On a Case of Reversion induced by Cross-Breeding and its Fixation. Ibid. Xxv. 1907, p. 15 r.

53. Colour-varieties of the Rabbit and of other Rodents. Ibid. XXvi. 1907 , p. 287 .

54. The production and fixation of new Breeds. Proc. Am. Breeders' Ass. III. 1907, p. 34 .

55. - and Forbes, A. Heredity of Hair-length in Guinea-pigs and its bearing on the theory of pure Gametes. Ibid. 1906.

56. - and Allen, G. M. The Heredity of Albinism. Proc. Am. Ac. Arts and Sci., Vol. xxxvili. 1903, No. 2 r, p. 603.

57. with Carpenter, Clark, Mast and Barrows. The effects of inbreeding, cross-breeding and selection upon the fertility and variability of Drosophila. Proc. Am. Acad. of Arts and Sci., Vol. XLI. No. 33. $\quad$ I 906.

58. - [and MacCurdy. See MacCurdy.]

58a. Correns, C. Untersuchungen über die Xenien bei Zea Mays. Ber. Deutsch. Bot. Gesellsch. I899, xvil. No. Io, p. 4 I 0.

59. - Über den Einfluss, welchen die Zahl der zur Bestäubung verwendeten Pollenkörner auf die Nachkommenschaft hat. Ber. Deutsch. Bot. Gesellsch. 1900, Xvili. p. 422 . 
60. Correns, C. G. Mendel's Regel über das Verhalten der Nachkommenschaft der Rassenbastarde. Ber. Deutsch. Bot. Gesellsch., xvir. Heft 4, p. ${ }_{58}$. 1900.

61 U Über Levkoyenbastarde. Zur Kenntnis der Grenzen der Mendel'schen Regeln. Bot. Centr.-Bl., Bd. Lxxxiv. p. 97. I900.

62. - Gregor Mendel's "Versuche über Pflanzenhybriden" und die Bestätigung ihrer Ergebnisse durch die neuesten Untersuchungen. Bot. Ztg., I 900 , Lvili. No. I 5, p. 230.

63. - Bastarde zwischen Maisrassen, mit besonderer Berücksichtigung der Xenien. Bibl. Bot. Original-Abh. a. d. Gesamtgebiete d. Bot., Heft 53. I 90 I.

64. - Die Ergebnisse der neuesten Bastardforschungen für die Vererbungslehre. Ber. Deutsch. Bot. Gesellsch. I90I, XIX. p. (7 I).

65. - Scheinbare Ausnahmen von der Mendels'schen Spaltungsregel für Bastarde. Ber. Deutsch. Bot. Gesellsch. I902, Xx. p. I 59.

66. - Über den Modus und den Zeitpunkt der Spaltung der Anlagen bei den Bastarden vom Erbsen-Typus. Bot. Ztg., I902, LX. No. $5 / 6$, p. 66 .

67. Über Bastardierungsversuche mit Mirabilis-Sippen. Erste Mitteilung. Ber. Deutsch. Bot. Gesellsch. 1902, Bd. Xx. 10, p. 594.

68. - Neue Untersuchung auf dem Gebiet der Bastardierungslehre. Bot. Ztg., I9०3, LXI. p. II3.

69. U. Über die dominierenden Merkmale der Bastarde. Ber. Deutsch. Bot. Gesellsch. I903, xxI. p. I33.

70. - Weitere Beiträge zur Kenntnis der dominierenden Merkmale und der Mosaikbildung der Bastarde. Ibid. p. I95.

71. —. Die Merkmalspaare beim Studium der Bastarde. Ibid. p. 202 .

72. Experimentelle Untersuchungen über die Gynodioecie. Ibid. 1904, XxII. p. 506.

73. - Ein typisch spaltender Bastard zwischen einer einjährigen und einer zweijährigen Sippe des Hyoscyamus niger. Ibid. p. 5 I 7 .

74. Zur Kenntnis der scheinbar neuen Merkmale der Bastarde. Zweite Mitteilung über Bastardierungsversuche mit Mirabilis-Sippen. Ber. Deutsch. Bot. Gesellsch. I905, Bd. xxıII. p. 70.

75. Weitere Untersuchungen über die Gynodioecie. Ber. Deutsch. Bot. Gesellsch., xxili. I905, p. $45^{2}$.

76. Einige Bastardierungsversuche mit normalen Sippen und ihre allgemeinen Ergebnisse. Jahrb. f. wissensch. Bot., xLI. Heft 3, p. $45^{8}$. 1905 .

77. Ü Über Vererbungsgesetze. Vortrag. Sept. I905. Berlin. Borntraeger.

78. - Gregor Mendel's Briefe an Carl Nägeli. Abh. d. K. S. Gesellsch. d. Wissensch., math.-phys. Kl., XxIx. 11. Leipzig I905, p. 189 . 
79. Correns, C. Ein Vererbungsversuch mit Dimorphatheca pluvialis. Das Keimen der beiderlei Früchte der $D$. pluvialis. Ber. d. Deut. Bot. Ges. 1906, xxiv. p. 162 .

8o. —. Zur Kenntniss d. Geschlechtsformen polygamer Blütenpflanzen u. ihrer Beeinflussbarkeit. Jahrb. Wiss. Bot., xLIv. I907, p. 124 .

81. —. Bestimmung u. Vererbung des Geschlechtes. Leipzig; Borntraeger, 1907.

82. - Die Vererbung der Geschlechtsformen bei den gynodiöcischen Pflanzen. Ber. Deut. Bot. Gesell. Jahrg. 1906, Bd. xxıv. p. 459 .

83. Coutagne, G. Recherches Expérimentales sur l'Hérédité chez les vers à soie. Bull. sci. de la France et Belgique, xxxvı. 1902.

83a. Crampe. Zucht-Versuche mit zahmen Wanderratten. Landwirths. Jahrb., XII. I883, p. 389 ; and XIII. 1884, p. 699.

84. Cuénot, L. La loi de Mendel et l'hérédité de la pigmentation chez les souris. Arch. Zool. exp. et gén. Notes et Revue. 1902.

85. - $2^{\text {me }}$ note. Ibid. 1903 .

86. - $3^{\text {me }}$ note. Ibid. 1904 .

87. - $4^{\text {me }}$ note. Ibid. 1905.

88. - $\quad 5^{\text {me }}$ note. Ibid. 1907 .

89. - Un paradoxe héréditaire chez les Souris. Bull. Mens. de la Réunion Biol. de Nancy, 1904, p. 1050.

90. Darbishire, A. D. Note on the Results of Crossing Japanese Waltzing Mice with European Albino Races. Biometrika, II. I902, IOI; continued, ibid. p. I65; ibid. p. 282 ; ibid. III. p. I.

91. —. On the supposed Antagonism of Mendelian to Biometric Theories of Heredity. Manchester Mem., xuIx. (I905), No. 6.

92. - On the Difference between Physiological and Statistical Laws of Heredity. Ibid., Vol. L. No. I I.

93. - Professor Lang's breeding experiments with Helix hortensis and $H$. nemoralis. The Journ. Conchol., Vol. xi. No. 7, p. 193.

94. - On the result of Crossing Round with Wrinkled Peas, with especial Reference to their Starch Grains. Proc. Roy. Soc., Vol. 8o, B., 1908 , p. 122.

95. Davenport, C. B. Review of von Guaita's Experiments in breeding Mice. Biol. Bull. 11. I900, p. I2r.

96. —- Mendel's Law of Dichotomy in Hybrids. Ibid. p. 307.

97. - Colour inheritance in Mice. Wonderhorses and Mendelism. Science, N.S., Vol. XIx. No. 472 , p. I10, I904; No. 473, p. 151 , 1904.

98. _- Report on the work of the Station for Exp. Evol., Cold Spring Harbour. 1905. [Details in regard to Cats.] Fourth Yearbook Carnegie Inst.

99. —- Report for 1906. Ibid. 1907. 
100. Davenport, C. B. Origin of black Sheep in the Flock. Science, N. S. XxiI. 1905, p. 674 .

101. - Inheritance in Poultry. Publ. Carnegie Inst., No. 52, r907.

102. - Dominance of Characteristics in Poultry. R. Hort. Soc. Conference on Genetics. I907.

ro3. - Heredity and Mendel's Law. Proc. Wash. Ac. Sci. IX. I907, p. 179 .

104. - Determination of Dominance in Mendelian Inheritance. Proc. Amer. Phil. Soc., XlviI. I908, p. 59.

105. - Inheritance in Canaries. Publ. Carnegie Instit. Washington, No. $95,1908$.

ı 6. - Degeneration, Albinism and Inbreeding. Sci., N. S. xxvilı. I 908 , p. 454 .

107. and Davenport, G. C. Heredity of Eye-Colour in Man. Sci., N. S. Xxvi. I907, p. 589.

108. —- Heredity of Hair-form in Man. Amer. Nat., xuIr. I908, p. 34I.

109. Doncaster, L. On the Inheritance of Tortoiseshell and Related Colours in Cats. Proc. Camb. Phil. Soc. Xil. pt. I, p. 35. 1904.

I 10. - On the Inheritance of Coat Colour in Rats. Proc. Camb. Phil. Soc., Vol. xIII. pt. IV. p. 2 I5. 1905.

II I. - and Raynor, G. H. Breeding Experiments with Lepidoptera. Proc. Zool. Soc. London, I906, Vol. I. p. I 25. See also Nature, I907, 76, p. 248.

I 2. - On the Maturation of the unfertilized egg, and the fate of the polar bodies in the Tenthredinidae (Saw-flies). Quart. Journ. Micros. Sci., xL1X. pt. IV. I906, p. 561 .

I 3. Collective Inquiry as to Progressive Melanism in Lepidoptera. Entom. Rec. xvil.

I14. Sex Inheritance in the moth Abraxas grossulariata and its var. lacticolor. Rep. Evol. Ctee. Iv. I 908.

Drinkard, A. W. [See Price, H. L.]

115. Drinkwater, $H$. An account of a Brachydactylous Family. Proc. Roy. Soc. Edinburgh, xxvill. r9o8, p. 35.

I16. Durham, F. M. A Preliminary Account of the Inheritance of Coat-colour in Mice. Rep. Evol. Ctee. Iv. p. 4I, I908.

I 7. - Note on Melanins. Jour. Physiol. xxxv. I907.

I 8. - and Marryat, D. C. E. Inheritance of Sex in Canaries. Rep. Evol. Ctee. IV. I 908.

I 19. East, E. M. The Relation of Certain Biological Principles to Plant-breeding. Bull. 158 Connecticut Agric. Exp. Station, Nov. 1907.

120. Emerson, R. A. Preliminary Account of Variation in Bean Hybrids. I $5^{\text {th }}$ Ann. Rep. Nebraska Agric. Exp. station, I902, p. 30.

121. Heredity in Bean Hybrids. I 7 th Ann. Rep. Agric. Exp. station of Nebraska, I904, p. 33 . 
12. Farabee, W. C. Inheritance of Digital Malformations in Man. Papers of Peabody Mus. of Am. Arch. and Ethn. Harvard Univ. III. 3, I905, p. 69.

I 23. Focke, W. O. Die Pflanzenmischlinge. Berlin, I88 I.

124. Foot, K. and Strobell, E. C. Study of Chromosomes in the spermatogenesis of Anasa tristis. Am. Journ. Anatomy, vir. r907, p. 279.

r25. Galton, F. The average contribution of each several ancestor to the total heritage of the offspring. Proc. Roy. Soc., Vol. 6r, r897, p. $40 \mathrm{I}$.

I 26. Garrod, A. E. The Incidence of Alkaptonuria, a study in chemical Individuality. Lancet, Dec. I3, I902.

I27. The Inborn Errors of Metabolism (Croonian Lectures). Lancet, Jan. 4, I I, I8, 25, I908.

I28. Gates, R. R. Pollen Development in Hybrids of Oenothera lata and $O$. Lamarckiana and its relation to Mutation. Bot. Gaz. 43, I907, p. 8r.

I 29. - Hybridization and germ-cells of Oenothera mutants. Ibid. 44, I907, p. I.

r30. - A study of Reduction in Oen. rubrinervis. Ibid. 46, r9o8, p. $\mathbf{I}$.

131. - Chromosomes of Oenothera. Science, N. S., xxvir. p. I93.

132. Gossage, A. M. The inheritance of certain human abnormalities. Quart. Journ. Med. 1908, p. 331.

133. Gregory, R. P. The abortive development of the Pollen in certain Sweet Peas. Proc. Camb. Phil. Soc. I 905, XIII. p. I 48.

I 34 . The seed characters of Pisum sativum. The New Phytologist, II. IO, 1903, p. 226.

[See also Bateson.]

135. Guaita, G. von. Versuche mit Kreuzungen von verschiedenen Rassen der Hausmaus. Ber. Naturf. Ges. Freiburg, X., XI. I898, I900.

136. Guyer, M. F. Spermatogenesis of Normal and Hybrid Pigeons. Chicago, I900. Diss.

137. Hybridism and the Germ-Cell. Bull. No. 2 I of Univ. of Cincinnati, 1902.

I38. Haacke, W. Die Gesetze der Rassenmischung und die Konstitution des Keimplasmas. Arch. f. Entwicklungsmech. d. Org. XxI. No. I, I 906 . "This remarkable paper gives details of Mendelian experiments made on a large scale with Mice, prior to the rediscovery of Mendel.]

139. Häcker, V. Über das Schicksal der elterlichen und grosselterlichen Kernanteile. Jen. Ztsch. 1. Naturwissens. XxxviI. I902, p. 297.

140. - Bastardierung und Geschlechtszellenbildung. Zool. Jahrb., Sup. vir. 1904, p. I6r. 
14I. Häcker, V. Bastardierung und Geschlechtszellenbildung: Ein kritisches Referat. Zool. Jahrb. 1904, p. I6r.

142. - Die Chromosomen als angenommene Vererbungsträger. Ergeb. u. Fortschritte Zool. I. Jena (Fischer), r907.

143. - Über Mendelsche Vererbung bei Axolotln. Zool. Anz. XXxi. 1907 , p. 99.

144. — Üb. Axolotlkreuzungen. Verh. d. Deutsch. Zool. Ges. r9o8, p. 194 .

145. Hagedoorn, A. L. Origin of two new retrogressive varieties by one Mutation in Mice. Univ. California Publications in Physiol. III. p. 87,1908 .

146. Hardy, G. H. Statistical Results of Mendelian Heredity. Sci., N. S. xxviII. p. 49.

147. Harris. Experiments with Hemerophila abruptaria. Proc. Ent. Soc. Lond. I904, p. Ixxii. and 1905, p. Ixiii.

Harrison, A. [See Main, H.]

148. Heider, K. Vererbung und Chromosomen. Vortrag. Versamml. d. Naturforscher u. Ärzte. Jena, r 906.

149. Henking, $H$. Unters. üb. d. ersten Entwickelungsvorgänge in d. Eiern der Insekten. Z. wiss. Zool. I 890.

1 50. Hildebrand, F. Über einige Pflanzenbastardierungen. Jen. Ztg. f. Naturw. r889, xxiI.

I 5 I. Hind, W. Taillessness in Manx Cat. Ann. Rep. N. Staffs. Field Club, r889, p. 8 r.

152. Hurst, C. C. Notes on some experiments in Hybridisation and Cross-Breeding. Journ. R. Hort. Soc. XxIv. 1900, p. 90.

r53. —. Mendel's "Law" applied to Orchid Hybrids. Ibid. Xxvi. pt. 4 and Xxvir. 1902, pts. 2 and 3 .

r54. - Mendel's Principles applied to Wheat Hybrids. Ibid. xxviI. 1903, pt. 4 .

155. - Experiments in the Heredity of Peas. Ibid. I904, XxvirI. p. 483 .

156. - Experiments with Poultry. Rep. Evol. Committee Roy. Soc. I905, II. p. I 3 I.

157. - Experimental Studies on Heredity in Rabbits. Linn. Soc. Journ. Zool. 1905, Xxix. p. 283.

158. On the Inheritance of Coat-Colour in Horses. Proc. Roy. Soc., Vol. 77, B., 1906, p. 388 .

I59. - Mendel's Principles applied to Orchid Hybrids. Journ. R. Hort. Soc. XXvil. I902.

160. - Mendelian Characters in Plants and Animals. Report Conf. on Genetics, R. Hortic. Soc. London, I 906, p. I I 4.

I61. On the Inheritance of Eye-colour in Man. Proc. Roy. Soc., Vol. 80, B., 1908, p. 85 . 
162. Hurst, C. C. Mendel's Law of Heredity and its application to Man. Leicester Lit. Phil. Soc. Trans., XII. I908, p. 35.

163. Jennings, H. S. Heredity, Variation, and Evolution in Protozoa. Jour. Exp. Zool. v. I908, p. 577 .

164. Johannsen, W. Über Erblichkeit in Populationen und in reinen Linien. Jena, 1903. Fischer.

165. —. Üb. Knospenmutation bei Phaseolus. Zt. f. Ind. Abstammungs- u. Vererbungslehre, I. I908, p. I.

166. Kammerer, P. Vererbung erzwungener Fortpflanzungsanpassungen. Die Nachkommen der spätgeborenen Salamandra maculosa und der frühgeborenen Salamandra atra. Arch. f. Entwm. xxv. 1907, p. 7.

r66a. Kennel, J. Üb. eine stummelschwänzige Hauskatze u. ihre Nachkommenschaft. Zool. JB., Syst., Xv. I90I, 2 I9.

167. Lang, A. Über Vorversuche zu Untersuchungen über die Varietätenbildung von Helix liortensis Müller und Helix nemoralis L. Festschr. z. 70. Geburtstage E. Haeckels, p. 439. Jena, I904.

168. —_. Über die Mendelschen Gesetze, Art- und Varietätenbildung, Mutation und Variation, insbesondere bei unseren Hain- und Gartenschnecken. Vortrag. Schweiz. Naturf. Gesellsch. Luzern, I905. H. Keller.

169. - Über d. Bastarde von Helix hortensis u. Helix nemoralis. Jena (Fischer), I908.

I 70. Leake, H. Martin. Studies in Experimental Breeding of Indian Cottons. J. R. As. Soc. Bengal, N. S., IV. I 908 , p. I3.

I7I. Lewis, T., and Embleton, D. Split-Hand and Split-Foot Deformities, their types, origin, and transmission. Biometrika, vi. I908, p. 26 .

172. Lock, R. H. Studies in Plant-Breeding in the Tropics, I. Ann. R. Bot. Gard. Peradeniya, I904, Vol. II. p. 301 .

I73. - Continued, II. Ibid. Vol. II. p. 357.

I74. - III. Experiments with Maize. Ibid. Vol. III. I906, p. 95.

175. The present state of knowledge of Heredity in Pisum. Ibid. Iv. I908, p. 93 .

I76. On the inheritance of certain invisible characters in Peas. Proc. Roy. Soc., Vol. 79, B., 1907, p. 28.

I77. Recent progress in the study of Variation, Heredity, and Evolution. London, Murray, I906.

I78. Lotsy, J. P. Vorlesungen über Deszendenztheorien mit besonderer Berücksichtigung der botanischen Seite der Frage. Jena, I 906. Fischer.

179. Lutz, A. M. Prelim. Note on Chromosomes of Oenothera Lamarckiana and one of its Mutants O. gigas. Science, N. S. xxvi., p. I5 $\mathrm{I}$.

180. Lutz, F. E. The tegminal position in Gryllus. Canad. Ent., June rgo6, p. 207. 
181. Lutz, F. E. Inheritance of the manner of clasping the Hands. Amer. Nat. I908, p. 195.

182. Note on Inheritance of Variations in Color-pattern of Crioceris asparagi. Psyche, June, 1908, p. 50.

183. MacCurdy, H. and Castle, W. E. Selection and CrossBreeding in relation to the Inheritance of Coat-pigments and Coatpatterns in Rats and Guinea-pigs. Publ. Carnegie Inst., No. 70, 1907.

184. Macdougal, D. T. Mutation in Plants. Contrib. N. Y. Bot. Garden, No. 48, 1903 .

185. - Heredity and the Origin of Species. Chicago, rgo5.

186. Mutants and Hybrids of the Oenotheras. Publ. Carnegie Inst., No. 24, 1905 .

187. - Discontinuous variation in Pedigree-Cultures. Pop. Sci. Monthly, rgo6, Sept.

187a. Mutations, Variations and Relationships of Oenothera. Carn. Publ., No. 8r.

188. McClung, C. E. The Accessory Chromosome-sex determinant? Biol. Bull. III. 1902, p. 43 .

189. McCracken, Isabel. A Study of the Inheritance of Dichromatism in Lina Lapponica. J. of Exp. Zool. I905, II. p. I I 7 .

190. - Inheritance of Dichromatism in Lina and Gastroidea. Ibid. 1906, III. p. 32 I.

191. - Occurrence of a Sport in Melasoma (Lina) scripta and its behaviour in Heredity. Jour. Exp. Zool. 1907, Iv. p. 22 .

192. Main, H. and Harrison, A. Experiments with Amphidasys betularia. Proc. Ent. Soc. Lond. 1905, p. vi.

193. Marryat, D. C. E. Notes on the Infection and Histology of two Wheats immune to the attacks of Puccinia glumarum, yellow rust. Journ. Agric. Sci. II. 1907, p. 129.

[See also Durham.]

194. Mayer, A. G. Some species of Partula from Tahiti. Memoirs Mus. Comp. Zool. Cambridge U.S.A. I902, p. Ir 7 .

195. Mendel, Gregor Johann. Versuche über Pflanzen-Hybriden. Verh. Naturf. Ver. in Brünn, Bd. Io, 1865, Abh. p. I ; reprinted in Flora, rgor, and in Ostwalds Klassiker d. exakten Wissensch. English Trans. in Journ. R. Hort. Soc. rgoI, XXvi.

196. — Über einige aus künstlicher Befruchtung gewonnene Hieracium-Bastarde. Ibid. vill. I869, Abh. p. 26.

197. — Briefe an Carl Nägeli. [See Correns (78).]

198. Millardet, A. Note sur l'hybridation sans croisement ou fausse hybridation. Mém. Soc. des Sci. phys. et nat. de Bordeaux, IV. $4^{\text {me }}$ Série, 1894.

199. Morgan, T. H. The Assumed Purity of the Germ-Cells in Mendelian Results. Science, 1905, xxIr. p. 877. 
200. Morgan, T. H. Are the Germ-Cells of Mendelian Hybrids "Pure"? Biol. Cblt. 1906, xxvi. p. 289.

20r. The cause of Gynandromorphism in Insects. Amer. Nat. XLI. 1907, p. 715 .

202. —- Production of two kinds of Spermatozoa in PhylloxeransFunctional "Female-producing and Rudimentary Spermatozoa." Proc. Soc. Exp. Biol. and Med. v. 1908 , p. 56.

203. - Some Experiments in Heredity in Mice. Science, N. S. xxvi1. I908, p. 493.

204. Mudge, G. P. On some features in the Hereditary Transmission of the Self-black and the "Irish" Coat Characters in Rats. Proc. Roy. Soc. B., Vol. 80, rgo8, p. 97. Also Ibid. p. 388.

205. - - On Intravascular Coagulation in Albinoes, \&c. Ibid. Vol. 79, I907, p. I03.

206. Nettleship, E. On heredity in the various forms of Cataract. Rep. Roy. Lond. Ophth. Hosp. Xvi. I905, p. I.

207. - Additional cases of Hereditary Cataract. Ibid. XvI.

208. - On Retinitis pigmentosa and allied diseases. Ibid. xviI. Pts. I, II, and III.

209. - Cases of colour-blindness in women. Ophth. Soc. Trans. XXVI. 1905 .

2ro. - Three new Pedigrees of Eye Disease. Ophth. Soc. Trans. Xxvili. 1908, p. 220.

$2 \mathrm{II}$. - A history of congenital stationary night-blindness in nine consecutive generations. Ibid. xxviI. 1907.

212. - and Ogilvie, F. M. A peculiar form of hereditary congenital cataract. Ibid. xxvi. 1906.

2I 3. Noorduyn, C. L. W. Iets over Kleuren, Kleurverandering der Vogels en paring van variëteiten. Album der Natuur, Dec. I903, p. 7I.

214. - Een Tegenspraak van Mendel's Wet der Erfelijkheid. Ibid. 1906. [Wild male Canaries bred with some domestic varieties gave some offspring more or less pied with white or yellow.]

215. - Die Erblichkeit der Farben bei Kanarienvögeln. Arch. Rassen u. Gesell. Biol., 5 Jahrg. 1908, p. r6r.

[Ogilvie, F. M., see Nettleship, E.]

216. Orton, W. A. The Wilt disease of Cotton and its control. U.S. Dep. Agric. Bull. 27, 1900.

217. Ostenfeld, C. H. Zur Kenntnis der Apogamie in der Gattung Hieracium. Ber. Deutsch. Bot. Gesellsch. 1904, xxir. 7.

2 r8. Castration and Hybridisation Experiments with some species of Hieracia. Bot. Tidsk. xxvir. 3. Kjobenhavn, 1906, p. 225. 
2rg. Pearson, $\mathbf{K}$. On the Inheritance of Characters not capable of exact Quantitative Measurement. Part II. On the Inheritance of Coat-Colour in Horses. Part III. On the Inheritance of Eye-Colour in Man. Phil. Trans. Roy. Soc., Vol. 195, A., I900, p. 79.

220. - On a generalised Theory of Alternative Inheritance, with special reference to Mendel's Laws. Ibid., Vol. 203, A., I904, p. 53.

221. Price, H. L. and Drinkard, A. W. Inheritance in Tomato Hybrids. Virginia Agric. Exp. Sta. Bull. I77, r908, p. I8.

222. Prout, L. B. On two variable broods of Triphaena comes from Forres. Ent. Rec. Xv. p. 2 I 7 and Xvi. p. I.

223. Xanthorrhoe ferrugata and the Mendelian hypothesis. Trans. Ent. Soc. Lond. 1906, p. 525. [The experiments proved that purple var. is dominant, black is recessive. Author's conclusion erroneous through mistake as to meaning of terms, as pointed out by Doncaster. Ibid., Proc. 1907 , p. xx.]

224. Przibram, H. Vererbungsversuche üb. asymmetrische Augenfärbung bei Angorakatzen. Arch. f. Entwm. xxv. 1907, p. 260.

225. Punnett, R. C. Mendelism. Macmillan and Bowes, Cambridge, 1905.

226. Mendelism in relation to disease. Proc. Roy. Soc. Med. 1908.

227. - Sex-determination in Hydatina with some remarks on Parthenogenesis. Proc. Roy. Soc., Vol. 78, B., I906, p. 223.

[See also Bateson.]

[See also Wood.]

228. Raunkiaer. Kimdannelse uden Befrugtning hos Maelkeb $\varphi$ tte (Taraxacum). Kjobenh. Bot. Tidsskr. xxv. 1903, p. 109. Abstr. in Bot. Cblt. xcin. I 903 , p. 8 I.

[Raynor, G. H., see Doncaster.]

229. Rosenberg, O. Das Verhalten der Chromosomen in einer hybriden Pflanze. Ber. Deutsch. Bot. Gesellsch. I903, XXI. p. Io.

230. - Über die Tetradenteilung eines Drosera-Bastardes. Ibid. I904, XXII. p. 47 .

231. Über die Reduktionsteilung in Drosera. Meddel. f. Stockholms Högsk. Bot. Inst. I 904 .

232. Zur Kenntnis der Reduktionsstellung in Pflanzen. Bot. Notiser. 1905 .

233. — Über die Embryobildung in der Gattung Hieracium. Ber. Deutsch. Bot. Gesellsch. XxIv. 3. 1906.

234. Erblichkeitsgesetze und Chromosomen. Bot. Studier. Uppsala, 1906, p. 237 .

235. Cytological Studies on the Apogamy in Hieracium. Bot. Tidsskrift, 28. Copenhagen, r907.

236. Russo, A. Modificazioni Sperimentali dell' Elemento epetheliale dell' Ovaia dei Mammiferi. Reale Accad. Lincei, I907, vi. p. 3 I3. 
Saunders, E. R. [See Bateson.]

237. Schuster, E. H. J. Results of crossing Grey (House) Mice with Albinos. Biometrika, IV. Igo6.

238. Shull, G. H. The Composition of a Field of Maize. Proc. American Breeders' Ass., Vol. IV.

239. - The Pedigree Culture. The Plant World, Ir. p. 2 I, I 908.

240. - Importance of the Mutation Theory in practical Breeding. Proc. American Breeders' Ass. III. p. 60, 1907.

24r. Some new cases of Mendelian Inheritance. Bot. Gaz. 45, 1908, p. 103.

242. - A new Mendelian Ratio and several types of latency. American Naturalist, XLII. p. 433, 1908.

243. - Elementary Species and Hybrids of Bursa. Science, N. S., xxv. 1907 .

244. - The Significance of Latent Characters. Ibid. p. 792.

245. - Some Latent Characters of a White Bean. Ibid. p. 828.

246. Spillmann; W. J. A Mendelian character in Cattle. Science, I906, N. S., xxIII. 549.

247. - Quantitative Studies on the Transmission of parental characters to Hybrid offspring. Proc. I5th Ann. Con. of Ass. Am. Agri. Coll. and Exp. Stations, p. 88.

248. Exceptions to Mendel's Law. Science, 1902, N. S., Ẋvi., No. 4 II, p. 794 .

249. - Standardizing Breed Characteristics. Soc. Prom. Agric. Sci. 1907.

250. - An interpretation of elementary species. Sci., N. S., xxvir. I908, p. 896.

251. Inheritance of the Belt in Hampshire Swine. Ibid. 1907, p. 54 I.

252. Inheritance of Coat-Colour in Swine. Ibid., N. S., xxiv. p. 614, i 906 .

253. - Origin of Varieties in Domesticated Species. Ibid., N. S., xxviII. I908, p. $25^{2}$.

253a. - Spurious Allelomorphism. Amer. Nat. XLII. I908, p. 6 г 2. 253b. Standfuss, M. Handb. d. pal. Gross-Schmetterl. Jena, 1896. 254. Staples-Browne, R. Note on Heredity in Pigeons. Proc. Zool. Soc. of London, 1905, Vol. II. p. $55^{\circ}$.

255. - On the Inheritance of Colour in Domestic Pigeons, with special reference to reversion. Proc. Zool. Soc. London, p. 67, 1908.

256. Stevens, N. M. Study of the Germ-cells of Aphis rosae and Aphis oenotherae. Journ. Exp. Zool. 1905.

257. —. Studies in Spermatogenesis with especial reference to the accessory chromosome. Carnegie Inșt. Washington, 1905, No. 36 . 
258. Stevens, N. M. Ditto, Part 11. with reference to Sex Determination. Ibid. I906, No. 36, II.

259. - Studies on the Germ-cells of Aphids. Ibid. 1906, No. 5 I.

260. Colour-inheritance and sex-inheritance in certain Aphids. Sci., N. S., Xxvi. 1907, p. 2 I6.

261. A Study of the Germ-cells of certain Diptera. J. Exp. Zool. I908, v. p. 359 .

Strobell, E. C. [See Foot, K.]

262. Sutton, A. W. Brassica Crosses. Jour. Linn. Soc., Botany, xxxvil. I908, p. 337 .

263. Sutton, W. S. On the Morphology of the Chromosome group in Brachystola magna. Biol. Bull. IV. I, I902, p. 24.

264. Tannreuther, G. W. History of the Germ-cells and early Embryology of certain Aphids. Zool. Jahrb. xxiv. 1907, p. 6 ro.

265. Tischler, G. Zellstudien an sterilen Bastardpflanzen. Arch. Zellforschung, I. 1908, p. 35 .

266. Tower, W. L. An Investigation of Evolution in Chrysomelid Beetles of the Genus Leptinotarsa. Publ. Carnegie Inst. No. 48, 1906.

267. Toyama, $\mathbf{K}$. On the hybridology of the Silk-worm. Report Sericult. Ass. Japan. May, 1906.

268. — - On some Silk-worm crosses with special reference to Mendel's Law of Heredity. Bull. Coll. Agric. Tokyo Imp. Univ. 1906, vil.

269. Tschermak, E. Über künstliche Kreuzung bei Pisum sativum. Zts. f. d. landw. Versuchsw. in Österr. I 900, III. Jahrgang, Hett 5.

270. - Über Züichtung neuer Getreiderassen mittels künstlicher Kreuzung. Ibid. I90I, IV. Jahrgang, Heft 2.

271. W. Weitere Beiträge über Verschiedenwertigkeit der Merkmale bei Kreuzung von Erbsen und Bohnen. Ber. Deutsch. Bot. Gesellsch. I 90I, XIX. Heft 2.

272. - Weitere Beiträge über Verschiedenwertigkeit der Merkmale bei Kreuzung von Erbsen und Bohnen. Zts. f. d. landw. Versuchsw. in Österr. I90I, IV. Jahrgang, Heft 6.

273. - Über die gesetzmässige Gestaltungsweise der Mischlinge. (Fortgesetzte Studien an Erbsen und Bohnen.) Zts. f. d. landw. Versuchsw. in Österr. I902.

274. —. Der gegenwärtige Stand der Mendel'schen Lehre. Ibid. 1902.

275. Die Theorie der Kryptomerie und des Kryptohybridismus. Beihefte z. Bot. Centralbl. I903, Bd. Xvi. Heft I.

276. - Über künstliche Auslösung des Blühens beim Roggen. Ber. Deutsch. Bot. Gesellsch. Xxil. I 904.

278. Weitere Kreuzungsstudien an Erbsen, Levkojen und Bohnen. Zts. f. d. landw. Versuchsw: in Österr. I904. 


\section{Bibliography}

279. Tschermak, E. Über Züchtung neuer Getreiderassen mittels künstlicher Kreuzung. II. Kreuzungsstudien am Roggen. Ibid. 1906.

280. —. Die Blüh- und Fruchtbarkeitsverhältnisse bei Roggen und Gerste und das Auftreten von Mutterkorn. Fühlings Landw. Ztg. Lv. I906, Heft 6, p. I94.

28r. - Der moderne Stand des Vererbungsproblems. Arch. Rassen- u. Gesell.-Biol., 5. Jahrgang, I908, p. 305.

282. - Über Bildung neuer Formen durch Kreuzung. Résultats sci. du Congrès intern. Bot., Wien, I906, p. 323.

283. - Die Mendelsche Lehre u. die Galtonsche Theorie vom Ahnenerbe. Arch. Rass.- u. Gesell.-Biol,, 2. Jahrgang, I905, p. 663.

284. Die Kreuzung im Dienste der Pflanzenzüchtung. Deut. Landw. Gesell., Bd. 20, 1905, p. 325.

285. - Die Lehre von den formbildenden Faktoren u. ihre Bedeutung f. die rationelle Pflanzenzüchtung. Jahrbuch für Pf. u. Tierzüchtung. 1903 .

286. — Die Kreuzungszüchtung des Getreides und die Frage nach den Ursachen der Mutation. Monatshefte f. Landw. I 908.

287. de Vries, H. Intracellulare Pangenesis. Jena, x889. G. Fischer. 288. - Monstruosités Héréditaires offertes en échange aux Jardins Botaniques. Bot. Jaarboek, IX. I897, pp. 62-93.

289. - Sur la loi de disjonction des hybrides. C. R. I900, Cxxx. 26. mars.

290. —- Das Spaltungsgesetz der Bastarde. Ber. Deutsch. Bot. Gesellsch. XviII. I900, p. 83.

29r. U Über erbungleiche Kreuzungen. Ber. Deutsch. Bot. Gesellsch. XviII. I900, p. 435 .

292. _- Sur la fécondation hybride de l'endosperme chez le mais. Rev. Gén. de Bot. I900, XII. p. I29. I 900.

293. Sur les unités des caractères spécifiques et leur application à l'étude des hybrides. Rev. Gén. de Bot. xI!̣. 1900, p. 257.

294. . - Die Mutationen und die Mutationsperioden bei der Entstehung der Arten. Vortrag. Hamburg, I901.

295. - Anwendung der Mutationslehre auf die Bastardierungsgesetze. Ber. Deutsch. Bot. Gesellsch. XXI. I903, p. 45.

296. . Befruchtung und Bastardierung. Vortrag in d. I5r. J.-Vers. d. Hol. Gesellsch. d. Wissensch. Leipzig, 1903. Veit \& Co.

297. _ La loi de Mendel et les caractères constants des hybrides. C. R. I903. 2. février.

298. - Die Mutationstheorie. 2 vols. Leipzig, 1901-3. Veit \& Co.

299. - Species and Varieties, their Origin by Mutation. Chicago, Open Court Publishing Co. 1905.

B. H. 
300. de Vries, H. On Twin Hybrids. Bot. Gaz., Vol. 44, 1907, p. 401.

301. - Die Svalöfer Methode zur Veredelung landwirthschaftlicher Kulturgewächse u. ihrer Bedeutung f. d. Selektionstheorie. Arch. Rass.- u. Gesells.-Biol. III. I906, p. 325 .

30ra. Walker, G. Remarkable cases of hereditary Anchyloses, or absence of various phalangeal joints with defects of the little and ring fingers. Johns Hopkins Hosp. Bull., XII. I90I.

302. Warburg, J. C. On some races of Lasiocampa quercus. Ent. Rec. XIII. 1901, p. 237.

303. Wheldale, M. Inheritance of Flower Colour in Antirrhinum majus. Proc. Roy. Soc. 79, B., 1907, p. 288.

304. Wichura, M. Die Bastardbefruchtung im Pflanzenreich. Breslau, 1865 .

305. Wilson, E. B. Mendel's Principles of Heredity and the Maturation of the Germ-cells. Science, Dec. I902, p. 99 r.

306. - Studies on Chromosomes. I. The Behaviour of the Idiochromosomes in Hemiptera. Journ. Exp. Zool. 11. 3, p. 37 r. Baltimore, r905.

307. - Studies on Chromosomes. II. The paired Microchromosomes, Idiochromosomes and Heterotropic chromosomes in Hemiptera. Ibid., Vol. II. 4. I905.

308. - Studies on Chromosomes. III. The sexual differences of the Chromosome-groups in Hemiptera, with some considerations on the determination and inheritance of sex. Ibid. III. I906. No. I.

3०9. Note on the Chromosome-groups of Metapodius and Banasa. Biol. Bull. XII. I907, p. 303.

310. - The Case of Anasa Tristis. Science, N. S., xxv. I907, p. I9r.

3II. Wilson, J. Mendelian Characters among Short-horn Cattle. Sci. Proc. Roy. Dublin Soc. Xı. (N. S.) 1908 (see also Nature, Apr. 2, I 908 , p. 509).

3r2. Wood, T. B. Note on the inheritance of horns and face-colour in sheep. Journ. Agri. Sci., Vol. I. pt. 3, p. 364 .

313. - and Punnett, R. C. Heredity in Plants and Animals. Trans. Highland Agric. Soc. Scotland, I908.

314. Woods, F. A. Mendel's Law and some records in Rabbit breeding. Biometrika, II. I903, p. 299.

315. Non-inheritance of Sex in Man. Biometrika, v. 1906, p. 73 .

316. Yule, G..Udny. Mendel's Laws and their probable relations to intra-racial Heredity. New Phytologist, I. 1902, Nos. 9 and 10. 


\section{INDEX OF SUBJECTS}

Abraxas grossulariata

colours 44

relations to var. lacticolor $\mathbf{I 7 4}$

Acidalia virgularia $\mathbf{2 5 2}$

Acquired characters 5

Adaptation 288

Aglia tau 44, 310

sex-limited descent 187,310

Agouti-colour 80, II7, 199

\section{ALBINOS}

axolotl, exceptional 43

cats 227

giving reversionary offspring $88,9^{6}$

guinea-pigs, smudged 113

man 4I, 226

orchids 96

rabbit 75

recessive 45

Alkaptonuria 227, 233

Allelomorphism $10, \mathrm{Ir}, \mathrm{I} 6$

spurious I5I, I53, 160, 195, 3I4

Alternation of generations $\quad 257$

Amphidasys betularia 44

Amphilepsis $\mathbf{2 4 8}$

Ancestry, theory of heredity based on, contrasted with Mendelian system 55,130

applied to Basset hounds 126

Anemone, hybrid 250

Angerona prunaria 44

Annual and biennial habit 25

Anthers 28

Anthocyan 98, 280

Anticipation, in age at which disease appears 218

Antirihinum

colours 19, 38, 269, 308

coupling 318

"Delilah" types 87

dwarf I9

golden-leaved 253

heredity compared with that of sweet pea 98

intermediate colours 236

Aphis

alternation $\quad 257$

chromosomes $27 \mathbf{I}$

colour 190

sex 189,258

Apogamy 246
Aquilegia, hybrid said to breed true

373

Arabis albida, double 197

Asymmetry compared with variation 276

Athene noctua 49, IIo

Atropa Belladonna 38, I35

Axils of sweet peas $\mathbf{I}_{\mathbf{5}} \mathbf{2}$

Axolotl albino 43

\section{BARLEY}

abortion of florets 26

colour 39

ears 22,27

hoods 21

Basset hounds 6, 126

Bean, see Phaseolus

Bees, Mendel's experiments with 329

Beet, "runners" 25

various characters 307

Begonia, doubling 198

Beta 25, 307

Biennial and annual habit 25

Biometry 6 misuse of statistics 235

"Bisexual" heredity 285

Black

fowls 136

pigeons 100

insects 137

Blue colour

in animals 83

in plants, dominant and recessive 135

in maize 256

Boarmia, wings I 70

Bombyx mori, see Silkworm

Brachydactyly 210, 214

Brassica 3r, 39, 308

Bryonia dioica and alba colour of fruits 135

sex 166, 203

Bud-sports 272

Bulbing, of turnip $\mathbf{3} \mathbf{I}$ of Beta 307

Bursa 25, 307

Callimorpha dominula 44, 135

Campanula hose-in-hose 26,200

CANARY

baldness 37

cinnamon II3, 178

colours 43

$26-2$ 


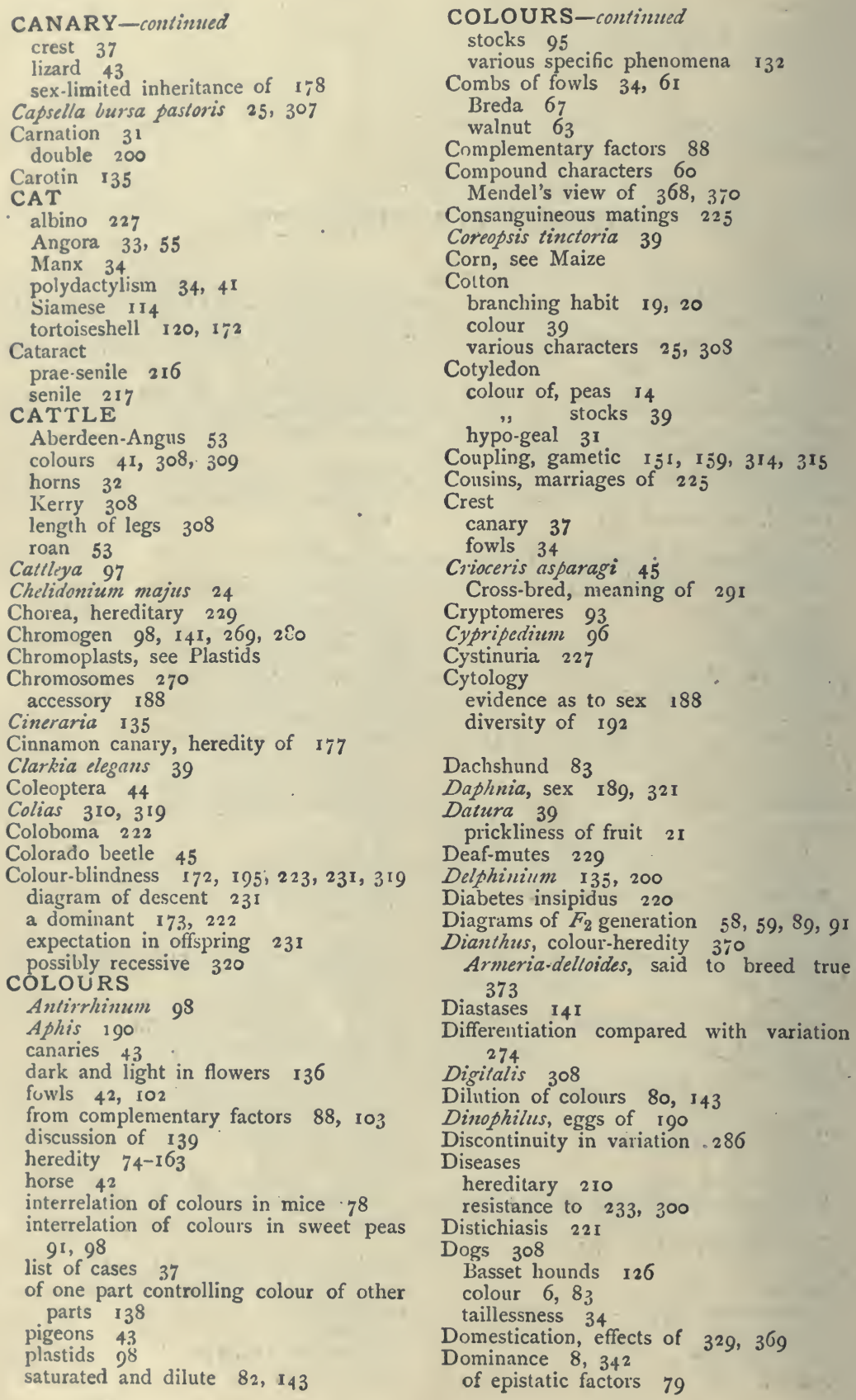




\section{Index of Subjects}

Dominance

imperfect 53

incident of special cases 13,50

irregularities of 255

and phylngeny $2 ; 8$

recognition of 231

in sex $16_{5}$

Dominant malformations and diseases 210

sex-limited 222

Dominant whites $\mathrm{rOI}, \mathrm{rO}_{4}$

in plants 105,299

DOUBLE FLOWERS 31, I96

Arabis 197

carnation 200

Petunia r98

Primula 199

stocks $20 \mathrm{I}$

Drosera, chromosomes $27 \mathrm{I}$

Drosophila 310, 310

Dutch pattern $84,85, \mathbf{I}_{42}$

Dwarfness $8,18,238,281$

Echinoderm hybrids 3 II

Ectopia lentis 222

Ectrodactylism 228

Environmental disturbance 246

Epidermolysis 220

Epistatic factors 79, 98, ror

Eugenics 304

"Ever-sporting" types 253

Evolution 283

Exceptional cases 245

in pigeon 36

Extracted types 92

EYE-COLOUR

canaries II3 $_{3}$

cats $4 \mathrm{I}$

fowls 43

guinea-pig II3

heredity, general 'account of I07

man 4I, 106

mice III

owl 49

Siamese cats II4

Eye-lashes, redupication of 22 I

$F_{1}$ and $F_{2}$ generations defined 8,57

FACTORS

carried by albino 77

for colour 75

complementary 88

epistatic and hypostatic 79

latent $93, I_{4} 6$

"False-hybrids" 248

Fancy points, often fructuational 300

Fasciation I $4_{4}$

Fat, excessive in yellow mice 163

Ferments 98, 233

Fertilisation

double 270

nature of 249

Ficaria 329
Fingers

abortion of 216

crooked 208

short 210

Fixing types 296

Fluctuational variation $239, \mathbf{2 8} 7$

FOWLS 34

Andalusians 5I

" eye-colour Iro

Aseel 102

barring 319

Brahma 102

Breda 35,36

broodiness 36

brown-breasted dominant $\quad 136$

buff 12 I

colours 42

comb shapes 34,6 I

crest 35

Dorking 63,255

down colour 42, 5 ז

duckwing 42

Egyptian 36, 187

eye-colour 43, I Io

Faverolles 35

feathered leg 35

fertility 36

frizzling 35

Hamburgh $6_{3}$

Houdan 36, 255

Indian game 62,186

Leghorn, black 187 .

" brown 102, 182

, white I02, I86

," $"$ eye-colour I ro

lobe 43

Malay 63

, eye-colour I ro

muff 35

pile 120

Plymouth Rock

Polish 36

polydactylism $35,228,255$

reversion in 103

rose-comb, white $5 \mathbf{I}, \mathbf{1 0} 3$

rumpless 35

sex-limited descent 43, 3 I9

shrieking 36

silky $34,35,43,103$

", eye-colour I I0

, heredity of pigmentation $\mathbf{I 8 I}$

skin yellow or white $\mathbf{1 8 2}$

Spanish I86

"vulture-hock" 35

white types dominant and recessive 102

Wyandottes, black 187

Gallus bankiva

colour 104

comb 6I

Galton's theory of heredity comipared with Mendelism 55, 129 
Gametes 11, 16

compared with zygotes 56

in groups of four 195

Gastroidea dissimilis 45

Genetics

geometrical aspect $28 \mathrm{I}$

study of I

Gerbera Jamesoni, red and yellow 136

Geum urbano-rivale, said to breed true $\mathbf{3 5 5}$

"Ghost"-markings in peas 145

Glands, Matthiola 21

Glaucoma 222

Gossypium, see Cotton

Gowers' disease 222, 225

Gradational forms 235

GUINEA-PIG

albino II 3

Angora hair 33

chocolate II 8

colour $4^{\text {I }}$

eye-colour $1 \mathrm{I} 3$

polydactylism 34

re-combinations in $\mathbf{7 r}$

rosetted 33

Gynandromorphs $32 \mathrm{I}$

Haemophilia 222, 224

Hair

curly 207

white lock 207

Hands

malformations of $21 \mathrm{I}, 216$

mode of clasping 48

Hapsburg lip, probably dominant 206

Hare-lip 220

Hawkweeds 246

Height

- Antirrhinum is

man 209

peas 8

sweet peas 9

Helianthus

branching habit I9

colour 39

red variety 308

Helix hortensis and nemoralis 45, $3 \mathrm{II}$

Hemerophila abruptaria 44

Heterostylism $27,48,68$

Heterozygote 15,16

permanent 5 I, I55, I $81,253,298$ in sex 190

Heterozygous forms 37, 52, 299

Hieracium 246

Mendel's experiments with 380

Homoeosis 196, 213,221

Homozygote 15,16

Hooded standard in sweet pea, genetics of 153

Hordeum, see Barley

Horns

cattle $\quad 32$

goats $\mathrm{I} 70$

sheep 169
Horse, race-

chestnut, a recessive 124

colour 42, 309, 3 II

trotters and pacers 32

Hose-in-hose flowers 26, 197, 200

"Hybrid-character" 347

Hybrids

breeding true $246,249,323,355$

some fenales permanently 193

sterility of $25 \mathrm{I}$

Hydatina 192

Hyoscyamus

annual 25

colour 39

Hypo-geal cotyledons, Phaseolus 3 I

Hypostatic factors 79

Incompatibility of characters

73

Insanity 229

Intermediates

misunderstanding in regard to $235,2+1$ various kinds of $236-239$

Inter-racial heredity not different from intra-racial 49

Irideremia $\mathbf{2 2 2}$

Iris, colours of 108

Lasiocampa quercus 44

"Latency" 93, I 45

Lathyrus odoratus, see Sweet Pea

Lavatera 373

Leaf-characters $22,24,25,72$

Lecithin, alleged effects of 193

Leopard, black, damask marks in $\mathbf{I} \mathbf{4 5}$

Lepidoptera colours 43,310

gynandromorphs 321

polymorphic females 319

Leplinotarsa 45

Lina lapponica 45

Lychnis

colour 39

hairiness 19

sex $\quad$ r69

Lymantria dispar $32 \mathrm{I}$

Maize

colour 4 I, 256

dent and flint 264

maternal characters $2 \sigma_{4}$

starchy endosperm 30

MAN 32, 41, 204 eye-colour 106, 206

hair-colour 206

interracial crossing 208

irregular numbers 254

mulatto 208

musical sense $\mathbf{2 2 5}$

recessive variations $\mathbf{2 2 5}$

stature 209

various dominants 207

Mangel, "runners" 25, 307

Maternal characters

in orchids 248 
Maternal characters in seeds 258

in snails $3 \mathrm{II}$

Matthiola, see Stocks sinulata $2 \mathrm{I}$

Medicago, twist of fruits 48

Megachile, crosses sweet peas $15 \mathrm{I}$

Melanic moths $44,137,187$

Melasoma scripta 45

Mendelian system contrasted with Galton's 55,129

Meristic characters 47

MICE

black-eyed white 87

colour 4I, 309

dominant pied 87

eye-colour III, 309

excessive fatness 113

hairless 32

interrelation of colours 78

reversion 112

sterility of 120

waltzing habit 33 , II I

yellow 79, I1 8

Mimulus, hose-in-hose $20 \mathrm{r}$

Mirabilis

colours $39,309,312$

intermediate colours in 236

Mollusca 45,48

Monilithrix 220

Monolepsis 248, 323

Mosaic seeds 249

Mulatto 208

Musical sense 225

Mutation 287

Myopia 221

Nectarine 20, 273

Negro 208

Nicotiana hybrids $37 \mathrm{I}$

Night-blindness

sex-limited 222

stationary $22 \mathrm{I}$

Novelties

made by re-combinations 60 raising 293

Numbers, aberrant 252,345

Nystagmus 227, 322

Oak-egger 44

Oats 3 II

Oedema, nervous 220

Oenothera 28, 31, 250, 286, 323 chromosomes $27 \mathrm{I}$

Orchids

reversion in 39

monolepsis . 248

Owl, eye-colour 49, I ro

Oxalis 47

Papaver 39

colour $24 \mathrm{I}$

double 200

Papilio, polymorphic females
Paralysis, familiar 229

peroneal 225

Paramoecium 277

Pararge egeria $25 \mathrm{I}$

Parthenogenesis 246, 323

Partula 48

PEA, edible

"Acacia" 315

"American Wonder" 244

blunt pods 22, 72

colour of cotyledon 14, 40, 72, 344

bleaching 346

particoloured 243

colour of flower 40

" of pod $14,3+6$

", of seed-coat $14,40,144,262,343$

"Continuity" 72

dwarfness 18,236

fasciated stem 25

fasciation $\mathbf{r}_{4}$

height $14,236,307$

indent 29,259

maple 144,263

Mendel's experiment 8,337

"nummy" 263

"Nain de Bretagne" 262

"Nonpareil" 72, 244

pod

colour of $\mathrm{I}_{4}$

parchment 22

pointed 22

shape of $\mathrm{I}_{4}$

soft 22

seed

particoloured 243

shape $14,28,260,267$

starch in seed 28

sugar peas 22, 260

tallness 18

thickness of stem 307

time of flowering 307

"Victoria" 263

Peach 20, 273

Pelargonium, leaf-colour $3^{\mathbf{1}} \mathbf{3}$

Periodicity 197

Petalody 197

Petals

imbricated 26

laciniated 24

Petunia, doubling 198

Phaseolus

colours 39

cotyledons $3 \mathrm{I}$

Mendel's experiments with $3^{6} 5$

pods 22

sterility in hybrids 348

tall and dwarf 18

weight of seeds ${ }^{\circ} 239$

Phylloxera 189

Phyteuma Halleri 40

Phytophaga 44

Pied varieties, dominant pied in mice and fowls 86 
Pied varieties, relation to whole-coloured 84

Pigs, colour 42
PIGEONS
colour 43,309
fantail 36
nun 36 100
reversion 100
sex-linited descent 194, 319
shell 36
webled foot 36
Piginents
of horses 125
of mice 116

Pisum sativum, see Peas, edible

Plant-characters I4

Plastid colours 95, 98, 204 white dominant to yellow

Polarity of zygote cell $3^{\text {I } 5}$

Polemonium 40

Pollen grains of sweet pea $2 S, 91$, I 50 figured 150

\section{POLYDAC'TYLISM}

cat 34

fowls $35,228,255$

guinea-pig 34

imperfect dominance of 53

man 228

Polyzoa 275

Poppies 39, 200, 24I

"Porcupine men" 219

Porokeratosis 220

Potato 307

Practical hints 231, 29r

Pre-Mendelian writings 5

Presence and absence, hypothesis of 54 , 65,76 bearing on pathology 232

Prickliness 2 I

PRIMULA 40, 309

colour in $F_{2} \quad 72,294$

colour in $F_{2}$ from two whites 105

coupling 315

crossing 294

dominant and recessive whites 105

double 31,199

heterostylism 27, 68, 295

"lavender" 299

palm-leaf and fern-leaf 24

special phenomena of colour in 138

stellate and imbricate petals 26,236

striping $\quad 3^{12}$

white-edged flowers $\quad 3_{3} 8$

Pseudo-hypertrophic muscular paralysis 222, 225

Ptosis 222

Puccinia ghtmarum 25, 233

"Pure lines" of Johannsen 239

Purity of type

critical meaning of 17,291

how produced by Selection of germ-cells 146

Pygaera 310

\section{RABBIT}

Angora hair 33

Belgian hare 119

colour $4 \mathrm{I}$

a dominant pied form 86

Dutch-marked $84,85,142,237$

"English" pattern 4I, 86, 239

Himalayan III, 123

interrelations of colours $\mathbf{7 5}$

lop-eared $25 \mathrm{I}, 323$

sex statistics 193

tortoiseshell II 7

yellow in 6

Ranunculus arvensis, fruits

Ratios in ficaria 329

$9: 3: 4$ explained 77,$80 ; 12: 3: 1,78$;

$9: 7, \quad 89 ; \quad 18: 18: 6: 6: 16, \quad 40$;

$27: 9: 28,9$ I

aberrant 252,318

various 79, 3 II

RATS

colours $4 \mathrm{I}$

"ghost"-markings in albino 145

hooded 85

Irish colour 85

no yellow known i 6

Recessive characters 8,342

recognition of 232

in man 225

Reciprocal crosses giving different results $167,174,186,203,323$

Re-combination

novelties through 60

source of new breeds $7 \dot{i}$

Red Indians 209

Reduplication in gametic series 318

Repetition of Parts and Heredity 275

Repulsion $\mathrm{I}_{51}, \mathrm{I}_{55}, 3^{14}$

Retinitis pigmentosa 225

REVERSION

nature of 99,278

fowls 103

mice 99 , III

orchids 96

pigeons 100

stocks 95

sweet peas 89

Right- and Left-handerlness 48

Rogueing crops 292

Round seed 28

Rust disease 25,233

Salix 249, 385

Salvia 4 I

Sap-colour 98

Saturation of colours 80,143

Seasonal forms 257

Seed shape peas $I_{4}$

character 15

maize 30

Seed skin, Pisum, colour of I4

Segregation $7,10,11,13$ 


\section{Index of Subjects}

Segregation-continued

absence of $246,256,373$

consequences of 15,356

nature of 268

time of 269,313

somatic 273,312

and species 283

Selection 240

natural 288

Selective mating between gametes 119 ,

$$
16 r, 195
$$

Serrated leaf, Urlica, Phyteuma 22

SEX

and chromosomes I88

and horns in sheep 169

and double flowers 197

and Spurious Allelomorphism 174, 195, 319

and wings 170

determination of $164,32 \mathrm{I}$

of Bryonia 166

Lychnis 169

of rabbits 192

summary of evidence 190

Sex-limited descent 169, 221, 222, 310, 319

in deaf-mutism 229

in tylosis 219

dominants $23 \mathrm{r}$

Sheep

colour 42

horns $\quad 169$

Shirley poppy 24I

Silene inflata 19

Silk, colours 137, 310

Silkworm colours $43,137,3$ Io

Silky fowl

comb 34

feathers 35

inheritance of pigmentation 185

white colour of $\mathrm{IO}_{3}$

Single flowers $3 \mathbf{I}$

Smooth foliage 22

Snapdragon, see Antirrhinum

Sociological application 303

Somatic segregation 273,312

Species

basal organisation perhaps not transferable 73

hybrids of $283,37 \mathrm{r}$

problem of 4,283

segregation between $\mathbf{2 8 4}$

Spleen, enlarged 220

Sports, bud 272

Standard

erect and hooded 26, I53-157

shape of, in sweet pea 153

Stature

man 209

peas $8,28 \mathrm{I}$

Stem, colour of, in Primula 138

Sterility of anthers in sweet pea 28 , 152
Sterility-continued

affecting descent 169

of Boarmia hybrids I 72

of clouble flowers 198, $20 \mathrm{r}$

of hybrids $25 \mathrm{I}, 366$

of yellow mice i63

self-sterility 242

Stigmas, colour of, in Primula

STOCKS

branching 19,20

"Brompton," peculiarities of 134

colours 95,98

cream 204

double 201

glands $2 \mathrm{I}$

"half-hoary" 238

hoariness $19,95,238$

, relation to colours $\mathbf{1} 33$

ovules and pollen dissimilar in doubles 166

pods, individuality of 254

ratios 314

reversion $95, \quad \mathrm{r} 33$

"sulphur" 204

white, peculiarity of 106

Straw 22

Strawberry 248

Striping, in Antirrhinum 99

in Mirabilis $3 \mathbf{1 2}$

Structural characters in animals 32

Style" $"$ in plants 18

Primula 27

Oenothera 28

Subtraction-stages of factors I 43, 237

Swedes, colour 135

SWEET PEA 8, 9, 39

bicolours dominant 87

bud-sport 274

"bush" variety 19 crossed with cupid" 28 r

colour in axils 152

colours, relation of $9 \mathrm{I}$.

"cupid" 19, 28r

gametic coupling in 140

height I8, 28I

heredity compared with that of Anlir. rhinum 98

pollen grains $28,89,9 \mathrm{I}, 150,{ }_{5} 6$

reversion in $89,28 \mathrm{I}$

seed-shape 265

"snapdragon" 26

spurious allelomorphism 153,155

Symmetry and heredity 274

Taillessness, Cat, Dog 34

Fowl 35

Tallness 8, i 8

Taraxacum 247

Technique 301

Teleangiectasis 220

Terms in $F_{2}$, number of 59

Todas, colour-blindness in 223 
Tomato 26,308

Tortoiseshell cats I 20

rabbits II7

Transformation, Gärtner's views on 376

Triphaena comes 44

Triticum, see Wheat

Tropacolum, variegated 313

Turnip

bulbing of $3 \mathrm{I}$

colour of 135

Turlur, sex-limited descent 194

Tylosis 219

Unfixable types 298

"Unisexual" heredity 285

Unil-character's 5, I5 nature of 266

Urlica 22

Variation

nature of $100,147,280$

confusion as to $30 \mathrm{I}$

Variegation $\mathbf{3} \mathbf{1 2}$

Verbascum, colour 41, I34

Viola 4I, $28_{4}$

Waltzing mice 33 , I I I

Weight of seed 239

WHEAT

beard 22

culour 4I, 3 II

ears 22

foliage $\mathbf{2 2}$
WHEAT - continucd

glutenous $3 \mathrm{I}, 258$

keel 22

maternal characters in seed 258

Polish 259

re-combination of characters $7 \mathrm{I}$

rust 25,233

White flowers in $F_{2}$ from cream $\times$ red $7 \mathrm{I}$

White types, not albinos

dominant IOI, 104, 145

in poultry, various types $\mathrm{IO2}$

properties of IOI

recessive 145

Wild species, Mendelian phenomena in

49

Wrinkled seed 28

Xanthoma 220

Xanthornoe ferrugata 44

Xenia 30

YELLOW

in certain animals $1 \mathbf{I 5}$

in fowls 120

discussion of 121

mice, peculiar phenomena in 118

silk I37

varieties of red moths 135

$Z$ ea, see Maize

Zysopetalum 248

Zygote II, I6

a double structure 56 


\section{INDEX OF AUTHORS}

Abderhalden $\quad 227$

Allen, mice, rats $4^{1}$

Anthony, Manx cat 34

Bacot

Triphaena comes 44

Lasiocampa quercus 44

Acidalia virgularia 252

Baehr, von, sex of Aphidae I89

Ballantyne 219

Balls, cotton 19, 20, 25, 28, 39, 300

Baur, E. $253,308,3$ r 3,318

Bell, W. 207

Bentham 333

Biffen

barley 22, 26, 39

Brassica 3r, 233

gluten $3 \mathbf{r}^{\circ}$

straw 22

wheat 20, 22, 4I

maternal character in 258

rust in 25

Blakston, canary 37

Bond, C. J. 229

Bonhote, pigeons 36, 309

Boveri 249

Boys-Smith, goats 32, 170

Brainerd, Viola 41, 284

Bruce, R. 53

Campbell, A., hairless mice 32

Castle

Drosophila 310, 3 II

guinea-pig $33,34,118$

mice 4I

rabbit 33,79, II 7

lop-eared $25 I$

rats 85

sex 165,187

Chapman, H. J. 96

Charlesworth, Messrs 96

Cockerell 308

Conklin 48

Cookson, N. C., orchids 96

Correns

Bryonia 166

Campanula 26, 200

Hyoscyamus 25, 39

Lychnis 39

Matthiola 20,39

maize $30,41,162,256$

Mendel 7

Mimulus 200

Mirabilis 309,312

Phyteuma 23, 40

\section{Correns-continuced \\ Pisum 28, 40 \\ Polemonium 40 \\ segregation 270 \\ sex 167,169}

Coutagne, silkworm 43, I37

Crampe, rats $4 \mathrm{r}, 85$

Crampton 48

Cuénot

mice $4 \mathrm{I}, 76,86$, II 2

yellow mice $1 \times 9$

Cunier, night-blindness 220

Darbishire 129

mice $33,4 \mathrm{I}, \mathrm{II} 2$

starch in peas 28,53

Darwin, C. 2, 35, 70, 289

reversion in pigeons 100

Himalayan rabbits III

peaches and nectarines 273

on segregation 334

Davenport

canary 37,43

eye-colour $4 \dot{t}$, , 106

fowls $34,35,36,43$

Manx cat 34

Denaiffe, Pisum 28

Doncaster

cats $4 \mathrm{I}$

colour-blindness 320

Abraxas grossulariata 44, 174

Angerona prunaria 44

rats 85

tortoiseshell cats I 20, 172

Downey 224

Drinkard, tomato 26, 47

Drinkwater, brachydactyly 210

Durham

action of tyrosinase 269

mice $4 \mathrm{I}, 78,87,309$

", eye-colour II3

canaries 43,177

, eye-colour II 3

pigments, identification of 116,125

yellow mice 120

Embleton, D. 216, 228

Emerson, Phaseolus 22, 40

Farabee, brachydactyly 2 10

Federley 310

Fischer, E. 257

Focke 249, 332

-Xenia 30

Forbes, rabbit and guinea-pig 33 
Fotherby 228

Fromherz 227

Fryer, C. I 2 I

Gaertner 7, 33 I, 37 I on Lychnis ${ }_{169}$

Galton 5

Basset hounds 126

eugenics 304

eye-colour 106

law of heredity $6,54,55$

stature 209

Garrod, A. E. 227, 233

Gaskoin 33

Gates 27 I

Gerould 3 I9

Giglioli 49, I 10

Godron 2, 34, 333

Goebel, double tlowers 196, 2 or

Goldschmidt $\quad 32$ I

Gossage, heredity of disease 207, 219 , 220

Gregory

doubles 109

Primula 24, 26, 27, 40, 309, 315

starch in Pea seed 28,68

Groth 308

Guaita, von 33

Häcker, axolotl 43

Hagedoorn 309

Harmer, S. F. 275

Harris 44

Harrison, Amphidasys betularia 44

Heape 192

Henking 188

Herringham 225

Herrlinger 225

Hildebrand 47

Hind, Manx cat

Hunter, J. I I 3

Hurst

eye-colour 41, 106

fowls 34,43

horse 42,124

mice 4 I

musical sense 225

orchids $39,96,24^{8}$

Pisum 28, 40

Himalayan rabbit III

yellow rabbit II 7, I I9

rabbit $33,75,79,84,86$

sex of 193

Janczewski 250

Jennings 277

Johannsen, pure lines 239

Jones 308 :

Kajanus 307,308

Keeble 307,308

Kellogg 310

Killby, E. 260
Kölreuter 349

Korschinsky 286

Kuttner 32 I

Lang, A., Helix 45, 308, 31 I -323

Lawrence, Sir W. 200, $219,30+$

I.eake 308

Lewis, T. 216,228

Lock

maize $30,41,53,256,264$

Pisum 28, 40, 260

"ghost"-markings on seed-coats

Lutz, A. M. 27 I

Lutz, F. E.

Crioceris 45

Drosophila 310

rights and lefts 48

McClung $\mathbf{1} 88$

McCracken, Coleoptera 44, 45, 137

MacCurdy 85

Magnus, V. 226

Main, Amphidasys 44

Marryat

rust in wheat 25

Mirabilis 236, 309, 312

Masters, double flowers 196

Mayer, A. G. $4^{8}$

Mendel

biography 327

experiments IO, I I, 335

fasciation 25

Hieracium 380

Pisum 18, 40, 339

rediscovery of method 7

round seed 28

segregation 13

sugar-pea 22,348

Merrifield 257

Millais, Sir E., Basset hounds 6, 126

Millardet 248 .

Morgan, T. H., Drosophila 3 ro sex of Aphis r89, I9I

Mudge 4I, 85, 209

Nägeli $54,329,332$

Naudin 333

Nettleship, E.

cataract 217 ; "anticipation" in 218 colour-blindness 224 night-blindness 220 nystagmus 322

Newman

Abraxas grossulariata

Callimorpha $4+$

Nilsson-Ehle 3 I I

Noorduijn, canaries 43

Nussbaum 192

Oberthut, Boarmia hybrids 170

Ogilvie 218

Ostenfeld 247

Overton 279 
Pearl 319

Pearson, K.

biometry 6

eye colour 106

horse-colours $\mathrm{I}_{2} 4$

colours of poppies 242

Pellew 307

Plate 310

Porritt 44

Potts, F. A. 192

Price, tomato 26, 47

Prout 44, 252

Przibram, eye colour in cats $4 \mathrm{~T}$

Punnett chocolate rabbit 116

- Clarkia elegans 39

coupling $3^{\mathrm{I}} 5$

fowls 36,102

Himalayan rabbit II I

Hydatina $\mathbf{9}^{2}$

polymorphic females 319

silky fowls r8I

sweet pea 89

yellow mice I 19

Raunkiaer 247

Raynor, G. H. I 74

Rivers, W. H. R. ${ }^{223}$

Rizzoli 207

Rosenberg 247, 271

Russo, sex of rabbits 192

Salaman 307

Saunders

Atropa belladonna $3^{8}$

Datura 39

Petunia 322

stocks (Matthiola) I9, 31, I32, 254 , $3^{\mathrm{I}} 4$

double 201, 322

Ranunculus $2 \mathrm{I}$

Salvia 4 I

Schröder, Baron 96

Shull 79, 307, 31 I

Capsella 25

Hilianthus 19,39

Phaseolus 40

ratios 79

Verbascum 4I

Smalley 309

Smith, Geoffrey 192

Sollas

guinea-pig 33

, , coat-colours of 118

Spillnian'

cattle 4 I

pigs 42

wheat 20, 22, 4 I

Standfuss 257
Standfuss, Aglia tau 44, I37, 187-3ro Callimorpha 44

Staples-Browne pigeons 36,43 , 100, 309, 319

pigs 42

Stedman 227

Stevens 27I sex of Aphis 190

Strasburger 249

Sturtevant 309

Surface 3 I9

Sutton, A. W., Brassica 3r, 39, 134

Sutton, L. I99

Thomson, J. A. Iз०

Toyama, silkworm 43, I37

Tschermak

barley 22, 26, 39

cryptomeres 93

Matthiola 39

Mendel 7

Phaseolus 22, 31, 39

Pisum 18, 22, 28, 259

wheat $20,22,41$

de Vilmorin $3^{1} \mathbf{5}$

de Vries

Antirrhinum 38, 87

Atropa belladonna 38

Chelidonium 24

Coreopsis 39

Datura 39

dominance 278

hybrids breeding true 249,323

Lychnis 19, 34

maize 30

Mendel 7

mutation and fluctuation 287

Oenothera 28, 249, 323

Papaver 39

species and variety $28 \mathbf{5}$

unit characters 5

Viola 4 I

Walker, G., brachydactyly 2 I 4

Wallace, A. R. 289

Walther 300

Warburg, Lasiocampa quercus 44

Weismann 5, 289

Weldon 243

Wheldale, Antirrhinum 38, 87, 98, 280, 308

colour of fruits 135

Whitman, C. O., pigeons 194

Wichura 249, 335

Wilson, E. B., sex and accessory chromosome 187 , I9I

Wilson, J., cattle 4 I, 53, 308

Wood, sheep 42, 169

Wootten I99 





BUNDING SECT. SEP $211 \mathrm{H}$

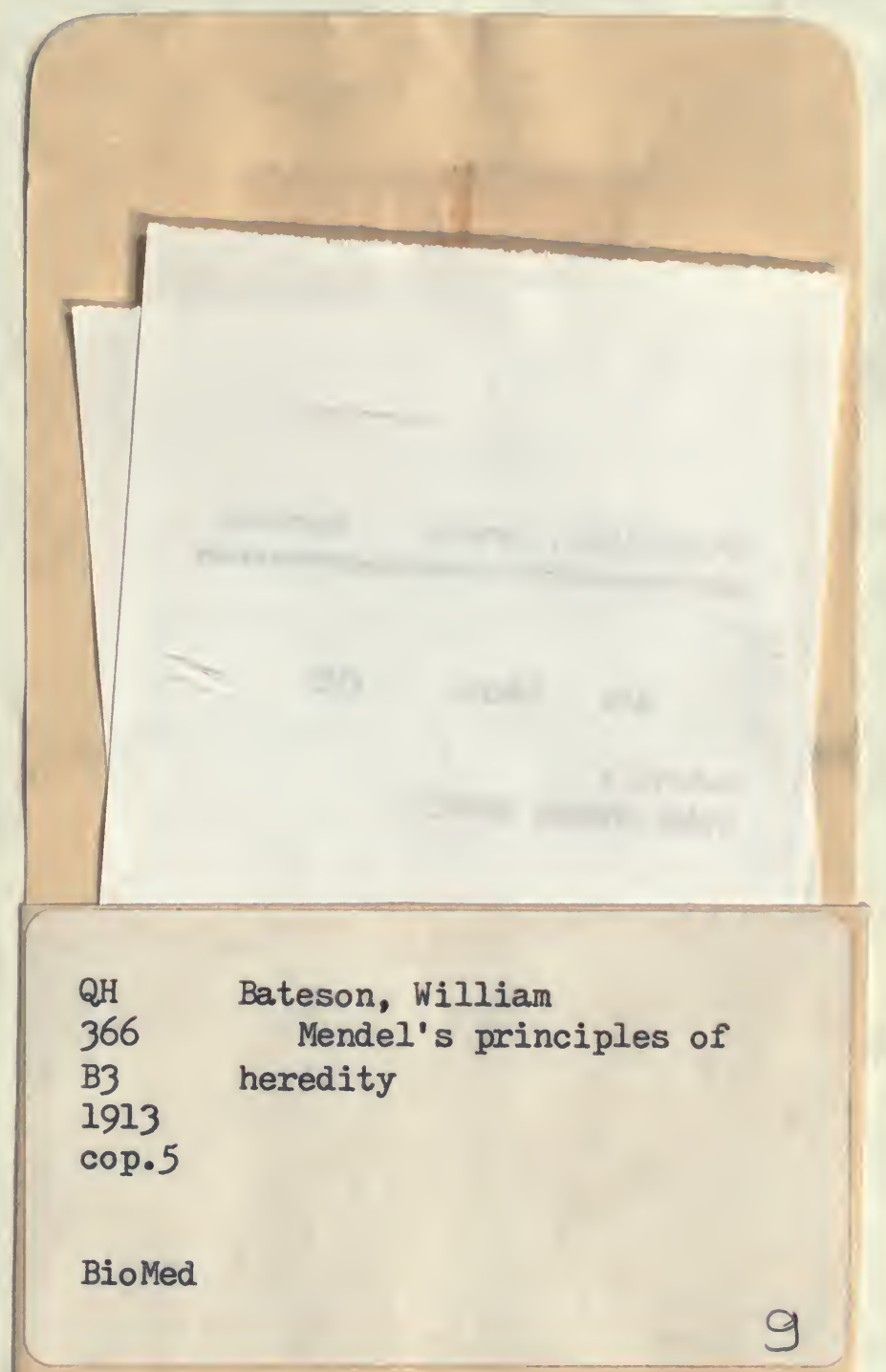




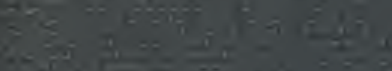

